ORP-56324

Revision 0

\title{
Final Report - DuraMelter 100 Tests to Support LAW Glass Formulation Correlation Development, VSL-06R6480-1, Rev. 0
}

Prepared for the U.S. Department of Energy

Assistant Secretary for Environmental Management

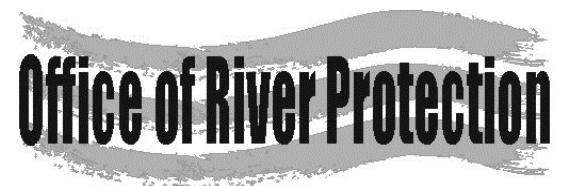

P.O. Box 450

Richland, Washington 99352 
ORP-56324

Revision 0

\section{Final Report - DuraMelter 100 Tests to Support LAW Glass} Formulation Correlation Development, VSL-06R6480-1, Rev. 0

A. A. Kruger

Department of Energy - Office of River Protection

I. S. Muller

The Catholic University of America

W. Gong

The Catholic University of America
I. L. Pegg

The Catholic University of America

K. S. Matlack

The Catholic University of America

Date Published

March 2006

Prepared for the U.S. Department of Energy

Assistant Secretary for Environmental Management

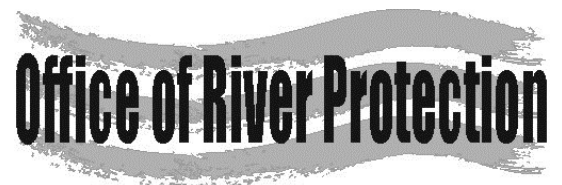

P.O. Box 450

Richland, Washington 99352

APPROVED

By Julia Raymer at 8:51 am, Dec 03, 2013

Release Approval

Date 
ORP-56324

Revision 0

TRADEMARK DISCLAIMER

Reference herein to any specific commercial product, process, or service by tradename, trademark, manufacturer, or otherwise, does not necessarily constitute or imply its endorsement, recommendation, or favoring by the United States Government or any agency thereof or its contractors or subcontractors.

This report has been reproduced from the best available copy.

Printed in the United States of America 
VSL-06R6480-1

\title{
Final Report
}

DuraMelter 100 Tests to Support LAW Glass Formulation Correlation Development

prepared by

Keith S. Matlack, Isabelle S. Muller, Weiliang Gong, and Ian L. Pegg

\author{
Vitreous State Laboratory \\ The Catholic University of America \\ Washington, DC 20064
}

for

Duratek, Inc.

and

Bechtel National, Inc.

February 7, 2006

Rev. 0, 3/21/06 
The Catholic University of America Vitreous State Laboratory DuraMelter100 Tests to Support LAW Glass Formulation Correlation Development Final Report, VSL-06R6480-1, Rev. 0

Document Title: DuraMelter100 Tests to Support LAW Glass Formulation Correlation Development

Document Number and Revision: VSL-06R6480-1, Rev. 0

Issue Date: $\quad 3 / 21 / 06$

Performing Organization: Vitreous State Laboratory, The Catholic University of America

Test Specification: 24590-LAW-TSP-RT-04-0004, Rev. 0

Test Exceptions: 24590-LAW-TEF-RT-05-00002

Test Plan: VSL-05T5480-1, Rev. 0

R\&T Focus Area(s): $\quad$ LAW Waste Form Qualification

Test Scoping Statement(s): VSL-24, LAW Glass Formulation to Support Melter Runs with Simulants. VL-04, LAW Small Scale Melter Tests.

\section{Completeness of Testing:}

This report describes the results of work and testing specified by the above-listed Test Specification(s), Test Plan(s), and Text Exception(s). The work and any associated testing followed established quality assurance requirements and was conducted as authorized. The descriptions provided in this test report are an accurate account of both the conduct of the work and the data collected. Results required by the Test Plan are reported. Also reported are any unusual or anomalous occurrences that are different from the starting hypotheses. The test results and this report have been reviewed and verified.

I.L. Pegg:

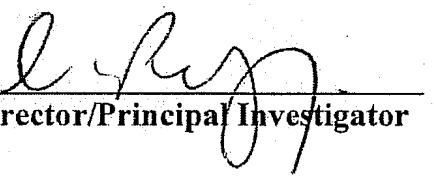

I. Joseph: frinocent tosetb Duratek Sub-Contract Manager

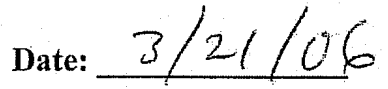

Date: $3 / 21 / 06$ 
The Catholic University of America Vitreous State Laboratory ests to Support LAW Glass Formulation Correl Final Report, VSL-06R6480-1, Rev. 0

\section{TABLE OF CONTENTS}

LIST OF TABLES.

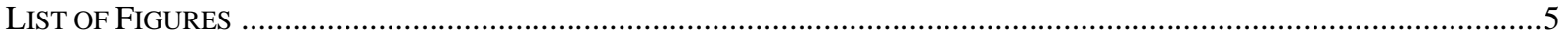

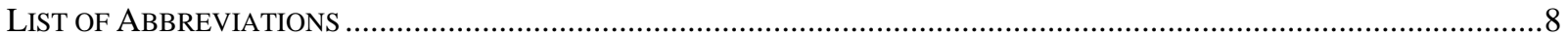

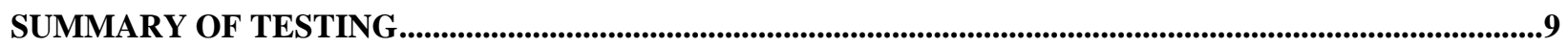

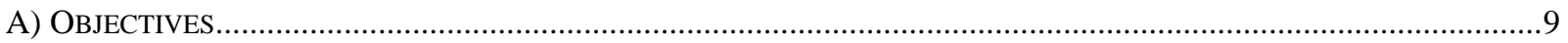

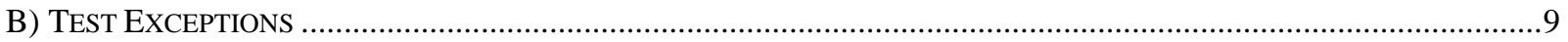

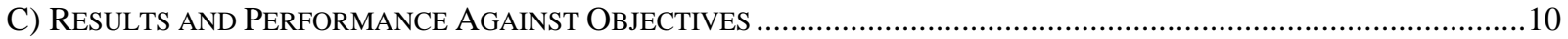

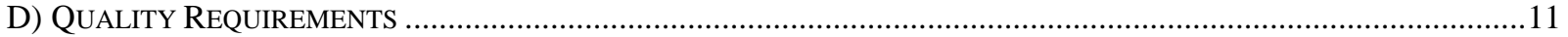

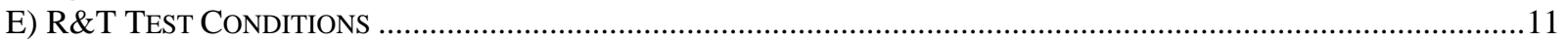

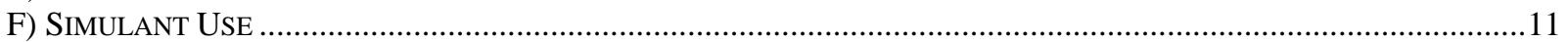

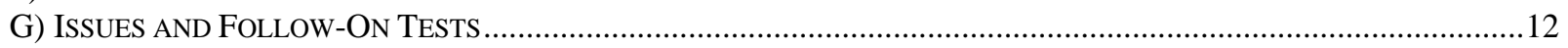

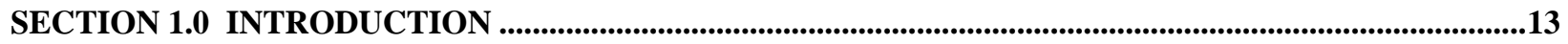

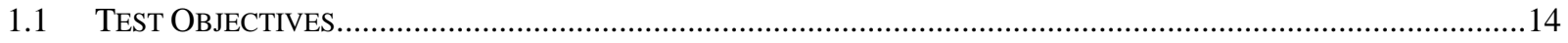

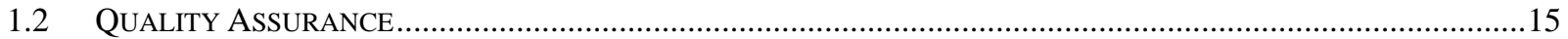

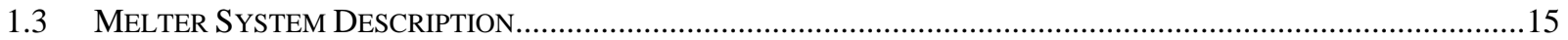

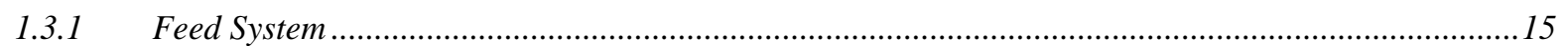

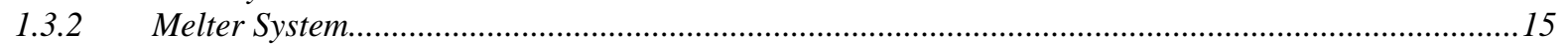

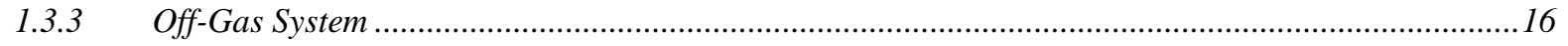

SECTION 2.0 WASTE SIMULANT AND GLASS FORMULATIONS ..................................................17

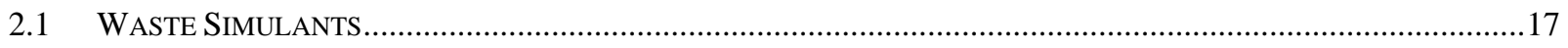

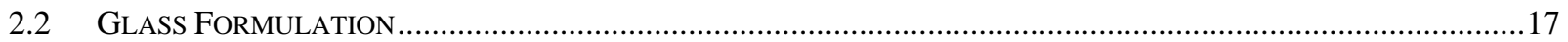

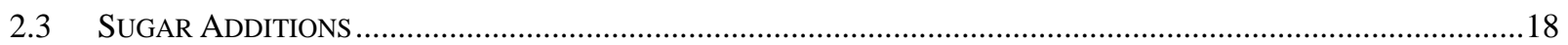

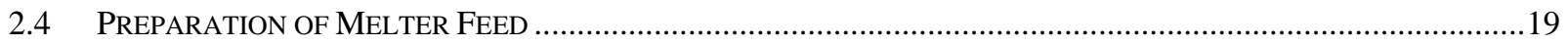

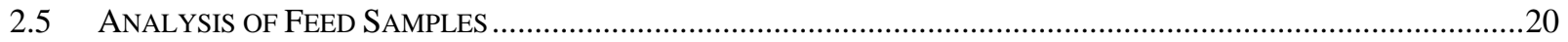

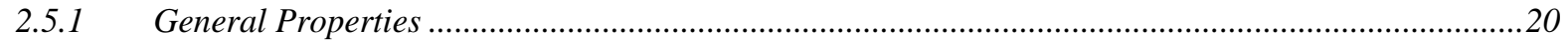

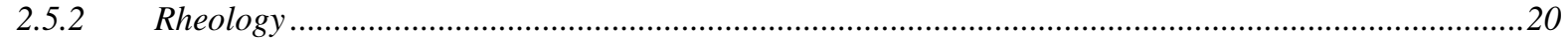

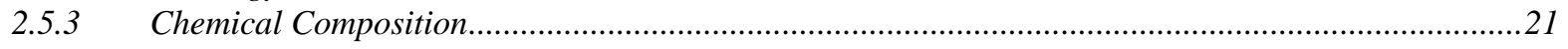

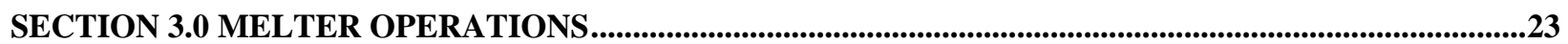

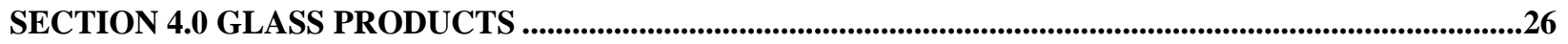

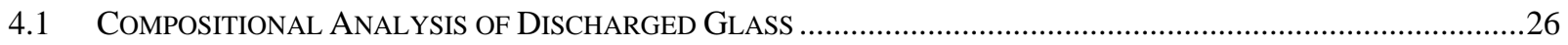

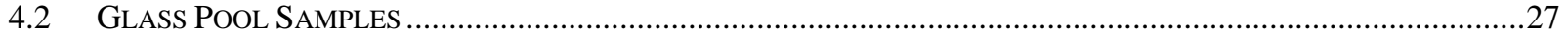

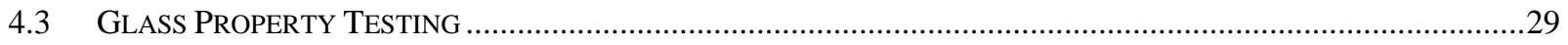

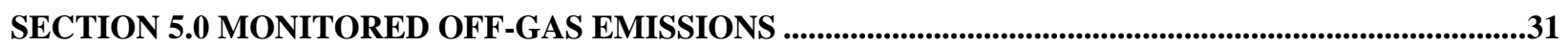

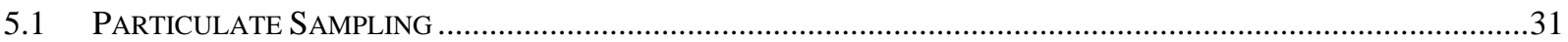

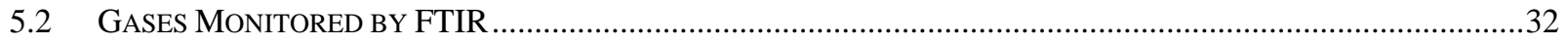

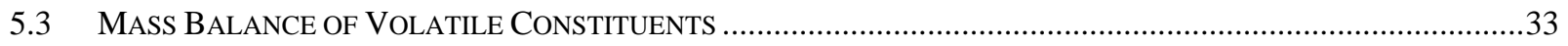

SECTION 6.0 SUMMARY AND CONCLUSIONS .......................................................................................34

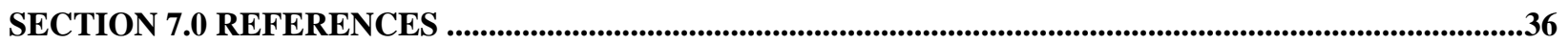




\section{List of Tables}

Table 2.1. Compositions of Glasses for DM100 Melter Testing (wt\%). T-1

Table 2.2. Test Matrix. $\quad$ T-2

Table 2.3. Composition of the Grouped Component “Others” for LAWEH Glasses. T-3

Table 2.4. $\quad$ LAWE3 Simulant Recipe at Nominal 8 Molar Sodium (Actual 8.46 Molar Sodium) based on AP-101 waste composition [32]. T-4

Table 2.5. $\quad$ LAWE4H Waste Simulant Recipe at Nominal 8 Molar Sodium (Actual 7.97 Molar Sodium) without Potassium based on AN-105 waste composition [32]. T-5

Table 2.6. $\quad$ LAWE5H Simulant Recipe at Nominal 7 Molar Sodium (Actual 7.13 Molar Sodium) based on AN-107 waste composition [32]. T-6

Table 2.7. $\quad$ LAWE6H Simulant Recipe at Nominal 7 Molar Sodium (Actual 6.86 Molar Sodium) based on AN-104 waste composition [32]. $\quad$ T-7

Table 2.8. $\quad$ LAWE7H Simulant Recipe at Nominal 5 Molar Sodium (Actual 5.02 Molar Sodium) based on AN-102 waste composition [32]. $\quad$ T-8

Table 2.9. $\quad$ LAWE9H Simulant Recipe at Nominal 4 Molar Sodium based on AZ-101 waste composition [32].

Table 2.10. $\quad$ LAWE10H Simulant Recipe at 2 Molar Sodium based on AZ-102 waste composition [32].

Table 2.11. Oxide Composition of LAWE3 Simulant and Corresponding Glass Composition.

Table 2.12.

Table 2.13.

Table 2.14.

Table 2.15.

Table 2.16.

Table 2.17.

Table 2.18. Oxide Composition of LAWE4H Simulant and Corresponding Glass Composition. Oxide Composition of LAWE5H Simulant and Corresponding Glass Composition. Oxide Composition of LAWE6H Simulant and Corresponding Glass Composition. Oxide Composition of LAWE7H Simulant and Corresponding Glass Composition. Oxide Composition of LAWE9H Simulant and Corresponding Glass Composition. Oxide Composition of LAWE10H Simulant and Corresponding Glass Compositions.

Glass Former Additives for 1 Liter of LAWE Waste Simulant and Corresponding Feed Properties in Crucible Scale Tests.

Adjustments to Feed Required for Tests Targeting $1.1 \mathrm{wt} \% \mathrm{Cl}$ and

Table 2.19. $1.3 \mathrm{wt} \% \mathrm{~F}$ in the Glass Product and Corresponding Feed Properties.

Table 2.20 Measured Properties of Melter Feed Samples.

Rheological Properties for Select Melter Feed Samples.

Table 2.21. XRF-Analyzed Compositions of Feed Samples (wt\%).

Table 2.22.

Table 2.23

Table 3.1.

Table 3.2.

Table 4.1.

Table 4.2 DCP and XRF Analyzed Compositions of Select Melter Feed Samples (wt\%).

Table 4.5 . Results on Dip Samples after Washing Procedure. 
Table 5.1. $\quad$ Results from Melter Off-Gas Emission Samples.

Table 5.2. Average and Range of Concentrations (ppmv) for Selected Species in Off-Gas Measured by FTIR Spectroscopy.

Table 5.3

Average $\mathrm{NO}_{\mathrm{x}}$ Fluxes in Off-Gas Measured by FTIR Spectroscopy.

\section{List of Figures}

Figure 3.1.a. (bounds from WTP-RPT-075, Rev. 0, Feb. 2003). 
Figure 3.7.c. Figure 3.7.d. Figure 3.7.e. Figure 3.7.f. Figure 3.8. Figure 4.1 . Figure 4.2. Figure 4.3. Figure 4.4 . Figure 4.5. Figure 4.6. Figure 4.7. Figure 4.8. Figure 4.9. Figure 4.10. Figure 4.11. Figure 4.12. Figure 4.13.a. Figure 4.13.b. Figure 4.14.a.

Figure 4.14.b. Figure 4.14.c. Figure 4.15

Figure 4.16

Figure 4.17

Figure 4.18

Figure 4.19.

Figure 5.1. Figure 5.2. Figure 5.3. Figure 5.4. Figure 5.5. Figure 5.6. Figure 5.7.a. Figure 5.7.b. Figure 5.7.c. Figure 5.7.d. Figure 5.7.e. Figure 5.7.f. Figure 5.8.a. Figure 5.8.b.
Melt pool resistance and electrode power during Test 3. Melt pool resistance and electrode power during Test 4.

Melt pool resistance and electrode power during Test 5. Melt pool resistance and electrode power during Test 6 .

F-40

F-41

F-42

F-43

Melt pool resistance and feed sodium content.

F-44

XRF analysis of $\mathrm{Na}_{2} \mathrm{O}$ and $\mathrm{SiO}_{2}$ in melter glasses.

F-45

$\begin{array}{ll}\text { XRF analysis of oxides in product glasses which are constant during testing. } & \text { F-46 }\end{array}$

$\begin{array}{ll}\text { XRF analysis of oxides in product glasses which vary during testing. } & \text { F-47 }\end{array}$

$\begin{array}{ll}\text { XRF analysis of sulfur in melter glasses. } & \text { F-48 }\end{array}$

$\begin{array}{ll}\text { XRF analysis of chlorine in melter glasses. } & \text { F-49 }\end{array}$

$\begin{array}{lr}\text { XRF analysis of fluorine in melter glasses. } & \text { F-50 }\end{array}$

$\begin{array}{ll}\text { XRF analysis of rhenium in melter glasses. } & \text { F-51 }\end{array}$

Dip samples from Test 6A. Note separated sulfur on two rods in the back right buckets. F-52

Separated sulfur on dip sample DWV-D-22A from Test 6A. F-53

Dip samples from Test 6A removed from rods and placed in Petri dishes for examination. F-54

Separated sulfur on dip sample DWV-D-22A from Test 6A after removal from rod. F-55

$\begin{array}{ll}\text { Separated sulfur remaining on rods after glass removal during Test 4B. } & \text { F-56 }\end{array}$

Back scattered electron image taken at 1000X magnification on WVZ-D-74C. $\quad$ F-57

Spectra ffom secondary phases in WVZ-D-74C.

SEM micrograph of secondary phases in BWV-D-68E. Note that the material consists

$\begin{array}{ll}\text { of two distinct phases: a substrate and crystals which protrude from the substrate. } & \text { F-59 }\end{array}$

$\begin{array}{ll}\text { Spectrum from substrate of secondary phases in BWV-D-68E. } & \text { F-60 }\end{array}$

$\begin{array}{ll}\text { Spectrum from protruding crystals on secondary phases of BWV-D-68E. } & \text { F-61 }\end{array}$

Melt viscosity (at $1100^{\circ} \mathrm{C}$ ) for formulations tested as crucible melts (LAWE3, LAWE7H, and LAWE10H) and DM100 melter glasses with nominal(WVZ-G-61D, AWV-G-78A, BWV-G-32B) and increased halides(DWV-G-51B, BWV-G-142B).

F-62

Melt electrical conductivity (at $1100^{\circ} \mathrm{C}$ ) for formulations tested as crucible melts

(LAWE3, LAWE7H, and LAWE10H) and DM100 melter glasses with nominal

(WVZ-G-61D, AWV-G-78A, BWV-G-32B) and increased halides

(DWV-G-51B, BWV-G-142B).

F-63

PCT normalized mass loss (for boron and sodium) for formulations tested as crucible

Glasses (LAWE3, LAWE7H, and LAWE10H) and DM100 melter glasses with nominal

(WVZ-G-61D, AWV-G-78A, BWV-G-32B) and increased halides

(DWV-G-51B, BWV-G-142B).

F-64

Alteration rate given by Vapor Hydration Test $\left(\right.$ at $200{ }^{\circ} \mathrm{C}$ ) for formulations tested as crucible glasses (LAWE3, LAWE7H, and LAWE10H) and DM100 melter glasses with nominal (WVZ-G-61D, AWV-G-78A, BWV-G-32B) and increased halides

(DWV-G-51B, BWV-G-142B).

TCLP zinc concentration for formulations tested as crucible glasses (LAWE3, LAWE7H, and LAWE10H) and DM100 melter glasses with nominal (WVZ-G-61D, AWV-G-78A, BWV-G-32B) and increased halides (DWV-G-51B, BWV-G-142B).

Measured particle emission rate from the DM100 as a function of feed sodium content. F-67

Measured sodium emission rate from the DM100 as a function of feed sodium content. F-68

Measured rhenium emission rate from the DM100 as a function of feed sodium content. F-69

Measured chlorine emission rate from the DM100 as a function of feed sodium content. F-70

Measured fluorine emission rate from the DM100 as a function of feed sodium content. F-71

$\begin{array}{ll}\text { Percent sulfur carryover from the DM100 as a function of feed sodium content. } & \text { F-72 }\end{array}$

$\begin{array}{ll}\text { Nitric oxide concentrations in off-gas from FTIR during Test } 1 . & \text { F-73 }\end{array}$

$\begin{array}{ll}\text { Nitric oxide concentrations in off-gas from FTIR during Test } 2 . & \text { F-74 }\end{array}$

$\begin{array}{ll}\text { Nitric oxide concentrations in off-gas from FTIR during Test } 3 . & \text { F-75 }\end{array}$

$\begin{array}{ll}\text { Nitric oxide concentrations in off-gas from FTIR during Test } 4 . & \text { F-76 }\end{array}$

$\begin{array}{ll}\text { Nitric oxide concentrations in off-gas from FTIR during Test } 5 . & \text { F-77 }\end{array}$

$\begin{array}{ll}\text { Nitric oxide concentrations in off-gas from FTIR during Test } 6 . & \text { F-78 }\end{array}$

$\begin{array}{ll}\mathrm{NO}_{2} \text { concentration in off-gas from FTIR during Test } 1 . & \text { F-79 }\end{array}$

$\begin{array}{ll}\mathrm{NO}_{2} \text { concentration in off-gas from FTIR during Test } 2 . & \text { F-80 }\end{array}$ 
Figure 5.8.c. $\quad \mathrm{NO}_{2}$ concentration in off-gas from FTIR during Test $3 . \quad$ F-81

Figure 5.8.d. $\quad \mathrm{NO}_{2}$ concentration in off-gas from FTIR during Test $4 . \quad$ F-82

$\begin{array}{lll}\text { Figure 5.8.e. } \quad \mathrm{NO}_{2} \text { concentration in off-gas from FTIR during Test } 5 . & \text { F-83 }\end{array}$

$\begin{array}{lll}\text { Figure 5.8.f. } & \mathrm{NO}_{2} \text { concentration in off-gas from FTIR during Test } 6 . & \text { F-84 }\end{array}$

Figure 5.9.a. $\quad$ CO concentration in off-gas from FTIR during Test $1 . \quad$ F-85

Figure 5.9.b. $\quad$ CO concentration in off-gas from FTIR during Test 2. $\quad$ F-86

$\begin{array}{lll}\text { Figure 5.9.c. } & \text { CO concentration in off-gas from FTIR during Test } 3 . & \text { F-87 }\end{array}$

Figure 5.9.d. $\quad$ CO concentration in off-gas from FTIR during Test $4 . \quad$ F-88

Figure 5.9.e. $\quad$ CO concentration in off-gas from FTIR during Test $5 . \quad$ F-89

Figure 5.9.f. $\quad$ CO concentration in off-gas from FTIR during Test $6 . \quad$ F-90

Figure 5.10.a. $\quad$ Ammonia concentration in off-gas from FTIR during Test $1 . \quad$ F-91

Figure 5.10.b. $\quad$ Ammonia concentration in off-gas from FTIR during Test 2. $\quad$ F-92

Figure 5.10.c. $\quad$ Ammonia concentration in off-gas from FTIR during Test 3. $\quad$ F-93

Figure 5.10.d. $\quad$ Ammonia concentration in off-gas from FTIR during Test $5 . \quad$ F-94

Figure 5.10.e. $\quad$ Ammonia concentration in off-gas from FTIR during Test $6 . \quad$ F-95

Figure 5.11.a. $\quad$ Hydrogen fluoride concentration in off-gas from FTIR during Test $4 . \quad$ F-96

Figure 5.11.b. $\quad$ Hydrogen fluoride concentration in off-gas from FTIR during Test $5 . \quad$ F-97

Figure 5.11.c. $\quad$ Hydrogen fluoride concentration in off-gas from FTIR during Test $6 . \quad$ F-98 


\title{
List of Abbreviations
}

\author{
AA Atomic Absorption Spectroscopy \\ ADS Air Displacement Slurry \\ DCP-AES Direct Current Plasma Atomic Emission Spectroscopy \\ DF Decontamination Factor \\ DM DuraMelter ${ }^{\circledR}$ \\ DOE Department of Energy \\ DRE Destruction \& Removal Efficiency \\ dscfm Dry standard cubic foot per minute \\ EDS Energy Dispersive X-ray Spectroscopy \\ EPA Environmental Protection Agency \\ FTIR Fourier Transform Infrared Spectroscopy \\ GC Gas Chromatography \\ HEPA High-Efficiency Particulate Air Filter \\ HLW High Level Waste \\ LAW Low Activity Waste \\ M Molarity \\ N Normality \\ PCT Product Consistency Test \\ QA Quality Assurance \\ QAPjP Quality Assurance Project Plan for Testing Programs Generating Environmental Regulatory Data \\ QAPP Quality Assurance Project Plan \\ QC Quality Control \\ RPP River Protection Project \\ RPP-WTP River Protection Project-Waste Treatment Plant \\ SEM Scanning Electron Microscope \\ SIPP Semi Integrated Pilot Plant \\ SRNL Savannah River National Laboratory \\ TCLP Toxicity Characteristic Leach Procedure \\ VHT Vapor Hydration Test \\ VSL Vitreous State Laboratory \\ WTP Waste Treatment and Immobilization Plant \\ XRF X-Ray Fluorescence
}




\section{SUMMARY OF TESTING}

\section{A) Objectives}

The DM100 melter tests described in this report were designed to assess sulfate phase separation during the processing of glass formulations calculated from the LAW glass formulation algorithm when estimated process uncertainties are included in the worst-case direction. In particular, the principal objective of the tests was to provide data on the formation or lack of formation of a persistent and accumulating separate sulfate phase on the glass melt while processing various glass formulations in the alkali-limited region through the sulfatelimited region with decreasing alkali content. Additional objectives included determination of the effect of halides on secondary phase formation, determination of technetium retention in the glass product over a range of LAW glass compositions using rhenium as a surrogate, and generation of glasses for product quality testing for compositions that were not previously available. Completion of the test objectives is detailed in the following table:

\begin{tabular}{|l|l|l|}
\hline \multicolumn{1}{|c|}{ Test Objective } & $\begin{array}{l}\text { Objective } \\
\text { Met? }\end{array}$ & \multicolumn{1}{|c|}{ Discussion Section } \\
\hline $\begin{array}{l}\text { Confirm that, in the alkali-limited } \\
\text { region where waste loading is } \\
\text { determined by sodium or sodium plus } \\
\text { potassium content, sulfate phase } \\
\text { accumulation does not occur across } \\
\text { that range of sodium and potassium } \\
\text { combinations. }\end{array}$ & Yes & $\begin{array}{l}\text { Sections } 3.0 \text { and 4.2 provide visual } \\
\text { observations and corroborative chemical } \\
\text { analysis demonstrating the lack of } \\
\text { secondary sulfate phase accumulation } \\
\text { across the range of sodium and potassium } \\
\text { combinations tested. }\end{array}$ \\
\hline $\begin{array}{l}\text { Demonstrate that, in the region where } \\
\text { sulfate phase accumulation can limit } \\
\text { waste loading, the proposed glass } \\
\text { formulation correlation yields glasses } \\
\text { without sulfate phase accumulation. }\end{array}$ & Yes & $\begin{array}{l}\text { Sections 3.0 and 4.2 provide visual } \\
\text { observations and corroborative chemical } \\
\text { analysis demonstrating the lack of } \\
\text { secondary sulfate phase accumulation } \\
\text { across the range of sodium and sulfur } \\
\text { concentrations tested. }\end{array}$ \\
\hline $\begin{array}{l}\text { Determine the effect of chlorine and } \\
\text { fluorine on sulfate phase accumulation. }\end{array}$ & Yes & $\begin{array}{l}\text { Sections 3.0 and 4.2 provide visual } \\
\text { observations and corroborative chemical } \\
\text { analysis demonstrating that secondary } \\
\text { sulfate phase accumulation did occur in } \\
\text { tests with higher concentrations of chlorine } \\
\text { and fluorine in the feed. }\end{array}$ \\
\hline
\end{tabular}

\section{B) Test Exceptions}

WTP Test Exception, 24590-LAW-TEF-RT-05-00002, K. Abel, River Protection Project, Waste Treatment Plant, 3000 George Washington Way, Richland, WA 99352, 3/31/05. 


\section{C) Results and Performance Against Objectives}

A series of six tests were conducted on the DM100-WV melter using a range of LAW Sub-Envelope simulants and corresponding melter feeds that produced glasses in the alkali-limited region through the sulfate-limited LAW glass composition region with varying alkali content. In addition, in all of the seven glass compositions that were used, the impact of higher levels of chlorine and fluorine on sulfate phase formation was also investigated. Rhenium as a surrogate for technetium was added to the feed in all tests to provide data on retention in the glass product, and the relative proportions of particulate and gaseous fractions in the melter exhaust. The results showed that each glass composition and transitions between glass compositions occurred without the formation of a separate sulfate phase on the melt pool surface. Conversely, separate sulfate phases were observed in most of the tests processing the same glass compositions containing high concentrations of halides. Separated sulfate phases were removed by bubbling the melt pool prior to continuing testing. Sulfate phases were identified by visual observations of multiple dip samples. Visual results were confirmed by chemical and microstructural analysis, which indicated the material was composed of two principal phases: an alkali and calcium sulfate phase with lesser amounts of chlorine, chromium, and rhenium in addition to a phase of radiating crystals composed of alkali sulfate, chlorine, and chromium. No difficulties were encountered in maintaining nominal operating conditions (glass temperature of $1150^{\circ} \mathrm{C}$ and a near complete cold cap while processing glass at $2000 \mathrm{~kg} / \mathrm{m}^{2} /$ day) in any of the tests.

Analysis of the feed samples and product glasses confirmed the composition of the feed and showed the intended transitions between the various compositions. Product glasses were selected to permit direct comparisons with the corresponding crucible melt results at high potassium and high sodium, moderate sodium, and low sodium levels, as well as to assess the effects of the increased halide levels on glass properties. All of the measured properties of the crucible and melter glasses are within acceptable limits for processing and product quality.

During the present tests, total particulate carry-over from the melter ranged from a quarter percent to over two percent and increased with increasing sodium, potassium, and halide feed content. Melter DFs were determined for most of the elements in the feed for each unique glass composition. The most volatile species were rhenium, halogens, and sulfur. Good mass balance closure was obtained for halides, sulfur, and rhenium for most of the test segments. Sulfur retention in the glass typically ranged from 75 - 95\% and increased with increased feed sodium content and decreased with feed halide content. Chlorine, fluorine, and rhenium retention in the glass was typically $50-60 \%$, $65-75 \%$, and $20-40 \%$, respectively, with no obvious relationship to concentrations of the other feed constituents. Rhenium melter emissions were entirely particulate, whereas chlorine, fluorine, and sulfur melter emissions were both particulate and gaseous. Consistent with previous tests conducted at 0.5 stoichiometeric carbon ratio, approximately half of the feed nitrogen oxides were emitted as $\mathrm{NO}$ and $\mathrm{NO}_{2}$ gases. Byproducts of incomplete combustion, such as carbon monoxide and ammonia increased with the amounts of carbon and nitrogen oxides in the feed. 


\section{D) Quality Requirements}

This work was conducted under a quality assurance program based on NQA-1 (1989) and NQA-2a (1990) Part 2.7 that is in place at the VSL. This program is supplemented by a VSL Quality Assurance Project Plan (QAPP) for RPP-WTP work. Test and procedure requirements by which the testing activities are planned and controlled are also defined in this plan. The program is supported by VSL standard operating procedures that were used for this work. This work was not subject to DOE/RW-0333P or the WTP QAPjP for environmental regulatory data.

\section{E) R\&T Test Conditions}

Melter tests were conducted using the DM100-WV melter, which has a melt surface area of $0.108 \mathrm{~m}^{2}$. The DM100-WV is a continuously fed, ceramic refractory-lined melter equipped with a lance bubbler for glass pool agitation. The glass-melt is joule-heated using a pair of opposing Inconel electrodes. Feed is introduced into the melter by a peristaltic pump to ensure consistent feeding. For operational simplicity the melter is equipped with a dry off-gas treatment system involving gas filtration operations only.

Sufficient LAW feed, based on the compositions of various waste streams, was procured to conduct 800 hours of DM100 testing. The target processing rate of $2000 \mathrm{~kg}$ glass $/ \mathrm{m}^{2} /$ day was maintained throughout the tests. Multiple dip samples were collected from the melt pool at the end and during the transition from each glass composition to determine whether a separate sulfate phase had formed on the melt surface. Glass samples were analyzed for composition to calculate elemental mass balances.

Melter emissions data were collected during the steady-state portions of the tests. The melter exhaust was sampled for metals/anions/particles according to 40-CFR-60 Methods 3, 5, 26, and 29. Particulate collection required isokinetic sampling. Melter emissions were monitored for a variety of gaseous components, notably $\mathrm{CO}$ and nitrogen species, by Fourier Transform Infra-Red Spectroscopy (FTIR). Feed samples and melter glass samples (both discharged glass samples and dip samples) were analyzed for their chemical composition. Rheological properties of the feed samples were measured.

\section{F) Simulant Use}

The LAW waste simulants for the DM100 melter tests are based on the composition data provided in the WTP Test Specification 24590-LAW-TSP-RT-02-012, Rev. 0. and Test Exception 24590-LAW-TEF-RT-05-00002. The waste simulant compositions are based on the compositions of LAW tanks AP-101, AN-105, AN-107, AN-104, AN-102, AZ-101, and AZ-102. The concentrations of $\mathrm{Cl}, \mathrm{Cr}, \mathrm{F}, \mathrm{Ni}, \mathrm{Pb}$, and $\mathrm{P}$ in the waste simulants were modified so that their target concentrations total half a weight percent in the glass product. All tests were conducted with the nominal halide concentrations, and also with $\mathrm{Cl}$ and $\mathrm{F}$ concentrations of $1.1 \mathrm{wt} \%$ and $1.3 \mathrm{wt} \%$ on a glass basis, respectively. The concentrations of potassium and sulfate 
in the simulants also were modified to achieve the required $\mathrm{K}_{2} \mathrm{O}$ and $\mathrm{SO}_{3}$ concentrations in the respective glass compositions.

\section{G) Issues and Follow-On Tests}

The results from these tests demonstrate that glass formulations generated by the LAW glass formulation algorithm (including process uncertainties in the worst-case direction) which span a wide range of sodium and sulfate concentrations can be processed without forming separate sulfur phases on the melt surface. However, the results also indicate that separate sulfur phases are likely to form on the melt pool surface if the same feeds contain high halide concentrations. Chemical analysis of this separated material indicates that rhenium, which was used as a surrogate for radioactive technetium, is sequestered into the soluble secondary phase. In the limited number of previous tests with LAW feeds containing high chlorine concentrations, the formation of separated secondary phases was not observed and, therefore, high chlorine concentrations do not appear to be the only determining factor for the formation of secondary phases on the melt pool surface. Consequently, it is recommended that further testing be performed to better understand the relationship between chlorine and fluorine concentrations and the formation of secondary sulfate phases, and to define acceptable limits. It is recommended that this work should include isolating the effect of each of the two halides, defining halide concentration thresholds for secondary phase formation, and processing glasses with high concentrations of halides that in past studies with nominal halide concentrations did not result in secondary sulfate phases. The results of these tests should then be incorporated into a revision of the LAW glass formulation algorithm. It is worth noting that some additional DM100 melter testing is already planned for FY07. 


\section{SECTION 1.0 INTRODUCTION}

Acceptable glass formulations for vitrification of Hanford LAW waste streams must meet a variety of product quality, processability, and waste loading requirements. To this end, a number of formulations were investigated during Part A [1], Part B1 [2] and Part B2 [3, 4] of the WTP development program. The testing resulted in the selection of target glass compositions for the processing of eight of the Phase I LAW tank wastes. The selected glass compositions were tested at the crucible scale to confirm their compliance with ILAW performance requirements. DM100 [5-16] and LAW Pilot Melter [17-28] tests were then conducted to demonstrate the viability of these glass compositions for LAW waste vitrification at high processing rates.

Glass formulation development work has been based on Hanford tank waste composition data [29-32] that have evolved over time. As a result, the target glass compositions for waste processing also have been adjusted to accommodate the changes in LAW waste compositions. The major components of interest in the LAW waste streams, from a waste loading perspective, are sodium, sulfur, and to a lesser extent potassium. Although schedule constraints associated with selection of glass compositions for melter testing did not always allow for extensive optimization of glass compositions as the waste composition data and WTP flow-sheet evolved, a range of acceptable glass formulations was selected for melter testing that met waste loading, melter processing, and ILAW performance requirements. Melter testing included composition variations involving the nominal feed and $\pm 15 \%$ variations in the amount of waste simulant added to the melter feed. As a result of these efforts, the WTP Project now has a series of glass compositions with different sodium, potassium, and sulfur concentrations that have been tested and validated over a range of melter scales, including the LAW Pilot Melter.

Results from glass formulation and melter testing have been analyzed to develop a glass formulation algorithm for WTP LAW glasses [33]. Given the composition of an LAW stream, the algorithm permits the calculation of a suitable glass composition and the required glass forming additives such that all processing, waste loading, and product quality requirements would be met. Such an algorithm has obvious value for the operation of the WTP LAW vitrification facility. Reference [33] provides recommendations for testing and confirmation of the proposed algorithm based on a set of glass compositions generated by the algorithm. The recommendations also include an assessment of the effects of process control uncertainties (in terms of composition variations) expected for the LAW vitrification process followed by crucible and melter testing to ensure that acceptable glass properties and processing behavior are maintained when likely process control uncertainties are included. Subsequently, WTP R\&T provided expected standard deviations for $\mathrm{Na}_{2} \mathrm{O}$ and $\mathrm{SO}_{3}$ concentrations based on process variations and analytical errors [34]. Based on that information, VSL/Duratek developed and recommended a series of test glass compositions and a series of DM100 melter tests ("Recommendations for Testing to Support LAW Glass Composition Correlation," VSL, 9/13/04) that were incorporated into a WTP Test Specification [35]. In response, a VSL Test

Plan [36] was issued which details melter testing at the DM100 scale to provide the data required 
to underpin the proposed algorithm. The data from the testing also serve to strengthen the correlation algorithm by producing LAW glasses that extend from the alkali-limited region through the sulfate-limited region with decreasing alkali contents.

Thus, the DM100 melter tests described in this report were designed to assess sulfate phase separation during the processing of glass formulations calculated from the LAW glass formulation algorithm when estimated process uncertainties are included in the worst-case direction. The DM100 melter testing is required since reliable information on sulfate incorporation and secondary phase accumulation in the dynamic melter environment cannot be obtained using laboratory crucible melts alone. Sulfate incorporation during real-time feed processing in an actual melter depends both on thermodynamic and kinetic factors. Crucible melts can provide information on saturation sulfate solubility, but are limited in their ability for assessing the practical effects of kinetic factors. Crucible melt testing to support the development of the correlation algorithm is being performed separately and therefore is not included in this report, which focuses on the results of melter testing.

\subsection{Test Objectives}

The principal objective of the DM100 tests was to provide data on the formation or lack of formation of a persistent and accumulating separate sulfate phase on the glass melt while processing various glass formulations in the alkali-limited region through the sulfate-limited region [33] with decreasing alkali content. The DM100-WV unit was selected for these tests. This melter platform was used for all of the initial LAW Envelopes A, B, and C tests [5-16] to demonstrate feasibility for the subsequent tests on the larger Pilot Melter [17-28]. The same melter was selected for the present tests in order to maintain comparisons between the data sets. Sufficient LAW feed was procured for this work to conduct 800 hours of DM100 testing with seven different glass compositions. In addition, transitions between the various compositions were also tested.

Per the WTP Test Specification for this work [35], the major objectives of these tests were to:

- Confirm that, in the alkali-limited region where waste loading is determined by sodium or sodium plus potassium content, sulfate phase accumulation does not occur across that range of sodium and potassium combinations.

- Demonstrate that, in the region where sulfate phase accumulation can limit waste loading, the proposed glass formulation correlation yields glasses without sulfate phase accumulation.

- Determine the effect of chlorine and fluorine on sulfate phase accumulation. 


\subsection{Quality Assurance}

This work was conducted under a quality assurance program based on NQA-1 (1989) and NQA-2a (1990) Part 2.7 that is in place at the VSL. This program is supplemented by a VSL Quality Assurance Project Plan (QAPP) for RPP-WTP work [37]. Test and procedure requirements by which the testing activities are planned and controlled are also defined in this plan. The program is supported by VSL standard operating procedures that were used for this work [38].

This work did not generate data to support waste form qualification activities; nor did it generate environmental regulatory data, or data to support permitting activities. This work is not subject to DOE/RW-0333P or the WTP QAPjP [39] for environmental regulatory data.

\subsection{Melter System Description}

\subsubsection{Feed System}

A schematic diagram of the DM100 vitrification system is shown in Figure 1.1. The melter feed is introduced in batches into a feed container that is mounted on a load cell for weight monitoring. The feed is stirred with a variable speed mixer and constantly recirculated except for periodic, momentary interruptions during which the weight is recorded. The recirculation loop extends to the top of the melter where feed is diverted from the recirculation loop through a peristaltic pump and into the melter through a Teflon-lined feed line and vertical water-cooled feed tube. The use of a peristaltic pump in place of the usual simulated ADS pump was requested by WTP R\&T for these tests because of the ability to reduce feed rate variations.

\subsubsection{Melter System}

Cross-sectional diagrams through the DM100-WV melter are shown in Figures 1.2a-c. The DM100-WV unit is a ceramic refractory-lined melter fitted with a pair of opposing Inconel 690 plate electrodes as well as a bottom electrode. The melter can be operated with either three-phase or single-phase power. However, the standard mode of operation, which was used for these tests, is single-phase with voltage applied to the side electrodes only. The bubbler used for stirring the melt pool enters from the top and is removable. The glass product is removed from the melter by means of an air-lift discharge system. The DM100-WV has a melt surface of $12 \times 14$ inches, giving a melt surface area of $0.108 \mathrm{~m}^{2}$. The nominal depth of the melt pool is about 19 inches, which gives a typical glass inventory of between 115 and $120 \mathrm{~kg}$. The plenum height is 27.5 inches. Temperatures are monitored by means of a series of thermocouples located in the melt pool, the electrodes, the plenum space, and the discharge chamber. 


\subsubsection{Off-Gas System}

For operational simplicity, the DM100-WV is equipped with a dry off-gas treatment system involving gas filtration operations only. Exhaust gases leave the melter plenum through a film cooler device that minimizes the formation of solid deposits. The film-cooler air has constant flow rate and its temperature is thermostatically controlled. Consequently, under steady-state operating conditions, the exhaust gases passing through the transition line (between the melter and the first filtration device) can be sampled at constant temperature and airflow rate. The geometry of the transition line conforms to the requirements of the 40-CFR-60 air sampling techniques. Immediately downstream of the transition line are cyclonic filters followed by conventional pre-filters and HEPA filters. The temperature of the cyclonic filters is maintained above $150^{\circ} \mathrm{C}$ while the temperatures in the HEPAs are kept sufficiently high to prevent moisture condensation. The entire train of gas filtration operations is duplicated and each train is used alternately. An induced draft fan completes the system. 


\section{SECTION 2.0 WASTE SIMULANT AND GLASS FORMULATIONS}

The DM100 melter tests described in this report were designed to assess sulfate phase separation during the processing of glass formulations calculated from the LAW glass formulation algorithm [33] when estimated process uncertainties are included in the worst-case direction. The compositions of the glasses selected for DM100 melter testing [35] and the order in which they were processed are given in Tables 2.1 and 2.2, respectively.

\subsection{Waste Simulants}

The LAW waste simulants for the DM100 melter tests are based on the composition data provided in the WTP Test Specification [32] and Test Exception [40]. The concentrations of Cl, $\mathrm{Cr}, \mathrm{F}, \mathrm{Ni}, \mathrm{Pb}$, and $\mathrm{P}$ in the waste simulants were modified so that their target concentrations in the glass equal the "others" concentrations given in Table 2.3. The concentrations of potassium and sulfate in the simulants also were modified to achieve the $\mathrm{K}_{2} \mathrm{O}$ and $\mathrm{SO}_{3}$ concentrations in the respective glass compositions. The potassium concentration in the LAWE4H simulant for Test 1 was set to zero so that the effect of potassium could be studied during the transition from LAWE3 to LAWE4H. The waste simulant compositions for LAWE3, LAWE4H, LAWE5H, LAWE6H, LAWE7H, LAWE9H, and LAWE10H are given in Tables 2.4-2.10. These compositions are based on the compositions of LAW tanks AP-101, AN-105, AN-107, AN-104, AN-102, AZ-101, and AZ-102. For Tests 1-3, the Cl and F concentrations were 0.20 wt\% and $0.08 \mathrm{wt} \%$, respectively. For Tests $4-6$, the $\mathrm{Cl}$ and $\mathrm{F}$ concentrations were $1.1 \mathrm{wt} \%$ and $1.3 \mathrm{wt} \%$, respectively. The halides were added as the respective sodium salts with a corresponding decrease in the sodium from other sources so that the target $\mathrm{Na}_{2} \mathrm{O}$ concentrations in the glass compositions were maintained at the values given in Table 2.1, prior to renormalization for the added halides.

The simulant concentrations for feed processing in terms of the sodium molarity are given in Tables 2.4-2.10. The feed concentrations were estimated based on the waste loading and results from previous testing of melter feeds [41, 42]. In some cases, these concentrations are substantially different from feed concentrations that have been used previously in melter testing. For example, the waste loading for LAWE9H is $11.34 \mathrm{wt} \%$ and, based on this waste loading, the projected feed concentration is 4 molar sodium. The closest waste loadings that have been used previously in melter tests are about $7 \mathrm{wt} \%$ and $14 \mathrm{wt} \%$, while the closest feed concentrations are 2.75 and 5.0 molar sodium.

\subsection{Glass Formulation}

The compositions of the glasses used for melter testing are given in Tables 2.1 and 2.3. The glass compositions along with the waste loadings and contributions from the waste 
simulants and glass former additives are given in Tables 2.11-2.17. Crucible melts of glasses LAWE3, LAWE4H, LAWE5H, LAWE7H, LAWE9H, and LAWE10H were prepared and tested at VSL for compliance with processing and product quality requirements. LAWE6H was not prepared because it is sufficiently close to another glass composition that has already been characterized. The results of the tests confirmed that all of the glasses meet all the processing and product quality requirements.

Glass former additives [43] (for one liter each of the waste simulants) and the measured feed properties are given for crucible batch feed samples in Table 2.18. These are based on the concentrations of the waste simulants given in Tables 2.4-2.10. The feed produced in crucible size batches had the benefit of rigorous density determination and therefore the sodium molarities exactly matched the desired targets. The feed recipes provided to Optima were based on estimated densities and therefore the sodium molarities sometimes vary slightly from target. This difference also results in melter feed and crucible batch preparations having slightly different glass yields although the product glass compositions are the same. In each case, feed was prepared on the crucible scale, characterized and verified to be within the WTP specified rheology bounds [44], prior to melter tests. Based on the results of these measurements, additional water was added to LAWE10H to provide a feed within the prescribed rheological boundaries. Samples of all of the melter feeds were prepared and characterized before their use in the respective melter tests. The highest tested feed concentrations with rheological properties within the WTP bounds were selected for melter testing.

\subsection{Sugar Additions}

With high nitrate feeds, the addition of reductants is necessary in order to control melt foaming. Sugar, which was used for this purpose at West Valley, has also been selected as the baseline reductant for the WTP. The amount of sugar required increases with the amount of nitrates present in the feed and decreases with the amount of waste organics present in the feed, which themselves act as reductants. Excessive additions of reductants can be deleterious, leading to over-reduction of the melt and formation of sulfides and molten metals. Consequently, the oxidants and reductants in the feed must be suitably balanced. The basis for achieving this balance was developed by VSL and Duratek for the vitrification of high-sodium-nitrate feeds at Savannah River's M-Area and has been successfully applied to the processing of a wide variety of simulated RPP-WTP feeds over the past eight years. In developing this approach, we elected to conservatively adopt the most reducing potential reaction as the basis for the definition of a "sugar ratio" of 1.0 as a result of concerns for over-reducing the melt. Such a reaction, using sodium salts as an example, is:

$$
\mathrm{C}_{12} \mathrm{H}_{22} \mathrm{O}_{11}+8 \mathrm{NaNO}_{3}=8 \mathrm{CO}_{2}+4 \mathrm{CO}+4 \mathrm{~N}_{2}+11 \mathrm{H}_{2} \mathrm{O}+4 \mathrm{Na}_{2} \mathrm{O}
$$

Fundamentally, the basis that is selected is simply a convention, since the precise stoichiometry of the reactions involved is neither known nor constant under the conditions prevailing in the melter. However, with this convention, a sugar ratio of 1.0 corresponds to one mole of sucrose per eight moles of nitrate or, more generally, 1.5 moles of organic carbon per mole of nitrate. It is then expected that significantly less sugar than this will be required in practice. The 
empirically determined amount required to successfully control melt foaming without significantly reducing the glass melt was found to correspond to a ratio of 0.5 when any nitrites present were counted as nitrates (i.e., 0.75 moles of organic carbon per mole of nitrate + nitrite). This approach has been employed for all WTP melter testing. It is, however, expected that slight variations around the nominal value of 0.5 may be necessary to account for differences in the reducing power of waste organics in comparison to sugar, particularly for LAW streams that are high in organics.

The calculation of the amount of sugar needed to achieve a stoichiometric sugar ratio of 0.5 for Test 1A (LAWE3) proceeds as follows:

- One liter of simulant contains $183067 \mathrm{mg}$ nitrate and $58110 \mathrm{mg}$ nitrite (see Table 2.4) which corresponds to 2.953 moles of nitrate and 1.263 moles of nitrite, giving a total of 4.216 moles of NOx

- Required total amount of organic carbon for a sugar ratio of 0.5 is $4.216 \times 0.75=3.162$ moles

- One liter of simulant contains $2718 \mathrm{mg}$ or 0.227 moles of organic carbon (see Table 2.4)

- $\quad$ Therefore, $3.162-0.227=2.935$ moles of organic carbon must be added.

- The molecular weight of sucrose is 342 g per mole, 2.935 x 342/12 = $83.65 \mathrm{~g}$ sugar must be added per liter of simulant, as shown in Table 2.18.

\subsection{Preparation of Melter Feed}

Optima Chemicals, which has supplied all of the LAW simulants for the previous DM100 and LAW Pilot Melter studies, provided the required amounts of the various feed blends to conduct all the tests shown in Table 2.2. The LAW feed from Optima was received at VSL complete except for sugar, a small portion of the sodium in the target composition, potassium in the LAWE4H formulation, the additional chlorine and fluorine to increase the target glass concentrations to 1.1 and 1.3 wt\% respectively in Tests 4-6, the rhenium spike, and a small portion of the feed water. Sugar was added to the feed at a stoichiometric ratio of 0.5 in the manner described in Section 2.3. The amount of sodium left out of the feed by Optima corresponded to the amount of sodium in the $\mathrm{NaF}$ and $\mathrm{NaCl}$ added to the feed in Tests 4-6 to achieve target glass concentrations of the halides and sodium. Adjustments made to the feed to accommodate the additional sodium halides are shown in Table 2.19. The majority of the sodium that was removed was in the form of sodium hydroxide. However in tests with the LAWE9H composition, sodium carbonate was removed to account for the sodium, and in tests with the LAWE10H composition, sodium carbonate and nitrate were removed. In Tests 1-3, sodium was added as a $50 \%$ sodium hydroxide solution to the feed to bring the sodium feed content to the target concentration. Additions of the halides to the target glass compositions in Tests 4-6 reduced the concentration of the other constituents in the target composition by a total of $2.12 \mathrm{wt} \%$ after renormalization. The addition of halides to the feed had little effect on the properties of the feed as shown by comparison of the measured feed properties of crucible batches given in Tables 2.18 and 2.19 for feeds with low and high halide concentrations, respectively. Elements with the highest concentrations in the target composition, such as silicon 
and sodium, are reduced by the largest absolute amounts. The potassium oxide target of $0.54 \mathrm{wt} \%$ in Tests $1 \mathrm{~A}$ and $6 \mathrm{~B}$ was achieved by adding potassium hydroxide to the feed. This addition resulted in a slight decrease in the concentrations of the other constituents in proportion to their feed concentrations. This was partially mitigated by the addition of sodium hydroxide to maintain the original sodium concentration, as shown in Table 2.12. A WTP Test Exception [40] was issued to specify the addition of rhenium (as a technetium surrogate) to the feed in all of the tests in order to track its fate in the process. The rhenium was added in the form of perrhenic acid to achieve a concentration of $0.1 \mathrm{wt} \%$ as rhenium oxide assuming total retention in the glass product. The rhenium spike, as with the halides, reduces the concentrations of the other constituents in proportion to their concentration.

\subsection{Analysis of Feed Samples}

\subsubsection{General Properties}

Feed samples from each test were analyzed to confirm physical properties and chemical composition. Samples were taken during melter testing from an inline sampling port in the feed line. Sample names and sampling dates are listed, and measured properties are compared to target in Table 2.20. Target values were obtained by measurements on crucible batch samples and, for glass yield $(\mathrm{kg} / \mathrm{kg})$, by calculation from the recipe. Measured water content, density, glass yield, and $\mathrm{pH}$ were similar for all samples from the same test, and were consistent with the target values. One exception is the $\mathrm{pH}$ for samples from Tests 2C through 3C, where the melter feed samples have $\mathrm{pH}$ values from 0.5 to 2.2 higher than the values measured on the crucible batch samples. This difference may be a result of absorption of atmospheric carbon dioxide, which can vary depending on the time at which the final amount of sodium hydroxide was added, the length of time the sample was mixed, and the time lapse before the $\mathrm{pH}$ is measured. The highest $\mathrm{pH}$ values were measured in tests using waste that contained the highest concentration of hydroxide, such as in Tests $1 \mathrm{~A}$ and 1B. For this reason, $\mathrm{pH}$ values were lower in tests with added sodium halides than comparable tests with sodium added mostly as sodium hydroxide. Small decreases in glass yield were observed due to the incorporation of reductants in the melter feed, which do not produce glass; crucible batch samples had no added reductants and therefore had higher glass yields. Also, small amounts of water are added during the transfer of melter feed, which reduces glass yields. Despite the addition of sugar and water to the melter feed, measured glass yields were within seven and a half percent of the target, validating the use of target glass yields $(\mathrm{kg} / \mathrm{kg})$ for glass production rates.

\subsubsection{Rheology}

Samples of the melter feeds that were used for these tests were also subjected to rheological characterization. The results from rheological characterization of a variety of other melter feeds and waste simulants, as well as the effects of a range of test variables, are described in detail in a separate report [41]. Melter feeds were characterized using a Haake RS75 rheometer, which was equipped with either a Z40DIN or a FL22-SZ40 sensor. A typical set of measurements consists of identifying the flow characteristics of the slurry by measuring the shear stress on the slurry at controlled shear rates and temperatures. In these measurements, the 
shear rate values are preset and are increased stepwise from $0.01 s^{-1}$ to $200 \mathrm{~s}^{-1}$ (70 $\mathrm{s}^{-1}$ for FL22-SZ40) with a sufficient delay (typically 15 to 30 seconds) between steps to ensure that shear stress is allowed to fully relax and therefore be measured at equilibrium. This approach is somewhat different from the "flow curve" approach in which the shear rate is ramped up to some maximum value and then ramped back down to produce a hysteresis curve that is dependent on the selected ramp rate. The viscosity of the sample as a function of the shear rate is then calculated as the ratio of the shear stress to the shear rate. The yield stress data for the melter feeds were measured using a controlled-stress mode in which the torque on the rotor was slowly increased while the resulting deformation of the fluid was monitored. The discontinuity in the measured deformation-torque curve was identified as the yield stress. It should be noted that this direct measurement of the yield stress can be quite different from the value obtained by extrapolation of the shear stress-shear rate curve to zero, the value often reported. All of the measurements in this work were made at $25^{\circ} \mathrm{C}$; previous work [41], which examined a range of temperatures, showed a relatively weak effect of temperature.

Rheograms for select melter feeds, which show the feed viscosity versus shear rate, are presented in Figures 2.1 and 2.2; measured values for viscosity at selected shear rates and the yield stress are shown in Table 2.21. Samples for rheological testing were selected at the extremes of feed sodium content and waste loading. An additional sample was tested to determine the effect of the added halides on rheological properties. Notice that melter feed with LAW Envelope B waste is considerably more viscous than the feed samples from LAW Envelope A waste. Higher viscosity in melter feed samples with LAW Envelope B wastes has also been observed in previous studies and is due to the addition of more glass forming additives and less of the aqueous waste, which results in a lower $\mathrm{pH}$. Halide additions to the feed had no discernable effect on the rheological properties of the feed. The rheological properties of all three feed samples are well within the Project bounds [44]. The LAWE10H feed was brought within these limits by the dilution of the feed with additional water prior to the melter test (see Section 2.2). The yield stress values exhibited the same trends as those described for the viscosity at low shear rates.

\subsubsection{Chemical Composition}

The chemical compositions of the feed samples were determined by first making a glass from the feed via crucible melt. The glass was subsequently crushed and analyzed directly by $\mathrm{X}$-Ray Fluorescence Spectroscopy (XRF). The boron oxide and lithium oxide target values were used for normalizing the XRF data since their concentrations were not determined by XRF. Data are compared to the target compositions in Table 2.22. Selected samples from the early tests were also subjected to DCP analysis of solutions generated by microwave aided acid dissolution; the results are compared to those obtained by XRF in Table 2.23. The XRF values reported for fluorine were generated using an analytical method which uses a polished solid monolith as opposed to the less than 200 mesh glass powders used for analysis of all other elements. The results generally corroborate the consistency of the feed composition and show good agreement with the target composition for the major components. Boron and lithium concentrations measured by DCP were within nine percent of the target, validating the use of the target value for normalizing the XRF data. Of the oxides with a target concentration of one percent or greater, 
only the XRF values for magnesium, titanium, zinc, and zirconium oxides had deviations of greater than $10 \%$ from target. These deviations were also observed at about the same frequency and magnitude in the product glasses (see Section 4.1). Only two tests had feed samples with measured magnesium oxide deficits greater than 10\%; the deficits for both these tests were less than $11 \%$. Surpluses in titanium oxide and deficits in zinc oxide between 10 and 18.5 percent were measured in samples from about half the tests. Similar observations with respect to titanium were made in previous tests with LAW melter feeds [7-10, 12, 14] due to its presence as a contaminant in the glass forming additives. The DCP measured values for zinc deviated from their respective target values significantly less than did the corresponding values measured by XRF, suggesting a potential low analytical bias for zinc using the XRF for these samples. Only three tests had feed samples with measured zirconium oxide deficits greater than 10\%; the deficits for both of these tests were less than $10.3 \%$. Since a low bias for sodium is not uncommon for the DCP analysis, the sodium values generated by XRF are used in preference. Volatile minor elements such as sulfur, rhenium, and halides are, as expected, below target due to loss during crucible melting.

The target concentrations in the feed are verified from the simulant vendor's batching sheets. The additional amounts of chemicals added by the VSL are calculated, checked, and weighed out using calibrated balances. 


\section{SECTION 3.0 MELTER OPERATIONS}

A series of six tests were conducted on the DM100-WV melter between 6/13/05 and 9/16/05. The tests used a range of LAW Sub-Envelope simulants and corresponding melter feeds which produced glasses in the alkali-limited region through the sulfate-limited LAW glass composition region with varying alkali content. In addition, in all of the seven glass compositions that were used, the impact of higher levels of chlorine and fluorine on sulfate phase formation was also investigated. These tests produced almost seven metric tons of glass from over fourteen metric tons of feed. Summaries for all the tests are provided in Table 3.1. The tests were divided as follows:

- Test 1: 100 hours, glass compositions LAWE3 and LAWE4H without K

- Test 2: 175 hours, glass compositions LAWE4H, LAWE5H, LAWE6H, and LAWE7H

- Test 3: 125 hours, glass compositions LAWE7H, LAWE9H, and LAWE10H

- Test 4: 115 hours, glass compositions LAWE10H, LAWE9H, and LAWE7H, all with Cl and $\mathrm{F}$ targeting 1.1 and $1.3 \mathrm{wt} \%$, respectively, in the glass product assuming total retention

- Test 5: 175 hours, LAWE7H, LAWE6H, LAWE5H, and LAWE4H, all with $\mathrm{Cl}$ and F targeting 1.1 and $1.3 \mathrm{wt} \%$, respectively, in the glass product assuming total retention

- Test 6: 100 hours, LAWE4H without K and LAWE3, both with $\mathrm{Cl}$ and F targeting 1.1 and $1.3 \mathrm{wt} \%$, respectively, in the glass product assuming total retention.

A primary objective of these melter tests was to determine if secondary sulfur phases result from processing or transitioning between a variety of glass compositions generated by the LAW glass formulation algorithm [33]. This was achieved using a sampling and analysis protocol designed to differentiate between a pervasive sulfate layer and transient localized patches of sulfate. Dip sampling was performed while each new glass composition was processed (typically after four melter turnovers) and during the transition between glass compositions when the glass composition has transitioned approximately half way to the new glass composition (0.69 melter turnovers or about 9 hours processing at $2,000 \mathrm{~kg} / \mathrm{m}^{2} / \mathrm{day}$ production rate). At each sampling event, feeding was interrupted and bubbling reduced while five dip samples were collected. The melt surface was considered free of a pervasive sulfate layer if at least four of the five samples had no visible secondary sulfate phases, in which case feeding of the melter was resumed. If only one of the five samples had visible secondary sulfate, it was considered transient (sulfate phase resulting from the consumption of the cold cap and/or the melting of salt deposits in the plenum and film cooler because radiant heat from the glass increases as the cold cap is consumed). If more than one of the five samples exhibited secondary sulfate, the cold cap was burned off entirely and the dip-sampling repeated. Inspection and analysis of the dip samples indicated that no secondary sulfate layers were present on the melt pool surface during any of the tests without the envelope maximum concentration of halides. Conversely, secondary layers were observed in the transitions and at the end of many of the tests in which the increased levels of halides were included in the feed. No specific pattern of sulfate layer formation with sulfur and sodium feed content was identified. The common compositional 
factor in the formation of secondary phases was the presence of the additional halides. After a sulfate layer was detected on the melt surface, the glass pool was bubbled until the dip samples indicated that the sulfate layer had dissipated, prior to commencing the subsequent test segment. A detailed list of all the individual dip samples, including visual results and analysis, is provided in Section 4.2.

Attempts were made to replicate the melter configuration and operating conditions used for the corresponding tests conducted earlier and the previous LAW Sub-Envelope [5-16] tests. These conditions include a near complete cold cap, which is between 80-95\% melt surface coverage for the DM100, since a 100\% cold cap tends to lead to "bridging" in smaller melters. Cold cap conditions were similar to those experienced in previous DM100 tests with LAW simulants [5-16]. The target glass production rate of $2000 \mathrm{~kg} / \mathrm{m}^{2} /$ day was approximated for all tests, as depicted in Figures 3.1.a - 3.1.f. No significant processing problems were observed as a result of the intended compositional changes or additions of halides to the feed. During the second test, three feed interruptions of up to 65 minutes in duration occurred as a result of clogging of the feed line. The tubing from the peristaltic pump to the melter feed nozzle was therefore changed to a wider diameter tube and shielded from heat radiating from the melter lid. No significant interruptions were experienced due to feed system clogging after these changes were made. The instantaneous production rates varied by about $25 \%$. The larger decreases in feed rate and gaps in the production rate curves shown in Figures 3.1.a - 3.1.f were the result of down-time for feed transfers, dip sampling, and in the case of Test 2, to change the tubing around the peristaltic pump.

Glass pool bubbling was adjusted over the course of all the tests to maintain the target production rate of $2000 \mathrm{~kg} / \mathrm{m}^{2} /$ day and, therefore, the bubbling rate used for each composition gives an indication of the relative rates the feed compositions can be processed. Bubbling rates used throughout the tests are shown in Figures 3.2.a - 3.2.f and average bubbling rates for each test segment are given in Table 3.2. During each test segment, bubbling rates were relatively constant except around feed interruptions for feed transfers and dip sampling events. Figure 3.3 shows the increase in the bubbling rate from about $7 \mathrm{lpm}$ to over $20 \mathrm{lpm}$ as the sodium content of feed increased over the course of testing. The increase in sodium concentration in these glass compositions coincides with increase in waste loading and therefore the higher bubbling rate could be in response to the lower proportion of glass forming additives in the feed. The same relationship of increasing ease of production with decreasing feed sodium content and waste loading was observed in tests on the LAW Pilot Melter [45]. Also apparent in Figure 3.3 is an up to two-fold decrease in bubbling required when the envelope maximum concentrations of halides are added to the feed. The increased ease of production with increased feed halide content observed with these LAW simulants was not observed in previous tests with HLW simulants [46], indicating that there are compositional effects on the production rate increases realizable through halide additions to the feed. Comparison of Test 1B with 2A and Test 5D with 6A permits an assessment of the effect of potassium on the bubbling rate needed to obtain the same glass production rate; the effect is small but in the direction of faster melting with potassium than without. 
The results of various operational measurements that were made during these tests are given in Table 3.2. Glass temperatures are shown in Figures 3.4.a - 3.4.f, plenum temperatures in Figures 3.5.a - 3.5.f, electrode temperatures in Figure 3.6.a - 3.6.f, and melt pool resistance in Figure 3.7.a - 3.7.f; electrode power is included in Figures 3.5.a - 3.7.f. Test segment average bulk glass temperatures $\left(1144-1155^{\circ} \mathrm{C}\right)$ approximated the target glass temperature of $1150^{\circ} \mathrm{C}$. Glass temperatures near the top of the melt pool are not reliable indicators of bulk glass temperatures as a result of gradients near the cold cap. Plenum temperatures typically ranged from 525 to $600^{\circ} \mathrm{C}$, within the 450 to $650^{\circ} \mathrm{C}$ target. This is deliberately somewhat higher than for the larger melters (DM1200 and LAW Pilot Melter) in order to reduce cold cap bridging that occurs in the smaller melters. Higher plenum temperatures occurred in Tests 4 - 6 during repeated dip sampling events and bubbling periods used to remove secondary phases from the melt surface, as can be observed in Figure 3.5.f at 50 hours run time. The east electrode temperatures averaged between $1125-1150^{\circ} \mathrm{C}$; that electrode was about $50-75^{\circ} \mathrm{C}$ hotter than the west electrode and was about $225^{\circ} \mathrm{C}$ hotter than the bottom electrode, which was not powered during these tests. The difference in temperature between the side electrodes is probably due to extreme sensitivity of thermocouple placement in the electrodes and therefore not reflective of an overall temperature difference between the sides of the melter. Power supplied to the electrodes typically varied by only $1 \mathrm{~kW}$ from the average values for most of the testing; however, much larger changes were observed during dip sampling events and extended bubbling periods to rid the melt surface of secondary phases. As expected, melt pool resistance increased with decreasing sodium content of the glass, as illustrated in Figure 3.8. Unlike the bubbler flow required to achieve the target production, the glass resistance is unaffected by the higher halide concentrations in the feed.

The gas temperature at the film cooler was about $300^{\circ} \mathrm{C}$ and depended on the plenum temperature, the amount of added film cooler air, and the temperature of the added film cooler air. A small drop of ten to fifteen degrees in gas temperature was observed across the (insulated) transition line; the high temperature is maintained in order to prevent condensation in the downstream filtration units. 


\section{SECTION 4.0 GLASS PRODUCTS}

Nearly seven metric tons of glass was produced in these tests. The glass was discharged from the melter periodically into 5-gallon carbon steel pails using an airlift system. The discharged product glass was sampled at the end of each test by removing sufficient glass from the top of the cans for total inorganic analysis. Product glass masses, discharge date, and analysis performed are given in Table 4.1. Sets of five glass samples were also obtained by dipping a rod into the glass pool during and at the end of each test. All of the "dip samples" underwent visual examination to detect the presence of a separate sulfate phase on the glass surface; select samples were also subjected to chemical and microstructural analysis to confirm the visual results.

\subsection{Compositional Analysis of Discharged Glass}

Discharge glass samples were crushed and analyzed directly by XRF. The target values for boron and lithium oxides, which are not determined by XRF, were used for normalizing the XRF data to $100 \mathrm{wt} \%$. The XRF analyzed compositions of all discharged glass samples are provided in Table 4.2. The XRF values reported for fluorine were generated using an analytical method which uses a polished solid monolith as opposed to the less than 200 mesh glass powders used for analysis of all other elements. Selected samples from the early tests were also subjected to DCP analysis of solutions generated by microwave aided acid dissolution; the results are compared to those obtained by XRF in Table 4.3. The majority of the XRF analysis results compared very favorably to their corresponding target values and also corroborated the feed sample analyses (see Section 2.5.3). Of the oxides with a target concentration of one percent or greater (not including halides spiked into the feed in Tests 4-6), only the XRF values for magnesium, sodium, titanium, zinc, and zirconium oxides had deviations of greater than $10 \%$ from target. Only one test had samples with measured magnesium oxide deficits greater than $10 \%$; the deficit for that test was less than $12 \%$. Surpluses in titanium oxide and deficits in zinc oxide of between 10 and 20 percent were measured in samples from about half the tests. Similar observations with respect to titanium were made in previous tests with LAW melter feeds [7-10, $12,14]$ due to its presence as a contaminant in the glass forming additives. The DCP measured values for zinc deviated from their respective target values significantly less than did the corresponding values measured by XRF, suggesting a potential low analytical bias for zinc using the XRF for these samples. Only two tests had samples with measured zirconium oxide deficits greater than 10\%; the deficits for both these tests were less than $12.5 \%$. Surpluses in sodium occurred in Tests 3C and 4A while processing feeds with the lowest sodium content, suggesting that the glass pool was not completely turned over with respect to this element. The absolute oxide deviations, for oxides with deviations greater than 10 relative percent, are relatively small and therefore would have no significant effect on glass properties. Values for all the major oxides analyzed by DCP compare favorably with the XRF analysis except for silicon and zinc. $\mathrm{SiO}_{2}$ concentrations measured by DCP are frequently lower than those measured by XRF, but the deviations are less than $10 \%$ from the target. Chromium was significantly higher than the target value for both DCP and XRF analysis due to leaching of chromium from melter components 
such as the refractories and Inconel. Volatile components, such as halides and rhenium, were depleted in the glass product, as expected. Rhenium values measured by XRF were on average $40 \%$ less than those measured by DCP, in contrast to a previous study which showed the methods providing comparable values [47]. Rhenium mass balances around the melter (glass + emissions) using the XRF glass values are between 82 and 114\% (see Section 5.3), suggesting that the XRF values can be reliably used. Using DCP glass values for the rhenium balance would result in rhenium surpluses for each test and, therefore, these were not used for mass balance determinations. The DCP analysis results for boron and lithium are within eight percent of the target values, validating the use of the target boron and lithium concentrations for normalizing the XRF data.

Compositional trends for most oxides measured by XRF with target concentrations greater than one percent are illustrated in Figures 4.1 - 4.3. Sodium, silicon, calcium, and magnesium glass concentrations show the intended transition from a high-sodium composition to a low-sodium, high alkaline earth composition and then transition back to the high-sodium concentration. Potassium oxide concentrations are near the target of about five weight percent in the first and last test segments, drop dramatically in the second and second-to-last test segments due to the intentional omission from the feed; the values are constant throughout the rest of the tests at about half a weight percent. Many oxide concentrations remain constant throughout testing including aluminum, iron, titanium, and zinc, which are shown in Figure 4.2, as well as zirconium and boron.

Compositional trends for the volatile elements sulfur, chlorine, fluorine, and rhenium measured by XRF are compared to target concentrations in Figures 4.4-4.7. The sulfur contents of the glasses follow the inverse trend to soda. At the higher target concentrations of sulfur, which correspond to the lowest soda concentrations, a larger proportion of the sulfur is volatilized. This is in keeping with several previous melter tests which showed that sulfur retention in the product glass as a percent of feed is highest for the LAW Sub-Envelope A compositions and lowest for LAW Sub-Envelope B compositions [8, 10, 12, 45]. During the tests with additional halides, chlorine and fluorine reach steady-state concentrations in the glass product at about 55 and 75 percent of their target values, respectively. Chlorine retention in the product glass of between 50 and 60 percent of feed has been typically observed in LAW melter tests $[8,10,12]$. The test results also show that rhenium showed even greater volatility than the halides, with only between 20 and 40 percent remaining in the glass product. It is also evident from Figures 4.4-4.7 that further depletions of the volatile constituents in the glass occurred during the idling periods in between tests.

\subsection{Glass Pool Samples}

Glass dip samples were obtained primarily to ascertain whether a secondary sulfate layer had formed on the surface of the glass melt. A list of all dip samples taken including sample names, target glass volatile concentration $\left(\mathrm{Cl}, \mathrm{F}, \mathrm{SO}_{3}, \mathrm{ReO}_{2}\right)$, sampling date, and visual observations, is given in Table 4.4. Samples were taken in groups of five by dipping metal rods into the melter and placing the rods with adhering glass into steel pails to cool. After about ten minutes, the glass was sufficiently cool to observe separated phases, if present, on the surface of 
the glass as well as adhering to the threads of the metal rod. An example of cooled glass on the metal rods is shown in Figure 4.8 and a closer view of separated phases on one of the samples is shown in Figure 4.9. After the glass cooled and observations of secondary phases on the rod were noted, the glass was broken off the rod and placed in Petri dishes for closer examination, as shown in Figure 4.10. Pictures were taken of the glass in Petri dishes, including detailed pictures of secondary phases like those shown in Figure 4.11. It is important to note that much of the secondary phases strongly adhere to the rod, as shown in Figure 4.12, and therefore not all the secondary phases are preserved with the archived glass sample.

Visual observations of the dip samples indicated that secondary phases were not present on the melt surface during or at the end of any of the tests with nominal halide concentrations. In contrast, however, secondary phases were observed in most of the tests with envelope maximum halide concentrations. In three of the fifteen dip sampling events during the tests with nominal halide concentrations, small amounts of secondary phases were observed in one of five samples. Given the established testing criteria, these solitary samples with secondary phases represent localized, transient sulfate patches resulting from the consumption of the cold cap and/or the melting of salt deposits in the plenum and film cooler as radiant heat from the glass increases as the cold cap is consumed. In ten of the fifteen dip sampling events during the tests with envelope maximum halide concentrations, at least two of five dip samples had evidence of secondary phases. These secondary phases occurred at the end of seven of the nine test segments and at half of the transitions between glass formulations. In the vast majority of the dip sampling events that indicated the presence of a secondary phase, three to five of the dip samples had visible secondary phases. No pattern was observed within sets of five dip samples, such as the first or last samples being more or less likely to contain secondary phases. Most of the secondary phases were removed from the melt surface after about a half hour of bubbling; however, in two instances, significantly longer bubbling periods were required (both dip sample events around Test 4C).

Chemical and morphological analysis of the secondary phases was conducted on select samples by Scanning Electron Microscope (SEM) equipped with Energy Dispersive X-ray Spectroscopy (EDS). The first sample analyzed was also the first sample to indicate the presence of a secondary phase: WVZ-D-74C from Test 1B. The results of the analysis, depicted in Figures 4.13.a and 4.13.b, indicate that the secondary phases are composed of alkali halides and alkali perrhenates with no sulfur species. Another sample with visible secondary phases, AWV-D147A from Test 3B, had similar secondary phases although no fluorine was observed and sulfate was present. It is important to note that for both of these samples, only a very small amount of material was available for analysis which may bias the results. Conversely, samples taken from tests conducted using feed enriched with halides produced more than sufficient amounts of secondary phase material for analysis. A typical example is shown in Figures 4.14.a - 4.14.c. The analysis reveals two distinct phases: a substrate consisting mainly of calcium and alkali sulfate with lesser amounts of chromium, chlorine, and rhenium, and crystals radiating out from the substrate consisting of alkali sulfate with lesser amounts of chromium and chlorine. The analysis indicating the presence of a phase containing calcium sulfate confirm observations of an insoluble component in the secondary phase that could not easily be washed from the sampling rods with water. 
Additional verification of the presence and composition of secondary phases was provided by a glass washing procedure. Two hundred and fifty milligrams of ground glass from select samples was placed in a bottle with two hundred and fifty milliliters of de-ionized water, agitated, and set aside until the particles settled to the bottom of the container. Subsequently, enough of the supernatant solution was removed for anion chromatography to measure sulfate, chloride, and fluoride concentrations, as well as for DCP to measure rhenium. The results of this procedure for the samples tested are compared to the respective targets in the glass product, and the visual observations of secondary phases, in Table 4.5. Also included are the results of this procedure performed on several glasses produced in previous tests [48-50], which did not contain halides, sulfur, or rhenium to provide estimates of background concentrations for each element. Based on analysis of these samples, concentrations of at least $1 \mathrm{ppm} \mathrm{F,} 23 \mathrm{ppm} \mathrm{Cl,} 2.1 \mathrm{ppm}$ sulfate, and $0.5 \mathrm{ppm}$ Re (2 X "blank" concentration) are required as an indication of soluble secondary phases. Other additional samples included in Table 4.5 are samples taken after idling periods which have definitive secondary phases, and off-gas particulate material removed from the cyclones which are known to be enriched in volatile feed components. Measured concentrations of sulfate, and halides in the cyclone solids sample, are much higher than the background indicating that the method can easily distinguish between samples with and without soluble secondary phases. The measured concentrations for the dip samples support the field observations of secondary phases and are also consistent with the results obtained from subsequent SEM-EDS analysis. Samples in which no secondary phases were observed in the field seldom have concentrations above the blank thresholds, whereas samples with visually observed secondary phases usually have at least one measured concentration above the blank values. It is important to note that often, much of the observed secondary phase remains on the rod, and therefore not all solutions generated from samples taken where secondary phases were observed should be expected to have high solution concentrations. Also, portions of the secondary phases are insoluble and therefore will not release anions into solution. Another point of interest is the amount of rhenium in many of the wash solutions. Rhenium was used as a surrogate for radioactive technetium present in the actual waste.

\subsection{Glass Property Testing}

Glass property measurements, including product quality testing, were performed on five glasses taken from the end of five selected test segments, as specified in the Test Specification [35]. These selections permit direct comparisons with the corresponding crucible melt results at high potassium and high sodium, moderate sodium, and low sodium levels; in addition, they permit an assessment of the effects of the increased halide levels on glass properties. In Figures 4.15 - 4.19, the melter glass data are compared to the corresponding data collected in crucible-scale glass formulation studies for melt viscosity, melt electrical conductivity, sodium and boron Product Consistency Test (PCT; ASTM C 1285) leach rates, Vapor Hydration Test (VHT) alteration rate, and zinc concentrations in Toxicity Characteristic Leach Procedure (TCLP) leachates (SW-846 Method 1311). The data are also presented in tabular form in Table 4.6 for viscosity and electrical conductivity, Table 4.7 for PCT and VHT, and Table 4.8 for TCLP. All of the measured properties of the crucible and melter glasses are within acceptable limits for processing and product quality. The viscosities and electrical conductivities of the crucible and melter glasses are similar and the higher halide concentrations do not show any 
noticeable effect. PCT releases for the high alkali (18.21 wt $\% \mathrm{Na}_{2} \mathrm{O}$ and $4.98 \mathrm{wt} \% \mathrm{~K}_{2} \mathrm{O}$ ) LAWE3 crucible and melter glasses are similar at the low halide concentrations, but show substantially higher $\mathrm{B}$ and $\mathrm{Na}$ releases for the melter glass sample with higher halide concentration. In contrast, PCT releases of the lower alkali, LAWE7 and LAWE10 crucible and melter glasses are similar, with no significant effect of higher halide concentrations. VHT alteration rates for the crucible and melter glasses are similar, with the differences within the normally large variations inherent in the VHT test results. Zinc concentrations in TCLP leachates for the LAWE7 and LAWE10 crucible and melter glasses are similar, with no noticeable effect of higher halide concentrations. TCLP results for the LAWE3 crucible and melter glasses, however, show higher $\mathrm{Zn}$ concentration in the TCLP leachate for the melter glass with higher halide concentration, as compared to the corresponding melter glass with lower halide concentration and the crucible glass. The analyzed composition of the LAWE3 melter glass with higher halide concentration, DWV-G-51B (see Table 4.2), does not show any deviation that would explain the higher PCT and TCLP leach rates. Based on these observations, it seems likely that the PCT and TCLP leach rates of glasses with high alkali concentrations increase as their $\mathrm{Cl}$ and $\mathrm{F}$ concentrations are increased.

Samples of the as-melted and heat treated crucible and melter glasses were examined for the presence of secondary phases. Glass samples were heat treated at $850^{\circ} \mathrm{C}$ for 20 hours, and according to the canister centerline cooling (CCC) curve [51]. Observations of the as-melted and heat treated samples are given in Table 4.9. The majority of the as-melted glass samples appeared clear and green in color. Traces of secondary sulfate phases were observed on melter discharge samples from tests with high $\mathrm{Cl}$ and $\mathrm{F}$. All of the heat treated glasses, except for LAWE10H samples, appeared clear and green in color, without the presence of any secondary phases. Heat treated crucible and melter samples of LAWE10H showed small amounts (0.1 to $0.6 \mathrm{vol} \%$ ) of augite crystals, mostly at the crucible contact surface. 


\section{SECTION 5.0 \\ MONITORED OFF-GAS EMISSIONS}

\subsection{Particulate Sampling}

The melter exhaust was sampled for metals/particles/acid gases according to 40-CFR-60 Methods 3, 5, 26, and 29 at steady-state operating conditions during each test segment. The concentrations of off-gas species that are present as particulates and gaseous species that are collected in impinger solutions were derived from laboratory data on solutions extracted from air samples (filters and various solutions) together with measurements of the volume of air sampled. Particulate collection required isokinetic sampling, which entails removing gas from the exhaust at the same velocity that the air is flowing in the duct (40-CFR-60, Methods 1-5). Typically, a sample size of $30 \mathrm{dscf}$ was taken at a rate of between 0.5 and $0.75 \mathrm{dscfm}$. Total particulate loading was determined by combining gravimetric analysis of the standard particle filter and chemical analysis of probe rinse solutions. An additional impinger containing $2 \mathrm{~N} \mathrm{NaOH}$ was added to the sampling train to ensure complete scrubbing of all acid gases and to distinguish between halogens in the form of acids or homonuclear molecular gases. The collected materials were analyzed using direct current plasma atomic emission spectroscopy (DCP-AES) for the majority of the constituents and ion chromatography (IC) for anions. Melter emission fluxes are compared to feed fluxes in Table 5.1. Notice the distinction that is made between constituents sampled as particles and as "gas". The "gaseous" constituents are operationally defined as those species that are scrubbed in the impinger solutions after the air stream has passed through a $0.3 \mu \mathrm{m}$ heated filter. All samples are well within the $90-110 \%$ limits for isokinetic sampling.

Solids carry-over from the melter ranged over about an order of magnitude, from a quarter percent to over two percent of feed solids, depending on feed sodium and halide content. This trend is illustrated in Figure 5.1, which depicts particulate melter emissions as a function of feed sodium content. Notice for each composition, the fifty to one hundred percent increase in particle emissions in tests with higher halide concentrations in the feed. The apparent decrease in emissions at the highest sodium concentrations resulted from omitting potassium in the feed during Tests $1 \mathrm{~B}$ and $6 \mathrm{~A}$ as well as the potassium oxide concentration in Tests $1 \mathrm{~A}$ and $6 \mathrm{~B}$ being an order of magnitude higher than for any other test; potassium is more volatile than sodium and hence contributes more to particle emissions relative to its concentration in the feed. The relationship between feed sodium content with sodium, rhenium, chlorine, fluorine, and sulfur emissions is illustrated in Figures 5.2 - 5.6, respectively. As expected, sodium emissions increase with increasing feed sodium content and also increase by a factor of two at the higher halide feed concentrations. Rhenium emissions appear to increase with feed sodium content in the lower sodium concentration range but are unaffected in the higher sodium range. Although rhenium emissions were mostly higher in tests with the halides added to the feed, the effect was significantly less than for sodium. Halide emissions increase with increasing feed concentration but are unaffected by the other feed constituents, including sodium. Sulfur retention in the glass was inversely proportional to the sodium content of the target glass; however, it should be noted that in these tests, the feed sulfur varies inversely with the feed sodium content. The additional 
halides added to the feed in Tests 4-6 result on average in about a twenty percent increase in sulfur emissions. Many of these trends, most notably the increase in sulfur emissions with feed sodium content and increased emissions with higher feed halide content, have been previously observed [5-16, 45]; however, the current tests provide a unique data set to evaluate the effect of feed halide and sodium content on emissions because of the systematic changes in glass compositions and the consistency of the test conditions.

As expected, the feed elements emitted at the lowest melter DF in ascending order were rhenium, chlorine, fluorine, sulfur, chromium, potassium, sodium, and boron. Measured rhenium carry-over between 52 and 78 percent of feed is consistent with previous tests conducted with LAW Envelope A simulants at similar feed and glass concentrations [47]. Emissions of chlorine, fluorine, and sulfur were mostly particulate at high sodium and lower halide feed contents. The proportion of these constituents emitted as acid gases increased with increasing feed content. Almost all of the halides present in gas phase were measured in the acidic impinger and not in the basic impinger solutions, indicating that the halides were present as acid gases $(\mathrm{HCl}+\mathrm{HF})$ rather than as homonuclear molecular gases $\left(\mathrm{Cl}_{2}+\mathrm{F}_{2}\right)$. Boron, sulfur, and the halides were the only elements detected in the impinger solutions collected downstream of the heated particle filter in the sampling train, which constitute the "gas" fraction of the melter emissions.

\subsection{Gases Monitored by FTIR}

Melter emissions were monitored in each test for a variety of gaseous components, most notably CO and nitrogen species, by Fourier Transform Infra-Red Spectroscopy (FTIR). The off-gas system temperature is maintained well above $100^{\circ} \mathrm{C}$ beyond the sampling port downstream of the HEPA filter to prevent analyte loss due to condensation prior to monitoring. A summary of average concentrations monitored during each test is provided in Table 5.2. Concentrations of various monitored species are plotted in Figures 5.7.a - 5.11.c. The analytes listed in Table 5.2 are those that were thought likely to be observed during the tests based on previous work; no other species were detected in the off-gas stream by FTIR. The most abundant nitrogen species monitored was NO, which is consistent with previous tests [5-16, 46, 47] in which nitrates and nitrites were present in the feed. The $\mathrm{NO}_{2}$ concentration was about 10 to 20 times less than NO, in keeping with previous DM100 tests with LAW simulants. Exceptions were Tests $3 \mathrm{C}$ and $4 \mathrm{~A}$ due to the very low concentrations of nitrogen oxides in the feed. As expected, nitrogen oxide concentrations were proportional to the nitrogen oxide content of the feed. Consequently, measured concentrations of by-products of incomplete organic combustion, such as ammonia and carbon monoxide, were proportional to nitrogen oxide concentrations since the feed organic content was adjusted to the feed nitrogen oxide content with additions of sugar (see Section 2.3). The measured water contents were typically between five and six percent and were about half the amount measured by the Method 5-type sampling discussed earlier as result of air inleakage between the two sampling points. This is the same amount of dilution observed in previous studies subsequent to the modification of the exhaust system in July of 2002 [8, 10, $12,14,16,47,50,52]$. As reported previously [16], an additional $50 \mathrm{scfm}$ of inleakage was measured prior to the modification resulting in exhaust dilutions of about 3 instead of about 2 in early tests $[7,9,11,13,15]$. A detailed discussion of melter exhaust dilution by film cooler air and off-gas system inleakage for the purposes of evaluating hydrogen concentrations has been 
given previously [53]. Little or no $\mathrm{HF}$ or $\mathrm{HCl}$ were observed by FTIR in tests conducted without the additional halides added to the feed, whereas HF was detected in the off-gas system exhaust throughout tests with higher halide concentrations, as shown in Figures 5.11.a - 5.11.c. These measurements are in keeping with the gaseous emission rates measured by the Method 5-type sampling and the dilution in between the two sampling points. Conversely, $\mathrm{HCl}$ was detected in significant quantities in tests processing high halide feed using the Method 5-type sampling method, but none was detected by the FTIR. The exhaust stream passes through three particulate filters in between the two sampling locations; $\mathrm{HCl}$ may react with or adhere to filtered particles thus removing it from the exhaust stream prior to measurement by the FTIR. Similar to previous DM100 LAW tests [7-13, 15, 47], little $\mathrm{SO}_{2}$ was detected throughout testing. Gaseous sulfur emissions detected using 40-CFR-60 Methods are probably sulfuric acid or $\mathrm{SO}_{2}$ which was oxidized prior to reaching the down-stream FTIR sampling port. The variations in emissions over the course of each test segment, which may be as high as an order of magnitude are due in part to changes in the melt pool cold cap.

A nitrogen mass balance is summarized in Table 5.3 for each test segment. In all tests but two, about half of the feed nitrogen oxides were reduced to diatomic nitrogen, in keeping with expectations for feed with a sugar ratio of 0.5 . No obvious difference in the nitrogen mass balance was observed as a function of simulant composition during these tests. The two test segments with higher relative emissions were Tests $3 \mathrm{C}$ and $4 \mathrm{~A}$. The feed for both these tests contained a simulant based on an AZ-102 waste composition with all the $\mathrm{NaNO}_{2}$ removed to accommodate the halide additions (see Table 2.10). As a result, the feed concentrations of nitrogen oxide are so small that contaminant amounts of nitrates in other simulant components and feed additives can significantly affect the mass balance.

\subsection{Mass Balance of Volatile Constituents}

Table 5.4 provides the elemental mass balance around the melter for sulfur and rhenium for all tests as well as chlorine and fluorine for tests with high feed halide concentrations. The total recovery of these volatile feed constituents was good for all tests except Test 4A. This test was short in duration and followed a long idling period which depleted volatile constituents from the glass and therefore the recoveries for this test were low. Except for one other chlorine value, mass balance closures were between 80 and 120\% and almost all of the closures for sulfur and chlorine were within 90 and 110\%. Fluorine closures were mostly between 80 and 90\%, probably due to a slight low bias in the glass analysis technique. As described in Section 4.1, chlorine, fluorine, and rhenium retention in the glass was typically 50-60\%, 65-75\%, and 20$40 \%$, respectively, with no obvious influence by the other feed constituents. Sulfur retention in the glass typically ranged from $75-95 \%$ depending on feed sodium, sulfur, and halide concentrations. Melter emissions of rhenium were exclusively particulate, whereas sulfur and halide emissions were both particulate and gaseous. The ratio of particulate to gaseous sulfur increased with increasing feed sodium content. Greater proportions of chlorine emissions were particulate, in contrast to fluorine, which had a higher proportion emitted as a gas. These proportions vary greatly between the tests with no obvious relationship to the other feed components. 


\section{SECTION 6.0 SUMMARY AND CONCLUSIONS}

A series of six tests were conducted on the DM100-WV melter using a range of LAW Sub-Envelope simulants and corresponding melter feeds that produced glasses in the alkali-limited region through the sulfate-limited LAW glass composition region with varying alkali content. In addition, in all of the seven glass compositions that were used, the impact of higher levels of chlorine and fluorine on sulfate phase formation was also investigated. Rhenium as a surrogate for technetium was added to the feed in all tests to provide data on retention in the glass product and the relative proportions of particulate and gaseous fractions in the melter exhaust. The results showed that each glass composition and transitions between glass compositions occurred without the formation of a separate sulfate phase on the melt pool surface. Conversely, separate sulfate phases were observed in most of the tests processing the same glass compositions containing high concentrations of halides. Separated sulfate phases were removed by bubbling the melt pool prior to continuing testing. Sulfate phases were identified by visual observations from multiple dip samples. Visual results were confirmed by chemical and microstructural analysis, which indicated that the material was composed of two principal phases: an alkali and calcium sulfate phase with lesser amounts of chlorine, chromium, and rhenium in addition to a phase of radiating crystals composed of alkali sulfate, chlorine, and chromium. No difficulties were encountered in maintaining nominal operating conditions (glass temperature of $1150^{\circ} \mathrm{C}$ and a near complete cold cap while processing glass at $2000 \mathrm{~kg} / \mathrm{m}^{2} /$ day) in any of the tests.

Analysis of the feed samples and product glasses confirmed the composition of the feed and showed the intended transitions between the various compositions. Product glasses were selected to permit direct comparisons with the corresponding crucible melt results at high potassium and high sodium, moderate sodium, and low sodium levels, as well as to assess the effects of the increased halide levels on glass properties. All of the measured properties of the crucible and melter glasses are within acceptable limits for processing and product quality.

During the present tests, total particulate carry-over from the melter ranged from a quarter percent to over two percent and increased with increasing sodium, potassium, and halide feed content. Melter DFs were determined for most of the elements in the feed for each unique glass composition. The most volatile species were rhenium, halogens, and sulfur. Good mass balance closure was obtained for halides, sulfur, and rhenium for most of the test segments. Sulfur retention in the glass typically ranged from 75 - 95\% and increased with increased feed sodium content and decreased with increased feed halide content. Chlorine, fluorine, and rhenium retention in the glass was typically 50 - 60\%, 65 - 75\%, and 20 - 40\%, respectively, with no obvious relationship to concentrations of the other feed constituents. Rhenium melter emissions were entirely particulate, whereas chlorine, fluorine, and sulfur melter emissions were both particulate and gaseous. Consistent with previous tests conducted at 0.5 stoichiometeric carbon, approximately half of the feed nitrogen oxides were emitted as $\mathrm{NO}$ and $\mathrm{NO}_{2}$ gases. 
Byproducts of incomplete combustion, such as carbon monoxide and ammonia increased with the amounts of carbon and nitrogen oxides in the feed.

The results from these tests demonstrate that glass formulations generated by the LAW glass formulation algorithm (including process uncertainties in the worst-case direction) which span a wide range of sodium and sulfate concentrations can be processed without forming separate sulfur phases on the melt surface. However, the results also indicate that separate sulfur phases are likely to form on the melt pool surface if the same feeds contain high halide concentrations. Chemical analysis of this separated material indicates that rhenium, which was used as a surrogate for radioactive technetium, is sequestered into the soluble secondary phase. In the limited number of previous tests with LAW feeds containing high chlorine concentrations, the formation of separated secondary phases was not observed and, therefore, high chlorine concentrations do not appear to be the only determining factor for the formation of secondary phases on the melt pool surface. Consequently, it is recommended that further testing be performed to better understand the relationship between chlorine and fluorine concentrations and the formation of secondary sulfate phases, and to define acceptable limits. It is recommended that this work should include isolating the effect of each of the two halides, defining halide concentration thresholds for secondary phase formation, and processing glasses with high concentrations of halides that in past studies with nominal halide concentrations did not result in secondary sulfate phases. The results of these tests should then be incorporated into a revision of the LAW glass formulation algorithm. 


\section{SECTION 7.0 \\ REFERENCES}

[1] “Glass Formulation and Testing with TWRS LAW Simulants,” Final Report to Duratek Inc. and BNFL Inc., I.S. Muller and I.L. Pegg, Vitreous State Laboratory, The Catholic University of America, Washington, DC, 1/16/98.

[2] "Glass Formulation and Testing With RPP-WTP LAW Simulants," I.S. Muller, A.C. Buechele, and I.L. Pegg, VSL-01R3560-2, Vitreous State Laboratory, The Catholic University of America, Washington, DC, 2/23/01.

[3] "Baseline LAW Glass Formulation Testing," I.S. Muller and I.L. Pegg, VSL-03R3460-1, Rev.0, Vitreous State Laboratory, The Catholic University of America, Washington, DC, 8/8/03.

[4] “Glass Formulations to Support Melter Testing”, I.S. Muller and I.L. Pegg, VSL03R3460-2, Rev.0, Vitreous State Laboratory, The Catholic University of America, Washington, DC, 2/6/04.

[5] "Melter Tests with LAW Envelope A and C Simulants to Support Enhanced Sulfate Incorporation,” K.S. Matlack, S.P. Morgan, and I.L. Pegg, VSL-01R3501-2, Rev. 0, Vitreous State Laboratory, The Catholic University of America, Washington, D.C., $1 / 26 / 01$.

[6] "Melter Tests with LAW Envelope B Simulants to Support Enhanced Sulfate Incorporation,” K.S. Matlack, S.P. Morgan, and I.L. Pegg, VSL-00R3501-1, Rev. 0, Vitreous State Laboratory, The Catholic University of America, Washington, D.C., $11 / 27 / 00$.

[7] "Compositional Variation Tests on DuraMelter 100 with LAW Sub-Envelope A1 Feed (LAWA44 Glass) in Support of the LAW Pilot Melter,” K.S. Matlack, W. Gong, and I.L. Pegg, VSL-02R62N0-4, Rev. 0, Vitreous State Laboratory, The Catholic University of America, Washington, D.C., 6/18/02.

[8] "DuraMelter 100 Sub-Envelope Changeover Testing Using LAW Sub-Envelope A1 and C1 Feeds in Support of the LAW Pilot Melter,” K.S. Matlack, W. Gong, and I.L. Pegg, VSL-02R62N0-6, Rev. 0, Vitreous State Laboratory, The Catholic University of America, Washington, D.C., 9/9/03.

[9] “Compositional Variation Tests on DuraMelter 100 with LAW Sub-Envelope A2 Feed (LAWA88) Glass in Support of the LAW Pilot Melter,” K. S. Matlack, W. Gong and I.L. Pegg, VSL-02R62N0-3, Rev. 0, Vitreous State Laboratory, The Catholic University of America, Washington, D.C., 11/1/02. 
[10] "DuraMelter 100 Sub-Envelope Changeover Testing Using LAW Sub-Envelope A2 and B1 Feeds in Support of the LAW Pilot Melter,” K.S. Matlack, W. Gong, and I.L. Pegg, VSL-03R3410-1, Rev. 0, Vitreous State Laboratory, The Catholic University of America, Washington, D.C., 8/22/03.

[11] "Compositional Variation Tests on DuraMelter 100 with LAW Sub-Envelope A3 Feed in Support of the LAW Pilot Melter,” K.S. Matlack, W. Gong, and I.L. Pegg, VSL01R62N0-1, Rev. 1, Vitreous State Laboratory, The Catholic University of America, Washington, D.C., 7/15/02.

[12] "DuraMelter 100 Sub-Envelope Changeover Testing Using LAW Sub-Envelope A3 and C2 Feeds in Support of the LAW Pilot Melter,” K.S. Matlack, W. Gong, and I.L. Pegg, VSL-03R3410-3, Rev. 0, Vitreous State Laboratory, The Catholic University of America, Washington, D.C., 10/17/03.

[13] "Compositional Variation Tests on DuraMelter 100 with LAW Sub-Envelope B1 Feed in Support of the LAW Pilot Melter,” K.S. Matlack, W. Gong, and I.L. Pegg, VSL02R62N0-5, Rev. 0, Vitreous State Laboratory, The Catholic University of America, Washington, D.C., 5/8/03.

[14] “Compositional Variation Tests on DuraMelter 100 with LAW Sub-Envelope B2 Feed in Support of the LAW Pilot Melter,” K.S. Matlack and I.L. Pegg, VSL-03R3410-2, Rev. 0, The Catholic University of America, Vitreous State Laboratory, Washington, D.C., 10/20/03.

[15] “Compositional Variation Tests on DuraMelter 100 with LAW Sub-Envelope C1 Feed (LAWC22 Glass) in Support of the LAW Pilot Melter,” K.S. Matlack, W. Gong, and I.L. Pegg, VSL-02R62N0-2, Rev. 1, Vitreous State Laboratory, The Catholic University of America, Washington, D.C., 9/23/02.

[16] “Compositional Variation Tests on DuraMelter 100 with LAW Sub-Envelope C2 Feed in Support of the LAW Pilot Melter,” K.S. Matlack, W. Gong, R.A. Callow and I.L. Pegg, VSL-04R4410-1, Rev. 0, Vitreous State Laboratory, The Catholic University of America, Washington, DC, 6/17/04.

[17] “RPP-WTP Pilot Melter Envelope B Throughput Test Results Report,” TRR-PLT-57, Duratek, Inc., Columbia, MD, 10/13/00.

[18] “RPP-WTP Pilot Melter Envelope A and C Throughput Test Results Report,” TRR-PLT54, Duratek, Inc., Columbia, MD, 10/13/00.

[19] “RPP-WTP Pilot Melter Sub-Envelope A1 Variation Test Results Report,” TRR-PLT071, Rev.0, Duratek, Inc., Columbia, MD, 4/28/03. 
[20] “RPP-WTP Pilot Melter Sub-Envelope C1-A1 Changeover Test Results Report,” TRRPLT-035, Rev.0, Duratek, Inc., Columbia, MD, 9/29/03.

[21] “RPP-WTP Pilot Melter Sub-Envelope A2 Variation Test Results Report,” TRR-PLT070, Rev.0, Duratek, Inc., Columbia, MD, 10/4/02.

[22] “RPP-WTP Pilot Melter Sub-Envelope A2-B1 Changeover Test Results Report,” TRRPLT-078, Rev.0, Duratek, Inc., Columbia, MD, 11/3/03.

[23] “RPP-WTP Pilot Melter Sub-Envelope A3 Variation Test Results Report,” TRR-PLT060, Rev.2, Duratek, Inc., Columbia, MD, 11/19/02.

[24] “RPP-WTP Pilot Melter Sub-Envelope C2-A3 Changeover Test Results Report,” TRRPLT-079, Rev.0, Duratek, Inc., Columbia, MD, 11/11/03.

[25] “RPP-WTP Pilot Melter Sub-Envelope B1 Variation Test Results Report,” TRR-PLT074, Rev.0, Duratek, Inc., Columbia, MD, 8/26/03.

[26] “RPP-WTP Pilot Melter Sub-Envelope B2 Variation Test Results Report,” TRR-PLT073, Rev.0, Duratek, Inc., Columbia, MD, 10/27/03.

[27] “RPP-WTP Pilot Melter Sub-Envelope C1 Variation Test Results Report,” TRR-PLT069, Rev.2, Duratek, Inc., Columbia, MD, 2/6/03.

[28] “RPP-WTP Pilot Melter Sub-Envelope C2 Variation Test Results Report,” TRR-PLT072, Rev.1, Duratek, Inc., Columbia, MD, 3/12/03.

[29] “Low Activity Waste Feed staging for Case 3S6D” in “Tank Farm Contributor Operation and Utilization Plan”, Kirkbride R.A. et al., HNF-SD-WM-SP-012, Rev. 2.

[30] Low Activity Waste Feed staging for Case 3S6E in "Tank Farm Contractor Operation and Utilization Plan,” R.A. Kirkbride et al., HNF-SD-WM-SP-012, Rev.2, 4/19/00.

[31] Data Extracted from Appendices C and D in "Tank Farm Contractor Operation and Utilization Plan,” R.A. Kirkbride et al., HNF-SD-WM-SP-012, Rev.3, email transmission from R.A. Kirkbride of Numatec Hanford Corporation to I.L. Pegg of VSL on 10/2/01.

[32] "LAW Pilot Melter and DM-100 Sub-Envelope Changeover Testing," E.V. Morrey, WTP Test Specification, 24590-LAW-TSP-RT-02-012, Rev. 0.

[33] “Proposed Approach for Development of LAW Glass Formulation Correlation”, I. S. Muller, G. Diener, I. Joseph and I. L. Pegg, VSL-03L4460-1, Rev. 2, Vitreous State Laboratory, The Catholic University of America, Washington, DC, 10/29/04.

[34] “Plot for Discussion,” e-mail from J.Vienna to I. L. Pegg and I. Joseph, 7/22/04. 
[35] "DM-100 Melter Testing to Support LAW Glass Formulation Correlation Development," K. Abel and J. Westsik, WTP Test Specification, 24590-WTP-TSP-RT-04-0004, Rev. 0, $1 / 12 / 05$.

[36] "DuraMelter 100 Tests to Support LAW Glass Formulation Correlation Development," K. S. Matlack, G. Diener, I.S. Muller, and I. L. Pegg, VSL-05T5480-1, Rev. 0, Vitreous State Laboratory, The Catholic University of America, Washington, DC, 5/9/05.

[37] “Quality Assurance Project Plan for RPP-WTP Support Activities Conducted by VSL,” Vitreous State Laboratory, QAPP Rev. 8, Vitreous State Laboratory, The Catholic University of America, Washington, DC, 6/2/05.

[38] “Master List of Controlled VSL Manuals and Standard Operating Procedures in Use," QA-MLCP, Rev. 15, Vitreous State Laboratory, The Catholic University of America, Washington, DC, 6/6/05.

[39] D. B. Blumenkranz, "Quality Assurance Project Plan for Testing Programs Generating Environmental Regulatory Data,” PL-24590-QA00001, Rev. 0, Bechtel National Inc., Richland, WA, 6/7/01.

[40] WTP Test Exception, 24590-LAW-TEF-RT-05-00002, K. Abel, River Protection Project, Waste Treatment Plant, 3000 George Washington Way, Richland, WA 99352, 3/31/05.

[41] "Physical and Rheological Properties of Waste Simulants and Melter Feeds for RPPWTP LAW Vitrification,” I.S. Muller, H. Gan, and I.L. Pegg, VSL-00R3520-1, Rev. 0, Vitreous State Laboratory, The Catholic University of America, Washington, DC, $1 / 16 / 01$.

[42] “Characterization of Simulated WTP LAW Melter Feeds,” H. Zhao, I.S. Muller, and I.L. Pegg, VSL-04R4500-1, Rev. 0, Vitreous State Laboratory, The Catholic University of America, Washington, DC, 5/26/04.

[43] “Selection of HLW and LAW Glass Formers,” SRT-RPP-2002-00034, 2/6/02.

[44] "Development of LAW and HLW Vitrification Physical Property Bounding Conditions and Simulant Verification Criteria,” A. Poloski, H. Smith, G. Smith, and B. Calloway, WTP-RPT-075, Rev. 0, 2/03.

[45] "Comparison of Off-Gas Emissions from Tests with LAW Simulants on the DM100, DM1200, and DM3300 Melters,” R.A. Callow, K.S. Matlack, and I.L. Pegg, VSL04S4850-1, Rev. 0, Vitreous State Laboratory, The Catholic University of America, Washington, DC, 4/19/04. 
[46] "Integrated DM1200 Melter Testing of Bubbler Configurations Using HLW AZ-101 Simulants," K.S. Matlack, W. Gong, T. Bardakci, N. D’Angelo, W. Lutze, R. A. Callow, M. Brandys, W.K. Kot, and I.L. Pegg, VSL-04R4800-4, Rev.0, Vitreous State Laboratory, The Catholic University of America, Washington, DC, 10/5/04.

[47] "DM100 HLW and LAW Tests of the Influence of Technetium on Cesium Volatility Using Rhenium as a Technetium Surrogate,” K.S. Matlack, W.K. Kot, and I.L. Pegg, VSL-04R4710-1, Rev. 0, Vitreous State Laboratory, The Catholic University of America, Washington, DC, 9/28/04.

[48] “DM1200 Tests with AZ-101 HLW Simulants,” K.S. Matlack, W. Gong, T. Bardakci, N.D'Angelo, W.K. Kot, and I.L. Pegg, VSL-03R3800-4, Rev. 0, Vitreous State Laboratory, The Catholic University of America, Washington, DC, 2/17/04.

[49] "Integrated DM1200 Melter Testing of HLW C-106/AY-102 Composition Using Bubblers," K.S. Matlack, W. Gong, T. Bardakci, N. D’Angelo, W. Kot and I.L. Pegg, VSL-03R3800-1, Rev. 0, Vitreous State Laboratory, The Catholic University of America, Washington, DC, 9/15/03.

[50] “DuraMelter 100 HLW Simulant Validation Tests with C-106/AY-102 Feeds,” K.S. Matlack, W. Gong and I.L. Pegg, VSL-05R5710-1, Rev. 0, Vitreous State Laboratory, The Catholic University of America, Washington, DC, 6/2/05.

[51] “LAW Container Centerline Cooling Data” RPP-WTP Memorandum, L. Petkus to C. Musick, CCN\# 074181, 10/16/03.

[52] "DuraMelter100 Tests with AP-101/AY-102 Blended Feed from the Semi-Integrated Pilot Plant," K. S. Matlack, W. Gong, and I. L. Pegg, VSL-05R5410-1, Rev. 0, Vitreous State Laboratory, The Catholic University of America, Washington, DC, 2/24/05.

[53] "Off-Gas Hydrogen Concentrations in Tests on the DuraMelter 100 Melter System Using LAW Sub-Envelope A1 and A2 Simulants," K. S. Matlack, T. S R. Schatz, and I. L. Pegg, VSL-02S4100-1, Rev. 0, Vitreous State Laboratory, The Catholic University of America, Washington, DC, 2/8/02. 
Table 2.1. Compositions of Glasses for DM100 Melter Testing (wt\%).

\begin{tabular}{|c|c|c|c|c|c|c|c|}
\hline Glass & LAWE3 & LAWE4H** & LAWE5H & LAWE6H & LAWE7H & LAWE9H & LAWE10H \\
\hline $\mathrm{Na}_{2} \mathrm{O}$ & 18.21 & 21.27 & 18.97 & 15.83 & 13.53 & 8.93 & 4.33 \\
\hline $\mathrm{SO}_{3}$ & 0.35 & 0.41 & 0.46 & 0.53 & 0.59 & 0.69 & 0.80 \\
\hline $\mathrm{K}_{2} \mathrm{O}$ & 4.98 & 0.00 & 0.54 & 0.54 & 0.54 & 0.54 & 0.54 \\
\hline $\mathrm{Al}_{2} \mathrm{O}_{3}$ & 6.10 & 5.97 & 5.99 & 6.01 & 6.02 & 6.05 & 6.07 \\
\hline $\mathrm{B}_{2} \mathrm{O}_{3}$ & 10.00 & 9.79 & 9.81 & 9.85 & 9.87 & 9.92 & 9.95 \\
\hline $\mathrm{Fe}_{2} \mathrm{O}_{3}$ & 5.50 & 5.38 & 5.40 & 5.42 & 5.43 & 5.45 & 5.48 \\
\hline $\mathrm{TiO}_{2}$ & 1.40 & 1.37 & 1.37 & 1.38 & 1.38 & 1.39 & 1.39 \\
\hline $\mathrm{ZnO}$ & 3.50 & 3.43 & 3.43 & 3.45 & 3.46 & 3.47 & 3.48 \\
\hline $\mathrm{ZrO}_{2}$ & 3.00 & 2.94 & 2.94 & 2.95 & 2.96 & 2.97 & 2.99 \\
\hline $\mathrm{CaO}$ & 2.02 & 2.46 & 3.61 & 5.46 & 6.31 & 6.86 & 6.96 \\
\hline $\mathrm{Li}_{2} \mathrm{O}$ & 0.00 & 0.00 & 0.49 & 2.38 & 3.17 & 4.08 & 4.26 \\
\hline $\mathrm{MgO}$ & 1.48 & 1.45 & 1.45 & 1.46 & 1.49 & 2.36 & 2.94 \\
\hline $\begin{array}{l}\text { Added } \\
\mathrm{Na}_{2} \mathrm{O}\end{array}$ & - & - & - & - & - & - & 1.39 \\
\hline Others* & 0.50 & 0.49 & 0.49 & 0.50 & 0.49 & 0.50 & 0.50 \\
\hline $\mathrm{SiO}_{2}$ & 42.95 & 45.04 & 45.04 & 44.25 & 44.75 & 46.79 & 48.92 \\
\hline Sum & 100.00 & 100.00 & 100.00 & 100.00 & 100.00 & 100.00 & 100.00 \\
\hline
\end{tabular}

* Composition of others is given in Table 2.3

"-" Empty data field

** Melter tests were conducted using LAWE4H glasses targeting 0.00 and $0.54 \mathrm{wt} \% \mathrm{~K}_{2} \mathrm{O}$. Sodium was held constant at $21.27 \mathrm{wt} \%$; however, increasing

$\mathrm{K}_{2} \mathrm{O}$ resulted in lower concentrations of other components. 
The Catholic University of America Vitreous State Laboratory
DuraMelter 100 Tests to Support LAW Glass Formulation Correlation Development Final Report, VSL-06R6480-1, Rev. 0

Table 2.2. Test Matrix.

\begin{tabular}{|c|c|c|c|c|}
\hline $\begin{array}{c}\text { Melter } \\
\text { Test }\end{array}$ & Objective & Feed Composition & $\begin{array}{c}\text { Duration } \\
\text { (hours) }\end{array}$ & $\begin{array}{c}\mathrm{Cl} \text { and F } \\
\text { Content (glass } \\
\text { basis) }\end{array}$ \\
\hline $1 \mathrm{~A}$ & \multirow{2}{*}{ K Effect } & LAWE3 & 50 & $0.20 \mathrm{wt} \% \mathrm{Cl}$ \\
\hline 1B & & LAWE4H with no $\mathrm{K}_{2} \mathrm{O}$ & 50 & $0.08 \mathrm{wt} \% \mathrm{~F}$ \\
\hline $2 \mathrm{~A}$ & \multirow{4}{*}{$\begin{array}{l}\text { Na-S Effects; } \\
\text { high } \mathrm{Na}\end{array}$} & LAWE4H & 25 & \multirow{4}{*}{$\begin{array}{l}0.20 \mathrm{wt} \% \mathrm{Cl} \\
0.08 \mathrm{wt} \% \mathrm{~F}\end{array}$} \\
\hline $2 \mathrm{~B}$ & & LAWE5H & 50 & \\
\hline $2 \mathrm{C}$ & & LAW6H & 50 & \\
\hline $2 \mathrm{D}$ & & LAWE7H & 50 & \\
\hline $3 \mathrm{~A}$ & \multirow{3}{*}{$\begin{array}{l}\text { Na-S Effects; } \\
\text { low Na }\end{array}$} & LAWE7H & 25 & \multirow{3}{*}{$\begin{array}{l}0.20 \mathrm{wt} \% \mathrm{Cl} \\
0.08 \mathrm{wt} \% \mathrm{~F}\end{array}$} \\
\hline $3 \mathrm{~B}$ & & LAWE9H & 50 & \\
\hline $3 \mathrm{C}$ & & LAWE10H & 50 & \\
\hline $4 \mathrm{~A}$ & \multirow{3}{*}{$\begin{array}{l}\text { Na-S Effects; } \\
\text { low Na at high } \\
\text { Cl and F }\end{array}$} & LAWE10H & 25 & \multirow{3}{*}{$\begin{array}{l}1.1 \mathrm{wt} \% \mathrm{Cl} \\
1.3 \mathrm{wt} \% \mathrm{~F}\end{array}$} \\
\hline $4 \mathrm{~B}$ & & LAWE9H & 50 & \\
\hline $4 \mathrm{C}$ & & LAWE7H & 50 & \\
\hline $5 \mathrm{~A}$ & \multirow{4}{*}{$\begin{array}{l}\text { Na-S Effects; } \\
\text { high Na at high } \\
\text { Cl and F }\end{array}$} & LAWE7H & 25 & \multirow{4}{*}{$\begin{array}{l}1.1 \mathrm{wt} \% \mathrm{Cl} \\
1.3 \mathrm{wt} \% \mathrm{~F}\end{array}$} \\
\hline $5 B$ & & LAW6H & 50 & \\
\hline $5 \mathrm{C}$ & & LAWE5H & 50 & \\
\hline $5 \mathrm{D}$ & & LAWE4H & 50 & \\
\hline $6 \mathrm{~A}$ & \multirow{2}{*}{$\begin{array}{l}\text { K Effect at high } \\
\mathrm{Cl} \text { and } \mathrm{F}\end{array}$} & LAWE4H with no $\mathrm{K}_{2} \mathrm{O}$ & 50 & \multirow{2}{*}{$\begin{array}{l}1.1 \mathrm{wt} \% \mathrm{Cl} \\
1.3 \mathrm{wt} \% \mathrm{~F}\end{array}$} \\
\hline $6 \mathrm{~B}$ & & LAWE3 & 50 & \\
\hline
\end{tabular}


The Catholic University of America Vitreous State Laboratory
DuraMelter 100 Tests to Support LAW Glass Formulation Correlation Development Final Report, VSL-06R6480-1, Rev. 0

Table 2.3. Composition of the Grouped Component “Others” for LAWEH Glasses.

\begin{tabular}{|c|c|c|}
\hline Components & Relative Concentrations (wt\%) & Concentration in LAWE Glasses (wt \%) \\
\hline $\mathrm{Cl}$ & 40.01 & 0.200 \\
\hline $\mathrm{Cr}_{2} \mathrm{O}_{3}$ & 16.07 & 0.080 \\
\hline $\mathrm{F}$ & 14.97 & 0.080 \\
\hline $\mathrm{NiO}$ & 2.00 & 0.008 \\
\hline $\mathrm{PbO}$ & 2.00 & 0.008 \\
\hline $\mathrm{P}_{2} \mathrm{O}_{5}$ & 24.95 & 0.124 \\
\hline \hline $\mathrm{Total}$ & 100.00 & 0.500 \\
\hline
\end{tabular}


Table 2.4. LAWE3 Simulant Recipe at Nominal 8 Molar Sodium (Actual 8.46 Molar Sodium) Based on AP-101 Waste Composition [32].

\begin{tabular}{|c|c|c|c|c|c|c|c|c|c|c|}
\hline $\begin{array}{c}\text { Envelope } \\
\text { Constituents }\end{array}$ & $\begin{array}{r}\text { Simula } \\
\text { Mod } \\
\text { Maxin } \\
\text { Molar R }\end{array}$ & $\begin{array}{l}\mathrm{P}-101 \\
\mathrm{H} \text { for } \\
\mathrm{K} / \mathrm{Na} \\
\text { of } 0.18\end{array}$ & $\begin{array}{l}\text { Glass } \\
\text { Oxides }\end{array}$ & $\begin{array}{c}\text { LAW E3 } \\
\text { Simulant as } \\
\text { Oxides (wt\%) }\end{array}$ & $\begin{array}{c}\text { Waste } \\
\text { Contribution to } \\
\text { Glass }\end{array}$ & Source in Simulant & $\begin{array}{l}\text { Order for } \\
\text { Addition }\end{array}$ & $\begin{array}{c}\text { Formula } \\
\text { Weight }\end{array}$ & Assay* & $\begin{array}{c}\text { Target } \\
\text { Weight } \\
\text { (g) }\end{array}$ \\
\hline- & $\mathrm{mg} / \mathrm{L}$ & Molarity & Loading & $100 \%$ & $25.41 \%$ & In $439 \mathrm{ml}^{2}$ water $^{\$}$ add foll & compound & e order lis & & \\
\hline $\mathrm{Al}$ & 9625 & 0.357 & $\mathrm{Al}_{2} \mathrm{O}_{3}$ & 5.26 & 1.34 & $\mathrm{Al}\left(\mathrm{NO}_{3}\right)_{3} \cdot 9 \mathrm{H}_{2} \mathrm{O}, 60 \%$ sol. & 1 & 375.14 & 0.61 & 220.51 \\
\hline $\mathrm{B}$ & 12 & 0.001 & $\mathrm{~B}_{2} \mathrm{O}_{3}$ & 0.01 & 0.00 & $\mathrm{H}_{3} \mathrm{BO}_{3}$ & 2 & 61.83 & 0.99 & 0.07 \\
\hline $\mathrm{Cr}$ & 749 & 0.014 & $\mathrm{Cr}_{2} \mathrm{O}_{3}$ & 0.32 & 0.08 & $\mathrm{Na}_{2} \mathrm{CrO}_{4} \cdot 4 \mathrm{H}_{2} \mathrm{O}$ & 8 & 234.04 & 0.99 & 3.42 \\
\hline $\mathrm{K}$ & 56301 & 1.440 & $\mathrm{~K}_{2} \mathrm{O}$ & 19.61 & 4.98 & $\mathrm{KOH}$ & 7 & 56.10 & 0.91 & 88.97 \\
\hline $\mathrm{Na}$ & 183920 & 8.000 & $\mathrm{Na}_{2} \mathrm{O}$ & 71.67 & 18.21 & $\mathrm{NaOH}, 50 \%$ sol. $\mathrm{d}=1.53$ & 6 & 40.00 & 0.50 & 226.00 \\
\hline $\mathrm{Ni}$ & 82 & 0.001 & $\mathrm{NiO}$ & 0.03 & 0.01 & $\mathrm{NiO}$ & 3 & 74.69 & 1.00 & 0.10 \\
\hline $\mathrm{Pb}$ & 95 & 0.0005 & $\mathrm{PbO}$ & 0.03 & 0.01 & $\mathrm{PbO}$ & 4 & 223.20 & 1.00 & 0.10 \\
\hline $\mathrm{Si}$ & 157 & 0.006 & $\mathrm{SiO}_{2}$ & 0.10 & 0.02 & $\mathrm{SiO}_{2}$ & 5 & 60.09 & 0.99 & 0.34 \\
\hline $\mathrm{Cl}$ & 2730 & 0.077 & $\mathrm{Cl}$ & 0.79 & 0.20 & $\mathrm{NaCl}$ & 10 & 58.45 & 0.99 & 4.55 \\
\hline $\mathrm{F}$ & 1083 & 0.057 & $\mathrm{~F}$ & 0.31 & 0.08 & $\mathrm{NaF}$ & 11 & 42.00 & 0.99 & 2.42 \\
\hline $\mathrm{PO}_{4}$ & 2251 & 0.024 & $\mathrm{P}_{2} \mathrm{O}_{5}$ & 0.49 & 0.12 & $\mathrm{Na}_{3} \mathrm{PO}_{4} \cdot 12 \mathrm{H}_{2} \mathrm{O}$ & 9 & 380.12 & 0.99 & 9.10 \\
\hline $\mathrm{SO}_{4}$ & 5764 & 0.060 & $\mathrm{SO}_{3}$ & 1.39 & 0.35 & $\mathrm{Na}_{2} \mathrm{SO}_{4}$ & 12 & 142.06 & 0.99 & 8.61 \\
\hline $\mathrm{NO}_{2}$ & 58110 & 1.263 & - & - & - & $\mathrm{NaNO}_{2}$ & 16 & 69.00 & 1.00 & 87.60 \\
\hline $\mathrm{NO}_{3}$ & 183067 & 2.953 & - & - & - & $\mathrm{NaNO}_{3}$ & 17 & 84.99 & 0.99 & 161.61 \\
\hline $\mathrm{CO}_{3}$ & 44775 & 0.746 & - & - & - & $\mathrm{Na}_{2} \mathrm{CO}_{3}$ & 18 & 105.99 & 1.00 & 79.08 \\
\hline Org.Carbon & 2718 & 0.227 & - & - & - & - & - & - & - & - \\
\hline Acetate & 3025 & 0.051 & - & - & - & Sodium Acetate (C2) & 13 & 136.08 & 0.99 & 7.04 \\
\hline Formate & 2213 & 0.049 & - & - & - & Sodium Formate (C1) & 14 & 68.01 & 0.99 & 3.38 \\
\hline Oxalate & 3321 & 0.038 & - & - & - & Sodium Oxalate (C2) & 15 & 134.00 & 0.99 & 5.11 \\
\hline- & - & - & - & - & - & \multicolumn{4}{|l|}{ Target Glass Weight } & 1361.45 \\
\hline- & - & - & SUM & 100.00 & 25.41 & \multicolumn{4}{|l|}{ Total simulant Weight } & 1346.99 \\
\hline
\end{tabular}

"-" Empty data field.

* Assay refers to the purity of the raw material as specified by the vendor.

\$-An actual 8 molar sodium simulant was produced at the crucible scale with $494.33 \mathrm{ml}$ water yielding a measured simulant total weight of $1402.32 \mathrm{~g}$. 
Table 2.5. LAWE4H Waste Simulant Recipe at Nominal 8 Molar Sodium (Actual 7.97 Molar Sodium) without Potassium Based on AN-105 Waste Composition [32].

\begin{tabular}{|c|c|c|c|c|c|c|c|c|c|c|}
\hline \multirow{2}{*}{\begin{tabular}{|c|}
$\begin{array}{c}\text { Envelope } \\
\text { Constituents }\end{array}$ \\
- \\
\end{tabular}} & \multicolumn{2}{|c|}{$\begin{array}{l}\text { Simulant AN-105 } \\
\text { Modified for E4H }\end{array}$} & \multirow{2}{*}{$\begin{array}{c}\begin{array}{c}\text { Glass } \\
\text { Oxides }\end{array} \\
\text { Loading } \\
\end{array}$} & \multirow[t]{2}{*}{$\begin{array}{c}\text { LAW E4H } \\
\text { Simulant as } \\
\text { Oxides (wt\%) }\end{array}$} & \multirow{2}{*}{\begin{tabular}{|c|}
$\begin{array}{c}\text { Waste } \\
\text { Contribution to } \\
\text { Glass }\end{array}$ \\
$27.18 \%$ \\
\end{tabular}} & $\begin{array}{l}\text { Source in } \\
\text { Simulant }\end{array}$ & \multirow{2}{*}{$\begin{array}{l}\text { Order for } \\
\text { Addition }\end{array}$} & \multirow{2}{*}{$\begin{array}{c}\text { Formula } \\
\text { Weight }\end{array}$} & \multirow[t]{2}{*}{ Assay* } & \multirow[t]{2}{*}{$\begin{array}{c}\text { Target } \\
\text { Weight (g) }\end{array}$} \\
\hline & $\mathrm{mg} / \mathrm{L}$ & $\mathrm{M}$ & & & & In $274.40 \mathrm{ml}$ water ${ }^{\$}$ add following compounds in the order listed below & & & & \\
\hline \multirow{2}{*}{$\mathrm{Al}$} & \multirow{2}{*}{30554} & \multirow{2}{*}{1.132} & \multirow{2}{*}{$\mathrm{Al}_{2} \mathrm{O}_{3}$} & \multirow{2}{*}{18.23} & \multirow{2}{*}{4.95} & $\mathrm{Al}\left(\mathrm{NO}_{3}\right)_{3} \cdot 9 \mathrm{H}_{2} \mathrm{O}, 60 \%$ sol. & 1 & 375.14 & 0.61 & 422.01 \\
\hline & & & & & & $\mathrm{Al}(\mathrm{OH})_{3}$ & 8 & 78.00 & 1.00 & 35.22 \\
\hline $\mathrm{B}$ & 79 & 0.007 & $\mathrm{~B}_{2} \mathrm{O}_{3}$ & 0.08 & 0.02 & $\mathrm{H}_{3} \mathrm{BO}_{3}$ & 2 & 61.83 & 0.99 & 0.45 \\
\hline $\mathrm{Cr}$ & 624 & 0.012 & $\mathrm{Cr}_{2} \mathrm{O}_{3}$ & 0.29 & 0.08 & $\mathrm{Na}_{2} \mathrm{CrO}_{4} \cdot 4 \mathrm{H}_{2} \mathrm{O}$ & 9 & 234.04 & 0.99 & 2.85 \\
\hline $\mathrm{K}$ & - & - & $\mathrm{K}_{2} \mathrm{O}$ & 0.00 & $0.00 * *$ & $\mathrm{KOH}$ & 7 & 56.10 & 0.91 & 0.00 \\
\hline $\mathrm{Na}$ & 183920 & 8.000 & $\mathrm{Na}_{2} \mathrm{O}$ & 78.26 & 21.27 & $\mathrm{NaOH}, 50 \%$ sol. $\mathrm{d}=1.53$ & 6 & 40.00 & 0.50 & 458.04 \\
\hline $\mathrm{Ni}$ & 70 & 0.001 & $\mathrm{NiO}$ & 0.03 & 0.01 & $\mathrm{NiO}$ & 3 & 74.69 & 1.00 & 0.09 \\
\hline $\mathrm{Pb}$ & 83 & 0.0004 & $\mathrm{PbO}$ & 0.03 & 0.01 & $\mathrm{PbO}$ & 4 & 223.20 & 1.00 & 0.09 \\
\hline $\mathrm{Si}$ & 157 & 0.006 & $\mathrm{SiO}_{2}$ & 0.11 & 0.03 & $\mathrm{SiO}_{2}$ & 5 & 60.09 & 0.99 & 0.34 \\
\hline $\mathrm{Cl}$ & 2304 & 0.065 & $\mathrm{Cl}$ & 0.73 & 0.20 & $\mathrm{NaCl}$ & 11 & 58.45 & 0.99 & 3.84 \\
\hline $\mathrm{F}$ & 912 & 0.048 & $\mathrm{~F}$ & 0.29 & 0.08 & $\mathrm{NaF}$ & 12 & 42.00 & 0.99 & 2.04 \\
\hline $\mathrm{PO}_{4}$ & 1899 & 0.020 & $\mathrm{P}_{2} \mathrm{O}_{5}$ & 0.45 & 0.12 & $\mathrm{Na}_{3} \mathrm{PO}_{4} \cdot 12 \mathrm{H}_{2} \mathrm{O}$ & 10 & 380.12 & 0.99 & 7.68 \\
\hline $\mathrm{SO}_{4}$ & 5764 & 0.060 & $\mathrm{SO}_{3}$ & 1.52 & 0.41 & $\mathrm{Na}_{2} \mathrm{SO}_{4}$ & 13 & 142.06 & 0.99 & 8.61 \\
\hline $\mathrm{NO}_{2}$ & 85428 & 1.857 & - & - & - & $\mathrm{NaNO}_{2}$ & 17 & 69.00 & 0.97 & 128.79 \\
\hline $\mathrm{NO}_{3}$ & 126988 & 2.048 & - & - & - & $\mathrm{NaNO}_{3}$ & - & 84.99 & 0.99 & 0.00 \\
\hline TOC & 2093 & 0.174 & - & - & - & - & - & - & - & - \\
\hline Acetate & 2251 & 0.038 & - & - & - & Sodium Acetate (C2) & 14 & 136.08 & 0.99 & 5.24 \\
\hline Formate & 2135 & 0.047 & - & - & - & Sodium Formate (C1) & 15 & 68.01 & 0.99 & 3.26 \\
\hline Glycolate & 1936 & 0.025 & - & - & - & Glycolic Acid (C2) & 16 & 76.05 & 0.71 & 2.73 \\
\hline- & - & - & - & - & - & \multicolumn{4}{|l|}{ Target Glass } & 1165.59 \\
\hline- & - & - & SUM & 100.00 & 27.18 & \multicolumn{4}{|l|}{ Total simulant wt. } & 1355.67 \\
\hline
\end{tabular}

"-" Empty data field.

* Assay refers to the purity of the raw material as specified by the vendor.

** Melter tests were conducted using LAWE4H glasses containing 0.00 and $0.54 \mathrm{wt} \% \mathrm{~K}_{2} \mathrm{O}$ (see Table 2.2)

$\${ }^{-}$An actual 8 molar sodium simulant was produced at the crucible scale with $270.42 \mathrm{ml}$ water and $8.25 \mathrm{~g}$ KOH yielding a measured simulant total weight of 1359.94 . 
Table 2.6. LAWE5H Simulant Recipe at Nominal 7 Molar Sodium (Actual 7.13 Molar Sodium) Based on AN-107 Waste Composition [32].

\begin{tabular}{|c|c|c|c|c|c|c|c|c|c|c|}
\hline \begin{tabular}{|c|} 
Envelope \\
Constituents
\end{tabular} & \multicolumn{2}{|c|}{$\begin{array}{l}\text { Simulant AN-107 } \\
\text { Modified for E5H }\end{array}$} & \multirow{2}{*}{\begin{tabular}{|c|}
$\begin{array}{c}\text { Glass } \\
\text { Oxides }\end{array}$ \\
Loading \\
\end{tabular}} & \multirow[t]{2}{*}{\begin{tabular}{|c|} 
LAW E5H \\
Simulant as \\
Oxides (wt\%) \\
\end{tabular}} & \multirow{2}{*}{\begin{tabular}{|c|}
$\begin{array}{c}\text { Waste } \\
\text { Contribution } \\
\text { To Glass }\end{array}$ \\
$20.64 \%$ \\
\end{tabular}} & $\begin{array}{l}\text { Source in } \\
\text { Simulant }\end{array}$ & \multirow{2}{*}{$\begin{array}{l}\text { Order for } \\
\text { Addition }\end{array}$} & \multirow{2}{*}{$\begin{array}{c}\text { Formula } \\
\text { Weight }\end{array}$} & \multirow[t]{2}{*}{ Assay* } & \multirow[t]{2}{*}{$\begin{array}{c}\text { Target } \\
\text { Weight (g) }\end{array}$} \\
\hline- & $\mathrm{mg} / \mathrm{L}$ & M & & & & In $645 \mathrm{ml}$ water ${ }^{\$}$ add following compounds in the order listed below & & & & \\
\hline $\mathrm{Al}$ & 140 & 0.005 & $\mathrm{Al}_{2} \mathrm{O}_{3}$ & 0.11 & 0.02 & $\mathrm{Al}\left(\mathrm{NO}_{3}\right)_{3} \cdot 9 \mathrm{H}_{2} \mathrm{O}-60 \%$ sol. & 1 & 375.14 & 0.61 & 3.21 \\
\hline Ca & 309 & 0.008 & $\mathrm{CaO}$ & 0.18 & 0.04 & $\mathrm{Ca}\left(\mathrm{NO}_{3}\right)_{2} \cdot 4 \mathrm{H}_{2} \mathrm{O}$ & 2 & 236.16 & 0.99 & 1.84 \\
\hline $\mathrm{Cr}$ & 613 & 0.012 & $\mathrm{Cr}_{2} \mathrm{O}_{3}$ & 0.38 & 0.08 & $\mathrm{Na}_{2} \mathrm{CrO}_{4} \cdot 4 \mathrm{H}_{2} \mathrm{O}$ & 7 & 234.04 & 0.99 & 2.80 \\
\hline $\mathrm{Fe}$ & 936 & 0.017 & $\mathrm{Fe}_{2} \mathrm{O}_{3}$ & 0.57 & 0.12 & $\mathrm{Fe}\left(\mathrm{NO}_{3}\right)_{3} \cdot 9 \mathrm{H}_{2} \mathrm{O}$ & 4 & 404.01 & 1.00 & 6.78 \\
\hline $\mathrm{K}$ & 5122 & 0.131 & $\mathrm{~K}_{2} \mathrm{O}$ & 2.62 & 0.54 & $\mathrm{KOH}$ & 6 & 56.10 & 0.91 & 8.09 \\
\hline $\mathrm{Na}$ & 160930 & 7.000 & $\mathrm{Na}_{2} \mathrm{O}$ & 91.92 & 18.97 & $\mathrm{NaOH}-50 \%$ sol. $d=1.53$ & 5 & 40.00 & 0.50 & 297.25 \\
\hline $\mathrm{Ni}$ & 68 & 0.001 & $\mathrm{NiO}$ & 0.04 & 0.01 & $\mathrm{NiO}$ & 8 & 74.69 & 1.00 & 0.09 \\
\hline $\mathrm{Pb}$ & 82 & 0.0004 & $\mathrm{PbO}$ & 0.04 & 0.01 & $\mathrm{PbO}$ & 9 & 223.20 & 1.00 & 0.09 \\
\hline $\mathrm{Cl}$ & 2246 & 0.063 & $\mathrm{Cl}$ & 0.95 & 0.20 & $\mathrm{NaCl}$ & 11 & 58.45 & 0.99 & 3.74 \\
\hline $\mathrm{F}$ & 887 & 0.047 & $\mathrm{~F}$ & 0.38 & 0.08 & $\mathrm{NaF}$ & 12 & 42.00 & 0.99 & 1.98 \\
\hline $\mathrm{PO}_{4}$ & 1861 & 0.020 & $\mathrm{P}_{2} \mathrm{O}_{5}$ & 0.59 & 0.12 & $\mathrm{Na}_{3} \mathrm{PO}_{4} \cdot 12 \mathrm{H}_{2} \mathrm{O}$ & 10 & 380.12 & 0.99 & 7.53 \\
\hline $\mathrm{SO}_{4}$ & 6310 & 0.066 & $\mathrm{SO}_{3}$ & 2.23 & 0.46 & $\mathrm{Na}_{2} \mathrm{SO}_{4}$ & 13 & 142.06 & 0.99 & 9.43 \\
\hline $\mathrm{NO}_{2}$ & 36013 & 0.783 & - & - & & $\mathrm{NaNO}_{2}$ & 23 & 69.00 & 1.00 & 54.29 \\
\hline $\mathrm{NO}_{3}$ & 116010 & 1.871 & - & - & - & $\mathrm{NaNO}_{3}$ & 24 & 84.99 & 0.99 & 153.65 \\
\hline Org.Carbon & 21597 & 1.800 & - & - & - & - & - & - & - & - \\
\hline EDTA & 5123 & 0.018 & - & - & - & $\mathrm{Na}_{2}$ EDTA. $2 \mathrm{H}_{2} \mathrm{O}(\mathrm{C} 10)$ & 14 & 372.24 & 0.99 & 6.64 \\
\hline HEDTA & 5123 & 0.019 & - & - & - & $\mathrm{Na}_{3} \mathrm{HEDTA}$ (C10) - 41\%sol. & 15 & 344.20 & 0.42 & 15.44 \\
\hline Acetate & 5123 & 0.087 & - & - & - & Sodium Acetate (C2) & 16 & 136.08 & 0.99 & 11.92 \\
\hline Formate & 5123 & 0.114 & - & - & - & Sodium Formate (C1) & 17 & 68.01 & 0.99 & 7.82 \\
\hline Oxalate & 2846 & 0.032 & - & - & - & Sodium Oxalate (C2) & 18 & 134.00 & 0.99 & 4.38 \\
\hline Gluconate & 4269 & 0.022 & - & - & - & Sodium Gluconate (C6) & 3 & 218.14 & 0.99 & 4.82 \\
\hline Glycolic & 11385 & 0.150 & - & - & - & Glycolic Acid (C2) & 19 & 76.05 & 0.71 & 16.06 \\
\hline NTA & 4554 & 0.024 & - & - & - & Nitrilotriacetic Acid (C6) & 20 & 191.14 & 0.98 & 4.65 \\
\hline Citric & 12524 & 0.065 & - & - & - & Citric Acid (C6) & 21 & 192.12 & 0.99 & 12.65 \\
\hline Iminodiacetic & 4013 & 0.030 & - & - & - & Iminodiacetic Acid (C4) & 22 & 133.10 & 0.98 & 4.10 \\
\hline- & - & - & - & - & - & \multicolumn{4}{|c|}{ Target Glass } & 1143.54 \\
\hline- & - & - & SUM & 100.00 & 20.64 & \multicolumn{4}{|c|}{ Total simulant weight } & 1284.23 \\
\hline
\end{tabular}

"-" Empty data field.

* Assay refers to the purity of the raw material as specified by the vendor.

$\${ }^{-}$An actual 7 molar sodium simulant was produced at the crucible scale with $664.01 \mathrm{ml}$ water yielding a measured simulant total weight of $1303.24 \mathrm{~g}$. 
Table 2.7. LAWE6H Simulant Recipe at Nominal 7 Molar Sodium (Actual 6.86 Molar Sodium) Based on AN-104 Waste Composition [32].

\begin{tabular}{|c|c|c|c|c|c|c|c|c|c|c|}
\hline $\begin{array}{c}\text { Envelope } \\
\text { Constituents }\end{array}$ & \multicolumn{2}{|c|}{$\begin{array}{l}\text { Simulant AN-104 } \\
\text { Modified for E6H }\end{array}$} & \multirow{2}{*}{$\begin{array}{c}\text { Glass } \\
\text { Oxides } \\
\text { Loading } \\
\end{array}$} & \multirow{2}{*}{$\begin{array}{c}\text { LAW E6H } \\
\text { Simulant as } \\
\text { Oxides (wt\%) } \\
-\end{array}$} & \multirow{2}{*}{$\begin{array}{c}\begin{array}{c}\text { Waste } \\
\text { Contribution } \\
\text { To Glass }\end{array} \\
20.88 \% \\
\end{array}$} & \multirow[t]{2}{*}{ Source in Simulant } & \multirow[t]{2}{*}{$\begin{array}{l}\text { Order for } \\
\text { Addition }\end{array}$} & \multirow[t]{2}{*}{$\begin{array}{c}\text { Formula } \\
\text { Weight }\end{array}$} & \multirow[t]{2}{*}{ Assay* } & \multirow[t]{2}{*}{$\begin{array}{c}\text { Target } \\
\text { Weight (g) }\end{array}$} \\
\hline- & $\mathrm{mg} / \mathrm{L}$ & Molarity & & & & & & & & \\
\hline $\mathrm{Al}$ & 25069 & 0.929 & $\mathrm{Al}_{2} \mathrm{O}_{3}$ & 16.55 & 3.46 & $\mathrm{Al}\left(\mathrm{NO}_{3}\right)_{3} \cdot 9 \mathrm{H}_{2} \mathrm{O}, 60 \%$ sol. & 1 & 375.14 & 0.61 & 412.76 \\
\hline- & - & - & - & - & - & $\mathrm{Al}(\mathrm{OH})_{3}$ & 4 & 78.00 & 1.00 & 20.47 \\
\hline $\mathrm{Cr}$ & 734 & 0.014 & $\mathrm{Cr}_{2} \mathrm{O}_{3}$ & 0.37 & 0.08 & $\mathrm{Na}_{2} \mathrm{CrO}_{4} \cdot 4 \mathrm{H}_{2} \mathrm{O}$ & 5 & 234.04 & 0.99 & 3.35 \\
\hline $\mathrm{K}$ & 6140 & 0.157 & $\mathrm{~K}_{2} \mathrm{O}$ & 2.58 & 0.54 & $\mathrm{KOH}$ & 3 & 56.10 & 0.91 & 9.70 \\
\hline $\mathrm{Na}$ & 160930 & 7.000 & $\mathrm{Na}_{2} \mathrm{O}$ & 75.81 & 15.83 & $\mathrm{NaOH}, 50 \%$ sol. $\mathrm{d}=1.53$ & 2 & 40.00 & 0.50 & 294.59 \\
\hline $\mathrm{Ni}$ & 84 & 0.001 & $\mathrm{NiO}$ & 0.04 & 0.01 & $\mathrm{NiO}$ & 6 & 74.69 & 1.00 & 0.11 \\
\hline $\mathrm{Pb}$ & 99 & 0.001 & $\mathrm{PbO}$ & 0.04 & 0.01 & $\mathrm{PbO}$ & 7 & 223.20 & 1.00 & 0.11 \\
\hline $\mathrm{Si}$ & 228 & 0.008 & $\mathrm{SiO}_{2}$ & 0.17 & 0.04 & $\mathrm{SiO}_{2}$ & 8 & 60.09 & 0.99 & 0.49 \\
\hline $\mathrm{Cl}$ & 2688 & 0.076 & $\mathrm{Cl}$ & 0.94 & 0.20 & $\mathrm{NaCl}$ & 10 & 58.45 & 0.99 & 4.48 \\
\hline $\mathrm{F}$ & 1064 & 0.056 & $\mathrm{~F}$ & 0.37 & 0.08 & $\mathrm{NaF}$ & 11 & 42.00 & 0.99 & 2.38 \\
\hline $\mathrm{PO}_{4}$ & 2238 & 0.024 & $\mathrm{P}_{2} \mathrm{O}_{5}$ & 0.58 & 0.12 & $\mathrm{Na}_{3} \mathrm{PO}_{4} \cdot 12 \mathrm{H}_{2} \mathrm{O}$ & 9 & 380.12 & 0.99 & 9.05 \\
\hline $\mathrm{SO}_{4}$ & 8708 & 0.091 & $\mathrm{SO}_{3}$ & 2.54 & 0.53 & $\mathrm{Na}_{2} \mathrm{SO}_{4}$ & 12 & 142.06 & 0.99 & 13.01 \\
\hline $\mathrm{NO}_{2}$ & 78634 & 1.709 & $\mathrm{NO}_{2}$ & - & - & $\mathrm{NaNO}_{2}$ & 15 & 69.00 & 1.00 & 118.54 \\
\hline $\mathrm{NO}_{3}$ & 124203 & 2.003 & $\mathrm{NO}_{3}$ & - & - & $\mathrm{NaNO}_{3}$ & & 84.99 & 0.99 & 0.00 \\
\hline $\mathrm{CO}_{3}$ & 31659 & 0.528 & $\mathrm{CO}_{3}$ & - & - & $\mathrm{Na}_{2} \mathrm{CO}_{3}$ & 16 & 105.99 & 1.00 & 55.92 \\
\hline Org.Carbon & 2044 & 0.170 & - & - & - & - & - & - & - & - \\
\hline Acetate & 2284 & 0.039 & - & - & - & Sodium Acetate (C2) & 13 & 136.08 & 0.99 & 5.31 \\
\hline Formate & 4176 & 0.093 & - & - & - & Sodium Formate (C1) & 14 & 68.01 & 0.99 & 6.37 \\
\hline- & - & - & - & - & - & \multicolumn{4}{|c|}{ Target Glass Weight } & 1370.37 \\
\hline- & - & - & SUM & 100.00 & 20.88 & \multicolumn{4}{|c|}{ Estimated $^{\$}$ Total Simulant Weight } & 1356.64 \\
\hline
\end{tabular}

"-" Empty data field.

* Assay refers to the purity of the raw material as specified by the vendor.

\$ An actual 7 molar sodium simulant was produced at the crucible scale with $380.21 \mathrm{ml}$ water yielding a measured simulant total weight of 1336.85 g. 
Table 2.8. LAWE7H Simulant Recipe at Nominal 5 Molar Sodium (Actual 5.02 Molar Sodium) Based on AN-102 Waste Composition [32].

\begin{tabular}{|c|c|c|c|c|c|c|c|c|c|c|}
\hline $\begin{array}{c}\text { Envelope } \\
\text { Constituents }\end{array}$ & \multicolumn{2}{|c|}{$\begin{array}{l}\text { Simulant AN-102 } \\
\text { Modified for E7H }\end{array}$} & $\begin{array}{l}\text { Glass } \\
\text { Oxides }\end{array}$ & $\begin{array}{c}\text { LAW E7H } \\
\text { Simulant as } \\
\text { Oxides (wt\%) }\end{array}$ & $\begin{array}{c}\text { Waste } \\
\text { Contribution to } \\
\text { Glass }\end{array}$ & Source in Simulant & $\begin{array}{l}\text { Order for } \\
\text { Addition }\end{array}$ & $\begin{array}{l}\text { Formula } \\
\text { Weight }\end{array}$ & Assay* & $\begin{array}{c}\text { Target } \\
\text { Weight (g) }\end{array}$ \\
\hline- & $\mathrm{mg} / \mathrm{L}$ & Molarity & Loading & - & $16.22 \%$ & In $686 \mathrm{ml}$ water ${ }^{\$}$ add follo & mpounds in & order list & below & \\
\hline $\mathrm{Al}$ & 6201 & 0.230 & $\mathrm{Al}_{2} \mathrm{O}_{3}$ & 6.31 & 1.02 & $\mathrm{Al}\left(\mathrm{NO}_{3}\right)_{3} \cdot 9 \mathrm{H}_{2} \mathrm{O}, 60 \%$ sol. & 1 & 375.14 & 0.61 & 142.07 \\
\hline $\mathrm{B}$ & 18 & 0.002 & $\mathrm{~B}_{2} \mathrm{O}_{3}$ & 0.03 & 0.01 & $\mathrm{H}_{3} \mathrm{BO}_{3}$ & 3 & 61.83 & 0.99 & 0.11 \\
\hline $\mathrm{Ca}$ & 248 & 0.006 & $\mathrm{CaO}$ & 0.19 & 0.03 & $\mathrm{Ca}\left(\mathrm{NO}_{3}\right)_{2} \cdot 4 \mathrm{H}_{2} \mathrm{O}$ & 2 & 236.16 & 0.99 & 1.47 \\
\hline $\mathrm{Cr}$ & 608 & 0.012 & $\mathrm{Cr}_{2} \mathrm{O}_{3}$ & 0.48 & 0.08 & $\mathrm{Na}_{2} \mathrm{CrO}_{4} \cdot 4 \mathrm{H}_{2} \mathrm{O}$ & 6 & 234.04 & 0.99 & 2.78 \\
\hline $\mathrm{K}$ & 5137 & 0.131 & $\mathrm{~K}_{2} \mathrm{O}$ & 3.33 & 0.54 & $\mathrm{KOH}$ & 5 & 56.10 & 0.91 & 8.12 \\
\hline $\mathrm{Na}$ & 114950 & 5.000 & $\mathrm{Na}_{2} \mathrm{O}$ & 83.41 & 13.53 & $\mathrm{NaOH}, 50 \%$ sol. $\mathrm{D}=1.53$ & 4 & 40.00 & 0.50 & 102.73 \\
\hline $\mathrm{Ni}$ & 70 & 0.001 & $\mathrm{NiO}$ & 0.05 & 0.01 & $\mathrm{NiO}$ & 7 & 74.69 & 1.00 & 0.09 \\
\hline $\mathrm{Pb}$ & 83 & 0.0004 & $\mathrm{PbO}$ & 0.05 & 0.01 & $\mathrm{PbO}$ & 8 & 223.20 & 1.00 & 0.09 \\
\hline $\mathrm{Si}$ & 46 & 0.002 & $\mathrm{SiO}_{2}$ & 0.05 & 0.01 & $\mathrm{SiO}_{2}$ & 9 & 60.09 & 0.99 & 0.10 \\
\hline $\mathrm{Cl}$ & 2248 & 0.063 & $\mathrm{Cl}$ & 1.21 & 0.20 & $\mathrm{NaCl}$ & 11 & 58.45 & 0.99 & 3.74 \\
\hline $\mathrm{F}$ & 893 & 0.047 & $\mathrm{~F}$ & 0.48 & 0.08 & $\mathrm{NaF}$ & 12 & 42.00 & 0.99 & 1.99 \\
\hline $\mathrm{PO}_{4}$ & 1871 & 0.020 & $\mathrm{P}_{2} \mathrm{O}_{5}$ & 0.75 & 0.12 & $\mathrm{Na}_{3} \mathrm{PO}_{4} \cdot 12 \mathrm{H}_{2} \mathrm{O}$ & 10 & 380.12 & 0.99 & 7.56 \\
\hline $\mathrm{SO}_{4}$ & 8165 & 0.085 & $\mathrm{SO}_{3}$ & 3.66 & 0.59 & $\mathrm{Na}_{2} \mathrm{SO}_{4}$ & 13 & 142.06 & 0.99 & 12.20 \\
\hline $\mathrm{NO}_{2}$ & 43205 & 0.939 & $\mathrm{NO}_{2}$ & - & - & $\mathrm{NaNO}_{2}$ & 18 & 69.00 & 1.00 & 65.13 \\
\hline $\mathrm{NO}_{3}$ & 111873 & 1.804 & $\mathrm{NO}_{3}$ & - & - & $\mathrm{NaNO}_{3}$ & 19 & 84.99 & 0.99 & 94.65 \\
\hline $\mathrm{CO}_{3}$ & 27723 & 0.462 & $\mathrm{CO}_{3}$ & - & - & $\mathrm{Na}_{2} \mathrm{CO}_{3}$ & 20 & 105.99 & 1.00 & 48.96 \\
\hline Org.Carbon & 14731 & 1.228 & - & - & - & - & - & - & - & - \\
\hline Formate & 16320 & 0.363 & - & - & - & Sodium Formate (C1) & 14 & 68.01 & 0.99 & 24.91 \\
\hline Oxalate & 938 & 0.011 & - & - & - & Sodium Oxalate (C2) & 15 & 134.00 & 0.99 & 1.44 \\
\hline Glycolate & 21420 & 0.282 & - & - & - & Glycolic Acid (C2) & 16 & 76.05 & 0.71 & 30.22 \\
\hline Citric Acid & 8976 & 0.047 & - & - & - & Citric Acid (C6) & 17 & 192.12 & 0.99 & 9.07 \\
\hline- & - & - & - & - & - & & lass Weight & & & 1145.23 \\
\hline- & - & - & SUM & 100.00 & 16.22 & & dlant Weigh & & & 1243.44 \\
\hline
\end{tabular}

"-" Empty data field.

* Assay refers to the purity of the raw material as specified by the vendor.

$\$$ - An actual 5 molar sodium simulant was produced at the crucible scale with $689.14 \mathrm{ml}$ water yielding a measured simulant total weight of $1246.58 \mathrm{~g}$. 
Table 2.9. LAWE9H Simulant Recipe at Nominal 4 Molar Sodium Based on AZ-101 Waste Composition [32].

\begin{tabular}{|c|c|c|c|c|c|c|c|c|c|c|}
\hline $\begin{array}{c}\text { Envelope } \\
\text { Constituents }\end{array}$ & \multicolumn{2}{|c|}{$\begin{array}{l}\text { Simulant AZ-101 } \\
\text { Modified for E9H }\end{array}$} & $\begin{array}{l}\text { Glass } \\
\text { Oxides }\end{array}$ & $\begin{array}{c}\text { LAW E9H } \\
\text { Simulant as } \\
\text { Oxides (wt } \% \text { ) }\end{array}$ & $\begin{array}{c}\text { Waste } \\
\text { Contribution to } \\
\text { Glass }\end{array}$ & $\begin{array}{l}\text { Source in } \\
\text { Simulant }\end{array}$ & $\begin{array}{l}\text { Order for } \\
\text { Addition }\end{array}$ & $\begin{array}{c}\text { Formula } \\
\text { Weight }\end{array}$ & Assay* & $\begin{array}{c}\text { Target } \\
\text { Weight (g) }\end{array}$ \\
\hline- & $\mathrm{mg} / \mathrm{L}$ & $\mathrm{M}$ & Loading & & $11.34 \%$ & In $806 \mathrm{ml}$ water add follov & npounds in & e order lis & $\mathrm{d}$ below & \\
\hline $\mathrm{Al}$ & 4761 & 0.176 & $\mathrm{Al}_{2} \mathrm{O}_{3}$ & 5.71 & 0.65 & $\mathrm{Al}\left(\mathrm{NO}_{3}\right)_{3} \cdot 9 \mathrm{H}_{2} \mathrm{O}, 60 \%$ sol. & 1 & 375.14 & 0.61 & 109.07 \\
\hline $\mathrm{Ca}$ & 360 & 0.009 & $\mathrm{CaO}$ & 0.32 & 0.04 & $\mathrm{Ca}\left(\mathrm{NO}_{3}\right)_{2} \cdot 4 \mathrm{H}_{2} \mathrm{O}$ & 2 & 236.16 & 0.99 & 2.14 \\
\hline $\mathrm{Cr}$ & 741 & 0.014 & $\mathrm{Cr}_{2} \mathrm{O}_{3}$ & 0.69 & 0.08 & $\mathrm{Na}_{2} \mathrm{CrO}_{4} \cdot 4 \mathrm{H}_{2} \mathrm{O}$ & 5 & 234.04 & 0.99 & 3.39 \\
\hline $\mathrm{K}$ & 6227 & 0.159 & $\mathrm{~K}_{2} \mathrm{O}$ & 4.77 & 0.54 & $\mathrm{KOH}$ & 4 & 56.10 & 0.91 & 9.84 \\
\hline $\mathrm{Na}$ & 91960 & 4.000 & $\mathrm{Na}_{2} \mathrm{O}$ & 78.74 & 8.93 & $\mathrm{NaOH}, 50 \%$ sol. $d=1.53$ & 3 & 40.00 & 0.50 & 185.67 \\
\hline $\mathrm{Ni}$ & 85 & 0.002 & $\mathrm{NiO}$ & 0.07 & 0.01 & $\mathrm{NiO}$ & 6 & 74.69 & 1.00 & 0.11 \\
\hline $\mathrm{Pb}$ & 101 & 0.001 & $\mathrm{PbO}$ & 0.07 & 0.01 & $\mathrm{PbO}$ & 7 & 223.20 & 1.00 & 0.11 \\
\hline $\mathrm{Si}$ & 53 & 0.002 & $\mathrm{SiO}_{2}$ & 0.07 & 0.01 & $\mathrm{SiO}_{2}$ & 8 & 60.09 & 0.99 & 0.12 \\
\hline $\mathrm{Cl}$ & 2723 & 0.077 & $\mathrm{Cl}$ & 1.73 & 0.20 & $\mathrm{NaCl}$ & 10 & 58.45 & 0.99 & 4.53 \\
\hline $\mathrm{F}$ & 1078 & 0.057 & $\mathrm{~F}$ & 0.69 & 0.08 & $\mathrm{NaF}$ & 11 & 42.00 & 0.99 & 2.41 \\
\hline $\mathrm{PO}_{4}$ & 2245 & 0.077 & $\mathrm{P}_{2} \mathrm{O}_{5}$ & 1.07 & 0.12 & $\mathrm{Na}_{3} \mathrm{PO}_{4} \cdot 12 \mathrm{H}_{2} \mathrm{O}$ & 9 & 380.12 & 0.99 & 9.08 \\
\hline $\mathrm{SO}_{4}$ & 11485 & 0.120 & $\mathrm{SO}_{3}$ & 6.08 & 0.69 & $\mathrm{Na}_{2} \mathrm{SO}_{4}$ & 12 & 142.06 & 0.99 & 17.16 \\
\hline $\mathrm{NO}_{2}$ & 48214 & 1.048 & $\mathrm{NO}_{2}$ & - & - & $\mathrm{NaNO}_{2}$ & 15 & 69.00 & 1.00 & 72.68 \\
\hline $\mathrm{NO}_{3}$ & 41345 & 0.667 & $\mathrm{NO}_{3}$ & - & - & $\mathrm{NaNO}_{3}$ & 16 & 84.99 & 0.99 & 10.26 \\
\hline $\mathrm{CO}_{3}$ & 38740 & 0.646 & $\mathrm{CO}_{3}$ & - & - & ${ }^{\#} \mathrm{Na}_{2} \mathrm{CO}_{3}$ & 17 & 105.99 & 1.00 & 0 \\
\hline Org.Carbon & 401 & 0.033 & - & - & - & - & - & - & - & - \\
\hline formate & 393 & 0.009 & - & - & - & Sodium Formate (C1) & 13 & 68.01 & 0.99 & 0.60 \\
\hline Oxalate & 1085 & 0.012 & - & - & - & Sodium Oxalate (C2) & 14 & 134.00 & 0.99 & 1.67 \\
\hline- & - & - & - & - & - & Target Glass Weight & & & & 1388.13 \\
\hline- & - & - & SUM & 100.00 & 11.34 & Total Simulant Weight & & & & 1200.23 \\
\hline
\end{tabular}

"-" Empty data field.

* Assay refers to the purity of the raw material as specified by the vendor.

${ }^{\#} \mathrm{Na}_{2} \mathrm{CO}_{3}$ was omitted to accommodate $\mathrm{NaF}$ and $\mathrm{NaCl}$ additions in Tests 4-6 and $\mathrm{NaOH}$ in Tests 1-3. 
Table 2.10. LAWE10H Simulant Recipe at 2 Molar Sodium Based on AZ-102 Waste Composition [32].

\begin{tabular}{|c|c|c|c|c|c|c|c|c|c|c|}
\hline $\begin{array}{c}\text { Envelope } \\
\text { Constituents }\end{array}$ & \multicolumn{2}{|c|}{$\begin{array}{l}\text { Simulant AZ-102 } \\
\text { Modified for E10H }\end{array}$} & \multirow{2}{*}{\begin{tabular}{|c} 
Glass \\
Oxides \\
Loading
\end{tabular}} & \multirow{2}{*}{$\begin{array}{c}\text { LAW E10H } \\
\text { Simulant as } \\
\text { Oxides (wt\%) } \\
\\
\end{array}$} & \multirow{2}{*}{$\begin{array}{c}\begin{array}{c}\text { Waste } \\
\text { Contribution } \\
\text { to Glass }\end{array} \\
6.20 \% \\
\end{array}$} & Source in Simulant & \multirow[t]{2}{*}{$\begin{array}{c}\text { Order } \\
\text { for } \\
\text { Addition }\end{array}$} & \multirow[t]{2}{*}{$\begin{array}{c}\text { Formula } \\
\text { Weight }\end{array}$} & \multirow[t]{2}{*}{ Assay } & \multirow[t]{2}{*}{$\begin{array}{c}\text { Target } \\
\text { Weight (g) }\end{array}$} \\
\hline- & $\mathrm{mg} / \mathrm{L}$ & Molarity & & & & In $970 \mathrm{ml}$ water ${ }^{\$}$ add following compounds in the order listed. & & & & \\
\hline $\mathrm{Al}$ & 123 & 0.005 & $\mathrm{Al}_{2} \mathrm{O}_{3}$ & 0.26 & 0.02 & $\mathrm{Al}\left(\mathrm{NO}_{3}\right)_{3} \cdot 9 \mathrm{H}_{2} \mathrm{O}, 60 \%$ sol. & 2 & 375.14 & 0.61 & 2.82 \\
\hline $\mathrm{Cr}$ & 760 & 0.015 & $\mathrm{Cr}_{2} \mathrm{O}_{3}$ & 1.25 & 0.08 & $\mathrm{Na}_{2} \mathrm{CrO}_{4} \cdot 4 \mathrm{H}_{2} \mathrm{O}$ & 5 & 234.04 & 0.99 & 3.47 \\
\hline $\mathrm{K}$ & 6412 & 0.164 & $\mathrm{~K}_{2} \mathrm{O}$ & 8.70 & 0.54 & $\mathrm{KOH}$ & 4 & 56.098 & 0.91 & 10.13 \\
\hline $\mathrm{Na}$ & 45980 & 2.000 & $\mathrm{Na}_{2} \mathrm{O}$ & 69.84 & 4.33 & $\mathrm{NaOH}, 50 \%$ sol. $\mathrm{d}=1.53$ & 3 & 40.00 & 0.50 & 108.59 \\
\hline $\mathrm{Ni}$ & 88 & 0.002 & $\mathrm{NiO}$ & 0.13 & 0.01 & $\mathrm{NiO}$ & 6 & 74.69 & 1.00 & 0.11 \\
\hline $\mathrm{Pb}$ & 103 & 0.001 & $\mathrm{PbO}$ & 0.13 & 0.01 & $\mathrm{PbO}$ & 7 & 223.20 & 1.00 & 0.11 \\
\hline $\mathrm{Si}$ & 171 & 0.006 & $\mathrm{SiO}_{2}$ & 0.41 & 0.03 & $\mathrm{SiO}_{2}$ & 8 & 60.09 & 0.99 & 0.37 \\
\hline $\mathrm{Cl}$ & 2809 & 0.079 & $\mathrm{Cl}$ & 3.17 & 0.20 & $\mathrm{NaCl}$ & 10 & 58.45 & 0.99 & 4.68 \\
\hline $\mathrm{F}$ & 1111 & 0.058 & $\mathrm{~F}$ & 1.25 & 0.08 & $\mathrm{NaF}$ & 11 & 42.00 & 0.99 & 2.48 \\
\hline $\mathrm{PO}_{4}$ & 2323 & 0.024 & $\mathrm{P}_{2} \mathrm{O}_{5}$ & 1.96 & 0.12 & $\mathrm{Na}_{3} \mathrm{PO}_{4} \cdot 12 \mathrm{H}_{2} \mathrm{O}$ & 9 & 380.12 & 0.99 & 9.39 \\
\hline $\mathrm{SO}_{4}$ & 13744 & 0.143 & $\mathrm{SO}_{3}$ & 12.91 & 0.80 & $\mathrm{Na}_{2} \mathrm{SO}_{4}$ & 12 & 142.06 & 0.99 & 20.53 \\
\hline $\mathrm{NO}_{2}$ & 23680 & 0.515 & $\mathrm{NO}_{2}$ & - & - & ${ }^{\#} \mathrm{NaNO}_{2}$ & 14 & 69.00 & 1.00 & 0 \\
\hline $\mathrm{NO}_{3}$ & 7837 & 0.126 & $\mathrm{NO}_{3}$ & - & - & $\mathrm{NaNO}_{3}$ & 15 & 84.99 & 0.99 & 9.67 \\
\hline $\mathrm{CO}_{3}$ & 23080 & 0.385 & $\mathrm{CO}_{3}$ & - & - & ${ }^{\#} \mathrm{Na}_{2} \mathrm{CO}_{3}$ & 1 & 105.99 & 1.00 & 0 \\
\hline Org.Carbon & 608 & 0.051 & - & - & - & - & - & - & - & - \\
\hline Oxalate & 2242 & 0.025 & - & - & - & Oxalic Acid (C2) & 13 & 126.00 & 1.00 & 3.21 \\
\hline- & - & - & - & - & - & \multicolumn{4}{|l|}{ Target Glass } & 1431.41 \\
\hline- & - & - & SUM & 100.00 & 6.20 & \multicolumn{4}{|l|}{ Total simulant wt. } & 1094.47 \\
\hline
\end{tabular}

-" Empty data field

* Assay refers to the purity of the raw material as specified by the vendor.

\# - $\mathrm{Na}_{2} \mathrm{CO}_{3}$ and $\mathrm{NaNO}_{2}$ were omitted to accommodate $\mathrm{NaF}$ and $\mathrm{NaCl}$ additions in Tests 4-6 and $\mathrm{NaOH}$ in Tests 1-3. 
The Catholic University of America Vitreous State Laboratory
ORP-56324, Rev. 0

DuraMelter 100 Tests to Support LAW Glass Formulation Correlation Development Final Report, VSL-06R6480-1, Rev. 0

Table 2.11. Oxide Composition of LAWE3 Simulant and Corresponding Glass Composition.

\begin{tabular}{|c|c|c|c|}
\hline GLASS & $\begin{array}{l}\text { AP-101 in LAWE3 Glass } \\
\text { at } 18.21 \mathrm{wt} \% \mathrm{Na}_{2} \mathrm{O}\end{array}$ & $\begin{array}{c}\text { Glass Former } \\
\text { Additives (wt } \% \text { ) }\end{array}$ & $\begin{array}{l}\text { LAWE3 } \\
\text { (wt\%) }\end{array}$ \\
\hline Loading & $25.41 \%$ & $74.59 \%$ & $25.41 \%$ \\
\hline $\mathrm{Al}_{2} \mathrm{O}_{3}$ & 1.34 & 4.76 & 6.10 \\
\hline $\mathrm{B}_{2} \mathrm{O}_{3}$ & 0.00 & 10.00 & 10.00 \\
\hline $\mathrm{CaO}$ & 0.00 & 2.02 & 2.02 \\
\hline $\mathrm{Cr}_{2} \mathrm{O}_{3}$ & 0.08 & 0.00 & 0.08 \\
\hline $\mathrm{Fe}_{2} \mathrm{O}_{3}$ & 0.00 & 5.50 & 5.50 \\
\hline $\mathrm{K}_{2} \mathrm{O}$ & 4.98 & 0.00 & 4.98 \\
\hline $\mathrm{Li}_{2} \mathrm{O}$ & 0.00 & 0.00 & 0.00 \\
\hline $\mathrm{MgO}$ & 0.00 & 1.48 & 1.48 \\
\hline $\mathrm{Na}_{2} \mathrm{O}$ & 18.21 & 0.00 & 18.21 \\
\hline $\mathrm{NiO}$ & 0.01 & 0.00 & 0.01 \\
\hline $\mathrm{PbO}$ & 0.01 & 0.00 & 0.01 \\
\hline $\mathrm{SiO}_{2}$ & 0.02 & 42.93 & 42.95 \\
\hline $\mathrm{TiO}_{2}$ & 0.00 & 1.40 & 1.40 \\
\hline $\mathrm{ZnO}$ & 0.00 & 3.50 & 3.50 \\
\hline $\mathrm{ZrO}_{2}$ & 0.00 & 3.00 & 3.00 \\
\hline $\mathrm{Cl}$ & 0.20 & 0.00 & 0.20 \\
\hline $\mathrm{F}$ & 0.08 & 0.00 & 0.08 \\
\hline $\mathrm{P}_{2} \mathrm{O}_{5}$ & 0.12 & 0.00 & 0.12 \\
\hline $\mathrm{SO}_{3}$ & 0.35 & 0.00 & 0.35 \\
\hline Sum & 25.41 & 74.59 & 100.00 \\
\hline
\end{tabular}


The Catholic University of America Vitreous State Laboratory
ORP-56324, Rev. 0

DuraMelter 100 Tests to Support LAW Glass Formulation Correlation Development Final Report, VSL-06R6480-1, Rev. 0

\section{Table 2.12. Oxide Composition of LAWE4H Simulant and Corresponding Glass Composition.}

\begin{tabular}{|c|c|c|c|c|}
\hline GLASS & $\begin{array}{c}\text { AN-105 in LAWE4H } \\
\text { Glass at } 21.27 \text { wt } \% \\
\mathrm{Na}_{2} \mathrm{O}\end{array}$ & $\begin{array}{c}\text { Glass Former } \\
\text { Additives (wt\%) }\end{array}$ & $\begin{array}{c}\text { LAWE4H } \\
\text { Without K } \\
\text { (wt\%) } \\
\end{array}$ & $\begin{array}{l}\text { LAWE4H } \\
\text { (wt\%) }\end{array}$ \\
\hline Loading & $27.18 \%$ & $72.82 \%$ & $27.18 \%$ & $27.72 \%$ \\
\hline $\mathrm{Al}_{2} \mathrm{O}_{3}$ & 4.95 & 1.02 & 5.97 & 5.93 \\
\hline $\mathrm{B}_{2} \mathrm{O}_{3}$ & 0.02 & 9.77 & 9.79 & 9.72 \\
\hline $\mathrm{CaO}$ & 0.00 & 2.46 & 2.46 & 2.44 \\
\hline $\mathrm{Cr}_{2} \mathrm{O}_{3}$ & 0.08 & 0.00 & 0.08 & 0.08 \\
\hline $\mathrm{Fe}_{2} \mathrm{O}_{3}$ & 0.00 & 5.38 & 5.38 & 5.34 \\
\hline $\mathrm{K}_{2} \mathrm{O} *$ & 0.00 (or 0.54$)$ & 0.00 & 0.00 & 0.54 \\
\hline $\mathrm{Li}_{2} \mathrm{O}$ & 0.00 & 0.00 & 0.00 & 0.00 \\
\hline $\mathrm{MgO}$ & 0.00 & 1.45 & 1.45 & 1.44 \\
\hline $\mathrm{Na}_{2} \mathrm{O}$ & 21.27 & 0.00 & 21.27 & 21.27 \\
\hline $\mathrm{NiO}$ & 0.01 & 0.00 & 0.01 & 0.01 \\
\hline $\mathrm{PbO}$ & 0.01 & 0.00 & 0.01 & 0.01 \\
\hline $\mathrm{SiO}_{2}$ & 0.03 & 45.01 & 45.04 & 44.73 \\
\hline $\mathrm{TiO}_{2}$ & 0.00 & 1.37 & 1.37 & 1.36 \\
\hline $\mathrm{ZnO}$ & 0.00 & 3.43 & 3.43 & 3.41 \\
\hline $\mathrm{ZrO}_{2}$ & 0.00 & 2.94 & 2.94 & 2.92 \\
\hline $\mathrm{Cl}$ & 0.20 & 0.00 & 0.20 & 0.20 \\
\hline $\mathrm{F}$ & 0.08 & 0.00 & 0.08 & 0.08 \\
\hline $\mathrm{P}_{2} \mathrm{O}_{5}$ & 0.12 & 0.00 & 0.12 & 0.12 \\
\hline $\mathrm{SO}_{3}$ & 0.41 & 0.00 & 0.41 & 0.41 \\
\hline Sum & 27.18 & 72.82 & 100.00 & 100.00 \\
\hline
\end{tabular}

* Melter tests were conducted using LAWE4H glasses targeting 0.00 and $0.54 \mathrm{wt} \% \mathrm{~K}_{2} \mathrm{O}$. Sodium was held constant at $21.27 \mathrm{wt} \%$; however, increasing $\mathrm{K}_{2} \mathrm{O}$ resulted in lower concentrations of other components. 
The Catholic University of America Vitreous State Laboratory
ORP-56324, Rev. 0

DuraMelter 100 Tests to Support LAW Glass Formulation Correlation Development Final Report, VSL-06R6480-1, Rev. 0

Table 2.13. Oxide Composition of LAWE5H Simulant and Corresponding Glass Composition.

\begin{tabular}{|c|c|c|c|}
\hline GLASS & $\begin{array}{l}\text { AN-107 in LAWE5H } \\
\text { Glass at } 18.97 \text { wt } \% \mathrm{Na}_{2} \mathrm{O}\end{array}$ & $\begin{array}{c}\text { Glass Former } \\
\text { Additives } \\
(\mathrm{wt} \%)\end{array}$ & $\begin{array}{l}\text { LAWE5H } \\
\text { (wt\%) }\end{array}$ \\
\hline Loading & $20.64 \%$ & $79.36 \%$ & $20.64 \%$ \\
\hline $\mathrm{Al}_{2} \mathrm{O}_{3}$ & 0.02 & 5.97 & 5.99 \\
\hline $\mathrm{B}_{2} \mathrm{O}_{3}$ & 0.00 & 9.81 & 9.81 \\
\hline $\mathrm{CaO}$ & 0.04 & 3.57 & 3.61 \\
\hline $\mathrm{Cr}_{2} \mathrm{O}_{3}$ & 0.08 & 0.00 & 0.08 \\
\hline $\mathrm{Fe}_{2} \mathrm{O}_{3}$ & 0.12 & 5.29 & 5.40 \\
\hline $\mathrm{K}_{2} \mathrm{O}$ & 0.54 & 0.00 & 0.54 \\
\hline $\mathrm{Li}_{2} \mathrm{O}$ & 0.00 & 0.49 & 0.49 \\
\hline $\mathrm{MgO}$ & 0.00 & 1.45 & 1.45 \\
\hline $\mathrm{Na}_{2} \mathrm{O}$ & 18.97 & 0.00 & 18.97 \\
\hline $\mathrm{NiO}$ & 0.01 & 0.00 & 0.01 \\
\hline $\mathrm{PbO}$ & 0.01 & 0.00 & 0.01 \\
\hline $\mathrm{SiO}_{2}$ & 0.00 & 45.04 & 45.04 \\
\hline $\mathrm{TiO}_{2}$ & 0.00 & 1.37 & 1.37 \\
\hline $\mathrm{ZnO}$ & 0.00 & 3.43 & 3.43 \\
\hline $\mathrm{ZrO}_{2}$ & 0.00 & 2.94 & 2.94 \\
\hline $\mathrm{Cl}$ & 0.20 & 0.00 & 0.20 \\
\hline $\mathrm{F}$ & 0.08 & 0.00 & 0.08 \\
\hline $\mathrm{P}_{2} \mathrm{O}_{5}$ & 0.12 & 0.00 & 0.12 \\
\hline $\mathrm{SO}_{3}$ & 0.46 & 0.00 & 0.46 \\
\hline Sum & 20.64 & 79.36 & 100.00 \\
\hline
\end{tabular}


The Catholic University of America Vitreous State Laboratory
ORP-56324, Rev. 0

DuraMelter 100 Tests to Support LAW Glass Formulation Correlation Development Final Report, VSL-06R6480-1, Rev. 0

Table 2.14. Oxide Composition of LAWE6H Simulant and Corresponding Glass Composition.

\begin{tabular}{|c|c|c|c|}
\hline GLASS & $\begin{array}{l}\text { AN-104 in LAWE6H } \\
\text { Glass at } 15.83 \text { wt } \% \mathrm{Na}_{2} \mathrm{O}\end{array}$ & $\begin{array}{c}\text { Glass Former } \\
\text { Additives (wt } \%)\end{array}$ & $\begin{array}{l}\text { LAWE6H } \\
\text { (wt\%) }\end{array}$ \\
\hline Loading & $20.88 \%$ & $79.12 \%$ & $20.88 \%$ \\
\hline $\mathrm{Al}_{2} \mathrm{O}_{3}$ & 3.46 & 2.55 & 6.01 \\
\hline $\mathrm{B}_{2} \mathrm{O}_{3}$ & 0.00 & 9.85 & 9.85 \\
\hline $\mathrm{CaO}$ & 0.00 & 5.46 & 5.46 \\
\hline $\mathrm{Cr}_{2} \mathrm{O}_{3}$ & 0.08 & 0.00 & 0.08 \\
\hline $\mathrm{Fe}_{2} \mathrm{O}_{3}$ & 0.00 & 5.42 & 5.42 \\
\hline $\mathrm{K}_{2} \mathrm{O}$ & 0.54 & 0.00 & 0.54 \\
\hline $\mathrm{Li}_{2} \mathrm{O}$ & 0.00 & 2.38 & 2.38 \\
\hline $\mathrm{MgO}$ & 0.00 & 1.46 & 1.46 \\
\hline $\mathrm{Na}_{2} \mathrm{O}$ & 15.83 & 0.00 & 15.83 \\
\hline $\mathrm{NiO}$ & 0.01 & 0.00 & 0.01 \\
\hline $\mathrm{PbO}$ & 0.01 & 0.00 & 0.01 \\
\hline $\mathrm{SiO}_{2}$ & 0.04 & 44.21 & 44.25 \\
\hline $\mathrm{TiO}_{2}$ & 0.00 & 1.38 & 1.38 \\
\hline $\mathrm{ZnO}$ & 0.00 & 3.45 & 3.45 \\
\hline $\mathrm{ZrO}_{2}$ & 0.00 & 2.95 & 2.95 \\
\hline $\mathrm{Cl}$ & 0.20 & 0.00 & 0.20 \\
\hline $\mathrm{F}$ & 0.08 & 0.00 & 0.08 \\
\hline $\mathrm{P}_{2} \mathrm{O}_{5}$ & 0.12 & 0.00 & 0.12 \\
\hline $\mathrm{SO}_{3}$ & 0.53 & 0.00 & 0.53 \\
\hline Sum & 20.88 & 79.12 & 100.00 \\
\hline
\end{tabular}


The Catholic University of America Vitreous State Laboratory
ORP-56324, Rev. 0

DuraMelter 100 Tests to Support LAW Glass Formulation Correlation Development Final Report, VSL-06R6480-1, Rev. 0

\section{Table 2.15. Oxide Composition of LAWE7H Simulant and Corresponding Glass Composition.}

\begin{tabular}{|l|c|c|c|}
\hline $\mathrm{GLASS}$ & $\begin{array}{c}\text { AN-102 in LAWE7H } \\
\text { Glass at } 13.53 \mathrm{wt} \% \mathrm{Na}_{2} \mathrm{O}\end{array}$ & $\begin{array}{c}\text { Glass Former } \\
\text { Additives (wt\%) }\end{array}$ & $\begin{array}{c}\text { LAWE7H } \\
\text { (wt\%) }\end{array}$ \\
\hline Loading & $16.22 \%$ & $83.78 \%$ & $16.22 \%$ \\
\hline $\mathrm{Al}_{2} \mathrm{O}_{3}$ & 1.02 & 5.00 & 6.02 \\
\hline $\mathrm{B}_{2} \mathrm{O}_{3}$ & 0.01 & 9.86 & 9.87 \\
\hline $\mathrm{CaO}$ & 0.03 & 6.28 & 6.31 \\
\hline $\mathrm{Cr}_{2} \mathrm{O}_{3}$ & 0.08 & 0.00 & 0.08 \\
\hline $\mathrm{Fe}_{2} \mathrm{O}_{3}$ & 0.00 & 5.43 & 5.43 \\
\hline $\mathrm{K}_{2} \mathrm{O}$ & 0.54 & 0.00 & 0.54 \\
\hline $\mathrm{Li}_{2} \mathrm{O}$ & 0.00 & 3.17 & 3.17 \\
\hline $\mathrm{MgO}^{2}$ & 0.00 & 1.49 & 1.49 \\
\hline $\mathrm{Na}_{2} \mathrm{O}$ & 13.53 & 0.00 & 13.53 \\
\hline $\mathrm{NiO}$ & 0.01 & 0.00 & 0.01 \\
\hline $\mathrm{PbO}$ & 0.01 & 0.00 & 0.01 \\
\hline $\mathrm{SiO}_{2}$ & 0.01 & 44.75 & 44.75 \\
\hline $\mathrm{TiO}_{2}$ & 0.00 & 1.38 & 1.38 \\
\hline $\mathrm{ZnO}_{\mathrm{ZrO}}$ & 0.00 & 3.46 & 3.46 \\
\hline $\mathrm{Cl}_{2}$ & 0.00 & 0.00 & 2.96 \\
\hline $\mathrm{F}$ & 0.20 & 0.00 & 0.20 \\
\hline $\mathrm{P}_{2} \mathrm{O}_{5}$ & 0.59 & 0.08 \\
\hline $\mathrm{SO}_{3}$ & 16.22 & 0.12 \\
\hline $\mathrm{Sum}$ & & 0.59 \\
\hline
\end{tabular}


The Catholic University of America Vitreous State Laboratory
ORP-56324, Rev. 0

DuraMelter 100 Tests to Support LAW Glass Formulation Correlation Development Final Report, VSL-06R6480-1, Rev. 0

Table 2.16. Oxide Composition of LAWE9H Simulant
and Corresponding Glass Composition.

\begin{tabular}{|c|c|c|c|}
\hline $\begin{array}{l}\text { GLASS } \\
\text { Oxides }\end{array}$ & $\begin{array}{l}\text { AZ-101 in LAWE9H } \\
\text { Glass at } 8.93 \mathrm{wt} \% \mathrm{Na}_{2} \mathrm{O}\end{array}$ & $\begin{array}{c}\text { Glass Former } \\
\text { Additives (wt\%) }\end{array}$ & $\begin{array}{l}\text { LAWE9H } \\
\text { (wt\%) }\end{array}$ \\
\hline Loading & $11.34 \%$ & $88.66 \%$ & $11.34 \%$ \\
\hline $\mathrm{Al}_{2} \mathrm{O}_{3}$ & 0.65 & 5.41 & 6.05 \\
\hline $\mathrm{B}_{2} \mathrm{O}_{3}$ & 0.00 & 9.92 & 9.92 \\
\hline $\mathrm{CaO}$ & 0.04 & 6.82 & 6.86 \\
\hline $\mathrm{Cr}_{2} \mathrm{O}_{3}$ & 0.08 & 0.00 & 0.08 \\
\hline $\mathrm{Fe}_{2} \mathrm{O}_{3}$ & 0.00 & 5.45 & 5.45 \\
\hline $\mathrm{K}_{2} \mathrm{O}$ & 0.54 & 0.00 & 0.54 \\
\hline $\mathrm{Li}_{2} \mathrm{O}$ & 0.00 & 4.08 & 4.08 \\
\hline $\mathrm{MgO}$ & 0.00 & 2.36 & 2.36 \\
\hline $\mathrm{Na}_{2} \mathrm{O}$ & 8.93 & 0.00 & 8.93 \\
\hline $\mathrm{NiO}$ & 0.01 & 0.00 & 0.01 \\
\hline $\mathrm{PbO}$ & 0.01 & 0.00 & 0.01 \\
\hline $\mathrm{SiO}_{2}$ & 0.01 & 46.79 & 46.79 \\
\hline $\mathrm{TiO}_{2}$ & 0.00 & 1.39 & 1.39 \\
\hline $\mathrm{ZnO}$ & 0.00 & 3.47 & 3.47 \\
\hline $\mathrm{ZrO}_{2}$ & 0.00 & 2.97 & 2.97 \\
\hline $\mathrm{Cl}$ & 0.20 & 0.00 & 0.20 \\
\hline $\mathrm{F}$ & 0.08 & 0.00 & 0.08 \\
\hline $\mathrm{P}_{2} \mathrm{O}_{5}$ & 0.12 & 0.00 & 0.12 \\
\hline $\mathrm{SO}_{3}$ & 0.69 & 0.00 & 0.69 \\
\hline Sum & 11.34 & 88.66 & 100.00 \\
\hline
\end{tabular}


The Catholic University of America Vitreous State Laboratory
ORP-56324, Rev. 0

DuraMelter 100 Tests to Support LAW Glass Formulation Correlation Development Final Report, VSL-06R6480-1, Rev. 0

Table 2.17. Oxide Composition of LAWE10H Simulant and Corresponding Glass Compositions.

\begin{tabular}{|c|c|c|c|}
\hline GLASS & $\begin{array}{l}\text { AZ-102 in LAWE10H } \\
\text { Glass at } 4.33 \mathrm{wt} \% \mathrm{Na}_{2} \mathrm{O}\end{array}$ & $\begin{array}{c}\text { Glass Former } \\
\text { Additives (wt\%) }\end{array}$ & $\begin{array}{l}\text { LAWE10H } \\
\text { (wt\%) }\end{array}$ \\
\hline Loading & $6.20 \%$ & $93.80 \%$ & $6.20 \%$ \\
\hline $\mathrm{Al}_{2} \mathrm{O}_{3}$ & 0.02 & 6.05 & 6.07 \\
\hline $\mathrm{B}_{2} \mathrm{O}_{3}$ & 0.00 & 9.95 & 9.95 \\
\hline $\mathrm{CaO}$ & 0.00 & 6.96 & 6.96 \\
\hline $\mathrm{Cr}_{2} \mathrm{O}_{3}$ & 0.08 & 0.00 & 0.08 \\
\hline $\mathrm{Fe}_{2} \mathrm{O}_{3}$ & 0.00 & 5.48 & 5.48 \\
\hline $\mathrm{K}_{2} \mathrm{O}$ & 0.54 & 0.00 & 0.54 \\
\hline $\mathrm{Li}_{2} \mathrm{O}$ & 0.00 & 4.26 & 4.26 \\
\hline $\mathrm{MgO}$ & 0.00 & 2.94 & 2.94 \\
\hline $\mathrm{Na}_{2} \mathrm{O}$ & 4.33 & 1.39 & 5.72 \\
\hline $\mathrm{NiO}$ & 0.01 & 0.00 & 0.01 \\
\hline $\mathrm{PbO}$ & 0.01 & 0.00 & 0.01 \\
\hline $\mathrm{SiO}_{2}$ & 0.02 & 48.90 & 48.92 \\
\hline $\mathrm{TiO}_{2}$ & 0.00 & 1.39 & 1.39 \\
\hline $\mathrm{ZnO}$ & 0.00 & 3.48 & 3.48 \\
\hline $\mathrm{ZrO}_{2}$ & 0.00 & 2.99 & 2.99 \\
\hline $\mathrm{Cl}$ & 0.20 & 0.00 & 0.20 \\
\hline $\mathrm{F}$ & 0.08 & 0.00 & 0.08 \\
\hline $\mathrm{P}_{2} \mathrm{O}_{5}$ & 0.12 & 0.00 & 0.12 \\
\hline $\mathrm{SO}_{3}$ & 0.80 & 0.00 & 0.80 \\
\hline Sum & 6.20 & 93.80 & 100.00 \\
\hline
\end{tabular}


Table 2.18. Glass Former Additives for 1 Liter of LAWE Waste Simulant and Corresponding Feed Properties in Crucible Scale Tests.

\begin{tabular}{|c|c|c|c|c|c|c|c|}
\hline Additive Source & Feed LAWE3 & Feed LAWE4H* & Feed LAWE5H & Feed LAWE6H & Feed LAWE7H & Feed LAWE9H & Feed LAWE10H \\
\hline Additives in Glass (wt\%) & $74.59 \%$ & $72.82 \%$ & $79.36 \%$ & $79.12 \%$ & $83.78 \%$ & $88.66 \%$ & $93.80 \%$ \\
\hline Kyanite $\left(\mathrm{Al}_{2} \mathrm{SiO}_{5}\right) 325$ Mesh (Kyanite Mining) (g) & 111.67 & 18.15 & 117.42 & 56.86 & 96.64 & 126.86 & 147.13 \\
\hline $\mathrm{H}_{3} \mathrm{BO}_{3}$ (US Borax - Technical Granular) (g) & 241.75 & 202.23 & 199.26 & 239.75 & 200.67 & 244.59 & 253.08 \\
\hline Wollanstonite NYAD 325 Mesh (NYCO Minerals) (g) & 60.51 & 63.09 & 89.88 & 164.63 & 158.24 & 208.41 & 219.20 \\
\hline $\mathrm{Fe}_{2} \mathrm{O}_{3}(97 \%$ Alfa $)(\mathrm{g})$ & 71.35 & 60.14 & 57.43 & 71.07 & 59.21 & 70.04 & 71.22 \\
\hline $\mathrm{Li}_{2} \mathrm{CO}_{3}$ (Chemetall Foote Co. Technical grade) & - & - & 14.01 & 81.55 & 90.77 & 141.60 & 152.46 \\
\hline Olivine $\left(\mathrm{Mg}_{2} \mathrm{SiO}_{4}\right) 325$ Mesh (\#180 Unimin) (g) & 41.36 & 34.48 & 33.34 & 39.26 & 33.18 & 65.34 & 84.78 \\
\hline $\mathrm{Na}_{2} \mathrm{CO}_{3}$ (Technical grade) (g) & - & - & - & - & - & - & 34.18 \\
\hline $\mathrm{SiO}_{2}$ (Sil-co-Sil 75 US Silica) (g) & 468.23 & 446.62 & 387.91 & 459.37 & 358.02 & 438.69 & 465.92 \\
\hline $\mathrm{TiO}_{2}$ (Rutile Airfloated Chemaloy) (g) & 20.20 & 16.92 & 16.60 & 20.04 & 16.75 & 20.45 & 21.08 \\
\hline ZnO (KADOX - 920 Zinc Corp. of America) (g) & 47.65 & 39.98 & 39.22 & 47.28 & 39.63 & 48.17 & 49.81 \\
\hline Zircon $\mathrm{ZrSiO}_{4}$ (Flour) Mesh 325 (AM. Mineral) (g) & 61.60 & 51.69 & 50.71 & 60.97 & 51.13 & 62.18 & 64.55 \\
\hline Sucrose as Reductant (added only to actual melter feed) (g) & 83.65 & 78.50 & 5.44 & 74.50 & 23.70 & 35.71 & 12.26 \\
\hline Measured Simulant Weight for 1 liter (g) & 1402 & 1360 & 1303 & 1337 & 1247 & 1178 & 1094 \\
\hline Sum of Additives (g) & 1124 & 933 & 1006 & 1241 & 1104 & 1426 & 1563 \\
\hline Sum of Complete Batch (g) & 2527 & 2293 & 2309 & 2578 & 2351 & 2604 & 2659 \\
\hline Final Volume (l) & 1.452 & 1.324 & 1.383 & 1.490 & 1.425 & 1.572 & 1.583 \\
\hline Measured Density on crucible batch (g/ml) & 1.74 & 1.69 & 1.67 & 1.73 & 1.65 & 1.67 & 1.68 \\
\hline Target Glass Produced (g/l simulant) & 1361 & 1166 & 1144 & 1370 & 1145 & 1388 & 1431 \\
\hline Measured Weight \% Water in Slurry Feed on crucible batch & $35 \%$ & $40 \%$ & $41 \%$ & $35 \%$ & $40 \%$ & $39 \%$ & $40 \%$ \\
\hline Target Weight \% Additives in Slurry & $45 \%$ & $48 \%$ & $44 \%$ & $48 \%$ & $47 \%$ & $54 \%$ & $59 \%$ \\
\hline Target Glass Yield for crucible batch (g/kg of Feed) & 539 & 508 & 495 & 532 & 487 & 529 & 538 \\
\hline Measured Glass Yield on crucible batch (g/kg of Feed) & 500 & 480 & 495 & 510 & 479 & 514 & 536 \\
\hline Target Glass Yield (g/l of Feed) & 938 & 859 & 827 & 920 & 804 & 883 & 1008 \\
\hline Target Total Solids (g/l of Feed) & 1126 & 1012 & 993 & 1127 & 988 & 1020 & 988 \\
\hline Target Additives (g/l of Feed) & 774 & 688 & 727 & 833 & 775 & 907 & 983 \\
\hline
\end{tabular}

* Includes $0.56 \mathrm{wt} \% \mathrm{~K}_{2} \mathrm{O}$, \# - target values given in $\mathrm{g} / \mathrm{l}$ are the product of a target $\mathrm{g} / \mathrm{kg}$ feed value and the measured density.

"-" Empty data field 
Table 2.19. Adjustments to Feed Required for Tests Targeting $1.1 \mathrm{wt} \% \mathrm{Cl}$ and $1.3 \mathrm{wt} \% \mathrm{~F}$ in the Glass Product and Corresponding Feed Properties.

\begin{tabular}{|c|c|c|c|c|c|c|c|}
\hline \multirow{2}{*}{ Test } & LAWE3 & LAWE4H & LAWE5H & LAWE6H & LAWE7H & LAWE9H & LAWE10H \\
\hline & $6 \mathrm{~B}$ & $6 \mathrm{~A}$ & $5 \mathrm{C}$ & $5 B$ & $4 \mathrm{C}$ and $5 \mathrm{~A}$ & $4 \mathrm{~B}$ & $4 \mathrm{~A}$ \\
\hline $\begin{array}{l}\text { Grams of } 50 \% \mathrm{NaOH} \text { solution per } \mathrm{kg} \text { feed } \\
\text { omitted from feed received from Optima }\end{array}$ & 41.82 & 38.49 & 36.61 & 38.92 & 36.54 & 40.52 & 40.95 \\
\hline Grams $\mathrm{NaCl}$ per kg feed & 8.65 & 7.97 & 7.58 & 8.07 & 7.57 & 8.40 & 8.48 \\
\hline Grams NaF per kg feed & 16.02 & 14.74 & 14.01 & 14.90 & 13.99 & 15.51 & 15.68 \\
\hline Grams water per kg feed & 20.91 & 19.25 & 18.30 & 19.46 & 18.27 & 20.26 & $55.52 *$ \\
\hline Measured Density (g/ml) & 1.66 & 1.68 & 1.66 & 1.70 & 1.62 & 1.73 & 1.60 \\
\hline Measured Weight \% Water in Slurry Feed & $37 \%$ & $39 \%$ & $40 \%$ & $36 \%$ & $46 \%$ & $34 \%$ & $41 \%$ \\
\hline Measured Glass Yield (g/kg of Feed) & 514 & 483 & 486 & 504 & 467 & 528 & 521 \\
\hline
\end{tabular}

* $35.05 \mathrm{~g}$ of the $55.52 \mathrm{~g}$ of water added was to obtain acceptable feed rheological properties 
The Catholic University of America Vitreous State Laboratory
DuraMelter 100 Tests to Support LAW Glass Formulation Correlation Development Final Report, VSL-06R6480-1, Rev. 0

Table 2.20. Measured Properties of Melter Feed Samples.

\begin{tabular}{|c|c|c|c|c|c|c|c|}
\hline \multirow{2}{*}{ Test } & \multirow{2}{*}{$\begin{array}{c}\text { Sampling } \\
\text { Date }\end{array}$} & \multirow{2}{*}{ Sample Name } & \multirow{2}{*}{$\%$ Water } & \multirow{2}{*}{$\begin{array}{c}\text { Density } \\
(\mathrm{g} / \mathrm{ml})\end{array}$} & \multicolumn{2}{|c|}{ Glass Yield } & \multirow{2}{*}{ pH } \\
\hline & & & & & $(\mathrm{kg} / \mathrm{kg})$ & $(g / l)$ & \\
\hline \multirow{4}{*}{$1 \mathrm{~A}$} & $6 / 13 / 05$ & WVZ-F-39A & NA & NA & NA & NA & NA \\
\hline & $6 / 14 / 05$ & WVZ-F-48A & 36.28 & 1.65 & 0.500 & 825 & 11.96 \\
\hline & $6 / 15 / 05$ & WVZ-F-65A & 36.04 & 1.62 & 0.516 & 836 & 11.83 \\
\hline & \multicolumn{2}{|c|}{ Target* } & 35.28 & 1.74 & 0.533 & 927 & 11.94 \\
\hline \multirow{3}{*}{ 1B } & $6 / 16 / 05$ & WVZ-F-80A & 40.39 & 1.66 & 0.474 & 787 & 12.88 \\
\hline & $6 / 17 / 05$ & WVZ-F-97A & 39.98 & 1.62 & 0.478 & 774 & 12.80 \\
\hline & \multicolumn{2}{|r|}{ Target } & 40.12 & 1.69 & 0.491 & 830 & 12.95 \\
\hline \multirow{2}{*}{$2 A$} & $6 / 22 / 05$ & WVZ-F-106A & 40.72 & 1.60 & 0.482 & 770 & 12.98 \\
\hline & \multicolumn{2}{|r|}{ Target } & 40.12 & 1.69 & 0.491 & 830 & 12.95 \\
\hline \multirow{3}{*}{ 2B } & $6 / 23 / 05$ & WVZ-F-140A & 41.03 & 1.68 & 0.485 & 814 & 12.63 \\
\hline & $6 / 24 / 05$ & WVZ-F-152A & 41.29 & 1.65 & 0.486 & 802 & 12.59 \\
\hline & \multicolumn{2}{|r|}{ Target } & 40.56 & 1.67 & 0.482 & 805 & 12.59 \\
\hline \multirow{3}{*}{$2 \mathrm{C}$} & $6 / 25 / 05$ & AWV-F-16A & 36.28 & 1.70 & 0.497 & 845 & 10.74 \\
\hline & $6 / 26 / 05$ & AWV-F-30A & 36.53 & 1.71 & 0.497 & 850 & 10.47 \\
\hline & \multicolumn{2}{|r|}{ Target } & 34.83 & 1.73 & 0.499 & 863 & 9.87 \\
\hline \multirow{4}{*}{ 2D } & $6 / 27 / 05$ & AWV-F-54A & 41.25 & 1.56 & 0.469 & 732 & 9.32 \\
\hline & $6 / 28 / 05$ & AWV-F-68A & 46.01 & 1.60 & 0.474 & 758 & 9.24 \\
\hline & $6 / 29 / 05$ & AWV-F-78A & 40.44 & 1.64 & 0.479 & 786 & 9.18 \\
\hline & \multicolumn{2}{|r|}{ Target } & 40.15 & 1.65 & 0.478 & 789 & 7.61 \\
\hline \multirow{2}{*}{$\mathbf{3 A}$} & 7/6/05 & AWV-F-97A & 41.98 & 1.62 & 0.479 & 775 & 9.37 \\
\hline & \multicolumn{2}{|r|}{ Target } & 40.15 & 1.65 & 0.478 & 789 & 7.61 \\
\hline \multirow{3}{*}{ 3B } & $7 / 7 / 05$ & AWV-F-118A & 40.89 & 1.66 & 0.504 & 837 & 9.93 \\
\hline & $7 / 8 / 05$ & AWV-F-135A & 40.36 & 1.67 & 0.506 & 846 & 9.89 \\
\hline & \multicolumn{2}{|r|}{ Target } & 38.91 & 1.67 & 0.526 & 878 & 7.84 \\
\hline & $7 / 9 / 05$ & AWV-F-148A & 40.59 & 1.66 & 0.533 & 884 & 9.88 \\
\hline $3 C$ & $7 / 10 / 05$ & BWV-F-14A & 41.32 & 1.66 & 0.525 & 872 & 9.85 \\
\hline $3 \mathrm{C}$ & $7 / 11 / 05$ & BWV-F-32A & 40.10 & 1.67 & 0.540 & 901 & 9.91 \\
\hline & & Target & 39.81 & 1.68 & 0.538 & 904 & 7.50 \\
\hline$\Delta \mathbf{A}$ & $8 / 15 / 05$ & BWV-F-58A & 40.66 & 1.50 & 0.484 & 726 & 8.77 \\
\hline 4A & & Target & 41.45 & 1.60 & 0.519 & 830 & 8.74 \\
\hline & $8 / 16 / 05$ & BWV-F-72A & NA & NA & NA & NA & NA \\
\hline $4 B$ & $8 / 17 / 05$ & BWV-F-86A & 39.88 & 1.67 & 0.516 & 862 & 8.22 \\
\hline & & Target & 38.84 & 1.69 & 0.524 & 886 & 8.54 \\
\hline & $8 / 18 / 05$ & BWV-F-111A & NA & NA & NA & NA & NA \\
\hline$\Delta \Gamma$ & $8 / 19 / 05$ & BWV-F-128A & 42.36 & 1.59 & 0.458 & 728 & 7.79 \\
\hline 40 & $8 / 20 / 05$ & BWV-F-142A & NA & NA & NA & NA & NA \\
\hline & & Target & 45.83 & 1.62 & 0.476 & 771 & 7.69 \\
\hline $5 \mathbf{A}$ & $8 / 24 / 05$ & CWV-F-7A & 42.27 & 1.61 & 0.466 & 750 & 7.88 \\
\hline SA & & Target & 45.83 & 1.62 & 0.476 & 771 & 7.69 \\
\hline & $8 / 25 / 05$ & CWV-F-35A & 30.28 & 1.68 & 0.495 & 831 & 9.28 \\
\hline $5 B$ & $8 / 26 / 05$ & CWV-F-45A & 36.69 & 1.70 & NA & NA & 9.09 \\
\hline & & Target & 35.68 & 1.70 & 0.497 & 845 & 8.19 \\
\hline
\end{tabular}

NA - Not analyzed

* - Values from tests of crucible batch feeds except glass yield $(\mathrm{kg} / \mathrm{kg})$ which is calculated from recipe. 
Table 2.20. Measured Properties of Melter Feed Samples (continued).

\begin{tabular}{|c|c|c|c|c|c|c|c|}
\hline \multirow{2}{*}{ Test } & \multirow{2}{*}{$\begin{array}{c}\text { Sampling } \\
\text { Date }\end{array}$} & \multirow{2}{*}{ Sample Name } & \multirow{2}{*}{ \%Water } & \multirow{2}{*}{$\begin{array}{c}\text { Density } \\
(\mathrm{g} / \mathrm{ml})\end{array}$} & \multicolumn{2}{|c|}{ Glass Yield } & \multirow{2}{*}{$\mathbf{p H}$} \\
\hline & & & & & (kg/kg) & (g/l) & \\
\hline \multirow{3}{*}{$5 C$} & 8/27/05 & CWV-F-61A & NA & NA & NA & NA & NA \\
\hline & $8 / 28 / 05$ & CWV-F-75A & 40.65 & 1.65 & 0.492 & 811 & 11.29 \\
\hline & \multicolumn{2}{|c|}{ Target } & 40.43 & 1.66 & 0.481 & 798 & 11.18 \\
\hline \multirow{4}{*}{$5 D$} & $8 / 29 / 05$ & CWV-F-97A & NA & NA & NA & NA & NA \\
\hline & $8 / 30 / 05$ & CWV-F-105A & 39.66 & 1.66 & 0.465 & 772 & 11.63 \\
\hline & $8 / 31 / 05$ & CWV-F-125A & 30.36 & 1.60 & 0.485 & 778 & 11.63 \\
\hline & \multicolumn{2}{|c|}{ Target } & 38.83 & 1.68 & 0.487 & 818 & 11.42 \\
\hline \multirow{3}{*}{$6 A$} & $9 / 12 / 05$ & CWV-F-132A & 40.04 & 1.66 & 0.467 & 775 & 11.49 \\
\hline & $9 / 13 / 05$ & CWV-F-155A & 33.53 & 1.58 & 0.469 & 740 & 11.61 \\
\hline & \multicolumn{2}{|c|}{$\begin{array}{l}\text { Target } \\
\end{array}$} & 38.83 & 1.68 & 0.489 & 822 & 11.42 \\
\hline \multirow{3}{*}{ 6B } & $9 / 14 / 05$ & DWV-F-39A & 38.04 & 1.74 & 0.517 & 899 & 11.38 \\
\hline & $9 / 15 / 05$ & DWV-F-52A & 32.52 & 1.75 & 0.520 & 910 & 10.97 \\
\hline & \multicolumn{2}{|c|}{ Target } & 33.86 & 1.66 & 0.531 & 891 & 11.01 \\
\hline
\end{tabular}

NA - Not analyzed

* - Values from tests of crucible batch feeds except glass yield (kg/kg) which is calculated from recipe. 
Table 2.21. Rheological Properties for Select Melter Feed Samples.

\begin{tabular}{|c|c|c|c|c|c|c|}
\hline \multirow{2}{*}{$\begin{array}{c}\text { Waste } \\
\text { Composition }\end{array}$} & \multirow{2}{*}{ Test } & \multirow{2}{*}{ Sample Name } & \multirow{2}{*}{\begin{tabular}{|c|} 
Yield Stress \\
$(\mathrm{Pa})$
\end{tabular}} & \multicolumn{3}{|c|}{ Viscosity (Poise) } \\
\hline & & & & @1/s & @10/s & @100/s \\
\hline \multirow{3}{*}{$\begin{array}{c}\text { LAW } \\
\text { Envelope A }\end{array}$} & $2 \mathrm{~A}$ & WVZ-F-97A & 0.5 & 2.10 & 0.49 & 0.31 \\
\hline & $5 \mathrm{D}$ & CWV-F-132A & 1.3 & 5.30 & 0.80 & 0.38 \\
\hline & Average from previous & DM100 Test [8] & 0.5 & 3.14 & 0.64 & 0.39 \\
\hline \multirow{2}{*}{$\begin{array}{c}\text { LAW } \\
\text { Envelope B }\end{array}$} & $3 C$ & BWV-F-14A & 17.9 & 46.9 & 5.49 & 1.34 \\
\hline & Average from previous & DM100 Test [13] & 13.8 & 23.2 & 4.4 & 1.4 \\
\hline
\end{tabular}


Table 2.22. XRF-Analyzed Compositions of Feed Samples (wt\%).

\begin{tabular}{|c|c|c|c|c|c|c|c|c|c|c|c|c|c|c|c|c|}
\hline \multirow{2}{*}{ Test } & \multicolumn{6}{|c|}{ 1A } & \multicolumn{5}{|c|}{ 1B } & \multicolumn{5}{|c|}{ 2B } \\
\hline & Target & WVZ-F-39A & WVZ-F-48A & WVZ-F-65A & Avg. & \%Dev. & Target & WVZ-F-80A & WVZ-F-97A & Avg. & \%Dev. & Target & WVZ-F-140 & AWVZ-F-152A & Avg. & $\%$ Dev. \\
\hline $\mathrm{Al}_{2} \mathrm{O}_{3}$ & 6.09 & 5.87 & 5.96 & 6.08 & 5.97 & -2.02 & 5.96 & 5.92 & 5.96 & 5.94 & -0.44 & 5.98 & 6.11 & 5.78 & 5.95 & -0.65 \\
\hline $\mathrm{B}_{2} \mathrm{O}^{*} *$ & 9.99 & 9.99 & 9.99 & 9.99 & 9.99 & $\mathrm{NC}$ & 9.78 & 9.78 & 9.78 & 9.78 & $\mathrm{NC}$ & 9.80 & 9.80 & 9.80 & 9.80 & $\mathrm{NC}$ \\
\hline $\mathrm{CaO}$ & 2.02 & 1.90 & 2.04 & 1.92 & 1.95 & -3.15 & 2.46 & 2.22 & 2.29 & 2.25 & -8.29 & 3.61 & 3.31 & 3.48 & 3.39 & -5.93 \\
\hline $\mathrm{Cl}$ & 0.20 & 0.07 & 0.12 & 0.07 & 0.09 & $\mathrm{NC}$ & 0.20 & 0.10 & 0.06 & 0.08 & $\mathrm{NC}$ & 0.20 & 0.05 & 0.05 & 0.05 & $\mathrm{NC}$ \\
\hline $\mathrm{Cr}_{2} \mathrm{O}_{3}$ & 0.08 & 0.13 & 0.15 & 0.14 & 0.14 & $\mathrm{NC}$ & 0.08 & 0.13 & 0.14 & 0.14 & NC & 0.08 & 0.14 & 0.14 & 0.14 & NC \\
\hline $\mathrm{F}$ & 0.08 & $<0.08$ & $<0.08$ & NA & $<0.08$ & $\mathrm{NC}$ & 0.08 & $<0.08$ & $<0.08$ & 0.00 & NC & 0.08 & $<0.08$ & $<0.08$ & $<0.08$ & $\mathrm{NC}$ \\
\hline $\mathrm{K}_{2} \mathrm{O}$ & 4.98 & 4.42 & 5.00 & 4.68 & 4.70 & -5.62 & $\S$ & 0.18 & 0.15 & 0.16 & $\mathrm{NC}$ & 0.54 & 0.61 & 0.61 & 0.61 & $\mathrm{NC}$ \\
\hline $\mathrm{Li}_{2} \mathrm{O}^{*}$ & $\S$ & $<0.01$ & $<0.01$ & $<0.01$ & $<0.01$ & $\mathrm{NC}$ & $\S$ & $<0.01$ & $<0.01$ & $<0.01$ & $\mathrm{NC}$ & 0.49 & 0.49 & 0.49 & 0.49 & NC \\
\hline $\mathrm{MgO}$ & 1.48 & 1.59 & 1.37 & 1.50 & 1.49 & 0.82 & 1.45 & 1.46 & 1.71 & 1.59 & 9.53 & 1.45 & 1.41 & 1.27 & 1.34 & -7.58 \\
\hline $\mathrm{Na}_{2} \mathrm{O}$ & 18.19 & 18.68 & 17.53 & 18.35 & \begin{tabular}{|l|}
18.19 \\
\end{tabular} & -0.03 & 21.25 & 22.70 & 21.32 & 22.01 & 3.60 & 18.95 & 18.99 & 20.08 & 19.53 & 3.08 \\
\hline $\mathrm{NiO}$ & 0.01 & $<0.01$ & $<0.01$ & $<0.01$ & $<0.01$ & $\mathrm{NC}$ & 0.01 & $<0.01$ & $<0.01$ & $<0.01$ & $\mathrm{NC}$ & 0.01 & $<0.01$ & $<0.01$ & $<0.01$ & $\mathrm{NC}$ \\
\hline $\mathrm{P}_{2} \mathrm{O}_{5}$ & 0.12 & 0.17 & 0.19 & 0.19 & 0.18 & $\mathrm{NC}$ & 0.12 & 0.17 & 0.17 & 0.17 & $\mathrm{NC}$ & 0.12 & 0.18 & 0.18 & 0.18 & NC \\
\hline $\mathrm{PbO}$ & 0.01 & 0.01 & 0.01 & 0.08 & 0.03 & $\mathrm{NC}$ & 0.01 & $<0.01$ & $<0.01$ & $<0.01$ & $\mathrm{NC}$ & 0.01 & 0.01 & 0.01 & 0.01 & $\mathrm{NC}$ \\
\hline $\mathrm{SiO}_{2}$ & 42.91 & 42.85 & 43.85 & 44.20 & 43.63 & 1.68 & 44.99 & 45.56 & 46.19 & 45.87 & 1.96 & 45.00 & 46.75 & 44.89 & 45.82 & 1.83 \\
\hline $\mathrm{SO}_{3}$ & 0.35 & 0.24 & 0.31 & 0.30 & 0.28 & $\mathrm{NC}$ & 0.41 & 0.32 & 0.29 & 0.31 & $\mathrm{NC}$ & 0.46 & 0.33 & 0.33 & 0.33 & $\mathrm{NC}$ \\
\hline $\mathrm{TiO}_{2}$ & 1.40 & 1.74 & 1.63 & 1.52 & 1.63 & 16.48 & 1.37 & 1.34 & 1.40 & 1.37 & -0.10 & 1.37 & 1.49 & 1.57 & 1.53 & 11.77 \\
\hline $\mathrm{V}_{2} \mathrm{O}_{5}$ & $\S$ & 0.01 & 0.01 & 0.01 & 0.01 & $\mathrm{NC}$ & $\S$ & 0.01 & 0.01 & 0.01 & $\mathrm{NC}$ & $\S$ & 0.01 & 0.01 & 0.01 & $\mathrm{NC}$ \\
\hline $\mathrm{ZnO}$ & 3.50 & 3.30 & 3.18 & 2.91 & 3.13 & -10.44 & 3.43 & 2.74 & 2.85 & 2.79 & -18.47 & 3.43 & 2.85 & 3.14 & 3.00 & -12.48 \\
\hline $\mathrm{ZrO}_{2}$ & 3.00 & 3.79 & 3.17 & 2.93 & 3.30 & 10.04 & 2.94 & 2.63 & 2.65 & 2.64 & -10.15 & 2.94 & 2.55 & 2.97 & 2.76 & -6.00 \\
\hline Sum 1 & 100.00 & 100.00 & 100.00 & 100.00 & 100.00 & $\mathrm{NC}$ & 100.00 & 100.00 & 100.00 & 100.00 & $\mathrm{NC}$ & 100.00 & 100.00 & 100.00 & 100.00 & NC \\
\hline
\end{tabular}


Table 2.22. XRF-Analyzed Compositions of Feed Samples (wt\%) (continued).

\begin{tabular}{|c|c|c|c|c|c|c|c|c|c|c|c|c|c|c|c|c|c|}
\hline \multirow[b]{2}{*}{ Test } & \multicolumn{5}{|c|}{$2 \mathrm{C}$} & \multicolumn{7}{|c|}{$2 D$ and $3 A$} & \multicolumn{5}{|c|}{ 3B } \\
\hline & Target & \begin{tabular}{|l|} 
AWV- \\
F-16A
\end{tabular} & $\begin{array}{l}\text { AWV- } \\
\text { F-30A }\end{array}$ & Avg. & \%Dev. & Target & $\begin{array}{l}\text { AWV- } \\
\text { F-54A } \\
\end{array}$ & $\begin{array}{l}\text { AWV- } \\
\text { F-68A }\end{array}$ & $\begin{array}{l}\text { AWV- } \\
\text { F-78A }\end{array}$ & $\begin{array}{l}\text { AWV- } \\
\text { F-97A }\end{array}$ & Avg. & $\%$ Dev. & Target & $\begin{array}{l}\text { AWV- } \\
\text { F-118A }\end{array}$ & $\begin{array}{l}\text { AWV- } \\
F-135 A\end{array}$ & Avg. & $\%$ Dev \\
\hline $\mathrm{Al}_{2} \mathrm{O}_{3}$ & 6.00 & 5.85 & 6.00 & 5.92 & -1.36 & 6.01 & 5.70 & 5.89 & 6.03 & 6.07 & 5.92 & -1.50 & 6.05 & 6.03 & 5.96 & 6.00 & -0.82 \\
\hline $\mathrm{B}_{2} \mathrm{O}_{3} *$ & 9.84 & 9.84 & 9.84 & 9.84 & $\mathrm{NC}$ & 9.86 & 9.86 & 9.86 & 9.86 & 9.86 & 9.86 & NC & 9.91 & 9.91 & 9.91 & 9.91 & $\mathrm{NC}$ \\
\hline $\mathrm{CaO}$ & 5.45 & 5.21 & 5.17 & 5.19 & -4.86 & 6.30 & 6.11 & 6.04 & 5.93 & 6.13 & 6.05 & -3.97 & 6.85 & 6.38 & 6.34 & 6.36 & -7.20 \\
\hline $\mathrm{Cl}$ & 0.20 & 0.01 & 0.02 & 0.01 & $\mathrm{NC}$ & 0.20 & 0.02 & 0.02 & 0.02 & 0.01 & 0.02 & $\mathrm{NC}$ & 0.20 & 0.06 & 0.07 & 0.07 & $\mathrm{NC}$ \\
\hline $\mathrm{Cr}_{2} \mathrm{O}_{3}$ & 0.08 & 0.15 & 0.15 & 0.15 & $\mathrm{NC}$ & 0.08 & 0.14 & 0.14 & 0.13 & 0.14 & 0.14 & NC & 0.08 & 0.16 & 0.15 & 0.15 & $\mathrm{NC}$ \\
\hline $\mathrm{F}$ & 0.08 & NA & NA & $\mathrm{NC}$ & $\mathrm{NC}$ & 0.08 & NA & NA & NA & NA & $\mathrm{NC}$ & $\mathrm{NC}$ & 0.08 & NA & NA & $\mathrm{NC}$ & $\mathrm{NC}$ \\
\hline $\mathrm{Fe}_{2} \mathrm{O}_{3}$ & 5.41 & 5.41 & 5.22 & 5.32 & -1.80 & 5.42 & 5.55 & 5.42 & 5.34 & 5.34 & 5.41 & -0.24 & 5.44 & 5.17 & 5.08 & 5.13 & -5.86 \\
\hline $\mathrm{K}_{2} \mathrm{O}$ & 0.54 & 0.59 & 0.60 & 0.60 & $\mathrm{NC}$ & 0.54 & 0.65 & 0.61 & 0.63 & 0.66 & 0.64 & NC & 0.54 & 0.65 & 0.62 & 0.63 & $\mathrm{NC}$ \\
\hline $\mathrm{Li}_{2} \mathrm{O}^{*}$ & 2.38 & 2.38 & 2.38 & 2.38 & $\mathrm{NC}$ & 3.17 & 3.17 & 3.17 & 3.17 & 3.17 & 3.17 & NC & 4.08 & 4.08 & 4.08 & 4.08 & NC \\
\hline $\mathrm{MgO}$ & 1.46 & 1.36 & 1.42 & 1.39 & -4.74 & 1.49 & 1.51 & 1.57 & 1.55 & 1.19 & 1.45 & -2.38 & 2.36 & 2.28 & 2.29 & 2.28 & -3.09 \\
\hline $\mathrm{Na}_{2} \mathrm{O}$ & 15.81 & 16.40 & 16.18 & 16.29 & 3.01 & 13.52 & 13.60 & 13.43 & 12.84 & 13.38 & 13.31 & -1.51 & 8.92 & 9.45 & 9.50 & 9.48 & 6.24 \\
\hline $\mathrm{NiO}$ & 0.01 & $<0.01$ & $<0.01$ & $<0.01$ & $\mathrm{NC}$ & 0.01 & $<0.01$ & $<0.01$ & $<0.01$ & $<0.01$ & 0.00 & $\mathrm{NC}$ & 0.01 & $<0.01$ & $<0.01$ & $<0.01$ & $\mathrm{NC}$ \\
\hline $\mathrm{P}_{2} \mathrm{O}_{5}$ & 0.12 & 0.17 & 0.17 & 0.17 & NC & 0.12 & 0.18 & 0.18 & 0.18 & 0.18 & 0.18 & NC & 0.12 & 0.19 & 0.18 & 0.19 & $\mathrm{NC}$ \\
\hline $\mathrm{PbO}$ & 0.01 & 0.01 & $<0.01$ & 0.01 & $\mathrm{NC}$ & 0.01 & $<0.01$ & $<0.01$ & $<0.01$ & $<0.01$ & $<0.01$ & NC & 0.01 & $<0.01$ & $<0.01$ & $<0.01$ & $\mathrm{NC}$ \\
\hline $\mathrm{ReO}_{2}$ & 0.10 & $<0.01$ & 0.01 & $<0.01$ & $\mathrm{NC}$ & 0.10 & $<0.01$ & $<0.01$ & $<0.01$ & $<0.01$ & $<0.01$ & $\mathrm{NC}$ & 0.10 & 0.02 & 0.02 & 0.02 & $\mathrm{NC}$ \\
\hline $\mathrm{SiO}_{2}$ & 44.21 & 44.40 & 45.00 & 44.70 & 1.12 & 44.71 & 45.17 & 45.84 & 46.44 & 45.50 & 45.74 & 2.30 & 46.75 & 47.79 & 48.01 & 47.90 & 2.46 \\
\hline $\mathrm{SO}_{3}$ & 0.53 & 0.29 & 0.28 & 0.28 & $\mathrm{NC}$ & 0.59 & 0.31 & 0.29 & 0.28 & 0.28 & 0.29 & NC & 0.69 & 0.33 & 0.33 & 0.33 & $\mathrm{NC}$ \\
\hline $\mathrm{TiO}_{2}$ & 1.38 & 1.48 & 1.43 & 1.45 & 5.45 & 1.38 & 1.57 & 1.53 & 1.50 & 1.62 & 1.55 & 12.76 & 1.39 & 1.56 & 1.56 & 1.56 & 12.28 \\
\hline $\mathrm{V}_{2} \mathrm{O}_{5}$ & $\S$ & 0.01 & 0.01 & 0.01 & $\mathrm{NC}$ & $\S$ & 0.01 & 0.01 & 0.01 & $<0.01$ & 0.01 & $\mathrm{NC}$ & $\S$ & 0.01 & $<0.01$ & 0.01 & $\mathrm{NC}$ \\
\hline $\mathrm{ZnO}$ & 3.45 & 3.28 & 3.13 & 3.21 & -7.00 & 3.46 & 3.29 & 3.11 & 3.06 & 3.35 & 3.20 & -7.37 & 3.47 & 3.01 & 2.99 & 3.00 & -13.41 \\
\hline $\mathrm{ZrO}_{2}$ & 2.95 & 3.17 & 3.00 & 3.08 & 4.63 & 2.96 & 3.16 & 2.92 & 3.02 & 3.12 & 3.05 & 3.27 & 2.97 & 2.91 & 2.90 & 2.90 & -2.10 \\
\hline Sum & 100.00 & 100.00 & 100.00 & 100.00 & $\mathrm{NC}$ & 100.00 & 100.00 & 100.00 & 100.00 & 100.00 & 100.00 & NC & 100.00 & 100.00 & 100.00 & 100.00 & NC \\
\hline
\end{tabular}

* Target values

$\S$ - Not a target constituent

NA - Not analyzed; NC - Not calculated 
Table 2.22. XRF-Analyzed Compositions of Feed Samples (wt\%) (continued).

\begin{tabular}{|c|c|c|c|c|c|c|c|c|c|c|c|c|c|c|c|c|c|}
\hline \multirow[b]{2}{*}{ Test } & \multicolumn{6}{|c|}{$3 C$} & \multicolumn{3}{|c|}{$4 A$} & \multicolumn{3}{|c|}{ 4B } & \multicolumn{5}{|c|}{$4 C$ and $5 A$} \\
\hline & Target & \begin{tabular}{|c|} 
AWV- \\
F-148A
\end{tabular} & \begin{tabular}{|l|} 
BWV- \\
F-14A
\end{tabular} & \begin{tabular}{|l|} 
BWV- \\
F-32A
\end{tabular} & Avg. & \%Dev. & Target & \begin{tabular}{|l|} 
BWV- \\
F-58A
\end{tabular} & $\%$ Dev. & Target & $\mid \begin{array}{l}\text { BWV- } \\
\text { F-86A }\end{array}$ & \%Dev. & Target & $\left|\begin{array}{c}\text { BWV- } \\
F-128 A\end{array}\right|$ & \begin{tabular}{|c|} 
CWV- \\
F-7A
\end{tabular} & Avg. & $\%$ Dev. \\
\hline $\mathrm{Al}_{2} \mathrm{O}_{3}$ & 6.06 & 6.11 & 6.07 & 6.02 & 6.07 & 0.04 & 5.93 & 6.20 & 4.48 & 5.92 & 6.07 & 2.59 & 5.89 & 5.91 & 6.07 & 5.99 & 1.76 \\
\hline $\mathrm{B}_{2} \mathrm{O}_{3} *$ & 9.94 & 9.94 & 9.94 & 9.94 & 9.94 & NC & 9.73 & 9.73 & NC & 9.70 & 9.70 & NC & 9.65 & 9.65 & 9.65 & 9.65 & NC \\
\hline $\mathrm{CaO}$ & 6.95 & 6.62 & 6.63 & 6.69 & 6.65 & -4.42 & 6.80 & 6.68 & -1.77 & 6.71 & 6.56 & -2.13 & 6.17 & 6.23 & 5.77 & 6.00 & -2.80 \\
\hline $\mathrm{Cl}$ & 0.20 & 0.09 & 0.09 & 0.11 & 0.10 & NC & 1.10 & 0.33 & NC & 1.10 & 0.15 & NC & 1.10 & 0.11 & 0.48 & 0.29 & NC \\
\hline $\mathrm{Cr}_{2} \mathrm{O}_{3}$ & 0.08 & 0.16 & 0.17 & 0.16 & 0.16 & $\mathrm{NC}$ & 0.08 & 0.15 & $\mathrm{NC}$ & 0.08 & 0.15 & NC & 0.08 & 0.13 & 0.14 & 0.14 & $\mathrm{NC}$ \\
\hline $\mathrm{F}$ & 0.08 & NA & NA & NA & $\mathrm{NC}$ & NC & 1.30 & 0.17 & $\mathrm{NC}$ & 1.30 & 0.26 & $\mathrm{NC}$ & 1.30 & 0.27 & 0.25 & 0.26 & NC \\
\hline $\mathrm{Fe}_{2} \mathrm{O}_{3}$ & 5.47 & 5.55 & 5.44 & 5.51 & 5.50 & 0.46 & 5.36 & 5.23 & -2.32 & 5.33 & 5.32 & -0.19 & 5.31 & 5.28 & 4.76 & 5.02 & -5.45 \\
\hline $\mathrm{K}_{2} \mathrm{O}$ & 0.54 & 0.66 & 0.69 & 0.66 & 0.67 & NC & 0.53 & 0.62 & NC & 0.53 & 0.59 & NC & 0.53 & 0.60 & 0.63 & 0.61 & NC \\
\hline $\mathrm{Li}_{2} \mathrm{O}^{*}$ & 4.26 & 4.26 & 4.26 & 4.26 & 4.26 & NC & 4.17 & 4.17 & NC & 3.99 & 3.99 & $\mathrm{NC}$ & 3.10 & 3.10 & 3.10 & 3.10 & NC \\
\hline $\mathrm{MgO}$ & 2.94 & 3.07 & 2.88 & 2.88 & 2.95 & 0.30 & 2.87 & 2.77 & -3.47 & 2.31 & 2.32 & 0.50 & 1.46 & 1.22 & 1.39 & 1.30 & -10.54 \\
\hline $\mathrm{Na}_{2} \mathrm{O}$ & 5.71 & 5.97 & 5.78 & 5.75 & 5.84 & 2.11 & 5.59 & 5.84 & 4.38 & 8.73 & 8.57 & -1.82 & 13.23 & 13.24 & 13.50 & 13.37 & 1.07 \\
\hline $\mathrm{NiO}$ & 0.01 & $<0.01$ & $<0.01$ & $<0.01$ & $<0.01$ & NC & 0.01 & $<0.01$ & $\mathrm{NC}$ & 0.01 & $<0.01$ & NC & 0.01 & $<0.01$ & $<0.01$ & $<0.01$ & NC \\
\hline $\mathrm{P}_{2} \mathrm{O}_{5}$ & 0.12 & 0.18 & 0.18 & 0.18 & 0.18 & NC & 0.12 & 0.22 & NC & 0.12 & 0.19 & NC & 0.12 & 0.16 & 0.18 & 0.17 & NC \\
\hline $\mathrm{PbO}$ & 0.01 & $<0.01$ & $<0.01$ & $<0.01$ & $<0.01$ & NC & 0.01 & $<0.01$ & NC & 0.01 & $<0.01$ & NC & 0.01 & $<0.01$ & $<0.01$ & $<0.01$ & NC \\
\hline $\mathrm{ReO}_{2}$ & 0.10 & 0.05 & 0.05 & 0.04 & 0.05 & NC & 0.10 & 0.03 & NC & 0.10 & 0.02 & NC & 0.10 & 0.01 & 0.02 & 0.01 & NC \\
\hline $\mathrm{SiO}_{2}$ & 48.88 & 48.95 & 49.38 & 49.19 & 49.17 & 0.61 & 47.83 & 49.93 & 4.37 & 45.75 & 48.17 & 5.29 & 43.76 & 46.27 & 46.70 & \begin{tabular}{|l|}
46.48 \\
\end{tabular} & 6.23 \\
\hline $\mathrm{SO}_{3}$ & 0.80 & 0.45 & 0.46 & 0.50 & 0.47 & NC & 0.78 & 0.39 & $\mathrm{NC}$ & 0.67 & 0.23 & $\mathrm{NC}$ & 0.58 & 0.25 & 0.61 & 0.43 & NC \\
\hline $\mathrm{TiO}_{2}$ & 1.39 & 1.59 & 1.59 & 1.60 & 1.59 & 14.60 & 1.36 & 1.58 & 16.55 & 1.36 & 1.61 & 18.17 & 1.35 & 1.63 & 1.46 & 1.55 & 14.69 \\
\hline $\mathrm{V}_{2} \mathrm{O}_{5}$ & $\S$ & 0.01 & 0.01 & 0.01 & 0.01 & NC & $\S$ & $<0.01$ & $\mathrm{NC}$ & $\S$ & 0.01 & $\mathrm{NC}$ & $\S$ & $<0.01$ & 0.01 & 0.01 & NC \\
\hline $\mathrm{ZnO}$ & 3.48 & 3.29 & 3.27 & 3.30 & 3.29 & -5.39 & 3.40 & 3.07 & -9.64 & 3.39 & 3.09 & -8.86 & 3.38 & 2.88 & 2.82 & 2.85 & -15.84 \\
\hline $\mathrm{ZrO}_{2}$ & 2.99 & 3.06 & 3.10 & 3.19 & 3.12 & 4.28 & 2.92 & 2.87 & -1.69 & 2.90 & 3.01 & 3.66 & 2.89 & 3.09 & 2.45 & 2.77 & -4.33 \\
\hline Sum & 100.00 & 100.00 & 100.00 & 100.00 & 100.00 & $\mathrm{NC}$ & 100.00 & 100.00 & $\mathrm{NC}$ & 100.00 & 100.00 & $\mathrm{NC}$ & 100.00 & 100.00 & 100.00 & 100.00 & NC \\
\hline
\end{tabular}


Table 2.22. XRF-Analyzed Compositions of Feed Samples (wt\%) (continued).

\begin{tabular}{|c|c|c|c|c|c|c|c|c|c|c|c|c|c|c|c|c|c|c|c|}
\hline \multirow[b]{2}{*}{ Test } & \multicolumn{5}{|c|}{$5 B$} & \multicolumn{3}{|c|}{$5 C$} & \multicolumn{3}{|c|}{$5 D$} & \multicolumn{3}{|c|}{ 6A } & \multicolumn{5}{|c|}{ 6B } \\
\hline & Target & $\begin{array}{l}\text { CWV- } \\
\text { F-35A }\end{array}$ & $\begin{array}{l}\text { CWV- } \\
\text { F-45A }\end{array}$ & Avg. & $\%$ Dev. & Target & $\begin{array}{l}\text { CWV- } \\
\text { F-75A }\end{array} \mid$ & \%Dev. & Target & \begin{tabular}{|c|} 
CWV- \\
F- \\
$125 \mathrm{~A}$ \\
\end{tabular} & $\%$ Dev. & Target & $\begin{array}{c}\text { CWV- } \\
\text { F- } \\
132 \mathrm{~A}\end{array}$ & $\%$ Dev. & Target & $\begin{array}{l}\text { DWV- } \\
\text { F-39A }\end{array}$ & $\begin{array}{l}\text { DWV- } \\
\text { F-52A }\end{array}$ & Avg. & $\%$ Dev. \\
\hline $\mathrm{Al}_{2} \mathrm{O}_{3}$ & 5.88 & 6.30 & 5.69 & 5.99 & 2.02 & 5.86 & 5.88 & 0.44 & 5.81 & 6.19 & 6.70 & 5.84 & 6.38 & 9.32 & 5.96 & 6.29 & 6.72 & 6.50 & 9.03 \\
\hline $\mathrm{B}_{2} \mathrm{O}_{3}{ }^{*}$ & 9.63 & 9.63 & 9.63 & 9.63 & $\mathrm{NC}$ & 9.59 & 9.59 & $\mathrm{NC}$ & 9.52 & 9.52 & NC & 9.57 & 9.57 & NC & 9.78 & 9.78 & 9.78 & 9.78 & $\mathrm{NC}$ \\
\hline $\mathrm{CaO}$ & 5.34 & 5.16 & 5.24 & 5.20 & -2.61 & 3.53 & 3.38 & -4.13 & 2.39 & 2.37 & -0.84 & 2.41 & 2.39 & -0.66 & 1.98 & 1.99 & 2.05 & 2.02 & 2.40 \\
\hline $\mathrm{Cl}$ & 1.10 & 0.04 & 0.50 & 0.27 & $\mathrm{NC}$ & 1.10 & 0.51 & $\mathrm{NC}$ & 1.10 & 0.37 & $\mathrm{NC}$ & 1.10 & 0.28 & $\mathrm{NC}$ & 1.10 & 0.19 & 0.20 & 0.20 & $\mathrm{NC}$ \\
\hline $\mathrm{Cr}_{2} \mathrm{O}_{3}$ & 0.08 & 0.14 & 0.15 & 0.14 & NC & 0.08 & 0.14 & $\mathrm{NC}$ & 0.08 & 0.13 & NC & 0.08 & 0.14 & $\mathrm{NC}$ & 0.08 & 0.11 & 0.11 & 0.11 & NC \\
\hline $\mathrm{F}$ & 1.30 & 0.42 & 0.42 & 0.42 & NC & 1.30 & 0.39 & $\mathrm{NC}$ & 1.30 & 0.13 & $\mathrm{NC}$ & 1.30 & 0.32 & $\mathrm{NC}$ & 1.30 & 0.30 & 0.30 & 0.30 & $\mathrm{NC}$ \\
\hline $\mathrm{Fe}_{2} \mathrm{O}_{3}$ & 5.30 & 5.38 & 5.35 & 5.37 & 1.30 & 5.28 & 4.97 & -5.94 & 5.23 & 5.34 & 2.14 & 5.26 & 5.09 & -3.31 & 5.38 & 5.30 & 5.38 & 5.34 & \begin{tabular}{|l|}
-0.69 \\
\end{tabular} \\
\hline $\mathrm{K}_{2} \mathrm{O}$ & 0.53 & 0.50 & 0.58 & 0.54 & NC & 0.53 & 0.60 & $\mathrm{NC}$ & 0.52 & $<0.01$ & NC & $\S$ & 0.12 & $\mathrm{NC}$ & 4.87 & 4.36 & 4.48 & 4.42 & -9.25 \\
\hline $\mathrm{Li}_{2} \mathrm{O}^{*}$ & 2.33 & 2.33 & 2.33 & 2.33 & NC & 0.48 & 0.48 & NC & $\S$ & $<0.01$ & NC & $\S$ & $<0.01$ & NC & $\S$ & $<0.01$ & $<0.01$ & $<0.01$ & NC \\
\hline $\mathrm{MgO}$ & 1.43 & 1.43 & 1.44 & 1.44 & 0.60 & 1.42 & 1.37 & -3.29 & 1.41 & 1.39 & -1.29 & 1.42 & 1.27 & -10.69 & 1.45 & 1.52 & 1.50 & 1.51 & 4.50 \\
\hline $\mathrm{Na}_{2} \mathrm{O}$ & 15.48 & $\mid 15.19$ & 15.24 & 15.21 & -1.70 & 18.55 & 18.84 & 1.56 & 20.68 & 20.20 & -2.33 & 20.80 & 20.69 & -0.49 & 17.81 & 18.42 & 17.69 & 18.06 & 1.42 \\
\hline $\mathrm{NiO}$ & 0.01 & 0.00 & $<0.01$ & 0.00 & NC & 0.01 & $<0.01$ & NC & 0.01 & 0.00 & NC & 0.01 & $<0.01$ & NC & 0.01 & $<0.01$ & $<0.01$ & $<0.01$ & NC \\
\hline $\mathrm{P}_{2} \mathrm{O}_{5}$ & 0.12 & 0.18 & 0.19 & 0.18 & NC & 0.12 & 0.19 & $\mathrm{NC}$ & 0.12 & 0.16 & $\mathrm{NC}$ & 0.12 & 0.16 & $\mathrm{NC}$ & 0.12 & 0.18 & 0.19 & 0.18 & $\mathrm{NC}$ \\
\hline $\mathrm{PbO}$ & 0.01 & 0.00 & $<0.01$ & 0.00 & NC & 0.01 & 0.01 & $\mathrm{NC}$ & 0.01 & $<0.01$ & NC & 0.01 & 0.01 & NC & 0.01 & 0.00 & 0.01 & $<0.01$ & NC \\
\hline $\mathrm{ReO}_{2}$ & 0.10 & 0.00 & 0.03 & 0.01 & NC & 0.10 & 0.02 & $\mathrm{NC}$ & 0.10 & 0.03 & $\mathrm{NC}$ & 0.10 & 0.01 & $\mathrm{NC}$ & 0.10 & 0.00 & 0.01 & $<0.01$ & $\mathrm{NC}$ \\
\hline $\mathrm{SiO}_{2}$ & 43.26 & 45.45 & 45.04 & 45.25 & 4.58 & 44.04 & 46.18 & 4.86 & 43.80 & 46.24 & 5.58 & 44.03 & 45.81 & 4.03 & 42.00 & 44.30 & 44.24 & 44.27 & 5.41 \\
\hline $\mathrm{SO}_{3}$ & 0.52 & 0.16 & 0.49 & 0.33 & NC & 0.45 & 0.45 & $\mathrm{NC}$ & 0.40 & 0.28 & NC & 0.40 & 0.29 & NC & 0.35 & 0.19 & 0.19 & 0.19 & $\mathrm{NC}$ \\
\hline $\mathrm{TiO}_{2}$ & 1.35 & 1.47 & 1.46 & 1.46 & 8.56 & 1.34 & 1.49 & 11.03 & 1.33 & 1.46 & 9.64 & 1.34 & 1.49 & 10.97 & 1.37 & 1.48 & 1.50 & 1.49 & 9.07 \\
\hline $\mathrm{V}_{2} \mathrm{O}_{5}$ & $\S$ & 0.01 & $<0.01$ & 0.00 & NC & $\S$ & 0.01 & $\mathrm{NC}$ & $\S$ & $<0.01$ & NC & $\S$ & 0.01 & NC & $\S$ & 0.01 & 0.01 & 0.01 & NC \\
\hline $\mathrm{ZnO}$ & 3.37 & 3.16 & 3.17 & 3.16 & -6.29 & 3.35 & 2.89 & -13.91 & 3.34 & 3.06 & -8.41 & 3.35 & 3.02 & -10.06 & 3.42 & 2.97 & 2.98 & 2.97 & \begin{tabular}{|l|}
-13.08 \\
\end{tabular} \\
\hline $\mathrm{ZrO}_{2}$ & 2.88 & 3.05 & 3.06 & 3.06 & 6.02 & 2.87 & 2.62 & -8.90 & 2.86 & 3.10 & 8.58 & 2.87 & 2.95 & 2.70 & 2.93 & 2.61 & 2.65 & 2.63 & -10.29 \\
\hline Sum & 100.00 & 100.00 & 100.00 & 100.00 & NC & 100.00 & 100.00 & NC & 100.00 & 100.00 & NC & 100.00 & 100.00 & $\mathrm{NC}$ & 100.00 & 100.00 & 100.00 & 100.00 & $\mathrm{NC}$ \\
\hline
\end{tabular}


The Catholic University of America Vitreous State Laboratory
ORP-56324, Rev. 0 DuraMelter 100 Tests to Support LAW Glass Formulation Correlation Development Final Report, VSL-06R6480-1, Rev. 0

Table 2.23. DCP and XRF Analyzed Compositions of Select Melter Feed Samples (wt\%).

\begin{tabular}{|c|c|c|c|c|c|c|c|c|c|c|c|}
\hline \multirow{3}{*}{ Test } & \multicolumn{3}{|c|}{$\mathbf{1 A}$} & \multicolumn{3}{|c|}{ 1B } & \multicolumn{5}{|c|}{ 2B } \\
\hline & \multirow{2}{*}{ Target } & \multicolumn{2}{|c|}{ WVZ-F-65A } & \multirow{2}{*}{ Target } & \multicolumn{2}{|c|}{ WVZ-F-80A } & \multirow{2}{*}{ Target } & \multicolumn{2}{|c|}{ WVZ-F-140A } & \multicolumn{2}{|c|}{ WVZ-F-152A } \\
\hline & & XRF & DCP & & XRF & DCP & & XRF & DCP & XRF & DCP \\
\hline $\mathrm{Al}_{2} \mathrm{O}_{3}$ & 6.09 & 6.08 & 5.97 & 5.96 & 5.92 & 5.70 & 5.98 & 6.11 & 5.61 & 5.78 & 5.51 \\
\hline $\mathrm{B}_{2} \mathrm{O}_{3}$ & 9.99 & 9.99* & 9.10 & 9.78 & 9.78* & 9.30 & 9.80 & 9.80* & 9.41 & 9.80* & 9.55 \\
\hline $\mathrm{CaO}$ & 2.02 & 1.92 & 2.05 & 2.46 & 2.22 & 2.35 & 3.61 & 3.31 & 3.29 & 3.48 & 3.33 \\
\hline $\mathrm{Cl}$ & 0.20 & 0.07 & NA & 0.20 & 0.10 & NA & 0.20 & 0.05 & NA & 0.05 & $\overline{\mathrm{NA}}$ \\
\hline $\mathrm{Cr}_{2} \mathrm{O}_{3}$ & 0.08 & 0.14 & 0.14 & 0.08 & 0.13 & 0.13 & 0.08 & 0.14 & 0.14 & 0.14 & 0.13 \\
\hline$F$ & 0.08 & NA & NA & 0.08 & NA & NA & 0.08 & $\overline{\mathrm{NA}}$ & NA & NA & NA \\
\hline $\mathrm{Fe}_{2} \mathrm{O}_{3}$ & 5.49 & 5.12 & 5.47 & 5.37 & 4.72 & 5.23 & 5.40 & 4.91 & 5.08 & 5.19 & 5.05 \\
\hline $\mathrm{K}_{2} \mathrm{O}$ & 4.98 & 4.68 & 4.95 & $\S$ & 0.18 & 0.13 & 0.54 & 0.61 & 0.65 & 0.61 & 0.64 \\
\hline $\mathrm{Li}_{2} \mathrm{O}$ & $\S$ & $0.00 *$ & 0.02 & $\S$ & $0.00 *$ & 0.02 & 0.49 & $0.49 *$ & 0.57 & $0.49 *$ & 0.56 \\
\hline $\mathrm{MgO}$ & 1.48 & 1.50 & 1.62 & 1.45 & 1.46 & 1.48 & 1.45 & 1.41 & 1.55 & 1.27 & 1.38 \\
\hline $\mathrm{Na}_{2} \mathrm{O}$ & 18.19 & 18.35 & 15.68 & 21.25 & 22.70 & 18.73 & 18.95 & 18.99 & 16.56 & 20.08 & 16.96 \\
\hline $\mathrm{NiO}$ & 0.01 & $<0.01$ & 0.02 & 0.01 & $<0.01$ & 0.02 & 0.01 & $<0.01$ & 0.02 & $<0.01$ & 0.02 \\
\hline $\mathrm{P}_{2} \mathrm{O}_{5}$ & 0.12 & 0.19 & 0.22 & 0.12 & 0.17 & 0.20 & 0.12 & 0.18 & 0.18 & 0.18 & 0.25 \\
\hline $\mathrm{PbO}$ & 0.01 & 0.08 & 0.02 & 0.01 & $<0.01$ & 0.01 & 0.01 & 0.01 & 0.01 & 0.01 & 0.01 \\
\hline $\mathrm{ReO}_{2}$ & 0.10 & 0.01 & 0.01 & 0.10 & 0.02 & 0.03 & 0.10 & 0.01 & 0.02 & 0.01 & 0.01 \\
\hline $\mathrm{SiO}_{2}$ & 42.91 & 44.20 & 40.84 & 44.99 & 45.56 & 43.22 & 45.00 & 46.75 & 43.61 & 44.89 & 43.85 \\
\hline $\mathrm{SO}_{3}$ & 0.35 & 0.30 & NA & 0.41 & 0.32 & NA & 0.46 & 0.33 & NA & 0.33 & NA \\
\hline $\mathrm{TiO}_{2}$ & 1.40 & 1.52 & 1.62 & 1.37 & 1.34 & 1.48 & 1.37 & 1.49 & 1.61 & 1.57 & 1.59 \\
\hline $\mathrm{ZnO}$ & 3.50 & 2.91 & 3.29 & 3.43 & 2.74 & 3.25 & 3.43 & 2.85 & 3.26 & 3.14 & 3.31 \\
\hline $\mathrm{ZrO}_{2}$ & 3.00 & 2.93 & 3.03 & 2.94 & 2.63 & 2.80 & 2.94 & 2.55 & 2.72 & 2.97 & 2.71 \\
\hline Sum & 100.00 & 100.00 & 94.05 & 100.00 & 100.00 & 94.07 & 100.00 & 100.00 & 94.29 & 100.00 & 94.86 \\
\hline
\end{tabular}

* - Target values used for normalizing XRF data.

$\S$ - Not a target constituent

NA - Not analyzed 
The Catholic University of America Vitreous State Laboratory
DuraMelter 100 Tests to Support LAW Glass Formulation Correlation Development Final Report, VSL-06R6480-1, Rev. 0

Table 2.23. DCP and XRF Analyzed Compositions of Select Melter Feed Samples (wt\%) (continued).

\begin{tabular}{|c|c|c|c|c|c|c|c|c|c|c|}
\hline \multirow{3}{*}{ Test } & \multicolumn{5}{|c|}{$2 \mathrm{C}$} & \multicolumn{5}{|c|}{$3 B$} \\
\hline & \multirow{2}{*}{ Target } & \multicolumn{2}{|c|}{ AWV-F-16A } & \multicolumn{2}{|c|}{ AWV-F-30A } & \multirow{2}{*}{ Target } & \multicolumn{2}{|c|}{ AWV-F-118A } & \multicolumn{2}{|c|}{ AWV-F-135A } \\
\hline & & XRF & DCP & XRF & DCP & & XRF & DCP & XRF & DCP \\
\hline $\mathrm{Al}_{2} \mathrm{O}_{3}$ & 6.00 & 5.85 & 5.97 & 6.00 & 6.19 & 6.05 & 6.03 & 5.97 & 5.96 & 5.87 \\
\hline $\mathrm{B}_{2} \mathrm{O}_{3}$ & 9.84 & 9.84* & 9.57 & $9.84 *$ & 9.28 & 9.91 & 9.91* & 9.59 & 9.91* & 9.97 \\
\hline $\mathrm{CaO}$ & 5.45 & 5.21 & 5.37 & 5.17 & 6.81 & 6.85 & 6.38 & 6.66 & 6.34 & 6.55 \\
\hline $\mathrm{Cl}$ & 0.20 & 0.01 & NA & 0.02 & NA & 0.20 & 0.06 & NA & 0.07 & $\mathrm{NA}$ \\
\hline $\mathrm{Cr}_{2} \mathrm{O}_{3}$ & 0.08 & 0.15 & 0.14 & 0.15 & 0.14 & 0.08 & 0.16 & 0.14 & 0.15 & 0.14 \\
\hline $\mathrm{F}$ & 0.08 & NA & NA & NA & NA & 0.08 & NA & NA & NA & $\mathrm{NA}$ \\
\hline $\mathrm{Fe}_{2} \mathrm{O}_{3}$ & 5.41 & 5.41 & 4.90 & 5.22 & 4.79 & 5.44 & 5.17 & 4.88 & 5.08 & 5.11 \\
\hline $\mathrm{K}_{2} \mathrm{O}$ & 0.54 & 0.59 & 0.60 & 0.60 & 0.61 & 0.54 & 0.65 & 0.66 & 0.62 & 0.65 \\
\hline $\mathrm{Li}_{2} \mathrm{O}$ & 2.38 & $2.38 *$ & 2.27 & $2.38^{*}$ & 2.27 & 4.08 & $4.08 *$ & 3.82 & $4.08^{*}$ & 3.75 \\
\hline $\mathrm{MgO}$ & 1.46 & 1.36 & 1.48 & 1.42 & 1.50 & 2.36 & 2.28 & 2.26 & 2.29 & 2.24 \\
\hline $\mathrm{Na}_{2} \mathrm{O}$ & 15.81 & 16.40 & 15.35 & 16.18 & 15.60 & 8.92 & 9.45 & 8.78 & 9.50 & 8.60 \\
\hline $\mathrm{NiO}$ & 0.01 & $<0.01$ & 0.02 & $<0.01$ & 0.02 & 0.01 & $<0.01$ & 0.03 & $<0.01$ & 0.03 \\
\hline $\mathrm{P}_{2} \mathrm{O}_{5}$ & 0.12 & 0.17 & 0.31 & 0.17 & 0.26 & 0.12 & 0.19 & 0.35 & 0.18 & 0.38 \\
\hline $\mathrm{PbO}$ & 0.01 & 0.01 & 0.02 & $<0.01$ & 0.01 & 0.01 & $<0.01$ & 0.01 & $<0.01$ & 0.01 \\
\hline $\mathrm{ReO}_{2}$ & 0.10 & $<0.01$ & 0.02 & 0.01 & 0.01 & 0.10 & 0.02 & 0.04 & 0.02 & 0.03 \\
\hline $\mathrm{SiO}_{2}$ & 44.21 & 44.40 & 41.44 & 45.00 & 41.36 & 46.75 & 47.79 & 43.72 & 48.01 & 43.26 \\
\hline $\mathrm{SO}_{3}$ & 0.53 & 0.29 & NA & 0.28 & NA & 0.69 & 0.33 & NA & 0.33 & NA \\
\hline $\mathrm{TiO}_{2}$ & 1.38 & 1.48 & 1.44 & 1.43 & 1.42 & 1.39 & 1.56 & 1.58 & 1.56 & 1.61 \\
\hline $\mathrm{ZnO}$ & 3.45 & 3.28 & 3.38 & 3.13 & 3.37 & 3.47 & 3.01 & 3.39 & 2.99 & 3.40 \\
\hline $\mathrm{ZrO}_{2}$ & 2.95 & 3.17 & 2.92 & 3.00 & 2.99 & 2.97 & 2.91 & 2.95 & 2.90 & 2.91 \\
\hline Sum & 100.00 & 100.00 & 95.20 & 100.00 & 96.63 & 100.00 & 100.00 & 94.83 & 100.00 & 94.51 \\
\hline
\end{tabular}

* - Target values used for normalizing XRF data.

$\S$ - Not a target constituent

NA - Not analyzed 
The Catholic University of America Vitreous State Laboratory
ORP-56324, Rev. 0 DuraMelter 100 Tests to Support LAW Glass Formulation Correlation Development Final Report, VSL-06R6480-1, Rev. 0

Table 3.1. Summary of Test Conditions and Results for DM100 Correlation Tests.

\begin{tabular}{|c|c|c|c|c|c|c|c|c|}
\hline & Test & $1 \mathrm{~A}$ & 1B & $2 \mathrm{~A}$ & 2B & $2 \mathrm{C}$ & 2D & $3 \mathrm{~A}$ \\
\hline \multirow{7}{*}{ Time } & Feed Start & $\begin{array}{c}6 / 13 / 2005 \\
12: 00 \\
\end{array}$ & $\begin{array}{c}6 / 15 / 2005 \\
15: 30 \\
\end{array}$ & $\begin{array}{c}6 / 22 / 2005 \\
6: 30 \\
\end{array}$ & $\begin{array}{c}6 / 23 / 2005 \\
9: 00 \\
\end{array}$ & $\begin{array}{c}6 / 25 / 2005 \\
12: 06 \\
\end{array}$ & $\begin{array}{c}6 / 27 / 2005 \\
14: 30 \\
\end{array}$ & $\begin{array}{c}7 / 6 / 2005 \\
6: 30 \\
\end{array}$ \\
\hline & \multirow[t]{2}{*}{ Feed End } & 6/15/2005 & $\begin{array}{c}6 / 17 / 2005 \\
18.00\end{array}$ & $\begin{array}{c}6 / 23 / 2005 \\
8 \cdot 30\end{array}$ & $\begin{array}{c}6 / 25 / 2005 \\
11 \cdot 30\end{array}$ & $6 / 27 / 2005$ & $6 / 29 / 2005$ & 7/7/2005 \\
\hline & & 15:00 & 18:00 & 8:30 & $\frac{11: 30}{0.0}$ & 14:00 & 19:15 & \\
\hline & Water Feeding (hr) & 1.0 & 0.0 & 1.0 & 0.0 & 0.0 & 0.0 & 1.0 \\
\hline & Idling Time (hr) & 0.0 & 0.9 & 0.0 & 0.4 & 0.4 & 2.5 & 0.0 \\
\hline & $\begin{array}{l}\text { Net Slurry Feeding } \\
\text { (hr) }\end{array}$ & 50.0 & 49.6 & 25.0 & 50.1 & 49.5 & 50.3 & 25.0 \\
\hline & $\begin{array}{l}\text { Cold Cap Burn-Off } \\
\text { (hr) }\end{array}$ & NA & 0.7 & NA & NA & NA & 1.5 & NA \\
\hline \multirow{8}{*}{ Feed } & Simulant & AP-101 & AN-105 & AN-105 & AN-107 & AN-104 & AN-102 & AN-102 \\
\hline & Target Glass & LAWE3 & $\begin{array}{l}\text { LAWE4H } \\
\text { without K }\end{array}$ & LAWE4H & LAWE5H & LAWE6H & LAWE7H & LAWE7H \\
\hline & Target wt $\% \mathrm{SO}_{3}$ & 0.35 & 0.41 & 0.41 & 0.46 & 0.53 & 0.59 & 0.59 \\
\hline & Target wt $\% \mathrm{Na}_{2} \mathrm{O}$ & 18.19 & 21.25 & 21.25 & 18.95 & 15.81 & 13.52 & 13.52 \\
\hline & Target wt\% Cl & 0.20 & 0.20 & 0.20 & 0.20 & 0.20 & 0.20 & 0.20 \\
\hline & Target wt\% F & 0.08 & 0.08 & 0.08 & 0.08 & 0.08 & 0.08 & 0.08 \\
\hline & $\begin{array}{l}\text { Target Glass Yield } \\
(\mathrm{kg} / \mathrm{kg})\end{array}$ & 0.533 & 0.491 & 0.488 & 0.482 & 0.499 & 0.478 & 0.478 \\
\hline & Used (kg) & 851 & 913 & 461 & 941 & 902 & 908 & 462 \\
\hline \multicolumn{2}{|c|}{ Avg. Production Rate $\left(\mathrm{kg} / \mathrm{m}^{2} /\right.$ day $)$} & 1997 & 1990 & 1981 & 1993 & 2002 & 1900 & 1945 \\
\hline \multicolumn{2}{|c|}{ Avg. Bubbling Rate (lpm) } & 10.4 & 20.7 & 17.6 & 15.6 & 12.0 & 9.5 & 8.9 \\
\hline \multirow{2}{*}{$\begin{array}{l}\text { Sulfate } \\
\text { Phases } \\
\text { on Melt } \\
\text { Surface }\end{array}$} & During Turnover & NA & No & NA & No & No & No & NA \\
\hline & End of Segment & No & No & No & No & No & No & No \\
\hline \multirow{3}{*}{ Product } & Measured wt $\% \mathrm{SO}_{3}$ & 0.33 & 0.36 & 0.36 & 0.43 & 0.45 & 0.54 & 0.51 \\
\hline & Measured wt\% Cl & 0.15 & 0.13 & 0.13 & 0.15 & 0.14 & 0.14 & 0.13 \\
\hline & Measured wt\% F & $<0.08$ & $<0.08$ & $<0.08$ & $<0.08$ & $<0.08$ & $<0.08$ & $<0.08$ \\
\hline
\end{tabular}

NA - Not applicable 
The Catholic University of America Vitreous State Laboratory

Table 3.1. Summary of Test Conditions and Results for DM100 Correlation Tests (continued).

\begin{tabular}{|c|c|c|c|c|c|c|c|c|}
\hline \multicolumn{2}{|r|}{ Test } & $3 B$ & $3 C$ & $4 \mathrm{~A}$ & $4 \mathrm{~B}$ & $4 \mathrm{C}$ & $5 \mathrm{~A}$ & $5 B$ \\
\hline \multirow{6}{*}{ Time } & Feed Start & $\begin{array}{c}\text { 7/7/2005 } \\
8: 51\end{array}$ & $\begin{array}{c}7 / 9 / 2005 \\
11: 36\end{array}$ & $\begin{array}{c}\text { 8/15/2005 } \\
16: 00\end{array}$ & $\begin{array}{c}8 / 16 / 2005 \\
10: 00\end{array}$ & $\begin{array}{c}8 / 18 / 2005 \\
14: 31\end{array}$ & $\begin{array}{c}8 / 24 / 2005 \\
6: 30\end{array}$ & $\begin{array}{c}8 / 25 / 2005 \\
9: 50\end{array}$ \\
\hline & Feed End & $\begin{array}{c}\text { 7/9/2005 } \\
11: 00\end{array}$ & $\begin{array}{c}\text { 7/11/2005 } \\
15: 00\end{array}$ & $\begin{array}{c}8 / 16 / 2005 \\
8: 30\end{array}$ & $\begin{array}{c}8 / 18 / 2005 \\
14: 00\end{array}$ & $\begin{array}{c}8 / 20 / 2005 \\
18: 00 \\
\end{array}$ & $\begin{array}{c}8 / 25 / 2005 \\
8: 30 \\
\end{array}$ & $\begin{array}{c}\text { 8/27/2005 } \\
13: 30 \\
\end{array}$ \\
\hline & Water Feeding (hr) & NA & NA & 1.0 & NA & NA & 1.0 & NA \\
\hline & Idling Time (hr) & 0.4 & 1.2 & 0.0 & 3.2 & 2.2 & 0.2 & 1.7 \\
\hline & Net Slurry Feeding (hr) & 49.8 & 50.2 & 15.5 & 48.8 & 49.3 & 24.8 & 50.0 \\
\hline & Cold Cap Burn-Off (hr) & NA & 0.8 & NA & NA & 0.2 & NA & NA \\
\hline \multirow{8}{*}{ Feed } & LAW Simulant & AZ-101 & AZ-102 & AZ-102 & AZ-101 & AN-102 & AN-102 & AN-104 \\
\hline & Target Glass & LAWE9H & LAWE10H & LAWE10H & LAWE9H & LAWE7H & LAWE7H & LAWE6H \\
\hline & Target wt $\% \mathrm{SO}_{3}$ & 0.69 & 0.80 & 0.80 & 0.69 & 0.59 & 0.59 & 0.53 \\
\hline & Target wt $\% \mathrm{Na}_{2} \mathrm{O}$ & 8.92 & 5.71 & 5.59 & 8.73 & 13.23 & 13.23 & 15.48 \\
\hline & Target wt\% Cl & 0.20 & 0.20 & 1.1 & 1.1 & 1.1 & 1.1 & 1.1 \\
\hline & Target wt\% F & 0.08 & 0.08 & 1.3 & 1.3 & 1.3 & 1.3 & 1.3 \\
\hline & $\begin{array}{c}\text { Target Glass Yield } \\
(\mathrm{kg} / \mathrm{kg})\end{array}$ & 0.526 & 0.538 & 0.519 & 0.524 & 0.476 & 0.476 & 0.497 \\
\hline & Used (kg) & 853 & 833 & 276 & 865 & 905 & 484 & 914 \\
\hline \multicolumn{2}{|c|}{ Avg. Production Rate $\left(\mathrm{kg} / \mathrm{m}^{2} /\right.$ day $)$} & 1984 & 1966 & 2034 & 2045 & 1924 & 2045 & 2000 \\
\hline \multicolumn{2}{|c|}{ Avg. Bubbling Rate (lpm) } & 9.0 & 8.5 & 7.6 & 5.9 & 5.0 & 4.6 & 5.0 \\
\hline \multirow{2}{*}{$\begin{array}{c}\text { Sulfate } \\
\text { Phases } \\
\text { on Melt } \\
\text { Surface }\end{array}$} & During Turnover & No & No & NA & Yes & Yes & NA & Yes \\
\hline & End of Segment & No & No & Yes & No & Yes & Yes & No \\
\hline \multirow{3}{*}{ Product } & Measured wt\% $\mathrm{SO}_{3}$ & 0.54 & 0.59 & 0.36 & 0.52 & 0.50 & 0.51 & 0.44 \\
\hline & Measured wt\% Cl & 0.13 & 0.13 & 0.41 & 0.67 & 0.63 & 0.55 & 0.63 \\
\hline & Measured wt\% F & $<0.08$ & $<0.08$ & 0.47 & 0.89 & 0.88 & 0.69 & 0.97 \\
\hline
\end{tabular}

NA - Not applicable 
The Catholic University of America Vitreous State Laboratory
DuraMelter 100 Tests to Support LAW Glass Formulation Correlation Development

Final Report, VSL-06R6480-1, Rev. 0

Table 3.1. Summary of Test Conditions and Results for DM100 Correlation Tests (continued).

\begin{tabular}{|c|c|c|c|c|c|}
\hline \multicolumn{2}{|r|}{ Test } & $5 \mathrm{C}$ & $5 \mathrm{D}$ & $6 \mathrm{~A}$ & $6 \mathrm{~B}$ \\
\hline \multirow{6}{*}{ Time } & Feed Start & $\begin{array}{c}8 / 27 / 2005 \\
14: 00\end{array}$ & $\begin{array}{c}8 / 29 / 2005 \\
17: 45\end{array}$ & $\begin{array}{c}\text { 9/12/2005 } \\
9: 35\end{array}$ & $\begin{array}{c}9 / 14 / 2005 \\
13: 45\end{array}$ \\
\hline & Feed End & $\begin{array}{c}8 / 29 / 2005 \\
16: 30\end{array}$ & $\begin{array}{c}\text { 8/31/2005 } \\
20: 30\end{array}$ & $\begin{array}{c}9 / 14 / 2005 \\
12: 30\end{array}$ & $\begin{array}{c}\text { 9/16/2005 } \\
16: 30\end{array}$ \\
\hline & Water Feeding (hr) & NA & NA & 0.9 & NA \\
\hline & Idling Time (hr) & 0.6 & 0.7 & 0.0 & 0.6 \\
\hline & Net Slurry Feeding (hr) & 49.9 & 50.1 & 50.0 & 50.2 \\
\hline & Cold Cap Burn-Off (hr) & NA & 0.2 & NA & ND \\
\hline \multirow{8}{*}{ Feed } & Simulant & AN-107 & AN-105 & AN-105 & AP-101 \\
\hline & Target Glass & LAWE5H & LAWE4H & $\begin{array}{l}\text { LAWE4H } \\
\text { without K }\end{array}$ & LAWE3 \\
\hline & Target wt $\% \mathrm{SO}_{3}$ & 0.46 & 0.41 & 0.41 & 0.35 \\
\hline & Target wt $\% \mathrm{Na}_{2} \mathrm{O}$ & 18.55 & 20.68 & 20.80 & 17.81 \\
\hline & Target wt\% Cl & 1.1 & 1.1 & 1.1 & 1.1 \\
\hline & Target wt\% F & 1.3 & 1.3 & 1.3 & 1.3 \\
\hline & $\begin{array}{c}\text { Target Glass Yield } \\
(\mathrm{kg} / \mathrm{kg})\end{array}$ & 0.481 & 0.487 & 0.489 & 0.531 \\
\hline & Used (kg) & 915 & 918 & 926 & 865 \\
\hline \multicolumn{2}{|c|}{ Avg. Production Rate (kg/m²/day) } & 1942 & 1965 & 1994 & 2015 \\
\hline \multicolumn{2}{|c|}{ Avg. Bubbling Rate (lpm) } & 7.1 & 8.6 & 9.4 & 4.7 \\
\hline \multirow{2}{*}{$\begin{array}{l}\text { Sulfate } \\
\text { Phases } \\
\text { on Melt } \\
\text { Surface }\end{array}$} & During Turnover & No & No & NA & No \\
\hline & End of Segment & Yes & Yes & Yes & Yes \\
\hline \multirow{3}{*}{ Product } & Measured wt\% $\mathrm{SO}_{3}$ & 0.41 & 0.34 & 0.34 & 0.33 \\
\hline & Measured wt\% Cl & 0.65 & 0.67 & 0.65 & 0.68 \\
\hline & Measured wt\% F & 0.94 & 0.98 & 0.97 & 0.98 \\
\hline
\end{tabular}

NA - Not applicable 
Table 3.2. Summary of Measured Melter Parameters for DM100 Correlation Tests.

\begin{tabular}{|c|c|c|c|c|c|c|c|c|c|c|c|c|c|c|c|c|c|c|c|c|}
\hline \multirow{2}{*}{\multicolumn{3}{|c|}{ Test }} & \multicolumn{3}{|c|}{$1 \mathrm{~A}$} & \multicolumn{3}{|c|}{ 1B } & \multicolumn{3}{|c|}{$2 \mathrm{~A}$} & \multicolumn{3}{|c|}{$2 B$} & \multicolumn{3}{|c|}{$2 \mathrm{C}$} & \multicolumn{3}{|c|}{$2 D$} \\
\hline & & & AVG & MIN & MAX & AVG & MIN & MAX & AVG & MIN & MAX & AVG & MIN & MAX & AVG & MIN & MAX & AVG & MIN & MAX \\
\hline \multirow{12}{*}{$\begin{array}{c}\mathrm{T} \\
\mathrm{E} \\
\mathrm{M} \\
\mathrm{P} \\
\mathrm{E} \\
\mathrm{R} \\
\mathrm{A} \\
\mathrm{T} \\
\mathrm{U} \\
\mathrm{R} \\
\mathrm{E} \\
\text { (C) }\end{array}$} & \multirow{3}{*}{ Electrode } & East & 1127 & 1107 & 1140 & 1125 & 1109 & 1155 & 1127 & 1109 & 1159 & 1129 & 1117 & 1139 & 1125 & 1113 & 1147 & 1129 & 1091 & 1148 \\
\hline & & West & 1071 & 1007 & 1088 & 1083 & 1063 & 1105 & 1088 & 1029 & 1106 & 1085 & 1070 & 1097 & 1075 & 1060 & 1090 & 1060 & 1029 & 1077 \\
\hline & & Bottom & 906 & 867 & 919 & 915 & 910 & 926 & 905 & 897 & 909 & 915 & 903 & 921 & 897 & 889 & 923 & 896 & 887 & 911 \\
\hline & \multirow{4}{*}{ Glass } & 19 ” from bottom & 982 & 814 & 1036 & 1007 & 898 & 1082 & 1008 & 628 & 1086 & 982 & 872 & 1071 & 975 & 884 & 1034 & 928 & 789 & 1103 \\
\hline & & 16 " from bottom & 1134 & 1085 & 1169 & 1133 & 1109 & 1190 & 1134 & 924 & 1163 & 1131 & 1091 & 1160 & 1127 & 1101 & 1156 & 1120 & 1074 & 1168 \\
\hline & & 10 " from bottom & 1150 & 1112 & 1178 & 1151 & 1135 & 1196 & 1155 & 1126 & 1189 & 1150 & 1133 & 1185 & 1149 & 1128 & 1175 & 1148 & 1100 & 1174 \\
\hline & & 4" from bottom & 1154 & 1112 & 1170 & 1153 & 1136 & 1191 & 1153 & 1121 & 1185 & 1153 & 1127 & 1180 & 1152 & 1123 & 1175 & 1150 & 1098 & 1169 \\
\hline & \multirow{2}{*}{ Plenum } & Exposed & 563 & 461 & 686 & 575 & 477 & 683 & 571 & 487 & 718 & 571 & 542 & 604 & 588 & 538 & 720 & 586 & 534 & 810 \\
\hline & & Thermowell & 555 & 531 & 667 & 563 & 528 & 643 & 567 & 520 & 709 & 548 & 528 & 574 & 566 & 545 & 628 & 575 & 535 & 768 \\
\hline & \multicolumn{2}{|c|}{ Discharge Chamber } & 1047 & 981 & 1071 & 1055 & 1016 & 1077 & 1046 & 1020 & 1067 & 987 & 957 & 1050 & 983 & 962 & 1018 & 976 & 948 & 1005 \\
\hline & \multicolumn{2}{|c|}{ Film Cooler Outlet } & 290 & 284 & 293 & 289 & 279 & 295 & 285 & 270 & 295 & 280 & 271 & 285 & 279 & 270 & 284 & 279 & 270 & 293 \\
\hline & \multicolumn{2}{|c|}{ Transition Line Outlet } & 285 & 271 & 311 & 289 & 279 & 297 & 282 & 272 & 307 & 279 & 268 & 284 & 276 & 271 & 283 & 276 & 267 & 289 \\
\hline \multicolumn{3}{|c|}{ Lance Bubbling (lpm) } & 10.4 & 1.5 & 11.6 & 20.7 & 1.6 & 28.0 & 17.6 & 1.6 & 25.7 & 15.6 & 1.5 & 17.1 & 12.0 & 1.6 & 15.1 & 9.5 & 1.6 & 13.1 \\
\hline \multicolumn{3}{|c|}{ Melter Pressure (inches water) } & -0.99 & -4.32 & 0.28 & -0.96 & -4.58 & 0.26 & -1.06 & -1.99 & 0.06 & -0.85 & -3.74 & 0.25 & -0.83 & -4.67 & 0.31 & -0.87 & -3.63 & 0.36 \\
\hline \multicolumn{3}{|c|}{ Electrode Voltage (V) } & 41.1 & 36.9 & 48.9 & 41.7 & 34.9 & 44.9 & 39.4 & 32.5 & 43.6 & 41.0 & 2.0 & 42.7 & 40.9 & 2.0 & 42.9 & 42.4 & 2.1 & 45.1 \\
\hline \multicolumn{3}{|c|}{ Total Power $(\mathrm{kW})$} & 21.5 & 19.7 & 30.9 & 22.3 & 17.9 & 23.9 & 21.9 & 16.8 & 26.9 & 23.6 & 0.3 & 24.4 & 22.3 & 0.3 & 23.9 & 23.1 & 0.3 & 25.0 \\
\hline \multicolumn{3}{|c|}{ Glass Pool Resistance (Ohms) } & 0.078 & 0.067 & 0.084 & 0.078 & 0.068 & 0.087 & 0.071 & 0.059 & 0.077 & 0.071 & 0.015 & 0.076 & 0.075 & 0.015 & 0.081 & 0.078 & 0.016 & 0.089 \\
\hline
\end{tabular}


Table 3.2. Summary of Measured Melter Parameters for DM100 Correlation Tests (continued).

\begin{tabular}{|c|c|c|c|c|c|c|c|c|c|c|c|c|c|c|c|c|c|c|c|c|}
\hline \multirow{2}{*}{\multicolumn{3}{|c|}{ Test }} & \multicolumn{3}{|c|}{$3 A$} & \multicolumn{3}{|c|}{ 3B } & \multicolumn{3}{|c|}{$3 C$} & \multicolumn{3}{|c|}{$4 \mathrm{~A}$} & \multicolumn{3}{|c|}{ 4B } & \multicolumn{3}{|c|}{ 4C } \\
\hline & & & AVG & MIN & MAX & AVG & MIN & MAX & AVG & MIN & MAX & AVG & MIN & MAX & AVG & MIN & MAX & AVG & MIN & MAX \\
\hline \multirow{12}{*}{$\begin{array}{c}\mathrm{T} \\
\mathrm{E} \\
\mathrm{M} \\
\mathrm{P} \\
\mathrm{E} \\
\mathrm{R} \\
\mathrm{A} \\
\mathrm{T} \\
\mathrm{U} \\
\mathrm{R} \\
\mathrm{E} \\
\text { (C) }\end{array}$} & \multirow{3}{*}{ Electrode } & East & 1127 & 1101 & 1136 & 1130 & 1113 & 1143 & 1132 & 1108 & 1143 & 1135 & 1113 & 1147 & 1137 & 1110 & 1148 & 1138 & 1108 & 1150 \\
\hline & & West & 1060 & 1029 & 1075 & 1053 & 1041 & 1066 & 1038 & 1026 & 1048 & 1050 & 1027 & 1065 & 1048 & 1015 & 1062 & 1048 & 1011 & 1063 \\
\hline & & Bottom & 888 & 880 & 894 & 901 & 890 & 914 & 908 & 895 & 921 & 919 & 898 & 932 & 905 & 897 & 920 & 899 & 889 & 906 \\
\hline & \multirow{4}{*}{ Glass } & 19 " from bottom & 879 & 707 & 969 & 891 & 787 & 987 & 886 & 718 & 1071 & 955 & 779 & 1139 & 967 & 796 & 1134 & 980 & 777 & 1140 \\
\hline & & 16 ” from bottom & 1116 & 1076 & 1140 & 1115 & 1054 & 1157 & 1134 & 1072 & 1163 & 1134 & 1054 & 1165 & 1141 & 1078 & 1168 & 1143 & 1107 & 1165 \\
\hline & & 10 " from bottom & 1146 & 1100 & 1168 & 1147 & 1116 & 1165 & 1153 & 1127 & 1171 & 1153 & 1127 & 1172 & 1154 & 1112 & 1174 & 1154 & 1117 & 1170 \\
\hline & & 4" from bottom & 1154 & 1107 & 1172 & 1154 & 1128 & 1174 & 1152 & 1129 & 1168 & 1144 & 1109 & 1163 & 1147 & 1107 & 1167 & 1145 & 1109 & 1161 \\
\hline & \multirow{2}{*}{ Plenum } & Exposed & 579 & 544 & 706 & 582 & 517 & 672 & 576 & 514 & 673 & 571 & 508 & 682 & 575 & 487 & 786 & 588 & 531 & 793 \\
\hline & & Thermowell & 577 & 550 & 708 & 557 & 432 & 641 & 544 & 504 & 619 & 578 & 522 & 693 & 568 & 506 & 751 & 570 & 534 & 758 \\
\hline & \multicolumn{2}{|c|}{ Discharge Chamber } & 972 & 941 & 1014 & 967 & 943 & 1000 & 964 & 944 & 995 & 972 & 938 & 1009 & 954 & 915 & 987 & 947 & 918 & 983 \\
\hline & \multicolumn{2}{|c|}{ Film Cooler Outlet } & 284 & 261 & 310 & 283 & 262 & 290 & 282 & 262 & 288 & 289 & 276 & 295 & 289 & 163 & 297 & 290 & 263 & 295 \\
\hline & \multicolumn{2}{|c|}{ Transition Line Outlet } & 280 & 276 & 310 & 278 & 260 & 287 & 276 & 260 & 287 & 275 & 266 & 280 & 274 & 176 & 294 & 271 & 254 & 291 \\
\hline \multicolumn{3}{|c|}{ Lance Bubbling (lpm) } & 8.9 & 1.5 & 10.1 & 9.0 & 1.6 & 27.9 & 8.5 & 1.5 & 26.7 & 7.6 & 2.1 & 11.2 & 5.9 & 1.6 & 15.0 & 5.0 & 1.6 & 7.1 \\
\hline \multicolumn{3}{|c|}{ Melter Pressure (inches water) } & -1.03 & -2.53 & 0.09 & -1.02 & -4.15 & 0.15 & -1.05 & -2.55 & 0.00 & -1.21 & -3.64 & -0.10 & -1.08 & -4.12 & 0.12 & -1.02 & -4.56 & 0.11 \\
\hline \multicolumn{3}{|c|}{ Electrode Voltage (V) } & 43.9 & 39.9 & 47.0 & 47.9 & 2.0 & 55.5 & 54.4 & 2.0 & 59.4 & 56.5 & 51.7 & 59.2 & 50.4 & 2.0 & 58.0 & 45.1 & 2.0 & 52.6 \\
\hline \multicolumn{3}{|c|}{ Total Power (kW) } & 23.3 & 20.2 & 24.9 & 23.3 & 0.3 & 26.9 & 23.0 & 0.3 & 24.4 & 23.7 & 19.8 & 25.3 & 22.7 & 0.3 & 27.8 & 23.6 & 0.3 & 27.9 \\
\hline \multicolumn{3}{|c|}{ Glass Pool Resistance (Ohms) } & 0.083 & 0.077 & 0.089 & 0.099 & 0.015 & 0.122 & 0.129 & 0.015 & 0.149 & 0.135 & 0.126 & 0.149 & 0.112 & 0.015 & 0.137 & 0.086 & 0.015 & 0.111 \\
\hline
\end{tabular}


Table 3.2. Summary of Measured Melter Parameters for DM100 Correlation Tests (continued).

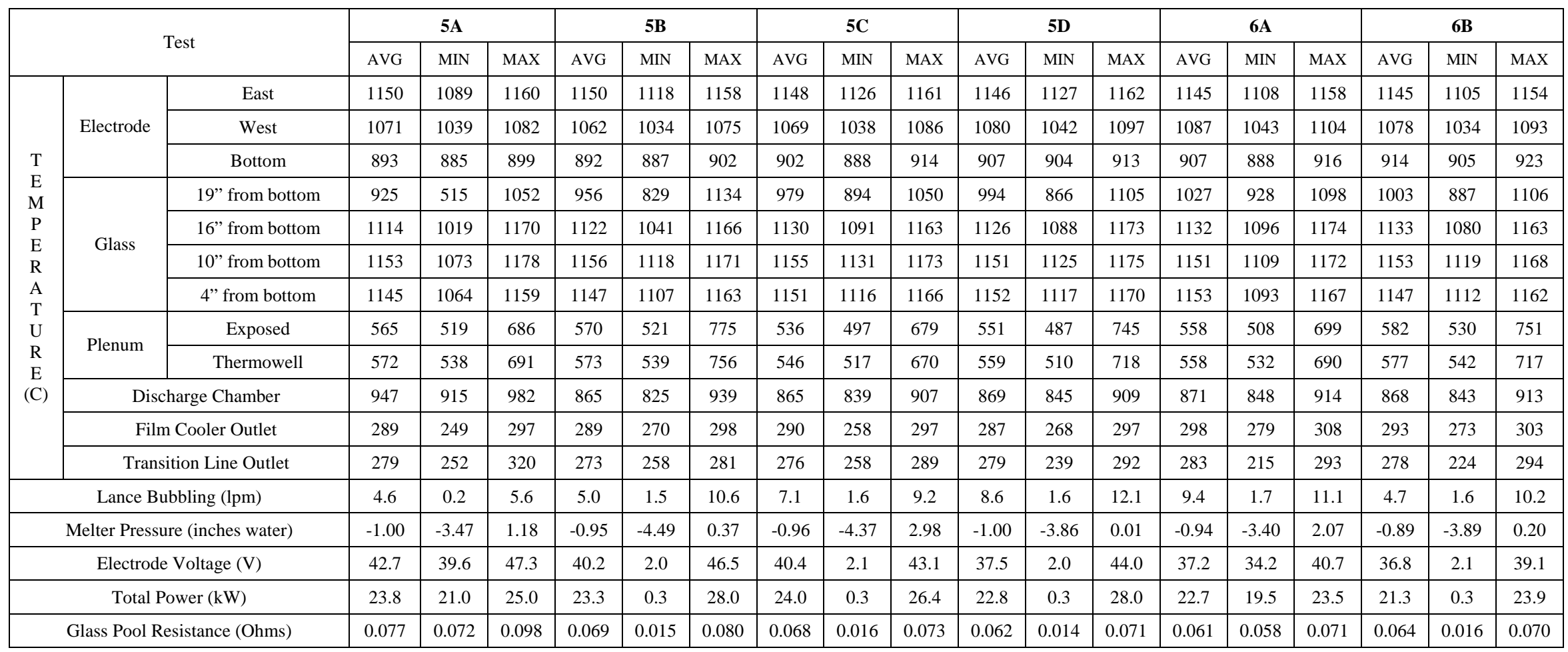


Table 4.1. Listing of DM100 Glasses Discharged, Analysis Performed, Masses, Target and Measured $\mathrm{SO}_{3}$ Concentrations.

\begin{tabular}{|c|c|c|c|c|c|c|c|}
\hline \multirow{2}{*}{ Test } & \multirow{2}{*}{ Date } & \multirow{2}{*}{ Sample Name } & \multirow{2}{*}{ Analysis } & \multirow{2}{*}{ Mass (kg) } & \multirow{2}{*}{$\begin{array}{c}\text { Cumulative } \\
\text { Mass (kg) }\end{array}$} & \multicolumn{2}{|c|}{$\mathrm{SO}_{3}$ wt \% } \\
\hline & & & & & & Target & Measured \\
\hline \multirow{33}{*}{$\mathbf{1 A}$} & \multirow{7}{*}{ 6/13/05 } & WVZ-G-40A & - & - & - & \multirow{33}{*}{0.35} & - \\
\hline & & WVZ-G-40B & XRF & 24.60 & 24.60 & & 0.53 \\
\hline & & WVZ-G-40C & - & - & - & & - \\
\hline & & WVZ-G-41A & XRF & 27.18 & 51.78 & & 0.51 \\
\hline & & WVZ-G-41B & - & - & - & & - \\
\hline & & WVZ-G-41C & XRF & 22.16 & 73.94 & & 0.47 \\
\hline & & WVZ-G-45A & - & - & - & & - \\
\hline & \multirow{16}{*}{$6 / 14 / 05$} & WVZ-G-45B & $\mathrm{XRF}$ & 30.08 & 104.02 & & 0.44 \\
\hline & & WVZ-G-46A & - & - & - & & - \\
\hline & & WVZ-G-46B & XRF & 23.24 & 127.26 & & 0.42 \\
\hline & & WVZ-G-46C & - & - & - & & - \\
\hline & & WVZ-G-46D & $\mathrm{XRF}$ & 21.92 & 149.18 & & 0.40 \\
\hline & & WVZ-G-46E & - & - & - & & - \\
\hline & & WVZ-G-48A & XRF & 19.26 & 168.44 & & 0.40 \\
\hline & & WVZ-G-48B & - & - & - & & - \\
\hline & & WVZ-G-48C & XRF & 23.30 & 191.74 & & 0.37 \\
\hline & & WVZ-G-51A & - & - & - & & - \\
\hline & & WVZ-G-51B & XRF & 25.92 & 217.66 & & 0.39 \\
\hline & & WVZ-G-51C & - & - & - & & - \\
\hline & & WVZ-G-51D & XRF & 23.80 & 241.46 & & 0.36 \\
\hline & & WVZ-G-51E & - & - & - & & - \\
\hline & & WVZ-G-56A & $\mathrm{XRF}$ & 27.54 & 269.00 & & 0.36 \\
\hline & & WVZ-G-56B & - & - & - & & - \\
\hline & \multirow{16}{*}{$6 / 15 / 05$} & WVZ-G-56C & XRF & 23.60 & 292.60 & & 0.35 \\
\hline & & WVZ-G-56D & - & - & - & & - \\
\hline & & WVZ-G-58A & $\mathrm{XRF}$ & 26.74 & 319.34 & & 0.35 \\
\hline & & WVZ-G-58B & - & - & - & & - \\
\hline & & WVZ-G-58C & XRF & 23.62 & 342.96 & & 0.35 \\
\hline & & WVZ-G-58D & - & - & - & & - \\
\hline & & WVZ-G-61A & $\mathrm{XRF}$ & 24.56 & 367.52 & & 0.35 \\
\hline & & WVZ-G-61B & - & - & - & & - \\
\hline & & WVZ-G-61C & $\mathrm{XRF}$ & 25.70 & 393.22 & & 0.33 \\
\hline & & WVZ-G-61D & $\begin{array}{c}\text { XRF, PCT, } \\
\text { TCLP, F, CCC }\end{array}$ & 13.84 & 407.06 & & 0.33 \\
\hline \multirow{8}{*}{$1 B$} & & WVZ-G-70A & - & - & - & \multirow{8}{*}{0.41} & - \\
\hline & & WVZ-G-70B & $\mathrm{XRF}$ & 31.14 & 438.20 & & 0.33 \\
\hline & & WVZ-G-72A & - & - & - & & - \\
\hline & & WVZ-G-72B & $\mathrm{XRF}$ & 30.20 & 468.40 & & 0.35 \\
\hline & & WVZ-G-72C & - & - & - & & - \\
\hline & & WVZ-G-74A & XRF & 22.74 & 491.14 & & 0.35 \\
\hline & \multirow{2}{*}{ 6/16/05 } & WVZ-G-75A & - & - & - & & - \\
\hline & & WVZ-G-75B & $\mathrm{XRF}$ & 20.58 & 511.72 & & 0.39 \\
\hline
\end{tabular}

"-" Empty data field 
Table 4.1. Listing of DM100 Glasses Discharged, Analysis Performed, Masses, Target and Measured $\mathrm{SO}_{3}$ Concentrations (continued).

\begin{tabular}{|c|c|c|c|c|c|c|c|}
\hline \multirow{2}{*}{ Test } & \multirow{2}{*}{ Date } & \multirow{2}{*}{ Sample Name } & \multirow{2}{*}{ Analysis } & \multirow{2}{*}{ Mass (kg) } & \multirow{2}{*}{$\begin{array}{c}\text { Cumulative } \\
\text { Mass (kg) }\end{array}$} & \multicolumn{2}{|c|}{$\mathrm{SO}_{3}$ wt \% } \\
\hline & & & & & & Target & Measured \\
\hline \multirow{31}{*}{$1 B$} & \multirow{17}{*}{ 6/16/05 } & WVZ-G-76A & - & - & - & \multirow{31}{*}{0.41} & - \\
\hline & & WVZ-G-76B & XRF & 20.54 & 532.26 & & 0.33 \\
\hline & & WVZ-G-76C & - & - & - & & - \\
\hline & & WVZ-G-79A & XRF & 21.08 & 553.34 & & 0.36 \\
\hline & & WVZ-G-80A & - & - & - & & - \\
\hline & & WVZ-G-80B & XRF & 20.32 & 573.66 & & 0.35 \\
\hline & & WVZ-G-80C & - & - & - & & - \\
\hline & & WVZ-G-81A & $\mathrm{XRF}$ & 20.06 & 593.72 & & 0.36 \\
\hline & & WVZ-G-81B & - & - & - & & - \\
\hline & & WVZ-G-81C & XRF & 23.80 & 617.52 & & 0.34 \\
\hline & & WVZ-G-86A & - & - & - & & - \\
\hline & & WVZ-G-86B & XRF & 21.82 & 639.34 & & 0.36 \\
\hline & & WVZ-G-87A & - & - & - & & - \\
\hline & & WVZ-G-87B & XRF & 18.82 & 658.16 & & 0.35 \\
\hline & & WVZ-G-87C & - & - & - & & - \\
\hline & & WVZ-G-89A & XRF & 23.24 & 681.40 & & 0.36 \\
\hline & & WVZ-G-89B & - & - & - & & - \\
\hline & \multirow{14}{*}{ 6/17/05 } & WVZ-G-89C & $\mathrm{XRF}$ & 16.78 & 698.18 & & 0.35 \\
\hline & & WVZ-G-90A & - & - & - & & - \\
\hline & & WVZ-G-90B & XRF & 26.64 & 724.82 & & 0.37 \\
\hline & & WVZ-G-90C & - & - & - & & - \\
\hline & & WVZ-G-90D & XRF & 27.48 & 752.30 & & 0.35 \\
\hline & & WVZ-G-95A & - & - & - & & - \\
\hline & & WVZ-G-95B & XRF & 22.20 & 774.50 & & 0.35 \\
\hline & & WVZ-G-95C & - & - & - & & - \\
\hline & & WVZ-G-95D & XRF & 22.26 & 796.76 & & 0.35 \\
\hline & & WVZ-G-96A & - & - & - & & - \\
\hline & & WVZ-G-96B & XRF & 20.72 & 817.48 & & 0.34 \\
\hline & & WVZ-G-96C & - & - & - & & - \\
\hline & & WVZ-G-97A & XRF & 25.86 & 843.34 & & 0.37 \\
\hline & & WVZ-G-97B & - & 11.86 & 855.20 & & 0.34 \\
\hline \multirow{10}{*}{$2 A$} & \multirow{10}{*}{$6 / 22 / 05$} & WVZ-G-118A & - & - & - & \multirow{10}{*}{0.41} & - \\
\hline & & WVZ-G-119A & XRF & 17.82 & 873.02 & & 0.36 \\
\hline & & WVZ-G-120A & - & - & - & & - \\
\hline & & WVZ-G-120B & XRF & 24.44 & 897.46 & & 0.37 \\
\hline & & WVZ-G-121A & - & - & - & & - \\
\hline & & WVZ-G-121B & XRF & 22.04 & 919.50 & & 0.37 \\
\hline & & WVZ-G-121C & - & - & - & & - \\
\hline & & WVZ-G-121D & XRF & 19.38 & 938.88 & & 0.38 \\
\hline & & WVZ-G-123A & - & - & - & & - \\
\hline & & WVZ-G-123B & XRF & 21.18 & 960.06 & & 0.37 \\
\hline
\end{tabular}

"-" Empty data field 
Table 4.1. Listing of DM100 Glasses Discharged, Analysis Performed, Masses, Target and Measured $\mathrm{SO}_{3}$ Concentrations (continued).

\begin{tabular}{|c|c|c|c|c|c|c|c|}
\hline \multirow{2}{*}{ Test } & \multirow{2}{*}{ Date } & \multirow{2}{*}{ Sample Name } & \multirow{2}{*}{ Analysis } & \multirow{2}{*}{ Mass (kg) } & \multirow{2}{*}{$\begin{array}{c}\text { Cumulative } \\
\text { Mass (kg) }\end{array}$} & \multicolumn{2}{|c|}{$\mathrm{SO}_{3}$ wt $\%$} \\
\hline & & & & & & Target & Measured \\
\hline \multirow{10}{*}{$2 A$} & \multirow{3}{*}{$6 / 22 / 05$} & WVZ-G-123C & - & - & - & \multirow{10}{*}{0.41} & - \\
\hline & & WVZ-G-123D & XRF & 22.74 & 982.80 & & 0.36 \\
\hline & & WVZ-G-126A & - & - & - & & - \\
\hline & \multirow{16}{*}{ 6/23/05 } & WVZ-G-126B & XRF & 25.18 & 1007.98 & & 0.37 \\
\hline & & WVZ-G-126C & - & - & - & & - \\
\hline & & WVZ-G-126D & XRF & 20.80 & 1028.78 & & 0.36 \\
\hline & & WVZ-G-129A & - & - & - & & - \\
\hline & & WVZ-G-129B & XRF & 22.66 & 1051.44 & & 0.38 \\
\hline & & WVZ-G-129C & - & - & - & & - \\
\hline & & WVZ-G-129D & XRF & 21.54 & 1072.98 & & 0.36 \\
\hline \multirow{32}{*}{ 2B } & & WVZ-G-139A & - & - & - & \multirow{32}{*}{0.46} & - \\
\hline & & WVZ-G-139B & XRF & 24.18 & 1097.16 & & 0.37 \\
\hline & & WVZ-G-140A & - & - & - & & - \\
\hline & & WVZ-G-140B & XRF & 25.66 & 1122.82 & & 0.36 \\
\hline & & WVZ-G-140C & - & - & - & & - \\
\hline & & WVZ-G-141A & XRF & 26.88 & 1149.70 & & 0.41 \\
\hline & & WVZ-G-141B & - & - & - & & - \\
\hline & & WVZ-G-143A & XRF & 22.74 & 1172.44 & & 0.41 \\
\hline & & WVZ-G-143B & - & - & - & & - \\
\hline & \multirow{19}{*}{$6 / 24 / 05$} & WVZ-G-143C & XRF & 27.96 & 1200.40 & & 0.43 \\
\hline & & WVZ-G-143D & - & - & - & & - \\
\hline & & WVZ-G-147A & XRF & 25.10 & 1225.50 & & 0.40 \\
\hline & & WVZ-G-147B & - & - & - & & - \\
\hline & & WVZ-G-147C & XRF & 23.36 & 1248.86 & & 0.41 \\
\hline & & WVZ-G-150A & - & - & - & & - \\
\hline & & WVZ-G-150B & XRF & 26.66 & 1275.52 & & 0.44 \\
\hline & & WVZ-G-151A & - & - & - & & - \\
\hline & & WVZ-G-151B & XRF & 23.60 & 1299.12 & & 0.45 \\
\hline & & WVZ-G-152A & - & - & - & & - \\
\hline & & WVZ-G-152B & XRF & 23.36 & 1322.48 & & 0.42 \\
\hline & & WVZ-G-152C & - & - & - & & - \\
\hline & & WVZ-G-152D & XRF & 26.32 & 1348.80 & & 0.41 \\
\hline & & WVZ-G-153A & - & - & - & & - \\
\hline & & WVZ-G-153B & XRF & 24.98 & 1373.78 & & 0.44 \\
\hline & & WVZ-G-153C & - & - & - & & - \\
\hline & & WVZ-G-153D & XRF & 21.84 & 1395.62 & & 0.43 \\
\hline & & AWV-G-5A & - & - & - & & - \\
\hline & & AWV-G-5B & XRF & 26.52 & 1422.14 & & 0.40 \\
\hline & \multirow{4}{*}{ 6/25/05 } & AWV-G-5C & - & - & - & & - \\
\hline & & AWV-G-5D & XRF & 24.22 & 1446.36 & & 0.42 \\
\hline & & AWV-G-6A & - & - & - & & - \\
\hline & & AWV-G-6B & XRF & 27.00 & 1473.36 & & 0.43 \\
\hline
\end{tabular}

"-" Empty data field 
Table 4.1. Listing of DM100 Glasses Discharged, Analysis Performed, Masses, Target and Measured $\mathrm{SO}_{3}$ Concentrations (continued).

\begin{tabular}{|c|c|c|c|c|c|c|c|}
\hline \multirow{2}{*}{ Test } & \multirow{2}{*}{ Date } & \multirow{2}{*}{ Sample Name } & \multirow{2}{*}{ Analysis } & \multirow{2}{*}{ Mass (kg) } & \multirow{2}{*}{$\begin{array}{c}\text { Cumulative } \\
\text { Mass (kg) }\end{array}$} & \multicolumn{2}{|c|}{$\mathrm{SO}_{3}$ wt \% } \\
\hline & & & & & & Target & Measured \\
\hline \multirow{3}{*}{ 2B } & \multirow{10}{*}{$6 / 25 / 05$} & AWV-G-6C & - & - & - & \multirow{3}{*}{0.46} & - \\
\hline & & AWV-G-6D & $\mathrm{XRF}$ & 27.40 & 1500.76 & & 0.44 \\
\hline & & AWV-G-14A & XRF, F & 12.68 & 1513.44 & & 0.43 \\
\hline \multirow{34}{*}{$2 \mathrm{C}$} & & AWV-G-16A & - & - & - & \multirow{34}{*}{0.53} & - \\
\hline & & AWV-G-21A & XRF & 28.68 & 1542.12 & & 0.44 \\
\hline & & AWV-G-21B & - & - & - & & - \\
\hline & & AWV-G-22A & XRF & 26.92 & 1569.04 & & 0.43 \\
\hline & & AWV-G-22B & - & - & - & & - \\
\hline & & AWV-G-22C & XRF & 24.74 & 1593.78 & & 0.44 \\
\hline & & AWV-G-24A & - & - & - & & - \\
\hline & \multirow{18}{*}{ 6/26/05 } & AWV-G-24B & XRF & 27.28 & 1621.06 & & 0.45 \\
\hline & & AWV-G-24C & - & - & - & & - \\
\hline & & AWV-G-27A & XRF & 24.84 & 1645.90 & & 0.44 \\
\hline & & AWV-G-27B & - & - & - & & - \\
\hline & & AWV-G-27C & XRF, DCP & 23.28 & 1669.18 & & 0.46 \\
\hline & & AWV-G-29A & - & - & - & & - \\
\hline & & AWV-G-29B & XRF, DCP & 23.68 & 1692.86 & & 0.46 \\
\hline & & AWV-G-30A & - & - & - & & - \\
\hline & & AWV-G-30B & XRF & 26.04 & 1718.90 & & 0.45 \\
\hline & & AWV-G-32A & - & - & - & & - \\
\hline & & AWV-G-32B & XRF & 25.14 & 1744.04 & & 0.45 \\
\hline & & AWV-G-35A & - & - & - & & - \\
\hline & & AWV-G-35B & XRF & 23.92 & 1767.96 & & 0.44 \\
\hline & & AWV-G-35C & - & - & - & & - \\
\hline & & AWV-G-35D & XRF & 23.70 & 1791.66 & & 0.45 \\
\hline & & AWV-G-36A & - & - & - & & - \\
\hline & & AWV-G-36B & XRF & 25.16 & 1816.82 & & 0.52 \\
\hline & & AWV-G-36C & - & - & - & & - \\
\hline & \multirow{13}{*}{ 6/27/05 } & AWV-G-36D & XRF & 28.08 & 1844.90 & & 0.46 \\
\hline & & AWV-G-41A & - & - & - & & - \\
\hline & & AWV-G-41B & XRF & 24.62 & 1869.52 & & 0.53 \\
\hline & & AWV-G-42A & - & - & - & & - \\
\hline & & AWV-G-42B & $\mathrm{XRF}$ & 27.14 & 1896.66 & & 0.45 \\
\hline & & AWV-G-42C & - & - & - & & - \\
\hline & & AWV-G-42D & XRF & 26.76 & 1923.42 & & 0.47 \\
\hline & & AWV-G-45A & - & - & - & & - \\
\hline & & AWV-G-45B & XRF, DCP, F & 26.46 & 1949.88 & & 0.45 \\
\hline \multirow{4}{*}{$2 \mathbf{D}$} & & AWV-G-49A & - & - & - & \multirow{4}{*}{0.59} & - \\
\hline & & AWV-G-54A & XRF & 29.28 & 1979.16 & & 0.47 \\
\hline & & AWV-G-54B & - & - & - & & - \\
\hline & & AWV-G-54C & XRF & 27.32 & 2006.48 & & 0.48 \\
\hline
\end{tabular}

"-" Empty data field 
Table 4.1. Listing of DM100 Glasses Discharged, Analysis Performed, Masses, Target and Measured $\mathrm{SO}_{3}$ Concentrations (continued).

\begin{tabular}{|c|c|c|c|c|c|c|c|}
\hline \multirow{2}{*}{ Test } & \multirow{2}{*}{ Date } & \multirow{2}{*}{ Sample Name } & \multirow{2}{*}{ Analysis } & \multirow{2}{*}{ Mass (kg) } & \multirow{2}{*}{$\begin{array}{c}\text { Cumulative } \\
\text { Mass (kg) }\end{array}$} & \multicolumn{2}{|c|}{$\mathrm{SO}_{3}$ wt \% } \\
\hline & & & & & & Target & Measured \\
\hline \multirow{28}{*}{ 2D } & \multirow{2}{*}{$6 / 27 / 05$} & AWV-G-54D & - & - & - & \multirow{42}{*}{0.59} & - \\
\hline & & AWV-G-55A & $\mathrm{XRF}$ & 25.48 & 2031.96 & & 0.50 \\
\hline & \multirow{13}{*}{ 6/28/05 } & AWV-G-56A & - & - & - & & - \\
\hline & & AWV-G-60A & XRF & 27.26 & 2059.22 & & 0.49 \\
\hline & & AWV-G-60B & - & - & - & & - \\
\hline & & AWV-G-60C & XRF, DCP & 28.84 & 2088.06 & & 0.51 \\
\hline & & AWV-G-61A & - & - & - & & \\
\hline & & AWV-G-61B & XRF & 23.54 & 2111.60 & & 0.51 \\
\hline & & AWV-G-63A & - & - & - & & - \\
\hline & & AWV-G-63B & XRF & 29.10 & 2140.70 & & 0.49 \\
\hline & & AWV-G-63C & - & - & - & & - \\
\hline & & AWV-G-67A & XRF & 25.04 & 2165.74 & & 0.52 \\
\hline & & AWV-G-67B & - & - & - & & - \\
\hline & & AWV-G-68A & XRF & 23.66 & 2189.40 & & 0.51 \\
\hline & & AWV-G-68B & - & - & - & & - \\
\hline & \multirow{13}{*}{$6 / 29 / 05$} & AWV-G-70A & XRF & 33.32 & 2222.72 & & 0.51 \\
\hline & & AWV-G-71A & - & - & - & & - \\
\hline & & AWV-G-71B & XRF & 25.22 & 2247.94 & & 0.51 \\
\hline & & AWV-G-71C & - & - & - & & - \\
\hline & & AWV-G-71D & XRF & 27.58 & 2275.52 & & 0.52 \\
\hline & & AWV-G-75A & - & - & - & & - \\
\hline & & AWV-G-75B & XRF & 26.48 & 2302.00 & & 0.54 \\
\hline & & AWV-G-75C & - & - & - & & - \\
\hline & & AWV-G-76A & XRF & 23.48 & 2325.48 & & 0.52 \\
\hline & & AWV-G-76B & - & - & - & & - \\
\hline & & AWV-G-76C & XRF & 25.04 & 2350.52 & & 0.53 \\
\hline & & AWV-G-76D & - & - & - & & - \\
\hline & & AWV-G-78A & \begin{tabular}{|c|} 
XRF, F, DCP, \\
PCT,TCLP, CCC \\
\end{tabular} & 24.86 & 2375.38 & & 0.54 \\
\hline \multirow{14}{*}{$\mathbf{3 A}$} & \multirow{11}{*}{$7 / 6 / 05$} & AWV-G-98A & - & - & - & & - \\
\hline & & AWV-G-98B & XRF & 31.40 & 2406.78 & & 0.45 \\
\hline & & AWV-G-99A & - & - & - & & - \\
\hline & & AWV-G-100A & XRF & 21.28 & 2428.06 & & 0.47 \\
\hline & & AWV-G-100B & - & - & - & & - \\
\hline & & AWV-G-100C & XRF & 25.64 & 2453.70 & & 0.48 \\
\hline & & AWV-G-101A & - & - & - & & - \\
\hline & & AWV-G-101B & XRF & 27.58 & 2481.28 & & 0.48 \\
\hline & & AWV-G-101C & - & - & - & & - \\
\hline & & AWV-G-106A & XRF & 29.08 & 2510.36 & & 0.49 \\
\hline & & AWV-G-106B & - & - & - & & - \\
\hline & \multirow{3}{*}{$7 / 7 / 05$} & AWV-G-106C & XRF & 23.64 & 2534.00 & & 0.51 \\
\hline & & AWV-G-106D & - & - & - & & - \\
\hline & & AWV-G-106E & XRF & 22.76 & 2556.76 & & 0.51 \\
\hline
\end{tabular}

"-" Empty data field 
Table 4.1. Listing of DM100 Glasses Discharged, Analysis Performed, Masses, Target and Measured $\mathrm{SO}_{3}$ Concentrations (continued).

\begin{tabular}{|c|c|c|c|c|c|c|c|}
\hline \multirow{2}{*}{ Test } & \multirow{2}{*}{ Date } & \multirow{2}{*}{ Sample Name } & \multirow{2}{*}{ Analysis } & \multirow{2}{*}{ Mass (kg) } & \multirow{2}{*}{$\begin{array}{c}\text { Cumulative } \\
\text { Mass (kg) }\end{array}$} & \multicolumn{2}{|c|}{$\mathrm{SO}_{3}$ wt \% } \\
\hline & & & & & & Target & Measured \\
\hline \multirow{4}{*}{ 3A } & \multirow{13}{*}{$7 / 7 / 05$} & AWV-G-108A & - & - & - & \multirow{4}{*}{0.59} & - \\
\hline & & AWV-G-108B & $\mathrm{XRF}$ & 25.24 & 2582.00 & & 0.51 \\
\hline & & AWV-G-108C & - & - & - & & - \\
\hline & & AWV-G-108D & XRF & 15.82 & 2597.82 & & 0.51 \\
\hline \multirow{33}{*}{ 3B } & & AWV-G-117A & - & - & - & \multirow{33}{*}{0.69} & - \\
\hline & & AWV-G-118A & XRF & 22.30 & 2620.12 & & 0.52 \\
\hline & & AWV-G-118B & - & - & - & & - \\
\hline & & AWV-G-118C & XRF & 28.10 & 2648.22 & & 0.53 \\
\hline & & AWV-G-119A & - & - & - & & - \\
\hline & & AWV-G-121A & XRF & 28.76 & 2676.98 & & 0.54 \\
\hline & & AWV-G-121B & - & - & - & & - \\
\hline & & AWV-G-122A & XRF & 25.16 & 2702.14 & & 0.57 \\
\hline & & AWV-G-122B & - & - & - & & - \\
\hline & \multirow{16}{*}{$7 / 8 / 05$} & AWV-G-125A & XRF & 27.82 & 2729.96 & & 0.57 \\
\hline & & AWV-G-125B & - & - & - & & - \\
\hline & & AWV-G-127A & XRF & 25.10 & 2755.06 & & 0.56 \\
\hline & & AWV-G-127B & - & - & - & & - \\
\hline & & AWV-G-128A & XRF & 29.82 & 2784.88 & & 0.55 \\
\hline & & AWV-G-128B & - & - & - & & - \\
\hline & & AWV-G-129A & XRF & 22.28 & 2807.16 & & 0.53 \\
\hline & & AWV-G-129B & - & - & - & & - \\
\hline & & AWV-G-129C & XRF & 24.38 & 2831.54 & & 0.52 \\
\hline & & AWV-G-130A & - & - & - & & - \\
\hline & & AWV-G-130B & XRF & 28.96 & 2860.50 & & 0.54 \\
\hline & & AWV-G-135A & - & - & - & & - \\
\hline & & AWV-G-135B & XRF & 24.26 & 2884.76 & & 0.54 \\
\hline & & AWV-G-137A & - & - & - & & - \\
\hline & & AWV-G-140A & XRF & 26.72 & 2911.48 & & 0.53 \\
\hline & & AWV-G-140B & - & - & - & & - \\
\hline & \multirow{12}{*}{ 7/9/05 } & AWV-G-140C & XRF & 25.32 & 2936.80 & & 0.52 \\
\hline & & AWV-G-141A & - & - & - & & - \\
\hline & & AWV-G-141B & XRF & 24.72 & 2961.52 & & 0.53 \\
\hline & & AWV-G-142A & - & - & - & & - \\
\hline & & AWV-G-142B & XRF & 27.42 & 2988.94 & & 0.52 \\
\hline & & AWV-G-142C & - & - & - & & - \\
\hline & & AWV-G-142D & XRF & 25.02 & 3013.96 & & 0.53 \\
\hline & & AWV-G-147A & XRF, F, DCP & 11.70 & 3025.66 & & 0.54 \\
\hline \multirow{4}{*}{$3 C$} & & AWV-G-148A & - & - & - & \multirow{4}{*}{0.80} & - \\
\hline & & AWV-G-153A & XRF & 26.12 & 3051.78 & & 0.58 \\
\hline & & AWV-G-153B & - & - & - & & - \\
\hline & & AWV-G-153C & XRF & 28.42 & 3080.20 & & 0.57 \\
\hline
\end{tabular}

"-" Empty data field 
Table 4.1. Listing of DM100 Glasses Discharged, Analysis Performed, Masses, Target and Measured $\mathrm{SO}_{3}$ Concentrations (continued).

\begin{tabular}{|c|c|c|c|c|c|c|c|}
\hline \multirow{2}{*}{ Test } & \multirow{2}{*}{ Date } & \multirow{2}{*}{ Sample Name } & \multirow{2}{*}{ Analysis } & \multirow{2}{*}{ Mass (kg) } & \multirow{2}{*}{\begin{tabular}{|c|} 
Cumulative \\
Mass (kg)
\end{tabular}} & \multicolumn{2}{|c|}{$\mathrm{SO}_{3}$ wt $\%$} \\
\hline & & & & & & Target & Measured \\
\hline \multirow{30}{*}{$3 C$} & \multirow{3}{*}{ 7/9/05 } & AWV-G-153D & - & - & - & \multirow{30}{*}{0.80} & - \\
\hline & & AWV-G-155A & XRF & 24.42 & 3104.62 & & 0.56 \\
\hline & & BWV-G-9A & - & - & - & & - \\
\hline & \multirow{18}{*}{ 7/10/05 } & BWV-G-10A & XRF & 25.32 & 3129.94 & & 0.56 \\
\hline & & BWV-G-10B & - & - & - & & - \\
\hline & & BWV-G-10C & XRF & 25.60 & 3155.54 & & 0.57 \\
\hline & & BWV-G-11A & - & - & - & & - \\
\hline & & BWV-G-12A & $\mathrm{XRF}$ & 24.62 & 3180.16 & & 0.55 \\
\hline & & BWV-G-12B & - & - & - & & - \\
\hline & & BWV-G-12C & XRF & 25.60 & 3205.76 & & 0.58 \\
\hline & & BWV-G-12D & - & - & - & & - \\
\hline & & BWV-G-12E & XRF & 23.30 & 3229.06 & & 0.58 \\
\hline & & BWV-G-14A & - & - & - & & - \\
\hline & & BWV-G-14B & XRF & 24.26 & 3253.32 & & 0.58 \\
\hline & & BWV-G-17A & - & - & - & & - \\
\hline & & BWV-G-17B & XRF & 27.48 & 3280.80 & & 0.56 \\
\hline & & BWV-G-19A & - & - & - & & - \\
\hline & & BWV-G-19B & XRF & 21.68 & 3302.48 & & 0.58 \\
\hline & & BWV-G-19C & - & - & - & & - \\
\hline & & BWV-G-21A & XRF & 26.54 & 3329.02 & & 0.59 \\
\hline & & BWV-G-24A & - & - & - & & - \\
\hline & \multirow{9}{*}{ 7/11/05 } & BWV-G-24B & XRF & 21.30 & 3350.32 & & 0.57 \\
\hline & & BWV-G-24C & - & - & - & & - \\
\hline & & BWV-G-26A & XRF & 27.52 & 3377.84 & & 0.58 \\
\hline & & BWV-G-26B & - & - & - & & - \\
\hline & & BWV-G-27A & XRF & 19.06 & 3396.90 & & 0.55 \\
\hline & & BWV-G-28A & - & - & - & & - \\
\hline & & BWV-G-28B & XRF & 29.74 & 3426.64 & & 0.58 \\
\hline & & BWV-G-32A & - & - & - & & - \\
\hline & & BWV-G-32B & $\begin{array}{c}\text { XRF,PCT,TCLP, } \\
\text { CCC }\end{array}$ & 23.38 & 3450.02 & & 0.59 \\
\hline \multirow{11}{*}{$4 \mathrm{~A}$} & \multirow{5}{*}{$8 / 15 / 05$} & BWV-G-58A & - & - & - & \multirow{11}{*}{0.78} & - \\
\hline & & BWV-G-59A & XRF, F & 26.08 & 3476.10 & & 0.11 \\
\hline & & BWV-G-60A & - & - & - & & - \\
\hline & & BWV-G-60B & XRF, F & 23.14 & 3499.24 & & 0.19 \\
\hline & & BWV-G-61A & - & - & - & & - \\
\hline & \multirow{6}{*}{$8 / 16 / 05$} & BWV-G-61B & XRF, F & 23.64 & 3522.88 & & 0.25 \\
\hline & & BWV-G-62A & - & - & - & & - \\
\hline & & BWV-G-62B & XRF, F & 21.16 & 3544.04 & & 0.30 \\
\hline & & BWV-G-66A & - & - & - & & - \\
\hline & & BWV-G-66B & XRF, F & 24.06 & 3568.10 & & 0.34 \\
\hline & & BWV-G-66C & XRF, CCC & 12.00 & 3580.10 & & 0.36 \\
\hline
\end{tabular}

"-" Empty data field 
Table 4.1. Listing of DM100 Glasses Discharged, Analysis Performed, Masses, Target and Measured $\mathrm{SO}_{3}$ Concentrations (continued).

\begin{tabular}{|c|c|c|c|c|c|c|c|}
\hline \multirow{2}{*}{ Test } & \multirow{2}{*}{ Date } & \multirow{2}{*}{ Sample Name } & \multirow{2}{*}{ Analysis } & \multirow{2}{*}{ Mass (kg) } & \multirow{2}{*}{$\begin{array}{c}\text { Cumulative } \\
\text { Mass (kg) }\end{array}$} & \multicolumn{2}{|c|}{$\mathrm{SO}_{3} \mathrm{wt} \%$} \\
\hline & & & & & & Target & Target \\
\hline \multirow{34}{*}{ 4B } & \multirow{8}{*}{$3 / 16 / 05$} & BWV-G-71A & - & - & - & \multirow{34}{*}{0.67} & - \\
\hline & & BWV-G-71B & XRF, F & 28.44 & 3608.54 & & 0.39 \\
\hline & & BWV-G-72A & - & - & - & & - \\
\hline & & BWV-G-72B & XRF & 25.72 & 3634.26 & & 0.40 \\
\hline & & BWV-G-73A & - & - & - & & - \\
\hline & & BWV-G-73B & XRF, F & 24.62 & 3658.88 & & 0.42 \\
\hline & & BWV-G-78A & - & - & - & & - \\
\hline & & BWV-G-79A & XRF & 27.20 & 3686.08 & & 0.43 \\
\hline & \multirow{16}{*}{$8 / 17 / 05$} & BWV-G-79B & - & - & - & & - \\
\hline & & BWV-G-81A & $\mathrm{XRF}, \mathrm{F}$ & 27.02 & 3713.10 & & 0.45 \\
\hline & & BWV-G-81B & - & - & - & & - \\
\hline & & BWV-G-81C & XRF & 26.08 & 3739.18 & & 0.46 \\
\hline & & BWV-G-81D & - & - & - & & - \\
\hline & & BWV-G-84A & XRF, F & 26.36 & 3765.54 & & 0.48 \\
\hline & & BWV-G-85A & - & - & - & & - \\
\hline & & BWV-G-85B & XRF & 28.42 & 3793.96 & & 0.48 \\
\hline & & BWV-G-86A & - & - & - & & - \\
\hline & & BWV-G-88A & XRF, F & 23.18 & 3817.14 & & 0.49 \\
\hline & & BWV-G-88B & - & - & - & & - \\
\hline & & BWV-G-88C & $\mathrm{XRF}$ & 26.10 & 3843.24 & & 0.50 \\
\hline & & BWV-G-89A & - & - & - & & - \\
\hline & & BWV-G-89B & XRF & 26.98 & 3870.22 & & 0.51 \\
\hline & & BWV-G-89C & - & - & - & & - \\
\hline & & BWV-G-93A & XRF & 26.48 & 3896.70 & & 0.51 \\
\hline & \multirow{16}{*}{ 8/18/05 } & BWV-G-93B & - & - & - & & - \\
\hline & & BWV-G-93C & XRF & 27.48 & 3924.18 & & 0.51 \\
\hline & & BWV-G-95A & - & - & - & & - \\
\hline & & BWV-G-95B & $\mathrm{XRF}$ & 26.94 & 3951.12 & & 0.53 \\
\hline & & BWV-G-98A & - & - & - & & - \\
\hline & & BWV-G-98B & $\mathrm{XRF}$ & 24.74 & 3975.86 & & 0.51 \\
\hline & & BWV-G-99A & - & - & - & & - \\
\hline & & BWV-G-99B & XRF & 25.42 & 4001.28 & & 0.51 \\
\hline & & BWV-G-102A & - & - & - & & - \\
\hline & & BWV-G-102B & XRF, F & 26.91 & 4028.19 & & 0.52 \\
\hline \multirow{6}{*}{$4 C$} & & BWV-G-113A & - & - & - & \multirow{6}{*}{0.58} & - \\
\hline & & BWV-G-113B & XRF & 26.66 & 4054.85 & & 0.53 \\
\hline & & BWV-G-113C & - & - & - & & - \\
\hline & & BWV-G-113D & XRF & 22.98 & 4077.83 & & 0.49 \\
\hline & & BWV-G-117A & - & - & - & & - \\
\hline & & BWV-G-117B & $\mathrm{XRF}$ & 26.58 & 4104.41 & & 0.55 \\
\hline
\end{tabular}

"-" Empty data field 
Table 4.1. Listing of DM100 Glasses Discharged, Analysis Performed, Masses, Target and Measured $\mathrm{SO}_{3}$ Concentrations (continued).

\begin{tabular}{|c|c|c|c|c|c|c|c|}
\hline \multirow{2}{*}{ Test } & \multirow{2}{*}{ Date } & \multirow{2}{*}{ Sample Name } & \multirow{2}{*}{ Analysis } & \multirow{2}{*}{ Mass (kg) } & \multirow{2}{*}{$\begin{array}{c}\text { Cumulative } \\
\text { Mass (kg) }\end{array}$} & \multicolumn{2}{|c|}{$\mathrm{SO}_{3}$ wt $\%$} \\
\hline & & & & & & Target & Measured \\
\hline \multirow{28}{*}{$4 C$} & \multirow{15}{*}{$8 / 19 / 05$} & BWV-G-119A & - & - & - & \multirow{40}{*}{0.58} & - \\
\hline & & BWV-G-121A & XRF & 25.78 & 4130.19 & & 0.54 \\
\hline & & BWV-G-121B & - & - & - & & - \\
\hline & & BWV-G-124A & XRF & 28.40 & 4158.59 & & 0.46 \\
\hline & & BWV-G-124B & - & - & - & & - \\
\hline & & BWV-G-125A & XRF & 28.48 & 4187.07 & & 0.45 \\
\hline & & BWV-G-125B & - & - & - & & - \\
\hline & & BWV-G-126A & XRF & 18.26 & 4205.33 & & 0.45 \\
\hline & & BWV-G-126B & - & - & - & & - \\
\hline & & BWV-G-128A & $\mathrm{XRF}, \mathrm{F}$ & 30.34 & 4235.67 & & 0.50 \\
\hline & & BWV-G-128B & - & - & - & & - \\
\hline & & BWV-G-128C & XRF & 28.10 & 4263.77 & & 0.43 \\
\hline & & BWV-G-129A & - & - & - & & - \\
\hline & & BWV-G-129B & XRF & 20.44 & 4284.21 & & 0.45 \\
\hline & & BWV-G-129C & - & - & - & & - \\
\hline & & BWV-G-129D & XRF & 25.62 & 4309.83 & & 0.45 \\
\hline & & BWV-G-134A & - & - & - & & - \\
\hline & & BWV-G-134B & XRF & 24.76 & 4334.59 & & 0.46 \\
\hline & & BWV-G-134C & - & - & - & & - \\
\hline & & BWV-G-136A & $\mathrm{XRF}$ & 25.20 & 4359.79 & & 0.46 \\
\hline & & BWV-G-136B & - & - & - & & - \\
\hline & 8/20/05 & BWV-G-140A & $\mathrm{XRF}$ & 23.86 & 4383.65 & & 0.46 \\
\hline & $8 / 20 / 05$ & BWV-G-140B & - & - & - & & - \\
\hline & & BWV-G-141A & XRF & 20.80 & 4404.45 & & 0.46 \\
\hline & & BWV-G-141B & - & - & - & & - \\
\hline & & BWV-G-141C & XRF & 25.98 & 4430.43 & & 0.48 \\
\hline & & BWV-G-142A & - & - & - & & - \\
\hline & & BWV-G-142B & $\begin{array}{c}\text { XRF, F, } \\
\text { PCT,TCLP, CCC }\end{array}$ & 24.40 & 4454.83 & & 0.50 \\
\hline \multirow{12}{*}{$5 \mathbf{A}$} & \multirow{12}{*}{$8 / 24 / 05$} & CWV-G-8A & - & - & - & & - \\
\hline & & CWV-G-8B & $\mathrm{XRF}, \mathrm{F}$ & 25.48 & 4480.31 & & 0.47 \\
\hline & & CWV-G-13A & - & - & - & & - \\
\hline & & CWV-G-13B & XRF & 25.18 & 4505.49 & & 0.49 \\
\hline & & CWV-G-15A & - & - & - & & - \\
\hline & & CWV-G-15B & XRF & 23.80 & 4529.29 & & 0.48 \\
\hline & & CWV-G-16A & - & - & - & & - \\
\hline & & CWV-G-16B & $\mathrm{XRF}$ & 24.06 & 4553.35 & & 0.48 \\
\hline & & CWV-G-16C & - & - & - & & - \\
\hline & & CWV-G-16D & $\mathrm{XRF}$ & 22.90 & 4576.25 & & 0.48 \\
\hline & & CWV-G-20A & - & - & - & & - \\
\hline & & CWV-G-20B & XRF, F & 29.20 & 4605.45 & & 0.52 \\
\hline
\end{tabular}

\footnotetext{
"-" Empty data field
} 
Table 4.1. Listing of DM100 Glasses Discharged, Analysis Performed, Masses, Target and Measured $\mathrm{SO}_{3}$ Concentrations (continued).

\begin{tabular}{|c|c|c|c|c|c|c|c|}
\hline \multirow{2}{*}{ Test } & \multirow{2}{*}{ Date } & \multirow{2}{*}{ Sample Name } & \multirow{2}{*}{ Analysis } & \multirow{2}{*}{ Mass (kg) } & \multirow{2}{*}{$\begin{array}{c}\text { Cumulative } \\
\text { Mass (kg) }\end{array}$} & \multicolumn{2}{|c|}{$\mathrm{SO}_{3}$ wt $\%$} \\
\hline & & & & & & Target & Measured \\
\hline \multirow{7}{*}{$5 \mathrm{~A}$} & \multirow{16}{*}{$8 / 25 / 05$} & CWV-G-20C & - & - & - & \multirow{7}{*}{0.58} & - \\
\hline & & CWV-G-20D & XRF & 24.60 & 4630.05 & & 0.50 \\
\hline & & CWV-G-22A & - & - & - & & - \\
\hline & & CWV-G-22B & XRF & 21.36 & 4651.41 & & 0.51 \\
\hline & & CWV-G-22C & - & - & - & & - \\
\hline & & CWV-G-22D & XRF & 23.20 & 4674.61 & & 0.49 \\
\hline & & CWV-G-26A & XRF, F & 11.08 & 4685.69 & & 0.51 \\
\hline \multirow{32}{*}{$5 B$} & & CWV-G-28A & - & - & - & \multirow{32}{*}{0.52} & - \\
\hline & & CWV-G-30A & XRF & 24.30 & 4709.99 & & 0.48 \\
\hline & & CWV-G-30B & - & - & - & & - \\
\hline & & CWV-G-30C & XRF & 19.26 & 4729.25 & & 0.46 \\
\hline & & CWV-G-31A & - & - & - & & - \\
\hline & & CWV-G-31B & XRF & 29.74 & 4758.99 & & 0.47 \\
\hline & & CWV-G-35A & - & - & - & & - \\
\hline & & CWV-G-35B & XRF & 28.88 & 4787.87 & & 0.46 \\
\hline & & CWV-G-37A & - & - & - & & - \\
\hline & \multirow{19}{*}{ 8/26/05 } & CWV-G-37B & XRF & 21.86 & 4809.73 & & 0.44 \\
\hline & & CWV-G-37C & - & - & - & & - \\
\hline & & CWV-G-38A & XRF & 20.36 & 4830.09 & & 0.44 \\
\hline & & CWV-G-38B & - & - & - & & - \\
\hline & & CWV-G-38C & XRF, F & 22.36 & 4852.45 & & 0.45 \\
\hline & & CWV-G-43A & - & - & - & & - \\
\hline & & CWV-G-43B & XRF & 29.42 & 4881.87 & & 0.47 \\
\hline & & CWV-G-44A & - & - & - & & - \\
\hline & & CWV-G-44B & XRF & 20.24 & 4902.11 & & 0.47 \\
\hline & & CWV-G-45A & - & - & - & & - \\
\hline & & CWV-G-45B & XRF & 28.82 & 4930.93 & & 0.46 \\
\hline & & CWV-G-45C & - & - & - & & - \\
\hline & & CWV-G-46A & XRF & 21.60 & 4952.53 & & 0.45 \\
\hline & & CWV-G-46B & - & - & - & & - \\
\hline & & CWV-G-46C & XRF & 20.50 & 4973.03 & & 0.45 \\
\hline & & CWV-G-50A & - & - & - & & - \\
\hline & & CWV-G-50B & XRF & 19.50 & 4992.53 & & 0.44 \\
\hline & & CWV-G-50C & - & - & - & & - \\
\hline & & CWV-G-50D & XRF, F & 22.16 & 5014.69 & & 0.46 \\
\hline & \multirow{4}{*}{$8 / 27 / 05$} & CWV-G-50E & - & - & - & & - \\
\hline & & CWV-G-50F & XRF & 20.14 & 5034.83 & & 0.44 \\
\hline & & CWV-G-52A & - & - & - & & - \\
\hline & & CWV-G-52B & XRF & 24.68 & 5059.51 & & 0.43 \\
\hline
\end{tabular}


Table 4.1. Listing of DM100 Glasses Discharged, Analysis Performed, Masses, Target and Measured $\mathrm{SO}_{3}$ Concentrations (continued).

\begin{tabular}{|c|c|c|c|c|c|c|c|}
\hline \multirow{2}{*}{ Test } & \multirow{2}{*}{ Date } & \multirow{2}{*}{ Sample Name } & \multirow{2}{*}{ Analysis } & \multirow{2}{*}{ Mass (kg) } & \multirow{2}{*}{$\begin{array}{c}\text { Cumulative } \\
\text { Mass (kg) }\end{array}$} & \multicolumn{2}{|c|}{$\mathrm{SO}_{3}$ wt \% } \\
\hline & & & & & & Target & Measured \\
\hline \multirow{7}{*}{$5 B$} & \multirow{14}{*}{$3 / 27 / 05$} & CWV-G-53C & - & - & - & \multirow{7}{*}{0.52} & - \\
\hline & & CWV-G-54A & $\mathrm{XRF}$ & 25.94 & 5085.45 & & 0.42 \\
\hline & & CWV-G-54B & - & - & - & & - \\
\hline & & CWV-G-54C & XRF & 23.62 & 5109.07 & & 0.42 \\
\hline & & CWV-G-54D & - & - & - & & - \\
\hline & & CWV-G-58A & XRF & 21.86 & 5130.93 & & 0.43 \\
\hline & & CWV-G-58B & XRF, F & 12.56 & 5143.49 & & 0.44 \\
\hline \multirow{34}{*}{$5 C$} & & CWV-G-60A & - & - & - & \multirow{34}{*}{0.45} & - \\
\hline & & CWV-G-61A & XRF & 20.18 & 5163.67 & & 0.43 \\
\hline & & CWV-G-61B & - & - & - & & - \\
\hline & & CWV-G-61C & XRF & 23.62 & 5187.29 & & 0.44 \\
\hline & & CWV-G-63A & - & - & - & & - \\
\hline & & CWV-G-63B & XRF & 23.86 & 5211.15 & & 0.44 \\
\hline & & CWV-G-67A & - & - & - & & - \\
\hline & \multirow{20}{*}{$8 / 28 / 05$} & CWV-G-68A & XRF & 25.82 & 5236.97 & & 0.42 \\
\hline & & CWV-G-68B & - & - & - & & - \\
\hline & & CWV-G-69A & XRF & 24.52 & 5261.49 & & 0.42 \\
\hline & & CWV-G-69B & - & - & - & & - \\
\hline & & CWV-G-69C & XRF, F & 19.90 & 5281.39 & & 0.43 \\
\hline & & CWV-G-69D & - & - & - & & - \\
\hline & & CWV-G-71A & XRF & 23.50 & 5304.89 & & 0.42 \\
\hline & & CWV-G-71B & - & - & - & & - \\
\hline & & CWV-G-71C & XRF & 20.98 & 5325.87 & & 0.42 \\
\hline & & CWV-G-71D & - & - & - & & - \\
\hline & & CWV-G-72A & XRF & 18.82 & 5344.69 & & 0.42 \\
\hline & & CWV-G-72B & - & - & - & & - \\
\hline & & CWV-G-72C & XRF & 23.52 & 5368.21 & & 0.42 \\
\hline & & CWV-G-75A & - & - & - & & - \\
\hline & & CWV-G-75B & XRF & 22.16 & 5390.37 & & 0.41 \\
\hline & & CWV-G-75C & - & - & - & & - \\
\hline & & CWV-G-79A & XRF & 25.04 & 5415.41 & & 0.41 \\
\hline & & CWV-G-79B & - & - & - & & - \\
\hline & & CWV-G-79C & $\mathrm{XRF}$ & 26.82 & 5442.23 & & 0.43 \\
\hline & & CWV-G-79D & - & - & - & & - \\
\hline & \multirow{7}{*}{ 8/29/05 } & CWV-G-81A & XRF, F & 21.86 & 5464.09 & & 0.42 \\
\hline & & CWV-G-81B & - & - & - & & - \\
\hline & & CWV-G-81C & XRF & 22.54 & 5486.63 & & 0.42 \\
\hline & & CWV-G-83A & - & - & - & & - \\
\hline & & CWV-G-83B & XRF & 20.80 & 5507.43 & & 0.40 \\
\hline & & CWV-G-83C & - & - & - & & - \\
\hline & & CWV-G-86A & XRF & 22.90 & 5530.33 & & 0.41 \\
\hline
\end{tabular}

"-" Empty data field 
Table 4.1. Listing of DM100 Glasses Discharged, Analysis Performed, Masses, Target and Measured $\mathrm{SO}_{3}$ Concentrations (continued).

\begin{tabular}{|c|c|c|c|c|c|c|c|}
\hline \multirow[t]{2}{*}{ Test } & \multirow{2}{*}{ Date } & \multirow{2}{*}{ Sample Name } & \multirow{2}{*}{ Analysis } & \multirow{2}{*}{ Mass (kg) } & \multirow{2}{*}{$\begin{array}{c}\text { Cumulative } \\
\text { Mass (kg) }\end{array}$} & \multicolumn{2}{|c|}{$\mathrm{SO}_{3}$ wt \% } \\
\hline & & & & & & Target & Measured \\
\hline \multirow{6}{*}{$5 C$} & \multirow{11}{*}{$3 / 29 / 05$} & CWV-G-86B & - & - & - & \multirow{6}{*}{0.45} & - \\
\hline & & CWV-G-86C & XRF & 21.18 & 5551.51 & & 0.41 \\
\hline & & CWV-G-88A & - & - & - & & - \\
\hline & & CWV-G-90A & XRF & 20.94 & 5572.45 & & 0.40 \\
\hline & & CWV-G-90B & - & - & - & & - \\
\hline & & CWV-G-90C & XRF, F, CCC & 25.50 & 5597.95 & & 0.41 \\
\hline \multirow{34}{*}{$5 D$} & & CWV-G-93A & - & - & - & \multirow{34}{*}{0.40} & - \\
\hline & & CWV-G-97A & XRF & 26.54 & 5624.49 & & 0.38 \\
\hline & & CWV-G-97B & - & - & - & & - \\
\hline & & CWV-G-97C & XRF & 20.68 & 5645.17 & & 0.38 \\
\hline & & CWV-G-98A & - & - & - & & - \\
\hline & \multirow{18}{*}{$8 / 30 / 05$} & CWV-G-98B & XRF & 19.82 & 5664.99 & & 0.39 \\
\hline & & CWV-G-99A & - & - & - & & - \\
\hline & & CWV-G-100A & XRF & 25.92 & 5690.91 & & 0.39 \\
\hline & & CWV-G-100B & - & - & - & & - \\
\hline & & CWV-G-101A & XRF & 21.30 & 5712.21 & & 0.39 \\
\hline & & CWV-G-101B & - & - & - & & - \\
\hline & & CWV-G-105A & $\mathrm{XRF}$ & 20.62 & 5732.83 & & 0.37 \\
\hline & & CWV-G-105B & - & - & - & & - \\
\hline & & CWV-G-105C & XRF & 21.58 & 5754.41 & & 0.39 \\
\hline & & CWV-G-107A & - & - & - & & - \\
\hline & & CWV-G-107B & XRF, F & 19.46 & 5773.87 & & 0.37 \\
\hline & & CWV-G-107C & - & - & - & & - \\
\hline & & CWV-G-107D & XRF & 22.32 & 5796.19 & & 0.36 \\
\hline & & CWV-G-109A & - & - & - & & - \\
\hline & & CWV-G-109B & XRF & 23.70 & 5819.89 & & 0.36 \\
\hline & & CWV-G-109C & - & - & - & & - \\
\hline & & CWV-G-112A & XRF & 24.21 & 5844.10 & & 0.36 \\
\hline & & CWV-G-112B & - & - & - & & - \\
\hline & \multirow{11}{*}{ 8/31/05 } & CWV-G-112C & XRF & 24.32 & 5868.42 & & 0.34 \\
\hline & & CWV-G-114A & - & - & - & & - \\
\hline & & CWV-G-114B & XRF & 19.60 & 5888.02 & & 0.34 \\
\hline & & CWV-G-114C & - & - & - & & - \\
\hline & & CWV-G-114D & XRF, F & 23.52 & 5911.54 & & 0.36 \\
\hline & & CWV-G-114E & - & - & - & & - \\
\hline & & CWV-G-118A & XRF & 21.52 & 5933.06 & & 0.35 \\
\hline & & CWV-G-118B & - & - & - & & - \\
\hline & & CWV-G-118C & XRF & 19.22 & 5952.28 & & 0.33 \\
\hline & & CWV-G-119A & - & - & - & & - \\
\hline & & CWV-G-119B & XRF & 20.82 & 5973.10 & & 0.33 \\
\hline
\end{tabular}

"-" Empty data field 
Table 4.1. Listing of DM100 Glasses Discharged, Analysis Performed, Masses, Target and Measured $\mathrm{SO}_{3}$ Concentrations (continued).

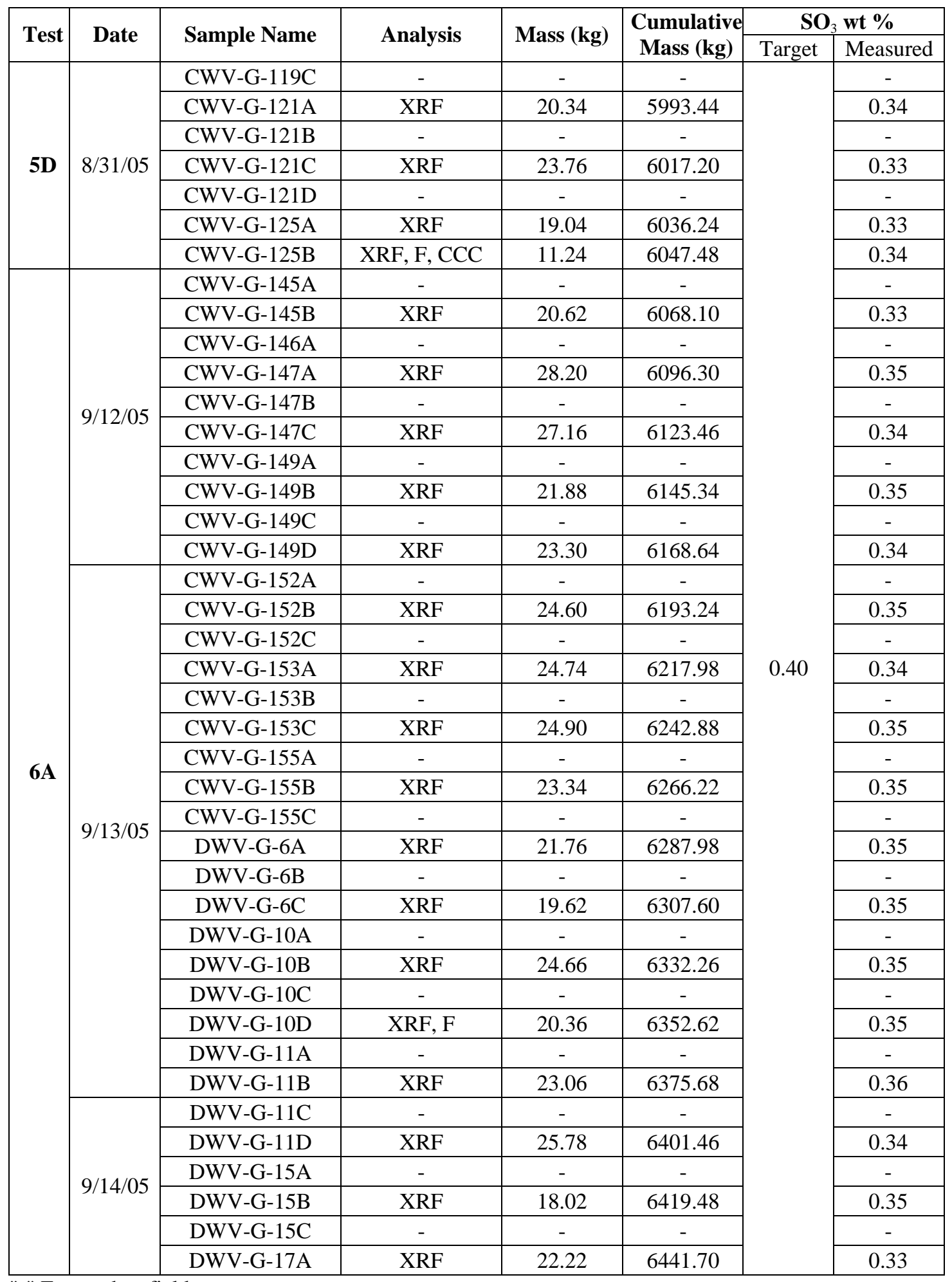

"-" Empty data field 
Table 4.1. Listing of DM100 Glasses Discharged, Analysis Performed, Masses, Target and Measured $\mathrm{SO}_{3}$ Concentrations (continued).

\begin{tabular}{|c|c|c|c|c|c|c|c|}
\hline \multirow{2}{*}{ Test } & \multirow{2}{*}{ Date } & \multirow{2}{*}{ Sample Name } & \multirow{2}{*}{ Analysis } & \multirow{2}{*}{ Mass (kg) } & \multirow{2}{*}{$\begin{array}{c}\text { Cumulative } \\
\text { Mass (kg) }\end{array}$} & \multicolumn{2}{|c|}{$\mathrm{SO}_{3}$ wt \% } \\
\hline & & & & & & Target & Measured \\
\hline \multirow{5}{*}{ 6A } & \multirow{11}{*}{$9 / 14 / 05$} & DWV-G-17B & - & - & - & \multirow{5}{*}{0.40} & - \\
\hline & & DWV-G-17C & XRF & 20.94 & 6462.64 & & 0.35 \\
\hline & & DWV-G-19A & - & - & - & & - \\
\hline & & DWV-G-19B & XRF, F & 18.14 & 6480.78 & & 0.35 \\
\hline & & DWV-G-19C & XRF, CCC & 11.68 & 6492.46 & & 0.34 \\
\hline \multirow{37}{*}{$6 B$} & & DWV-G-23A & - & - & - & \multirow{37}{*}{0.35} & - \\
\hline & & DWV-G-23B & XRF & 20.26 & 6512.72 & & 0.33 \\
\hline & & DWV-G-25A & - & - & - & & - \\
\hline & & DWV-G-25B & XRF & 24.00 & 6536.72 & & 0.33 \\
\hline & & DWV-G-25C & - & - & - & & - \\
\hline & & DWV-G-25D & $\mathrm{XRF}$ & 29.62 & 6566.34 & & 0.33 \\
\hline & \multirow{19}{*}{$9 / 15 / 0$} & DWV-G-29A & - & - & - & & - \\
\hline & & DWV-G-29B & XRF & 24.48 & 6590.82 & & 0.34 \\
\hline & & DWV-G-29C & - & - & - & & - \\
\hline & & DWV-G-29D & XRF, F & 25.26 & 6616.08 & & 0.33 \\
\hline & & DWV-G-32A & - & - & - & & - \\
\hline & & DWV-G-32B & XRF & 21.24 & 6637.32 & & 0.32 \\
\hline & & DWV-G-32C & - & - & - & & - \\
\hline & & DWV-G-33A & XRF & 21.24 & 6658.56 & & 0.31 \\
\hline & & DWV-G-33B & - & - & - & & - \\
\hline & & DWV-G-34A & XRF & 18.84 & 6677.40 & & 0.34 \\
\hline & & DWV-G-34B & - & - & - & & - \\
\hline & & DWV-G-34C & XRF & 21.88 & 6699.28 & & 0.32 \\
\hline & & DWV-G-38A & - & - & - & & - \\
\hline & & DWV-G-38B & XRF & 26.32 & 6725.60 & & 0.31 \\
\hline & & DWV-G-39A & - & - & - & & - \\
\hline & & DWV-G-39B & XRF, F & 25.24 & 6750.84 & & 0.32 \\
\hline & & DWV-G-43A & - & - & - & & - \\
\hline & & DWV-G-43B & XRF & 23.88 & 6774.72 & & 0.31 \\
\hline & & DWV-G-43C & - & - & - & & - \\
\hline & \multirow{12}{*}{ 9/16/05 } & DWV-G-43D & XRF & 23.30 & 6798.02 & & 0.30 \\
\hline & & DWV-G-45A & - & - & - & & - \\
\hline & & DWV-G-45B & XRF & 30.32 & 6828.34 & & 0.32 \\
\hline & & DWV-G-45C & - & - & - & & - \\
\hline & & DWV-G-46A & XRF & 20.81 & 6849.15 & & 0.31 \\
\hline & & DWV-G-46B & - & - & - & & - \\
\hline & & DWV-G-46C & XRF & 25.68 & 6874.83 & & 0.31 \\
\hline & & DWV-G-48A & - & - & - & & - \\
\hline & & DWV-G-48B & XRF & 25.06 & 6899.89 & & 0.32 \\
\hline & & DWV-G-48C & - & - & - & & - \\
\hline & & DWV-G-51A & XRF & 26.92 & 6926.81 & & 0.31 \\
\hline & & DWV-G-51B & $\begin{array}{c}\text { XRF,PCT,TCLP, } \\
\text { CCC }\end{array}$ & 10.80 & 6937.61 & & 0.33 \\
\hline
\end{tabular}


Table 4.2. XRF Analyzed Compositions for Glass Samples Discharged During DM100 Tests (wt\%).

\begin{tabular}{|c|c|c|c|c|c|c|c|c|c|c|c|c|c|c|c|c|}
\hline Test & \multicolumn{16}{|c|}{ 1A } \\
\hline \multicolumn{2}{|c|}{ Glass (kg) } & 24.60 & 51.78 & 73.94 & 104.02 & 127.26 & 149.18 & 168.44 & 191.74 & 217.66 & 241.46 & 269.00 & 292.60 & 319.34 & 342.96 & 367.52 \\
\hline Constituent & Target & \begin{tabular}{|l|} 
WVZ- \\
G-40B \\
\end{tabular} & \begin{tabular}{|l} 
WVZ- \\
G-41A
\end{tabular} & \begin{tabular}{|l} 
WVZ- \\
G-41C
\end{tabular} & \begin{tabular}{|l|} 
WVZ- \\
G-45B \\
\end{tabular} & \begin{tabular}{|l|} 
WVZ- \\
G-46B \\
\end{tabular} & \begin{tabular}{|l} 
WVZ- \\
G-46D \\
\end{tabular} & \begin{tabular}{|l|} 
WVZ- \\
G-48A \\
\end{tabular} & \begin{tabular}{|l|} 
WVZ- \\
G-48C \\
\end{tabular} & \begin{tabular}{|l} 
WVZ- \\
G-51B
\end{tabular} & \begin{tabular}{|l|} 
WVZ- \\
G-51D
\end{tabular} & $\begin{array}{l}\text { WVZ- } \\
\text { G-56A }\end{array}$ & \begin{tabular}{|l|} 
WVZ- \\
G-56C \\
\end{tabular} & \begin{tabular}{|l} 
WVZ- \\
G-58A \\
\end{tabular} & \begin{tabular}{|l|} 
WVZ- \\
G-58C \\
\end{tabular} & $\begin{array}{l}\text { WVZ- } \\
\text { G-61A }\end{array}$ \\
\hline $\mathrm{Al}_{2} \mathrm{O}_{3}$ & 6.09 & 7.18 & 6.96 & 6.66 & 6.57 & 6.58 & 6.32 & 6.40 & 6.25 & 6.28 & 6.15 & 6.16 & 6.10 & 6.26 & 6.17 & 5.96 \\
\hline $\mathrm{B}_{2} \mathrm{O}_{3} *$ & 9.99 & 10.63 & 10.50 & 10.42 & 10.32 & 10.26 & 10.22 & 10.18 & 10.15 & 10.12 & 10.10 & 10.07 & 10.06 & 10.05 & 10.04 & 10.03 \\
\hline $\mathrm{CaO}$ & 2.02 & 3.68 & 3.32 & 3.04 & 2.87 & 2.76 & 2.79 & 2.63 & 2.45 & 2.52 & 2.33 & 2.17 & 2.23 & 2.15 & 2.14 & 2.20 \\
\hline $\mathrm{CdO}$ & $\S$ & 0.09 & 0.08 & 0.06 & 0.06 & $<0.01$ & $<0.01$ & $<0.01$ & $<0.01$ & $<0.01$ & $<0.01$ & $<0.01$ & $<0.01$ & $<0.01$ & $<0.01$ & $<0.01$ \\
\hline $\mathrm{Cl}$ & 0.20 & 0.18 & 0.18 & 0.17 & 0.17 & 0.17 & 0.16 & 0.16 & 0.16 & 0.15 & 0.16 & 0.16 & 0.16 & 0.16 & 0.15 & 0.17 \\
\hline $\mathrm{Cr}_{2} \mathrm{O}_{3}$ & 0.08 & 0.47 & 0.42 & 0.38 & 0.36 & 0.34 & 0.35 & 0.33 & 0.30 & 0.32 & 0.29 & 0.26 & 0.27 & 0.26 & 0.26 & 0.27 \\
\hline $\mathrm{F}^{\#}$ & 0.08 & $<0.08$ & $<0.08$ & $<0.08$ & $<0.08$ & $<0.08$ & $<0.08$ & $<0.08$ & $<0.08$ & $<0.08$ & $<0.08$ & $<0.08$ & $<0.08$ & $<0.08$ & $<0.08$ & $<0.08$ \\
\hline $\mathrm{Fe}_{2} \mathrm{O}_{3}$ & 5.49 & 4.17 & 4.38 & 4.38 & 4.59 & 4.79 & 5.09 & 5.06 & 4.92 & 4.99 & 5.11 & 4.78 & 5.12 & 5.03 & 4.99 & 5.39 \\
\hline $\mathrm{K}_{2} \mathrm{O}$ & 4.98 & 2.38 & 2.85 & 3.09 & 3.36 & 3.66 & 3.80 & 3.92 & 3.98 & 3.97 & 4.27 & 4.23 & 4.45 & 4.46 & 4.40 & 4.68 \\
\hline $\mathrm{Li}_{2} \mathrm{O}^{*}$ & $\S$ & $<0.01$ & $<0.01$ & $<0.01$ & $<0.01$ & $<0.01$ & $<0.01$ & $<0.01$ & $<0.01$ & $<0.01$ & $<0.01$ & $<0.01$ & $<0.01$ & $<0.01$ & $<0.01$ & $<0.01$ \\
\hline $\mathrm{MgO}$ & 1.48 & 1.31 & 1.33 & 1.36 & 1.39 & 1.37 & 1.40 & 1.43 & 1.45 & 1.44 & 1.43 & 1.52 & 1.41 & 1.48 & 1.52 & 1.44 \\
\hline $\mathrm{Na}_{2} \mathrm{O}$ & 18.19 & 19.00 & 19.00 & 19.20 & 18.99 & 18.31 & 18.26 & 17.94 & 18.83 & 18.78 & 18.68 & 18.94 & 18.56 & 18.04 & 18.85 & 18.21 \\
\hline $\mathrm{NiO}$ & 0.01 & 0.01 & 0.01 & $<0.01$ & $<0.01$ & $<0.01$ & $<0.01$ & $<0.01$ & $<0.01$ & $<0.01$ & $<0.01$ & $<0.01$ & $<0.01$ & $<0.01$ & $<0.01$ & $<0.01$ \\
\hline $\mathrm{P}_{2} \mathrm{O}_{5}$ & 0.12 & 0.26 & 0.24 & 0.23 & 0.22 & 0.21 & 0.22 & 0.20 & 0.21 & 0.19 & 0.19 & 0.21 & 0.20 & 0.20 & 0.20 & 0.19 \\
\hline $\mathrm{PbO}$ & 0.01 & 0.07 & $<0.01$ & 0.01 & 0.01 & 0.01 & $<0.01$ & $<0.01$ & $<0.01$ & 0.01 & 0.01 & 0.01 & 0.01 & $<0.01$ & 0.01 & 0.01 \\
\hline $\mathrm{ReO}_{2}$ & 0.10 & 0.00 & 0.01 & 0.01 & 0.02 & 0.02 & 0.02 & 0.03 & 0.02 & 0.03 & 0.02 & 0.02 & 0.02 & 0.03 & 0.02 & 0.02 \\
\hline $\mathrm{SiO}_{2}$ & 42.91 & 41.97 & 41.92 & 42.40 & 42.17 & 42.46 & 41.83 & 42.26 & 42.23 & 42.08 & 42.31 & 43.34 & 42.72 & 43.60 & 43.03 & 42.71 \\
\hline $\mathrm{SO}_{3}$ & 0.35 & 0.53 & 0.51 & 0.47 & 0.44 & 0.42 & 0.40 & 0.40 & 0.37 & 0.39 & 0.36 & 0.36 & 0.35 & 0.35 & 0.35 & 0.35 \\
\hline $\mathrm{TiO}_{2}$ & 1.40 & 1.53 & 1.55 & 1.53 & 1.59 & 1.64 & 1.70 & 1.69 & 1.67 & 1.64 & 1.65 & 1.52 & 1.61 & 1.59 & 1.56 & 1.64 \\
\hline $\mathrm{V}_{2} \mathrm{O}_{5}$ & $\S$ & 0.38 & 0.30 & 0.26 & 0.22 & 0.19 & 0.19 & 0.15 & 0.13 & 0.13 & 0.09 & 0.08 & 0.07 & 0.06 & 0.06 & 0.06 \\
\hline $\mathrm{ZnO}$ & 3.50 & 3.00 & 3.04 & 2.98 & 3.10 & 3.19 & 3.40 & 3.35 & 3.21 & 3.27 & 3.25 & 2.95 & 3.19 & 3.08 & 3.02 & 3.28 \\
\hline $\mathrm{ZrO}_{2}$ & 3.00 & 3.23 & 3.40 & 3.35 & 3.54 & 3.61 & 3.84 & 3.87 & 3.68 & 3.70 & 3.59 & 3.21 & 3.45 & 3.21 & 3.22 & 3.40 \\
\hline Sum & 100.00 & 100.00 & 100.00 & 100.00 & 100.00 & 100.00 & 100.00 & 100.00 & 100.00 & 100.00 & 100.00 & 100.00 & 100.00 & 100.00 & 100.00 & 100.00 \\
\hline
\end{tabular}

* - Target values calculated based on simple well-stirred tank model;

\# - Fluorine values measured directly by XRF given in bold, other values interpolated between measured values.

$\S$ Not a target component 
Table 4.2. XRF Analyzed Compositions for Glass Samples Discharged During DM100 Tests (wt\%) (continued).

\begin{tabular}{|c|c|c|c|c|c|c|c|c|c|c|c|c|c|c|c|c|}
\hline Test & \multicolumn{4}{|c|}{ 1A } & \multicolumn{12}{|c|}{ 1B } \\
\hline \multicolumn{2}{|c|}{ Glass (kg) } & 393.22 & 407.06 & 407.06 & \multicolumn{2}{|c|}{438.20} & 468.40 & 491.14 & 511.72 & 532.26 & 553.34 & 573.66 & 593.72 & 617.52 & 639.34 & 658.16 \\
\hline Constituent & Target & $\begin{array}{l}\text { WVZ- } \\
\text { G-61C }\end{array}$ & $\begin{array}{l}\text { WVZ- } \\
\text { G-61D }\end{array}$ & \%Dev. & Target & \begin{tabular}{|l} 
WVZ- \\
G-70B
\end{tabular} & $\begin{array}{l}\text { WVZ- } \\
\text { G-72B }\end{array}$ & $\begin{array}{l}\text { WVZ- } \\
\text { G-74A }\end{array}$ & $\begin{array}{l}\text { WVZ- } \\
\text { G-75B }\end{array}$ & $\begin{array}{l}\text { WVZ- } \\
\text { G-76B }\end{array}$ & $\begin{array}{l}\text { WVZ- } \\
\text { G-79A }\end{array}$ & \begin{tabular}{|l} 
WVZ- \\
G-80B
\end{tabular} & \begin{tabular}{|l} 
WVZ- \\
G-81A
\end{tabular} & $\begin{array}{l}\text { WVZ- } \\
\text { G-81C }\end{array}$ & $\begin{array}{l}\text { WVZ- } \\
\text { G-86B }\end{array}$ & $\begin{array}{l}\text { WVZ- } \\
\text { G-87B }\end{array}$ \\
\hline $\mathrm{Al}_{2} \mathrm{O}_{3}$ & 6.09 & 6.25 & 6.00 & -1.61 & 5.96 & 6.02 & 6.10 & 6.06 & 6.54 & 6.04 & 5.98 & 5.93 & 6.18 & 6.15 & 6.05 & 6.12 \\
\hline $\mathrm{B}_{2} \mathrm{O}_{3} *$ & 9.99 & 10.02 & 10.02 & $\mathrm{NC}$ & 9.78 & 9.96 & 9.92 & 9.90 & 9.88 & 9.86 & 9.85 & 9.84 & 9.83 & 9.82 & 9.81 & 9.81 \\
\hline $\mathrm{CaO}$ & 2.02 & 2.30 & 2.16 & 7.09 & 2.46 & 2.15 & 2.19 & 2.27 & 2.37 & 2.27 & 2.31 & 2.32 & 2.31 & 2.31 & 2.39 & 2.34 \\
\hline $\mathrm{CdO}$ & $\S$ & $<0.01$ & $<0.01$ & $\mathrm{NC}$ & $\S$ & $<0.01$ & $<0.01$ & $<0.01$ & $<0.01$ & $<0.01$ & $<0.01$ & $<0.01$ & $<0.01$ & $<0.01$ & $<0.01$ & $<0.01$ \\
\hline $\mathrm{Cl}$ & 0.20 & 0.15 & 0.15 & $\mathrm{NC}$ & 0.20 & 0.15 & 0.15 & 0.14 & 0.11 & 0.13 & 0.14 & 0.14 & 0.13 & 0.14 & 0.13 & 0.13 \\
\hline $\mathrm{Cr}_{2} \mathrm{O}_{3}$ & 0.08 & 0.32 & 0.27 & $\mathrm{NC}$ & 0.08 & 0.26 & 0.26 & 0.27 & 0.35 & 0.26 & 0.27 & 0.27 & 0.27 & 0.25 & 0.28 & 0.27 \\
\hline $\mathrm{F}^{\#}$ & 0.08 & $<0.08$ & $<0.08$ & $\mathrm{NC}$ & 0.08 & $<0.08$ & $<0.08$ & $<0.08$ & $<0.08$ & $<0.08$ & $<0.08$ & $<0.08$ & $<0.08$ & $<0.08$ & $<0.08$ & $<0.08$ \\
\hline $\mathrm{Fe}_{2} \mathrm{O}_{3}$ & 5.49 & 5.36 & 5.45 & -0.85 & 5.37 & 5.28 & 5.42 & 5.43 & 4.77 & 5.38 & 5.45 & 5.41 & 5.36 & 5.34 & 5.50 & 5.37 \\
\hline $\mathrm{K}_{2} \mathrm{O}$ & 4.98 & 4.48 & 4.78 & -4.03 & $\S$ & 4.16 & 3.52 & 3.16 & 2.53 & 2.37 & 2.19 & 1.89 & 1.61 & 1.39 & 1.25 & 1.07 \\
\hline $\mathrm{Li}_{2} \mathrm{O}^{*}$ & $\S$ & $<0.01$ & $<0.01$ & $\mathrm{NC}$ & $\S$ & $<0.01$ & $<0.01$ & $<0.01$ & $<0.01$ & $<0.01$ & $<0.01$ & $<0.01$ & $<0.01$ & $<0.01$ & $<0.01$ & $<0.01$ \\
\hline $\mathrm{MgO}$ & 1.48 & 1.43 & 1.39 & -5.68 & 1.45 & 1.46 & 1.43 & 1.46 & 1.47 & 1.45 & 1.47 & 1.44 & 1.53 & 1.45 & 1.44 & 1.46 \\
\hline $\mathrm{Na}_{2} \mathrm{O}$ & 18.19 & 18.40 & 18.27 & 0.41 & 21.25 & 18.94 & 18.97 & 18.71 & 19.70 & 19.76 & 19.56 & 20.25 & 20.12 & 20.59 & 20.06 & 20.85 \\
\hline $\mathrm{NiO}$ & 0.01 & 0.01 & 0.00 & $\mathrm{NC}$ & 0.01 & $<0.01$ & $<0.01$ & $<0.01$ & 0.02 & 0.01 & 0.01 & $<0.01$ & $<0.01$ & $<0.01$ & $<0.01$ & $<0.01$ \\
\hline $\mathrm{P}_{2} \mathrm{O}_{5}$ & 0.12 & 0.18 & 0.18 & $\mathrm{NC}$ & 0.12 & 0.19 & 0.18 & 0.19 & 0.19 & 0.18 & 0.18 & 0.17 & 0.17 & 0.17 & 0.18 & 0.16 \\
\hline $\mathrm{PbO}$ & 0.01 & 0.01 & 0.01 & $\mathrm{NC}$ & 0.01 & $<0.01$ & 0.01 & 0.01 & $<0.01$ & 0.01 & 0.01 & 0.01 & 0.01 & $<0.01$ & 0.01 & $<0.01$ \\
\hline $\mathrm{ReO}_{2}$ & 0.10 & 0.02 & 0.02 & $\mathrm{NC}$ & 0.10 & 0.02 & 0.02 & 0.03 & 0.02 & 0.03 & 0.03 & 0.03 & 0.03 & 0.04 & 0.04 & 0.03 \\
\hline $\mathrm{SiO}_{2}$ & 42.91 & 42.38 & 42.65 & -0.61 & 44.99 & 43.04 & 43.34 & 43.78 & 44.41 & 43.90 & 44.19 & 44.00 & 44.27 & 44.19 & 44.36 & 44.19 \\
\hline $\mathrm{SO}_{3}$ & 0.35 & 0.33 & 0.33 & -6.84 & 0.41 & 0.33 & 0.35 & 0.35 & 0.39 & 0.33 & 0.36 & 0.35 & 0.36 & 0.34 & 0.36 & 0.35 \\
\hline $\mathrm{TiO}_{2}$ & 1.40 & 1.62 & 1.63 & 16.82 & 1.37 & 1.59 & 1.59 & 1.60 & 1.41 & 1.56 & 1.56 & 1.54 & 1.53 & 1.52 & 1.56 & 1.51 \\
\hline $\mathrm{V}_{2} \mathrm{O}_{5}$ & $\S$ & 0.07 & 0.04 & $\mathrm{NC}$ & $\S$ & 0.04 & 0.03 & 0.03 & 0.06 & 0.02 & 0.02 & 0.02 & 0.02 & 0.01 & 0.02 & 0.01 \\
\hline $\mathrm{ZnO}$ & 3.50 & 3.24 & 3.28 & -6.23 & 3.43 & 3.17 & 3.22 & 3.26 & 2.86 & 3.22 & 3.23 & 3.24 & 3.15 & 3.17 & 3.28 & 3.19 \\
\hline $\mathrm{ZrO}_{2}$ & 3.00 & 3.43 & 3.37 & 12.50 & 2.94 & 3.24 & 3.29 & 3.35 & 2.92 & 3.21 & 3.19 & 3.14 & 3.12 & 3.10 & 3.29 & 3.12 \\
\hline Sum & 100.00 & 100.00 & 100.00 & 0.00 & 100.00 & 100.00 & 100.00 & 100.00 & 100.00 & 100.00 & 100.00 & 100.00 & 100.00 & 100.00 & 100.00 & 100.00 \\
\hline
\end{tabular}

* - Target values calculated based on simple well-stirred tank mode

\# - Fluorine values measured directly by XRF given in bold, other values interpolated between measured values.

NC - Not calculated

$\S$ - Not a target component 
Table 4.2. XRF Analyzed Compositions for Glass Samples Discharged During DM100 Tests (wt\%) (continued).

\begin{tabular}{|c|c|c|c|c|c|c|c|c|c|c|c|c|c|c|c|c|}
\hline Test & \multicolumn{11}{|c|}{ 1B } & \multicolumn{5}{|c|}{$2 \mathrm{~A}$} \\
\hline Glass (kg) & \multicolumn{2}{|c|}{681.40} & 698.18 & 724.82 & 752.30 & 774.50 & 796.76 & 817.48 & 843.34 & 855.20 & 855.20 & \multicolumn{2}{|c|}{873.02} & 897.46 & 919.50 & 938.88 \\
\hline Constituent & Target & \begin{tabular}{|l} 
WVZ- \\
G-89A
\end{tabular} & \begin{tabular}{|l|} 
WVZ- \\
G-89C
\end{tabular} & \begin{tabular}{|l|} 
WVZ- \\
G-90B
\end{tabular} & \begin{tabular}{|l} 
WVZ- \\
G-90D
\end{tabular} & \begin{tabular}{|l|} 
WVZ- \\
G-95A
\end{tabular} & \begin{tabular}{|l|} 
WVZ- \\
G-95D
\end{tabular} & $\begin{array}{l}\text { WVZ- } \\
\text { G-96B }\end{array}$ & \begin{tabular}{|l} 
WVZ- \\
G-97A
\end{tabular} & $\begin{array}{l}\text { WVZ- } \\
\text { G-97B }\end{array}$ & $\%$ Dev & Target & \begin{tabular}{|c|} 
WVZ- \\
G-119A
\end{tabular} & \begin{tabular}{|c|} 
WVZ- \\
G-120B
\end{tabular} & \begin{tabular}{|c|} 
WVZ- \\
G-121B
\end{tabular} & $\begin{array}{c}\text { WVZ- } \\
\text { G-121D }\end{array}$ \\
\hline $\mathrm{Al}_{2} \mathrm{O}_{3}$ & 5.96 & 6.35 & 5.96 & 6.00 & 5.95 & 5.88 & 6.15 & 5.98 & 5.92 & 6.20 & 4.52 & 5.93 & 6.15 & 6.10 & 6.14 & 6.12 \\
\hline $\mathrm{B}_{2} \mathrm{O}_{3} *$ & 9.78 & 9.80 & 9.80 & 9.80 & 9.79 & 9.79 & 9.79 & 9.79 & 9.79 & 9.79 & $\mathrm{NC}$ & 9.73 & 9.78 & 9.77 & 9.76 & 9.76 \\
\hline $\mathrm{CaO}$ & 2.46 & 2.34 & 2.36 & 2.39 & 2.35 & 2.35 & 2.33 & 2.42 & 2.40 & 2.28 & -6.88 & 2.44 & 2.49 & 2.42 & 2.39 & 2.44 \\
\hline $\mathrm{CdO}$ & $\S$ & $<0.01$ & $<0.01$ & $<0.01$ & $<0.01$ & $<0.01$ & $<0.01$ & $<0.01$ & $<0.01$ & $<0.01$ & $\mathrm{NC}$ & $\S$ & $<0.01$ & $<0.01$ & $<0.01$ & $<0.01$ \\
\hline $\mathrm{Cl}$ & .20 & 0.14 & 0.13 & 0.13 & 0.12 & 0.14 & 0.13 & 0.14 & 0.13 & 0.12 & $\mathrm{NC}$ & 0.20 & 0.07 & 0.08 & 0.09 & 0.10 \\
\hline $\mathrm{Cr}_{2} \mathrm{O}_{3}$ & 0.08 & 0.25 & 0.26 & 0.27 & 0.26 & 0.26 & 0.25 & 0.26 & 0.25 & 0.24 & $\mathrm{NC}$ & 0.08 & 0.31 & 0.29 & 0.28 & 0.28 \\
\hline$F^{\#}$ & 0.08 & $<0.08$ & $<0.08$ & $<0.08$ & $<0.08$ & $<0.08$ & $<0.08$ & $<0.08$ & $<0.08$ & $<0.08$ & $-\mathrm{NC}$ & 0.08 & $<0.08$ & $<0.08$ & $<0.08$ & $<0.08$ \\
\hline $\mathrm{Fe}_{2} \mathrm{O}_{3}$ & 5.37 & 5.31 & 5.34 & 5.37 & 5.31 & 5.35 & 5.33 & 5.57 & 5.41 & 4.89 & -8.43 & 5.35 & 5.25 & 5.03 & 5.07 & 5.22 \\
\hline $\mathrm{K}_{2} \mathrm{O}$ & $\S$ & 0.97 & 0.83 & 0.74 & 0.65 & 0.55 & 0.52 & 0.47 & 0.40 & 0.38 & $\mathrm{NC}$ & 0.54 & 0.47 & 0.47 & 0.48 & 0.51 \\
\hline $\mathrm{Li}_{2} \mathrm{O}^{*}$ & $\S$ & $<0.01$ & $<0.01$ & $<0.01$ & $<0.01$ & $<0.01$ & $<0.01$ & $<0.01$ & $<0.01$ & $<0.01$ & $\mathrm{NC}$ & $\S$ & $<0.01$ & $<0.01$ & $<0.01$ & $<0.01$ \\
\hline $\mathrm{MgO}$ & 1.45 & 1.51 & 1.50 & 1.48 & 1.47 & 1.49 & 1.49 & 1.53 & 1.54 & 1.52 & 5.17 & 1.44 & 1.46 & 1.52 & 1.46 & 1.48 \\
\hline $\mathrm{Na}_{2} \mathrm{O}$ & 21.25 & 20.41 & 20.90 & 20.85 & 21.25 & 21.30 & 21.03 & 20.72 & 21.25 & 21.41 & 1.29 & 21.13 & 21.39 & 21.93 & 22.46 & 21.38 \\
\hline $\mathrm{NiO}$ & 0.01 & $<0.01$ & $<0.01$ & 0.01 & 0.01 & $<0.01$ & $<0.01$ & $<0.01$ & & 0.03 & $\mathrm{NC}$ & & 0.02 & 01 & 1 & 0.01 \\
\hline $\mathrm{P}_{2} \mathrm{O}_{5}$ & 0.12 & 0.17 & 0.18 & 0.17 & 0.17 & 0.17 & 0.17 & 0.18 & 0.17 & 0.17 & $\mathrm{NC}$ & 0.12 & 0.19 & 0.17 & 0.16 & 0.16 \\
\hline $\mathrm{PbO}$ & 0.01 & 0.01 & $<0.01$ & $<0.01$ & 0.01 & 0.01 & 0.01 & 0.01 & $<0.01$ & 0.01 & NC & 0.01 & $<0.01$ & 0.01 & $<0.01$ & 0.01 \\
\hline $\mathrm{ReO}_{2}$ & 0.10 & 0.03 & 0.04 & 0.02 & 0.04 & 0.04 & 0.04 & 0.04 & 0.03 & 0.03 & $\mathrm{NC}$ & 0.10 & 0.01 & 0.02 & 0.02 & 0.03 \\
\hline $\mathrm{SiO}_{2}$ & 44.99 & 44.51 & 44.52 & 44.69 & 44.64 & 44.66 & 44.80 & 44.60 & 44.53 & 45.75 & 2.23 & 44.75 & 44.44 & 44.52 & 44.09 & 44.55 \\
\hline $\mathrm{SO}_{3}$ & 0.41 & 0.36 & 0.35 & 0.37 & 0.35 & 0.35 & 0.35 & 0.34 & 0.37 & 0.34 & $\mathrm{NC}$ & 0.41 & 0.36 & 0.37 & 0.37 & 0.38 \\
\hline $\mathrm{TiO}_{2}$ & 1.37 & 1.50 & 1.51 & 1.51 & 1.48 & 1.47 & 1.48 & 1.51 & 1.50 & 1.39 & 2.44 & 1.36 & 1.46 & 1.43 & 1.42 & 1.46 \\
\hline $\mathrm{V}_{2} \mathrm{O}_{5}$ & $\S$ & 0.01 & 0.01 & 0.01 & $<0.01$ & 0.01 & 0.01 & 0.01 & 0.01 & $<0.01$ & $\mathrm{NC}$ & $\S$ & 0.04 & 0.03 & 0.03 & 0.02 \\
\hline $\mathrm{ZnO}$ & 3.43 & 3.16 & 3.16 & 3.16 & 3.15 & 3.13 & 3.11 & 3.25 & 3.17 & 2.81 & -17.62 & 3.41 & 3.12 & 2.95 & 2.97 & 3.08 \\
\hline $\mathrm{ZrO}_{2}$ & 2.94 & 3.16 & 3.14 & 3.03 & 2.98 & 3.05 & 3.00 & 3.20 & 3.14 & 2.65 & -9.38 & 2.92 & 3.00 & 2.90 & 2.80 & 3.01 \\
\hline Sum & 100.00 & 100.00 & 00.00 & 100.00 & 100.00 & 100.00 & 100.00 & 100.00 & 100.00 & 100.00 & $\mathrm{NC}$ & 100.00 & 100.00 & 100.00 & 100.00 & 100.00 \\
\hline
\end{tabular}

* - Target values calculated based on simple well-stirred tank model

\# - Fluorine values measured directly by XRF given in bold, other values interpolated between measured values.

$\mathrm{NC}$ - Not calculated

$\S$ - Not a target component 
Table 4.2. XRF Analyzed Compositions for Glass Samples Discharged During DM100 Tests (wt\%) (continued).

\begin{tabular}{|c|c|c|c|c|c|c|c|c|c|c|c|c|c|c|c|c|}
\hline Test & & & & & A & & & & & & & 2 & B & & & \\
\hline Glass (kg) & 960 & 0.06 & 982.80 & 1007.98 & 1028.78 & 1051.44 & 1072.98 & 1072.98 & 109 & 7.16 & 1122.82 & 1149.70 & 1172.44 & 1200.40 & 1225.50 & 1248.86 \\
\hline Constituent & Target & $\begin{array}{l}\text { WVZ- } \\
\text { G-123B }\end{array}$ & \begin{tabular}{|c|} 
WVZ- \\
G-123D
\end{tabular} & \begin{tabular}{|l|} 
WVZ- \\
G-126B
\end{tabular} & \begin{tabular}{|c|} 
WVZ- \\
G-126D
\end{tabular} & \begin{tabular}{|c|} 
WVZ- \\
G-129B
\end{tabular} & \begin{tabular}{|l|} 
WVZ- \\
G-129D
\end{tabular} & $\%$ Dev. & Target & \begin{tabular}{|c|} 
WVZ- \\
G-139B
\end{tabular} & \begin{tabular}{|c|} 
WVZ- \\
G-140B
\end{tabular} & \begin{tabular}{|c|} 
WVZ- \\
G-141A
\end{tabular} & \begin{tabular}{|c|} 
WVZ- \\
G-143A
\end{tabular} & \begin{tabular}{|c|} 
WVZ- \\
G-143C
\end{tabular} & $\begin{array}{c}\text { WVZ- } \\
\text { G-147A }\end{array}$ & $\begin{array}{l}\text { WVZ- } \\
\text { G-147C }\end{array}$ \\
\hline $\mathrm{Al}_{2} \mathrm{O}_{3}$ & 5.93 & 6.07 & 6.15 & 6.10 & 6.12 & 5.89 & 6.34 & 6.36 & 5.98 & 6.41 & 6.69 & 6.39 & 6.01 & 5.98 & 6.07 & 6.30 \\
\hline $\mathrm{B}_{2} \mathrm{O}_{3} *$ & 9.73 & 9.75 & 9.75 & 9.74 & 9.74 & 9.74 & 9.74 & $\mathrm{NC}$ & 9.80 & 9.75 & 9.76 & 9.77 & 9.77 & 9.78 & 9.78 & 9.79 \\
\hline $\mathrm{CaO}$ & 2.44 & 2.49 & 2.39 & 2.35 & 2.41 & 2.47 & 2.41 & -1.77 & 3.61 & 2.50 & 2.56 & 2.75 & 2.92 & 3.03 & 2.96 & 3.13 \\
\hline $\mathrm{CdO}$ & $\S$ & $<0.01$ & $<0.01$ & $<0.01$ & $<0.01$ & $<0.01$ & $<0.01$ & NC & $\S$ & $<0.01$ & $<0.01$ & $<0.01$ & $<0.01$ & $<0.01$ & $<0.01$ & $<0.01$ \\
\hline $\mathrm{Cl}$ & 0.20 & 0.11 & 0.11 & 0.12 & 0.13 & 0.14 & 0.13 & $\mathrm{NC}$ & 0.20 & 0.13 & 0.12 & 0.15 & 0.14 & 0.14 & 0.14 & 0.14 \\
\hline $\mathrm{Cr}_{2} \mathrm{O}_{3}$ & 0.08 & 0.29 & 0.29 & 0.27 & 0.28 & 0.29 & 0.28 & NC & 0.08 & 0.26 & 0.24 & 0.25 & 0.25 & 0.25 & 0.23 & 0.21 \\
\hline $\mathrm{F}^{\#}$ & 0.08 & $<0.08$ & $<0.08$ & $<0.08$ & $<0.08$ & $<0.08$ & $<0.08$ & NC & 0.08 & $<0.08$ & $<0.08$ & $<0.08$ & $<0.08$ & $<0.08$ & $<0.08$ & $<0.08$ \\
\hline $\mathrm{Fe}_{2} \mathrm{O}_{3}$ & 5.35 & 5.36 & 5.18 & 5.04 & 5.27 & 5.44 & 5.26 & -2.04 & 5.40 & 5.08 & 4.74 & 5.14 & 5.18 & 5.22 & 4.87 & 4.67 \\
\hline $\mathrm{K}_{2} \mathrm{O}$ & 0.54 & 0.52 & 0.54 & 0.56 & 0.58 & 0.56 & 0.57 & NC & 0.54 & 0.55 & 0.56 & 0.57 & 0.61 & 0.62 & 0.61 & 0.59 \\
\hline $\mathrm{Li}_{2} \mathrm{O}^{*}$ & $\S$ & $<0.01$ & $<0.01$ & $<0.01$ & $<0.01$ & $<0.01$ & $<0.01$ & NC & 0.49 & 0.09 & 0.17 & 0.23 & 0.28 & 0.32 & 0.35 & 0.38 \\
\hline $\mathrm{MgO}$ & 1.44 & 1.35 & 1.40 & 1.40 & 1.37 & 1.42 & 1.35 & -6.88 & 1.45 & 1.39 & 1.42 & 1.41 & 1.33 & 1.42 & 1.43 & 1.42 \\
\hline $\mathrm{Na}_{2} \mathrm{O}$ & 21.13 & 21.18 & 21.27 & 21.79 & 21.39 & 21.54 & 20.98 & -1.25 & 18.95 & 21.85 & 20.99 & 20.51 & 20.48 & 19.68 & 20.68 & 19.78 \\
\hline $\mathrm{NiO}$ & 0.01 & 0.01 & 0.01 & $<0.01$ & 0.01 & 0.01 & 0.01 & NC & 0.01 & 0.01 & $<0.01$ & $<0.01$ & $<0.01$ & $<0.01$ & $<0.01$ & $<0.01$ \\
\hline $\mathrm{P}_{2} \mathrm{O}_{5}$ & 0.12 & 0.17 & 0.17 & 0.17 & 0.16 & 0.18 & 0.16 & NC & 0.12 & 0.17 & 0.17 & 0.17 & 0.17 & 0.17 & 0.17 & 0.62 \\
\hline $\mathrm{PbO}$ & 0.01 & $<0.01$ & 0.01 & 0.01 & 0.01 & 0.01 & $<0.01$ & NC & 0.01 & 0.01 & $<0.01$ & 0.01 & 0.01 & 0.01 & 0.01 & 0.01 \\
\hline $\mathrm{ReO}_{2}$ & 0.10 & 0.03 & 0.03 & 0.03 & 0.04 & 0.04 & 0.03 & NC & 0.10 & 0.03 & 0.03 & 0.04 & 0.03 & 0.03 & 0.04 & 0.03 \\
\hline $\mathrm{SiO}_{2}$ & 44.75 & 44.68 & 44.78 & 44.81 & 44.59 & 44.17 & 44.67 & -0.72 & 45.00 & 44.03 & 45.52 & 44.89 & 44.89 & 45.24 & 45.17 & 46.02 \\
\hline $\mathrm{SO}_{3}$ & 0.41 & 0.37 & 0.36 & 0.37 & 0.36 & 0.38 & 0.36 & NC & 0.46 & 0.37 & 0.36 & 0.40 & 0.40 & 0.43 & 0.40 & 0.41 \\
\hline $\mathrm{TiO}_{2}$ & 1.36 & 1.51 & 1.46 & 1.42 & 1.46 & 1.52 & 1.49 & 8.59 & 1.37 & 1.46 & 1.40 & 1.49 & 1.50 & 1.53 & 1.44 & 1.43 \\
\hline $\mathrm{V}_{2} \mathrm{O}_{5}$ & $\S$ & 0.02 & 0.02 & 0.02 & 0.02 & 0.01 & 0.01 & NC & $\S$ & 0.01 & 0.01 & 0.01 & 0.01 & 0.01 & 0.01 & 0.01 \\
\hline $\mathrm{ZnO}$ & 3.41 & 3.15 & 3.07 & 2.94 & 3.08 & 3.23 & 3.11 & -9.21 & 3.43 & 3.02 & 2.71 & 2.98 & 3.02 & 3.10 & 2.88 & 2.68 \\
\hline $\mathrm{ZrO}_{2}$ & 2.92 & 2.93 & 3.01 & 2.87 & 2.98 & 2.97 & 3.10 & 5.48 & 2.94 & 2.91 & 2.55 & 2.83 & 2.97 & 3.04 & 2.75 & 2.39 \\
\hline Sum & 100.00 & 100.00 & 100.00 & 100.00 & 100.00 & 100.00 & 100.00 & NC & 100.00 & 100.00 & 100.00 & 100.00 & 100.00 & 100.00 & 100.00 & 100.00 \\
\hline
\end{tabular}

* - Target values calculated based on simple well-stirred tank model

\# - Fluorine values measured directly by XRF given in bold, other values interpolated between measured values.

$\mathrm{NC}$ - Not calculated

$\S$ - Not a target component 
Table 4.2. XRF Analyzed Compositions for Glass Samples Discharged During DM100 Tests (wt\%) (continued).

\begin{tabular}{|c|c|c|c|c|c|c|c|c|c|c|c|c|c|c|c|c|}
\hline Test & \multicolumn{13}{|c|}{$2 B$} & \multicolumn{3}{|c|}{$2 \mathrm{C}$} \\
\hline Glass (kg) & & 1275.52 & 1299.12 & 1322.48 & \begin{tabular}{|l|l|}
348.80 \\
\end{tabular} & 1373.78 & 1395.62 & 1422.14 & 1446.36 & 1473.36 & 1500.76 & 1513.44 & 1513.44 & & 1542.12 & 1569.04 \\
\hline Constituent & Target & $\begin{array}{r}\text { WVZ- } \\
\text { G-150B }\end{array}$ & \begin{tabular}{|r|} 
WVZ- \\
G-151B
\end{tabular} & $\begin{array}{r}\text { WVZ- } \\
\text { G-152B }\end{array}$ & $\begin{array}{r}\text { WVZ- } \\
\text { G-152D }\end{array}$ & $\begin{array}{r}\text { WVZ- } \\
\text { G-153B }\end{array}$ & $\begin{array}{l}\text { WVZ- } \\
\text { G-153D }\end{array}$ & $\begin{array}{r}\text { AWV- } \\
\text { G-5B }\end{array}$ & $\begin{array}{r}\text { AWV- } \\
\text { G-5D }\end{array}$ & $\begin{array}{r}\text { AWV- } \\
\text { G-6B }\end{array}$ & $\begin{array}{r}\text { AWV- } \\
\text { G-6D }\end{array}$ & $\begin{array}{l}\text { AWV- } \\
\text { G-14A }\end{array}$ & $\%$ Dev. & Target & $\begin{array}{l}\text { AWV- } \\
\text { G-21A }\end{array}$ & $\begin{array}{l}\text { AWV- } \\
\text { G-22A }\end{array}$ \\
\hline $\mathrm{Al}_{2} \mathrm{O}_{3}$ & 5.98 & 5.85 & 5.84 & 5.84 & 5.91 & 5.77 & 5.97 & 5.88 & 5.93 & 5.81 & 5.82 & 5.81 & -2.89 & 6.00 & 5.82 & 5.78 \\
\hline $\mathrm{B}_{2} \mathrm{O}_{3} *$ & 9.80 & 9.79 & 9.79 & 9.79 & 9.79 & 9.80 & 9.80 & 9.80 & 9.80 & 9.80 & 9.80 & 9.80 & $\mathrm{NC}$ & 9.84 & 9.81 & 9.81 \\
\hline $\mathrm{CaO}$ & 3.61 & 3.13 & 3.27 & 3.26 & 3.32 & 3.32 & 3.19 & 3.36 & 3.28 & 3.34 & 3.43 & 3.39 & -5.95 & 5.45 & 3.63 & 3.88 \\
\hline $\mathrm{CdO}$ & $\S$ & $<0.01$ & $<0.01$ & $<0.01$ & $<0.01$ & $<0.01$ & $<0.01$ & $<0.01$ & $<0.01$ & $<0.01$ & $<0.01$ & $<0.01$ & NC & $\S$ & $<0.01$ & $<0.01$ \\
\hline $\mathrm{Cl}$ & 0.20 & 0.15 & 0.15 & 0.14 & 0.15 & 0.15 & 0.14 & 0.15 & 0.14 & 0.15 & 0.15 & 0.14 & $\mathrm{NC}$ & 0.20 & 0.15 & 0.15 \\
\hline $\mathrm{Cr}_{2} \mathrm{O}_{3}$ & .08 & 0.23 & 0.23 & 0.24 & 0.23 & 0.23 & 0.21 & 0.23 & 0.22 & 0.22 & 0.23 & 0.21 & $\mathrm{NC}$ & 0.08 & 0.22 & 0.22 \\
\hline$F^{\#}$ & 0.08 & $<0.08$ & $<0.08$ & $<0.08$ & $<0.08$ & $<0.08$ & $<0.08$ & $<0.08$ & $<0.08$ & $<0.08$ & $<0.08$ & $<0.08$ & $\mathrm{NC}$ & 0.08 & $<0.08$ & $<0.08$ \\
\hline $\mathrm{Fe}_{2} \mathrm{O}_{3}$ & 5.40 & 5.09 & 5.27 & 5.30 & 5.29 & 5.33 & 4.94 & 5.28 & 5.07 & 5.14 & 5.30 & 5.20 & -3.69 & 5.41 & 5.22 & 5.25 \\
\hline $\mathrm{K}_{2} \mathrm{O}$ & 0.54 & 0.59 & 0.61 & 0.61 & 0.62 & 0.61 & 0.61 & 0.64 & 0.64 & 0.61 & 0.62 & 0.62 & $\mathrm{NC}$ & 0.54 & 0.61 & 0.61 \\
\hline $\mathrm{Li}_{2} \mathrm{O}^{*}$ & 0.49 & 0.40 & 0.42 & 0.43 & 0.44 & 0.45 & 0.46 & 0.46 & 0.47 & 0.47 & 0.48 & 0.48 & $\mathrm{NC}$ & 2.38 & 0.88 & 1.18 \\
\hline $\mathrm{MgO}$ & 1.45 & 1.47 & 1.45 & 1.51 & 1.41 & 1.44 & 1.37 & 1.37 & 1.37 & 1.37 & 1.34 & 1.45 & -0.11 & 1.46 & 1.32 & 1.39 \\
\hline $\mathrm{Na}_{2} \mathrm{O}$ & 18.95 & 20.48 & 19.73 & 19.75 & 19.74 & 19.67 & 20.16 & 19.49 & 19.80 & 19.99 & 19.38 & 19.79 & 4.45 & 15.81 & 19.23 & 18.87 \\
\hline $\mathrm{NiO}$ & 0.01 & $<0.01$ & $<0.01$ & $<0.01$ & $<0.01$ & $<0.01$ & $<0.01$ & $<0.01$ & $<0.01$ & $<0.01$ & $<0.01$ & $<0.01$ & $\mathrm{NC}$ & 0.01 & $<0.01$ & $<0.01$ \\
\hline $\mathrm{P}_{2} \mathrm{O}_{5}$ & 0.12 & 0.18 & 0.17 & 0.17 & 0.18 & 0.18 & 0.18 & 0.17 & 0.19 & 0.17 & 0.18 & 0.18 & $\mathrm{NC}$ & 0.12 & 0.18 & 0.17 \\
\hline $\mathrm{PbO}$ & 0.01 & 0.01 & 0.01 & 0.01 & $<0.01$ & $<0.01$ & 0.01 & 0.01 & 0.01 & 0.01 & 0.01 & 0.01 & $\mathrm{NC}$ & 0.01 & 0.01 & 0.01 \\
\hline $\mathrm{ReO}_{2}$ & 0.10 & 0.03 & 0.04 & 0.04 & 0.03 & 0.03 & 0.03 & 0.03 & 0.03 & 0.03 & 0.04 & 0.04 & $\mathrm{NC}$ & 0.10 & 0.03 & 0.03 \\
\hline $\mathrm{SiO}_{2}$ & 45.00 & 44.84 & 44.98 & 44.69 & 44.67 & 44.76 & 45.32 & 45.04 & 45.19 & 45.11 & 44.99 & 44.84 & -0.35 & 44.21 & 44.83 & 44.56 \\
\hline $\mathrm{SO}_{3}$ & 0.46 & 0.44 & 0.45 & 0.42 & 0.41 & 0.44 & 0.43 & 0.40 & 0.42 & 0.43 & 0.44 & 0.43 & $\mathrm{NC}$ & 0.53 & 0.44 & 0.43 \\
\hline $\mathrm{TiO}_{2}$ & 1.37 & 1.52 & 1.57 & 1.56 & 1.56 & 1.57 & 1.48 & 1.56 & 1.52 & 1.55 & 1.58 & 1.57 & 14.36 & 1.38 & 1.54 & 1.53 \\
\hline $\mathrm{V}_{2} \mathrm{O}_{5}$ & $\S$ & 0.01 & 0.01 & $<0.01$ & 0.01 & 0.01 & 0.01 & 0.01 & 0.01 & 0.01 & 0.01 & 0.01 & $\mathrm{NC}$ & $\S$ & 0.01 & $<0.01$ \\
\hline $\mathrm{ZnO}$ & 3.43 & 3.00 & 3.10 & 3.15 & 3.15 & 3.17 & 2.88 & 3.13 & 2.99 & 3.02 & 3.18 & 3.09 & -9.89 & 3.45 & 3.10 & 3.11 \\
\hline $\mathrm{ZrO}_{2}$ & 2.94 & 2.80 & 2.93 & 3.08 & 3.08 & 3.05 & 2.82 & 3.00 & 2.93 & 2.78 & 3.02 & 2.94 & 0.21 & 2.95 & 2.97 & 3.03 \\
\hline Sum & 100.00 & 100.00 & 100.00 & 100.00 & 100.00 & 100.00 & 100.00 & 100.00 & 100.00 & 100.00 & 100.00 & 100.00 & $\mathrm{NC}$ & 100.00 & 100.00 & 100.00 \\
\hline
\end{tabular}

* - Target values calculated based on simple well-stirred tank model

\# - Fluorine values measured directly by XRF given in bold, other values interpolated between measured values.

NC - Not calculated

$\S$ - Not a target component 
Table 4.2. XRF Analyzed Compositions for Glass Samples Discharged During DM100 Tests (wt\%) (continued).

\begin{tabular}{|c|c|c|c|c|c|c|c|c|c|c|c|c|c|c|}
\hline Test & \multicolumn{14}{|c|}{$2 C$} \\
\hline \multicolumn{2}{|c|}{ Glass (kg) } & 1593.78 & 1621.06 & 1645.9 & 1669.18 & 1692.86 & 1718.9 & 1744.04 & 1767.96 & 1791.66 & 1816.82 & 1844.9 & 1869.52 & 1896.66 \\
\hline Constituent & Taroet & AWV-G- & AWV-G- & AWV-G- & AWV-G- & AWV-G- & AWV-G- & AWV-G- & AWV-G- & AWV-G- & AWV-G- & AWV-G- & AWV-G- & AWV-G- \\
\hline comstituent & 1arget & $22 \mathrm{C}$ & $24 B$ & 27A & $27 C$ & 29B & $30 \mathrm{~B}$ & $32 B$ & $35 B$ & $35 \mathrm{D}$ & $36 \mathrm{~B}$ & $36 \mathrm{D}$ & $41 B$ & 42B \\
\hline $\mathrm{Al}_{2} \mathrm{O}_{3}$ & 6.00 & 5.90 & 5.84 & 5.80 & 5.87 & 5.83 & 5.85 & 5.82 & 5.82 & 5.80 & 5.96 & 5.99 & 5.94 & 6.03 \\
\hline $\mathrm{B}_{2} \mathrm{O}_{3} *$ & 9.84 & 9.82 & 9.82 & 9.83 & 9.83 & 9.83 & 9.83 & 9.83 & 9.84 & 9.84 & 9.84 & 9.84 & 9.84 & 9.84 \\
\hline $\mathrm{CaO}$ & 5.45 & 4.03 & 4.25 & 4.38 & 4.56 & 4.56 & 4.59 & 4.66 & 4.84 & 4.90 & 4.81 & 4.91 & 4.72 & 4.85 \\
\hline $\mathrm{CdO}$ & $\S$ & $<0.01$ & $<0.01$ & $<0.01$ & $<0.01$ & $<0.01$ & $<0.01$ & $<0.01$ & $<0.01$ & $<0.01$ & $<0.01$ & $<0.01$ & $<0.01$ & $<0.01$ \\
\hline $\mathrm{Cl}$ & 0.20 & 0.14 & 0.13 & 0.13 & 0.14 & 0.14 & 0.14 & 0.13 & 0.13 & 0.13 & 0.13 & 0.13 & 0.13 & 0.14 \\
\hline $\mathrm{Cr}_{2} \mathrm{O}_{3}$ & 0.08 & 0.21 & 0.21 & 0.21 & 0.20 & 0.20 & 0.20 & 0.19 & 0.20 & 0.20 & 0.19 & 0.20 & 0.19 & 0.20 \\
\hline $\mathrm{F}^{\#}$ & 0.08 & $<0.08$ & $<0.08$ & $<0.08$ & $<0.08$ & $<0.08$ & $<0.08$ & $<0.08$ & $<0.08$ & $<0.08$ & $<0.08$ & $<0.08$ & $<0.08$ & $<0.08$ \\
\hline $\mathrm{Fe}_{2} \mathrm{O}_{3}$ & 5.41 & 5.15 & 5.22 & 5.23 & 5.32 & 5.23 & 5.16 & 5.10 & 5.30 & 5.24 & 5.05 & 5.10 & 4.82 & 5.03 \\
\hline $\mathrm{K}_{2} \mathrm{O}$ & 0.54 & 0.63 & 0.63 & 0.63 & 0.62 & 0.61 & 0.61 & 0.60 & 0.62 & 0.60 & 0.62 & 0.64 & 0.61 & 0.60 \\
\hline $\mathrm{Li}_{2} \mathrm{O}^{*}$ & 2.38 & 1.41 & 1.60 & 1.75 & 1.86 & 1.95 & 2.04 & 2.10 & 2.15 & 2.19 & 2.23 & 2.26 & 2.28 & 2.30 \\
\hline $\mathrm{MgO}$ & 1.46 & 1.36 & 1.41 & 1.38 & 1.37 & 1.43 & 1.45 & 1.35 & 1.39 & 1.39 & 1.30 & 1.39 & 1.43 & 1.44 \\
\hline $\mathrm{Na}_{2} \mathrm{O}$ & 15.81 & 18.36 & 17.73 & 17.99 & 17.53 & 17.94 & 17.92 & 17.96 & 17.32 & 17.55 & 17.10 & 17.03 & 17.13 & 17.28 \\
\hline $\mathrm{NiO}$ & 0.01 & $<0.01$ & $<0.01$ & $<0.01$ & $<0.01$ & $<0.01$ & $<0.01$ & $<0.01$ & $<0.01$ & $<0.01$ & $<0.01$ & $<0.01$ & $<0.01$ & $<0.01$ \\
\hline $\mathrm{P}_{2} \mathrm{O}_{5}$ & 0.12 & 0.18 & 0.19 & 0.18 & 0.18 & 0.18 & 0.16 & 0.17 & 0.17 & 0.18 & 0.17 & 0.18 & 0.17 & 0.18 \\
\hline $\mathrm{PbO}$ & 0.01 & 0.01 & $<0.01$ & $<0.01$ & 0.01 & 0.01 & $<0.01$ & $<0.01$ & $<0.01$ & $<0.01$ & $<0.01$ & $<0.01$ & $<0.01$ & 0.01 \\
\hline $\mathrm{ReO}_{2}$ & 0.10 & 0.04 & 0.03 & 0.03 & 0.03 & 0.03 & 0.03 & 0.03 & 0.03 & 0.03 & 0.03 & 0.03 & 0.03 & 0.03 \\
\hline $\mathrm{SiO}_{2}$ & 44.21 & 44.80 & 44.85 & 44.47 & 44.23 & 44.15 & 44.06 & 44.14 & 44.00 & 44.06 & 44.76 & 44.50 & 45.26 & 44.52 \\
\hline $\mathrm{SO}_{3}$ & 0.53 & 0.44 & 0.45 & 0.44 & 0.46 & 0.46 & 0.45 & 0.45 & 0.44 & 0.45 & 0.52 & 0.46 & 0.53 & 0.45 \\
\hline $\mathrm{TiO}_{2}$ & 1.38 & 1.48 & 1.51 & 1.50 & 1.52 & 1.47 & 1.46 & 1.43 & 1.49 & 1.48 & 1.42 & 1.45 & 1.37 & 1.40 \\
\hline $\mathrm{V}_{2} \mathrm{O}_{5}$ & $\S$ & 0.01 & $<0.01$ & $<0.01$ & $<0.01$ & 0.01 & 0.01 & 0.01 & 0.01 & 0.01 & 0.01 & 0.01 & 0.01 & $<0.01$ \\
\hline $\mathrm{ZnO}$ & 3.45 & 3.06 & 3.10 & 3.09 & 3.19 & 3.07 & 3.04 & 3.05 & 3.18 & 3.12 & 3.00 & 3.02 & 2.82 & 2.97 \\
\hline $\mathrm{ZrO}_{2}$ & 2.95 & 2.98 & 3.02 & 2.94 & 3.09 & 2.90 & 3.00 & 2.95 & 3.06 & 2.84 & 2.86 & 2.88 & 2.72 & 2.73 \\
\hline Sum & 100.00 & 100.00 & 100.00 & 100.00 & 100.00 & 100.00 & 100.00 & 100.00 & 100.00 & 100.00 & 100.00 & 100.00 & 100.00 & 100.00 \\
\hline
\end{tabular}

* - Target values calculated based on simple well-stirred tank model

\# - Fluorine values measured directly by XRF given in bold, other values interpolated between measured values.

$\mathrm{NC}$ - Not calculated

$\S$ - Not a target component 
Table 4.2. XRF Analyzed Compositions for Glass Samples Discharged During DM100 Tests (wt\%) (continued).

\begin{tabular}{|c|c|c|c|c|c|c|c|c|c|c|c|c|c|c|c|c|}
\hline Test & \multicolumn{4}{|c|}{$2 \mathrm{C}$} & \multicolumn{12}{|c|}{$2 D$} \\
\hline Glass (kg) & \multicolumn{4}{|c|}{\begin{tabular}{|l|l|l|l}
1923.42 & 1949.88 & 1949.88 \\
\end{tabular}} & \multicolumn{2}{|r|}{1979.16} & 2006.48 & \multicolumn{2}{|c|}{\begin{tabular}{|l|l|}
2031.96 & 2059.22 \\
\end{tabular}} & 2088.06 & 2111.6 & 2140.7 & 2165.74 & \multicolumn{2}{|c|}{\begin{tabular}{|l|l|}
2189.4 & 2222.72 \\
\end{tabular}} & 2247.94 \\
\hline Constituent & Target & AWV- & AWV- & \%Dev. & Target & AWV- & AWV- & AWV- & AWV- & AWV- & AWV- & AWV- & AWV- & AWV- & AWV- & AWV- \\
\hline constituent & 1 arget & G-42D & G-45B & \%Dev. & rarget & G-54A & G-54C & G-55A & G-60A & G-60C & G-61B & G-63B & G-67A & G-68A & G-70A & G-71B \\
\hline $\mathrm{Al}_{2} \mathrm{O}_{3}$ & 6.00 & 5.92 & 5.86 & -2.43 & 6.01 & 5.95 & 6.06 & 5.89 & 5.90 & 6.20 & 6.10 & 5.75 & 5.90 & 5.94 & 6.01 & 6.02 \\
\hline $\mathrm{B}_{2} \mathrm{O}_{3} *$ & 9.84 & 9.84 & 9.84 & NC & 9.86 & 9.84 & 9.85 & 9.85 & 9.85 & 9.85 & 9.85 & 9.86 & 9.86 & 9.86 & 9.86 & 9.86 \\
\hline $\mathrm{CaO}$ & 5.45 & 4.98 & 5.01 & -8.11 & 6.30 & 5.08 & 4.96 & 5.26 & 5.42 & 5.49 & 5.59 & 5.74 & 5.71 & 5.77 & 5.71 & 5.70 \\
\hline $\mathrm{CdO}$ & $\S$ & $<0.01$ & $<0.01$ & NC & $\S$ & $<0.01$ & $<0.01$ & $<0.01$ & $<0.01$ & $<0.01$ & $<0.01$ & $<0.01$ & $<0.01$ & $<0.01$ & $<0.01$ & $<0.01$ \\
\hline $\mathrm{Cl}$ & 0.20 & 0.13 & 0.14 & NC & 0.20 & 0.13 & 0.14 & 0.13 & 0.13 & 0.14 & 0.14 & 0.14 & 0.14 & 0.14 & 0.14 & 0.13 \\
\hline $\mathrm{Cr}_{2} \mathrm{O}_{3}$ & 0.08 & 0.20 & 0.20 & $\mathrm{NC}$ & 0.08 & 0.20 & 0.19 & 0.20 & 0.20 & 0.20 & 0.20 & 0.21 & 0.20 & 0.20 & 0.20 & 0.20 \\
\hline$F^{\#}$ & 0.08 & $<0.08$ & $<0.08$ & NC & 0.08 & $<0.08$ & $<0.08$ & $<0.08$ & $<0.08$ & $<0.08$ & $<0.08$ & $<0.08$ & $<0.08$ & $<0.08$ & $<0.08$ & $<0.08$ \\
\hline $\mathrm{Fe}_{2} \mathrm{O}_{3}$ & 5.41 & 5.14 & 5.16 & -4.78 & 5.42 & 5.16 & 4.82 & 5.12 & 5.20 & 5.23 & 5.31 & 5.51 & 5.40 & 5.40 & 5.24 & 5.24 \\
\hline $\mathrm{K}_{2} \mathrm{O}$ & 0.54 & 0.60 & 0.59 & $\mathrm{NC}$ & 0.54 & 0.61 & 0.61 & 0.64 & 0.63 & 0.62 & 0.62 & 0.63 & 0.62 & 0.65 & 0.66 & 0.66 \\
\hline $\mathrm{Li}_{2} \mathrm{O}^{*}$ & 2.38 & 2.32 & 2.33 & NC & 3.17 & 2.51 & 2.64 & 2.74 & 2.83 & 2.90 & 2.95 & 3.00 & 3.03 & 3.05 & 3.08 & 3.10 \\
\hline $\mathrm{MgO}$ & 1.46 & 1.45 & 1.54 & 5.31 & 1.49 & 1.48 & 1.55 & 1.46 & 1.42 & 1.50 & 1.45 & 1.47 & 1.51 & 1.44 & 1.51 & 1.56 \\
\hline $\mathrm{Na}_{2} \mathrm{O}$ & 15.81 & 17.13 & 16.96 & 7.22 & 13.52 & 16.50 & 16.40 & 15.78 & 15.40 & 15.49 & 14.86 & 14.93 & 14.41 & 14.36 & 14.83 & 14.37 \\
\hline $\mathrm{NiO}$ & 0.01 & $<0.01$ & $<0.01$ & NC & 0.01 & $<0.01$ & $<0.01$ & $<0.01$ & $<0.01$ & $<0.01$ & $<0.01$ & 0.01 & $<0.01$ & $<0.01$ & $<0.01$ & $<0.01$ \\
\hline $\mathrm{P}_{2} \mathrm{O}_{5}$ & 0.12 & 0.18 & 0.18 & NC & 0.12 & 0.18 & 0.18 & 0.17 & 0.17 & 0.17 & 0.17 & 0.18 & 0.18 & 0.17 & 0.17 & 0.17 \\
\hline $\mathrm{PbO}$ & 0.01 & 0.01 & $<0.01$ & NC & 0.01 & $<0.01$ & $<0.01$ & $<0.01$ & $<0.01$ & 0.01 & $<0.01$ & 0.01 & $<0.01$ & $<0.01$ & $<0.01$ & $<0.01$ \\
\hline $\mathrm{ReO}_{2}$ & 0.10 & 0.02 & 0.02 & NC & 0.10 & 0.02 & 0.02 & 0.03 & 0.03 & 0.03 & 0.02 & 0.02 & 0.02 & 0.03 & 0.02 & 0.02 \\
\hline $\mathrm{SiO}_{2}$ & 44.21 & 44.28 & 44.31 & 0.23 & 44.71 & 44.40 & 45.26 & 44.92 & 44.86 & 44.29 & 44.61 & 44.09 & 44.85 & 44.73 & 44.54 & 45.02 \\
\hline $\mathrm{SO}_{3}$ & 0.53 & 0.47 & 0.45 & $\mathrm{NC}$ & 0.59 & 0.47 & 0.48 & 0.50 & 0.49 & 0.51 & 0.51 & 0.49 & 0.52 & 0.51 & 0.51 & 0.51 \\
\hline $\mathrm{TiO}_{2}$ & 1.38 & 1.42 & 1.43 & 3.74 & 1.38 & 1.45 & 1.35 & 1.43 & 1.47 & 1.48 & 1.50 & 1.54 & 1.51 & 1.51 & 1.49 & 1.48 \\
\hline $\mathrm{V}_{2} \mathrm{O}_{5}$ & $\S$ & 0.01 & $<0.01$ & $\mathrm{NC}$ & $\S$ & 0.01 & 0.01 & 0.01 & 0.01 & 0.01 & 0.01 & $<0.01$ & 0.01 & $<0.01$ & 0.01 & 0.01 \\
\hline $\mathrm{ZnO}$ & 3.45 & 3.04 & 3.06 & -11.16 & 3.46 & 3.06 & 2.81 & 3.01 & 3.07 & 3.12 & 3.15 & 3.29 & 3.14 & 3.22 & 3.10 & 3.08 \\
\hline $\mathrm{ZrO}_{2}$ & 2.95 & 2.86 & 2.92 & -0.90 & 2.96 & 2.96 & 2.68 & 2.86 & 2.92 & 2.76 & 2.93 & 3.14 & 2.99 & 3.01 & 2.92 & 2.85 \\
\hline Sum & 100.00 & 100.00 & 100.00 & NC & 100.00 & 100.00 & 100.00 & 100.00 & 100.00 & 100.00 & 100.00 & 100.00 & 100.00 & 100.00 & 100.00 & 100.00 \\
\hline
\end{tabular}

* - Target values calculated based on simple well-stirred tank model

\# - Fluorine values measured directly by XRF given in bold, other values interpolated between measured values.

NC - Not calculated

$\S$ - Not a target component 
Table 4.2. XRF Analyzed Compositions for Glass Samples Discharged During DM100 Tests (wt\%) (continued).

\begin{tabular}{|c|c|c|c|c|c|c|c|c|c|c|c|c|c|c|c|c|c|}
\hline Test & \multicolumn{7}{|c|}{ 2D } & \multicolumn{10}{|c|}{ 3A } \\
\hline Glass (kg) & & 2275.52 & 2302 & 2325.48 & 2350.52 & 2375.38 & 2375.38 & 2406.78 & 2428.06 & 2453.7 & 2481.28 & 2510.36 & 2534 & 2556.76 & 2582 & 2597.82 & 2597.82 \\
\hline Constituent & Target & $\begin{array}{l}\text { AWV- } \\
\text { G-71D }\end{array}$ & $\begin{array}{l}\text { AWV- } \\
\text { G-75B } \\
\end{array}$ & $\begin{array}{l}\text { AWV- } \\
\text { G-76A }\end{array}$ & $\begin{array}{l}\text { AWV- } \\
\text { G-76C }\end{array}$ & $\begin{array}{l}\mathrm{AWV}- \\
\mathrm{G}-78 \mathrm{~A}\end{array}$ & \%Dev & $\begin{array}{l}\text { AWV- } \\
\text { G-98B }\end{array}$ & $\begin{array}{r}\text { AWV- } \\
\text { G-100A }\end{array}$ & $\begin{array}{r}\text { AWV- } \\
\text { G-100C }\end{array}$ & $\begin{array}{r}\text { AWV- } \\
\text { G-101B }\end{array}$ & \begin{tabular}{|r|} 
AWV- \\
G-106A \\
\end{tabular} & \begin{tabular}{|r|} 
AWV- \\
G-106C
\end{tabular} & \begin{tabular}{|r|} 
AWV- \\
G-106E \\
\end{tabular} & \begin{tabular}{|r|} 
AWV- \\
G-108B \\
\end{tabular} & \begin{tabular}{|r|} 
AWV- \\
G-108D \\
\end{tabular} & $\%$ Dev. \\
\hline $\mathrm{Al}_{2} \mathrm{O}_{3}$ & 6.01 & 6.00 & 5.97 & 5.99 & 6.00 & 6.08 & 1.03 & 6.37 & 6.11 & 5.93 & 6.06 & 5.93 & 5.90 & 5.82 & 6.06 & 6.02 & 0.15 \\
\hline $\mathrm{B}_{2} \mathrm{O}_{3} *$ & 9.86 & 9.86 & 9.86 & 9.86 & 9.86 & 9.86 & $\mathrm{NC}$ & 9.86 & 9.86 & 9.86 & 9.86 & 9.86 & 9.86 & 9.86 & 9.86 & 9.86 & $\mathrm{NC}$ \\
\hline $\mathrm{CaO}$ & 6.30 & 5.71 & 5.79 & 5.69 & 5.77 & 5.76 & -8.63 & 6.02 & 5.93 & 6.02 & 6.07 & 5.99 & 5.96 & 6.04 & 5.75 & 6.09 & -3.35 \\
\hline $\mathrm{CdO}$ & $\S$ & $<0.01$ & $<0.01$ & $<0.01$ & $<0.01$ & $<0.01$ & $\mathrm{NC}$ & $<0.01$ & $<0.01$ & $<0.01$ & $<0.01$ & $<0.01$ & $<0.01$ & $<0.01$ & $<0.01$ & $<0.01$ & $\mathrm{NC}$ \\
\hline $\mathrm{Cl}$ & 0.20 & 0.13 & 0.14 & 0.14 & 0.13 & 0.14 & $\mathrm{NC}$ & 0.04 & 0.06 & 0.09 & 0.09 & 0.11 & 0.11 & 0.12 & 0.13 & 0.13 & $\mathrm{NC}$ \\
\hline $\mathrm{Cr}_{2} \mathrm{O}_{3}$ & 0.08 & 0.22 & 0.20 & 0.19 & 0.20 & 0.19 & $\mathrm{NC}$ & 0.27 & 0.25 & 0.25 & 0.24 & 0.23 & 0.22 & 0.22 & 0.21 & 0.22 & $\mathrm{NC}$ \\
\hline $\mathrm{F}^{\#}$ & 0.08 & $<0.08$ & $<0.08$ & $<0.08$ & $<0.08$ & $<0.08$ & NC & $<0.08$ & $<0.08$ & $<0.08$ & $<0.08$ & $<0.08$ & $<0.08$ & $<0.08$ & $<0.08$ & $<0.08$ & $\mathrm{NC}$ \\
\hline $\mathrm{Fe}_{2} \mathrm{O}_{3}$ & 5.42 & 5.23 & 5.22 & 5.22 & 5.23 & 5.20 & -4.14 & 5.73 & 5.54 & 5.50 & 5.48 & 5.41 & 5.33 & 5.51 & 4.98 & 5.39 & -0.68 \\
\hline $\mathrm{K}_{2} \mathrm{O}$ & 0.54 & 0.63 & 0.62 & 0.62 & 0.63 & 0.63 & NC & 0.66 & 0.65 & 0.65 & 0.68 & 0.67 & 0.67 & 0.67 & 0.65 & 0.66 & $\mathrm{NC}$ \\
\hline $\mathrm{Li}_{2} \mathrm{O}^{*}$ & 3.17 & 3.11 & 3.12 & 3.13 & 3.14 & 3.14 & $\mathrm{NC}$ & 3.15 & 3.15 & 3.15 & 3.16 & 3.16 & 3.16 & 3.16 & 3.16 & 3.16 & $\mathrm{NC}$ \\
\hline $\mathrm{MgO}$ & 1.49 & 1.54 & 1.54 & 1.67 & 1.59 & 1.58 & 6.04 & 1.49 & 1.52 & 1.52 & 1.44 & 1.47 & 1.48 & 1.37 & 1.46 & 1.43 & -4.18 \\
\hline $\mathrm{Na}_{2} \mathrm{O}$ & 13.52 & 14.56 & 14.01 & 14.96 & 14.65 & 14.52 & 7.41 & 13.61 & 14.05 & 14.11 & 13.38 & 14.48 & 14.05 & 13.99 & 14.24 & 13.81 & 2.17 \\
\hline $\mathrm{NiO}$ & 0.01 & $<0.01$ & $<0.01$ & $<0.01$ & $<0.01$ & $<0.01$ & $\mathrm{NC}$ & 0.03 & 0.02 & 0.01 & 0.01 & 0.01 & 0.01 & $<0.01$ & $<0.01$ & $<0.01$ & $\mathrm{NC}$ \\
\hline $\mathrm{P}_{2} \mathrm{O}_{5}$ & 0.12 & 0.18 & 0.19 & 0.18 & 0.18 & 0.19 & $\mathrm{NC}$ & 0.17 & 0.18 & 0.16 & 0.17 & 0.17 & 0.17 & 0.18 & 0.17 & 0.18 & $\mathrm{NC}$ \\
\hline $\mathrm{PbO}$ & 0.01 & $<0.01$ & $<0.01$ & 0.01 & $<0.01$ & 0.01 & $\mathrm{NC}$ & $<0.01$ & 0.01 & $<0.01$ & 0.01 & 0.01 & $<0.01$ & $<0.01$ & 0.01 & $<0.01$ & $\mathrm{NC}$ \\
\hline $\mathrm{ReO}_{2}$ & 0.10 & 0.02 & 0.02 & 0.02 & 0.02 & 0.02 & $\mathrm{NC}$ & 0.01 & 0.01 & 0.01 & 0.01 & 0.02 & 0.02 & 0.02 & 0.03 & 0.02 & $\mathrm{NC}$ \\
\hline $\mathrm{SiO}_{2}$ & 44.71 & 44.80 & 45.35 & 44.48 & 44.64 & 44.81 & 0.23 & 43.84 & 44.02 & 44.02 & 44.73 & 43.96 & 44.63 & 44.42 & 45.39 & 44.37 & -0.76 \\
\hline $\mathrm{SO}_{3}$ & 0.59 & 0.52 & 0.54 & 0.52 & 0.53 & 0.54 & $\mathrm{NC}$ & 0.45 & 0.47 & 0.48 & 0.48 & 0.49 & 0.51 & 0.51 & 0.51 & 0.51 & $\mathrm{NC}$ \\
\hline $\mathrm{TiO}_{2}$ & 1.38 & 1.50 & 1.49 & 1.47 & 1.49 & 1.48 & 7.69 & 1.60 & 1.58 & 1.59 & 1.58 & 1.56 & 1.55 & 1.57 & 1.47 & 1.57 & 13.70 \\
\hline $\mathrm{V}_{2} \mathrm{O}_{5}$ & $\S$ & 0.01 & 0.01 & $<0.01$ & 0.01 & 0.01 & $\mathrm{NC}$ & 0.02 & 0.01 & 0.02 & 0.02 & 0.01 & 0.01 & 0.01 & 0.01 & 0.01 & $\mathrm{NC}$ \\
\hline $\mathrm{ZnO}$ & 3.46 & 3.09 & 3.05 & 3.01 & 3.09 & 3.03 & -12.33 & 3.41 & 3.31 & 3.33 & 3.35 & 3.32 & 3.28 & 3.35 & 3.03 & 3.35 & -2.95 \\
\hline $\mathrm{ZrO}_{2}$ & 2.96 & 2.89 & 2.86 & 2.85 & 2.84 & 2.80 & -5.27 & 3.27 & 3.27 & 3.27 & 3.18 & 3.15 & 3.08 & 3.17 & 2.88 & 3.20 & 8.33 \\
\hline Sum & 100.00 & 100.00 & 100.00 & 100.00 & 100.00 & 100.00 & $\mathrm{NC}$ & 100.00 & 100.00 & 100.00 & 100.00 & 100.00 & 100.00 & 100.00 & 100.00 & 100.00 & $\mathrm{NC}$ \\
\hline
\end{tabular}

* - Target values calculated based on simple well-stirred tank model

\# - Fluorine values measured directly by XRF given in bold, other values interpolated between measured values.

$\mathrm{NC}$ - Not calculated

$\S$ - Not a target component 
Table 4.2. XRF Analyzed Compositions for Glass Samples Discharged During DM100 Tests (wt\%) (continued).

\begin{tabular}{|c|c|c|c|c|c|c|c|c|c|c|c|c|c|c|c|}
\hline Test & \multicolumn{15}{|c|}{ 3B } \\
\hline Glass (kg) & & 2620.12 & 2648.22 & 2676.98 & 2702.14 & 2729.96 & 2755.06 & 2784.88 & 2807.16 & 2831.54 & 2860.5 & 2911.48 & 2936.8 & 2961.52 & 2988.94 \\
\hline Constituent & Target & $\begin{array}{r}\text { AWV- } \\
\text { G-118A }\end{array}$ & $\begin{array}{r}\text { AWV- } \\
\text { G-118C }\end{array}$ & $\begin{array}{r}\text { AWV- } \\
\text { G-121A }\end{array}$ & $\begin{array}{r}\text { AWV- } \\
\text { G-122A }\end{array}$ & $\begin{array}{r}\mathrm{AWV}- \\
\mathrm{G}-125 \mathrm{~A}\end{array}$ & $\begin{array}{r}\text { AWV- } \\
\text { G-127A }\end{array}$ & $\begin{array}{r}\text { AWV- } \\
\text { G-128A }\end{array}$ & $\begin{array}{r}\text { AWV- } \\
\text { G-129A }\end{array}$ & $\begin{array}{r}\text { AWV- } \\
\text { G-129C }\end{array}$ & $\begin{array}{r}\text { AWV- } \\
\text { G-130B }\end{array}$ & $\begin{array}{r}\text { AWV- } \\
\text { G-140A }\end{array}$ & $\begin{array}{r}\text { AWV- } \\
\text { G-140C }\end{array}$ & $\begin{array}{r}\text { AWV- } \\
\text { G-141B }\end{array}$ & $\begin{array}{r}\text { AWV- } \\
\text { G-142B }\end{array}$ \\
\hline $\mathrm{Al}_{2} \mathrm{O}_{3}$ & 6.05 & 6.58 & 6.93 & 6.49 & 5.98 & 5.84 & 5.86 & 5.85 & 6.01 & 6.22 & 6.25 & 6.32 & 6.58 & 6.20 & 6.04 \\
\hline $\mathrm{B}_{2} \mathrm{O}_{3} *$ & 9.91 & 9.87 & 9.88 & 9.88 & 9.89 & 9.89 & 9.90 & 9.90 & 9.90 & 9.90 & 9.90 & 9.91 & 9.91 & 9.91 & 9.91 \\
\hline $\mathrm{CaO}$ & 6.85 & 5.97 & 6.09 & 6.24 & 6.11 & 6.47 & 6.19 & 6.57 & 6.22 & 6.30 & 6.33 & 6.15 & 6.28 & 6.24 & 6.55 \\
\hline $\mathrm{CdO}$ & $\S$ & $<0.01$ & $<0.01$ & $<0.01$ & $<0.01$ & $<0.01$ & $<0.01$ & $<0.01$ & $<0.01$ & $<0.01$ & $<0.01$ & $<0.01$ & $<0.01$ & $<0.01$ & $<0.01$ \\
\hline $\mathrm{Cl}$ & 0.20 & 0.13 & 0.14 & 0.14 & 0.13 & 0.14 & 0.14 & 0.14 & 0.13 & 0.13 & 0.13 & 0.13 & 0.12 & 0.13 & 0.14 \\
\hline $\mathrm{Cr}_{2} \mathrm{O}_{3}$ & 0.08 & 0.22 & 0.21 & 0.21 & 0.19 & 0.21 & 0.18 & 0.20 & 0.18 & 0.19 & 0.18 & 0.17 & 0.17 & 0.17 & 0.18 \\
\hline$F^{\#}$ & 0.08 & $<0.08$ & $<0.08$ & $<0.08$ & $<0.08$ & $<0.08$ & $<0.08$ & $<0.08$ & $<0.08$ & $<0.08$ & $<0.08$ & $<0.08$ & $<0.08$ & $<0.08$ & $<0.08$ \\
\hline $\mathrm{Fe}_{2} \mathrm{O}_{3}$ & 5.44 & 5.30 & 5.36 & 5.39 & 5.13 & 5.63 & 5.24 & 5.71 & 5.25 & 5.27 & 5.20 & 5.02 & 5.24 & 5.06 & 5.43 \\
\hline $\mathrm{K}_{2} \mathrm{O}$ & 0.54 & 0.66 & 0.66 & 0.65 & 0.65 & 0.68 & 0.65 & 0.64 & 0.62 & 0.63 & 0.64 & 0.65 & 0.64 & 0.66 & 0.64 \\
\hline $\mathrm{Li}_{2} \mathrm{O}^{*}$ & 4.08 & 3.32 & 3.48 & 3.60 & 3.69 & 3.77 & 3.83 & 3.88 & 3.92 & 3.95 & 3.97 & 4.01 & 4.02 & 4.03 & 4.04 \\
\hline $\mathrm{MgO}$ & 2.36 & 1.40 & 1.58 & 1.64 & 1.91 & 1.92 & 2.08 & 2.11 & 2.22 & 2.23 & 2.20 & 2.24 & 2.22 & 2.28 & 2.31 \\
\hline $\mathrm{Na}_{2} \mathrm{O}$ & 8.92 & 13.66 & 12.48 & 11.76 & 12.06 & 10.81 & 11.27 & 11.18 & 10.92 & 10.55 & 10.34 & 9.70 & 10.06 & 10.15 & 9.90 \\
\hline $\mathrm{NiO}$ & 0.01 & $<0.01$ & $<0.01$ & $<0.01$ & $<0.01$ & $<0.01$ & $<0.01$ & $<0.01$ & $<0.01$ & $<0.01$ & $<0.01$ & $<0.01$ & $<0.01$ & $<0.01$ & $<0.01$ \\
\hline $\mathrm{P}_{2} \mathrm{O}_{5}$ & 0.12 & 0.18 & 0.18 & 0.17 & 0.18 & 0.19 & 0.18 & 0.18 & 0.17 & 0.17 & 0.17 & 0.18 & 0.17 & 0.18 & 0.18 \\
\hline $\mathrm{PbO}$ & 0.01 & $<0.01$ & 0.01 & $<0.01$ & $<0.01$ & $<0.01$ & $<0.01$ & $<0.01$ & 0.01 & $<0.01$ & $<0.01$ & $<0.01$ & $<0.01$ & 0.01 & $<0.01$ \\
\hline $\mathrm{ReO}_{2}$ & 0.10 & 0.03 & 0.03 & 0.03 & 0.03 & 0.04 & 0.03 & 0.03 & 0.04 & 0.04 & 0.04 & 0.03 & 0.04 & 0.04 & 0.03 \\
\hline $\mathrm{SiO}_{2}$ & 46.75 & 44.28 & 44.48 & 45.19 & 45.88 & 45.43 & 46.12 & 44.83 & 46.18 & 46.28 & 46.46 & 47.88 & 46.37 & 47.08 & 46.12 \\
\hline $\mathrm{SO}_{3}$ & 0.69 & 0.52 & 0.53 & 0.54 & 0.57 & 0.57 & 0.56 & 0.55 & 0.53 & 0.52 & 0.54 & 0.53 & 0.52 & 0.53 & 0.52 \\
\hline $\mathrm{TiO}_{2}$ & 1.39 & 1.57 & 1.57 & 1.61 & 1.52 & 1.65 & 1.55 & 1.69 & 1.56 & 1.60 & 1.59 & 1.52 & 1.57 & 1.53 & 1.66 \\
\hline $\mathrm{V}_{2} \mathrm{O}_{5}$ & $\S$ & 0.01 & 0.01 & 0.01 & 0.01 & 0.01 & 0.01 & 0.01 & 0.01 & $<0.01$ & 0.01 & $<0.01$ & 0.01 & 0.01 & 0.01 \\
\hline $\mathrm{ZnO}$ & 3.47 & 3.25 & 3.28 & 3.29 & 3.08 & 3.41 & 3.14 & 3.41 & 3.12 & 3.15 & 3.10 & 2.95 & 3.12 & 2.97 & 3.26 \\
\hline $\mathrm{ZrO}_{2}$ & 2.97 & 3.05 & 3.11 & 3.15 & 2.97 & 3.32 & 3.04 & 3.12 & 3.01 & 2.86 & 2.95 & 2.62 & 2.94 & 2.84 & 3.07 \\
\hline Sum & 100.00 & 100.00 & 100.00 & 100.00 & 100.00 & 100.00 & 100.00 & 100.00 & 100.00 & 100.00 & 100.00 & 100.00 & 100.00 & 100.00 & 100.00 \\
\hline
\end{tabular}

* - Target values calculated based on simple well-stirred tank model

\# - Fluorine values measured directly by XRF given in bold, other values interpolated between measured values.

NC - Not calculated

$\S$ - Not a target component 
Table 4.2. XRF Analyzed Compositions for Glass Samples Discharged During DM100 Tests (wt\%) (continued).

\begin{tabular}{|c|c|c|c|c|c|c|c|c|c|c|c|c|c|c|c|c|}
\hline Test & & 3 & B & & & & & & & 3 & & & & & & \\
\hline Glass (kg) & 301 & 3.96 & 3025.66 & 3025.66 & 305 & 1.78 & 3080.2 & 3104.62 & 3129.94 & 3155.54 & 3180.16 & 3205.76 & 3229.06 & 3253.32 & 3280.8 & 3302.48 \\
\hline Constituent & Target & $\begin{array}{c}\text { AWV- } \\
\text { G-142D }\end{array}$ & $\begin{array}{c}\text { AWV- } \\
\text { G-147A }\end{array}$ & \%Dev. & Target & $\begin{array}{l}\text { AWV- } \\
\text { G-153A } \\
\end{array}$ & $\begin{array}{c}\text { AWV- } \\
\text { G-153C }\end{array}$ & $\begin{array}{l}\text { AWV- } \\
\text { G-155A }\end{array}$ & $\begin{array}{l}\text { BWV- } \\
\text { G-10A }\end{array}$ & $\begin{array}{l}\text { BWV- } \\
\text { G-10C } \\
\end{array}$ & $\begin{array}{l}\text { BWV- } \\
\text { G-12A }\end{array}$ & $\begin{array}{l}\text { BWV- } \\
\text { G-12C }\end{array}$ & $\begin{array}{l}\text { BWV- } \\
\text { G-12E }\end{array}$ & $\begin{array}{l}\text { BWV- } \\
\text { G-14B }\end{array}$ & $\begin{array}{l}\text { BWV- } \\
\text { G-17B }\end{array}$ & $\begin{array}{l}\text { BWV- } \\
\text { G-19B }\end{array}$ \\
\hline $\mathrm{Al}_{2} \mathrm{O}_{3}$ & 6.05 & 6.44 & 6.29 & 3.95 & 6.06 & 5.94 & 6.02 & 6.34 & 6.02 & 6.22 & 6.47 & 6.35 & 6.21 & 6.08 & 6.28 & 6.34 \\
\hline $\mathrm{B}_{2} \mathrm{O}_{3} *$ & 9.91 & 9.91 & 9.91 & $\mathrm{NC}$ & 9.94 & 9.92 & 9.92 & 9.93 & 9.93 & 9.93 & 9.93 & 9.94 & 9.94 & 9.94 & 9.94 & 9.94 \\
\hline $\mathrm{CaO}$ & 6.85 & 6.34 & 6.34 & -7.50 & 6.95 & 6.49 & 6.48 & 6.24 & 6.58 & 6.26 & 6.53 & 6.50 & 6.46 & 6.51 & 6.39 & 6.11 \\
\hline $\mathrm{CdO}$ & $\S$ & $<0.01$ & $<0.01$ & $\mathrm{NC}$ & $\S$ & $<0.01$ & $<0.01$ & $<0.01$ & $<0.01$ & $<0.01$ & $<0.01$ & $<0.01$ & $<0.01$ & $<0.01$ & $<0.01$ & $<0.01$ \\
\hline $\mathrm{Cl}$ & 0.20 & 0.13 & 0.13 & $\mathrm{NC}$ & 0.20 & 0.14 & 0.14 & 0.13 & 0.13 & 0.12 & 0.13 & 0.14 & 0.13 & 0.13 & 0.13 & 0.13 \\
\hline $\mathrm{Cr}_{2} \mathrm{O}_{3}$ & 0.08 & 0.17 & 0.18 & $\mathrm{NC}$ & 0.08 & 0.17 & 0.17 & 0.17 & 0.17 & 0.16 & 0.17 & 0.17 & 0.17 & 0.17 & 0.16 & 0.15 \\
\hline $\mathrm{F}^{\#}$ & 0.08 & $<0.08$ & $<0.08$ & $\mathrm{NC}$ & 0.08 & $<0.08$ & $<0.08$ & $<0.08$ & $<0.08$ & $<0.08$ & $<0.08$ & $<0.08$ & $<0.08$ & $<0.08$ & $<0.08$ & $<0.08$ \\
\hline $\mathrm{Fe}_{2} \mathrm{O}_{3}$ & 5.44 & 5.26 & 5.25 & -3.50 & 5.47 & 5.25 & 5.25 & 5.11 & 5.35 & 4.92 & 5.46 & 5.32 & 5.35 & 5.36 & 5.24 & 4.85 \\
\hline $\mathrm{K}_{2} \mathrm{O}$ & 0.54 & 0.64 & 0.65 & $\mathrm{NC}$ & 0.54 & 0.63 & 0.63 & 0.66 & 0.65 & 0.63 & 0.64 & 0.63 & 0.63 & 0.63 & 0.63 & 0.64 \\
\hline $\mathrm{Li}_{2} \mathrm{O}^{*}$ & 4.08 & 4.05 & 4.05 & $\mathrm{NC}$ & 4.26 & 4.09 & 4.13 & 4.15 & 4.17 & 4.19 & 4.20 & 4.21 & 4.22 & 4.23 & 4.23 & 4.24 \\
\hline $\mathrm{MgO}$ & 2.36 & 2.31 & 2.30 & -2.51 & 2.94 & 2.44 & 2.50 & 2.49 & 2.59 & 2.78 & 2.77 & 2.82 & 2.88 & 2.91 & 3.11 & 3.07 \\
\hline $\mathrm{Na}_{2} \mathrm{O}$ & 8.92 & 9.90 & 9.81 & 9.97 & 5.71 & 9.18 & 9.10 & 8.18 & 8.13 & 7.87 & 7.22 & 7.29 & 7.02 & 7.13 & 7.05 & 7.10 \\
\hline $\mathrm{NiO}$ & 0.01 & $<0.01$ & $<0.01$ & $\mathrm{NC}$ & 0.01 & $<0.01$ & $<0.01$ & $<0.01$ & $<0.01$ & $<0.01$ & $<0.01$ & $<0.01$ & $<0.01$ & $<0.01$ & $<0.01$ & $<0.01$ \\
\hline $\mathrm{P}_{2} \mathrm{O}_{5}$ & 0.12 & 0.18 & 0.18 & $\mathrm{NC}$ & 0.12 & 0.19 & 0.20 & 0.18 & 0.18 & 0.20 & 0.18 & 0.19 & 0.19 & 0.19 & 0.18 & 0.19 \\
\hline $\mathrm{PbO}$ & 0.01 & $<0.01$ & 0.01 & $\mathrm{NC}$ & 0.01 & 0.01 & $<0.01$ & $<0.01$ & $<0.01$ & $<0.01$ & $<0.01$ & $<0.01$ & $<0.01$ & $<0.01$ & $<0.01$ & $<0.01$ \\
\hline $\mathrm{ReO}_{2}$ & 0.10 & 0.04 & 0.04 & $\mathrm{NC}$ & 0.10 & 0.03 & 0.04 & 0.03 & 0.04 & 0.04 & 0.03 & 0.04 & 0.04 & 0.03 & 0.05 & 0.04 \\
\hline $\mathrm{SiO}_{2}$ & 46.75 & 46.43 & 46.58 & -0.37 & 48.88 & 47.18 & 47.29 & 48.42 & 47.67 & 49.03 & 47.64 & 48.17 & 48.40 & 48.22 & 48.31 & 49.66 \\
\hline $\mathrm{SO}_{3}$ & 0.69 & 0.53 & 0.54 & $\mathrm{NC}$ & 0.80 & 0.58 & 0.57 & 0.56 & 0.56 & 0.57 & 0.55 & 0.58 & 0.58 & 0.58 & 0.56 & 0.58 \\
\hline $\mathrm{TiO}_{2}$ & 1.39 & 1.58 & 1.59 & 14.41 & 1.39 & 1.61 & 1.60 & 1.52 & 1.60 & 1.49 & 1.62 & 1.59 & 1.59 & 1.58 & 1.58 & 1.45 \\
\hline $\mathrm{V}_{2} \mathrm{O}_{5}$ & $\S$ & 0.01 & 0.01 & $\mathrm{NC}$ & $\S$ & 0.01 & 0.01 & 0.01 & 0.01 & 0.01 & 0.01 & 0.01 & 0.01 & 0.01 & 0.01 & 0.01 \\
\hline $\mathrm{ZnO}$ & 3.47 & 3.08 & 3.12 & -9.91 & 3.48 & 3.13 & 3.11 & 3.02 & 3.19 & 2.89 & 3.29 & 3.16 & 3.20 & 3.22 & 3.16 & 2.86 \\
\hline $\mathrm{ZrO}_{2}$ & 2.97 & 2.99 & 3.03 & 2.15 & 2.99 & 3.01 & 2.84 & 2.84 & 3.04 & 2.69 & 3.14 & 2.92 & 2.98 & 3.09 & 3.00 & 2.65 \\
\hline Sum & 100.00 & 100.00 & 100.00 & $\mathrm{NC}$ & 100.00 & 100.00 & 100.00 & 100.00 & 100.00 & 100.00 & 100.00 & 100.00 & 100.00 & 100.00 & 100.00 & 100.00 \\
\hline
\end{tabular}

* - Target values calculated based on simple well-stirred tank model

\# - Fluorine values measured directly by XRF given in bold, other values interpolated between measured values.

NC - Not calculated

$\S$ - Not a target component 
Table 4.2. XRF Analyzed Compositions for Glass Samples Discharged During DM100 Tests (wt\%) (continued).

\begin{tabular}{|c|c|c|c|c|c|c|c|c|c|c|c|c|c|c|c|c|c|}
\hline Test & & & & 3 & $\mathrm{C}$ & & & & & & & & $4 A$ & & & & \\
\hline Glass (kg) & 332 & 9.02 & 3350.32 & 3377.84 & 3396.9 & 3426.64 & 3450.02 & 3450.02 & 347 & 76.1 & 3499.24 & 3522.88 & 3544.04 & 3568.1 & 3580.1 & 3580.10 & 3580.10 \\
\hline Constituent & Target & \begin{tabular}{|l} 
BWV- \\
G-21A
\end{tabular} & $\begin{array}{l}\text { BWV- } \\
\text { G-24B }\end{array}$ & $\begin{array}{l}\text { BWV- } \\
\text { G-26A }\end{array}$ & $\begin{array}{l}\text { BWV- } \\
\text { G-27A }\end{array}$ & $\begin{array}{l}\text { BWV- } \\
\text { G-28B }\end{array}$ & $\begin{array}{l}\text { BWV- } \\
\text { G-32B }\end{array}$ & $\%$ Dev. & Target & \begin{tabular}{|l} 
BWV- \\
G-59A
\end{tabular} & $\begin{array}{l}\text { BWV- } \\
\text { G-60B }\end{array}$ & \begin{tabular}{|l|} 
BWV- \\
G-61B
\end{tabular} & $\begin{array}{l}\text { BWV- } \\
\text { G-62B }\end{array}$ & $\begin{array}{l}\text { BWV- } \\
\text { G-66B }\end{array}$ & \begin{tabular}{|l|} 
BWV- \\
G-66C
\end{tabular} & $\begin{array}{l}\text { BWV- } \\
\text { G-66C }\end{array}$ & \%Dev. \\
\hline $\mathrm{Al}_{2} \mathrm{O}_{3}$ & 6.06 & 6.15 & 6.24 & 6.13 & 6.20 & 5.93 & 6.28 & 3.60 & 5.93 & 6.45 & 6.63 & 6.08 & 6.30 & 6.23 & 6.13 & 6.13 & 3.36 \\
\hline $\mathrm{B}_{2} \mathrm{O}_{3} *$ & 9.94 & 9.94 & 9.94 & 9.94 & 9.94 & 9.94 & 9.94 & $\mathrm{NC}$ & 9.73 & 9.90 & 9.87 & 9.85 & 9.83 & 9.81 & 9.80 & 9.80 & $\mathrm{NC}$ \\
\hline $\mathrm{CaO}$ & 6.95 & 6.35 & 6.36 & 6.43 & 6.63 & 6.62 & 6.30 & -9.44 & 6.80 & 6.54 & 6.59 & 6.63 & 6.49 & 6.57 & 6.35 & 6.35 & -6.72 \\
\hline $\mathrm{CdO}$ & $\S$ & $<0.01$ & $<0.01$ & $<0.01$ & $<0.01$ & $<0.01$ & $<0.01$ & $\mathrm{NC}$ & $\S$ & $<0.01$ & $<0.01$ & $<0.01$ & $<0.01$ & $<0.01$ & $<0.01$ & $<0.01$ & $\mathrm{NC}$ \\
\hline $\mathrm{Cl}$ & 0.20 & 0.13 & 0.14 & 0.13 & 0.14 & 0.13 & 0.13 & $\mathrm{NC}$ & 1.10 & 0.05 & 0.17 & 0.27 & 0.32 & 0.38 & 0.41 & 0.41 & $\mathrm{NC}$ \\
\hline $\mathrm{Cr}_{2} \mathrm{O}_{3}$ & 0.08 & 0.16 & 0.16 & 0.16 & 0.17 & 0.16 & 0.16 & $\mathrm{NC}$ & 0.08 & 0.16 & 0.16 & 0.16 & 0.16 & 0.16 & 0.16 & 0.16 & $\mathrm{NC}$ \\
\hline $\mathrm{F}^{\#}$ & 0.08 & $<0.08$ & $<0.08$ & $<0.08$ & $<0.08$ & $<0.08$ & $<0.08$ & $\mathrm{NC}$ & 1.30 & 0.08 & 0.18 & 0.29 & 0.35 & 0.44 & 0.47 & 0.47 & $\mathrm{NC}$ \\
\hline $\mathrm{Fe}_{2} \mathrm{O}_{3}$ & 5.47 & 5.14 & 5.20 & 5.25 & 5.52 & 5.46 & 5.15 & -5.95 & 5.36 & 5.33 & 5.47 & 5.50 & 5.37 & 5.39 & 5.19 & 5.19 & -3.19 \\
\hline $\mathrm{K}_{2} \mathrm{O}$ & 0.54 & 0.64 & 0.66 & 0.66 & 0.66 & 0.63 & 0.66 & $\mathrm{NC}$ & 0.53 & 0.67 & 0.64 & 0.66 & 0.66 & 0.67 & 0.65 & 0.65 & $\mathrm{NC}$ \\
\hline $\mathrm{Li}_{2} \mathrm{O}^{*}$ & 4.26 & 4.24 & 4.24 & 4.25 & 4.25 & 4.25 & 4.25 & $\mathrm{NC}$ & 4.17 & 4.23 & 4.22 & 4.21 & 4.20 & 4.20 & 4.19 & 4.19 & $\mathrm{NC}$ \\
\hline $\mathrm{MgO}$ & 2.94 & 2.94 & 2.96 & 3.00 & 2.92 & 3.02 & 3.04 & 3.54 & 2.87 & 2.91 & 2.93 & 2.94 & 2.87 & 2.89 & 3.02 & 3.02 & 5.23 \\
\hline $\mathrm{Na}_{2} \mathrm{O}$ & 5.71 & 6.60 & 6.61 & 6.60 & 6.51 & 6.83 & 6.50 & 13.80 & 5.59 & 6.73 & 6.51 & 6.60 & 6.72 & 6.03 & 6.78 & 6.78 & 21.26 \\
\hline $\mathrm{NiO}$ & 0.01 & $<0.01$ & $<0.01$ & $<0.01$ & $<0.01$ & $<0.01$ & $<0.01$ & $\mathrm{NC}$ & 0.01 & 0.01 & 0.01 & 0.01 & 0.01 & 0.01 & $<0.01$ & $<0.01$ & $\mathrm{NC}$ \\
\hline $\mathrm{P}_{2} \mathrm{O}_{5}$ & 0.12 & 0.18 & 0.17 & 0.19 & 0.18 & 0.18 & 0.18 & $\mathrm{NC}$ & 0.12 & 0.19 & 0.18 & 0.17 & 0.18 & 0.18 & 0.18 & 0.18 & $\mathrm{NC}$ \\
\hline $\mathrm{PbO}$ & 0.01 & $<0.01$ & $<0.01$ & $<0.01$ & $<0.01$ & $<0.01$ & $<0.01$ & $\mathrm{NC}$ & 0.01 & $<0.01$ & $<0.01$ & $<0.01$ & 0.01 & $<0.01$ & $<0.01$ & $<0.01$ & $\mathrm{NC}$ \\
\hline $\mathrm{ReO}_{2}$ & 0.10 & 0.04 & 0.04 & 0.04 & 0.05 & 0.04 & 0.04 & NC & 0.10 & $<0.01$ & 0.01 & 0.02 & 0.02 & 0.03 & 0.03 & 0.03 & NC \\
\hline $\mathrm{SiO}_{2}$ & 48.88 & 49.35 & 49.08 & 49.01 & 48.21 & 48.44 & 49.29 & 0.84 & 47.83 & 48.86 & 48.23 & 48.46 & 48.49 & 48.79 & 48.80 & 48.80 & 2.01 \\
\hline $\mathrm{SO}_{3}$ & 0.80 & 0.59 & 0.57 & 0.58 & 0.55 & 0.58 & 0.59 & $\mathrm{NC}$ & 0.78 & 0.11 & 0.19 & 0.25 & 0.30 & 0.34 & 0.36 & 0.36 & $\mathrm{NC}$ \\
\hline $\mathrm{TiO}_{2}$ & 1.39 & 1.52 & 1.55 & 1.56 & 1.61 & 1.60 & 1.51 & 8.82 & 1.36 & 1.58 & 1.63 & 1.63 & 1.57 & 1.59 & 1.53 & 1.53 & 12.63 \\
\hline $\mathrm{V}_{2} \mathrm{O}_{5}$ & $\S$ & 0.01 & 0.01 & 0.01 & 0.01 & 0.01 & 0.01 & $\mathrm{NC}$ & $\S$ & 0.01 & 0.01 & 0.01 & 0.01 & 0.01 & 0.01 & 0.01 & $\mathrm{NC}$ \\
\hline $\mathrm{ZnO}$ & 3.48 & 3.08 & 3.12 & 3.16 & 3.34 & 3.27 & 3.07 & -11.76 & 3.40 & 3.08 & 3.19 & 3.20 & 3.12 & 3.17 & 3.03 & 3.03 & -10.89 \\
\hline $\mathrm{ZrO}_{2}$ & 2.99 & 2.93 & 2.95 & 2.92 & 3.11 & 2.89 & 2.90 & -3.02 & 2.92 & 3.11 & 3.16 & 3.06 & 3.02 & 3.12 & 2.89 & 2.89 & -1.10 \\
\hline Sum & 100.00 & \begin{tabular}{|l|}
100.00 \\
\end{tabular} & 100.00 & 100.00 & 100.00 & 100.00 & 100.00 & $\mathrm{NC}$ & 100.00 & 100.00 & 100.00 & 100.00 & \begin{tabular}{|l|}
100.00 \\
\end{tabular} & 100.00 & \begin{tabular}{|l|}
100.00 \\
\end{tabular} & 100.00 & $\mathrm{NC}$ \\
\hline
\end{tabular}

* - Target values calculated based on simple well-stirred tank model

\# - Fluorine values measured directly by XRF given in bold, other values interpolated between measured values.

$\mathrm{NC}$ - Not calculated

$\S$ - Not a target component 
Table 4.2. XRF Analyzed Compositions for Glass Samples Discharged During DM100 Tests (wt\%) (continued).

\begin{tabular}{|c|c|c|c|c|c|c|c|c|c|c|c|c|c|c|c|c|}
\hline Test & \multicolumn{16}{|c|}{$4 B$} \\
\hline \multicolumn{2}{|c|}{ Glass (kg) } & 3608.54 & 3634.26 & 3658.88 & 3686.08 & 3713.10 & 3739.18 & 3765.54 & 3793.96 & 3817.14 & 3843.24 & 3870.22 & 3896.70 & 3924.18 & 3951.12 & 3975.86 \\
\hline Constituent & Target & $\begin{array}{l}\text { BWV- } \\
\text { G-66C }\end{array}$ & $\begin{array}{l}\text { BWV- } \\
\text { G-72B }\end{array}$ & \begin{tabular}{|l|} 
BWV- \\
G-73B
\end{tabular} & \begin{tabular}{|l|} 
BWV- \\
G-79A
\end{tabular} & $\begin{array}{l}\text { BWV- } \\
\text { G-81A }\end{array}$ & \begin{tabular}{|l|} 
BWV- \\
G-81C
\end{tabular} & $\begin{array}{l}\text { BWV- } \\
\text { G-84A }\end{array}$ & \begin{tabular}{|l|} 
BWV- \\
G-85B
\end{tabular} & \begin{tabular}{|l|} 
BWV- \\
G-88A
\end{tabular} & \begin{tabular}{l|} 
BWV- \\
G-88C
\end{tabular} & \begin{tabular}{|l|} 
BWV- \\
G-89B
\end{tabular} & $\begin{array}{l}\text { BWV- } \\
\text { G-93A }\end{array}$ & $\begin{array}{l}\text { BWV- } \\
\text { G-93C }\end{array}$ & $\begin{array}{l}\text { BWV- } \\
\text { G-95B }\end{array}$ & $\begin{array}{l}\text { BWV- } \\
\text { G-98B }\end{array}$ \\
\hline $\mathrm{Al}_{2} \mathrm{O}_{3}$ & 5.92 & 6.08 & 6.11 & 6.01 & 6.14 & 5.96 & 5.99 & 6.05 & 6.32 & 6.15 & 6.01 & 5.80 & 5.87 & 5.86 & 5.95 & 5.92 \\
\hline $\mathrm{B}_{2} \mathrm{O}_{3} *$ & 9.70 & .78 & 9.77 & 9.75 & 9.74 & 9.74 & 9.73 & 9.72 & 9.72 & 9.72 & 9.71 & 9.71 & 9.71 & 9.71 & 9.71 & 9.70 \\
\hline $\mathrm{CaO}$ & 6.71 & 49 & 6.45 & 6.57 & 6.42 & 6.59 & 6.48 & 6.53 & 6.36 & 6.41 & 6.51 & 6.45 & 6.43 & 6.44 & 6.33 & 6.46 \\
\hline $\mathrm{CdO}$ & $\S$ & $<0.01$ & $<0.01$ & $<0.01$ & $<0.01$ & $<0.01$ & $<0.01$ & $<0.01$ & $<0.01$ & $<0.01$ & $<0.01$ & $<0.01$ & $<0.01$ & $<0.01$ & $<0.01$ & $<0.01$ \\
\hline $\mathrm{Cl}$ & 10 & 0.42 & 0.46 & 0.51 & 0.52 & 0.57 & 0.59 & 0.61 & 0.63 & 0.62 & 0.67 & 0.66 & 0.65 & 0.64 & 0.65 & 0.64 \\
\hline $\mathrm{Cr}_{2} \mathrm{O}_{3}$ & .08 & 0.17 & 0.16 & 0.16 & 0.16 & 0.17 & 0.17 & 0.17 & 0.17 & 0.16 & 0.17 & 0.17 & 0.17 & 0.17 & 0.17 & 0.17 \\
\hline $\mathrm{F}^{\#}$ & 30 & 54 & 0.61 & 0.68 & 0.73 & 0.77 & 0.77 & \begin{tabular}{|c|}
0.78 \\
\end{tabular} & 0.82 & 0.86 & 0.86 & 0.87 & 0.87 & 0.88 & \begin{tabular}{l|}
0.88 \\
\end{tabular} & 0.88 \\
\hline $\mathrm{Fe}_{2} \mathrm{O}_{3}$ & 33 & 37 & 5.40 & 5.48 & 5.27 & 5.53 & 5.40 & 5.51 & 5.27 & 5.41 & 5.49 & 5.48 & 5.47 & .46 & 5.28 & 5.49 \\
\hline $\mathrm{K}_{2} \mathrm{O}$ & 53 & 66 & 0.64 & 0.66 & 0.65 & 0.64 & 0.64 & 0.66 & 0.62 & 0.62 & 0.62 & 0.63 & 0.64 & 0.64 & 0.62 & 0.63 \\
\hline $\mathrm{Li}_{2} \mathrm{O}^{*}$ & 99 & 4.15 & 4.12 & 4.10 & 4.07 & 4.06 & 4.04 & 4.03 & 4.02 & 4.02 & 4.01 & 4.01 & 4.00 & 4.00 & 4.00 & 4.00 \\
\hline $\mathrm{MgO}$ & 31 & 91 & 2.82 & 2.73 & 2.74 & 2.63 & 2.57 & 2.53 & 2.59 & 2.52 & 2.47 & 2.54 & 2.42 & 2.55 & 2.46 & 2.38 \\
\hline $\mathrm{Na}_{2} \mathrm{O}$ & 73 & 78 & 6.87 & 6.93 & 7.06 & 7.15 & 7.99 & 7.47 & 8.48 & 8.22 & 8.09 & 8.35 & 8.50 & 8.36 & 8.47 & 8.56 \\
\hline $\mathrm{NiO}$ & 01 & 0.01 & $<0.01$ & 0.01 & 0.00 & $<0.01$ & $<0.01$ & $<0.01$ & $<0.01$ & $<0.01$ & $<0.01$ & $<0.01$ & $<0.01$ & $<0.01$ & $<0.01$ & $<0.01$ \\
\hline $\mathrm{P}_{2} \mathrm{O}_{5}$ & & 8 & & 0.17 & 0.19 & 0.18 & 0.17 & 0.18 & 0.19 & 0.18 & 0.19 & 0.19 & 0.19 & .19 & 0.18 & 0.18 \\
\hline $\mathrm{PbO}$ & 0.01 & 0.01 & 0.01 & 0.01 & $<0.01$ & $<0.01$ & $<0.01$ & $<0.01$ & $<0.01$ & $<0.01$ & $<0.01$ & $<0.01$ & $<0.01$ & $<0.01$ & $<0.01$ & $<0.01$ \\
\hline $\mathrm{ReO}_{2}$ & 10 & 0.03 & 0.03 & 0.02 & 0.02 & 0.03 & 0.03 & 0.03 & 0.03 & 0.04 & 0.03 & 0.03 & 0.04 & 0.04 & 0.04 & 0.03 \\
\hline $\mathrm{SiO}_{2}$ & 45.75 & 48.21 & 48.19 & 47.88 & 48.23 & 47.65 & 47.15 & 47.28 & 46.85 & 46.78 & 46.75 & 46.78 & 46.64 & 46.76 & 47.15 & 46.55 \\
\hline $\mathrm{SO}_{3}$ & 0.67 & 0.39 & 0.40 & 0.42 & 0.43 & 0.45 & 0.46 & 0.48 & 0.48 & 0.49 & 0.50 & 0.51 & 0.51 & 0.51 & 0.53 & 0.51 \\
\hline $\mathrm{TiO}_{2}$ & 1.36 & 1.57 & 1.57 & 1.59 & 1.57 & 1.60 & 1.59 & 1.62 & 1.56 & 1.59 & 1.61 & 1.59 & 1.58 & 1.58 & 1.56 & 1.60 \\
\hline $\mathrm{V}_{2} \mathrm{O}_{5}$ & $\S$ & 0.01 & 0.01 & 0.01 & 0.01 & 0.01 & 0.01 & 0.01 & 0.01 & 0.01 & 0.01 & 0.01 & 0.01 & 0.01 & 0.01 & 0.01 \\
\hline $\mathrm{ZnO}$ & 39 & 3.17 & 3.12 & 3.22 & 3.05 & 3.21 & 3.15 & 3.22 & 3.03 & 3.15 & 3.20 & 3.17 & 3.17 & 3.12 & 3.05 & 3.16 \\
\hline $\mathrm{ZrO}_{2}$ & 2.90 & 3.09 & 3.09 & 3.08 & 3.00 & 3.05 & 3.06 & 3.11 & 2.84 & 3.03 & 3.10 & 3.05 & 3.12 & 3.07 & 2.98 & 3.11 \\
\hline Sum & 100.00 & 100.00 & 100.00 & 100.00 & 100.00 & 100.00 & 100.00 & 100.00 & 100.00 & 100.00 & 100.00 & 100.00 & 100.00 & 100.00 & 100.00 & 100.00 \\
\hline
\end{tabular}

* - Target values calculated based on simple well-stirred tank model

\# - Fluorine values measured directly by XRF given in bold, other values interpolated between measured values.

$\mathrm{NC}$ - Not calculated

$\S$ - Not a target component 
Table 4.2 XRF Analyzed Compositions for Glass Samples Discharged During DM100 Tests (wt\%) (continued).

\begin{tabular}{|c|c|c|c|c|c|c|c|c|c|c|c|c|c|c|c|c|c|}
\hline Test & \multicolumn{4}{|c|}{ 4B } & \multicolumn{13}{|c|}{$4 \mathrm{C}$} \\
\hline \multicolumn{2}{|c|}{ Glass (kg) } & \multicolumn{3}{|c|}{\begin{tabular}{|l|l|l|}
4001.28 & 4028.19 & 4028.19 \\
\end{tabular}} & \multicolumn{4}{|c|}{\begin{tabular}{|l|l|l|}
4054.85 & 4077.83 & 4104.41 \\
\end{tabular}} & \multicolumn{2}{|c|}{\begin{tabular}{|l|l|}
4130.19 & 4158.59 \\
\end{tabular}} & \multirow{2}{*}{\begin{tabular}{|r}
4187.07 \\
BWV- \\
$G-125 A$
\end{tabular}} & \multirow{2}{*}{\begin{tabular}{|r|}
4205.33 \\
BWV- \\
$G-126 A$
\end{tabular}} & \multirow{2}{*}{\begin{tabular}{|r|}
4235.67 \\
BWV- \\
$G-128 A$
\end{tabular}} & \multicolumn{2}{|c|}{\begin{tabular}{|l|l|l|}
7263.77 & 4284.21 \\
\end{tabular}} & 14309.83 & \multirow{2}{*}{$\begin{array}{c}4334.59 \\
\text { BWV- } \\
\text { G-134B }\end{array}$} \\
\hline Constituent & Target & $\begin{array}{l}\text { BWV- } \\
\text { G-99B }\end{array}$ & $\begin{array}{r}\text { BWV- } \\
\text { G-102B }\end{array}$ & \%Dev. & Target & \begin{tabular}{|r|} 
BWV- \\
G-113B \\
\end{tabular} & $\begin{array}{r}\text { BWV- } \\
\text { G-113D }\end{array}$ & \begin{tabular}{|r|} 
BWV- \\
G-117B \\
\end{tabular} & \begin{tabular}{|r|} 
BWV- \\
G-121A \\
\end{tabular} & $\begin{array}{r}\text { BWV- } \\
\text { G-124A }\end{array}$ & & & & \begin{tabular}{|r|} 
BWV- \\
G-128C \\
\end{tabular} & \begin{tabular}{|c|}
$\mathrm{BWV}-$ \\
$\mathrm{G}-129 \mathrm{~B}$ \\
\end{tabular} & $\begin{array}{c}\text { BWV- } \\
\text { G-129D }\end{array}$ & \\
\hline $\mathrm{Al}_{2} \mathrm{O}_{3}$ & 5.92 & 5.99 & 5.87 & -0.82 & 5.89 & 6.01 & 5.95 & 5.84 & 6.47 & 5.96 & 5.84 & 6.01 & 5.91 & 6.24 & 5.99 & 5.92 & 5.91 \\
\hline $\mathrm{B}_{2} \mathrm{O}_{3} *$ & 9.70 & 9.70 & 9.70 & $\mathrm{NC}$ & 9.65 & 9.69 & 9.69 & 9.68 & 9.67 & 9.67 & 9.67 & 9.66 & 9.66 & 9.66 & 9.66 & 9.66 & 9.66 \\
\hline $\mathrm{CaO}$ & 6.71 & 6.44 & 6.54 & -2.42 & 6.17 & 6.44 & 6.43 & 6.28 & 6.04 & 6.10 & 6.14 & 6.17 & 6.00 & 6.18 & 6.12 & 6.15 & 6.09 \\
\hline $\mathrm{CdO}$ & $\S$ & $<0.01$ & $<0.01$ & $\mathrm{NC}$ & $\S$ & $<0.01$ & $<0.01$ & $<0.01$ & $<0.01$ & $<0.01$ & $<0.01$ & $<0.01$ & $<0.01$ & $<0.01$ & $<0.01$ & $<0.01$ & $<0.01$ \\
\hline $\mathrm{Cl}$ & 1.10 & 0.65 & 0.67 & $\mathrm{NC}$ & 1.10 & 0.64 & 0.62 & 0.65 & 0.58 & 0.61 & 0.59 & 0.60 & 0.60 & 0.61 & 0.61 & 0.63 & 0.61 \\
\hline $\mathrm{Cr}_{2} \mathrm{O}_{3}$ & 0.08 & 0.17 & 0.17 & $\mathrm{NC}$ & 0.08 & 0.17 & 0.17 & 0.17 & 0.18 & 0.17 & 0.17 & 0.17 & 0.18 & 0.18 & 0.18 & 0.19 & 0.19 \\
\hline $\mathrm{F}^{\#}$ & 1.30 & 0.89 & 0.89 & NC & 1.30 & 0.88 & 0.88 & 0.87 & 0.87 & 0.86 & 0.85 & 0.85 & 0.84 & 0.85 & 0.85 & 0.85 & 0.86 \\
\hline $\mathrm{Fe}_{2} \mathrm{O}_{3}$ & 5.33 & 5.46 & 5.57 & 4.52 & 5.31 & 5.44 & 5.43 & 5.23 & 5.04 & 5.10 & 5.21 & 5.29 & 5.11 & 5.49 & 5.36 & 5.43 & 5.43 \\
\hline $\mathrm{K}_{2} \mathrm{O}$ & 0.53 & 0.61 & 0.61 & $\mathrm{NC}$ & 0.53 & 0.61 & 0.65 & 0.62 & 0.60 & 0.62 & 0.61 & 0.62 & 0.61 & 0.61 & 0.60 & 0.61 & 0.64 \\
\hline $\mathrm{Li}_{2} \mathrm{O}^{*}$ & 3.99 & 4.00 & 3.99 & NC & 3.10 & 3.82 & 3.69 & 3.57 & 3.48 & 3.40 & 3.34 & 3.30 & 3.26 & 3.23 & 3.21 & 3.19 & 3.17 \\
\hline $\mathrm{MgO}$ & 2.31 & 2.51 & 2.44 & 5.64 & 1.46 & 2.37 & 2.09 & 1.97 & 1.92 & 1.81 & 1.76 & 1.68 & 1.73 & 1.57 & 1.60 & 1.57 & 1.47 \\
\hline $\mathrm{Na}_{2} \mathrm{O}$ & 8.73 & 8.73 & 8.36 & -4.23 & 13.23 & 8.74 & 9.20 & 10.33 & 11.23 & 10.79 & 11.02 & 11.08 & 11.80 & 11.49 & 12.03 & 11.96 & 12.10 \\
\hline $\mathrm{NiO}$ & 0.01 & $<0.01$ & $<0.01$ & $\mathrm{NC}$ & 0.01 & $<0.01$ & $<0.01$ & $<0.01$ & $<0.01$ & $<0.01$ & $<0.01$ & $<0.01$ & $<0.01$ & $<0.01$ & $<0.01$ & $<0.01$ & $<0.01$ \\
\hline $\mathrm{P}_{2} \mathrm{O}_{5}$ & 0.12 & 0.19 & 0.18 & NC & 0.12 & 0.17 & 0.19 & 0.17 & 0.17 & 0.19 & 0.17 & 0.18 & 0.17 & 0.17 & 0.18 & 0.17 & 0.17 \\
\hline $\mathrm{PbO}$ & 0.01 & $<0.01$ & $<0.01$ & NC & 0.01 & 0.01 & 0.01 & $<0.01$ & $<0.01$ & 0.01 & 0.01 & $<0.01$ & $<0.01$ & $<0.01$ & $<0.01$ & $<0.01$ & 0.01 \\
\hline $\mathrm{ReO}_{2}$ & 0.10 & 0.03 & 0.04 & NC & 0.10 & 0.03 & 0.02 & 0.02 & 0.02 & 0.03 & 0.01 & 0.01 & 0.02 & 0.02 & 0.03 & 0.02 & 0.02 \\
\hline $\mathrm{SiO}_{2}$ & 45.75 & 46.34 & 46.39 & 1.40 & 43.76 & 46.57 & 46.61 & 46.42 & 45.86 & 46.95 & 46.59 & 46.23 & 46.25 & 45.27 & 45.27 & 45.12 & 45.28 \\
\hline $\mathrm{SO}_{3}$ & 0.67 & 0.51 & 0.52 & NC & 0.58 & 0.53 & 0.49 & 0.55 & 0.54 & 0.46 & 0.45 & 0.45 & 0.50 & 0.43 & 0.45 & 0.45 & 0.46 \\
\hline $\mathrm{TiO}_{2}$ & 1.36 & 1.60 & 1.63 & 19.84 & 1.35 & 1.60 & 1.61 & 1.56 & 1.50 & 1.52 & 1.54 & 1.55 & 1.51 & 1.61 & 1.58 & 1.59 & 1.55 \\
\hline $\mathrm{V}_{2} \mathrm{O}_{5}$ & $\S$ & 0.01 & 0.01 & $\mathrm{NC}$ & $\S$ & 0.01 & $<0.01$ & $<0.01$ & 0.02 & 0.01 & 0.01 & $<0.01$ & $<0.01$ & $<0.01$ & 0.01 & 0.01 & 0.01 \\
\hline $\mathrm{ZnO}$ & 3.39 & 3.14 & 3.22 & -5.06 & 3.38 & 3.15 & 3.20 & 3.10 & 2.97 & 2.99 & 3.09 & 3.14 & 3.02 & 3.29 & 3.25 & 3.30 & 3.28 \\
\hline $\mathrm{ZrO}_{2}$ & 2.90 & 3.04 & 3.18 & 9.44 & 2.89 & 3.11 & 3.07 & 2.94 & 2.85 & 2.76 & 2.93 & 2.98 & 2.84 & 3.10 & 3.03 & 3.17 & 3.11 \\
\hline Sum & 100.00 & 100.00 & 100.00 & $\mathrm{NC}$ & 100.00 & 100.00 & 100.00 & 100.00 & 100.00 & 100.00 & 100.00 & 100.00 & 100.00 & 100.00 & 100.00 & \begin{tabular}{|l|l|}
0 & 100.00 \\
\end{tabular} & 100.00 \\
\hline
\end{tabular}

* - Target values calculated based on simple well-stirred tank model

\# - Fluorine values measured directly by XRF given in bold, other values interpolated between measured values.

$\mathrm{NC}$ - Not calculated

$\S$ - Not a target component 
Table 4.2. XRF Analyzed Compositions for Glass Samples Discharged During DM100 Tests (wt\%) (continued).

\begin{tabular}{|c|c|c|c|c|c|c|c|c|c|c|c|c|c|c|c|}
\hline Test & \multicolumn{7}{|c|}{$4 C$} & \multicolumn{8}{|c|}{$5 A$} \\
\hline \multicolumn{2}{|c|}{ Glass (kg) } & 4359.79 & 4383.65 & 4404.45 & 4430.43 & 4454.83 & 4454.83 & 4480.31 & 4505.49 & 4529.29 & 4553.35 & 4576.25 & 4605.45 & 4630.05 & 4651.41 \\
\hline & & BWV-G- & BWV-G- & BWV-G- & BWV-G- & BWV-G- & & CWV-G- & CWV-G- & CWV-G- & CWV-G- & CWV-G- & CWV-G- & CWV-G- & CWV-G \\
\hline Constituent & 1 arget & $136 \mathrm{~A}$ & $140 \mathrm{~A}$ & $141 \mathrm{~A}$ & $141 \mathrm{C}$ & 142B & \%Dev. & $8 \mathrm{~B}$ & 13B & $15 B$ & $16 \mathrm{~B}$ & $16 \mathrm{D}$ & 20B & $20 \mathrm{D}$ & $22 B$ \\
\hline $\mathrm{Al}_{2} \mathrm{O}_{3}$ & 5.89 & 5.73 & 5.95 & 5.91 & 5.92 & 5.92 & 0.57 & 6.16 & 6.54 & 6.52 & 6.39 & 5.77 & 5.96 & 5.88 & 5.92 \\
\hline $\mathrm{B}_{2} \mathrm{O}_{3} *$ & 9.65 & 9.66 & 9.65 & 9.65 & 9.65 & 9.65 & $\mathrm{NC}$ & 9.65 & 9.65 & 9.65 & 9.65 & 9.65 & 9.65 & 9.65 & 9.65 \\
\hline $\mathrm{CaO}$ & 6.17 & 6.09 & 5.88 & 5.94 & 5.72 & 5.82 & -5.64 & 6.03 & 5.93 & 5.96 & 5.67 & 6.06 & 5.92 & 5.85 & 5.80 \\
\hline $\mathrm{CdO}$ & $\S$ & $<0.01$ & $<0.01$ & $<0.01$ & $<0.01$ & $<0.01$ & $\mathrm{NC}$ & $<0.01$ & $<0.01$ & $<0.01$ & $<0.01$ & $<0.01$ & $<0.01$ & $<0.01$ & $<0.01$ \\
\hline $\mathrm{Cl}$ & 1.10 & 0.62 & 0.62 & 0.62 & 0.62 & 0.63 & $\mathrm{NC}$ & 0.24 & 0.31 & 0.41 & 0.44 & 0.49 & 0.52 & 0.52 & 0.54 \\
\hline $\mathrm{Cr}_{2} \mathrm{O}_{3}$ & 0.08 & 0.19 & 0.18 & 0.18 & 0.18 & 0.18 & $\mathrm{NC}$ & 0.24 & 0.22 & 0.22 & 0.19 & 0.21 & 0.21 & 0.21 & 0.21 \\
\hline $\mathrm{F}^{\#}$ & 1.30 & 0.86 & 0.87 & 0.87 & 0.88 & 0.88 & $\mathrm{NC}$ & 0.69 & 0.71 & 0.72 & 0.74 & 0.75 & 0.77 & 0.75 & 0.72 \\
\hline $\mathrm{Fe}_{2} \mathrm{O}_{3}$ & 5.31 & 5.45 & 5.18 & 5.30 & 4.98 & 5.04 & -5.06 & 5.28 & 5.32 & 5.41 & 4.84 & 5.55 & 5.33 & 5.23 & 5.15 \\
\hline $\mathrm{K}_{2} \mathrm{O}$ & 0.53 & 0.63 & 0.60 & 0.62 & 0.59 & 0.61 & $\mathrm{NC}$ & 0.58 & 0.60 & 0.59 & 0.59 & 0.63 & 0.63 & 0.62 & 0.61 \\
\hline $\mathrm{Li}_{2} \mathrm{O}^{*}$ & 3.10 & 3.16 & 3.15 & 3.14 & 3.13 & 3.13 & $\mathrm{NC}$ & 3.12 & 3.12 & 3.11 & 3.11 & 3.11 & 3.11 & 3.11 & 3.10 \\
\hline $\mathrm{MgO}$ & 1.46 & 1.48 & 1.49 & 1.49 & 1.58 & 1.53 & 5.04 & 1.57 & 1.55 & 1.52 & 1.53 & 1.49 & 1.50 & 1.50 & 1.49 \\
\hline $\mathrm{Na}_{2} \mathrm{O}$ & 13.23 & 12.33 & 12.63 & 12.50 & 13.07 & 12.34 & -6.72 & 12.85 & 12.79 & 12.72 & 13.43 & 12.65 & 12.77 & 12.77 & 12.75 \\
\hline $\mathrm{NiO}$ & 0.01 & $<0.01$ & $<0.01$ & $<0.01$ & $<0.01$ & $<0.01$ & $\mathrm{NC}$ & 0.02 & 0.02 & 0.01 & 0.01 & 0.01 & 0.01 & 0.01 & $<0.01$ \\
\hline $\mathrm{P}_{2} \mathrm{O}_{5}$ & 0.12 & 0.17 & 0.17 & 0.18 & 0.17 & 0.17 & $\mathrm{NC}$ & 0.17 & 0.17 & 0.17 & 0.18 & 0.17 & 0.17 & 0.17 & 0.18 \\
\hline $\mathrm{PbO}$ & 0.01 & $<0.01$ & $<0.01$ & $<0.01$ & 0.01 & 0.00 & $\mathrm{NC}$ & $<0.01$ & 0.01 & $<0.01$ & $<0.01$ & $<0.01$ & $<0.01$ & $<0.01$ & 0.01 \\
\hline $\mathrm{ReO}_{2}$ & 0.10 & 0.03 & 0.02 & 0.02 & 0.02 & 0.02 & $\mathrm{NC}$ & 0.00 & 0.01 & 0.01 & 0.01 & 0.02 & 0.02 & 0.03 & 0.02 \\
\hline $\mathrm{SiO}_{2}$ & 43.76 & 45.19 & 45.74 & 45.57 & 45.84 & 46.42 & 6.08 & 45.54 & 44.89 & 44.84 & 45.90 & 44.90 & 45.19 & 45.67 & 45.99 \\
\hline $\mathrm{SO}_{3}$ & 0.58 & 0.46 & 0.46 & 0.46 & 0.48 & 0.50 & $\mathrm{NC}$ & 0.47 & 0.49 & 0.48 & 0.48 & 0.48 & 0.52 & 0.50 & 0.51 \\
\hline $\mathrm{TiO}_{2}$ & 1.35 & 1.58 & 1.49 & 1.53 & 1.44 & 1.47 & 9.08 & 1.53 & 1.53 & 1.55 & 1.45 & 1.56 & 1.53 & 1.51 & 1.47 \\
\hline $\mathrm{V}_{2} \mathrm{O}_{5}$ & $\S$ & 0.01 & 0.01 & 0.01 & 0.01 & 0.01 & $\mathrm{NC}$ & 0.01 & 0.01 & 0.01 & 0.01 & 0.01 & $<0.01$ & 0.01 & 0.01 \\
\hline $\mathrm{ZnO}$ & 3.38 & 3.26 & 3.05 & 3.16 & 2.96 & 2.98 & -11.82 & 3.09 & 3.16 & 3.23 & 2.83 & 3.29 & 3.15 & 3.10 & 3.00 \\
\hline $\mathrm{ZrO}_{2}$ & 2.89 & 3.12 & 2.85 & 2.87 & 2.75 & 2.69 & -6.90 & 2.74 & 2.97 & 2.85 & 2.54 & 3.19 & 3.03 & 2.93 & 2.88 \\
\hline Sum & 100.00 & 100.00 & 100.00 & 100.00 & 100.00 & 100.00 & $\mathrm{NC}$ & 100.00 & 100.00 & 100.00 & 100.00 & 100.00 & 100.00 & 100.00 & 100.00 \\
\hline
\end{tabular}

* - Target values calculated based on simple well-stirred tank model

\# - Fluorine values measured directly by XRF given in bold, other values interpolated between measured values.

NC - Not calculated

$\S$ - Not a target component 
Table 4.2. XRF Analyzed Compositions for Glass Samples Discharged During DM100 Tests (wt\%) (continued).

\begin{tabular}{|c|c|c|c|c|c|c|c|c|c|c|c|c|c|c|c|c|c|}
\hline Test & \multicolumn{4}{|c|}{$5 A$} & \multicolumn{13}{|c|}{$5 B$} \\
\hline \multicolumn{2}{|c|}{ Glass (kg) } & 4674.61 & 4685.69 & 4685.69 & & 4709.99 & 4729.25 & 4758.99 & 4787.87 & 4809.73 & 4830.09 & 4852.45 & 4881.87 & 4902.11 & 4930.93 & 4952.53 & 4973.03 \\
\hline Constituent & Target & $\begin{array}{l}\text { CWV- } \\
\text { G-22D }\end{array}$ & $\begin{array}{l}\mathrm{CWV}- \\
\mathrm{G}-26 \mathrm{~A}\end{array}$ & \%Dev. & Target & $\begin{array}{l}\text { CWV- } \\
\text { G-30A }\end{array}$ & $\begin{array}{l}\text { CWV- } \\
\text { G-30C }\end{array}$ & $\begin{array}{l}\text { CWV- } \\
\text { G-31B }\end{array}$ & $\begin{array}{l}\text { CWV- } \\
\text { G-35B }\end{array}$ & $\begin{array}{l}\text { CWV- } \\
\text { G-37B }\end{array}$ & $\begin{array}{l}\text { CWV- } \\
\text { G-38A }\end{array}$ & $\begin{array}{l}\text { CWV- } \\
\text { G-38C }\end{array}$ & $\begin{array}{l}\text { CWV- } \\
\text { G-43B }\end{array}$ & $\begin{array}{l}\text { CWV- } \\
\text { G-44B }\end{array}$ & $\begin{array}{l}\text { CWV- } \\
\text { G-45B }\end{array}$ & $\begin{array}{l}\text { CWV- } \\
\text { G-46A }\end{array}$ & $\begin{array}{l}\text { CWV- } \\
\text { G-46C }\end{array}$ \\
\hline $\mathrm{Al}_{2} \mathrm{O}_{3}$ & 5.89 & 5.91 & 5.85 & -0.64 & 5.88 & 5.90 & 5.97 & 5.84 & 5.83 & 5.93 & 6.11 & 5.98 & 5.82 & 5.78 & 5.95 & 6.00 & 5.77 \\
\hline $\mathrm{B}_{2} \mathrm{O}_{3} *$ & 9.65 & 9.65 & 9.65 & NC & 9.63 & 9.65 & 9.65 & 9.64 & 9.64 & 9.64 & 9.64 & 9.64 & 9.64 & 9.64 & 9.63 & 9.63 & 9.63 \\
\hline $\mathrm{CaO}$ & 6.17 & 5.96 & 5.89 & -4.51 & 5.34 & 5.76 & 5.62 & 5.68 & 5.44 & 5.25 & 5.35 & 5.09 & 5.19 & 5.31 & 5.01 & 5.14 & 5.28 \\
\hline $\mathrm{CdO}$ & $\S$ & $<0.01$ & $<0.01$ & $\mathrm{NC}$ & $\S$ & $<0.01$ & $<0.01$ & $<0.01$ & $<0.01$ & $<0.01$ & $<0.01$ & $<0.01$ & $<0.01$ & $<0.01$ & $<0.01$ & $<0.01$ & $<0.01$ \\
\hline $\mathrm{Cl}$ & 1.10 & 0.54 & 0.55 & $\mathrm{NC}$ & 1.10 & 0.54 & 0.56 & 0.57 & 0.58 & 0.56 & 0.60 & 0.60 & 0.64 & 0.64 & 0.64 & 0.64 & 0.66 \\
\hline $\mathrm{Cr}_{2} \mathrm{O}_{3}$ & 0.08 & 0.21 & 0.21 & $\mathrm{NC}$ & 0.08 & 0.20 & 0.20 & 0.21 & 0.23 & 0.20 & 0.21 & 0.19 & 0.20 & 0.20 & 0.19 & 0.20 & 0.21 \\
\hline $\mathrm{F}^{\#}$ & 1.30 & 0.70 & 0.69 & $\mathrm{NC}$ & 1.30 & 0.72 & 0.75 & 0.79 & 0.83 & 0.86 & 0.89 & 0.92 & 0.92 & 0.92 & 0.92 & 0.91 & 0.91 \\
\hline $\mathrm{Fe}_{2} \mathrm{O}_{3}$ & 5.31 & 5.36 & 5.24 & -1.27 & 5.30 & 5.16 & 5.17 & 5.28 & 5.15 & 5.05 & 5.19 & 4.97 & 5.04 & 5.36 & 5.04 & 5.20 & 5.41 \\
\hline $\mathrm{K}_{2} \mathrm{O}$ & 0.53 & 0.60 & 0.61 & $\mathrm{NC}$ & 0.53 & 0.59 & 0.58 & 0.61 & 0.60 & 0.57 & 0.62 & 0.58 & 0.57 & 0.58 & 0.57 & 0.58 & 0.58 \\
\hline $\mathrm{Li}_{2} \mathrm{O}^{*}$ & 3.10 & 3.10 & 3.10 & $\mathrm{NC}$ & 2.33 & 2.96 & 2.87 & 2.75 & 2.66 & 2.60 & 2.56 & 2.52 & 2.48 & 2.45 & 2.43 & 2.41 & 2.40 \\
\hline $\mathrm{MgO}$ & 1.46 & 1.48 & 1.50 & 3.03 & 1.43 & 1.47 & 1.55 & 1.45 & 1.51 & 1.48 & 1.47 & 1.54 & 1.54 & 1.46 & 1.54 & 1.49 & 1.46 \\
\hline $\mathrm{Na}_{2} \mathrm{O}$ & 13.23 & 13.01 & 12.81 & -3.16 & 15.48 & 13.97 & 14.31 & 13.94 & 14.69 & 15.23 & 14.36 & 14.85 & 14.36 & 14.57 & 14.96 & 14.84 & 14.68 \\
\hline $\mathrm{NiO}$ & 0.01 & 0.01 & $<0.01$ & $\mathrm{NC}$ & 0.01 & $<0.01$ & $<0.01$ & 0.01 & 0.01 & $<0.01$ & $<0.01$ & $<0.01$ & $<0.01$ & $<0.01$ & $<0.01$ & $<0.01$ & $<0.01$ \\
\hline $\mathrm{P}_{2} \mathrm{O}_{5}$ & 0.12 & 0.17 & 0.18 & $\mathrm{NC}$ & 0.12 & 0.17 & 0.16 & 0.17 & 0.17 & 0.15 & 0.17 & 0.18 & 0.18 & 0.17 & 0.17 & 0.17 & 0.17 \\
\hline $\mathrm{PbO}$ & 0.01 & 0.01 & $<0.01$ & $\mathrm{NC}$ & 0.01 & $<0.01$ & $<0.01$ & $<0.01$ & $<0.01$ & 0.01 & 0.01 & 0.01 & 0.01 & $<0.01$ & $<0.01$ & $<0.01$ & 0.01 \\
\hline $\mathrm{ReO}_{2}$ & 0.10 & 0.02 & 0.02 & NC & 0.10 & 0.02 & 0.02 & 0.02 & 0.02 & 0.03 & 0.02 & 0.03 & 0.03 & 0.03 & 0.02 & 0.03 & 0.02 \\
\hline $\mathrm{SiO}_{2}$ & 43.76 & 44.91 & 45.73 & 4.51 & 43.26 & 44.98 & 44.72 & 45.00 & 44.86 & 44.78 & 44.98 & 45.48 & 45.82 & 44.79 & 45.44 & 44.84 & 44.63 \\
\hline $\mathrm{SO}_{3}$ & 0.58 & 0.49 & 0.51 & $\mathrm{NC}$ & 0.52 & 0.48 & 0.46 & 0.47 & 0.46 & 0.44 & 0.44 & 0.45 & 0.47 & 0.47 & 0.46 & 0.45 & 0.45 \\
\hline $\mathrm{TiO}_{2}$ & 1.35 & 1.55 & 1.49 & 10.70 & 1.35 & 1.47 & 1.46 & 1.48 & 1.42 & 1.39 & 1.43 & 1.35 & 1.39 & 1.44 & 1.34 & 1.41 & 1.45 \\
\hline $\mathrm{V}_{2} \mathrm{O}_{5}$ & $\S$ & 0.01 & 0.01 & $\mathrm{NC}$ & $\S$ & 0.01 & 0.01 & 0.01 & 0.01 & 0.01 & 0.01 & 0.01 & 0.01 & 0.01 & $<0.01$ & $<0.01$ & 0.01 \\
\hline $\mathrm{ZnO}$ & 3.38 & 3.20 & 3.06 & -9.56 & 3.37 & 3.05 & 3.03 & 3.12 & 3.03 & 2.95 & 3.03 & 2.86 & 2.90 & 3.13 & 2.89 & 3.04 & 3.17 \\
\hline $\mathrm{ZrO}_{2}$ & 2.89 & 3.10 & 2.88 & -0.54 & 2.88 & 2.87 & 2.90 & 2.96 & 2.87 & 2.87 & 2.93 & 2.77 & 2.81 & 3.05 & 2.78 & 2.99 & 3.09 \\
\hline Sum & 100.00 & 100.00 & 100.00 & $\mathrm{NC}$ & 100.00 & 100.00 & 100.00 & 100.00 & 100.00 & 100.00 & 100.00 & 100.00 & 100.00 & 100.00 & 100.00 & 100.00 & 100.00 \\
\hline
\end{tabular}

* - Target values calculated based on simple well-stirred tank model

\# - Fluorine values measured directly by XRF given in bold, other values interpolated between measured values.

$\mathrm{NC}$ - Not calculated

$\S$ - Not a target component 
Table 4.2. XRF Analyzed Compositions for Glass Samples Discharged During DM100 Tests (wt\%) (continued).

\begin{tabular}{|c|c|c|c|c|c|c|c|c|c|c|c|c|c|c|c|c|c|}
\hline Test & \multicolumn{10}{|c|}{$5 B$} & \multicolumn{7}{|c|}{$5 C$} \\
\hline \multicolumn{2}{|c|}{ Glass (kg) } & 4992.53 & 5014.69 & 5034.83 & 5059.51 & 5085.45 & 5109.07 & 5130.93 & 5143.49 & 5143.49 & \multicolumn{2}{|c|}{5163.67} & 5187.29 & 5211.15 & 5236.97 & 5261.49 & 5281.39 \\
\hline Constituent & Target & $\begin{array}{l}\text { CWV- } \\
\text { G-50B }\end{array}$ & $\begin{array}{l}\text { CWV- } \\
\text { G-50D }\end{array}$ & $\begin{array}{l}\text { CWV- } \\
\text { G-50F }\end{array}$ & $\begin{array}{l}\text { CWV- } \\
\text { G-52B }\end{array}$ & $\begin{array}{l}\text { CWV- } \\
\text { G-54A }\end{array}$ & $\begin{array}{l}\text { CWV- } \\
\text { G-54C }\end{array}$ & $\begin{array}{l}\text { CWV- } \\
\text { G-58A }\end{array}$ & $\begin{array}{l}\text { CWV- } \\
\text { G-58B } \\
\end{array}$ & \%Dev. & Target & $\begin{array}{l}\text { CWV- } \\
\text { G-61A } \\
\end{array}$ & $\begin{array}{l}\text { CWV- } \\
\text { G-61C }\end{array}$ & $\begin{array}{l}\text { CWV- } \\
\text { G-63B }\end{array}$ & $\begin{array}{l}\text { CWV- } \\
\text { G-68A }\end{array}$ & $\begin{array}{l}\text { CWV- } \\
\text { G-69A }\end{array}$ & $\begin{array}{l}\text { CWV- } \\
\text { G-69C }\end{array}$ \\
\hline $\mathrm{Al}_{2} \mathrm{O}_{3}$ & 5.88 & 5.91 & 5.88 & 5.98 & 5.88 & 5.94 & 5.82 & 5.82 & 5.78 & -1.61 & 5.86 & 6.09 & 5.97 & 5.92 & 5.81 & 5.88 & 5.95 \\
\hline $\mathrm{B}_{2} \mathrm{O}_{3} *$ & 9.63 & 9.63 & 9.63 & 9.63 & 9.63 & 9.63 & 9.63 & 9.63 & 9.63 & NC & 9.59 & 9.63 & 9.62 & 9.62 & 9.61 & 9.61 & 9.61 \\
\hline $\mathrm{CaO}$ & 5.34 & 5.14 & 5.13 & 5.01 & 4.99 & 5.06 & 5.13 & 5.14 & 5.07 & -5.10 & 3.53 & 4.84 & 4.84 & 4.38 & 4.35 & 4.07 & 3.93 \\
\hline $\mathrm{CdO}$ & $\S$ & $<0.01$ & $<0.01$ & $<0.01$ & $<0.01$ & $<0.01$ & $<0.01$ & $<0.01$ & $<0.01$ & $\mathrm{NC}$ & $\S$ & $<0.01$ & $<0.01$ & $<0.01$ & $<0.01$ & $<0.01$ & $<0.01$ \\
\hline $\mathrm{Cl}$ & 1.10 & 0.63 & 0.65 & 0.62 & 0.61 & 0.61 & 0.62 & 0.64 & 0.63 & $\mathrm{NC}$ & 1.10 & 0.62 & 0.66 & 0.66 & 0.65 & 0.64 & 0.64 \\
\hline $\mathrm{Cr}_{2} \mathrm{O}_{3}$ & 0.08 & 0.20 & 0.20 & 0.20 & 0.20 & 0.20 & 0.20 & 0.20 & 0.20 & $\mathrm{NC}$ & 0.08 & 0.19 & 0.21 & 0.19 & 0.20 & 0.20 & 0.18 \\
\hline $\mathrm{F}^{\#}$ & 1.30 & 0.91 & 0.91 & 0.92 & 0.93 & 0.94 & 0.95 & 0.96 & 0.97 & $\mathrm{NC}$ & 1.30 & 0.97 & 0.98 & 0.98 & 0.99 & 1.00 & 1.00 \\
\hline $\mathrm{Fe}_{2} \mathrm{O}_{3}$ & 5.30 & 5.30 & 5.29 & 5.17 & 5.28 & 5.36 & 5.32 & 5.37 & 5.29 & -0.23 & 5.28 & 5.10 & 5.43 & 4.93 & 5.22 & 4.98 & 4.77 \\
\hline $\mathrm{K}_{2} \mathrm{O}$ & 0.53 & 0.59 & 0.60 & 0.61 & 0.59 & 0.59 & 0.60 & 0.57 & 0.57 & $\mathrm{NC}$ & 0.53 & 0.56 & 0.59 & 0.58 & 0.60 & 0.59 & 0.57 \\
\hline $\mathrm{Li}_{2} \mathrm{O}^{*}$ & 2.33 & 2.39 & 2.38 & 2.37 & 2.36 & 2.35 & 2.35 & 2.35 & 2.34 & $\mathrm{NC}$ & 0.48 & 2.06 & 1.77 & 1.54 & 1.33 & 1.18 & 1.07 \\
\hline $\mathrm{MgO}$ & 1.43 & 1.56 & 1.46 & 1.42 & 1.53 & 1.46 & 1.42 & 1.45 & 1.50 & 4.90 & 1.42 & 1.49 & 1.51 & 1.55 & 1.42 & 1.44 & 1.51 \\
\hline $\mathrm{Na}_{2} \mathrm{O}$ & 15.48 & 14.47 & 14.85 & 15.21 & 15.35 & 15.26 & 14.93 & 15.17 & 15.44 & -0.27 & 18.55 & 15.54 & 15.51 & 16.51 & 16.67 & 17.55 & 17.66 \\
\hline $\mathrm{NiO}$ & 0.01 & $<0.01$ & $<0.01$ & $<0.01$ & $<0.01$ & 0.01 & $<0.01$ & 0.01 & $<0.01$ & $\mathrm{NC}$ & 0.01 & $<0.01$ & 0.01 & $<0.01$ & 0.01 & $<0.01$ & $<0.01$ \\
\hline $\mathrm{P}_{2} \mathrm{O}_{5}$ & 0.12 & 0.18 & 0.17 & 0.17 & 0.18 & 0.17 & 0.17 & 0.17 & 0.18 & NC & 0.12 & 0.16 & 0.18 & 0.18 & 0.16 & 0.16 & 0.17 \\
\hline $\mathrm{PbO}$ & 0.01 & $<0.01$ & $<0.01$ & 0.01 & $<0.01$ & $<0.01$ & $<0.01$ & $<0.01$ & $<0.01$ & NC & 0.01 & $<0.01$ & $<0.01$ & $<0.01$ & 0.01 & 0.01 & 0.01 \\
\hline $\mathrm{ReO}_{2}$ & 0.10 & 0.03 & 0.02 & 0.02 & 0.03 & 0.03 & 0.03 & 0.03 & 0.02 & $\mathrm{NC}$ & 0.10 & 0.02 & 0.03 & 0.03 & 0.03 & 0.02 & 0.02 \\
\hline $\mathrm{SiO}_{2}$ & 43.26 & 45.13 & 44.85 & 44.91 & 44.60 & 44.40 & 44.80 & 44.35 & 44.46 & 2.77 & 44.04 & 45.06 & 44.50 & 45.50 & 45.08 & 45.18 & 45.76 \\
\hline $\mathrm{SO}_{3}$ & 0.52 & 0.44 & 0.46 & 0.44 & 0.43 & 0.42 & 0.42 & 0.43 & 0.44 & $\mathrm{NC}$ & 0.45 & 0.43 & 0.44 & 0.44 & 0.42 & 0.42 & 0.43 \\
\hline $\mathrm{TiO}_{2}$ & 1.35 & 1.40 & 1.39 & 1.39 & 1.38 & 1.39 & 1.41 & 1.43 & 1.40 & 3.64 & 1.34 & 1.39 & 1.46 & 1.36 & 1.43 & 1.38 & 1.34 \\
\hline $\mathrm{V}_{2} \mathrm{O}_{5}$ & $\S$ & 0.01 & $<0.01$ & $<0.01$ & $<0.01$ & 0.01 & $<0.01$ & $<0.01$ & $<0.01$ & $\mathrm{NC}$ & $\S$ & $<0.01$ & $<0.01$ & $<0.01$ & 0.01 & 0.01 & 0.01 \\
\hline $\mathrm{ZnO}$ & 3.37 & 3.08 & 3.10 & 2.99 & 3.03 & 3.11 & 3.12 & 3.16 & 3.05 & -9.44 & 3.35 & 2.95 & 3.17 & 2.85 & 3.03 & 2.88 & 2.71 \\
\hline $\mathrm{ZrO}_{2}$ & 2.88 & 2.98 & 3.01 & 2.94 & 2.99 & 3.05 & 3.07 & 3.10 & 3.02 & 4.77 & 2.87 & 2.88 & 3.12 & 2.78 & 2.99 & 2.80 & 2.64 \\
\hline Sum & 100.00 & 100.00 & 100.00 & 100.00 & 100.00 & 100.00 & 100.00 & 100.00 & 100.00 & $\mathrm{NC}$ & 100.00 & 100.00 & 100.00 & 100.00 & 100.00 & 100.00 & 100.00 \\
\hline
\end{tabular}

* - Target values calculated based on simple well-stirred tank model

\# - Fluorine values measured directly by XRF given in bold, other values interpolated between measured values.

$\mathrm{NC}$ - Not calculated

$\S$ - Not a target component 
Table 4.2. XRF Analyzed Compositions for Glass Samples Discharged During DM100 Tests (wt\%) (continued).

\begin{tabular}{|c|c|c|c|c|c|c|c|c|c|c|c|c|c|c|c|c|}
\hline Test & \multicolumn{16}{|c|}{$5 C$} \\
\hline \multicolumn{2}{|c|}{ Glass (kg) } & 5304.89 & 5325.87 & 5344.69 & 5368.21 & 5390.37 & 5410.61 & 5442.23 & 5464.09 & 5486.63 & 5507.43 & 5530.33 & 5551.51 & 5572.45 & 5597.95 & 5597.95 \\
\hline Constituent & Target & $\begin{array}{l}\text { CWV- } \\
\text { G-71A }\end{array}$ & \begin{tabular}{|l|} 
CWV- \\
G-71C \\
\end{tabular} & $\begin{array}{l}\text { CWV- } \\
\text { G-72A }\end{array}$ & $\begin{array}{l}\text { CWV- } \\
\text { G-72C }\end{array}$ & $\begin{array}{l}\text { CWV- } \\
\text { G-75B }\end{array}$ & $\begin{array}{l}\text { CWV- } \\
\text { G-79A }\end{array}$ & $\begin{array}{l}\text { CWV- } \\
\text { G-79C }\end{array}$ & $\begin{array}{l}\text { CWV- } \\
\text { G-81A } \\
\end{array}$ & $\begin{array}{l}\text { CWV- } \\
\text { G-81C }\end{array}$ & $\begin{array}{l}\text { CWV- } \\
\text { G-83B }\end{array}$ & \begin{tabular}{|l|} 
CWV- \\
G-86A
\end{tabular} & $\begin{array}{l}\text { CWV- } \\
\text { G-86C }\end{array}$ & $\begin{array}{l}\text { CWV- } \\
\text { G-90A }\end{array}$ & $\begin{array}{l}\text { CWV- } \\
\text { G-90C } \\
\end{array}$ & \%Dev. \\
\hline $\mathrm{Al}_{2} \mathrm{O}_{3}$ & 5.86 & 5.97 & 5.90 & 5.92 & 5.78 & 5.70 & 5.79 & 5.99 & 5.83 & 5.90 & 6.11 & 6.00 & 5.94 & 5.95 & 5.83 & -0.43 \\
\hline $\mathrm{B}_{2} \mathrm{O}_{3} *$ & 9.59 & 9.60 & 9.60 & 9.60 & 9.60 & 9.60 & 9.60 & 9.60 & 9.60 & 9.60 & 9.59 & 9.59 & 9.59 & 9.59 & 9.59 & $\mathrm{NC}$ \\
\hline $\mathrm{CaO}$ & 3.53 & 4.12 & 4.06 & 4.01 & 3.82 & 3.81 & 3.75 & 3.57 & 3.52 & 3.38 & 3.45 & 3.34 & 3.49 & 3.42 & 3.38 & -4.24 \\
\hline $\mathrm{CdO}$ & $\S$ & $<0.01$ & $<0.01$ & $<0.01$ & $<0.01$ & $<0.01$ & $<0.01$ & $<0.01$ & $<0.01$ & $<0.01$ & $<0.01$ & $<0.01$ & $<0.01$ & $<0.01$ & $<0.01$ & $\mathrm{NC}$ \\
\hline $\mathrm{Cl}$ & 1.10 & 0.66 & 0.66 & 0.68 & 0.67 & 0.68 & 0.66 & 0.66 & 0.66 & 0.67 & 0.64 & 0.64 & 0.66 & 0.65 & 0.65 & $\mathrm{NC}$ \\
\hline $\mathrm{Cr}_{2} \mathrm{O}_{3}$ & 0.08 & 0.21 & 0.20 & 0.21 & 0.21 & 0.21 & 0.21 & 0.20 & 0.19 & 0.19 & 0.20 & 0.19 & 0.21 & 0.20 & 0.20 & $\mathrm{NC}$ \\
\hline$F^{\#}$ & 1.30 & 1.00 & 1.00 & 1.00 & 1.00 & 1.01 & 1.01 & 1.01 & 1.01 & 1.00 & 0.99 & 0.98 & 0.96 & 0.95 & 0.94 & $\mathrm{NC}$ \\
\hline $\mathrm{Fe}_{2} \mathrm{O}_{3}$ & 5.28 & 5.29 & 5.37 & 5.47 & 5.22 & 5.36 & 5.33 & 5.02 & 5.03 & 4.80 & 5.14 & 4.84 & 5.18 & 5.13 & 5.03 & -4.75 \\
\hline $\mathrm{K}_{2} \mathrm{O}$ & 0.53 & 0.60 & 0.61 & 0.59 & 0.59 & 0.58 & 0.59 & 0.61 & 0.60 & 0.57 & 0.61 & 0.58 & 0.61 & 0.58 & 0.58 & $\mathrm{NC}$ \\
\hline $\mathrm{Li}_{2} \mathrm{O}^{*}$ & 0.48 & 0.96 & 0.89 & 0.83 & 0.77 & 0.72 & 0.68 & 0.63 & 0.61 & 0.59 & 0.57 & 0.55 & 0.54 & 0.53 & 0.52 & $\mathrm{NC}$ \\
\hline $\mathrm{MgO}$ & 1.42 & 1.39 & 1.46 & 1.55 & 1.52 & 1.51 & 1.45 & 1.46 & 1.47 & 1.51 & 1.42 & 1.46 & 1.35 & 1.42 & 1.42 & 0.00 \\
\hline $\mathrm{Na}_{2} \mathrm{O}$ & 18.55 & 16.65 & 16.75 & 16.64 & 17.90 & 17.64 & 17.71 & 17.51 & 18.04 & 18.38 & 17.83 & 18.42 & 17.68 & 18.67 & 18.78 & 1.28 \\
\hline $\mathrm{NiO}$ & 0.01 & 0.01 & 0.01 & 0.01 & 0.01 & 0.01 & 0.01 & $<0.01$ & $<0.01$ & $<0.01$ & 0.01 & $<0.01$ & 0.01 & 0.01 & $<0.01$ & $\mathrm{NC}$ \\
\hline $\mathrm{P}_{2} \mathrm{O}_{5}$ & 0.12 & 0.18 & 0.17 & 0.17 & 0.18 & 0.17 & 0.17 & 0.19 & 0.18 & 0.18 & 0.18 & 0.18 & 0.18 & 0.17 & 0.18 & $\mathrm{NC}$ \\
\hline $\mathrm{PbO}$ & 0.01 & 0.01 & 0.01 & 0.01 & 0.01 & 0.01 & 0.01 & $<0.01$ & $<0.01$ & $<0.01$ & 0.01 & 0.01 & $<0.01$ & 0.01 & 0.01 & $\mathrm{NC}$ \\
\hline $\mathrm{ReO}_{2}$ & 0.10 & 0.03 & 0.03 & 0.03 & 0.04 & 0.03 & 0.03 & 0.03 & 0.03 & 0.03 & 0.03 & 0.02 & 0.03 & 0.03 & 0.03 & $\mathrm{NC}$ \\
\hline $\mathrm{SiO}_{2}$ & 44.04 & 45.30 & 45.14 & 44.93 & 44.73 & 44.81 & 44.90 & 45.95 & 45.61 & 45.99 & 45.57 & 45.91 & 45.79 & 44.92 & 45.25 & 2.74 \\
\hline $\mathrm{SO}_{3}$ & 0.45 & 0.42 & 0.42 & 0.42 & 0.42 & 0.41 & 0.41 & 0.43 & 0.42 & 0.42 & 0.40 & 0.41 & 0.41 & 0.40 & 0.41 & $\mathrm{NC}$ \\
\hline $\mathrm{TiO}_{2}$ & 1.34 & 1.48 & 1.50 & 1.53 & 1.47 & 1.52 & 1.52 & 1.44 & 1.44 & 1.41 & 1.46 & 1.39 & 1.48 & 1.49 & 1.44 & 7.44 \\
\hline $\mathrm{V}_{2} \mathrm{O}_{5}$ & $\S$ & $<0.01$ & $<0.01$ & 0.01 & 0.01 & 0.01 & 0.01 & 0.01 & 0.01 & 0.01 & 0.01 & 0.01 & $<0.01$ & 0.01 & $<0.01$ & $\mathrm{NC}$ \\
\hline $\mathrm{ZnO}$ & 3.35 & 3.08 & 3.14 & 3.21 & 3.03 & 3.11 & 3.12 & 2.88 & 2.90 & 2.74 & 2.93 & 2.77 & 2.99 & 2.96 & 2.90 & -13.59 \\
\hline $\mathrm{ZrO}_{2}$ & 2.87 & 3.04 & 3.10 & 3.18 & 3.04 & 3.09 & 3.04 & 2.82 & 2.84 & 2.66 & 2.86 & 2.69 & 2.91 & 2.92 & 2.85 & -0.72 \\
\hline Sum & 100.00 & 100.00 & 100.00 & 100.00 & 100.00 & 100.00 & 100.00 & 100.00 & 100.00 & 100.00 & 100.00 & 100.00 & 100.00 & 100.00 & 100.00 & $\mathrm{NC}$ \\
\hline
\end{tabular}

* - Target values calculated based on simple well-stirred tank model

\# - Fluorine values measured directly by XRF given in bold, other values interpolated between measured values.

$\mathrm{NC}$ - Not calculated

$\S$ - Not a target component 
Table 4.2. XRF Analyzed Compositions for Glass Samples Discharged During DM100 Tests (wt\%) (continued).

\begin{tabular}{|c|c|c|c|c|c|c|c|c|c|c|c|c|c|c|c|c|c|}
\hline Test & \multicolumn{17}{|c|}{ 5D } \\
\hline \multicolumn{2}{|c|}{ Glass (kg) } & 5624.49 & 5645.17 & 5664.99 & 5690.91 & 5712.21 & 5732.83 & 5754.41 & 5773.87 & 5796.19 & 5819.89 & 5844.10 & 5868.42 & 5888.02 & 5911.54 & 5933.06 & 5952.28 \\
\hline Constituent & Target & $\begin{array}{l}\text { CWV- } \\
\text { G-97A }\end{array}$ & $\begin{array}{l}\mathrm{CWV}- \\
\mathrm{G}-97 \mathrm{C}\end{array}$ & $\begin{array}{l}\text { CWV- } \\
\text { G-98B }\end{array}$ & $\begin{array}{r}\mathrm{CWV}- \\
\mathrm{G}-100 \mathrm{~A}\end{array}$ & $\begin{array}{r}\text { CWV- } \\
\text { G-101A }\end{array}$ & $\begin{array}{r}\text { CWV- } \\
\text { G-105A }\end{array}$ & $\begin{array}{r}\text { CWV- } \\
\text { G-105C }\end{array}$ & $\begin{array}{r}\text { CWV- } \\
\mathrm{G}-107 \mathrm{~B}\end{array}$ & $\begin{array}{r}\mathrm{CWV}- \\
\mathrm{G}-107 \mathrm{D}\end{array}$ & $\begin{array}{r}\text { CWV- } \\
G-109 B\end{array}$ & $\begin{array}{r}\mathrm{CWV}- \\
\mathrm{G}-112 \mathrm{~A}\end{array}$ & $\begin{array}{r}\mathrm{CWV}- \\
\mathrm{G}-112 \mathrm{C}\end{array}$ & $\begin{array}{r}\text { CWV } \\
G-114 B\end{array}$ & $\begin{array}{r}\mathrm{CWV} \\
\mathrm{G}-114 \mathrm{D}\end{array}$ & \begin{tabular}{|r|}
$\mathrm{CWV}-$ \\
$\mathrm{G}-118 \mathrm{~A}$ \\
\end{tabular} & $\begin{array}{r}\text { CWV- } \\
\text { G-118C }\end{array}$ \\
\hline $\mathrm{Al}_{2} \mathrm{O}_{3}$ & 5.81 & 5.76 & 5.95 & 5.86 & 5.87 & 5.87 & 5.99 & 5.91 & 5.97 & 5.93 & 5.75 & 5.88 & 5.79 & 5.89 & 5.95 & 5.95 & 5.74 \\
\hline $\mathrm{B}_{2} \mathrm{O}_{3} *$ & 9.52 & 9.58 & 9.57 & 9.56 & 9.56 & 9.55 & 9.55 & 9.54 & 9.54 & 9.54 & 9.53 & 9.53 & 9.53 & 9.53 & 9.53 & 9.53 & 9.53 \\
\hline $\mathrm{CaO}$ & 2.39 & 3.42 & 3.07 & 2.99 & 2.92 & 2.89 & 2.66 & 2.71 & 2.66 & 2.59 & 2.63 & 2.46 & 2.46 & 2.44 & 2.41 & 2.45 & 2.22 \\
\hline $\mathrm{CdO}$ & $\S$ & $<0.01$ & $<0.01$ & $<0.01$ & $<0.01$ & $<0.01$ & $<0.01$ & $<0.01$ & $<0.01$ & $<0.01$ & $<0.01$ & $<0.01$ & $<0.01$ & $<0.01$ & $<0.01$ & $<0.01$ & $<0.01$ \\
\hline $\mathrm{Cl}$ & 1.10 & 0.65 & 0.63 & 0.65 & 0.65 & 0.66 & 0.64 & 0.67 & 0.66 & 0.65 & 0.65 & 0.66 & 0.64 & 0.66 & 0.65 & 0.66 & 0.60 \\
\hline $\mathrm{Cr}_{2} \mathrm{O}_{3}$ & 0.08 & 0.21 & 0.19 & 0.19 & 0.19 & 0.20 & 0.19 & 0.21 & 0.21 & 0.21 & 0.21 & 0.21 & 0.21 & 0.21 & 0.21 & 0.21 & 0.20 \\
\hline $\mathrm{F}^{\#}$ & 1.30 & 0.95 & 0.96 & 0.97 & 0.98 & 0.99 & 0.99 & 1.00 & 1.01 & 1.01 & 1.00 & 1.00 & 1.00 & 0.99 & 0.99 & 0.99 & 0.99 \\
\hline $\mathrm{Fe}_{2} \mathrm{O}_{3}$ & 5.23 & 5.36 & 4.78 & 4.86 & 4.84 & 5.04 & 4.80 & 5.06 & 5.23 & 5.06 & 5.28 & 4.88 & 5.05 & 5.04 & 4.90 & 5.22 & 4.75 \\
\hline $\mathrm{K}_{2} \mathrm{O}$ & 0.52 & 0.58 & 0.57 & 0.56 & 0.57 & 0.58 & 0.56 & 0.56 & 0.55 & 0.54 & 0.55 & 0.56 & 0.56 & 0.57 & 0.56 & 0.57 & 0.51 \\
\hline $\mathrm{Li}_{2} \mathrm{O}^{*}$ & $\S$ & 0.42 & 0.35 & 0.30 & 0.24 & 0.20 & 0.17 & 0.14 & 0.12 & 0.10 & 0.08 & 0.07 & 0.05 & 0.05 & 0.04 & 0.03 & 0.03 \\
\hline $\mathrm{MgO}$ & 1.41 & 1.45 & 1.47 & 1.49 & 1.53 & 1.46 & 1.60 & 1.49 & 1.50 & 1.57 & 1.51 & 1.46 & 1.46 & 1.50 & 1.50 & 1.49 & 1.57 \\
\hline $\mathrm{Na}_{2} \mathrm{O}$ & 20.68 & 18.29 & 19.85 & 19.39 & 19.65 & 19.31 & 20.33 & 19.59 & 19.80 & 20.48 & 20.00 & 20.24 & 20.28 & 20.14 & 20.13 & 19.77 & 23.47 \\
\hline $\mathrm{NiO}$ & 0.01 & 0.01 & $<0.01$ & $<0.01$ & $<0.01$ & 0.01 & 0.01 & 0.01 & 0.01 & 0.01 & 0.01 & 0.01 & 0.01 & 0.01 & 0.01 & 0.01 & 0.01 \\
\hline $\mathrm{P}_{2} \mathrm{O}_{5}$ & 0.12 & 0.17 & 0.17 & 0.17 & 0.17 & 0.18 & 0.17 & 0.17 & 0.17 & 0.17 & 0.17 & 0.17 & 0.16 & 0.16 & 0.18 & 0.16 & 0.15 \\
\hline $\mathrm{PbO}$ & 0.01 & 0.01 & 0.01 & 0.01 & $<0.01$ & 0.01 & $<0.01$ & 0.01 & 0.01 & 0.01 & $<0.01$ & 0.01 & 0.01 & 0.01 & 0.01 & 0.01 & 0.01 \\
\hline $\mathrm{ReO}_{2}$ & 0.10 & 0.04 & 0.03 & 0.03 & 0.03 & 0.03 & 0.03 & 0.02 & 0.04 & 0.04 & 0.04 & 0.03 & 0.04 & 0.03 & 0.04 & 0.03 & 0.03 \\
\hline $\mathrm{SiO}_{2}$ & 43.80 & 44.97 & 45.30 & 45.74 & 45.61 & 45.52 & 45.23 & 45.38 & 44.74 & 44.63 & 44.76 & 45.64 & 45.30 & 45.32 & 45.69 & 45.16 & 43.29 \\
\hline $\mathrm{SO}_{3}$ & 0.40 & 0.38 & 0.38 & 0.39 & 0.39 & 0.39 & 0.37 & 0.39 & 0.37 & 0.36 & 0.36 & 0.36 & 0.34 & 0.34 & 0.36 & 0.35 & 0.33 \\
\hline $\mathrm{TiO}_{2}$ & 1.33 & 1.53 & 1.36 & 1.38 & 1.37 & 1.42 & 1.34 & 1.41 & 1.43 & 1.40 & 1.44 & 1.33 & 1.37 & 1.37 & 1.37 & 1.41 & 1.28 \\
\hline $\mathrm{V}_{2} \mathrm{O}_{5}$ & $\S$ & 0.01 & 0.01 & 0.01 & $<0.01$ & $<0.01$ & 0.01 & 0.01 & 0.01 & $<0.01$ & $<0.01$ & $<0.01$ & $<0.01$ & $<0.01$ & $<0.01$ & $<0.01$ & 0.01 \\
\hline $\mathrm{ZnO}$ & 3.34 & 3.15 & 2.72 & 2.77 & 2.76 & 2.89 & 2.72 & 2.90 & 3.00 & 2.88 & 3.02 & 2.79 & 2.89 & 2.87 & 2.76 & 3.00 & 2.68 \\
\hline $\mathrm{ZrO}_{2}$ & 2.86 & 3.07 & 2.65 & 2.70 & 2.68 & 2.82 & 2.65 & 2.82 & 2.99 & 2.84 & 3.01 & 2.71 & 2.87 & 2.85 & 2.72 & 2.99 & 2.62 \\
\hline Sum & 100.00 & 100.00 & 100.00 & 100.00 & 100.00 & 100.00 & 100.00 & 100.00 & 100.00 & 100.00 & 100.00 & 100.00 & 100.00 & 100.00 & 100.00 & 100.00 & 100.00 \\
\hline
\end{tabular}

* - Target values calculated based on simple well-stirred tank model

\# - Fluorine values measured directly by XRF given in bold, other values interpolated between measured values.

$\mathrm{NC}$ - Not calculated

$\S$ - Not a target component 
Table 4.2. XRF Analyzed Compositions for Glass Samples Discharged During DM100 Tests (wt\%) (continued).

\begin{tabular}{|c|c|c|c|c|c|c|c|c|c|c|c|c|c|c|c|c|c|}
\hline Test & \multicolumn{7}{|c|}{ 5D } & \multicolumn{10}{|c|}{ 6A } \\
\hline \multicolumn{2}{|c|}{ Glass (kg) } & \multicolumn{6}{|c|}{\begin{tabular}{|l|l|l|l|l|l|}
5973.10 & 5993.44 & 6017.20 & 6036.24 & 6047.48 & 6047.48 \\
\end{tabular}} & \multirow[b]{2}{*}{ Target } & \multicolumn{2}{|c|}{\begin{tabular}{|c|c|}
6068.10 & 6096.30 \\
\end{tabular}} & \multirow{2}{*}{\begin{tabular}{|r|}
6123.46 \\
$\mathrm{CWV}-$ \\
$\mathrm{G}-147 \mathrm{C}$
\end{tabular}} & \multirow{2}{*}{\begin{tabular}{|r|}
6145.34 \\
$\mathrm{CWV}-$ \\
$\mathrm{G}-149 \mathrm{~B}$
\end{tabular}} & \multirow{2}{*}{\begin{tabular}{|r|}
6168.64 \\
CWV- \\
G-149D \\
\end{tabular}} & \multirow{2}{*}{\begin{tabular}{|r|}
6193.24 \\
$\mathrm{CWV}-$ \\
$\mathrm{G}-152 \mathrm{~B}$
\end{tabular}} & \multirow{2}{*}{\multicolumn{2}{|c|}{\begin{tabular}{|r|r|}
6217.98 & 6242.88 \\
CWV- & CWV- \\
G-153A & G-153C \\
\end{tabular}}} & \multirow{2}{*}{\begin{tabular}{|r|}
6266.22 \\
$\mathrm{CWV}-$ \\
$\mathrm{G}-155 \mathrm{~B}$
\end{tabular}} \\
\hline Constituent & Target & $\begin{array}{r}\text { CWV- } \\
\text { G-119B }\end{array}$ & $\begin{array}{r}\text { CWV- } \\
\text { G-121A }\end{array}$ & $\begin{array}{r}\text { CWV- } \\
\text { G-121C }\end{array}$ & $\begin{array}{r}\text { CWV- } \\
\text { G-125A }\end{array}$ & $\begin{array}{r}\text { CWV- } \\
\text { G-125B }\end{array}$ & $\%$ Dev. & & \begin{tabular}{|r|} 
CWV- \\
G-145B
\end{tabular} & $\begin{array}{r}\text { CWV- } \\
\text { G-147A }\end{array}$ & & & & & & & \\
\hline $\mathrm{Al}_{2} \mathrm{O}_{3}$ & 5.81 & 6.28 & 6.07 & 5.85 & 5.85 & 5.81 & 0.08 & 5.84 & 6.45 & 6.22 & 6.13 & 6.04 & 6.35 & 6.19 & 5.88 & 5.90 & 5.88 \\
\hline $\mathrm{B}_{2} \mathrm{O}_{3} *$ & 9.52 & 9.53 & 9.53 & 9.53 & 9.52 & 9.52 & $\mathrm{NC}$ & 9.57 & 9.53 & 9.54 & 9.55 & 9.55 & 9.56 & 9.56 & 9.56 & 9.56 & 9.57 \\
\hline $\mathrm{CaO}$ & 2.39 & 2.41 & 2.42 & 2.31 & 2.38 & 2.30 & -3.66 & 2.41 & 2.48 & 2.52 & 2.51 & 2.49 & 2.44 & 2.49 & 2.36 & 2.33 & 2.36 \\
\hline $\mathrm{CdO}$ & $\S$ & $<0.01$ & $<0.01$ & $<0.01$ & $<0.01$ & $<0.01$ & NC & $\S$ & $<0.01$ & $<0.01$ & $<0.01$ & $<0.01$ & $<0.01$ & $<0.01$ & $<0.01$ & $<0.01$ & $<0.01$ \\
\hline $\mathrm{Cl}$ & 1.10 & 0.65 & 0.65 & 0.63 & 0.65 & 0.67 & NC & 1.10 & 0.15 & 0.26 & 0.39 & 0.44 & 0.47 & 0.52 & 0.56 & 0.57 & 0.58 \\
\hline $\mathrm{Cr}_{2} \mathrm{O}_{3}$ & 0.08 & 0.22 & 0.22 & 0.20 & 0.22 & 0.21 & NC & 0.08 & 0.30 & 0.30 & 0.28 & 0.28 & 0.27 & 0.28 & 0.25 & 0.23 & 0.24 \\
\hline $\mathrm{F}^{\#}$ & 1.30 & 0.99 & 0.98 & 0.98 & 0.98 & 0.98 & $\mathrm{NC}$ & 1.30 & 0.98 & 0.98 & 0.98 & 0.97 & 0.97 & 0.97 & 0.97 & 0.97 & 0.97 \\
\hline $\mathrm{Fe}_{2} \mathrm{O}_{3}$ & 5.23 & 5.21 & 5.31 & 5.10 & 5.31 & 4.97 & -4.93 & 5.26 & 5.25 & 5.47 & 5.45 & 5.48 & 5.38 & 5.47 & 5.21 & 5.06 & 5.21 \\
\hline $\mathrm{K}_{2} \mathrm{O}$ & 0.52 & 0.55 & 0.56 & 0.52 & 0.58 & 0.55 & NC & $\S$ & 0.50 & 0.44 & 0.38 & 0.33 & 0.33 & 0.33 & 0.28 & 0.24 & 0.22 \\
\hline $\mathrm{Li}_{2} \mathrm{O}^{*}$ & $\S$ & 0.02 & 0.02 & 0.02 & 0.01 & 0.01 & NC & $\S$ & 0.01 & 0.01 & 0.01 & 0.01 & $<0.01$ & $<0.01$ & $<0.01$ & $<0.01$ & $<0.01$ \\
\hline $\mathrm{MgO}$ & 1.41 & 1.55 & 1.54 & 1.59 & 1.56 & 1.57 & 11.56 & 1.42 & 1.55 & 1.53 & 1.60 & 1.57 & 1.53 & 1.48 & 1.52 & 1.59 & 1.56 \\
\hline $\mathrm{Na}_{2} \mathrm{O}$ & 20.68 & 19.91 & 19.98 & 21.88 & 19.99 & 20.58 & -0.52 & 20.80 & 19.81 & 19.88 & 19.89 & 19.93 & 20.10 & 19.87 & 20.50 & 20.97 & 20.70 \\
\hline $\mathrm{NiO}$ & 0.01 & 0.01 & 0.01 & 0.01 & 0.01 & 0.01 & NC & 0.01 & 0.05 & 0.04 & 0.04 & 0.03 & 0.03 & 0.03 & 0.02 & 0.02 & 0.02 \\
\hline $\mathrm{P}_{2} \mathrm{O}_{5}$ & 0.12 & 0.15 & 0.16 & 0.16 & 0.16 & 0.17 & NC & 0.12 & 0.18 & 0.16 & 0.16 & 0.17 & 0.17 & 0.17 & 0.18 & 0.17 & 0.16 \\
\hline $\mathrm{PbO}$ & 0.01 & 0.01 & $<0.01$ & 0.01 & 0.01 & $<0.01$ & NC & 0.01 & 0.01 & 0.01 & 0.01 & $<0.01$ & 0.01 & 0.01 & 0.01 & 0.01 & $<0.01$ \\
\hline $\mathrm{ReO}_{2}$ & 0.10 & 0.03 & 0.03 & 0.03 & 0.04 & 0.03 & NC & 0.10 & 0.01 & 0.02 & 0.02 & 0.03 & 0.02 & 0.03 & 0.03 & 0.03 & 0.03 \\
\hline $\mathrm{SiO}_{2}$ & 43.80 & 44.73 & 44.61 & 43.88 & 44.95 & 45.37 & 3.60 & 44.03 & 44.99 & 44.57 & 44.63 & 44.51 & 44.43 & 44.63 & 45.08 & 44.82 & 44.74 \\
\hline $\mathrm{SO}_{3}$ & 0.40 & 0.33 & 0.34 & 0.33 & 0.33 & 0.34 & NC & 0.40 & 0.33 & 0.35 & 0.34 & 0.35 & 0.34 & 0.35 & 0.34 & 0.35 & 0.35 \\
\hline $\mathrm{TiO}_{2}$ & 1.33 & 1.42 & 1.45 & 1.37 & 1.42 & 1.35 & 1.42 & 1.34 & 1.44 & 1.49 & 1.49 & 1.48 & 1.47 & 1.51 & 1.42 & 1.39 & 1.44 \\
\hline $\mathrm{V}_{2} \mathrm{O}_{5}$ & $\S$ & $<0.01$ & $<0.01$ & 0.01 & 0.01 & $<0.01$ & NC & $\S$ & 0.01 & 0.01 & 0.01 & $<0.01$ & $<0.01$ & 0.01 & $<0.01$ & 0.01 & $<0.01$ \\
\hline $\mathrm{ZnO}$ & 3.34 & 2.98 & 3.05 & 2.90 & 3.07 & 2.82 & -15.38 & 3.35 & 2.99 & 3.15 & 3.14 & 3.17 & 3.11 & 3.18 & 2.97 & 2.89 & 3.01 \\
\hline $\mathrm{ZrO}_{2}$ & 2.86 & 3.01 & 3.07 & 2.69 & 2.94 & 2.73 & -4.55 & 2.87 & 2.98 & 3.05 & 3.00 & 3.17 & 3.03 & 2.93 & 2.85 & 2.86 & 2.97 \\
\hline Sum & 100.00 & 100.00 & 100.00 & 100.00 & 100.00 & 100.00 & NC & 100.00 & 100.00 & 100.00 & 100.00 & 100.00 & 100.00 & 100.00 & 100.00 & 100.00 & 100.00 \\
\hline
\end{tabular}

* - Target values calculated based on simple well-stirred tank model

\# - Fluorine values measured directly by XRF given in bold, other values interpolated between measured values.

$\mathrm{NC}$ - Not calculated

$\S$ - Not a target component 
Table 4.2. XRF Analyzed Compositions for Glass Samples Discharged During DM100 Tests (wt\%) (continued).

\begin{tabular}{|c|c|c|c|c|c|c|c|c|c|c|c|c|c|c|c|c|c|}
\hline Test & \multicolumn{13}{|c|}{ 6A } & \multicolumn{4}{|c|}{ 6B } \\
\hline \multicolumn{2}{|c|}{ Glass (kg) } & 6287.98 & 6307.60 & 6332.26 & 6352.62 & 6375.68 & 6401.46 & 6419.48 & 6441.70 & 6462.64 & 6480.78 & 6492.46 & 6492.46 & \multicolumn{2}{|c|}{6512.72} & 6536.72 & 6566.34 \\
\hline Constituent & Target & $\begin{array}{l}\text { DWV- } \\
\text { G-6A }\end{array}$ & \begin{tabular}{|c|} 
DWV- \\
G-6C
\end{tabular} & $\begin{array}{l}\text { DWV- } \\
\text { G-10B }\end{array}$ & \begin{tabular}{|l|} 
DWV- \\
G-10D
\end{tabular} & \begin{tabular}{|l|} 
DWV- \\
G-11B
\end{tabular} & $\begin{array}{l}\text { DWV- } \\
\text { G-11D }\end{array}$ & \begin{tabular}{|l|} 
DWV- \\
G-15B
\end{tabular} & $\begin{array}{l}\text { DWV- } \\
\text { G-17A }\end{array}$ & \begin{tabular}{|l|} 
DWV- \\
G-17C
\end{tabular} & $\begin{array}{l}\text { DWV- } \\
\text { G-19B }\end{array}$ & \begin{tabular}{|l|} 
DWV- \\
G-19C
\end{tabular} & \%Dev. & Target & \begin{tabular}{|l|} 
DWV- \\
G-23B
\end{tabular} & $\begin{array}{l}\text { DWV- } \\
\text { G-25B }\end{array}$ & $\begin{array}{l}\text { DWV- } \\
\text { G-25D }\end{array}$ \\
\hline $\mathrm{Al}_{2} \mathrm{O}_{3}$ & 5.84 & 5.80 & 6.02 & 6.05 & 5.89 & 5.94 & 5.75 & 5.93 & 6.13 & 5.93 & 5.97 & 6.21 & 6.38 & 5.96 & 5.95 & 6.02 & 5.94 \\
\hline $\mathrm{B}_{2} \mathrm{O}_{3} *$ & 9.57 & 9.57 & 9.57 & 9.57 & 9.57 & 9.57 & 9.57 & 9.57 & 9.57 & 9.57 & 9.57 & 9.57 & NC & 9.78 & 9.60 & 9.64 & 9.67 \\
\hline $\mathrm{CaO}$ & 2.41 & 2.38 & 2.40 & 2.32 & 2.30 & 2.30 & 2.34 & 2.29 & 2.41 & 2.35 & 2.36 & 2.35 & -2.47 & 1.98 & 2.32 & 2.20 & 2.25 \\
\hline $\mathrm{CdO}$ & $\S$ & $<0.01$ & $<0.01$ & $<0.01$ & $<0.01$ & $<0.01$ & $<0.01$ & $<0.01$ & $<0.01$ & $<0.01$ & $<0.01$ & $<0.01$ & $\mathrm{NC}$ & $\S$ & $<0.01$ & $<0.01$ & $<0.01$ \\
\hline $\mathrm{Cl}$ & 1.10 & 0.61 & 0.63 & 0.62 & 0.63 & 0.63 & 0.62 & 0.64 & 0.64 & 0.65 & 0.64 & 0.65 & $\mathrm{NC}$ & 1.10 & 0.64 & 0.65 & 0.65 \\
\hline $\mathrm{Cr}_{2} \mathrm{O}_{3}$ & 0.08 & 0.24 & 0.24 & 0.22 & 0.23 & 0.22 & 0.23 & 0.22 & 0.23 & 0.23 & 0.22 & 0.22 & $\mathrm{NC}$ & 0.08 & 0.22 & 0.21 & 0.21 \\
\hline $\mathrm{F}^{\#}$ & 1.30 & 0.97 & 0.97 & 0.96 & 0.96 & 0.96 & 0.96 & 0.97 & 0.97 & 0.97 & 0.97 & 0.97 & $\mathrm{NC}$ & 1.30 & 0.98 & 0.98 & 0.99 \\
\hline $\mathrm{Fe}_{2} \mathrm{O}_{3}$ & 5.26 & 5.27 & 5.29 & 5.10 & 5.03 & 4.98 & 5.26 & 5.07 & 5.42 & 5.26 & 5.27 & 5.23 & -0.65 & 5.38 & 5.15 & 4.97 & 5.18 \\
\hline $\mathrm{K}_{2} \mathrm{O}$ & $\S$ & 0.21 & 0.18 & 0.17 & 0.18 & 0.16 & 0.15 & 0.16 & 0.15 & 0.13 & 0.14 & 0.13 & $\mathrm{NC}$ & 4.87 & 0.56 & 1.12 & 1.44 \\
\hline $\mathrm{Li}_{2} \mathrm{O}^{*}$ & $\S$ & $<0.01$ & $<0.01$ & $<0.01$ & $<0.01$ & $<0.01$ & $<0.01$ & $<0.01$ & $<0.01$ & $<0.01$ & $<0.01$ & $<0.01$ & $\mathrm{NC}$ & $\S$ & $<0.01$ & $<0.01$ & $<0.01$ \\
\hline $\mathrm{MgO}$ & 1.42 & 1.52 & 1.50 & 1.54 & 1.62 & 1.54 & 1.51 & 1.49 & 1.47 & 1.50 & 1.46 & 1.53 & 7.65 & 1.45 & 1.42 & 1.57 & 1.49 \\
\hline $\mathrm{Na}_{2} \mathrm{O}$ & 20.80 & 20.78 & 20.39 & 21.05 & 20.91 & 20.75 & 21.17 & 20.70 & 19.92 & 20.58 & 20.43 & 20.00 & -3.82 & 17.81 & 20.40 & 19.92 & 19.92 \\
\hline $\mathrm{NiO}$ & 0.01 & 0.01 & 0.01 & 0.01 & 0.01 & 0.01 & 0.01 & 0.01 & 0.01 & 0.01 & 0.01 & 0.01 & $\mathrm{NC}$ & 0.01 & 0.01 & 0.01 & 0.01 \\
\hline $\mathrm{P}_{2} \mathrm{O}_{5}$ & 0.12 & 0.17 & 0.17 & 0.17 & 0.16 & 0.17 & 0.17 & 0.17 & 0.17 & 0.15 & 0.17 & 0.18 & $\mathrm{NC}$ & 0.12 & 0.17 & 0.18 & 0.18 \\
\hline $\mathrm{PbO}$ & 0.01 & 0.01 & $<0.01$ & 0.01 & $<0.01$ & $<0.01$ & 0.01 & $<0.01$ & 0.01 & 0.01 & $<0.01$ & 0.01 & $\mathrm{NC}$ & 0.01 & $<0.01$ & 0.01 & 0.01 \\
\hline $\mathrm{ReO}_{2}$ & 0.10 & 0.04 & 0.03 & 0.03 & 0.03 & 0.03 & 0.04 & 0.04 & 0.04 & 0.04 & 0.04 & 0.04 & $\mathrm{NC}$ & 0.10 & 0.03 & 0.03 & 0.02 \\
\hline $\mathrm{SiO}_{2}$ & 44.03 & 44.61 & 44.77 & 44.68 & 45.07 & 45.39 & 44.46 & 45.27 & 44.86 & 44.76 & 44.92 & 45.27 & 2.81 & 42.00 & 44.88 & 45.26 & 44.56 \\
\hline $\mathrm{SO}_{3}$ & 0.40 & 0.35 & 0.35 & 0.35 & 0.35 & 0.36 & 0.34 & 0.35 & 0.33 & 0.35 & 0.35 & 0.34 & $\mathrm{NC}$ & 0.35 & 0.33 & 0.33 & 0.33 \\
\hline $\mathrm{TiO}_{2}$ & 1.34 & 1.43 & 1.46 & 1.42 & 1.38 & 1.37 & 1.43 & 1.39 & 1.48 & 1.45 & 1.45 & 1.43 & 6.75 & 1.37 & 1.45 & 1.39 & 1.44 \\
\hline $\mathrm{V}_{2} \mathrm{O}_{5}$ & $\S$ & 0.01 & $<0.01$ & 0.01 & $<0.01$ & $<0.01$ & $<0.01$ & $<0.01$ & $<0.01$ & 0.01 & 0.01 & 0.01 & NC & $\S$ & 0.01 & 0.01 & 0.01 \\
\hline $\mathrm{ZnO}$ & 3.35 & 3.03 & 3.06 & 2.91 & 2.90 & 2.84 & 3.02 & 2.90 & 3.11 & 3.04 & 3.05 & 3.01 & -10.16 & 3.42 & 2.99 & 2.83 & 2.96 \\
\hline $\mathrm{ZrO}_{2}$ & 2.87 & 3.00 & 2.94 & 2.80 & 2.78 & 2.77 & 2.94 & 2.84 & 3.07 & 2.99 & 2.97 & 2.84 & -1.06 & 2.93 & 2.90 & 2.69 & 2.73 \\
\hline Sum & 100.00 & 100.00 & 100.00 & 100.00 & 100.00 & 100.00 & 100.00 & 100.00 & 100.00 & 100.00 & 100.00 & 100.00 & $\mathrm{NC}$ & 100.00 & 100.00 & 100.00 & 100.00 \\
\hline
\end{tabular}

* - Target values calculated based on simple well-stirred tank model

\# - Fluorine values measured directly by XRF given in bold, other values interpolated between measured values.

$\mathrm{NC}$ - Not calculated

$\S$ - Not a target component 
Table 4.2. XRF Analyzed Compositions for Glass Samples Discharged During DM100 Tests (wt\%) (continued).

\begin{tabular}{|c|c|c|c|c|c|c|c|c|c|c|c|c|c|c|c|c|c|c|}
\hline Test & \multicolumn{18}{|c|}{ 6B } \\
\hline \multicolumn{2}{|c|}{ Glass (kg) } & 6590.82 & 6616.08 & 6637.32 & 6658.56 & 6677.40 & 6699.28 & 6725.60 & 6750.84 & 6774.72 & 6798.02 & 6828.34 & 6849.15 & 6874.83 & 6899.89 & 6926.81 & 6937.61 & 6937.61 \\
\hline Constituent & Target & $\begin{array}{l}\text { DWV- } \\
\text { G-29B }\end{array}$ & $\begin{array}{l}\text { DWV- } \\
\text { G-29D }\end{array}$ & $\begin{array}{l}\text { DWV- } \\
\text { G-32B }\end{array}$ & $\begin{array}{l}\text { DWV- } \\
\text { G-33A }\end{array}$ & $\begin{array}{l}\text { DWV- } \\
\text { G-34A }\end{array}$ & \begin{tabular}{|l|} 
DWV- \\
G-34C
\end{tabular} & $\begin{array}{l}\text { DWV- } \\
\text { G-38B }\end{array}$ & $\begin{array}{l}\text { DWV- } \\
\text { G-39B }\end{array}$ & $\begin{array}{l}\text { DWV- } \\
\text { G-43B }\end{array}$ & \begin{tabular}{|l|} 
DWV- \\
G-43D
\end{tabular} & $\begin{array}{l}\text { DWV- } \\
\text { G-45B }\end{array}$ & \begin{tabular}{|l|} 
DWV- \\
G-46A
\end{tabular} & \begin{tabular}{|l|} 
DWV- \\
G-46C
\end{tabular} & $\begin{array}{l}\text { DWV- } \\
\text { G-48B }\end{array}$ & \begin{tabular}{|l|} 
DWV- \\
G-51A
\end{tabular} & $\begin{array}{l}\text { DWV- } \\
\text { G-51B }\end{array}$ & \%Dev \\
\hline $\mathrm{Al}_{2} \mathrm{O}_{3}$ & 5.96 & 5.97 & 5.90 & 6.11 & 6.12 & 6.03 & 6.11 & 6.06 & 6.02 & 6.07 & 6.04 & 6.13 & 6.53 & 6.15 & 6.17 & 5.94 & 6.08 & 1.96 \\
\hline $\mathrm{B}_{2} \mathrm{O}_{3} *$ & 9.78 & 9.69 & 9.71 & 9.72 & 9.73 & 9.74 & 9.74 & 9.75 & 9.76 & 9.76 & 9.76 & 9.77 & 9.77 & 9.77 & 9.77 & 9.77 & 9.77 & $\mathrm{NC}$ \\
\hline $\mathrm{CaO}$ & 1.98 & 2.12 & 2.21 & 2.22 & 2.17 & 2.18 & 2.09 & 2.08 & 2.08 & 2.04 & 2.06 & 2.01 & 2.07 & 2.08 & 1.96 & 2.02 & 2.05 & 3.91 \\
\hline $\mathrm{CdO}$ & $\S$ & $<0.01$ & $<0.01$ & $<0.01$ & $<0.01$ & $<0.01$ & $<0.01$ & $<0.01$ & $<0.01$ & $<0.01$ & $<0.01$ & $<0.01$ & $<0.01$ & $<0.01$ & $<0.01$ & $<0.01$ & $<0.01$ & $\mathrm{NC}$ \\
\hline $\mathrm{Cl}$ & 1.10 & 0.65 & 0.68 & 0.68 & 0.66 & 0.67 & 0.68 & 0.67 & 0.67 & 0.67 & 0.66 & 0.67 & 0.68 & 0.68 & 0.66 & 0.69 & 0.68 & $\mathrm{NC}$ \\
\hline $\mathrm{Cr}_{2} \mathrm{O}_{3}$ & 0.08 & 0.21 & 0.22 & 0.22 & 0.21 & 0.24 & 0.21 & 0.20 & 0.21 & 0.20 & 0.21 & 0.20 & 0.21 & 0.21 & 0.20 & 0.21 & 0.21 & $\mathrm{NC}$ \\
\hline $\mathrm{F}^{\#}$ & 1.30 & 0.99 & 1.00 & 1.00 & 0.99 & 0.99 & 0.99 & 0.98 & 0.98 & 0.98 & 0.98 & 0.98 & 0.98 & 0.98 & 0.98 & 0.98 & 0.98 & $\mathrm{NC}$ \\
\hline $\mathrm{Fe}_{2} \mathrm{O}_{3}$ & 5.38 & 4.94 & 5.33 & 5.54 & 5.38 & 5.46 & 5.17 & 5.16 & 5.30 & 5.18 & 5.23 & 5.09 & 5.42 & 5.48 & 5.08 & 5.30 & 5.39 & 0.28 \\
\hline $\mathrm{K}_{2} \mathrm{O}$ & 4.87 & 2.02 & 2.50 & 2.96 & 3.18 & 3.38 & 3.56 & 3.74 & 3.90 & 4.11 & 4.16 & 4.23 & 4.39 & 4.50 & 4.39 & 4.56 & 4.62 & -5.18 \\
\hline $\mathrm{Li}_{2} \mathrm{O}^{*}$ & $\S$ & $<0.01$ & $<0.01$ & $<0.01$ & $<0.01$ & $<0.01$ & $<0.01$ & $<0.01$ & $<0.01$ & $<0.01$ & $<0.01$ & $<0.01$ & $<0.01$ & $<0.01$ & $<0.01$ & $<0.01$ & $<0.01$ & $\mathrm{NC}$ \\
\hline $\mathrm{MgO}$ & 1.45 & 1.45 & 1.41 & 1.42 & 1.52 & 1.54 & 1.51 & 1.59 & 1.56 & 1.40 & 1.45 & 1.43 & 1.44 & 1.45 & 1.40 & 1.49 & 1.47 & 1.70 \\
\hline $\mathrm{Na}_{2} \mathrm{O}$ & 17.81 & 19.93 & 18.91 & 18.10 & 18.54 & 18.22 & 18.69 & 18.90 & 18.21 & 18.06 & 18.28 & 18.16 & 17.48 & 17.76 & 18.16 & 18.08 & 17.44 & -2.04 \\
\hline $\mathrm{NiO}$ & 0.01 & $<0.01$ & 0.01 & 0.01 & 0.01 & 0.01 & $<0.01$ & 0.01 & $<0.01$ & 0.01 & $<0.01$ & $<0.01$ & 0.01 & 0.01 & $<0.01$ & 0.01 & 0.01 & $\mathrm{NC}$ \\
\hline $\mathrm{P}_{2} \mathrm{O}_{5}$ & 0.12 & 0.17 & 0.17 & 0.17 & 0.17 & 0.17 & 0.18 & 0.18 & 0.18 & 0.17 & 0.18 & 0.18 & 0.18 & 0.16 & 0.18 & 0.19 & 0.18 & $\mathrm{NC}$ \\
\hline $\mathrm{PbO}$ & 0.01 & $<0.01$ & 0.01 & 0.01 & 0.01 & 0.01 & 0.00 & 0.01 & 0.01 & $<0.01$ & $<0.01$ & $<0.01$ & 0.01 & $<0.01$ & .01 & 0.01 & 0.01 & $\mathrm{NC}$ \\
\hline $\mathrm{ReO}_{2}$ & 0.10 & 0.02 & 0.02 & 0.02 & 0.02 & 0.02 & 0.02 & 0.02 & 0.02 & 0.02 & 0.02 & 0.02 & 0.03 & 0.02 & 0.02 & 0.02 & 0.02 & $\mathrm{NC}$ \\
\hline $\mathrm{SiO}_{2}$ & 42.00 & 44.67 & 44.32 & 43.87 & 43.60 & 43.51 & 44.00 & 43.46 & 43.68 & 44.05 & 43.69 & 44.13 & 43.18 & 43.07 & 44.04 & 43.35 & 43.62 & 3.87 \\
\hline $\mathrm{SO}_{3}$ & 0.35 & 0.34 & 0.33 & 0.32 & 0.31 & 0.34 & 0.32 & 0.31 & 0.32 & 0.31 & 0.30 & 0.32 & 0.31 & 0.31 & 0.32 & 0.31 & 0.33 & $\mathrm{NC}$ \\
\hline $\mathrm{TiO}_{2}$ & 1.37 & 1.38 & 1.48 & 1.53 & 1.50 & 1.51 & 1.43 & 1.44 & 1.47 & 1.45 & 1.46 & 1.44 & 1.48 & 1.51 & 1.41 & 1.48 & 1.49 & 9.17 \\
\hline $\mathrm{V}_{2} \mathrm{O}_{5}$ & $\S$ & $<0.01$ & 0.01 & $<0.01$ & 0.01 & 0.01 & 0.01 & $<0.01$ & $<0.01$ & 0.01 & 0.01 & $<0.01$ & 0.01 & $<0.01$ & $<0.01$ & $<0.01$ & $<0.01$ & $\mathrm{NC}$ \\
\hline $\mathrm{ZnO}$ & 3.42 & 2.81 & 3.02 & 3.17 & 3.05 & 3.09 & 2.86 & 2.87 & 2.98 & 2.89 & 2.93 & 2.83 & 3.06 & 3.10 & 2.79 & 2.97 & 3.02 & -11.69 \\
\hline $\mathrm{ZrO}_{2}$ & 2.93 & 2.64 & 2.77 & 2.94 & 2.81 & 2.90 & 2.42 & 2.57 & 2.67 & 2.62 & 2.57 & 2.42 & 2.76 & 2.75 & 2.46 & 2.62 & 2.61 & -11.04 \\
\hline Sum & 100.00 & 100.00 & 100.00 & 100.00 & 100.00 & 100.00 & 100.00 & 100.00 & 100.00 & 100.00 & 100.00 & 100.00 & 100.00 & 100.00 & 100.00 & 100.00 & 100.00 & NC \\
\hline
\end{tabular}

* - Target values calculated based on simple well-stirred tank model

\# - Fluorine values measured directly by XRF given in bold, other values interpolated between measured values.

$\mathrm{NC}$ - Not calculated

$\S$ - Not a target component 
Table 4.3. Comparison of XRF and DCP Analyzed Compositions for Select Glass Samples (wt\%).

\begin{tabular}{|c|c|c|c|c|c|c|c|c|c|c|c|c|c|c|c|}
\hline \multirow{3}{*}{$\begin{array}{c}\text { Test } \\
\text { Constituent }\end{array}$} & \multicolumn{7}{|c|}{$2 \mathrm{C}$} & \multicolumn{5}{|c|}{$2 D$} & \multicolumn{3}{|c|}{ 3B } \\
\hline & \multirow{2}{*}{ Target } & \multicolumn{2}{|c|}{ AWV-G-27C } & \multicolumn{2}{|c|}{ AWV-G-29B } & \multicolumn{2}{|c|}{ AWV-G-45B } & \multirow{2}{*}{ Target } & \multicolumn{2}{|c|}{ AWV-G-60C } & \multicolumn{2}{|c|}{ AWV-G-78A } & \multirow{2}{*}{ Target } & \multicolumn{2}{|c|}{ AWV-G-147A } \\
\hline & & XRF & DCP & XRF & DCP & XRF & DCP & & XRF & DCP & XRF & DCP & & XRF & DCP \\
\hline $\mathrm{Al}_{2} \mathrm{O}_{3}$ & 6.00 & 5.87 & 6.06 & 5.83 & 6.35 & 5.86 & 6.34 & 6.01 & 6.20 & 6.19 & 6.08 & 6.28 & 6.05 & 6.29 & 6.28 \\
\hline $\mathrm{B}_{2} \mathrm{O}_{3} *$ & 9.84 & 9.83 & 9.74 & 9.83 & 9.27 & 9.84 & 9.21 & 9.86 & 9.85 & 9.19 & 9.86 & 9.22 & 9.91 & 9.91 & 9.27 \\
\hline $\mathrm{CaO}$ & 5.45 & 4.56 & 4.65 & 4.56 & 4.86 & 5.01 & 5.34 & 6.30 & 5.49 & 5.80 & 5.76 & 6.23 & 6.85 & 6.34 & 6.85 \\
\hline $\mathrm{Cl}$ & 0.20 & 0.14 & NA & 0.14 & NA & 0.14 & NA & 0.20 & 0.14 & NA & 0.14 & NA & 0.20 & 0.13 & NA \\
\hline $\mathrm{Cr}_{2} \mathrm{O}_{3}$ & 0.08 & 0.20 & 0.19 & 0.20 & 0.19 & 0.20 & 0.20 & 0.08 & 0.20 & 0.19 & 0.19 & 0.19 & 0.08 & 0.18 & 0.17 \\
\hline $\mathrm{F}$ & 0.08 & $<0.08$ & NA & $<0.08$ & NA & $<0.08$ & NA & 0.08 & $<0.08$ & NA & $<0.08$ & NA & 0.08 & $<0.08$ & $\mathrm{NA}$ \\
\hline $\mathrm{Fe}_{2} \mathrm{O}_{3}$ & 5.41 & 5.32 & 4.90 & 5.23 & 4.75 & 5.16 & 4.74 & 5.42 & 5.23 & 4.78 & 5.20 & 4.79 & 5.44 & 5.25 & 4.73 \\
\hline $\mathrm{K}_{2} \mathrm{O}$ & 0.54 & 0.62 & 0.64 & 0.61 & 0.68 & 0.59 & 0.65 & 0.54 & 0.62 & 0.67 & 0.63 & 0.66 & 0.54 & 0.65 & 0.64 \\
\hline $\mathrm{Li}_{2} \mathrm{O}^{*}$ & 2.38 & 1.86 & 1.89 & 1.95 & 1.98 & 2.33 & 2.18 & 3.17 & 2.90 & 2.65 & 3.14 & 2.99 & 4.08 & 4.05 & 3.96 \\
\hline $\mathrm{MgO}$ & 1.46 & 1.37 & 1.48 & 1.43 & 1.51 & 1.54 & 1.56 & 1.49 & 1.50 & 1.57 & 1.58 & 1.62 & 2.36 & 2.30 & 2.36 \\
\hline $\mathrm{Na}_{2} \mathrm{O}$ & 15.81 & 17.53 & 16.03 & 17.94 & 16.63 & 16.96 & 15.59 & 13.52 & 15.49 & 14.09 & 14.52 & 13.18 & 8.92 & 9.81 & 9.42 \\
\hline $\mathrm{NiO}$ & 0.01 & $<0.01$ & 0.03 & $<0.01$ & 0.03 & $<0.01$ & 0.03 & 0.01 & $<0.01$ & 0.03 & $<0.01$ & 0.03 & 0.01 & $<0.01$ & 0.03 \\
\hline $\mathrm{P}_{2} \mathrm{O}_{5}$ & 0.12 & 0.18 & 0.30 & 0.18 & 0.29 & 0.18 & 0.31 & 0.12 & 0.17 & 0.22 & 0.19 & 0.25 & 0.12 & 0.18 & 0.32 \\
\hline $\mathrm{PbO}$ & 0.01 & 0.01 & 0.02 & 0.01 & 0.02 & $<0.01$ & 0.02 & 0.01 & 0.01 & 0.01 & 0.01 & 0.01 & 0.01 & 0.01 & 0.01 \\
\hline $\mathrm{ReO}_{2}$ & 0.10 & 0.03 & 0.05 & 0.03 & 0.05 & 0.02 & 0.05 & 0.10 & 0.03 & 0.05 & 0.02 & 0.04 & 0.10 & 0.04 & 0.05 \\
\hline $\mathrm{SiO}_{2}$ & 44.21 & 44.23 & 42.35 & 44.15 & 41.66 & 44.31 & 41.51 & 44.71 & 44.29 & 41.73 & 44.81 & 41.82 & 46.75 & 46.58 & 42.62 \\
\hline $\mathrm{SO}_{3}$ & 0.53 & 0.46 & NA & 0.46 & NA & 0.45 & NA & 0.59 & 0.51 & NA & 0.54 & NA & 0.69 & 0.54 & NA \\
\hline $\mathrm{TiO}_{2}$ & 1.38 & 1.52 & 1.48 & 1.47 & 1.49 & 1.43 & 1.48 & 1.38 & 1.48 & 1.49 & 1.48 & 1.55 & 1.39 & 1.59 & 1.62 \\
\hline $\mathrm{V}_{2} \mathrm{O}_{5}$ & $\S$ & $<0.01$ & 0.02 & 0.01 & 0.01 & $<0.01$ & 0.01 & $\S$ & 0.01 & 0.01 & 0.01 & 0.01 & $\S$ & 0.01 & 0.01 \\
\hline $\mathrm{ZnO}$ & 3.45 & 3.19 & 3.35 & 3.07 & 3.28 & 3.06 & 3.31 & 3.46 & 3.12 & 3.36 & 3.03 & 3.46 & 3.47 & 3.12 & 3.42 \\
\hline $\mathrm{ZrO}_{2}$ & 2.95 & 3.09 & 2.85 & 2.90 & 2.94 & 2.92 & 2.96 & 2.96 & 2.76 & 2.94 & 2.80 & 2.97 & 2.97 & 3.03 & 3.03 \\
\hline Sum & 100.00 & 100.00 & 96.03 & 100.00 & 95.99 & 100.00 & 95.49 & 100.00 & 100.00 & 94.97 & 100.00 & 95.30 & 100.00 & 100.00 & 94.79 \\
\hline
\end{tabular}

* Target B and Li values used for normalizing XRF data calculated based on simple well-stirred tank model and the feed target composition

NA - Not analyzed

$\S$ Not a target component 
Table 4.4. Listing of Dip Samples Taken During DM100 Correlation Testing and Information on Secondary Phase.

\begin{tabular}{|c|c|c|c|c|c|c|c|}
\hline \multirow{2}{*}{ Test } & \multirow{2}{*}{$\begin{array}{c}\text { Sampling } \\
\text { Date }\end{array}$} & \multirow{2}{*}{ Sample Name } & \multirow{2}{*}{$\begin{array}{c}\text { Glass } \\
\text { (kg) }\end{array}$} & \multicolumn{3}{|c|}{ Target (wt\%) } & \multirow{2}{*}{$\begin{array}{c}\text { Secondary Phase } \\
\text { Observed }\end{array}$} \\
\hline & & & & $\mathrm{SO}_{3}$ & F & $\mathrm{Cl}$ & \\
\hline \multirow{6}{*}{ 1A } & $6 / 13 / 05$ & WVZ-D-28A & 0.00 & \multirow{6}{*}{0.35} & \multirow{6}{*}{0.08} & \multirow{6}{*}{0.20} & No \\
\hline & \multirow{5}{*}{ 6/15/05 } & WVZ-D-65A & \multirow{5}{*}{407.06} & & & & No \\
\hline & & WVZ-D-65B & & & & & No \\
\hline & & WVZ-D-65C & & & & & No \\
\hline & & WVZ-D-65D & & & & & No \\
\hline & & WVZ-D-65E & & & & & No \\
\hline \multirow{10}{*}{ 1B } & \multirow{5}{*}{ 6/16/05 } & WVZ-D-74A & \multirow{5}{*}{491.14} & \multirow{16}{*}{0.41} & \multirow{16}{*}{0.08} & \multirow{16}{*}{0.20} & No \\
\hline & & WVZ-D-74B & & & & & No \\
\hline & & WVZ-D-74C & & & & & Yes \\
\hline & & WVZ-D-74D & & & & & No \\
\hline & & WVZ-D-74E & & & & & No \\
\hline & \multirow{5}{*}{ 6/17/05 } & WVZ-D-97A & \multirow{6}{*}{855.20} & & & & No \\
\hline & & WVZ-D-97B & & & & & No \\
\hline & & WVZ-D-97C & & & & & No \\
\hline & & WVZ-D-97D & & & & & No \\
\hline & & WVZ-D-97E & & & & & No \\
\hline \multirow{6}{*}{$2 \mathrm{~A}$} & $6 / 22 / 05$ & WVZ-D-106A & & & & & No \\
\hline & & WVZ-D-133A & & & & & No \\
\hline & & WVZ-D-133B & & & & & No \\
\hline & & WVZ-D-133C & 1072.98 & & & & No \\
\hline & & WVZ-D-133D & & & & & No \\
\hline & $6 / 23 / 05$ & WVZ-D-133E & & & & & No \\
\hline & $0 / \angle J / U S$ & WVZ-D-141A & & & & & No \\
\hline & & WVZ-D-141B & & & & & No \\
\hline & & WVZ-D-141C & 1149.70 & & & & No \\
\hline & & WVZ-D-141D & & & & & No \\
\hline PP & & WVZ-D-141E & & 046 & $\cap 08$ & 0 & No \\
\hline $2 \mathbf{D}$ & & AWV-D-14A & & 0.40 & 0.00 & 0.20 & No \\
\hline & & AWV-D-14B & & & & & No \\
\hline & & AWV-D-14C & 1513.44 & & & & No \\
\hline & & AWV-D-14D & & & & & No \\
\hline & $6 / 25$ & AWV-D-14E & & & & & No \\
\hline & $0 / \angle J / 0 J$ & AWV-D-22A & & & & & No \\
\hline & & AWV-D-22B & & & & & No \\
\hline & & AWV-D-22C & 1593.78 & & & & No \\
\hline & & AWV-D-22D & & & & & No \\
\hline 25 & & AWV-D-22E & & 053 & $\cap 08$ & 0 & No \\
\hline 20 & & AWV-D-49A & & 0.35 & 0.00 & 0.20 & No \\
\hline & & AWV-D-49B & & & & & No \\
\hline & & AWV-D-49C & 1949.88 & & & & No \\
\hline & & AWV-D-49D & & & & & No \\
\hline & $6 / 27 / 05$ & AWV-D-49E & & & & & No \\
\hline & $\begin{array}{l}0 \\
0\end{array}$ & AWV-D-55A & & & & & No \\
\hline & & AWV-D-55B & & & & & No \\
\hline 2D & & AWV-D-55C & 2031.96 & 0.59 & 0.08 & 0.20 & No \\
\hline & & AWV-D-55D & & & & & No \\
\hline & & AWV-D-55E & & & & & No \\
\hline
\end{tabular}


Table 4.4 Listing of Dip Samples Taken During DM100 Correlation Testing and Information on Secondary Phase (continued).

\begin{tabular}{|c|c|c|c|c|c|c|c|}
\hline \multirow{2}{*}{ Test } & \multirow{2}{*}{$\begin{array}{c}\text { Sampling } \\
\text { Date }\end{array}$} & \multirow{2}{*}{ Sample Name } & \multirow{2}{*}{$\begin{array}{c}\text { Glass } \\
\text { (kg) }\end{array}$} & \multicolumn{3}{|c|}{ Target (wt\%) } & \multirow{2}{*}{ Secondary Phase? } \\
\hline & & & & $\mathrm{SO}_{3}$ & $\mathrm{~F}$ & $\mathrm{Cl}$ & \\
\hline \multirow{5}{*}{ 2D } & \multirow{5}{*}{ 6/29/05 } & AWV-D-78A & \multirow{6}{*}{2375.38} & \multirow{16}{*}{0.59} & \multirow{16}{*}{0.08} & \multirow{16}{*}{0.20} & No \\
\hline & & AWV-D-78B & & & & & No \\
\hline & & AWV-D-78C & & & & & No \\
\hline & & AWV-D-78D & & & & & No \\
\hline & & AWV-D-78E & & & & & Yes \\
\hline \multirow{11}{*}{ 3A } & $7 / 6 / 05$ & AWV-D-86A & & & & & No \\
\hline & \multirow{5}{*}{ 7/7/05 } & AWV-D-112A & \multirow{5}{*}{2597.82} & & & & No \\
\hline & & AWV-D-112B & & & & & No \\
\hline & & AWV-D-112C & & & & & No \\
\hline & & AWV-D-112D & & & & & No \\
\hline & & AWV-D-112E & & & & & No \\
\hline & \multirow{5}{*}{ 7/7/05 } & AWV-D-121A & \multirow{5}{*}{2676.98} & & & & No \\
\hline & & AWV-D-121B & & & & & No \\
\hline & & AWV-D-121C & & & & & No \\
\hline & & AWV-D-121D & & & & & No \\
\hline & & AWV-D-121E & & & & & No \\
\hline \multirow{10}{*}{ 3B } & \multirow{10}{*}{ 7/9/05 } & AWV-D-147A & \multirow{5}{*}{3025.66} & \multirow{10}{*}{0.69} & & & Yes \\
\hline & & AWV-D-147B & & & & & No \\
\hline & & AWV-D-147C & & & & & No \\
\hline & & AWV-D-147D & & & & & No \\
\hline & & AWV-D-147E & & & 008 & $0>0$ & No \\
\hline & & AWV-D-155A & & & 0.00 & 0.20 & No \\
\hline & & AWV-D-155B & & & & & No \\
\hline & & AWV-D-155C & 3104.62 & & & & No \\
\hline & & AWV-D-155D & & & & & No \\
\hline & & AWV-D-155E & & & & & No \\
\hline & & BWV-D-32A & & & & & No \\
\hline & & BWV-D-32B & & & & & No \\
\hline $3 C$ & 7/11/05 & BWV-D-32C & רחת 2450 & 0.80 & 0.08 & 0.20 & No \\
\hline & & BWV-D-32D & 3450.02 & & & & No \\
\hline & & BWV-D-32E & & & & & No \\
\hline & $8 / 15 / 05$ & BWV-D-38A & & & & & No \\
\hline & & BWV-D-68A & & & & & Yes \\
\hline & & BWV-D-68B & & & & & Yes \\
\hline & & BWV-D-68C & & & & & Yes \\
\hline & & BWV-D-68D & & & & & Yes \\
\hline 4A & & BWV-D-68E & 358010 & 0.78 & 1.30 & 1.1 & Yes \\
\hline & & BWV-D-68F & 3500.10 & & & & No \\
\hline & & BWV-D-68G & & & & & No \\
\hline & 8/16/05 & BWV-D-68H & & & & & No \\
\hline & & BWV-D-68I & & & & & No \\
\hline & & BWV-D-68J & & & & & No \\
\hline & & BWV-D-73A & & & & & Yes \\
\hline & & BWV-D-73B & & & & & Yes \\
\hline 4B & & BWV-D-73C & 3658.88 & 0.67 & 1.30 & 1.1 & Yes \\
\hline & & BWV-D-73D & & & & & Yes \\
\hline & & BWV-D-73E & & & & & Yes \\
\hline
\end{tabular}


Table 4.4. Listing of Dip Samples Taken During DM100 Correlation Testing and Information on Secondary Phase (continued).

\begin{tabular}{|c|c|c|c|c|c|c|c|}
\hline \multirow{2}{*}{ Test } & \multirow{2}{*}{$\begin{array}{l}\text { Sampling } \\
\text { Date }\end{array}$} & \multirow{2}{*}{ Sample Name } & \multirow{2}{*}{$\begin{array}{l}\text { Glass } \\
\text { (kg) }\end{array}$} & \multicolumn{3}{|c|}{ Target (wt\%) } & \multirow{2}{*}{$\begin{array}{c}\text { Secondary Phase } \\
\text { Observed }\end{array}$} \\
\hline & & & & $\mathrm{SO}_{3}$ & $\mathrm{~F}$ & $\mathrm{Cl}$ & \\
\hline \multirow{10}{*}{ 4B } & \multirow{5}{*}{ 8/16/05 } & BWV-D-78A & \multirow{5}{*}{3658.88} & \multirow{10}{*}{0.67} & \multirow{10}{*}{1.30} & \multirow{10}{*}{1.1} & Trace Sulfate \\
\hline & & BWV-D-78B & & & & & No \\
\hline & & BWV-D-78C & & & & & No \\
\hline & & BWV-D-78D & & & & & No \\
\hline & & BWV-D-78E & & & & & No \\
\hline & \multirow{10}{*}{ 8/18/05 } & BWV-D-102A & \multirow{5}{*}{4028.19} & & & & No \\
\hline & & BWV-D-102B & & & & & No \\
\hline & & BWV-D-102C & & & & & No \\
\hline & & BWV-D-102D & & & & & No \\
\hline & & BWV-D-102E & & & & & No \\
\hline \multirow{24}{*}{ 4C } & & BWV-D-118A & \multirow{13}{*}{4104.41} & \multirow{35}{*}{0.58} & \multirow{35}{*}{1.30} & & Yes \\
\hline & & BWV-D-118B & & & & & Yes \\
\hline & & BWV-D-118C & & & & & Yes \\
\hline & & BWV-D-118D & & & & & Yes \\
\hline & & BWV-D-118E & & & & & Yes \\
\hline & \multirow{8}{*}{ 8/19/05 } & BWV-D-118F & & & & & Yes \\
\hline & & BWV-D-118G & & & & & Yes \\
\hline & & BWV-D-118H & & & & & No \\
\hline & & BWV-D-119A & & & & & No \\
\hline & & BWV-D-119B & & & & & No \\
\hline & & BWV-D-119C & & & & & No \\
\hline & & BWV-D-119D & & & & & No \\
\hline & & BWV-D-119E & & & & & No \\
\hline & \multirow{11}{*}{$8 / 20 / 05$} & BWV-D-142A & \multirow{12}{*}{4454.83} & & & & Yes \\
\hline & & BWV-D-142B & & & & & Yes \\
\hline & & BWV-D-142C & & & & & Yes \\
\hline & & BWV-D-142D & & & & & No \\
\hline & & BWV-D-142E & & & & 1.1 & Yes \\
\hline & & BWV-D-143A & & & & & Yes \\
\hline & & BWV-D-143B & & & & & No \\
\hline & & BWV-D-143C & & & & & Yes \\
\hline & & BWV-D-143D & & & & & No \\
\hline & & BWV-D-143E & & & & & No \\
\hline & & BWV-D-143F & & & & & No \\
\hline \multirow{11}{*}{$5 \mathrm{~A}$} & $8 / 23 / 05$ & CWV-D-7A & & & & & No \\
\hline & & CWV-D-26A & & & & & No \\
\hline & & CWV-D-26B & & & & & Yes \\
\hline & & CWV-D-26C & & & & & No \\
\hline & & CWV-D-26D & & & & & Yes \\
\hline & $8 / 25 / 05$ & CWV-D-26E & 468569 & & & & No \\
\hline & 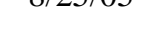 & CWV-D-27A & (4000.00 & & & & Yes \\
\hline & & CWV-D-27B & & & & & No \\
\hline & & CWV-D-27C & & & & & No \\
\hline & & CWV-D-27D & & & & & No \\
\hline & & CWV-D-27E & & & & & No \\
\hline
\end{tabular}


Table 4.4. Listing of Dip Samples Taken During DM100 Correlation Testing and Information on Secondary Phase (continued).

\begin{tabular}{|c|c|c|c|c|c|c|c|}
\hline \multirow[b]{2}{*}{ Test } & \multirow{2}{*}{$\begin{array}{l}\text { Sampling } \\
\text { Date }\end{array}$} & \multirow{2}{*}{ Sample Name } & \multirow{2}{*}{$\begin{array}{l}\text { Glass } \\
\text { (kg) }\end{array}$} & \multicolumn{3}{|c|}{ Target (wt\%) } & \multirow{2}{*}{$\begin{array}{c}\text { Secondary Phase } \\
\text { Observed }\end{array}$} \\
\hline & & & & $\mathrm{SO}_{3}$ & $\mathrm{~F}$ & $\mathrm{Cl}$ & \\
\hline \multirow{15}{*}{$5 B$} & \multirow{10}{*}{$8 / 25 / 05$} & CWV-D-32A & \multirow{10}{*}{4758.99} & \multirow{15}{*}{0.52} & \multirow{15}{*}{1.30} & \multirow{15}{*}{1.1} & Yes \\
\hline & & CWV-D-32B & & & & & No \\
\hline & & CWV-D-32C & & & & & Yes \\
\hline & & CWV-D-32D & & & & & No \\
\hline & & CWV-D-32E & & & & & Yes \\
\hline & & CWV-D-32F & & & & & No \\
\hline & & CWV-D-32G & & & & & No \\
\hline & & CWV-D-32H & & & & & No \\
\hline & & CWV-D-32I & & & & & No \\
\hline & & CWV-D-32J & & & & & Trace \\
\hline & \multirow{10}{*}{ 8/27/05 } & CWV-D-58A & \multirow{5}{*}{5143.49} & & & & No \\
\hline & & CWV-D-58B & & & & & No \\
\hline & & CWV-D-58C & & & & & No \\
\hline & & CWV-D-58D & & & & & No \\
\hline & & CWV-D-58E & & & & & No \\
\hline \multirow{15}{*}{$5 C$} & & CWV-D-67A & \multirow{5}{*}{5211.15} & \multirow{15}{*}{0.45} & \multirow{15}{*}{1.30} & \multirow{15}{*}{1.1} & No \\
\hline & & CWV-D-67B & & & & & No \\
\hline & & CWV-D-67C & & & & & Trace \\
\hline & & CWV-D-67D & & & & & No \\
\hline & & CWV-D-67E & & & & & No \\
\hline & \multirow{10}{*}{ 8/29/05 } & CWV-D-92A & \multirow{10}{*}{5597.95} & & & & Yes \\
\hline & & CWV-D-92B & & & & & Yes \\
\hline & & CWV-D-92C & & & & & Yes \\
\hline & & CWV-D-92D & & & & & No \\
\hline & & CWV-D-92E & & & & & No \\
\hline & & CWV-D-92F & & & & & No \\
\hline & & CWV-D-92G & & & & & No \\
\hline & & CWV-D-92H & & & & & No \\
\hline & & CWV-D-92I & & & & & No \\
\hline & & CWV-D-92J & & & & & No \\
\hline \multirow{15}{*}{ 5D } & & CWV-D-99A & & & & & No \\
\hline & & CWV-D-99B & & & & & No \\
\hline & 8/30/05 & CWV-D-99C & 5664.99 & & & & No \\
\hline & & CWV-D-99D & & & & & No \\
\hline & & CWV-D-99E & & & & & No \\
\hline & & CWV-D-125A & & & & & No \\
\hline & & CWV-D-125B & & & & & Yes \\
\hline & & CWV-D-125C & & 0.40 & 1.30 & 1.1 & Yes \\
\hline & & CWV-D-125D & & & & & No \\
\hline & $8 / 31 / 05$ & CWV-D-125E & 604748 & & & & Yes \\
\hline & 8/31/05 & CWV-D-126A & $604 / .48$ & & & & No \\
\hline & & CWV-D-126B & & & & & No \\
\hline & & CWV-D-126C & & & & & No \\
\hline & & CWV-D-126D & & & & & No \\
\hline & & CWV-D-126E & & & & & No \\
\hline
\end{tabular}


Table 4.4. Listing of Dip Samples Taken During DM100 Correlation Testing and Information on Secondary Phase (continued).

\begin{tabular}{|c|c|c|c|c|c|c|c|}
\hline \multirow{2}{*}{ Test } & \multirow{2}{*}{$\begin{array}{c}\text { Sampling } \\
\text { Date }\end{array}$} & \multirow{2}{*}{ Sample Name } & \multirow{2}{*}{$\begin{array}{l}\text { Glass } \\
\text { (kg) }\end{array}$} & \multicolumn{3}{|c|}{ Target (wt\%) } & \multirow{2}{*}{$\begin{array}{c}\text { Secondary Phase } \\
\text { Observed }\end{array}$} \\
\hline & & & & $\mathrm{SO}_{3}$ & $\mathrm{~F}$ & $\mathrm{Cl}$ & \\
\hline \multirow{11}{*}{ 6A } & 9/12/05 & CWV-D-132A & 6047.48 & \multirow{11}{*}{0.40} & \multirow{11}{*}{1.30} & \multirow{11}{*}{1.1} & No \\
\hline & \multirow{15}{*}{ 9/14/05 } & DWV-D-22A & \multirow{10}{*}{6492.46} & & & & Yes \\
\hline & & DWV-D-22B & & & & & Yes \\
\hline & & DWV-D-22C & & & & & No \\
\hline & & DWV-D-22D & & & & & No \\
\hline & & DWV-D-22E & & & & & No \\
\hline & & DWV-D-22F & & & & & No \\
\hline & & DWV-D-22G & & & & & No \\
\hline & & DWV-D-22H & & & & & No \\
\hline & & DWV-D-22I & & & & & No \\
\hline & & DWV-D-22J & & & & & No \\
\hline \multirow{15}{*}{ 6B } & & DWV-D-28A & \multirow{5}{*}{6566.34} & \multirow{15}{*}{0.35} & \multirow{15}{*}{1.30} & \multirow{15}{*}{1.1} & No \\
\hline & & DWV-D-28B & & & & & No \\
\hline & & DWV-D-28C & & & & & No \\
\hline & & DWV-D-28D & & & & & No \\
\hline & & DWV-D-28E & & & & & No \\
\hline & \multirow{10}{*}{ 9/16/05 } & DWV-D-52A & \multirow{10}{*}{6937.61} & & & & Yes \\
\hline & & DWV-D-52B & & & & & Yes \\
\hline & & DWV-D-52C & & & & & Yes \\
\hline & & DWV-D-52D & & & & & Yes \\
\hline & & DWV-D-52E & & & & & Yes \\
\hline & & DWV-D-52F & & & & & No \\
\hline & & DWV-D-52G & & & & & No \\
\hline & & DWV-D-52H & & & & & No \\
\hline & & DWV-D-52I & & & & & Yes \\
\hline & & DWV-D-52J & & & & & No \\
\hline
\end{tabular}


Table 4.5. Results on Dip Samples after Washing Procedure.

\begin{tabular}{|c|c|c|c|c|c|c|c|c|c|c|}
\hline \multirow[t]{2}{*}{ Test } & \multirow[t]{2}{*}{ Name } & \multirow{2}{*}{$\begin{array}{c}\text { Secondary Phases } \\
\text { Observed }\end{array}$} & \multicolumn{4}{|c|}{ Target Glass (wt\%) } & \multicolumn{4}{|c|}{$\begin{array}{c}\text { Concentration in Wash Solution, } \\
\mathrm{mg} / \mathrm{L}\end{array}$} \\
\hline & & & $\mathbf{F}$ & Cl & $\mathrm{SO}_{3}$ & $\mathbf{R e O}_{2}$ & $\mathbf{F}$ & Cl & Sulfate & $\mathbf{R e}$ \\
\hline LAWA3 [11]* & WVC-D-40A & Yes & 0.03 & 0.33 & 0.44 & 0 & 0.38 & 17.73 & 45.80 & $<0.25$ \\
\hline LAWC1[8]* & WVL-D-53A & Yes & 0.05 & 0.11 & 0.47 & 0 & 0.49 & 5.83 & 537.09 & $<0.25$ \\
\hline LAWA1[8]* & WVL-D-142A & Yes & $<0.01$ & 1.17 & 0.19 & 0 & 0.38 & 15.92 & 45.47 & $<0.25$ \\
\hline HLW AZ-101[48] & BLE-D-71A & No & 0.04 & $<0.01$ & 0.07 & 0 & 0.41 & 11.57 & 8.89 & $<0.25$ \\
\hline HLW C-106/AY-102 [49] & BLF-D-110B & No & $<0.01$ & 0.11 & $<0.01$ & 0 & 0.06 & 0.45 & 1.05 & $<0.25$ \\
\hline HLW SIPP [50] & BLJ-D-81A & No & $<0.01$ & $<0.01$ & 0.19 & 0 & 0.21 & 3.58 & 2.41 & $<0.25$ \\
\hline \multirow{4}{*}{ 1A } & WVZ-G-61C & No & 0.08 & 0.20 & 0.35 & 0.10 & 0.09 & 1.41 & 2.36 & $<0.25$ \\
\hline & WVZ-D-65A & No & 0.08 & 0.20 & 0.35 & 0.10 & 0.10 & 1.13 & 1.35 & $<0.25$ \\
\hline & WVZ-D-65C & No & 0.08 & 0.20 & 0.35 & 0.10 & 0.18 & 1.32 & 2.51 & $<0.25$ \\
\hline & WVZ-D-65E & No & 0.08 & 0.20 & 0.35 & 0.10 & 0.14 & 0.22 & 0.65 & $<0.25$ \\
\hline \multirow{8}{*}{$1 B$} & WVZ-G-72B & No & 0.08 & 0.20 & 0.41 & 0.10 & 0.14 & 0.38 & 0.67 & $<0.25$ \\
\hline & WVZ-D-74A & No & 0.08 & 0.20 & 0.41 & 0.10 & 0.25 & 2.06 & 2.33 & $<0.25$ \\
\hline & WVZ-D-74C & Yes & 0.08 & 0.20 & 0.41 & 0.10 & 6.77 & 41.44 & 10.96 & $<0.25$ \\
\hline & WVZ-D-74E & No & 0.08 & 0.20 & 0.41 & 0.10 & 0.17 & 0.43 & 0.59 & $<0.25$ \\
\hline & WVZ-G-96B & No & 0.08 & 0.20 & 0.41 & 0.10 & 0.25 & 0.86 & 2.61 & $<0.25$ \\
\hline & WVZ-D-97A & No & 0.08 & 0.20 & 0.41 & 0.10 & 0.42 & 2.94 & 2.01 & $<0.25$ \\
\hline & WVZ-D-97C & No & 0.08 & 0.20 & 0.41 & 0.10 & 0.11 & 3.15 & 3.44 & $<0.25$ \\
\hline & WVZ-D-97E & No & 0.08 & 0.20 & 0.41 & 0.10 & 0.15 & 0.49 & 1.36 & $<0.25$ \\
\hline \multirow{2}{*}{$2 A$} & WVZ-D-133C & No & 0.08 & 0.20 & 0.41 & 0.10 & 0.40 & 0.45 & 0.55 & $<0.25$ \\
\hline & WVZ-D-141C & No & 0.08 & 0.20 & 0.41 & 0.10 & 0.08 & 0.31 & 0.63 & $<0.25$ \\
\hline $2 B$ & AWV-D-14D & No & 0.08 & 0.20 & 0.46 & 0.10 & 0.13 & 0.49 & 1.17 & $<0.25$ \\
\hline \multirow{3}{*}{$2 \mathrm{C}$} & AWV-D-22C & No & 0.08 & 0.20 & 0.53 & 0.10 & 0.18 & 0.42 & 0.60 & $<0.25$ \\
\hline & AWV-D-49C & No & 0.08 & 0.20 & 0.53 & 0.10 & 0.12 & 0.50 & 1.28 & $<0.25$ \\
\hline & AWV-D-55C & No & 0.08 & 0.20 & 0.53 & 0.10 & 0.26 & 0.43 & 0.61 & $<0.25$ \\
\hline
\end{tabular}

* - samples often taken after extended idling periods and therefore observations of secondary phases may not be indicative of feed or test conditions. 
Table 4.5. Results on Dip Samples after Washing Procedure (continued).

\begin{tabular}{|c|c|c|c|c|c|c|c|c|c|c|}
\hline \multirow[t]{2}{*}{ Test } & \multirow[t]{2}{*}{ Name } & \multirow{2}{*}{$\begin{array}{c}\text { Secondary Phases } \\
\text { Observed }\end{array}$} & \multicolumn{4}{|c|}{ Target in Glass (wt\%) } & \multicolumn{4}{|c|}{$\begin{array}{c}\text { Concentration in Wash Solution, } \\
\text { mg/L }\end{array}$} \\
\hline & & & $\mathbf{F}$ & $\mathbf{C l}$ & $\mathrm{SO}_{3}$ & $\mathrm{ReO}_{2}$ & $\mathbf{F}$ & $\mathrm{Cl}$ & Sulfate & $\mathbf{R e}$ \\
\hline \multirow{3}{*}{$2 D$} & AWV-D-78C & No & 0.08 & 0.20 & 0.59 & 0.10 & 0.35 & 0.92 & 1.49 & $<0.25$ \\
\hline & AWV-D-78E & Yes & 0.08 & 0.20 & 0.59 & 0.10 & 0.83 & 21.29 & 14.24 & $<0.25$ \\
\hline & AWV-G-78A & No & 0.08 & 0.20 & 0.59 & 0.10 & 0.46 & 0.78 & 0.75 & $<0.25$ \\
\hline Cyclone Solids & AWV-O-86A & Yes & N/A & N/A & N/A & 0.10 & 134.73 & 450.66 & 211.42 & $<0.25$ \\
\hline \multirow{3}{*}{$\mathbf{3 A}$} & AWV-G-108D & No & 0.08 & 0.20 & 0.59 & 0.10 & 0.26 & 1.40 & 1.26 & $<0.25$ \\
\hline & AWV-D-112D & No & 0.08 & 0.20 & 0.59 & 0.10 & 0.17 & 2.39 & 1.28 & $<0.25$ \\
\hline & AWV-D-121C & No & 0.08 & 0.20 & 0.59 & 0.10 & 0.08 & 0.24 & 1.19 & $<0.25$ \\
\hline \multirow{3}{*}{ 3B } & AWV-D-147A & Yes & 0.08 & 0.20 & 0.69 & 0.10 & 0.38 & 23.25 & 15.96 & 14.05 \\
\hline & AWV-D-147C & No & 0.08 & 0.20 & 0.69 & 0.10 & 0.18 & 0.52 & 1.12 & 0.70 \\
\hline & AWV-D-155C & No & 0.08 & 0.20 & 0.69 & 0.10 & 0.10 & 0.43 & 0.81 & $<0.25$ \\
\hline $3 C$ & BWV-D-32D & No & 0.08 & 0.20 & 0.80 & 0.10 & 0.02 & 0.07 & 0.48 & $<0.25$ \\
\hline $4 \mathrm{~A}$ & BWV-D-68D & Yes & 1.30 & 1.10 & 0.78 & 0.10 & 1.62 & 15.41 & 60.37 & 3.82 \\
\hline \multirow{4}{*}{ 4B } & BWV-D-68H & No & 1.30 & 1.10 & 0.67 & 0.10 & 0.17 & 0.34 & 1.55 & $<0.25$ \\
\hline & BWV-D-73C & Yes & 1.30 & 1.10 & 0.67 & 0.10 & 0.84 & 10.78 & 33.24 & 2.00 \\
\hline & BWV-D-78C & No & 1.30 & 1.10 & 0.67 & 0.10 & 0.18 & 0.26 & 1.14 & $<0.25$ \\
\hline & BWV-D-102C & No & 1.30 & 1.10 & 0.67 & 0.10 & 0.16 & 0.24 & 0.99 & $<0.25$ \\
\hline \multirow{4}{*}{$4 C$} & BWV-D-118C & Yes & 1.30 & 1.10 & 0.58 & 0.10 & 14.91 & 116.34 & 220.57 & 18.63 \\
\hline & BWV-D-119C & No & 1.30 & 1.10 & 0.58 & 0.10 & 0.44 & 1.00 & 1.90 & $<0.25$ \\
\hline & BWV-D-142C & Yes & 1.30 & 1.10 & 0.58 & 0.10 & 0.56 & 4.07 & 10.40 & 0.67 \\
\hline & BWV-D-143D & No & 1.30 & 1.10 & 0.58 & 0.10 & 0.43 & 2.38 & 3.72 & 0.26 \\
\hline \multirow{2}{*}{$5 A$} & CWV-D-26C & No & 1.30 & 1.10 & 0.58 & 0.10 & 0.23 & 0.39 & 0.85 & $<0.25$ \\
\hline & CWV-D-27C & No & 1.30 & 1.10 & 0.58 & 0.10 & 0.18 & 0.67 & 0.99 & $<0.25$ \\
\hline \multirow{3}{*}{$5 B$} & CWV-D-32C & Yes & 1.30 & 1.10 & 0.52 & 0.10 & 14.70 & 142.00 & 372.79 & 25.99 \\
\hline & CWV-D-32H & No & 1.30 & 1.10 & 0.52 & 0.10 & 0.69 & 0.59 & 1.12 & $<0.25$ \\
\hline & CWV-D-58C & No & 1.30 & 1.10 & 0.52 & 0.10 & 0.33 & 1.36 & 2.96 & $<0.25$ \\
\hline
\end{tabular}

NA - Not applicable 
Table 4.5. Results on Dip Samples after Washing Procedure (continued).

\begin{tabular}{|c|c|c|c|c|c|c|c|c|c|c|}
\hline \multirow{2}{*}{ Test } & \multirow{2}{*}{ Name } & \multirow{2}{*}{$\begin{array}{c}\text { Secondary Phases } \\
\text { Observed }\end{array}$} & \multicolumn{4}{|c|}{ Target in Glass (wt \%) } & \multicolumn{4}{|c|}{ Concentration in Wash Solution, mg/L } \\
\hline & & & $\mathbf{F}$ & $\mathbf{C l}$ & $\mathrm{SO}_{3}$ & $\mathrm{ReO}_{2}$ & $\mathbf{F}$ & $\mathbf{C l}$ & Sulfate & $\mathbf{R e}$ \\
\hline \multirow{2}{*}{$5 C$} & CWV-D-92C & Yes & 1.30 & 1.10 & 0.45 & 0.10 & 0.95 & 6.55 & 17.54 & 2.58 \\
\hline & CWV-D-92H & No & 1.30 & 1.10 & 0.45 & 0.10 & 0.23 & 0.59 & 0.69 & $<0.25$ \\
\hline \multirow{3}{*}{$5 D$} & CWV-D-99C & No & 1.30 & 1.10 & 0.40 & 0.10 & 0.23 & 0.57 & 0.63 & $<0.25$ \\
\hline & CWV-D-125C & Yes & 1.30 & 1.10 & 0.40 & 0.10 & 2.87 & 17.19 & 40.80 & 6.14 \\
\hline & CWV-D-126C & No & 1.30 & 1.10 & 0.40 & 0.10 & 0.31 & 0.41 & 0.52 & $<0.25$ \\
\hline \multirow{2}{*}{ 6A } & DWV-D-22C & No & 1.30 & 1.10 & 0.40 & 0.10 & 0.26 & 0.47 & 0.63 & $<0.25$ \\
\hline & DWV-D-22H & No & 1.30 & 1.10 & 0.40 & 0.10 & 0.31 & 0.49 & 0.32 & $<0.25$ \\
\hline \multirow{3}{*}{$6 B$} & DWV-D-28C & No & 1.30 & 1.10 & 0.35 & 0.10 & 0.40 & 0.86 & 0.72 & $<0.25$ \\
\hline & DWV-D-52C & Yes & 1.30 & 1.10 & 0.35 & 0.10 & 0.57 & 3.80 & 1.24 & $<0.25$ \\
\hline & DWV-D-52I & Yes & 1.30 & 1.10 & 0.35 & 0.10 & 0.62 & 0.45 & 0.63 & $<0.25$ \\
\hline
\end{tabular}


Table 4.6. Viscosity and Electrical Conductivity Data for Crucible and Melter Glasses.

\begin{tabular}{|c|c|c|c|c|c|c|c|c|}
\hline Glass ID & $\begin{array}{c}\text { LAWE3 } \\
\text { Crucible } \\
\text { Glass }\end{array}$ & $\begin{array}{c}\text { WVZ-G-61D } \\
\text { DM100 } \\
\text { Melter Glass } \\
\text { Test 1A } \\
\text { (LAWE3 } \\
\text { low Cl, F) } \\
\end{array}$ & $\begin{array}{c}\text { DWV-G-51B } \\
\text { DM100 } \\
\text { Melter Glass } \\
\text { Test 6B } \\
\text { (LAWE3 } \\
\text { high Cl, F) } \\
\end{array}$ & $\begin{array}{c}\text { LAWE7H } \\
\text { Crucible } \\
\text { Glass }\end{array}$ & $\begin{array}{c}\text { AWV-G-78A } \\
\text { DM100 } \\
\text { Melter Glass } \\
\text { Test 2D } \\
\text { (LAWE7H } \\
\text { low Cl, F) } \\
\end{array}$ & $\begin{array}{c}\text { BWV-G-142B } \\
\text { DM100 Melter } \\
\text { Glass } \\
\text { Test 4C } \\
\text { (LAWE7H } \\
\text { high Cl, F) } \\
\end{array}$ & $\begin{array}{c}\text { LAWE10H } \\
\text { Crucible } \\
\text { Glass }\end{array}$ & $\begin{array}{c}\text { BWV-G-32B } \\
\text { DM100 } \\
\text { Melter Glass } \\
\text { Test 3C } \\
\text { (LAWE10H } \\
\text { low Cl, F) } \\
\end{array}$ \\
\hline \multicolumn{9}{|c|}{ Viscosity (poise) } \\
\hline $950^{\circ} \mathrm{C}$ & 544 & 496 & 408 & 144 & 217 & 240 & 364 & 546 \\
\hline $1000^{\circ} \mathrm{C}$ & 267 & 252 & 208 & 81 & 113 & 124 & 196 & 262 \\
\hline $1050^{\circ} \mathrm{C}$ & 145 & 139 & 116 & 49 & 65 & 70 & 112 & 140 \\
\hline $1100^{\circ} \mathrm{C}$ & 84 & 82 & 69 & 32 & 40 & 42 & 67 & 81 \\
\hline $1150^{\circ} \mathrm{C}$ & 52 & 52 & 44 & 21 & 26 & 27 & 42 & 50 \\
\hline $1200^{\circ} \mathrm{C}$ & 34 & 34 & 29 & 15 & 18 & 18 & 27 & 33 \\
\hline $1250^{\circ} \mathrm{C}$ & 24 & 24 & 20 & 11 & 13 & 13 & 18 & 22 \\
\hline \multicolumn{9}{|c|}{ Electrical Conductivity (S/cm) } \\
\hline $950^{\circ} \mathrm{C}$ & 0.136 & 0.120 & 0.128 & 0.103 & 0.101 & 0.102 & 0.049 & 0.054 \\
\hline $950^{\circ} \mathrm{C}$ & 0.178 & 0.170 & 0.172 & 0.141 & 0.148 & 0.144 & 0.068 & 0.074 \\
\hline $1000^{\circ} \mathrm{C}$ & 0.227 & 0.226 & 0.222 & 0.189 & 0.199 & 0.193 & 0.092 & 0.100 \\
\hline $1050^{\circ} \mathrm{C}$ & 0.281 & 0.286 & 0.277 & 0.247 & 0.253 & 0.248 & 0.122 & 0.132 \\
\hline $1100^{\circ} \mathrm{C}$ & 0.340 & 0.348 & 0.338 & 0.317 & 0.308 & 0.309 & 0.158 & 0.170 \\
\hline $1150^{\circ} \mathrm{C}$ & 0.404 & 0.410 & 0.404 & 0.400 & 0.362 & 0.374 & 0.201 & 0.216 \\
\hline $1200^{\circ} \mathrm{C}$ & 0.472 & 0.473 & 0.473 & 0.496 & 0.415 & 0.442 & 0.252 & 0.270 \\
\hline $1250^{\circ} \mathrm{C}$ & 0.543 & 0.535 & 0.546 & 0.607 & 0.467 & 0.513 & 0.311 & 0.332 \\
\hline
\end{tabular}


Table 4.7. Results of PCT (ASTM C1285, 7-days at $90^{\circ} \mathrm{C}$ ) and VHT (at $200^{\circ} \mathrm{C}$ for 24 days) for Crucible and Melter Glasses.

\begin{tabular}{|c|c|c|c|c|}
\hline Glass Sample & $\begin{array}{c}\text { LAWE3 } \\
\text { Crucible Glass }\end{array}$ & $\begin{array}{c}\text { WVZ-G-61D } \\
\text { DM100 Melter Glass } \\
\text { Test 1A (LAWE3 low CI, F) }\end{array}$ & $\begin{array}{c}\text { DWV-G-51B } \\
\text { DM100 Melter Glass } \\
\text { Test 6B (LAWE3 high Cl, F) }\end{array}$ & $\begin{array}{l}\text { WTP Contract } \\
\text { Limit }\end{array}$ \\
\hline \multicolumn{5}{|c|}{ 7-Day PCT, Stainless Steel Vessel; S/V=2000 $\mathrm{m}^{-1}$} \\
\hline \multicolumn{5}{|l|}{ Concentration (ppm) } \\
\hline $\mathrm{B}$ & 59.18 & 44.15 & 85.16 & - \\
\hline $\mathrm{Na}$ & 191.00 & 185.20 & 292.80 & - \\
\hline $\mathrm{Si}$ & 73.49 & 70.76 & 82.66 & - \\
\hline \multicolumn{5}{|l|}{ 7-Day PCT Normalized Concentrations (g/L) } \\
\hline $\mathrm{B}$ & 1.91 & 1.42 & 2.81 & - \\
\hline $\mathrm{Na}$ & 1.41 & 1.37 & 2.27 & - \\
\hline $\mathrm{Si}$ & 0.37 & 0.36 & 0.41 & - \\
\hline $\mathrm{pH}$ & 11.24 & 11.20 & 11.12 & - \\
\hline \multicolumn{5}{|l|}{ 7-Day PCT Normalized Mass Loss $\left(\mathrm{g} / \mathrm{m}^{2}\right)$} \\
\hline B & 0.95 & 0.71 & 1.40 & $<2.0$ \\
\hline $\mathrm{Na}$ & 0.71 & 0.68 & 1.13 & $<2.0$ \\
\hline $\mathrm{Si}$ & 0.18 & 0.18 & 0.20 & $<2.0$ \\
\hline \multicolumn{5}{|l|}{ 7-Day PCT Normalized Loss Rate $\left(\mathrm{g} / \mathrm{m}^{2} / \mathrm{d}\right)$} \\
\hline $\mathrm{B}$ & 0.14 & 0.10 & 0.20 & - \\
\hline $\mathrm{Na}$ & 0.10 & 0.10 & 0.16 & - \\
\hline $\mathrm{Si}$ & 0.03 & 0.03 & 0.03 & - \\
\hline \multicolumn{5}{|l|}{ VHT Alteration (24 days at $\left.200^{\circ} \mathrm{C}\right)$} \\
\hline Duration (days) & 24 & 24 & 24 & - \\
\hline Alteration depth $(\mu \mathrm{m})$ & 129 & 175 & 100 & - \\
\hline $\begin{array}{l}\text { Alteration rate }\left(\mathrm{g} / \mathrm{m}^{2} / \mathrm{d}\right) \text { calculated using } \\
\text { averaged density of } 2.65 \mathrm{~g} / \mathrm{cc}\end{array}$ & 14.3 & 19.4 & 11.1 & $<50.0$ \\
\hline
\end{tabular}

- Empty data field 
Table 4.7. Results of PCT (ASTM C1285, 7-days at $90^{\circ} \mathrm{C}$ ) and VHT (at $200^{\circ} \mathrm{C}$ for 24 Days) for Crucible and Melter Glasses (continued).

\begin{tabular}{|c|c|c|c|c|}
\hline Glass Sample & $\begin{array}{c}\text { LAWE7H } \\
\text { Crucible Glass }\end{array}$ & $\begin{array}{c}\text { AWV-G-78A } \\
\text { DM100 Melter Glass } \\
\text { Test 1A (LAWE7H low Cl, F) }\end{array}$ & $\begin{array}{c}\text { BWV-G-142B } \\
\text { DM100 Melter Glass } \\
\text { Test 6B (LAWE7H high Cl, F) } \\
\end{array}$ & $\begin{array}{l}\text { WTP Contract } \\
\text { Limit }\end{array}$ \\
\hline \multicolumn{5}{|c|}{ 7-Day PCT, Stainless Steel Vessel; S/V=2000m } \\
\hline \multicolumn{5}{|l|}{ Concentration (ppm) } \\
\hline $\mathrm{B}$ & 32.72 & 33.18 & 29.24 & - \\
\hline $\mathrm{Na}$ & 103.20 & 109.30 & 98.56 & - \\
\hline $\mathrm{Si}$ & 81.85 & 88.04 & 73.89 & - \\
\hline \multicolumn{5}{|l|}{ 7-Day PCT Normalized Concentrations (g/L) } \\
\hline $\mathrm{B}$ & 1.07 & 1.08 & 0.98 & - \\
\hline $\mathrm{Na}$ & 1.03 & 1.02 & 1.07 & - \\
\hline $\mathrm{Si}$ & 0.39 & 0.42 & 0.34 & - \\
\hline $\mathrm{pH}$ & 11.14 & 10.98 & 10.72 & - \\
\hline \multicolumn{5}{|l|}{ 7-Day PCT Normalized Mass Loss (g/m²) } \\
\hline $\mathrm{B}$ & 0.53 & 0.54 & 0.49 & $<2.0$ \\
\hline $\mathrm{Na}$ & 0.51 & 0.51 & 0.54 & $<2.0$ \\
\hline Si & 0.20 & 0.21 & 0.17 & $<2.0$ \\
\hline \multicolumn{5}{|l|}{ 7-Day PCT Normalized Loss Rate (g/m²/d) } \\
\hline $\mathrm{B}$ & 0.08 & 0.08 & 0.07 & - \\
\hline $\mathrm{Na}$ & 0.07 & 0.07 & 0.08 & - \\
\hline $\mathrm{Si}$ & 0.03 & 0.03 & 0.02 & - \\
\hline \multicolumn{5}{|l|}{ VHT Alteration $\left(24\right.$ days at $200^{\circ} \mathrm{C}$ ) } \\
\hline Duration (days) & 24 & 24 & 24 & - \\
\hline Alteration depth $(\mu \mathrm{m})$ & 152 & 87 & 63 & - \\
\hline $\begin{array}{l}\text { Alteration rate }\left(\mathrm{g} / \mathrm{m}^{2} / \mathrm{d}\right) \text { calculated using } \\
\text { averaged density of } 2.65 \mathrm{~g} / \mathrm{cc}\end{array}$ & 16.8 & 9.6 & 7.0 & $<50.0$ \\
\hline
\end{tabular}

- Empty data field 
Table 4.7. Results of PCT (ASTM C1285, 7-days at $90^{\circ} \mathrm{C}$ ) and VHT (at $200^{\circ} \mathrm{C}$ for 24 Days) for Crucible and Melter Glasses (continued).

\begin{tabular}{|c|c|c|c|}
\hline Glass Sample & $\begin{array}{c}\text { LAWE10H } \\
\text { Crucible Glass }\end{array}$ & $\begin{array}{c}\text { BWV-G-32B } \\
\text { DM100 Melter Glass } \\
\text { Test 1A (LAWE10H low Cl, F) }\end{array}$ & $\begin{array}{c}\text { WTP Contract } \\
\text { Limit }\end{array}$ \\
\hline \multicolumn{4}{|l|}{ 7-Day PCT, Stainless Steel Vessel; S/V=2000 m ${ }^{-1}$} \\
\hline \multicolumn{4}{|l|}{ Concentration (ppm) } \\
\hline B & 14.08 & 15.28 & - \\
\hline $\mathrm{Na}$ & 18.38 & 25.13 & - \\
\hline $\mathrm{Si}$ & 47.87 & 56.22 & - \\
\hline \multicolumn{4}{|l|}{ 7-Day PCT Normalized Concentrations (g/L) } \\
\hline B & 0.46 & 0.49 & - \\
\hline $\mathrm{Na}$ & 0.43 & 0.52 & - \\
\hline $\mathrm{Si}$ & 0.21 & 0.24 & - \\
\hline $\mathrm{pH}$ & 10.25 & 10.22 & - \\
\hline \multicolumn{4}{|l|}{ 7-Day PCT Normalized Mass Loss $\left(\mathrm{g} / \mathrm{m}^{2}\right)$} \\
\hline $\mathrm{B}$ & 0.23 & 0.25 & $<2.0$ \\
\hline $\mathrm{Na}$ & 0.22 & 0.26 & $<2.0$ \\
\hline $\mathrm{Si}$ & 0.10 & 0.12 & $<2.0$ \\
\hline \multicolumn{4}{|l|}{ 7-Day PCT Normalized Loss Rate (g/m²/d) } \\
\hline $\mathrm{B}$ & 0.03 & 0.04 & - \\
\hline $\mathrm{Na}$ & 0.03 & 0.04 & - \\
\hline Si & 0.01 & 0.02 & - \\
\hline \multicolumn{4}{|l|}{ VHT Alteration ( 24 days at $200^{\circ} \mathrm{C}$ ) } \\
\hline Duration (days) & 24 & 24 & - \\
\hline Alteration depth $(\mu \mathrm{m})$ & 17 & 20 & - \\
\hline $\begin{array}{l}\text { Alteration rate }\left(\mathrm{g} / \mathrm{m}^{2} / \mathrm{d}\right) \text { calculated using averaged } \\
\text { density of } 2.65 \mathrm{~g} / \mathrm{cc}\end{array}$ & 1.9 & 2.2 & $<50.0$ \\
\hline
\end{tabular}

- Empty data field 
Table 4.8. TCLP Results (in mg/L) for Crucible and Melter Glasses.

\begin{tabular}{|c|c|c|c|c|c|c|c|c|c|c|}
\hline & Blank & LAWE3 & WVZ-G-61D & DWV-G-51B & LAWE7H & AWV-G-78A & BWV-G-142B & LAWE10HR1 & BWV-G-32B & $\begin{array}{c}\text { UTS } \\
\text { Limits }\end{array}$ \\
\hline Ag & $<0.070$ & $<\mathbf{0 . 0 7 0}$ & $<0.070$ & $<0.070$ & $<\mathbf{0 . 0 7 0}$ & $<0.070$ & $<\mathbf{0 . 0 7 0}$ & $<0.070$ & $<0.070$ & 0.14 \\
\hline As & $<0.200$ & $<0.200$ & $<0.200$ & $<0.200$ & $<0.200$ & $<0.200$ & $<0.200$ & $<0.200$ & $<0.200$ & 5 \\
\hline Ba & $<0.000$ & $<1^{*}$ & $<1^{*}$ & $<1^{*}$ & $<1^{*}$ & $<1^{*}$ & $<1^{*}$ & $<1^{*}$ & $<1^{*}$ & 21 \\
\hline Cd & $<0.030$ & $<0.030$ & $<0.030$ & $<0.030$ & $<0.030$ & $<0.030$ & $<0.030$ & $<0.030$ & $<0.030$ & 0.11 \\
\hline $\mathrm{Cr}$ & $<\mathbf{0 . 0 1 0}$ & $<0.010$ & 0.082 & 0.062 & $<0.010$ & 0.034 & 0.047 & 0.029 & 0.031 & 0.6 \\
\hline $\mathbf{N i}$ & $<0.040$ & $<0.040$ & 0.099 & 0.085 & $<0.040$ & $<0.040$ & 0.057 & $<0.040$ & 0.054 & 11 \\
\hline $\mathbf{P b}$ & $<0.100$ & $<0.100$ & $<0.100$ & $<0.100$ & $<0.100$ & $<0.100$ & $<0.100$ & 0.132 & $<0.100$ & 0.75 \\
\hline Se & $<0.900$ & $<0.900$ & $<0.900$ & $<0.900$ & $<0.900$ & $<0.900$ & $<0.900$ & $<0.900$ & $<0.900$ & 5.7 \\
\hline Zn & $<0.020$ & 2.202 & 2.185 & 2.631 & 1.307 & 1.583 & 1.502 & 0.748 & 0.786 & 4.3 \\
\hline $\mathrm{Al}$ & $<0.030$ & 0.505 & 0.415 & 0.869 & 0.449 & 0.277 & 0.557 & 0.19 & 0.206 & N/A \\
\hline $\mathrm{B}$ & $<0.200$ & 2.421 & 2.413 & 3.186 & 1.437 & 1.837 & 1.573 & 0.723 & 0.782 & N/A \\
\hline $\mathrm{Ca}$ & $<0.000$ & 1.657 & 1.228 & 1.521 & 2.516 & 2.847 & 2.509 & 1.267 & 1.432 & N/A \\
\hline $\mathrm{Fe}$ & $<0.050$ & 0.299 & 1.138 & 0.847 & 0.342 & 0.432 & 1.21 & 0.248 & 0.34 & N/A \\
\hline $\mathrm{K}$ & $<0.080$ & 3.523 & 3.205 & 4.007 & 0.648 & 0.487 & 0.312 & 0.394 & 0.292 & N/A \\
\hline $\mathrm{Li}$ & $<0.050$ & $<0.050$ & $<0.050$ & $<0.050$ & 0.744 & 0.908 & 0.782 & 0.479 & 0.566 & N/A \\
\hline $\mathrm{Mg}$ & $<0.010$ & 0.677 & 0.66 & 0.869 & 0.403 & 0.563 & 0.483 & 0.38 & 0.485 & N/A \\
\hline $\mathrm{P}$ & $<0.600$ & $<0.600$ & $<0.600$ & $<0.600$ & $<0.600$ & $<0.600$ & $<0.600$ & $<0.600$ & $<0.600$ & N/A \\
\hline $\mathrm{Si}$ & $<0.040$ & 2.343 & 2.653 & 3.05 & 2.012 & 2.001 & 2.39 & 1.076 & 1.122 & N/A \\
\hline $\mathrm{Ti}$ & $<0.020$ & 0.029 & 0.041 & 0.039 & 0.021 & $<0.020$ & 0.039 & 0.023 & 0.023 & N/A \\
\hline $\mathrm{Zr}$ & $<0.020$ & 0.077 & 0.102 & 0.079 & 0.057 & 0.030 & 0.082 & 0.043 & 0.041 & N/A \\
\hline
\end{tabular}

* Measured Ba concentration in glass TCLP extracts is relatively high due to a suspected interference.

N/A - Not applicable 
Table 4.9 Descriptions of As-Melted and Heat Treated Crucible and Melter Glasses.

\begin{tabular}{|c|c|c|c|c|}
\hline Formulation & $\begin{array}{l}\text { Glass ID } \\
\text { and source }\end{array}$ & Glass as-melted & $\begin{array}{c}\text { Glass remelted at } 1200^{\circ} \mathrm{C} \\
\text { for } 1 \text { hour, heat treated for } \\
20 \text { hours at } 850^{\circ} \mathrm{C} \text {, and } \\
\text { quenched. }\end{array}$ & $\begin{array}{c}\text { Glass remelted at } 1200^{\circ} \mathrm{C} \text { for } \\
1 \text { hour, and cooled according } \\
\text { to Centerline Canister } \\
\text { Cooling profile [51]. }\end{array}$ \\
\hline \multirow{3}{*}{ LAWE3 } & $\begin{array}{c}\text { LAWE3 } \\
\text { Crucible melt }\end{array}$ & Clear green glass & Clear green glass & Clear green glass \\
\hline & $\begin{array}{c}\text { WVZ-G-61D } \\
\text { DM100 Test 1A - Nominal Cl and F }\end{array}$ & Clear green glass & Clear green glass & Clear green glass \\
\hline & $\begin{array}{c}\text { DWV-G-51B } \\
\text { DM100 Test 6B - High Cl and F }\end{array}$ & $\begin{array}{c}\text { Clear green glass } \\
\text { Traces of sulfate observed }\end{array}$ & Clear green glass & Clear green glass \\
\hline \multirow{3}{*}{$\begin{array}{l}\text { LAWE4H and } \\
\text { LAWE4HK0 }\end{array}$} & $\begin{array}{c}\text { LAWE4H } \\
\text { Crucible melt } \\
\end{array}$ & Clear green glass & Clear green glass & Clear green glass \\
\hline & $\begin{array}{ll}\text { CWV-G-125B } \\
\text { DM100 } & \text { Test 5D- High Cl and F }\end{array}$ & $\begin{array}{c}\text { Clear green glass } \\
\text { Traces of sulfate observed }\end{array}$ & NA & Clear green glass \\
\hline & $\begin{array}{c}\text { DWV-G-19C } \\
\text { DM100 Test 6A- High Cl and F }\end{array}$ & $\begin{array}{c}\text { Clear green glass } \\
\text { Traces of sulfate observed }\end{array}$ & NA & Clear green glass \\
\hline \multirow{2}{*}{ LAWE5H } & $\begin{array}{c}\text { LAWE5H } \\
\text { Crucible melt }\end{array}$ & Clear green glass & Clear green glass & Clear green glass \\
\hline & $\begin{array}{c}\text { CWV-G-90C } \\
\text { DM100 Test 5C- High Cl and F }\end{array}$ & $\begin{array}{c}\text { Clear green glass } \\
\text { Traces of sulfate observed }\end{array}$ & NA & Clear green glass \\
\hline \multirow{3}{*}{ LAWE7H } & $\begin{array}{c}\text { LAWE7H } \\
\text { Crucible melt }\end{array}$ & Clear green glass & Clear green glass & Clear green glass \\
\hline & $\begin{array}{c}\text { AWV-G-78A } \\
\text { DM100 Test 2D - Nominal Cl and F }\end{array}$ & Clear green glass & Clear green glass & Clear green glass \\
\hline & $\begin{array}{c}\text { BWV-G-142B } \\
\text { DM100 Test 4C - High Cl and F }\end{array}$ & $\begin{array}{c}\text { Clear green glass } \\
\text { Traces of sulfate observed }\end{array}$ & Clear green glass & Clear green glass \\
\hline \multirow{3}{*}{ LAWE10H } & $\begin{array}{c}\text { LAWE10H } \\
\text { Crucible melt } \\
\end{array}$ & Clear green glass & $\begin{array}{l}\sim 0.6 \text { vol.\% of large augite } \\
\text { crystals }\end{array}$ & $\begin{array}{l}\sim 0.1 \text { vol. } \% \text { of small augite } \\
\text { crystals at crucible contact }\end{array}$ \\
\hline & $\begin{array}{c}\text { BWV-G-32B } \\
\text { DM100 Test 3C - Nominal Cl and F }\end{array}$ & Clear green glass & $\begin{array}{l}\sim 0.4 \text { vol.\% of small augite } \\
\text { crystals at crucible contact }\end{array}$ & $\begin{array}{l}\sim 0.2 \text { vol.\% of small augite } \\
\text { crystals at crucible contact }\end{array}$ \\
\hline & $\begin{array}{c}\text { BWV-G-66C } \\
\text { DM100 Test 4A - High Cl and F }\end{array}$ & $\begin{array}{c}\text { Clear green glass } \\
\text { Traces of sulfate observed }\end{array}$ & NA & $\begin{array}{l}\sim 0.4 \text { vol. } \% \text { of small augite } \\
\text { crystals at crucible contact }\end{array}$ \\
\hline
\end{tabular}

NA - Not analyzed 
The Catholic University of America Vitreous State Laboratory
DuraMelter 100 Tests to Support LAW Glass Formulation Correlation Developmen

Final Report, VSL-06R6480-1, Rev. 0

Table 5.1. Results from Melter Off-Gas Emission Samples.

\begin{tabular}{|c|c|c|c|c|c|c|c|c|c|}
\hline & \multicolumn{4}{|c|}{ Test $1 \mathrm{~A}$} & \multicolumn{4}{|c|}{ Test 1B } \\
\hline & & \multicolumn{4}{|c|}{$\begin{array}{c}06 / 15 / 05 \text { 09:24 - 10:24 } \\
\text { 95.98\% Isokinetic, } 10.9 \% \text { Moisture, } 56.9 \mathrm{dscfm}\end{array}$} & \multicolumn{4}{|c|}{$\begin{array}{c}06 / 17 / 0510: 35-11: 35 \\
\text { 93.8\% Isokinetic, } 12.3 \% \text { Moisture, } 61.2 \mathrm{dscfm}\end{array}$} \\
\hline & & $\begin{array}{c}\text { Feed } \\
\text { Rate }^{\#} \\
(\mathrm{mg} / \mathrm{min})\end{array}$ & $\begin{array}{c}\text { Emissions } \\
\text { Rate } \\
\text { (mg/min) }\end{array}$ & $\%$ Feed & DF & $\begin{array}{c}\text { Feed } \\
\text { Rate }{ }^{\#} \\
(\mathrm{mg} / \mathrm{min})\end{array}$ & $\begin{array}{c}\text { Emissions } \\
\text { Rate } \\
\text { (mg/min) }\end{array}$ & $\%$ Feed & DF \\
\hline \multirow{21}{*}{ 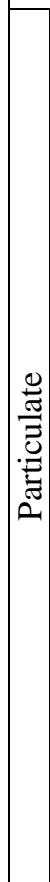 } & Total $^{\$}$ & 125235 & 1258 & 1.00 & 99.6 & 123130 & 878 & 0.71 & 140 \\
\hline & $\mathrm{Al}$ & 4877 & 4.20 & 0.09 & 1160 & 4773 & 9.36 & 0.20 & 510 \\
\hline & B & 4694 & 66.3 & 1.41 & 70.8 & 4595 & 34.4 & 0.75 & 133 \\
\hline & $\mathrm{Ca}$ & 2186 & 1.44 & 0.07 & 1519 & 2662 & 2.98 & 0.11 & 893 \\
\hline & $\mathrm{Cl}^{*}$ & 303 & 158 & 52.0 & 1.9 & 303 & 154 & 50.7 & 2.0 \\
\hline & $\mathrm{Cr}$ & 83 & 6.84 & 8.26 & 12.1 & 83 & 2.71 & 3.27 & 30.6 \\
\hline & $\mathrm{F}^{*}$ & 121 & 34.9 & 28.8 & 3.5 & 121 & 25.0 & 20.7 & 4.8 \\
\hline & $\mathrm{Fe}$ & 5812 & 2.38 & 0.04 & 2442 & 5685 & 1.85 & 0.03 & 3079 \\
\hline & $\mathrm{K}$ & 6259 & 182 & 2.91 & 34.4 & 0 & 15.4 & NC & NC \\
\hline & $\mathrm{Li}$ & 0 & $<0.10$ & NC & NC & 0 & $<0.10$ & $\mathrm{NC}$ & NC \\
\hline & $\mathrm{Mg}$ & 1351 & 0.13 & 0.01 & 10395 & 1324 & 0.18 & 0.01 & 7547 \\
\hline & $\mathrm{Na}$ & 20431 & 181 & 0.88 & 113 & 23868 & 202 & 0.84 & 118 \\
\hline & $\mathrm{Ni}$ & 12 & $<0.10$ & $<1.0$ & $>120$ & 12 & $<0.10$ & $<1.0$ & $>120$ \\
\hline & $\mathrm{P}$ & 79 & 0.41 & 0.52 & 192 & 79 & 0.37 & 0.46 & 216 \\
\hline & $\mathrm{Pb}$ & 14 & 0.19 & 1.35 & 74.3 & 14 & 0.18 & 1.31 & 76.3 \\
\hline & $\operatorname{Re}$ & 129 & 82.2 & 63.7 & 1.6 & 129 & 74.33 & 57.54 & 1.7 \\
\hline & S* & 212 & 15.2 & 7.17 & 13.9 & 249 & 18.7 & 7.51 & 13.3 \\
\hline & Si & 30367 & 11.0 & 0.04 & 2769 & 31839 & 13.81 & 0.04 & 2306 \\
\hline & $\mathrm{Ti}$ & 1271 & 3.05 & 0.24 & 417 & 1243 & 3.08 & 0.25 & 404 \\
\hline & $\mathrm{Zn}$ & 4257 & 8.32 & 0.20 & 512 & 4172 & 9.38 & 0.22 & 445 \\
\hline & $\mathrm{Zr}$ & 3362 & 0.64 & 0.02 & 5280 & 3295 & 0.55 & 0.02 & 6009 \\
\hline \multirow{4}{*}{ 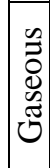 } & B & 4694 & 1.11 & 0.02 & 4245 & 4595 & 9.77 & 0.21 & 470 \\
\hline & $\mathrm{Cl}$ & 303 & 1.35 & 0.45 & 224 & 303 & 0.37 & 0.12 & 813 \\
\hline & $\mathrm{F}$ & 121 & 0.40 & 0.33 & 300 & 121 & 2.37 & 1.95 & 51.2 \\
\hline & $S$ & 212 & 0.19 & 0.09 & 1106 & 249 & 0.18 & 0.07 & 1364 \\
\hline
\end{tabular}

$\$$ - From gravimetric analysis of filters and particulate nitric acid rinses.

* - Based on analysis of water dissolution of filter particulate and acid dissolution of front-half rinse.

\# - Feed rate calculated from target composition and production rate. 
The Catholic University of America Vitreous State Laboratory
DuraMelter 100 Tests to Support LAW Glass Formulation Correlation Developmen

Final Report, VSL-06R6480-1, Rev. 0

Table 5.1. Results from Melter Off-Gas Emission Samples (continued).

\begin{tabular}{|c|c|c|c|c|c|c|c|c|c|}
\hline & \multicolumn{4}{|c|}{ Test 2A } & \multicolumn{4}{|c|}{ Test 2B } \\
\hline & & \multicolumn{4}{|c|}{$\begin{array}{c}06 / 22 / 0518: 48-19: 48 \\
\text { 97.4\% Isokinetic, } 11.9 \% \text { Moisture, } 68.8 \mathrm{dscfm}\end{array}$} & \multicolumn{4}{|c|}{$\begin{array}{c}06 / 24 / 0518: 22-19: 22 \\
\text { 96.7\% Isokinetic, } 11.7 \% \text { Moisture, } 62.4 \mathrm{dscfm} \\
\end{array}$} \\
\hline & & $\begin{array}{c}\text { Feed } \\
\text { Rate }^{\#} \\
(\mathrm{mg} / \mathrm{min})\end{array}$ & $\begin{array}{c}\text { Emissions } \\
\text { Rate } \\
\text { (mg/min) }\end{array}$ & $\%$ Feed & DF & $\begin{array}{c}\text { Feed } \\
\text { Rate }^{\#} \\
(\mathrm{mg} / \mathrm{min})\end{array}$ & $\begin{array}{c}\text { Emissions } \\
\text { Rate } \\
\text { (mg/min) }\end{array}$ & $\%$ Feed & DF \\
\hline \multirow{21}{*}{ 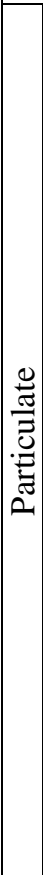 } & Total $^{\$}$ & 123482 & 945 & 0.77 & 131 & 122876 & 992 & 0.81 & 124 \\
\hline & $\mathrm{Al}$ & 4749 & 9.55 & 0.20 & 498 & 4789 & 4.83 & 0.10 & 992 \\
\hline & B & 4571 & 37.3 & 0.82 & 123 & 4604 & 43.7 & 0.95 & 105 \\
\hline & $\mathrm{Ca}$ & 2640 & 2.64 & 0.10 & 1002 & 3906 & 4.71 & 0.12 & 830 \\
\hline & $\mathrm{Cl}^{*}$ & 303 & 141 & 46.5 & 2.1 & 303 & 142 & 46.9 & 2.1 \\
\hline & $\mathrm{Cr}$ & 83 & 3.22 & 3.88 & 25.8 & 83 & 4.04 & 4.88 & 20.5 \\
\hline & $\mathrm{F}^{*}$ & 121 & 26.7 & 22.1 & 4.5 & 121 & 25.8 & 21.3 & 4.7 \\
\hline & $\mathrm{Fe}$ & 5663 & 3.07 & 0.05 & 1844 & 5716 & 4.74 & 0.08 & 1206 \\
\hline & $\mathrm{K}$ & 679 & 20.7 & 3.05 & 32.7 & 679 & 23.8 & 3.50 & 28.6 \\
\hline & $\mathrm{Li}$ & 0 & $<0.10$ & NC & $\mathrm{NC}$ & 345 & 2.31 & 0.67 & 149 \\
\hline & $\mathrm{Mg}$ & 1314 & 0.15 & 0.01 & 8666 & 1324 & 0.27 & 0.02 & 4975 \\
\hline & $\mathrm{Na}$ & 23733 & 208 & 0.88 & 114 & 21285 & 209 & 0.98 & 102 \\
\hline & $\mathrm{Ni}$ & 12 & $<0.10$ & $<1.00$ & $>120$ & 12 & $<0.10$ & $<1.00$ & $>120$ \\
\hline & $\mathrm{P}$ & 79 & 0.35 & 0.44 & 228 & 79 & 0.45 & 0.57 & 176 \\
\hline & $\mathrm{Pb}$ & 14 & 0.14 & 0.99 & 101 & 14 & 0.16 & 1.14 & 87.5 \\
\hline & $\operatorname{Re}$ & 129 & 77.5 & 59.97 & 1.7 & 129 & 70.3 & 54.44 & 1.8 \\
\hline & $\mathrm{S}^{*}$ & 249 & 21.5 & 8.63 & 11.6 & 279 & 22.6 & 8.10 & 12.3 \\
\hline & $\mathrm{Si}$ & 31669 & 18.5 & 0.06 & 1713 & 31846 & 27.9 & 0.09 & 1143 \\
\hline & $\mathrm{Ti}$ & 1234 & 3.16 & 0.26 & 391 & 1243 & 5.72 & 0.46 & 218 \\
\hline & Zn & 4147 & 8.98 & 0.22 & 462 & 4172 & 16.5 & 0.39 & 253 \\
\hline & $\mathrm{Zr}$ & 3273 & 0.75 & 0.02 & 4368 & 3295 & 1.51 & 0.05 & 2176 \\
\hline \multirow{4}{*}{ 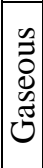 } & B & 4571 & 9.32 & 0.20 & 491 & 4604 & 9.93 & 0.22 & 464 \\
\hline & $\mathrm{Cl}$ & 303 & 0.28 & 0.09 & 1084 & 303 & 0.24 & 0.08 & 1244 \\
\hline & $F$ & 121 & 1.60 & 1.32 & 75.8 & 121 & 2.43 & 2.01 & 449.8 \\
\hline & $S$ & 249 & 0.13 & 0.05 & 1948 & 279 & 0.14 & 0.05 & 2039 \\
\hline
\end{tabular}

$\$$ - From gravimetric analysis of filters and particulate nitric acid rinses.

* - Based on analysis of water dissolution of filter particulate and acid dissolution of front-half rinse.

\# - Feed rate calculated from target composition and production rate. 
The Catholic University of America Vitreous State Laboratory
DuraMelter 100 Tests to Support LAW Glass Formulation Correlation Development

Final Report, VSL-06R6480-1, Rev. 0

Table 5.1. Results from Melter Off-Gas Emission Samples (continued).

\begin{tabular}{|c|c|c|c|c|c|c|c|c|c|}
\hline & \multicolumn{4}{|c|}{ Test 2C } & \multicolumn{4}{|c|}{ Test 2D } \\
\hline & & \multicolumn{4}{|c|}{$\begin{array}{c}\text { 06/27/05 09:03 - 10:06 } \\
\text { 95.0\% Isokinetic, 10.8\% Moisture, } 69.5 \mathrm{dscfm}\end{array}$} & \multicolumn{4}{|c|}{$\begin{array}{c}06 / 29 / 05 \text { 14:05 - 15:05 } \\
\text { 97.4\% Isokinetic, } 11.5 \% \text { Moisture, } 68.7 \mathrm{dscfm} \\
\end{array}$} \\
\hline & & $\begin{array}{c}\text { Feed } \\
\text { Rate }^{\#} \\
(\mathrm{mg} / \mathrm{min})\end{array}$ & $\begin{array}{c}\text { Emissions } \\
\text { Rate } \\
\text { (mg/min) }\end{array}$ & $\%$ Feed & DF & $\begin{array}{c}\text { Feed } \\
\text { Rate }^{\#} \\
(\mathrm{mg} / \mathrm{min})\end{array}$ & $\begin{array}{c}\text { Emissions } \\
\text { Rate } \\
\text { (mg/min) }\end{array}$ & $\%$ Feed & DF \\
\hline \multirow{21}{*}{ 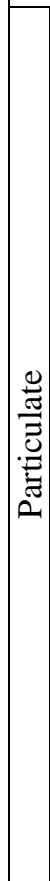 } & Total $^{\$}$ & 117707 & 1062 & 0.90 & 111 & 124848 & 839 & 0.67 & 149 \\
\hline & $\mathrm{Al}$ & 4805 & 15.0 & 0.31 & 322 & 4813 & 3.95 & 0.08 & 1217 \\
\hline & B & 4623 & 31.2 & 0.68 & 148 & 4633 & 11.9 & 0.26 & 389 \\
\hline & $\mathrm{Ca}$ & 5898 & 12.0 & 0.20 & 491 & 6817 & 5.84 & 0.09 & 1166 \\
\hline & $\mathrm{Cl}^{*}$ & 303 & 161 & 53.1 & 1.9 & 303 & 152 & 50.2 & 2.0 \\
\hline & $\mathrm{Cr}$ & 83 & 2.91 & 3.51 & 28.5 & 83 & 5.01 & 6.05 & 16.5 \\
\hline & $\mathrm{F}^{*}$ & 121 & 13.3 & 11.0 & 9.1 & 121 & 12.5 & 10.3 & 9.7 \\
\hline & $\mathrm{Fe}$ & 5727 & 7.36 & 0.13 & 778 & 5738 & 2.99 & 0.05 & 1920 \\
\hline & $\mathrm{K}$ & 679 & 24.9 & 3.67 & 27.2 & 679 & 28.8 & 4.24 & 23.6 \\
\hline & $\mathrm{Li}$ & 1674 & 6.34 & 0.38 & 264 & 2229 & 8.64 & 0.39 & 258 \\
\hline & $\mathrm{Mg}$ & 1333 & 0.47 & 0.04 & 2832 & 1360 & 0.20 & 0.01 & 6816 \\
\hline & $\mathrm{Na}$ & 17758 & 191 & 1.08 & 92.9 & 15186 & 159 & 1.05 & 95.5 \\
\hline & $\mathrm{Ni}$ & 12 & $<0.10$ & $<1.00$ & $>120$ & 12 & $<0.10$ & $<1.00$ & $>120$ \\
\hline & $\mathrm{P}$ & 79 & 0.42 & 0.53 & 187 & 79 & 0.14 & 0.17 & 576 \\
\hline & $\mathrm{Pb}$ & 14 & 0.14 & 1.02 & 97.8 & 14 & $<0.10$ & $<1.00$ & $>140$ \\
\hline & $\operatorname{Re}$ & 129 & 87.1 & 67.40 & 1.5 & 129 & 88.3 & 68.35 & 1.5 \\
\hline & S* & 322 & 26.6 & 8.26 & 12.1 & 358 & 41.0 & 11.5 & 8.7 \\
\hline & Si & 31287 & 50.6 & 0.16 & 618 & 31641 & 20.1 & 0.06 & 1575 \\
\hline & $\mathrm{Ti}$ & 1252 & 6.45 & 0.51 & 194 & 1252 & 2.64 & 0.21 & 475 \\
\hline & $\mathrm{Zn}$ & 4196 & 18.8 & 0.45 & 224 & 4208 & 7.78 & 0.18 & 541 \\
\hline & $\mathrm{Zr}$ & 3306 & 2.34 & 0.07 & 1413 & 3317 & 0.59 & 0.02 & 5634 \\
\hline \multirow{4}{*}{ 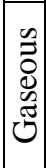 } & B & 4623 & 25.1 & 0.54 & 184 & 4633 & 28.2 & 0.61 & 164 \\
\hline & $\mathrm{Cl}$ & 303 & 0.87 & 0.29 & 349 & 303 & 4.03 & 1.33 & 75.1 \\
\hline & $\mathrm{F}$ & 121 & 19.9 & 16.42 & 6.1 & 121 & 25.4 & 20.93 & 4.8 \\
\hline & $S$ & 322 & 2.84 & 0.88 & 113 & 358 & 2.03 & 0.57 & 177 \\
\hline
\end{tabular}

$\$$ - From gravimetric analysis of filters and particulate nitric acid rinses.

* - Based on analysis of water dissolution of filter particulate and acid dissolution of front-half rinse.

\# - Feed rate calculated from target composition and production rate. 
DuraMelter 100 Tests to Support LAW Glass Formulation Correlation Developmen Final Report, VSL-06R6480-1, Rev. 0

Table 5.1. Results from Melter Off-Gas Emission Samples (continued).

\begin{tabular}{|c|c|c|c|c|c|c|c|c|c|}
\hline & \multicolumn{4}{|c|}{ Test 3B } & \multicolumn{4}{|c|}{ Test 3C } \\
\hline & & \multicolumn{4}{|c|}{$\begin{array}{c}07 / 08 / 0518: 10 \text { - 19:10 } \\
96.0 \% \text { Isokinetic, } 10.6 \% \text { Moisture, } 69.8 \mathrm{dscfm}\end{array}$} & \multicolumn{4}{|c|}{$\begin{array}{c}\text { 07/10/05 13:35 - 14:37 } \\
\text { 94.7\% Isokinetic, 8.1\% Moisture, } 69.9 \text { dscfm }\end{array}$} \\
\hline & & $\begin{array}{c}\text { Feed } \\
\text { Rate }^{\#} \\
(\mathrm{mg} / \mathrm{min})\end{array}$ & $\begin{array}{c}\text { Emissions } \\
\text { Rate } \\
\text { (mg/min) }\end{array}$ & \% Feed & DF & $\begin{array}{c}\text { Feed } \\
\text { Rate }^{\#} \\
(\mathrm{mg} / \mathrm{min})\end{array}$ & $\begin{array}{c}\text { Emissions } \\
\text { Rate } \\
\text { (mg/min) }\end{array}$ & $\%$ Feed & DF \\
\hline \multirow{21}{*}{ 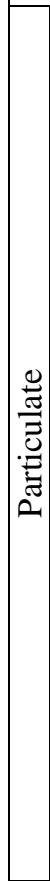 } & Total $^{\$}$ & 132549 & 514 & 0.39 & 258 & 136019 & 340 & 0.25 & 400 \\
\hline & $\mathrm{Al}$ & 4845 & 3.52 & 0.07 & 1375 & 4853 & 2.49 & 0.05 & 1952 \\
\hline & B & 4656 & 10.7 & 0.23 & 435 & 4670 & 5.95 & 0.13 & 785 \\
\hline & $\mathrm{Ca}$ & 7413 & 6.45 & 0.09 & 1149 & 7521 & 6.89 & 0.09 & 1092 \\
\hline & $\mathrm{Cl}^{*}$ & 303 & 67.4 & 22.2 & 4.5 & 303 & 21.8 & 7.19 & 13.9 \\
\hline & $\mathrm{Cr}$ & 83 & 1.71 & 2.06 & 48.5 & 83 & 1.19 & 1.44 & 69.5 \\
\hline & $\mathrm{F}^{*}$ & 121 & 3.0 & 2.48 & 40.3 & 121 & 2.3 & 1.90 & 52.6 \\
\hline & $\mathrm{Fe}$ & 5759 & 3.25 & 0.06 & 1771 & 5790 & 1.52 & 0.03 & 3802 \\
\hline & $\mathrm{K}$ & 679 & 13.2 & 1.95 & 51.4 & 679 & 10.1 & 1.49 & 66.9 \\
\hline & $\mathrm{Li}$ & 2869 & 6.23 & 0.22 & 461 & 2996 & 5.88 & 0.20 & 510 \\
\hline & $\mathrm{Mg}$ & 2154 & 0.32 & 0.01 & 6800 & 2684 & 0.28 & 0.01 & 9460 \\
\hline & $\mathrm{Na}$ & 10019 & 66.7 & 0.67 & 150 & 6414 & 35.7 & 0.56 & 180 \\
\hline & $\mathrm{Ni}$ & 12 & $<0.10$ & $<1.0$ & $>120$ & 12 & $<0.10$ & $<1.0$ & $>120$ \\
\hline & $\mathrm{P}$ & 79 & $<0.10$ & $<0.15$ & $>790$ & 79 & $<0.10$ & $<0.15$ & $>790$ \\
\hline & $\mathrm{Pb}$ & 14 & 0.12 & 0.88 & 114 & 14 & $<0.10$ & $<1.0$ & $>140$ \\
\hline & $\mathrm{Re}$ & 129 & 74.9 & 57.94 & 1.7 & 129 & 57.4 & 44.46 & 2.2 \\
\hline & $\mathrm{S}^{*}$ & 419 & 20.6 & 4.92 & 20.3 & 485 & 17.5 & 3.61 & 27.7 \\
\hline & $\mathrm{Si}$ & 33085 & 28.0 & 0.08 & 1181 & 34592 & 14.0 & 0.04 & 2466 \\
\hline & $\mathrm{Ti}$ & 1262 & 2.90 & 0.23 & 436 & 1262 & 2.27 & 0.18 & 555 \\
\hline & $\mathrm{Zn}$ & 4220 & 8.59 & 0.20 & 492 & 4233 & 8.20 & 0.19 & 516 \\
\hline & $\mathrm{Zr}$ & 3329 & 0.58 & 0.02 & 5715 & 3351 & 0.36 & 0.01 & 9239 \\
\hline \multirow{4}{*}{ 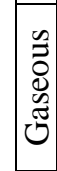 } & $\mathrm{B}$ & 4656 & 35.9 & 0.77 & 130 & 4670 & 43.1 & 0.92 & 109 \\
\hline & $\mathrm{Cl}$ & 303 & 55.5 & 18.34 & 5.5 & 303 & 146 & 48.30 & 2.1 \\
\hline & $\mathrm{F}$ & 121 & 37.6 & 31.06 & 3.2 & 121 & 41.3 & 34.12 & 2.9 \\
\hline & $\mathrm{S}$ & 419 & 45.4 & 10.85 & 9.2 & 485 & 62.7 & 12.92 & 7.7 \\
\hline
\end{tabular}

$\$$ - From gravimetric analysis of filters and particulate nitric acid rinses.

* - Based on analysis of water dissolution of filter particulate and acid dissolution of front-half rinse.

\# - Feed rate calculated from target composition and production rate. 
DuraMelter 100 Tests to Support LAW Glass Formulation Correlation Developmen Final Report, VSL-06R6480-1, Rev. 0

Table 5.1. Results from Melter Off-Gas Emission Samples (continued).

\begin{tabular}{|c|c|c|c|c|c|c|c|c|c|}
\hline & \multicolumn{4}{|c|}{ Test 4A } & \multicolumn{4}{|c|}{ Test 4B } \\
\hline & & \multicolumn{4}{|c|}{$\begin{array}{c}08 / 16 / 05 \text { 06:00 - 07:00 } \\
\text { 98.1\% Isokinetic, } 12.4 \% \text { Moisture, } 69.3 \mathrm{dscfm}\end{array}$} & \multicolumn{4}{|c|}{$\begin{array}{c}\text { 08/18/05 12:13 - 13:13 } \\
\text { 98.3\% Isokinetic, } 11.0 \% \text { Moisture, } 67.6 \mathrm{dscfm} \\
\end{array}$} \\
\hline & & $\begin{array}{c}\text { Feed } \\
\text { Rate }^{\#} \\
(\mathrm{mg} / \mathrm{min})\end{array}$ & $\begin{array}{c}\text { Emissions } \\
\text { Rate } \\
\text { (mg/min) }\end{array}$ & \% Feed & DF & $\begin{array}{c}\text { Feed } \\
\text { Rate }^{\#} \\
(\mathrm{mg} / \mathrm{min})\end{array}$ & $\begin{array}{c}\text { Emissions } \\
\text { Rate } \\
\text { (mg/min) }\end{array}$ & \% Feed & DF \\
\hline \multirow{21}{*}{ 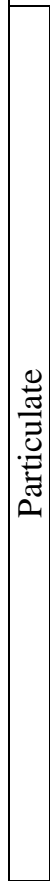 } & Total $^{\$}$ & 131193 & 587 & 0.45 & 223 & 130739 & 938 & 0.72 & 139 \\
\hline & $\mathrm{Al}$ & 4749 & 1.38 & 0.03 & 3437 & 4741 & 0.85 & 0.02 & 5578 \\
\hline & B & 4571 & 11.6 & 0.25 & 393 & 4557 & 19.7 & 0.43 & 231 \\
\hline & $\mathrm{Ca}$ & 7358 & 2.81 & 0.04 & 2616 & 7261 & 1.33 & 0.02 & 5450 \\
\hline & $\mathrm{Cl}^{*}$ & 1665 & 88.5 & 5.32 & 18.8 & 1665 & 266 & 16.0 & 6.3 \\
\hline & $\mathrm{Cr}$ & 83 & 2.06 & 2.48 & 40.3 & 83 & 1.91 & 2.30 & 43.5 \\
\hline & $\mathrm{F}^{*}$ & 1968 & 36.8 & 1.87 & 53.5 & 1968 & 42.5 & 2.16 & 46.3 \\
\hline & $\mathrm{Fe}$ & 5674 & 1.54 & 0.03 & 3686 & 5642 & 0.77 & 0.01 & 7283 \\
\hline & $\mathrm{K}$ & 666 & 23.5 & 3.53 & 28.3 & 666 & 38.8 & 5.83 & 17.2 \\
\hline & $\mathrm{Li}$ & 2933 & 13.0 & 0.44 & 226 & 2806 & 17.4 & 0.62 & 161.0 \\
\hline & $\mathrm{Mg}$ & 2620 & 0.18 & 0.01 & 14632 & 2109 & $<0.10$ & $<0.01$ & $>21090$ \\
\hline & $\mathrm{Na}$ & 6279 & 82.6 & 1.32 & 76.0 & 9806 & 162 & 1.66 & 60.4 \\
\hline & $\mathrm{Ni}$ & 12 & $<0.10$ & $<1.0$ & $>120$ & 12 & $<0.10$ & $<1.0$ & $>120$ \\
\hline & $\mathrm{P}$ & 79 & 0.29 & 0.36 & 278 & 79 & 0.39 & 0.49 & 204 \\
\hline & $\mathrm{Pb}$ & 14 & 0.35 & 2.48 & 40.3 & 14 & 0.54 & 3.86 & 25.9 \\
\hline & $\mathrm{Re}$ & 129 & 68.1 & 52.67 & 1.9 & 129 & 90.3 & 69.91 & 1.4 \\
\hline & S* & 473 & 28.3 & 5.98 & 16.7 & 406 & 40.8 & 10.0 & 10.0 \\
\hline & $\mathrm{Si}$ & 33849 & 9.91 & 0.03 & 3415 & 32377 & 5.39 & 0.02 & 6006 \\
\hline & $\mathrm{Ti}$ & 1234 & 1.19 & 0.10 & 1035 & 1234 & 0.67 & 0.05 & 1833 \\
\hline & $\mathrm{Zn}$ & 4135 & 19.7 & 0.48 & 210 & 4123 & 17.2 & 0.42 & 240 \\
\hline & $\mathrm{Zr}$ & 3273 & 0.20 & 0.01 & 16459 & 3250 & 0.13 & 0.00 & 24629 \\
\hline \multirow{4}{*}{ 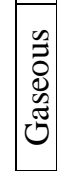 } & $\mathrm{B}$ & 4571 & 53.0 & 1.16 & 86.3 & 4557 & 16.4 & 0.36 & 278 \\
\hline & $\mathrm{Cl}$ & 1665 & 651 & 39.09 & 2.6 & 1665 & 444 & 26.66 & 3.8 \\
\hline & $\mathrm{F}$ & 1968 & 231 & 11.76 & 8.5 & 1968 & 221 & 11.23 & 8.9 \\
\hline & $\mathrm{S}$ & 473 & 66.9 & 14.12 & 7.1 & 406 & 43.6 & 10.74 & 9.3 \\
\hline
\end{tabular}

$\$$ - From gravimetric analysis of filters and particulate nitric acid rinses.

* - Based on analysis of water dissolution of filter particulate and acid dissolution of front-half rinse.

\# - Feed rate calculated from target composition and production rate. 
DuraMelter 100 Tests to Support LAW Glass Formulation Correlation Developmen Final Report, VSL-06R6480-1, Rev. 0

Table 5.1. Results from Melter Off-Gas Emission Samples (continued).

\begin{tabular}{|c|c|c|c|c|c|c|c|c|c|}
\hline & \multirow{2}{*}{\multicolumn{4}{|c|}{$\begin{array}{c}\text { Test 4C } \\
\text { 08/20/05 15:55 - 16:22 } \\
\text { 99.1\% Isokinetic, 12.6\% Moisture, } 69.4 \mathrm{dscfm}\end{array}$}} & \\
\hline & & & & & & \multicolumn{4}{|c|}{$\begin{array}{c}08 / 26 / 0520: 59-21: 29 \\
99.0 \% \text { Isokinetic, } 10.8 \% \text { Moisture, } 70.6 \mathrm{dscfm} \\
\end{array}$} \\
\hline & & $\begin{array}{c}\text { Feed } \\
\text { Rate }^{\#} \\
(\mathrm{mg} / \mathrm{min})\end{array}$ & $\begin{array}{c}\text { Emissions } \\
\text { Rate } \\
\text { (mg/min) }\end{array}$ & \% Feed & DF & $\begin{array}{c}\text { Feed } \\
\text { Rate }^{\#} \\
(\mathrm{mg} / \mathrm{min})\end{array}$ & $\begin{array}{c}\text { Emissions } \\
\text { Rate } \\
\text { (mg/min) }\end{array}$ & $\%$ Feed & DF \\
\hline \multirow{21}{*}{ 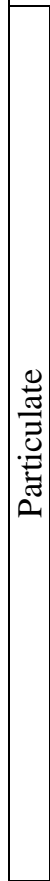 } & Total $^{\$}$ & 123872 & 1609 & 1.30 & 77.0 & 117754 & 1732 & 1.47 & 68.0 \\
\hline & $\mathrm{Al}$ & 4717 & 1.99 & 0.04 & 2368 & 4709 & 2.42 & 0.05 & 1950 \\
\hline & B & 4534 & 29.2 & 0.64 & 155 & 4524 & 43.9 & 0.97 & 103 \\
\hline & $\mathrm{Ca}$ & 6677 & 2.38 & 0.04 & 2803 & 5779 & 1.68 & 0.03 & 3445 \\
\hline & $\mathrm{Cl}^{*}$ & 1665 & 549 & 33.0 & 3.0 & 1665 & 627 & 37.66 & 2.7 \\
\hline & $\mathrm{Cr}$ & 83 & 5.73 & 6.91 & 14.5 & 83 & 3.66 & 4.42 & 22.6 \\
\hline & $\mathrm{F}^{*}$ & 1968 & 56.6 & 2.88 & 34.8 & 1968 & $\begin{array}{l}77.1 \\
\end{array}$ & 3.92 & 25.5 \\
\hline & $\mathrm{Fe}$ & 5621 & 0.77 & 0.01 & 7320 & 5610 & 0.87 & 0.02 & 6413 \\
\hline & $\mathrm{K}$ & 666 & 66.4 & 9.97 & 10.0 & 666 & 63.8 & 9.57 & 10.4 \\
\hline & $\mathrm{Li}$ & 2180 & 21.4 & 0.98 & 102 & 1639 & 14.9 & 0.91 & 110 \\
\hline & $\mathrm{Mg}$ & 1333 & 0.19 & 0.01 & 7057 & 1305 & $<0.10$ & $<0.01$ & $>13050$ \\
\hline & $\mathrm{Na}$ & 14860 & 373 & 2.51 & 39.8 & 17387 & 404 & 2.32 & 43.1 \\
\hline & $\mathrm{Ni}$ & 12 & 0.17 & 1.44 & 69.4 & 12 & 0.12 & 1.03 & 97.4 \\
\hline & $\mathrm{P}$ & 79 & 4.12 & 5.19 & 19.3 & 79 & 2.32 & 2.93 & 34.2 \\
\hline & $\mathrm{Pb}$ & 14 & 0.29 & 2.09 & 47.9 & 14 & 0.26 & 1.85 & 54.1 \\
\hline & $\mathrm{Re}$ & 129 & 95.1 & 73.64 & 1.4 & 129 & 93.8 & 72.62 & 1.4 \\
\hline & S* & 352 & 49.0 & 13.92 & 7.2 & 315 & 45.2 & 14.35 & 7.0 \\
\hline & $\mathrm{Si}$ & 30969 & 7.32 & 0.02 & 4234 & 30615 & 7.44 & 0.02 & 4115 \\
\hline & $\mathrm{Ti}$ & 1225 & 1.24 & 0.10 & 989 & 1225 & 1.31 & 0.11 & 939 \\
\hline & $\mathrm{Zn}$ & 4111 & 8.72 & 0.21 & 472 & 4099 & 6.75 & 0.16 & 607 \\
\hline & $\mathrm{Zr}$ & 3239 & 0.33 & 0.01 & 9901 & 3228 & 0.26 & 0.01 & 12417 \\
\hline \multirow{4}{*}{$\begin{array}{l}0 \\
0 \\
d \\
0 \\
0 \\
0\end{array}$} & B & 4534 & 16.0 & 0.35 & 284 & 4524 & 9.73 & 0.22 & 465 \\
\hline & $\mathrm{Cl}$ & 1665 & 272 & 16.34 & 6.1 & 1665 & 185 & 11.13 & 9.0 \\
\hline & $\mathrm{F}$ & 1968 & 309 & 15.68 & 6.4 & 1968 & 292 & 14.86 & 6.7 \\
\hline & $\mathrm{S}$ & 352 & 4.55 & 1.29 & 77.4 & 315 & 6.56 & 2.08 & 48.1 \\
\hline
\end{tabular}

$\$$ - From gravimetric analysis of filters and particulate nitric acid rinses.

* - Based on analysis of water dissolution of filter particulate and acid dissolution of front-half rinse.

\# - Feed rate calculated from target composition and production rate. 
DuraMelter 100 Tests to Support LAW Glass Formulation Correlation Developmen Final Report, VSL-06R6480-1, Rev. 0

Table 5.1. Results from Melter Off-Gas Emission Samples (continued).

\begin{tabular}{|c|c|c|c|c|c|c|c|c|c|}
\hline & \multicolumn{4}{|c|}{ Test 5C } & \multicolumn{4}{|c|}{ Test 5D } \\
\hline & & \multicolumn{4}{|c|}{$\begin{array}{c}\text { 08/29/05 13:49 - 14:29 } \\
\text { 99.3\% Isokinetic, } 11.8 \% \text { Moisture, } 67.4 \mathrm{dscfm}\end{array}$} & \multicolumn{4}{|c|}{$\begin{array}{c}08 / 31 / 0516: 04-16: 39 \\
\text { 99.1\% Isokinetic, 11.0\% Moisture, } 69.4 \mathrm{dscfm}\end{array}$} \\
\hline & & $\begin{array}{c}\text { Feed } \\
\text { Rate }^{\#} \\
(\mathrm{mg} / \mathrm{min})\end{array}$ & $\begin{array}{c}\text { Emissions } \\
\text { Rate } \\
\text { (mg/min) }\end{array}$ & \% Feed & DF & $\begin{array}{c}\text { Feed } \\
\text { Rate }^{\#} \\
(\mathrm{mg} / \mathrm{min})\end{array}$ & $\begin{array}{c}\text { Emissions } \\
\text { Rate } \\
\text { (mg/min) }\end{array}$ & \% Feed & DF \\
\hline \multirow{21}{*}{ 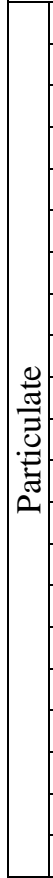 } & Total $^{\$}$ & 121567 & 2747 & 2.26 & 44.2 & 121064 & 1891 & 1.56 & 64.0 \\
\hline & $\mathrm{Al}$ & 4693 & 12.7 & 0.27 & 370 & 4653 & 9.55 & 0.21 & 487 \\
\hline & B & 4506 & 57.9 & 1.29 & 77.8 & 4473 & 54.7 & 1.22 & 81.8 \\
\hline & $\mathrm{Ca}$ & 3820 & 20.5 & 0.54 & 187 & 2586 & 3.07 & 0.12 & 844 \\
\hline & $\mathrm{Cl}^{*}$ & 1665 & 692 & 41.6 & 2.4 & 1665 & 644 & 38.7 & 2.59 \\
\hline & $\mathrm{Cr}$ & 83 & 7.61 & 9.18 & 10.9 & 83 & 3.93 & 4.74 & 21.1 \\
\hline & $\mathrm{F}^{*}$ & 1968 & 77.9 & 3.96 & 25.3 & 1968 & 84.4 & 4.29 & 23.3 \\
\hline & $\mathrm{Fe}$ & 5589 & 17.4 & 0.31 & 322 & 5536 & 3.69 & 0.07 & 1501 \\
\hline & $\mathrm{K}$ & 666 & 81.2 & 12.19 & 8.2 & 654 & 51.1 & 7.82 & 12.8 \\
\hline & $\mathrm{Li}$ & 338 & 10.5 & 3.12 & 32.1 & 0 & 0.53 & NC & NC \\
\hline & $\mathrm{Mg}$ & 1296 & 0.92 & 0.07 & 1403 & 1287 & 0.15 & 0.01 & 8406 \\
\hline & $\mathrm{Na}$ & 20836 & 636 & 3.05 & 32.7 & 23228 & 496 & 2.13 & 46.9 \\
\hline & $\mathrm{Ni}$ & 12 & 0.23 & 1.96 & 50.9 & 12 & $<0.10$ & $<1.0$ & $>120$ \\
\hline & $\mathrm{P}$ & 79 & 2.14 & 2.70 & 37.0 & 79 & 1.50 & 1.89 & 53.0 \\
\hline & $\mathrm{Pb}$ & 14 & 0.50 & 3.59 & 27.9 & 14 & 0.23 & 1.64 & 60.9 \\
\hline & $\operatorname{Re}$ & 129 & 107 & 83.06 & 1.2 & 129 & 73.0 & 56.52 & 1.8 \\
\hline & S* & 273 & 42.8 & 15.68 & 6.4 & 243 & 26.7 & 10.99 & 9.1 \\
\hline & $\mathrm{Si}$ & 31167 & 95.7 & 0.31 & 326 & 30997 & 23.2 & 0.07 & 1338 \\
\hline & $\mathrm{Ti}$ & 1216 & 10.2 & 0.84 & 119 & 1207 & 4.53 & 0.38 & 266 \\
\hline & $\mathrm{Zn}$ & 4074 & 32.6 & 0.80 & 125 & 4062 & 12.5 & 0.31 & 325 \\
\hline & $\mathrm{Zr}$ & 3217 & 1.62 & 0.05 & 1985 & 3205 & 0.68 & 0.02 & 4685 \\
\hline \multirow{4}{*}{ ט } & B & 4506 & 12.2 & 0.27 & 371 & 4473 & 9.75 & 0.22 & 459 \\
\hline & $\mathrm{Cl}$ & 1665 & 32.2 & 1.93 & 51.7 & 1665 & 33.3 & 2.00 & 50.0 \\
\hline & $\mathrm{F}$ & 1968 & 151 & 7.65 & 13.1 & 1968 & 147 & 7.45 & 13.4 \\
\hline & $S$ & 273 & 1.76 & 0.64 & 155 & 243 & 0.80 & 0.33 & 305 \\
\hline
\end{tabular}

$\$$ - From gravimetric analysis of filters and particulate nitric acid rinses.

* - Based on analysis of water dissolution of filter particulate and acid dissolution of front-half rinse.

\# - Feed rate calculated from target composition and production rate. 
DuraMelter 100 Tests to Support LAW Glass Formulation Correlation Developmen Final Report, VSL-06R6480-1, Rev. 0

Table 5.1. Results from Melter Off-Gas Emission Samples (continued).

\begin{tabular}{|c|c|c|c|c|c|c|c|c|c|}
\hline & \multicolumn{4}{|c|}{ Test 6A } & \multicolumn{4}{|c|}{ Test 6B } \\
\hline & & \multicolumn{4}{|c|}{$\begin{array}{c}09 / 14 / 0510: 00-10: 35 \\
\text { 103.5\% Isokinetic, } 11.6 \% \text { Moisture, } 67.1 \mathrm{dscfm}\end{array}$} & \multicolumn{4}{|c|}{$\begin{array}{c}09 / 16 / 0513: 10-13: 50 \\
102.4 \% \text { Isokinetic, 10.5\% Moisture, } 65.3 \mathrm{dscfm}\end{array}$} \\
\hline & & $\begin{array}{c}\text { Feed } \\
\text { Rate }^{\#} \\
(\mathrm{mg} / \mathrm{min})\end{array}$ & $\begin{array}{c}\text { Emissions } \\
\text { Rate } \\
\text { (mg/min) }\end{array}$ & $\%$ Feed & DF & $\begin{array}{c}\text { Feed } \\
\text { Rate }^{\#} \\
(\mathrm{mg} / \mathrm{min})\end{array}$ & $\begin{array}{c}\text { Emissions } \\
\text { Rate } \\
\text { (mg/min) }\end{array}$ & \% Feed & DF \\
\hline \multirow{21}{*}{ 莞 } & Total $^{\$}$ & 122332 & 2032 & 1.66 & 60.2 & 118035 & 2382 & 2.02 & 49.6 \\
\hline & $\mathrm{Al}$ & 4677 & 11.4 & 0.24 & 409 & 4773 & 2.98 & 0.06 & 1603 \\
\hline & B & 4496 & 56.1 & 1.25 & 80.1 & 4595 & 54.0 & 1.17 & 85.2 \\
\hline & $\mathrm{Ca}$ & 2608 & 4.05 & 0.16 & 644 & 2143 & 1.54 & 0.07 & 1395 \\
\hline & $\mathrm{Cl}^{*}$ & 1665 & 675 & 40.54 & 2.47 & 1665 & 693 & 41.6 & 2.4 \\
\hline & $\mathrm{Cr}$ & 83 & 4.06 & 4.90 & 20.4 & 83 & 6.88 & 8.30 & 12.0 \\
\hline & $\mathrm{F}^{*}$ & 1968 & 47.5 & 2.41 & 41.4 & 1968 & 102 & 5.18 & 19.3 \\
\hline & $\mathrm{Fe}$ & 5568 & 4.44 & 0.08 & 1253 & 5695 & 1.60 & 0.03 & 3566 \\
\hline & K & 0 & 5.77 & NC & NC & 6120 & 414 & 6.76 & 14.8 \\
\hline & $\mathrm{Li}$ & 0 & 0.23 & NC & NC & 0 & 0.14 & NC & NC \\
\hline & $\mathrm{Mg}$ & 1296 & 0.20 & 0.02 & 6517 & 1324 & 0.14 & 0.01 & 9476 \\
\hline & $\mathrm{Na}$ & 23363 & 520 & 2.23 & 44.9 & 20004 & 413 & 2.07 & 48.4 \\
\hline & $\mathrm{Ni}$ & 12 & 0.13 & 1.07 & 93.7 & 12 & 0.11 & 0.89 & 112 \\
\hline & $\mathrm{P}$ & 79 & 2.14 & 2.70 & 37.0 & 79 & 2.61 & 3.29 & 30.4 \\
\hline & $\mathrm{Pb}$ & 14 & 0.26 & 1.82 & 54.9 & 14 & 0.24 & 1.71 & 58.6 \\
\hline & $\operatorname{Re}$ & 129 & 79.3 & 61.39 & 1.6 & 129 & 99.8 & 77.23 & 1.3 \\
\hline & $\mathrm{S}^{*}$ & 243 & 25.3 & 10.41 & 9.6 & 212 & 18.3 & 8.63 & 11.6 \\
\hline & $\mathrm{Si}$ & 31160 & 30.9 & 0.10 & 1010 & 29723 & 11.2 & 0.04 & 2666 \\
\hline & $\mathrm{Ti}$ & 1216 & 4.95 & 0.41 & 246 & 1243 & 1.86 & 0.15 & 670 \\
\hline & $\mathrm{Zn}$ & 4074 & 15.0 & 0.37 & 271 & 4160 & 6.01 & 0.14 & 692 \\
\hline & $\mathrm{Zr}$ & 3217 & 0.72 & 0.02 & 4443 & 3284 & 0.29 & 0.01 & 11176 \\
\hline \multirow{4}{*}{ 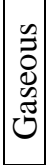 } & B & 4496 & 10.7 & 0.24 & 422 & 4595 & 7.86 & 0.17 & 585 \\
\hline & $\mathrm{Cl}$ & 1665 & 36.5 & 2.19 & 45.7 & 1665 & 29.5 & 1.77 & 56.5 \\
\hline & $\mathrm{F}$ & 1968 & 189 & 9.61 & 10.4 & 1968 & 74.5 & 3.78 & 26.4 \\
\hline & $\mathrm{S}$ & 243 & 1.12 & 0.46 & 217 & 212 & 0.60 & 0.28 & 354 \\
\hline
\end{tabular}

$\$$ - From gravimetric analysis of filters and particulate nitric acid rinses.

* - Based on analysis of water dissolution of filter particulate and acid dissolution of front-half rinse.

\# - Feed rate calculated from target composition and production rate. 
Table 5.2. Average and Range of Concentrations (ppmv) for Selected Species in Off-Gas Measured by FTIR Spectroscopy.

\begin{tabular}{|c|c|c|c|c|c|c|c|c|c|c|c|c|}
\hline \multirow{2}{*}{$\begin{array}{c}\text { Test } \\
-\end{array}$} & \multicolumn{2}{|r|}{$1 \mathrm{~A}$} & \multicolumn{2}{|r|}{$1 \mathrm{~B}$} & \multicolumn{2}{|r|}{$2 \mathrm{~A}$} & \multicolumn{2}{|r|}{$2 B$} & \multicolumn{2}{|r|}{$2 \mathrm{C}$} & \multicolumn{2}{|r|}{$2 \mathrm{D}$} \\
\hline & Avg. & Range & Avg. & Range & Avg. & Range & Avg. & Range & Avg. & Range & Avg. & Range \\
\hline $\mathrm{N}_{2} \mathrm{O}$ & 96.4 & $65.9-139$ & 112 & $<1.0-277$ & 121 & $<1.0-538$ & 67.2 & $<1.0-95.7$ & 84.6 & $4.7-138$ & 79.7 & $22.1-186$ \\
\hline NO & 1179 & $914-1511$ & 1187 & $2.4-2792$ & 1225 & $2.8-4013$ & 922 & $4.3-1346$ & 840 & $54.5-1282$ & 1085 & $603-2053$ \\
\hline $\mathrm{NO}_{2}$ & 132 & $71.7-178$ & 119 & $<1.0-412$ & 126 & $3.2-781$ & 89.6 & $<1.0-153$ & 52.1 & $2.5-108$ & 116 & $46.0-330$ \\
\hline $\mathrm{NH}_{3}$ & 39.9 & $21.0-65.0$ & 45.0 & $3.2-147$ & 48.4 & $5.1-351$ & 51.6 & $5.7-80.0$ & 58.1 & $2.9-116$ & 21.9 & $<1.0-57.5$ \\
\hline $\mathrm{H}_{2} \mathrm{O}[\%]$ & 5.4 & $4.4-7.1$ & 6.1 & $1.1-9.7$ & 6.1 & $3.8-15.5$ & 5.2 & $1.6-6.0$ & 5.0 & $1.9-6.9$ & 5.3 & $1.5-10.3$ \\
\hline $\mathrm{CO}_{2}$ & 3000 & $2000-4000$ & 2000 & $0-5000$ & 3000 & $1000-9000$ & 2000 & $0-7000$ & 3000 & $1000-5000$ & 3000 & $2000-6000$ \\
\hline Nitrous Acid & $<1.0$ & $<1-1.2$ & $<1.0$ & $<1-1.7$ & $<1.0$ & $<1.0-3.4$ & $<1.0$ & $<1.0-1.0$ & $<1.0$ & NA & $<1.0$ & $<1.0-1.4$ \\
\hline Nitric Acid & $<1.0$ & NA & $<1.0$ & NA & $<1.0$ & $<1.0-1.3$ & $<1.0$ & NA & $<1.0$ & NA & $<1.0$ & NA \\
\hline $\mathrm{HCN}$ & $<1.0$ & NA & $<1.0$ & NA & $<1.0$ & NA & $<1.0$ & NA & $<1.0$ & NA & $<1.0$ & NA \\
\hline $\mathrm{SO}_{2}$ & $<5.0$ & NA & $<5.0$ & NA & $<5.0$ & NA & $<5.0$ & NA & $<5.0$ & NA & $<5.0$ & NA \\
\hline Acetonitrile & $<1.0$ & NA & $<1.0$ & NA & $<1.0$ & NA & $<1.0$ & NA & $<1.0$ & NA & $<1.0$ & NA \\
\hline Acrylonitrile & $<1.0$ & NA & $<1.0$ & NA & $<1.0$ & NA & $<1.0$ & NA & $<1.0$ & NA & $<1.0$ & NA \\
\hline $\mathrm{CO}$ & 41.8 & $28.7-60.6$ & 45.2 & $3.7-101$ & 41.9 & $1.1-137$ & 25.5 & $<1.0-35.7$ & 42.2 & $1.0-62.6$ & 36.3 & $2.1-90.3$ \\
\hline $\mathrm{HCl}$ & $<1.0$ & NA & $<1.0$ & NA & $<1.0$ & NA & $<1.0$ & NA & $<1.0$ & NA & $<1.0$ & NA \\
\hline $\mathrm{HF}$ & $<1.0$ & NA & $<1.0$ & NA & $<1.0$ & $<1-11.2$ & $<1.0$ & $<1-12.1$ & $<1.0$ & NA & $<1.0$ & $<1.0-1.3$ \\
\hline
\end{tabular}

"-" Empty data field 
Table 5.2. Average and Range of Concentrations (ppmv) for Selected Species in Off-Gas Measured by FTIR Spectroscopy (continued).

\begin{tabular}{|c|c|c|c|c|c|c|c|c|c|c|c|c|}
\hline \multirow{2}{*}{$\begin{array}{c}\text { Test } \\
- \\
\end{array}$} & \multicolumn{2}{|r|}{$3 \mathrm{~A}$} & \multicolumn{2}{|r|}{$3 \mathrm{~B}$} & \multicolumn{2}{|r|}{$3 C$} & \multicolumn{2}{|r|}{$4 \mathrm{~A}$} & \multicolumn{2}{|c|}{$4 \mathrm{~B}$} & \multicolumn{2}{|r|}{$4 \mathrm{C}$} \\
\hline & Avg. & Range & Avg. & Range & Avg. & Range & Avg. & Range & Avg. & Range & Avg. & Range \\
\hline $\mathrm{N}_{2} \mathrm{O}$ & 78.7 & $<1.0-251$ & 41.4 & $17.8-136$ & 0.7 & $<1.0-1.7$ & 0.6 & $<1.0-1.9$ & $<47.5$ & $<1.0-138$ & 73.9 & $<1.0-204$ \\
\hline $\mathrm{NO}$ & 1059 & $8.5-2135$ & 439 & $215-1156$ & 65 & 28.1 - 192 & 48.2 & $<1.0-158$ & 379 & $14.8-1064$ & 975 & $4.6-2021$ \\
\hline $\mathrm{NO}_{2}$ & 109 & $2.3-292$ & 17.4 & $4.1-132$ & 2.6 & $<1.0-12.1$ & $<1.0$ & $<1.0-8.6$ & 11.3 & $<1.0-61.5$ & 90.7 & $6.6-244$ \\
\hline $\mathrm{NH}_{3}$ & 22.1 & $1.8-80.3$ & 11.2 & $<1.0-65.7$ & 1.7 & $<1.0-3.3$ & $<1.0$ & NA & $<1.0$ & NA & $<1.0$ & NA \\
\hline $\mathrm{H}_{2} \mathrm{O}[\%]$ & 5.4 & $4.2-15.0$ & 5.2 & $1.8-8.6$ & 5.1 & $1.4-9.6$ & 6.0 & $4.6-11.5$ & 5.2 & $2.2-10.7$ & 5.5 & $1.7-11.1$ \\
\hline $\mathrm{CO}_{2}$ & 3000 & $1000-6000$ & 2000 & $1000-6000$ & 2000 & $1000-5000$ & 2000 & $1000-4000$ & $<2000$ & $<1.0-6000$ & 2000 & $1000-5000$ \\
\hline Nitrous Acid & $<1.0$ & $<1.0-1.3$ & $<1.0$ & NA & $<1.0$ & NA & $<1.0$ & NA & $<1.0$ & NA & $<1.0$ & $<1.0-1.3$ \\
\hline Nitric Acid & $<1.0$ & NA & $<1.0$ & NA & $<1.5$ & $<1.0-3.6$ & 3.1 & $<1.0-6.2$ & $<1.0$ & $<1.0-5.1$ & $<1.0$ & $<1.0-1.1$ \\
\hline $\mathrm{HCN}$ & $<1.0$ & NA & $<1.0$ & NA & $<1.0$ & NA & $<1.0$ & NA & $<1.0$ & NA & $<1.0$ & NA \\
\hline $\mathrm{SO}_{2}$ & $<5.0$ & NA & $<5.0$ & NA & $<5.0$ & NA & $<5.0$ & NA & $<5.0$ & NA & $<5.0$ & NA \\
\hline Acetonitrile & $<1.0$ & NA & $<1.0$ & NA & $<1.0$ & NA & $<1.0$ & NA & $<1.0$ & NA & $<1.0$ & NA \\
\hline Acrylonitrile & $<1.0$ & NA & $<1.0$ & NA & $<1.0$ & NA & $<1.0$ & NA & $<1.0$ & NA & $<1.0$ & NA \\
\hline $\mathrm{CO}$ & 37.8 & $2.1-113$ & 12.9 & $2.1-37.6$ & $<1 . .0$ & $<1.0-3.6$ & $<1.0$ & $<1.0-4.0$ & 14.4 & $3.1-51.9$ & 47.6 & $5.7-128$ \\
\hline $\mathrm{HCl}$ & $<1.0$ & NA & $<1.0$ & NA & $<1.0$ & NA & $<1.0$ & NA & $<1.0$ & NA & $<1.0$ & NA \\
\hline $\mathrm{HF}$ & $<1.0$ & $<1.0-1.5$ & $<1.0$ & $<1.0-1.2$ & 5.0 & $1.1-7.9$ & 10.6 & $<1.0-26.0$ & 19.7 & $4.9-39.5$ & 19.8 & $12.7-32.2$ \\
\hline
\end{tabular}

"-" Empty data field 
Table 5.2. Average and Range of Concentrations (ppmv) for Selected Species in Off-Gas Measured by FTIR Spectroscopy (continued).

\begin{tabular}{|c|c|c|c|c|c|c|c|c|c|c|c|c|}
\hline \multirow{2}{*}{$\begin{array}{c}\text { Test } \\
-\end{array}$} & \multicolumn{2}{|r|}{$5 \mathrm{~A}$} & \multicolumn{2}{|r|}{$5 B$} & \multicolumn{2}{|r|}{$5 \mathrm{C}$} & \multicolumn{2}{|r|}{$5 \mathrm{D}$} & \multicolumn{2}{|r|}{$6 \mathrm{~A}$} & \multicolumn{2}{|r|}{$6 \mathrm{~B}$} \\
\hline & Avg. & Range & Avg. & Range & Avg. & Range & Avg. & Range & Avg. & Range & Avg. & Range \\
\hline $\mathrm{N}_{2} \mathrm{O}$ & 81.7 & $33.6-233$ & 93.1 & $<1.0-267$ & 59.8 & $1.7-182$ & 124 & $4.6-727$ & 128 & $18.1-658$ & 107 & $<1.0-218$ \\
\hline $\mathrm{NO}$ & 1187 & $674-2368$ & 1025 & $35.0-2243$ & 936 & $31.6-2286$ & 813 & $63.3-3750$ & 1169 & $237-4144$ & 1223 & $1.1-2084$ \\
\hline $\mathrm{NO}_{2}$ & 123 & $56.8-317$ & 100 & $<1.0-403$ & 190 & $1.5-579$ & 407 & $3.3-920$ & 97.9 & $13.9-738$ & 166 & $3.3-543$ \\
\hline $\mathrm{NH}_{3}$ & $<1.0$ & NA & $<1.0$ & $<1.0-22.6$ & $<1.3$ & $<1.0-21.2$ & 7.5 & $<1.0-167$ & 24.0 & $<1.0-172$ & 4.8 & $<1.0-29.1$ \\
\hline $\mathrm{H}_{2} \mathrm{O}[\%]$ & 6.0 & $4.6-12.1$ & 5.5 & $1.4-10.3$ & 5.7 & $2.0-12.1$ & 5.8 & $2.1-11.7$ & 5.8 & $1.8-13.2$ & 5.3 & $2.1-8.0$ \\
\hline $\mathrm{CO}_{2}$ & 3000 & $2000-8000$ & 3000 & $1000-7000$ & 2000 & $1000-5000$ & 2000 & $<1.0-8000$ & 2000 & $1000-9000$ & 3000 & $1000-9000$ \\
\hline Nitrous Acid & $<1.0$ & $<1.0-2.0$ & $<1.0$ & $<1.0-1.4$ & $<1.0$ & $<1.0-2.6$ & 1.7 & $<1.0-5.0$ & 1.2 & $<1.0-4.2$ & $<1.0$ & $<1.0-3.0$ \\
\hline Nitric Acid & $<1.0$ & $<1.0-2.0$ & $<1.0$ & NA & $<1.0$ & NA & $<1.0$ & $<1.0-2.2$ & 1.7 & $<1.0-3.1$ & $<1.0$ & $<1.0-4.4$ \\
\hline HCN & $<1.0$ & NA & $<1.0$ & NA & $<1.0$ & NA & $<1.0$ & NA & $<1.0$ & NA & $<1.0$ & NA \\
\hline $\mathrm{SO}_{2}$ & $<5.0$ & NA & $<5.0$ & NA & $<5.0$ & NA & $<5.0$ & NA & $<5.0$ & NA & $<5.0$ & NA \\
\hline Acetonitrile & $<1.0$ & NA & $<1.0$ & NA & $<1.0$ & NA & $<1.0$ & NA & $<1.0$ & NA & $<1.0$ & NA \\
\hline Acrylonitrile & $<1.0$ & NA & $<1.0$ & NA & $<1.0$ & NA & $<1.0$ & NA & $<1.0$ & NA & $<1.0$ & NA \\
\hline $\mathrm{CO}$ & 51.7 & $14.7-148$ & 52.1 & $15.2-121$ & 36.4 & $<1.0-95.3$ & 52.2 & $3.2-178$ & 48.6 & $5.6-168$ & 40.6 & $3.2-94$ \\
\hline $\mathrm{HCl}$ & $<1.0$ & NA & $<1.0$ & NA & $<1.0$ & NA & $<1.0$ & NA & $<1.0$ & NA & $<1.0$ & NA \\
\hline $\mathrm{HF}$ & 16.7 & $5.9-26.1$ & 19.3 & $5.6-34.9$ & 8.0 & $<1.0-17.0$ & 11.4 & $<1.0-18.9$ & 12.9 & $1.2-27.4$ & 13.2 & $<1.0-49.1$ \\
\hline
\end{tabular}

"-" Empty data field 
Table 5.3. Average $\mathrm{NO}_{\mathrm{x}}$ Fluxes in Off-Gas Measured by FTIR Spectroscopy.

\begin{tabular}{|c|c|c|c|c|c|c|}
\hline \multirow{2}{*}{ Test } & \multirow{2}{*}{$\begin{array}{c}\text { Feed } \\
{[\mathrm{mol} / \mathrm{hr}]}\end{array}$} & $\begin{array}{c}\text { Wet gas flow } \\
\text { rate }[\mathrm{scfm}]\end{array}$ & \multirow{2}{*}{$\mathrm{N}_{2} \mathrm{O}[\mathrm{mol} / \mathrm{hr}]$} & $\mathrm{NO}[\mathrm{mol} / \mathrm{hr}]$ & $\mathrm{NO}_{2}[\mathrm{~mol} / \mathrm{hr}]$ & $\begin{array}{c}\text { \% Feed } \\
\text { Emitted as } \\
\text { Nitrogen Oxides }\end{array}$ \\
\hline 1A & 29.0 & 145 & 1.0 & 12.0 & 1.3 & 49.4 \\
\hline 1B & 31.4 & 143 & 1.1 & 12.0 & 1.2 & 45.6 \\
\hline 2A & 31.4 & 153 & 1.3 & 13.2 & 1.4 & 50.5 \\
\hline 2B & 21.8 & 158 & 0.7 & 10.3 & 1.0 & 55.1 \\
\hline 2C & 26.0 & 160 & 1.0 & 9.4 & 0.6 & 42.1 \\
\hline 2D & 21.1 & 161 & 0.9 & 12.3 & 1.3 & 68.7 \\
\hline 3A & 21.6 & 159 & 0.9 & 11.9 & 1.2 & 64.7 \\
\hline 3B & 11.2 & 151 & 0.4 & 4.7 & 0.2 & 47.3 \\
\hline 3C & 0.8 & 155 & $<0.1$ & 0.7 & $<0.1$ & 121.0 \\
\hline 4A & 0.8 & 156 & $<0.1$ & 0.5 & $<0.1$ & 89.3 \\
\hline 4B & 11.6 & 152 & 0.5 & 4.1 & 0.1 & 40.4 \\
\hline 4C & 21.4 & 145 & 0.8 & 9.9 & 0.9 & 54.1 \\
\hline 5A & 22.8 & 158 & 0.9 & 13.2 & 1.4 & 67.8 \\
\hline 5B & 26.1 & 149 & 1.0 & 10.7 & 1.0 & 48.8 \\
\hline 5C & 21.3 & 156 & 0.7 & 10.2 & 2.1 & 61.1 \\
\hline 5D & 31.2 & 158 & 1.4 & 9.0 & 4.5 & 47.7 \\
\hline 6A & 31.6 & 167 & 1.5 & 13.8 & 1.2 & 52.0 \\
\hline 6B & 29.4 & 164 & 1.2 & 14.1 & 1.9 & 58.7 \\
\hline
\end{tabular}


Table 5.4. Mass Balances for Rhenium, Sulfur, Chlorine, and Fluorine During DM100 Tests (\% of Feed).

\begin{tabular}{|c|c|c|c|c|c|c|}
\hline Test & Glass & Element & Glass & $\begin{array}{c}\text { Particle } \\
\text { Emissions }\end{array}$ & $\begin{array}{l}\text { Gaseous } \\
\text { Emissions }\end{array}$ & Total \\
\hline \multirow{2}{*}{$1 \mathrm{~A}$} & \multirow{2}{*}{ LAWE3 } & Rhenium & 20 & 63.7 & $<0.1$ & 83.7 \\
\hline & & Sulfur & 94.3 & 7.2 & 0.1 & 101.6 \\
\hline \multirow{2}{*}{$1 \mathrm{~B}$} & \multirow{2}{*}{$\begin{array}{l}\text { LAWE4H } \\
\text { without K }\end{array}$} & Rhenium & 35 & 57.5 & $<0.1$ & 92.5 \\
\hline & & Sulfur & 87.8 & 7.5 & 0.2 & 95.5 \\
\hline \multirow{2}{*}{$2 \mathrm{~A}$} & \multirow{2}{*}{ LAWE4H } & Rhenium & 35 & 60.0 & $<0.1$ & 95 \\
\hline & & Sulfur & 87.8 & 8.6 & 0.1 & 96.5 \\
\hline \multirow{2}{*}{$2 \mathrm{~B}$} & \multirow{2}{*}{ LAWE5H } & Rhenium & 35 & 54.4 & $<0.1$ & 89.4 \\
\hline & & Sulfur & 93.4 & 8.1 & 0.1 & 101.6 \\
\hline \multirow{2}{*}{$2 \mathrm{C}$} & \multirow{2}{*}{ LAWE6H } & Rhenium & 25 & 67.4 & $<0.1$ & 92.4 \\
\hline & & Sulfur & 84.9 & 8.3 & 0.9 & 94.1 \\
\hline \multirow{2}{*}{$2 \mathrm{D}$} & \multirow{2}{*}{ LAWE7H } & Rhenium & 20 & 68.4 & $<0.1$ & 88.4 \\
\hline & & Sulfur & 91.5 & 11.5 & 0.6 & 103.1 \\
\hline \multirow{2}{*}{$3 \mathrm{~B}$} & \multirow{2}{*}{ LAWE7H } & Rhenium & 35 & 57.9 & $<0.1$ & 92.9 \\
\hline & & Sulfur & 78.3 & 4.9 & 10.9 & 94.1 \\
\hline \multirow{2}{*}{$3 C$} & \multirow{2}{*}{ LAWE9H } & Rhenium & 40 & 44.5 & $<0.1$ & 84.5 \\
\hline & & Sulfur & 73.8 & 3.6 & 12.9 & 90.3 \\
\hline
\end{tabular}


The Catholic University of America Vitreous State Laboratory
DuraMelter 100 Tests to Support LAW Glass Formulation Correlation Development Final Report, VSL-06R6480-1, Rev. 0

Table 5.4. Mass Balances for Rhenium, Sulfur, Chlorine, and Fluorine During DM100 Tests (\% of Feed) (continued).

\begin{tabular}{|c|c|c|c|c|c|c|}
\hline Test & & Element & Glass & $\begin{array}{c}\text { Particle } \\
\text { Emissions }\end{array}$ & $\begin{array}{c}\text { Gaseous } \\
\text { Emissions }\end{array}$ & Total \\
\hline \multirow{4}{*}{$4 \mathrm{~A}$} & \multirow{4}{*}{ LAWE10H } & Rhenium & 30 & 52.7 & $<0.1$ & 82.7 \\
\hline & & Sulfur & 45 & 6.0 & 14.1 & 65.1 \\
\hline & & Chlorine & 37.3 & 5.3 & 39.1 & 81.7 \\
\hline & & Fluorine & 36.2 & 1.9 & 11.8 & 50.0 \\
\hline \multirow{4}{*}{$4 \mathrm{~B}$} & \multirow{4}{*}{ LAWE9H } & Rhenium & 35 & 69.1 & $<0.1$ & 104.1 \\
\hline & & Sulfur & 75.4 & 10.0 & 10.7 & 96.1 \\
\hline & & Chlorine & 60.9 & 16.0 & 26.7 & 103.6 \\
\hline & & Fluorine & 68.5 & 2.2 & 11.2 & 81.9 \\
\hline \multirow{4}{*}{$4 \mathrm{C}$} & \multirow{4}{*}{ LAWE7H } & Rhenium & 20 & 73.6 & $<0.1$ & 93.6 \\
\hline & & Sulfur & 84.7 & 13.9 & 1.3 & 99.9 \\
\hline & & Chlorine & 57.3 & 33.0 & 16.3 & 106.6 \\
\hline & & Fluorine & 68.0 & 2.9 & 15.7 & 86.3 \\
\hline \multirow{4}{*}{$5 \mathrm{~B}$} & \multirow{4}{*}{ LAWE6H } & Rhenium & 25 & 72.6 & $<0.1$ & 97.6 \\
\hline & & Sulfur & 83.0 & 14.4 & 0.1 & 97.5 \\
\hline & & Chlorine & 57.3 & 4.4 & 11.1 & 72.8 \\
\hline & & Fluorine & 74.6 & 3.9 & 14.9 & 93.4 \\
\hline \multirow{4}{*}{$5 \mathrm{C}$} & \multirow{4}{*}{ LAWE5H } & Rhenium & 30 & 83.1 & $<0.1$ & 113.1 \\
\hline & & Sulfur & 89.1 & 15.7 & 0.6 & 105.4 \\
\hline & & Chlorine & 59.1 & 41.6 & 1.9 & 102.6 \\
\hline & & Fluorine & 72.3 & 4.0 & 7.7 & 84.0 \\
\hline \multirow{4}{*}{$5 \mathrm{D}$} & \multirow{4}{*}{ LAWE4H } & Rhenium & 35 & 56.5 & $<0.1$ & 91.5 \\
\hline & & Sulfur & 82.9 & 11.0 & 0.6 & 94.5 \\
\hline & & Chlorine & 60.9 & 38.7 & 2.0 & 101.6 \\
\hline & & Fluorine & 75.4 & 4.3 & 7.5 & 87.2 \\
\hline \multirow{4}{*}{$6 \mathrm{~A}$} & \multirow{4}{*}{$\begin{array}{l}\text { LAWE4H } \\
\text { without K }\end{array}$} & Rhenium & 40 & 61.4 & $<0.1$ & 101.4 \\
\hline & & Sulfur & 82.9 & 10.4 & 10.9 & 104.2 \\
\hline & & Chlorine & 59.1 & 40.5 & 2.2 & 101.8 \\
\hline & & Fluorine & 74.6 & 2.4 & 9.6 & 86.6 \\
\hline \multirow{4}{*}{$6 \mathrm{~B}$} & \multirow{4}{*}{ LAWE3H } & Rhenium & 20 & 77.2 & $<0.1$ & 97.2 \\
\hline & & Sulfur & 94.3 & 8.6 & 12.9 & 115.8 \\
\hline & & Chlorine & 61.8 & 41.6 & 1.8 & 105.2 \\
\hline & & Fluorine & 75.4 & 5.2 & 3.8 & 84.4 \\
\hline
\end{tabular}




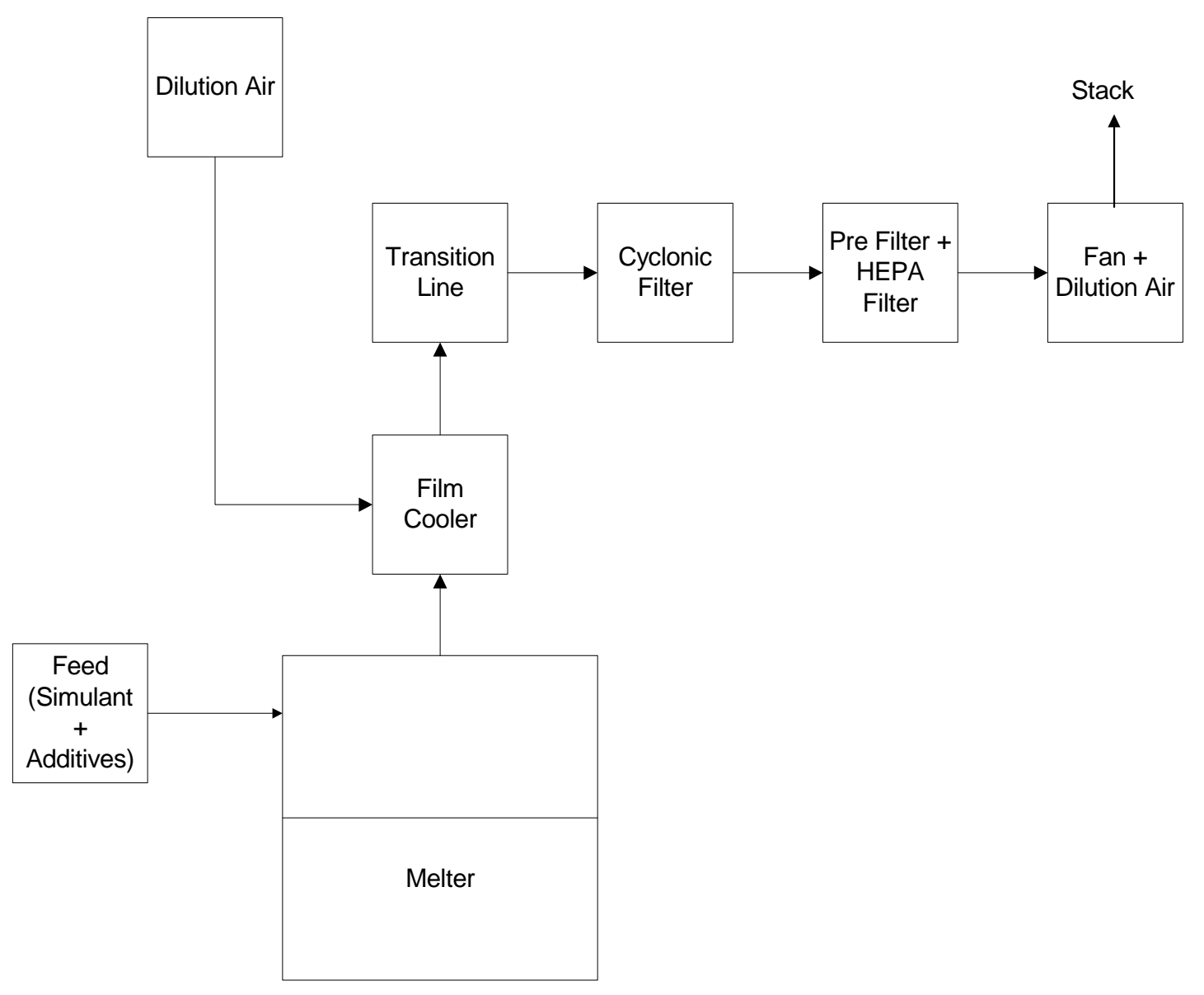

Figure 1.1. Schematic diagram of DuraMelter 100-WV vitrification system. 
The Catholic University of America Vitreous State Laboratory
ORP-56324, Rev. 0

DuraMelter 100 Tests to Support LAW Glass Formulation Correlation Development Final Report, VSL-06R6480-1, Rev. 0

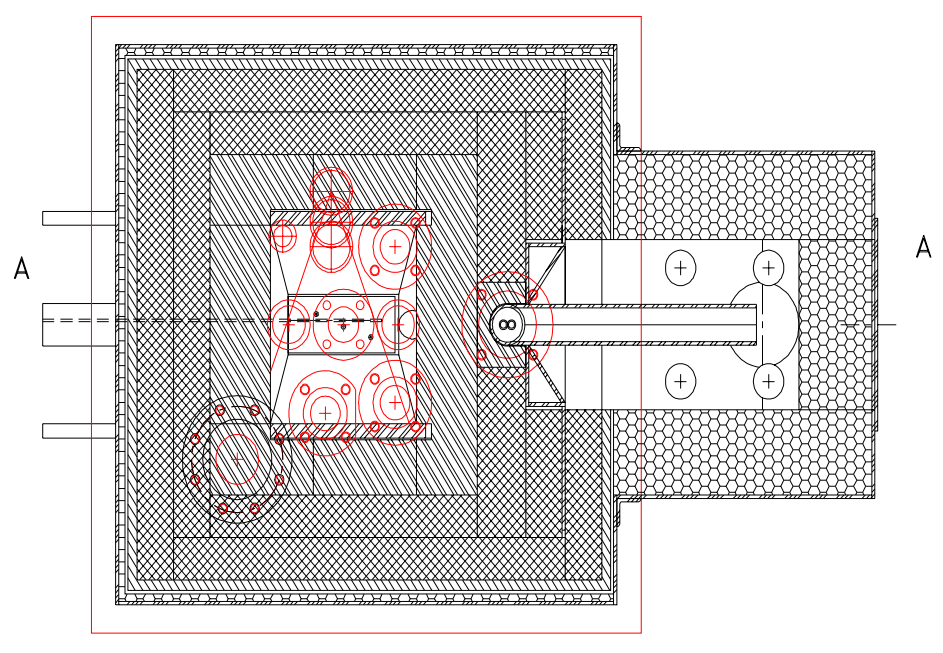

SECTION B-B

(BACKGROUND OMITED)

Figure 1.2(a). Cross-section through the DM100-WV melter-Plan View. 


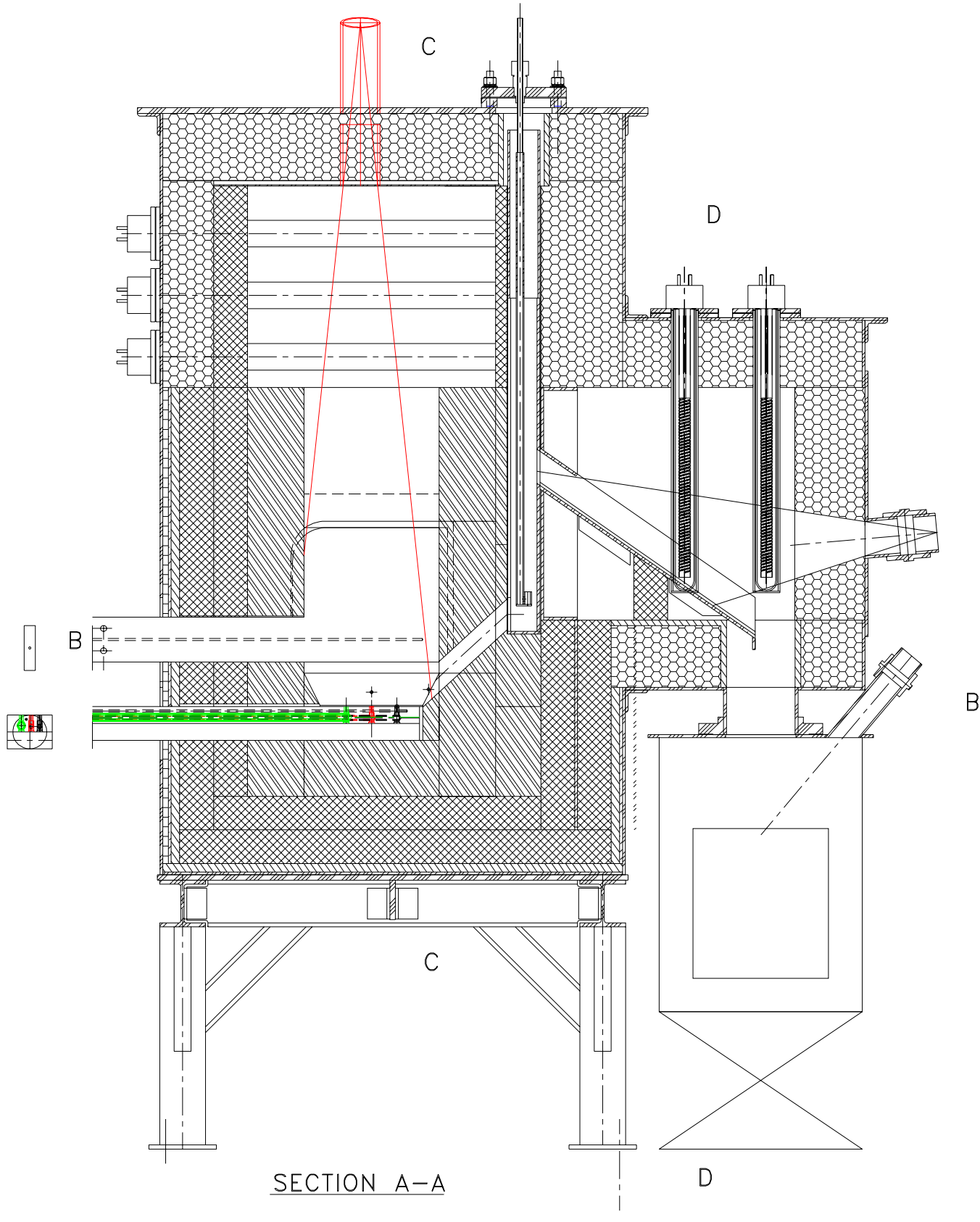

Figure 1.2(b). Cross-section through the DM100-WV melter-Section AA. 
The Catholic University of America Vitreous State Laboratory
ORP-56324, Rev. 0

DuraMelter 100 Tests to Support LAW Glass Formulation Correlation Development Final Report, VSL-06R6480-1, Rev. 0

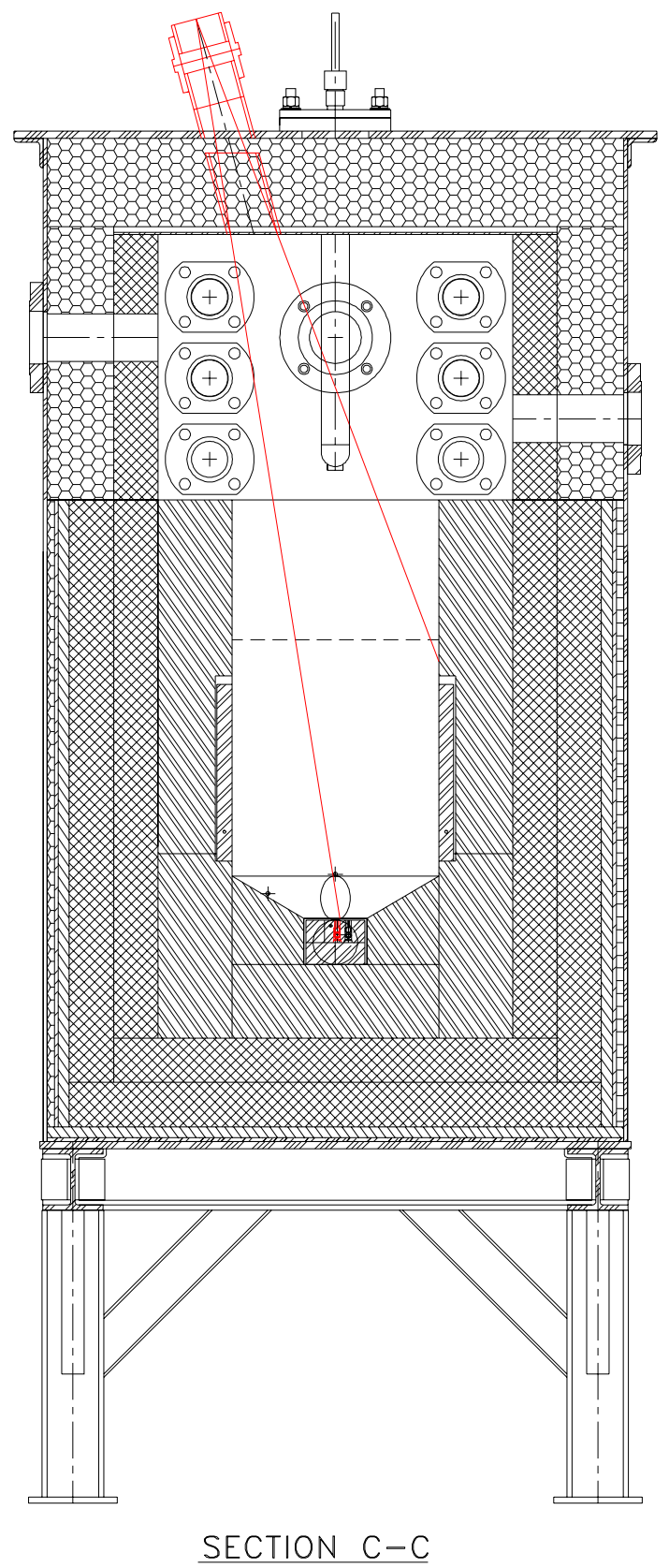

Figure 1.2(c). Cross-section through the DM100-WV melter-Section CC. 


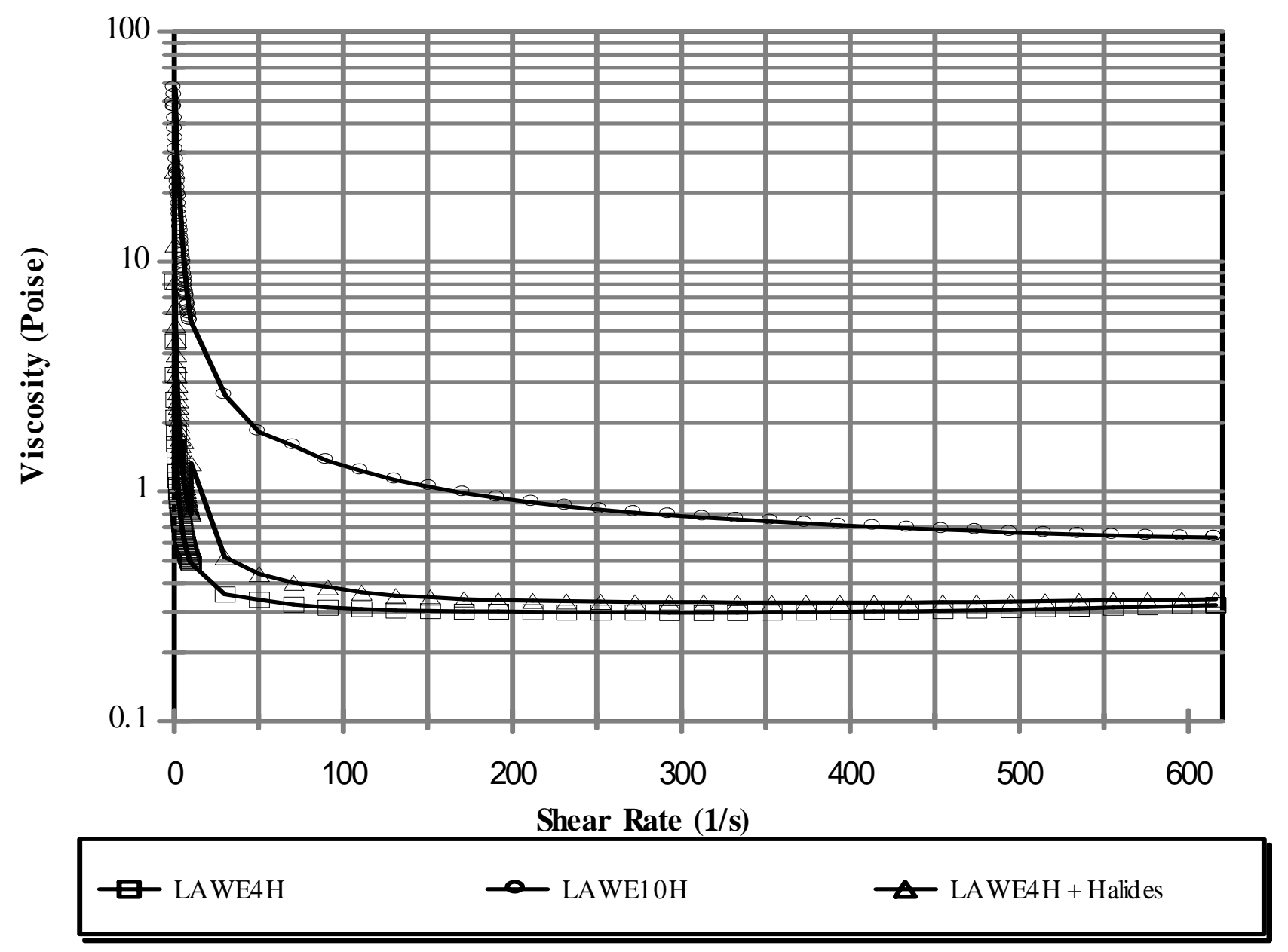

Figure 2.1. Measured viscosity of select LAW DM100 feed samples. 


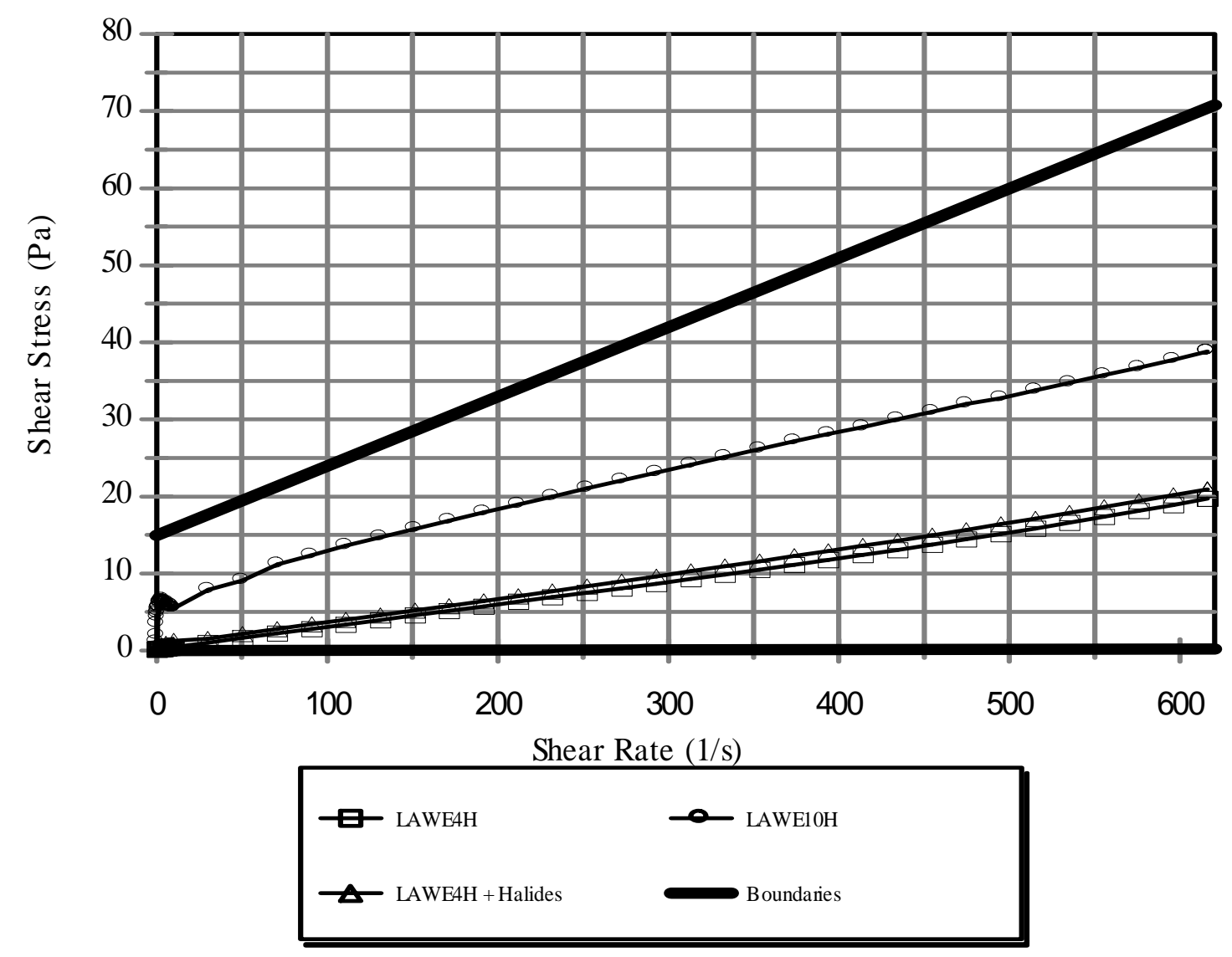

Figure 2.2. Comparison of measured feed rheology with proposed WTP bounds (bounds from WTP-RPT-075, Rev. 0, Feb. 2003). 


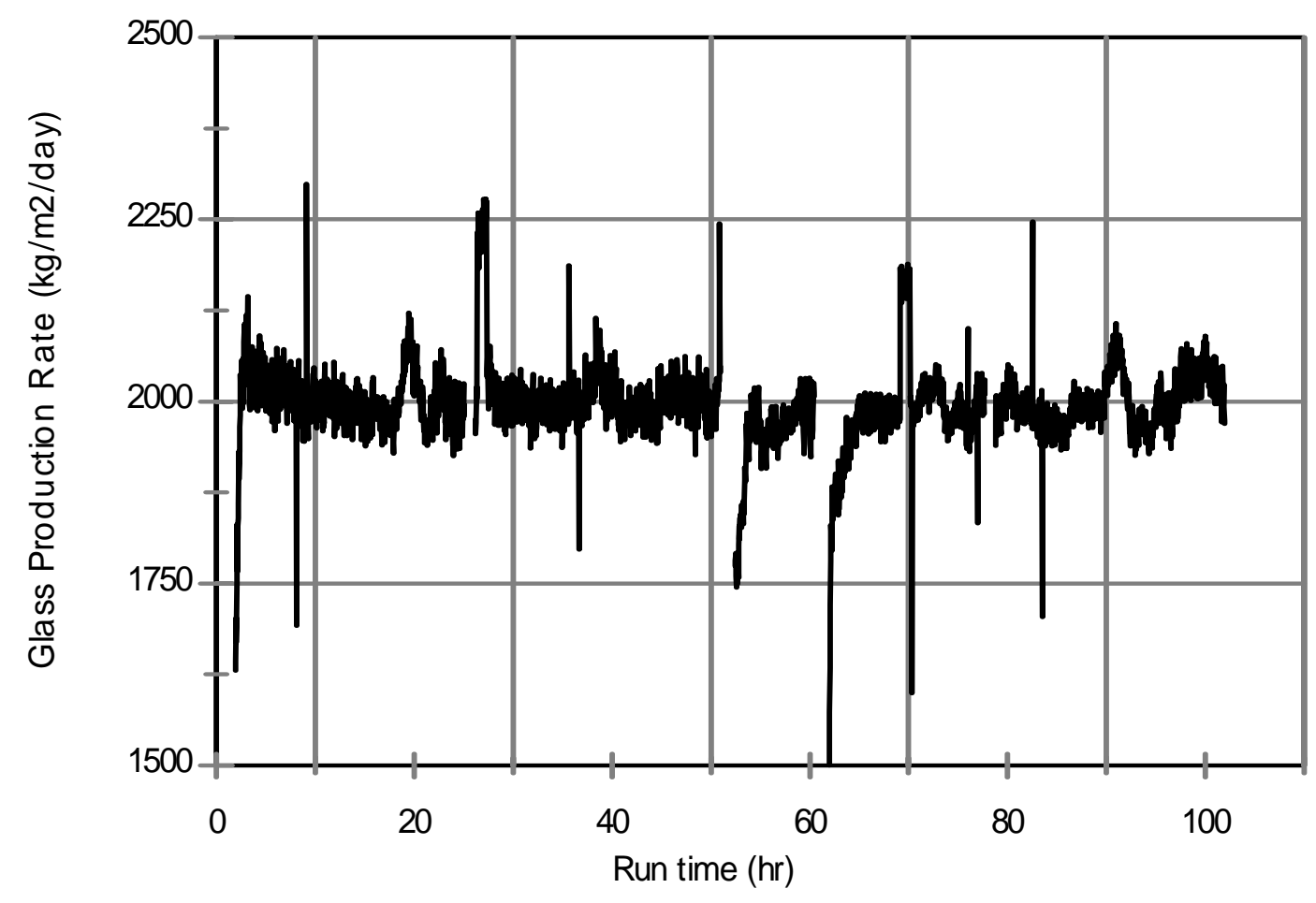

Figure 3.1.a. Glass production rates during Test 1. 


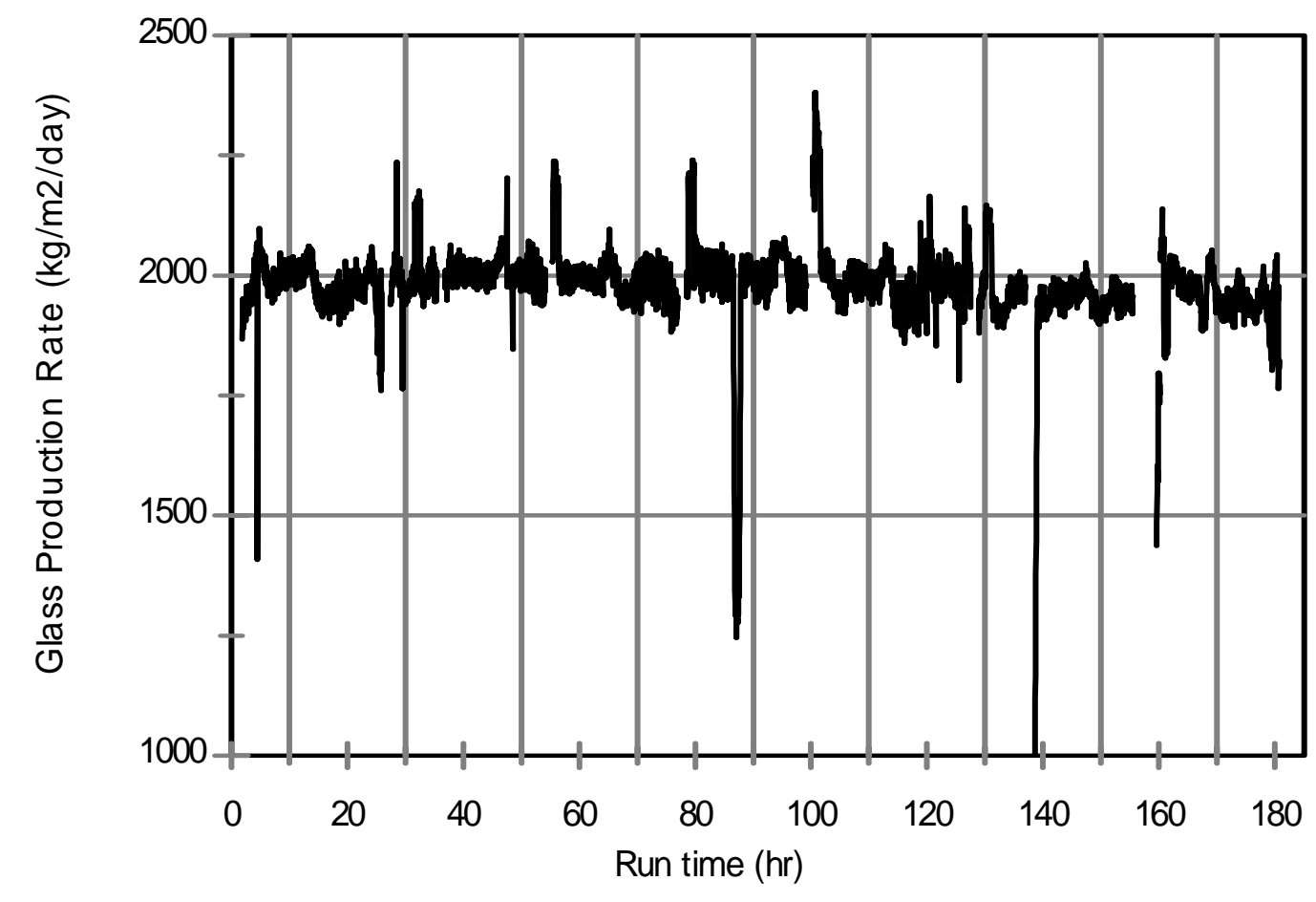

Figure 3.1.b. Glass production rates during Test 2. 


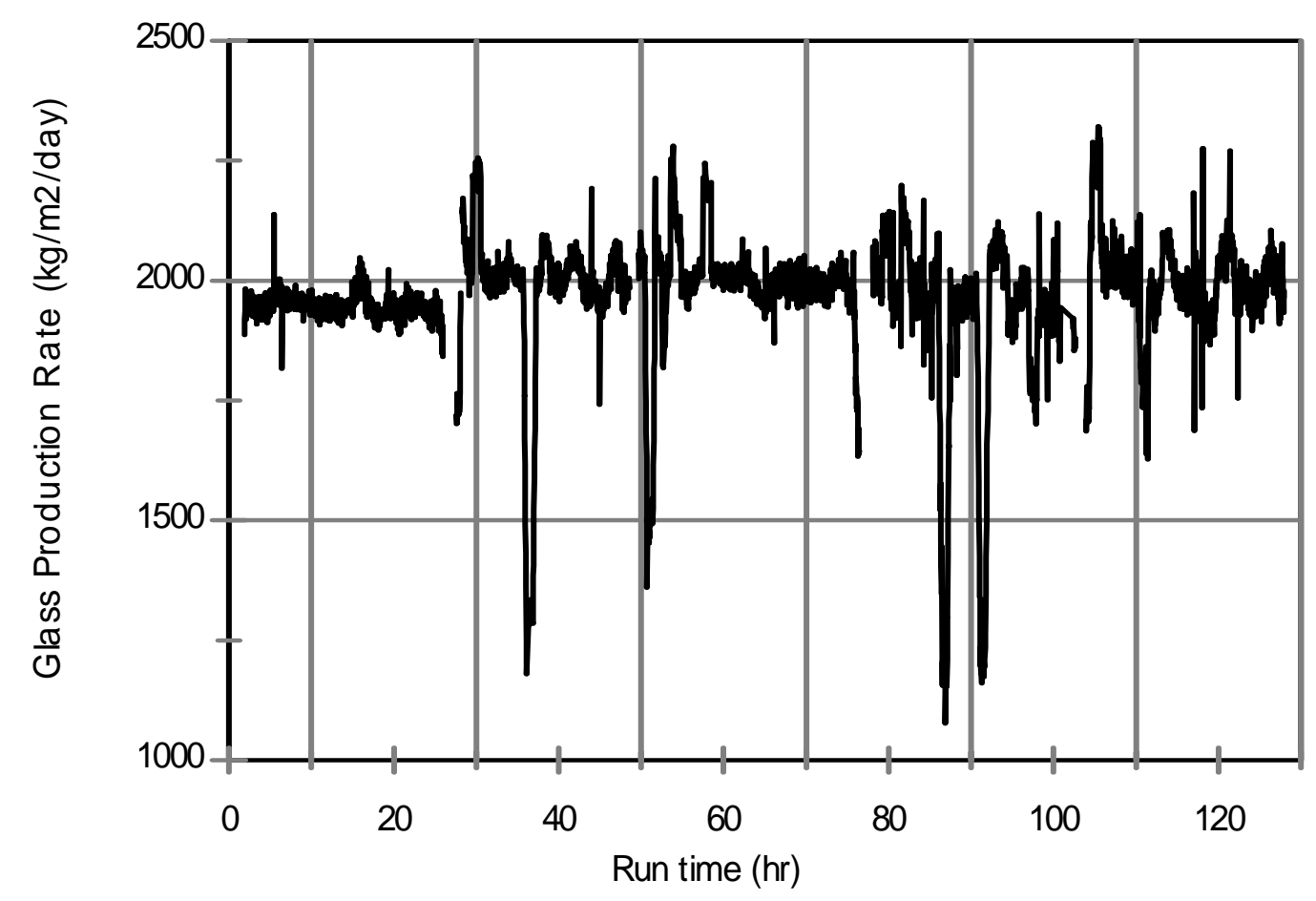

Figure 3.1.c. Glass production rates during Test 3. 


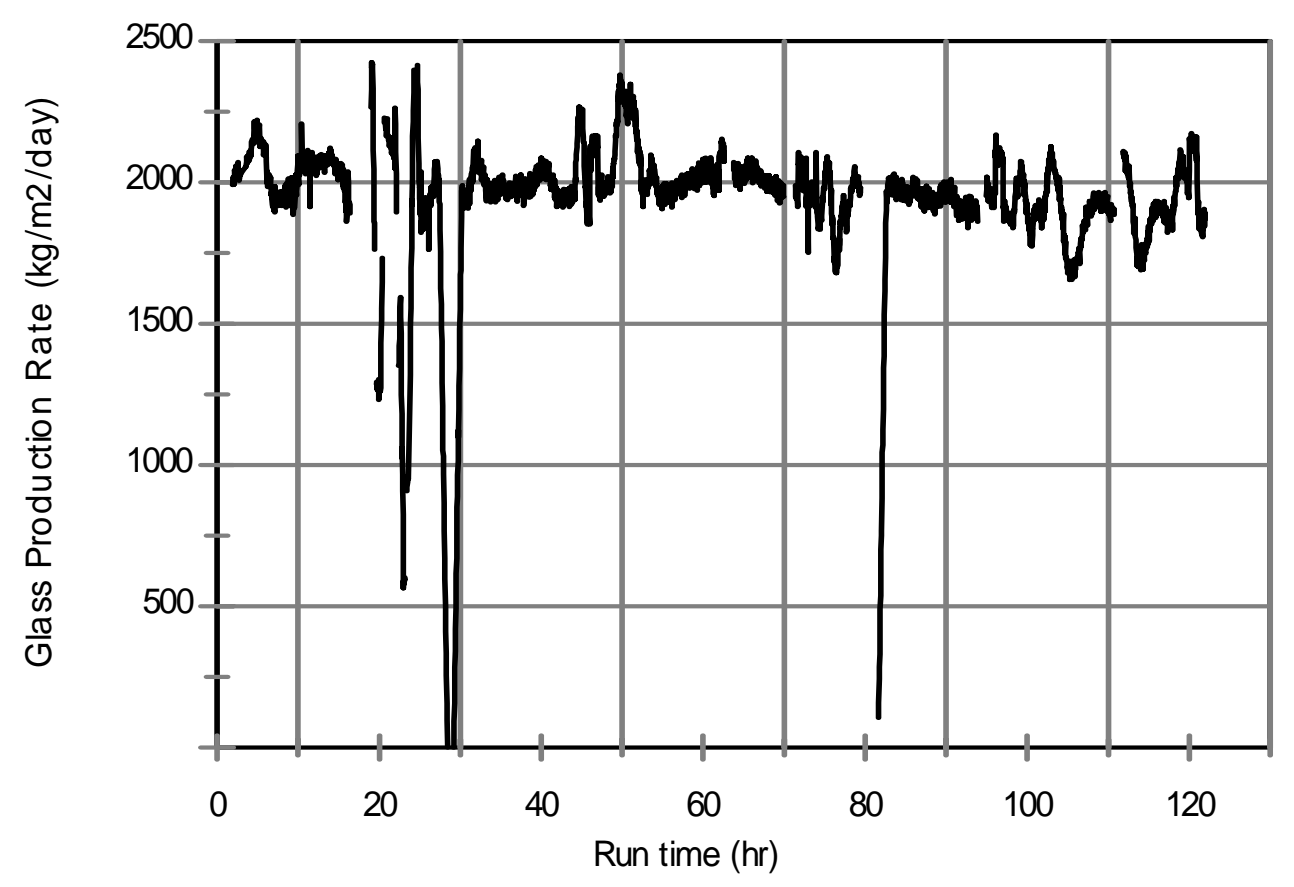

Figure 3.1.d. Glass production rates during Test 4. 


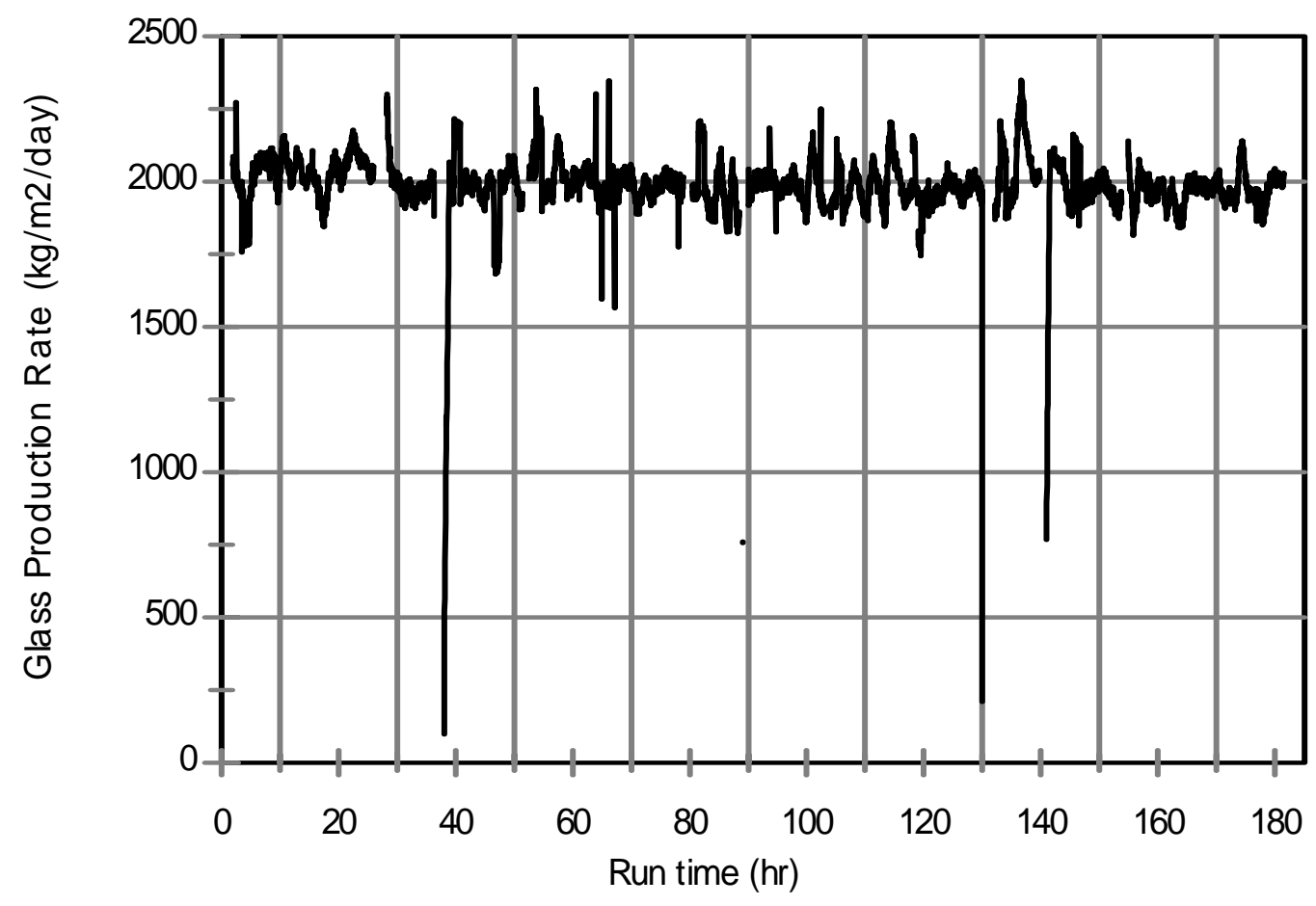

Figure 3.1.e. Glass production rates during Test 5. 


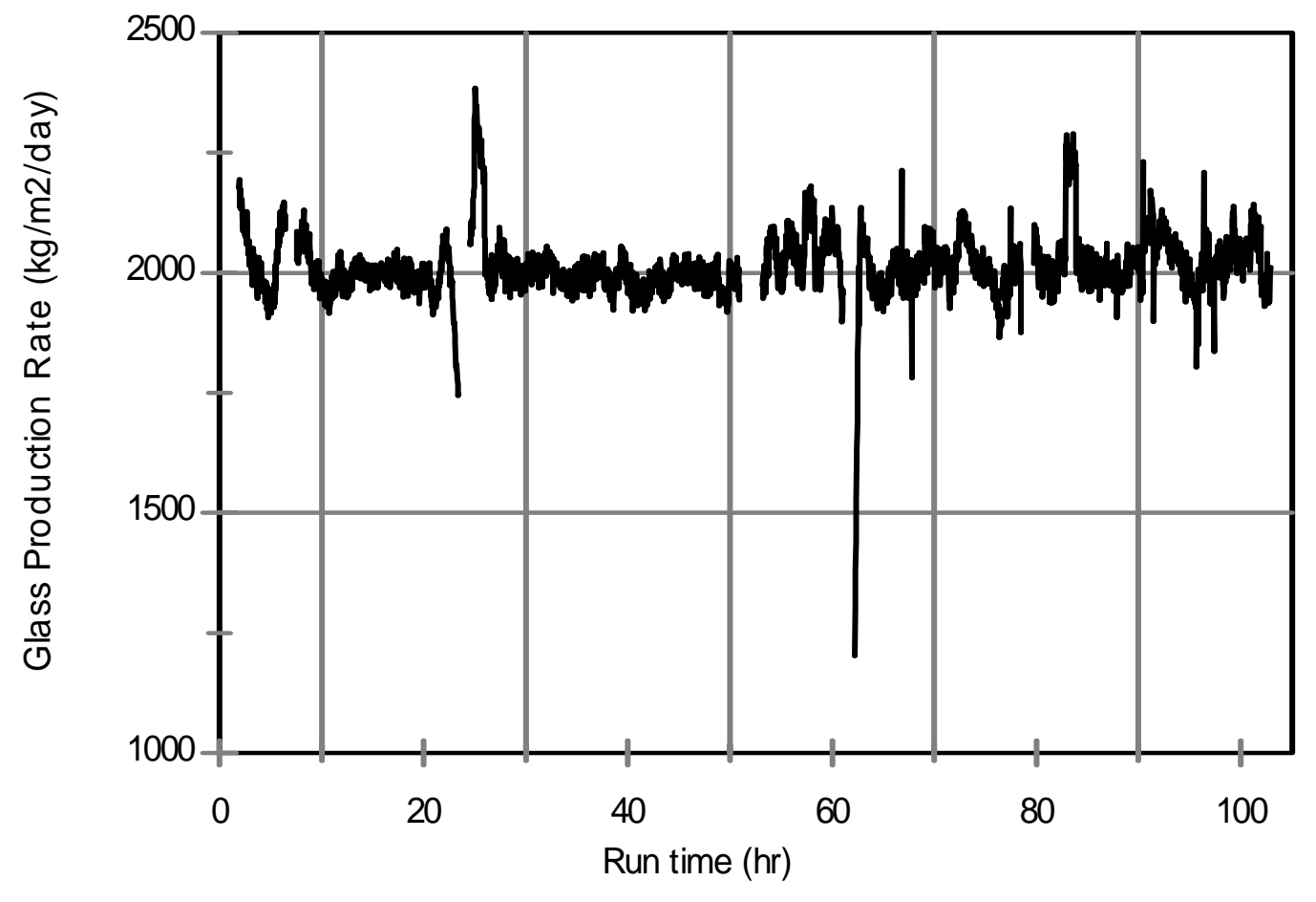

Figure 3.1.f. Glass production rates during Test 6. 


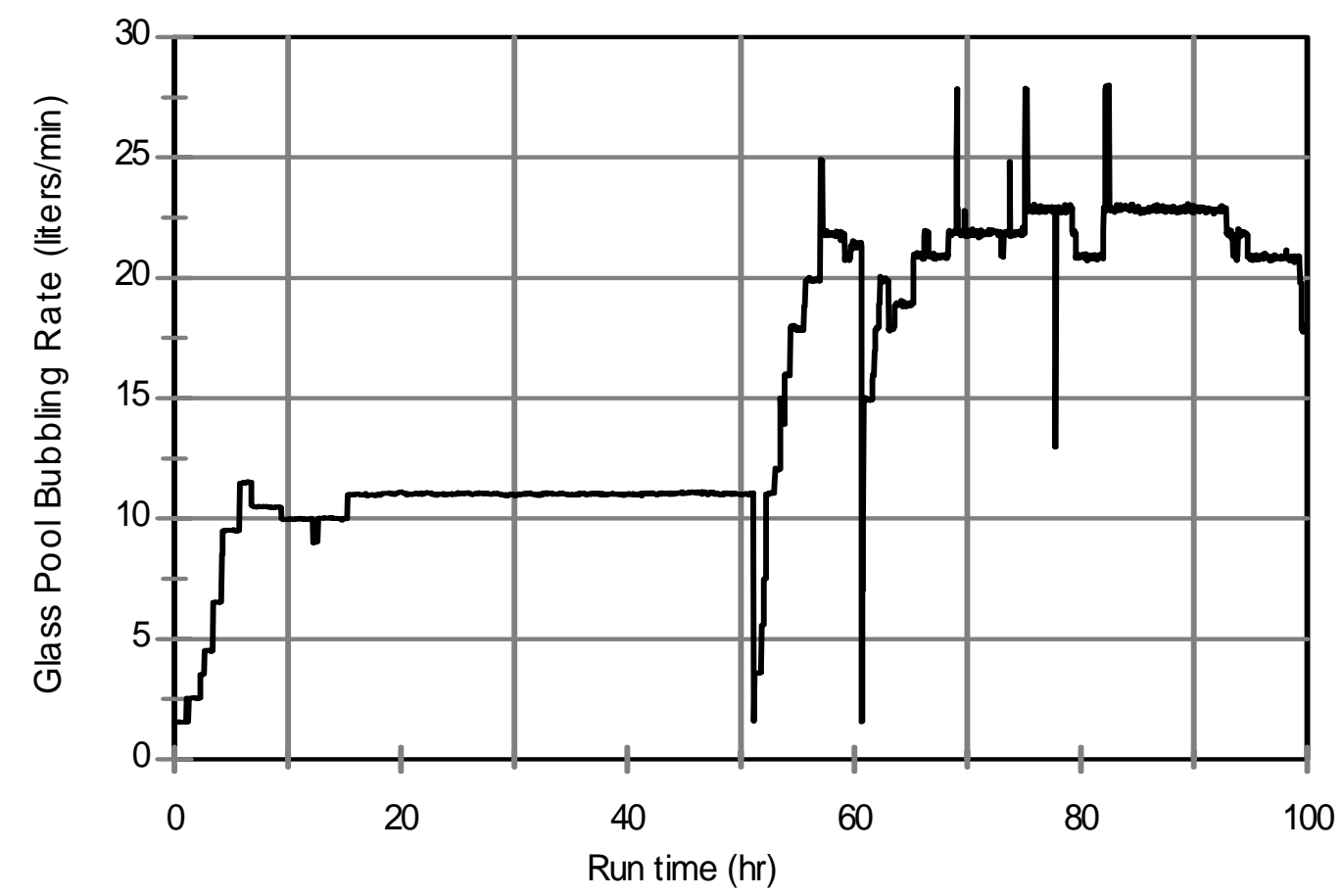

Figure 3.2.a. Glass pool bubbling during Test 1. 


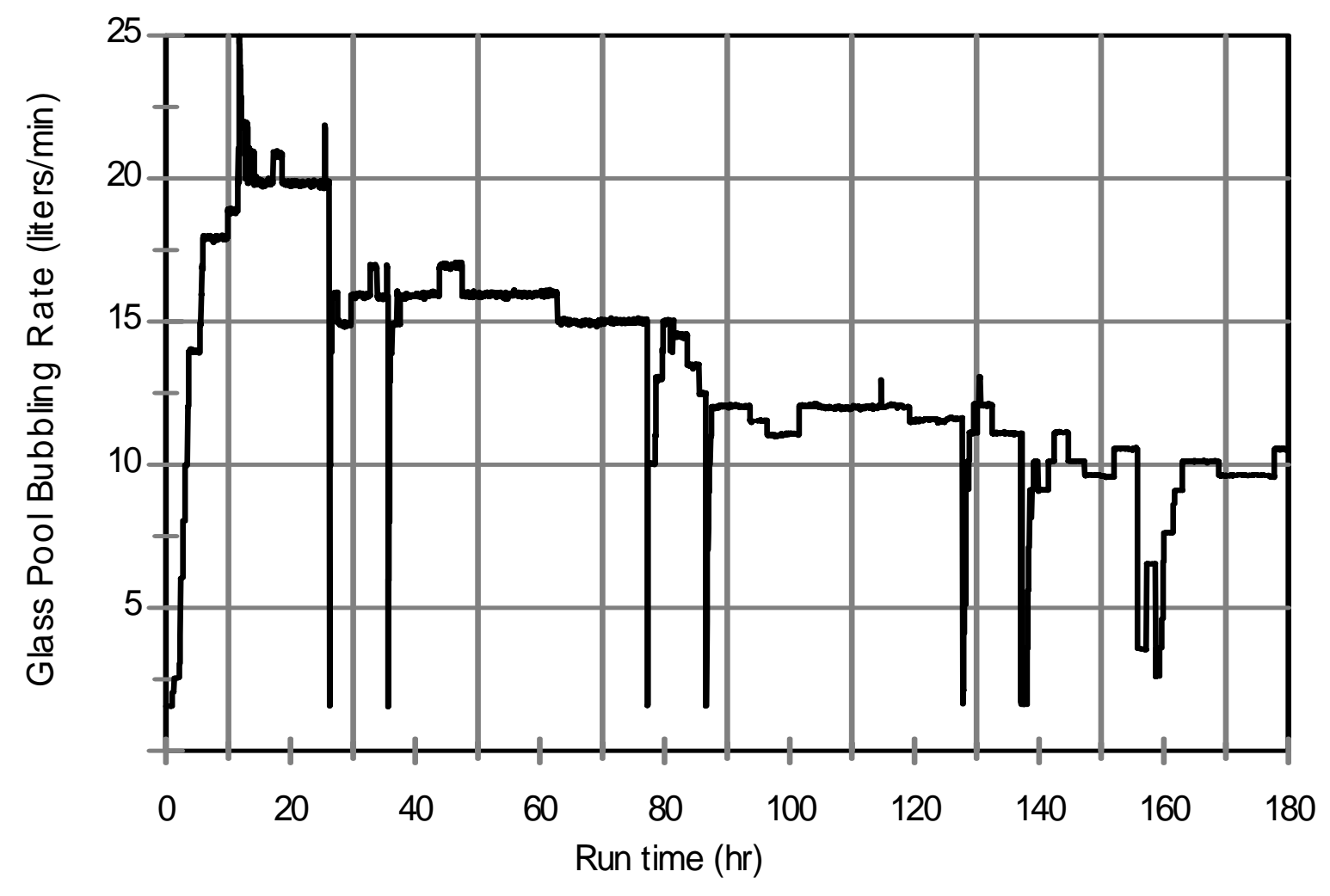

Figure 3.2.b. Glass pool bubbling during Test 2. 


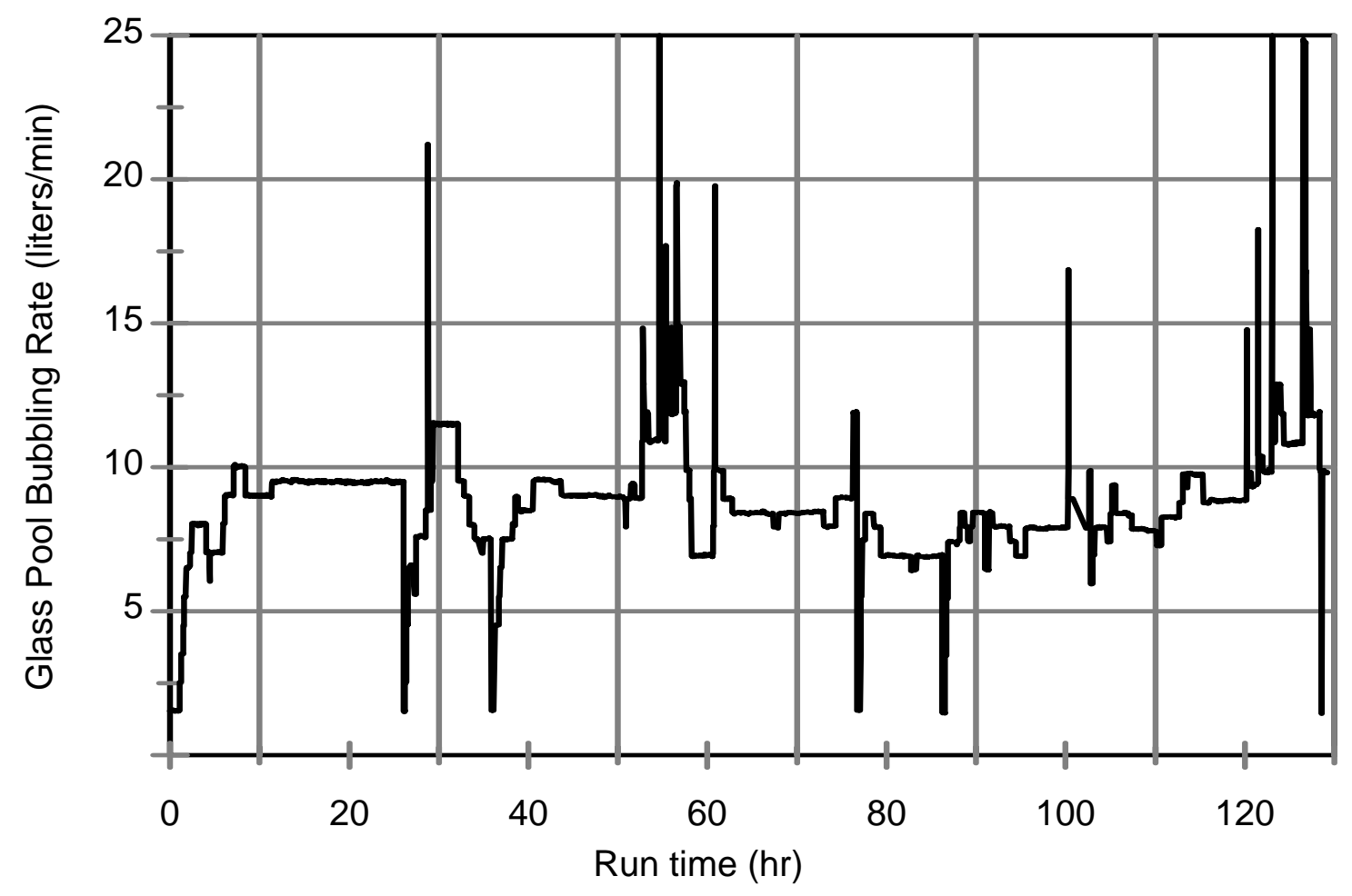

Figure 3.2.c. Glass pool bubbling during Test 3. 


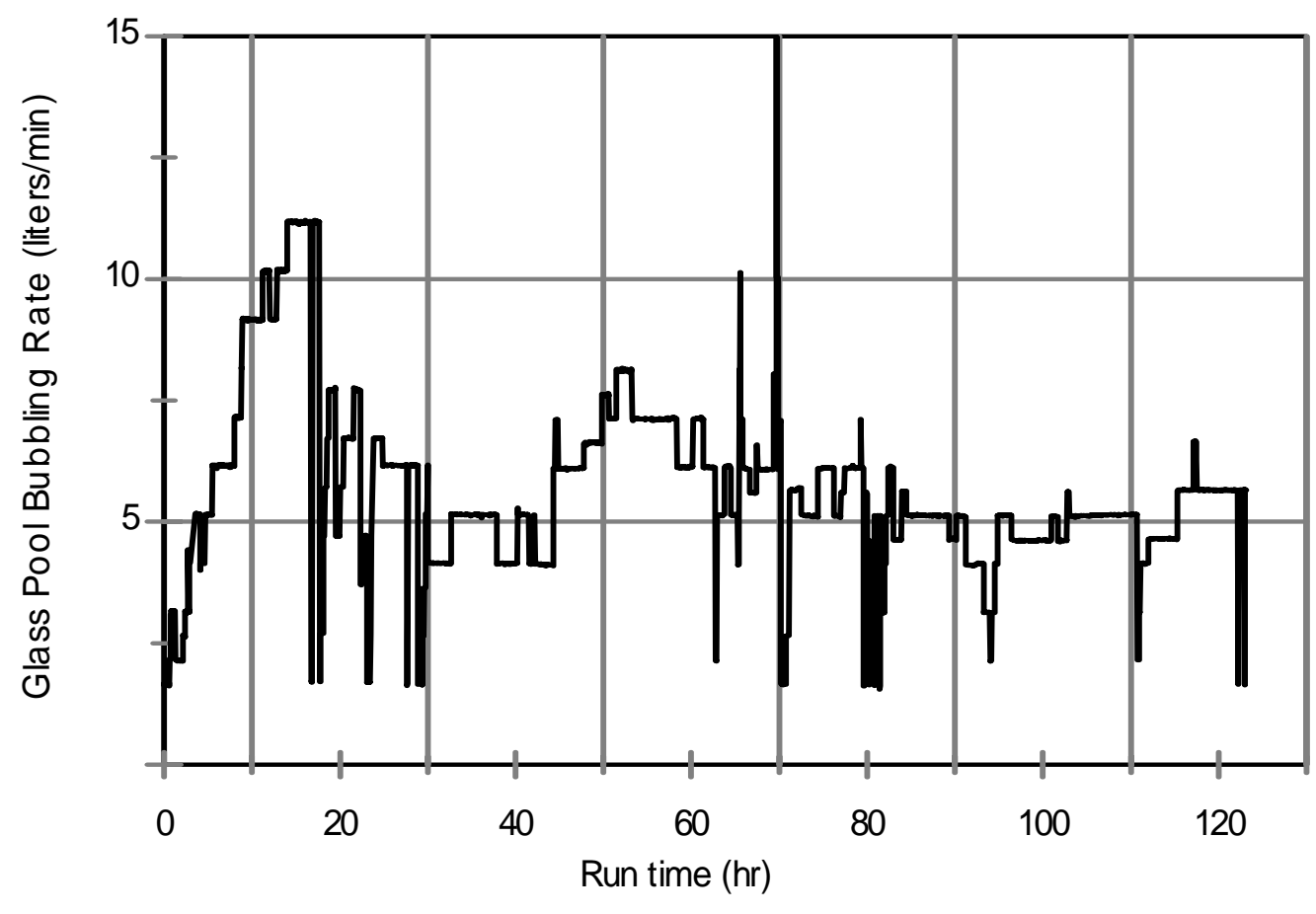

Figure 3.2.d. Glass pool bubbling during Test 4 . 


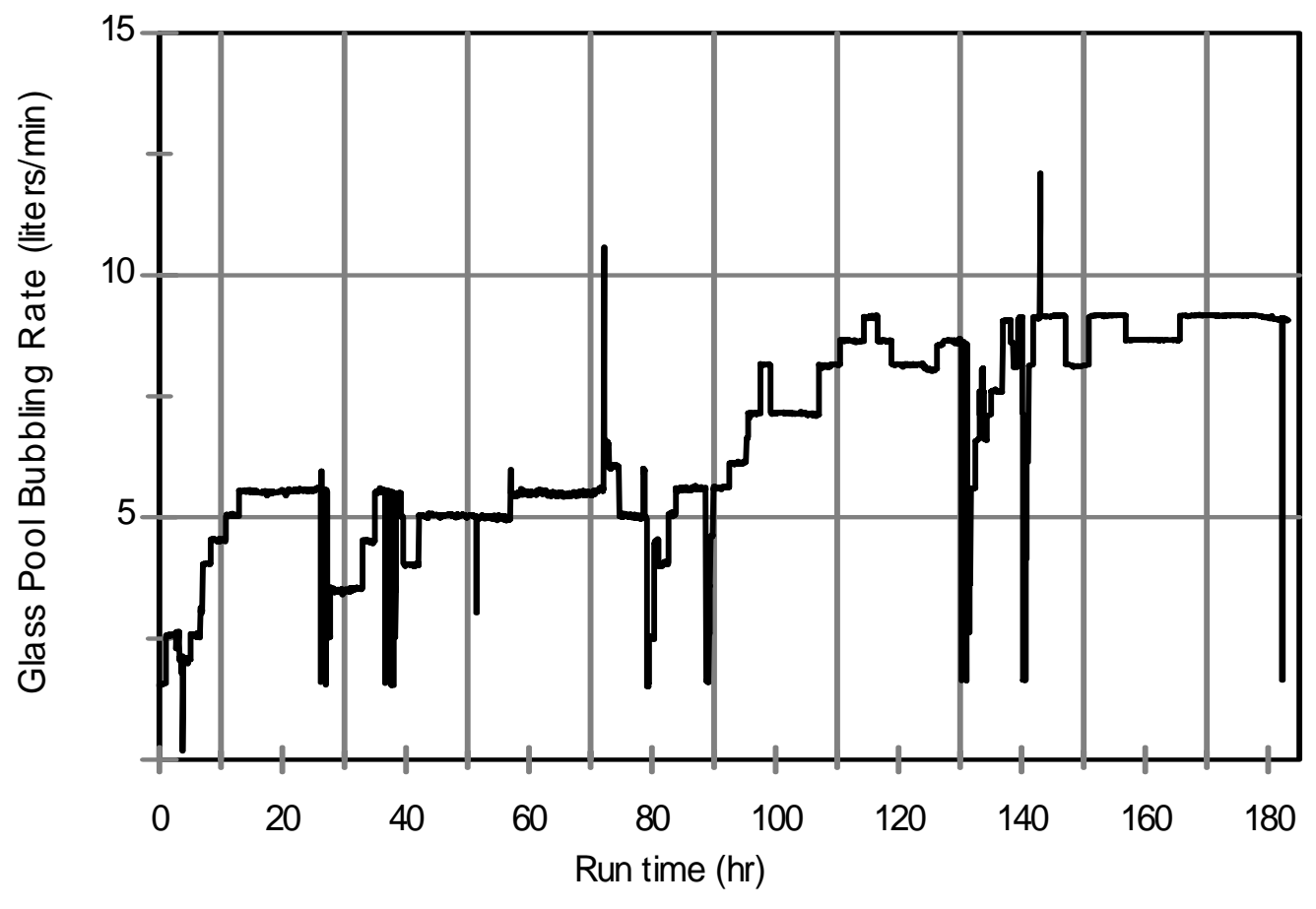

Figure 3.2.e. Glass pool bubbling during Test 5. 


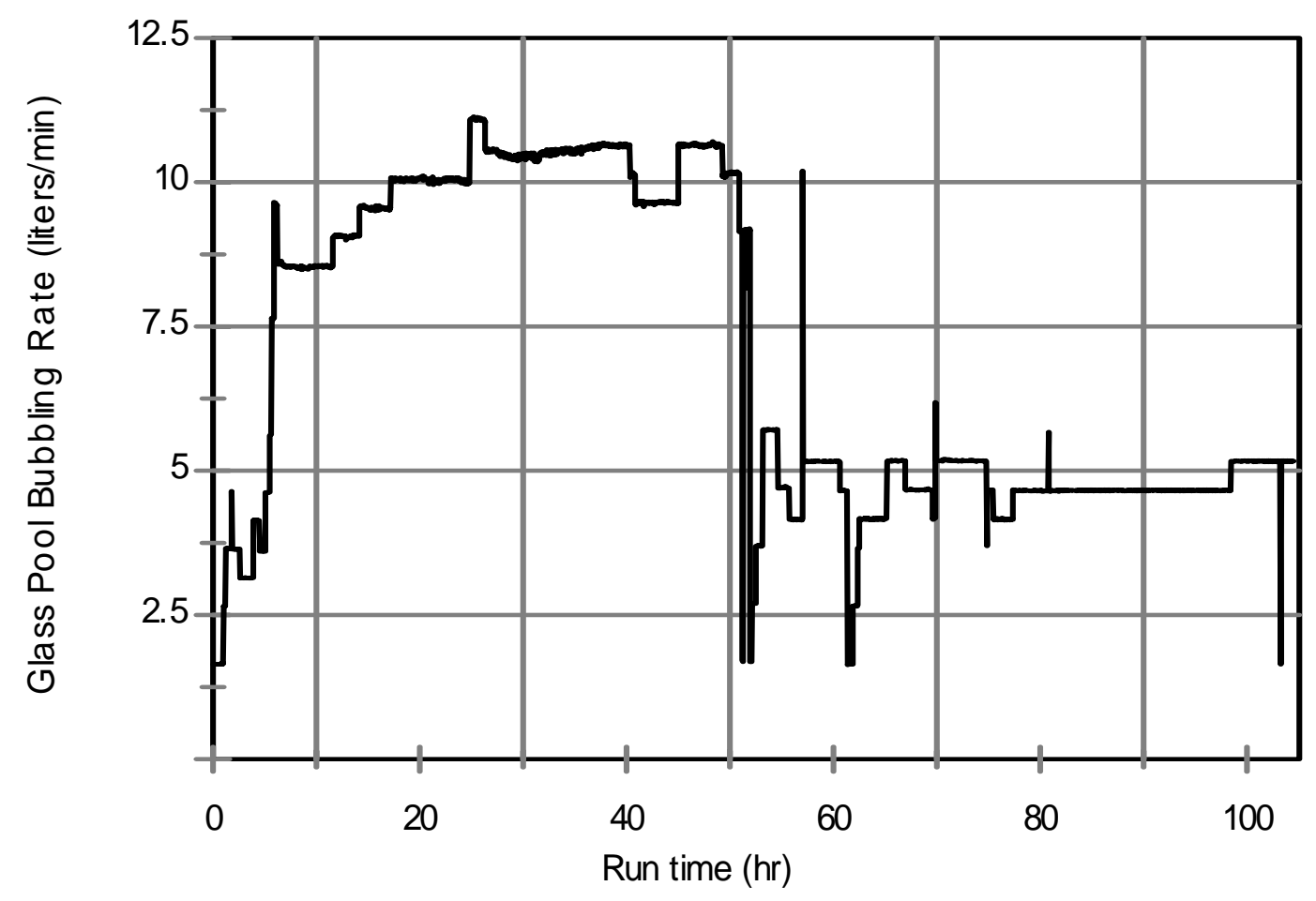

Figure 3.2.f. Glass pool bubbling during Test 6. 

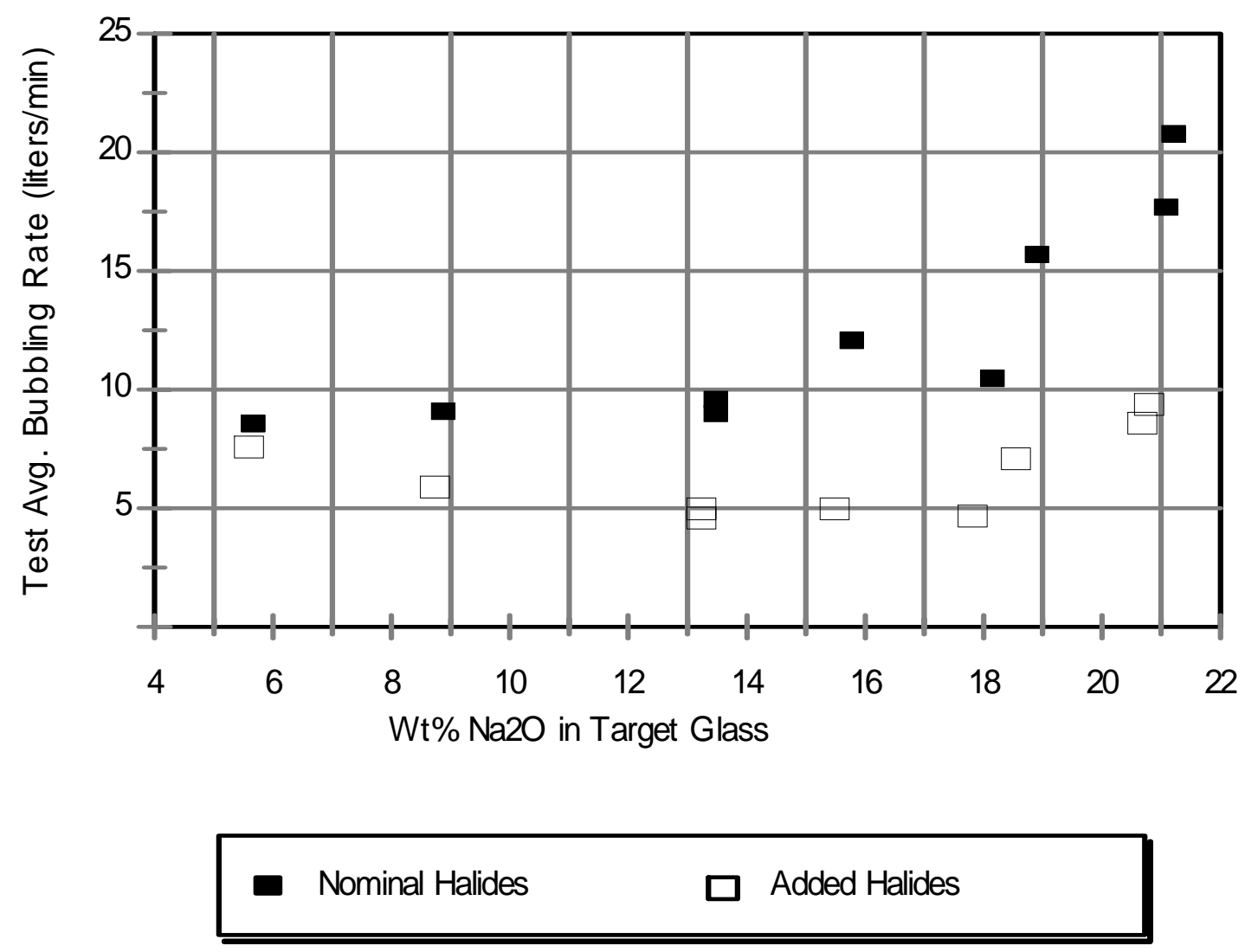

Figure 3.3. Glass pool bubbling and glass sodium content. 


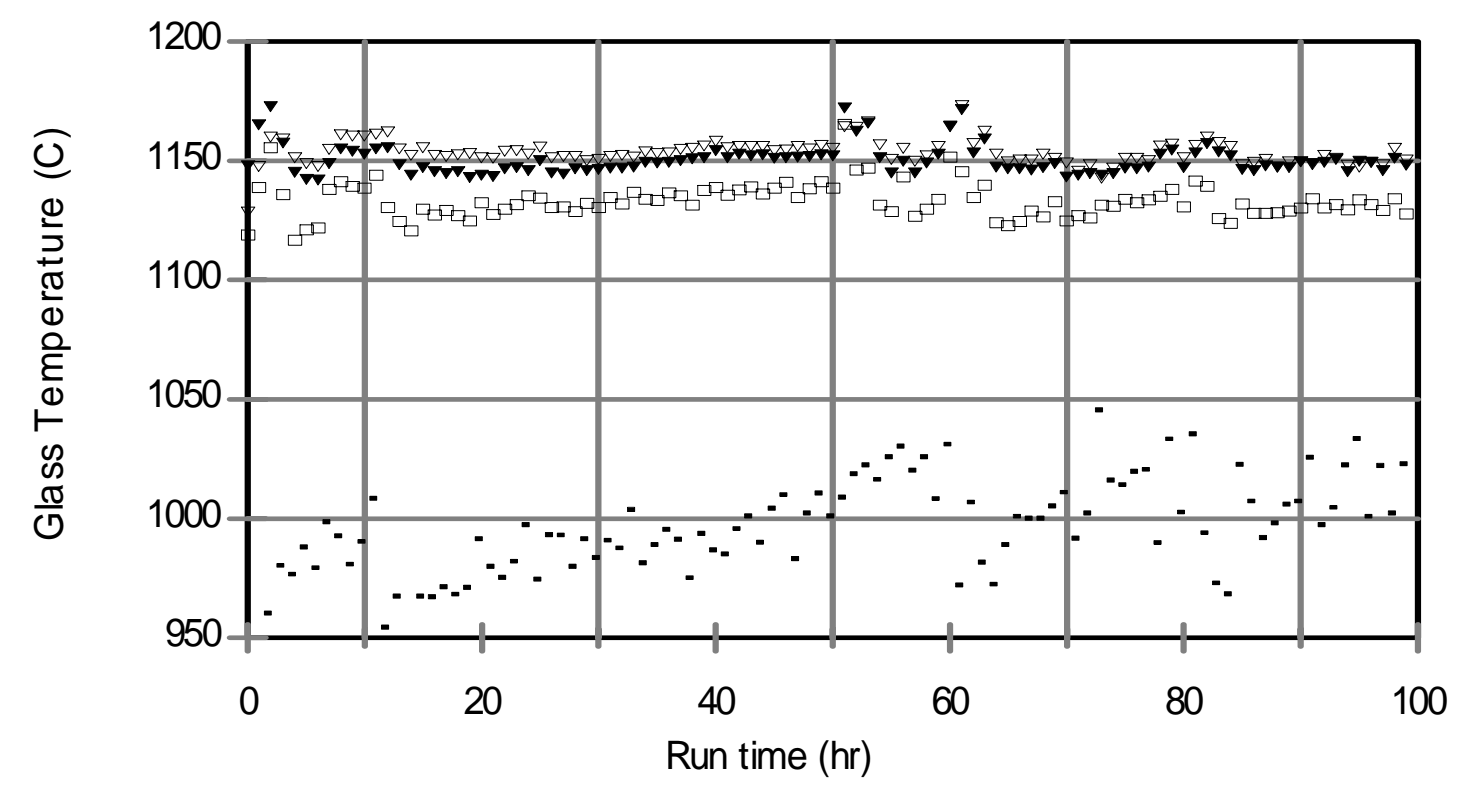
- 19 " from floor
$\square \quad 16$ " from floor
- $10^{\prime \prime}$ from floor
$\nabla \quad$ 4" from floor

Figure 3.4.a. Glass temperatures during Test 1 (hourly average data). 


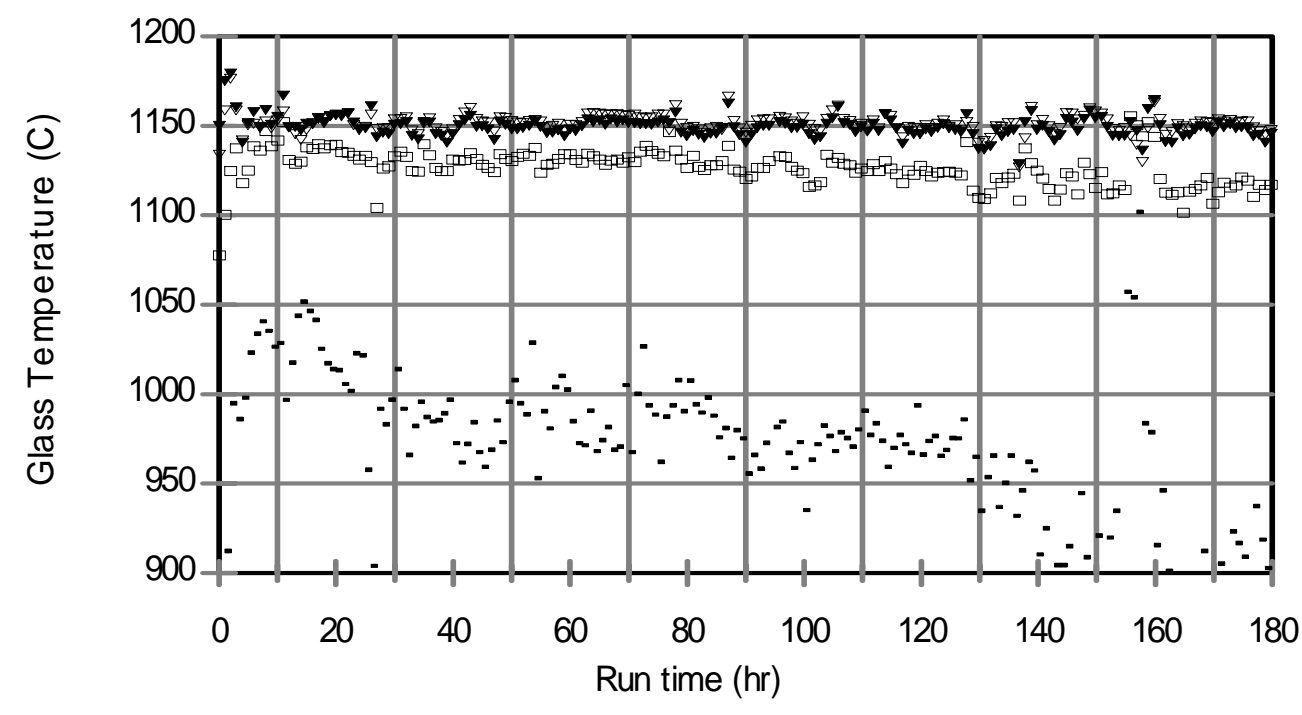

- $19^{\prime \prime}$ from floor $\quad \square 16^{\prime \prime}$ from floor

- $10^{\prime \prime}$ from floor $\quad \nabla \quad 4^{\prime \prime}$ from floor

Figure 3.4.b. Glass temperatures during Test 2 (hourly average data). 


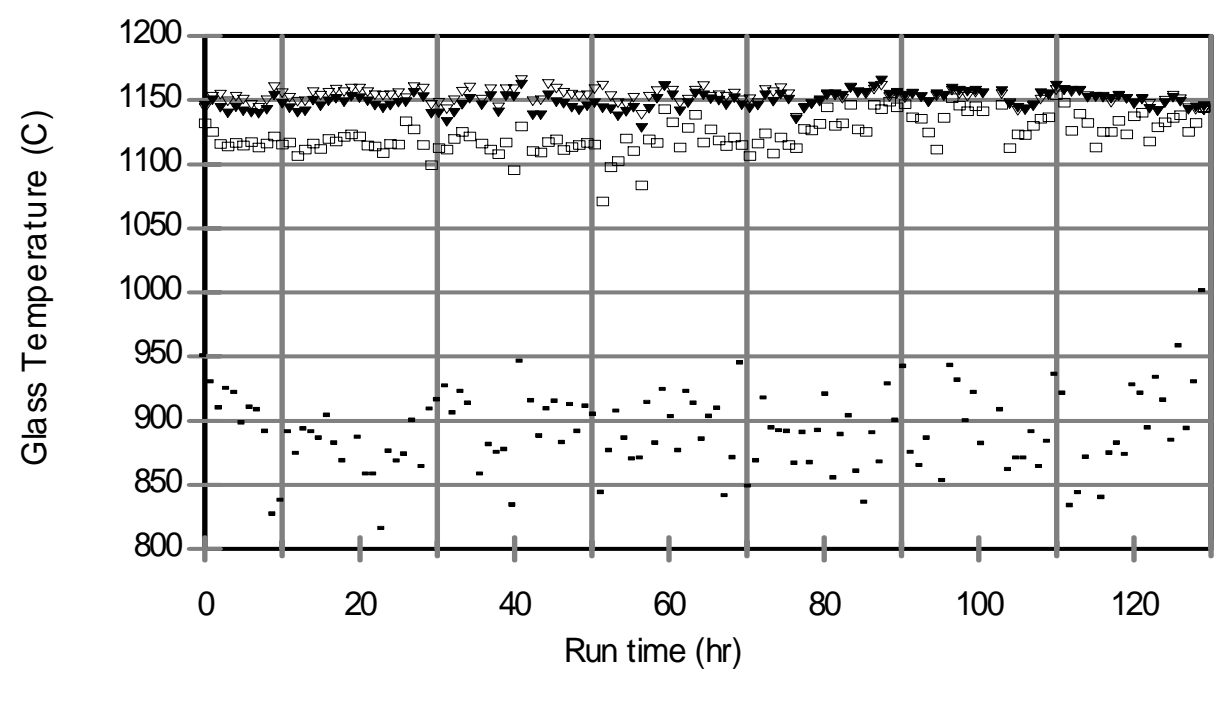
- 19 " from floor
$\square 16$ "from floor
- 10 " from floor
$\nabla \quad$ 4" from floor

Figure 3.4.c. Glass temperatures during Test 3 (hourly average data). 


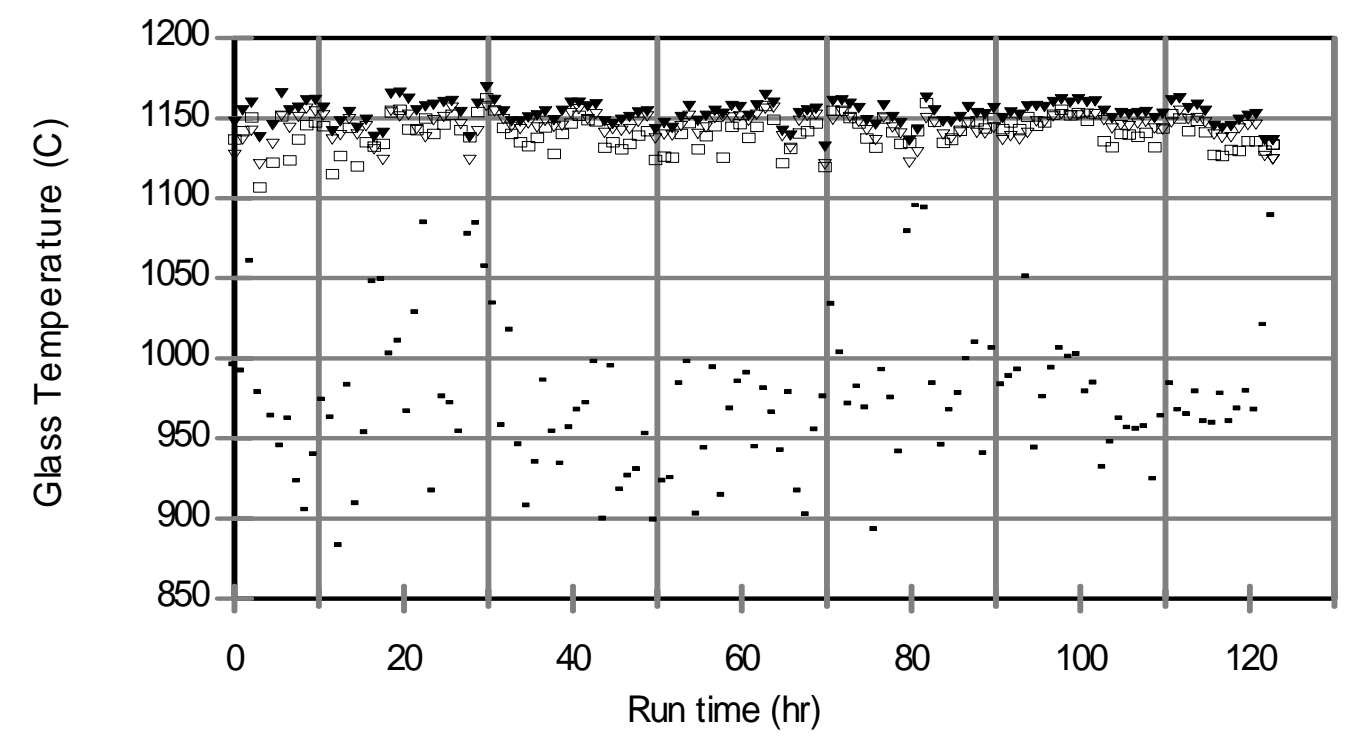

Figure 3.4.d. Glass temperatures during Test 4 (hourly average data). 


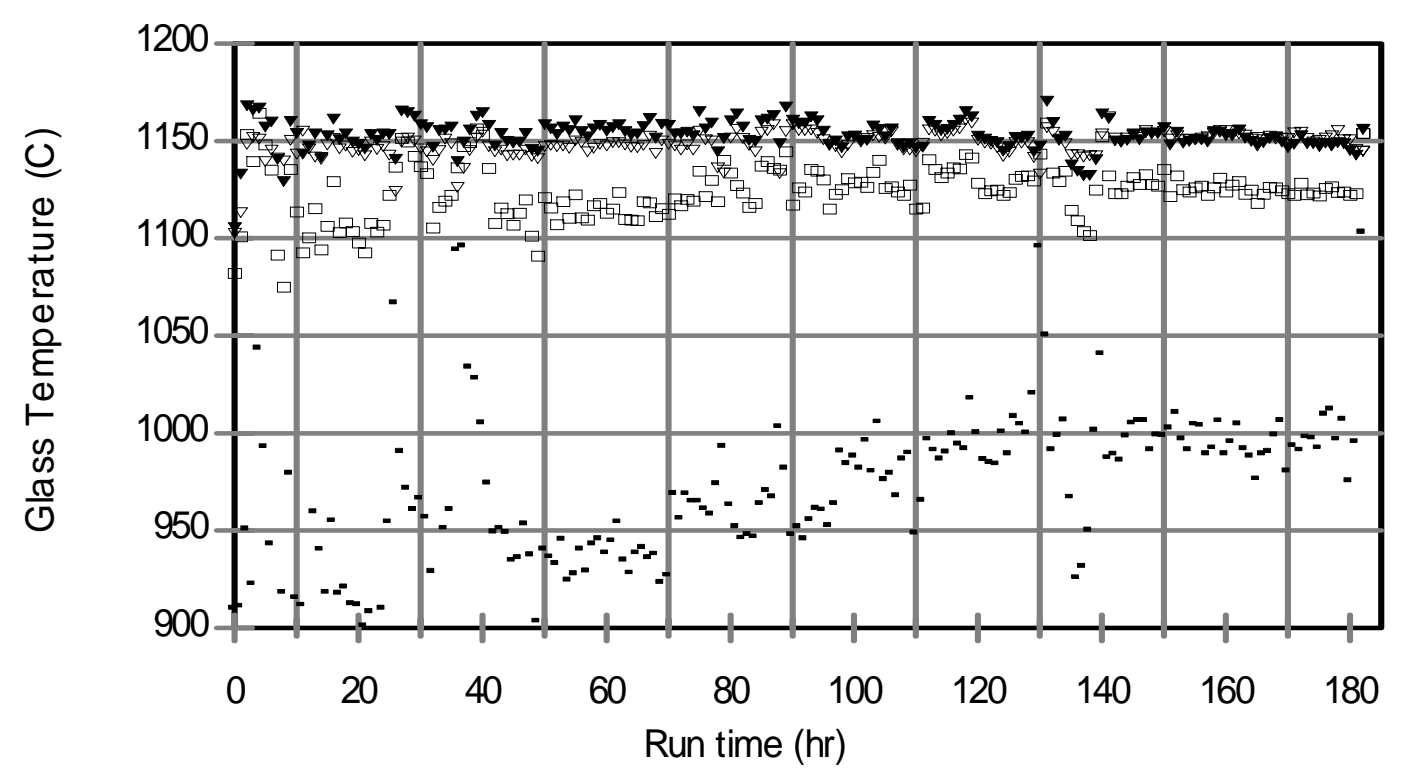

Figure 3.4.e. Glass temperatures during Test 5 (hourly average data). 


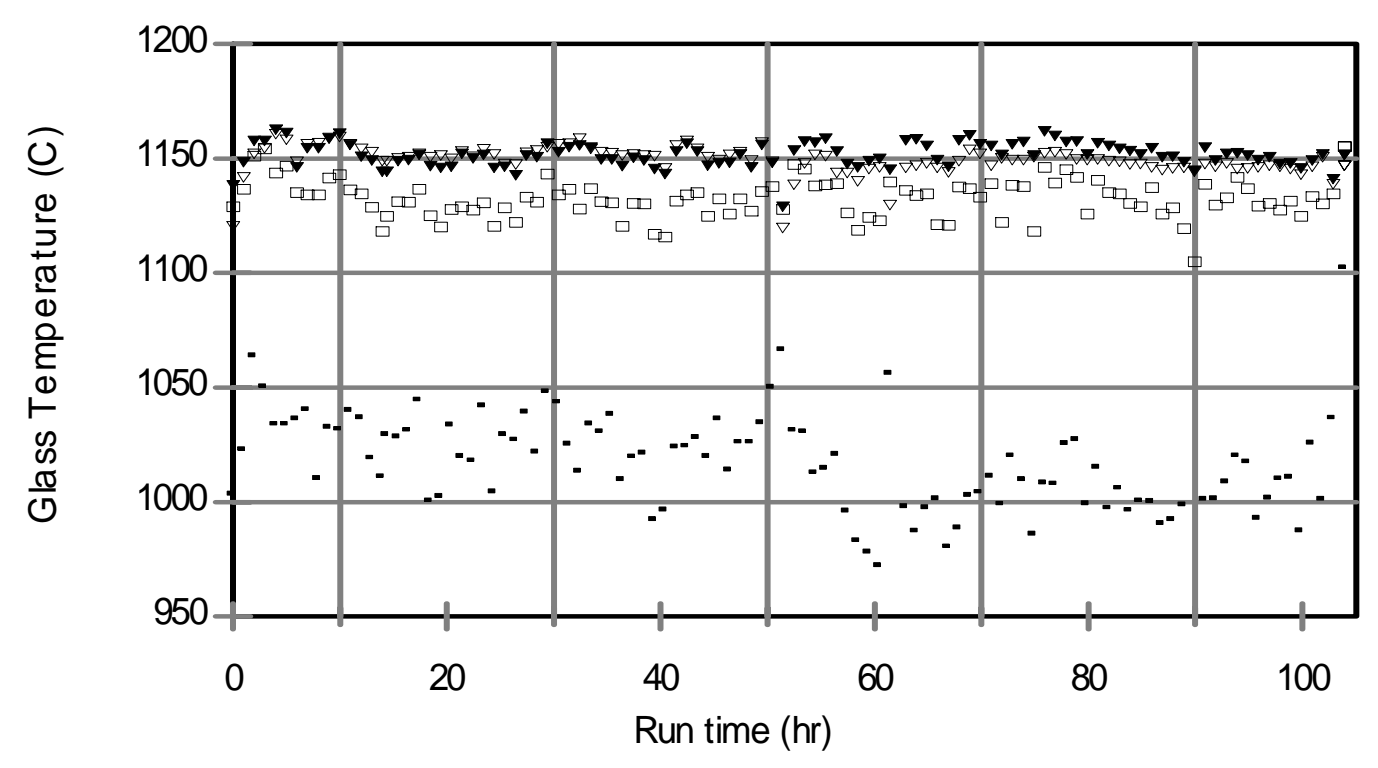

Figure 3.4.f. Glass temperatures during Test 6 (hourly average data). 


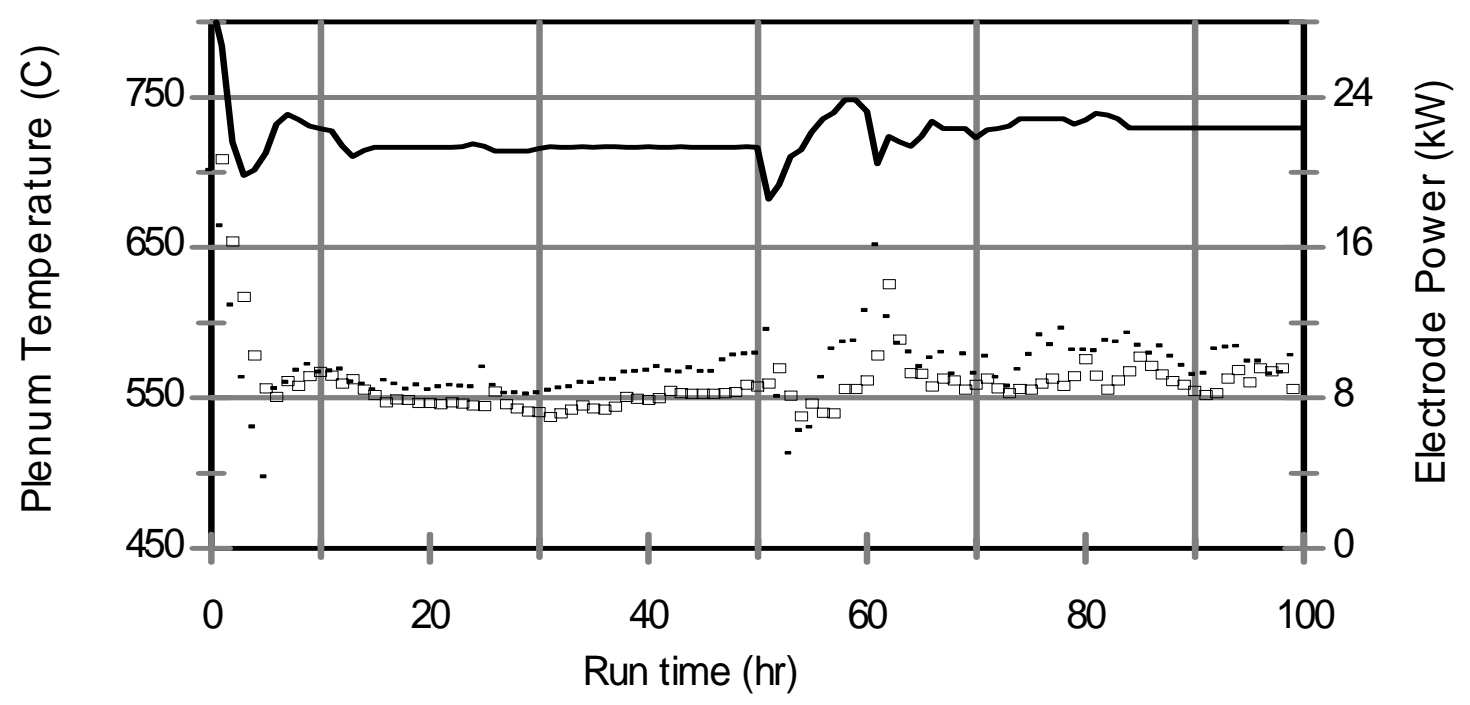

- 16" below ceiling (exposed)

$\square \quad 16$ " below ceiling (thermowell)

Electrode Power

Figure 3.5.a. Plenum temperatures and electrode power during Test 1 (hourly average data). 


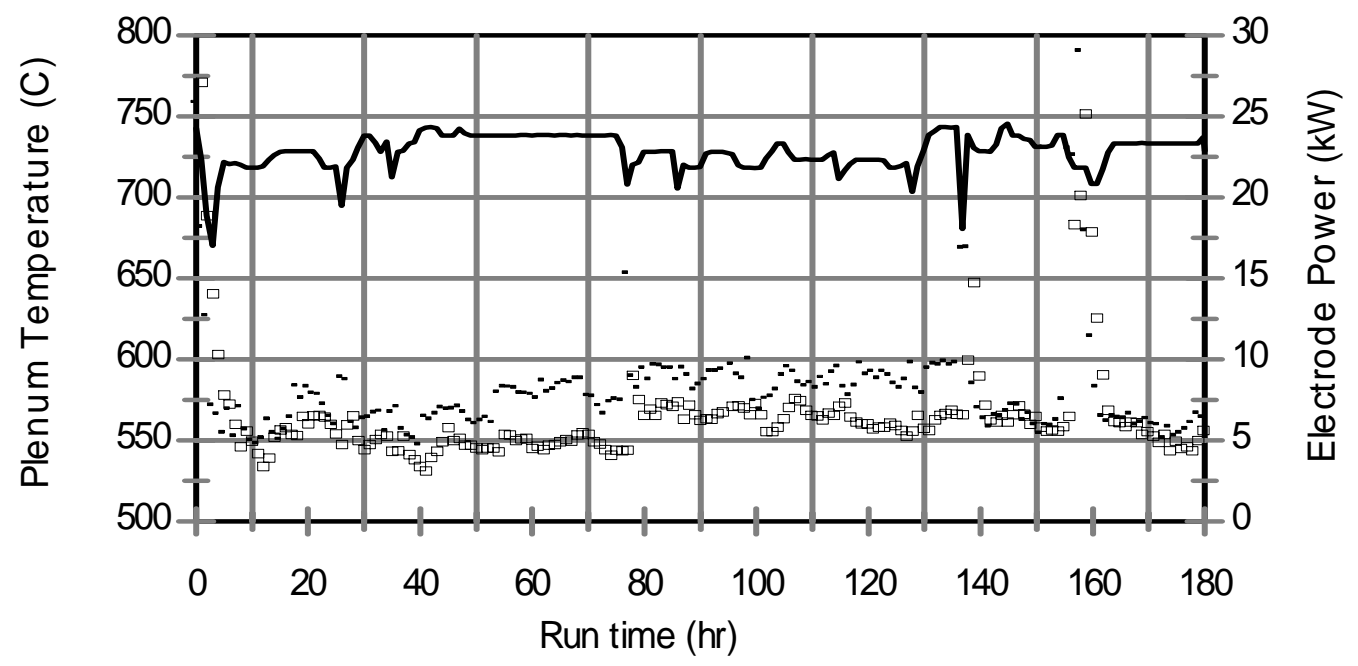

$$
\begin{aligned}
& -\quad 16 \text { " below ceiling (exposed) } \\
& \square \quad 16 \text { " below ceiling (thermowell) } \\
& \square \text { Electrode Power }
\end{aligned}
$$

Figure 3.5.b. Plenum temperatures and electrode power during Test 2 (hourly average data). 


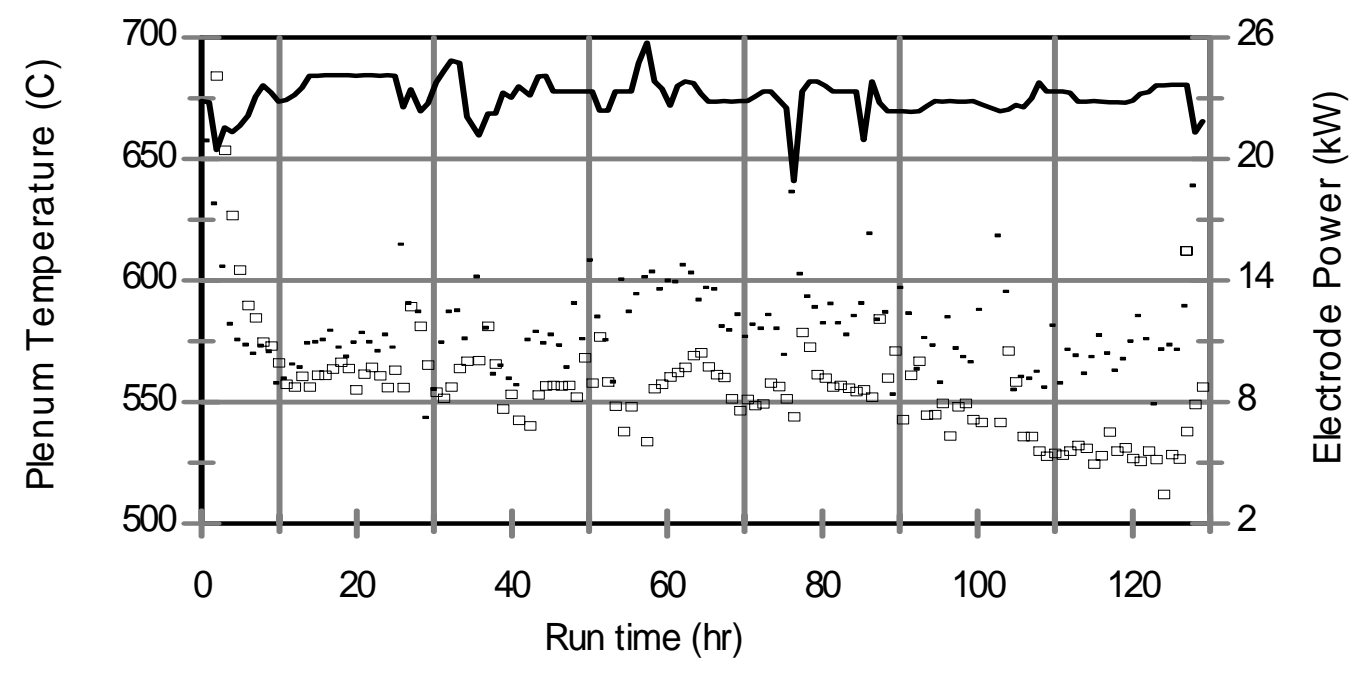

- $\quad 16 "$ below ceiling (exposed)

$\square \quad 16$ " below ceiling (thermowell) Electrode Power

Figure 3.5.c. Plenum temperatures and electrode power during Test 3 (hourly average data). 


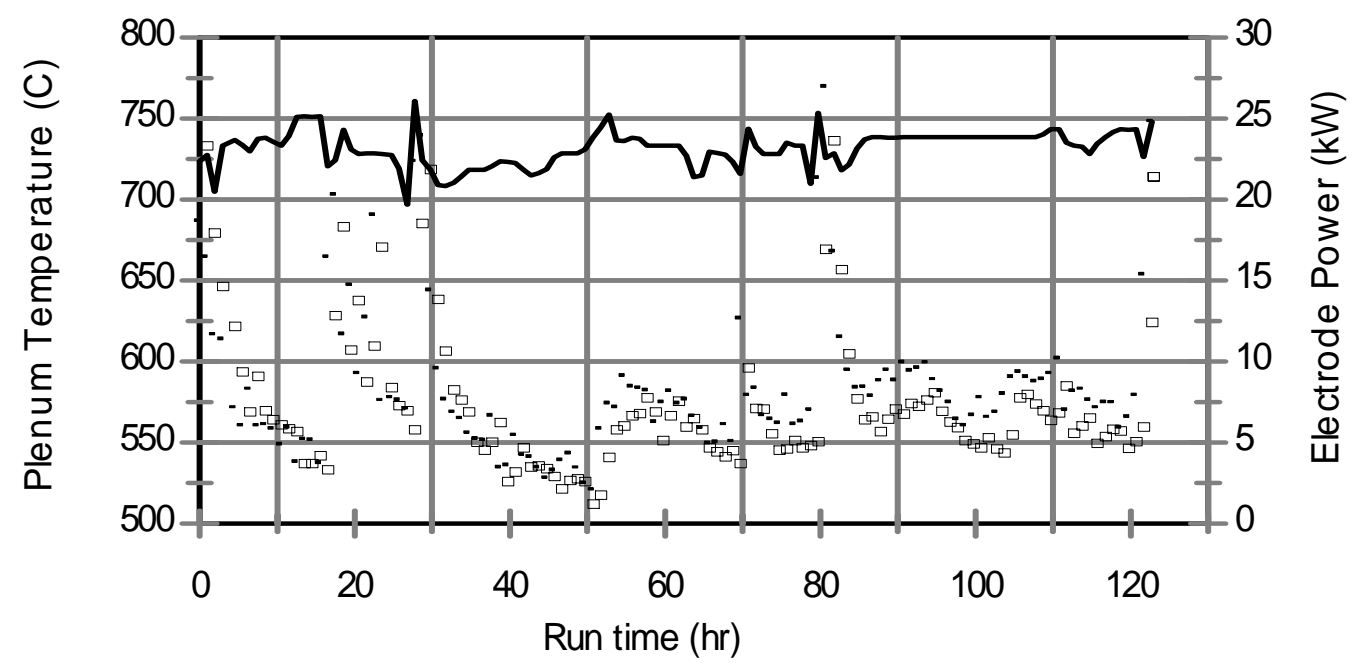

- $\quad$ 16" below ceiling (exposed)

$\square 16$ " below ceiling (thermowell)

Electrode Power

Figure 3.5.d. Plenum temperatures and electrode power during Test 4 (hourly average data). 


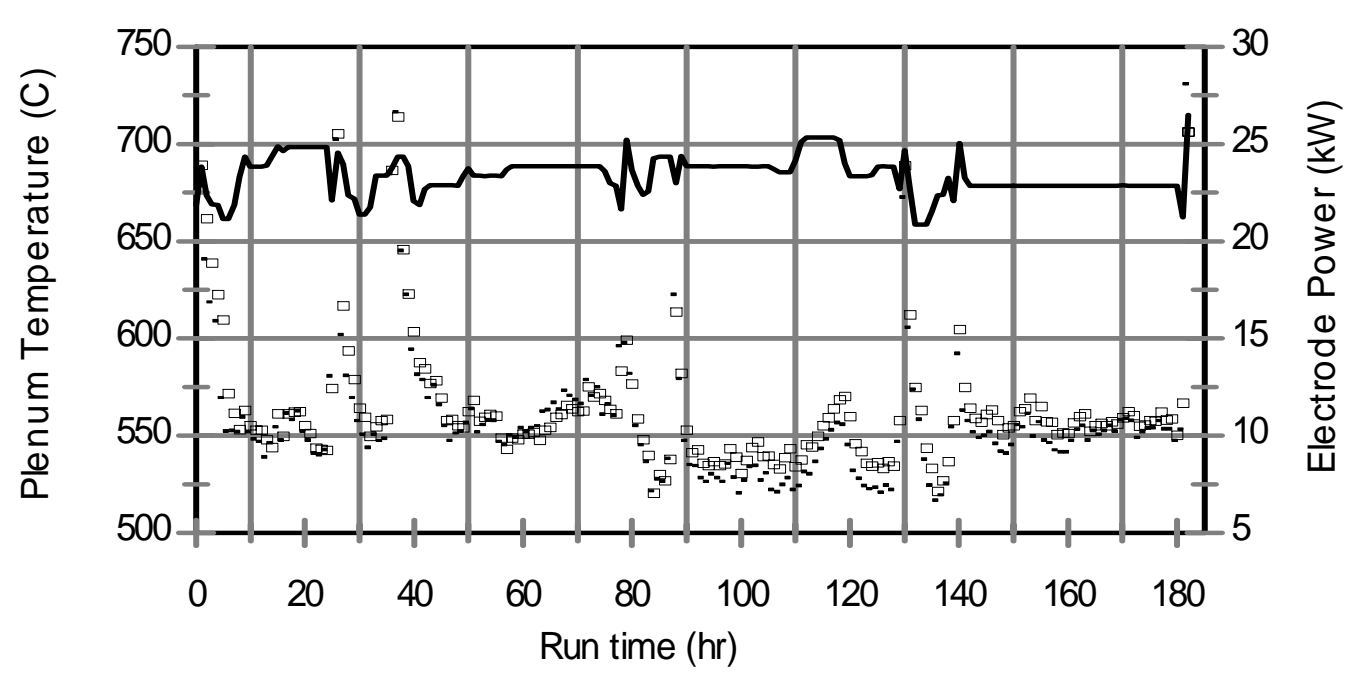

$$
\begin{aligned}
& \text { - } 16 \text { " below ceiling (exposed) } \\
& \square \quad 16^{\prime \prime} \text { below ceiling (thermowell) } \\
& \square \text { Electrode Power }
\end{aligned}
$$

Figure 3.5.e. Plenum temperatures and electrode power during Test 5 (hourly average data). 


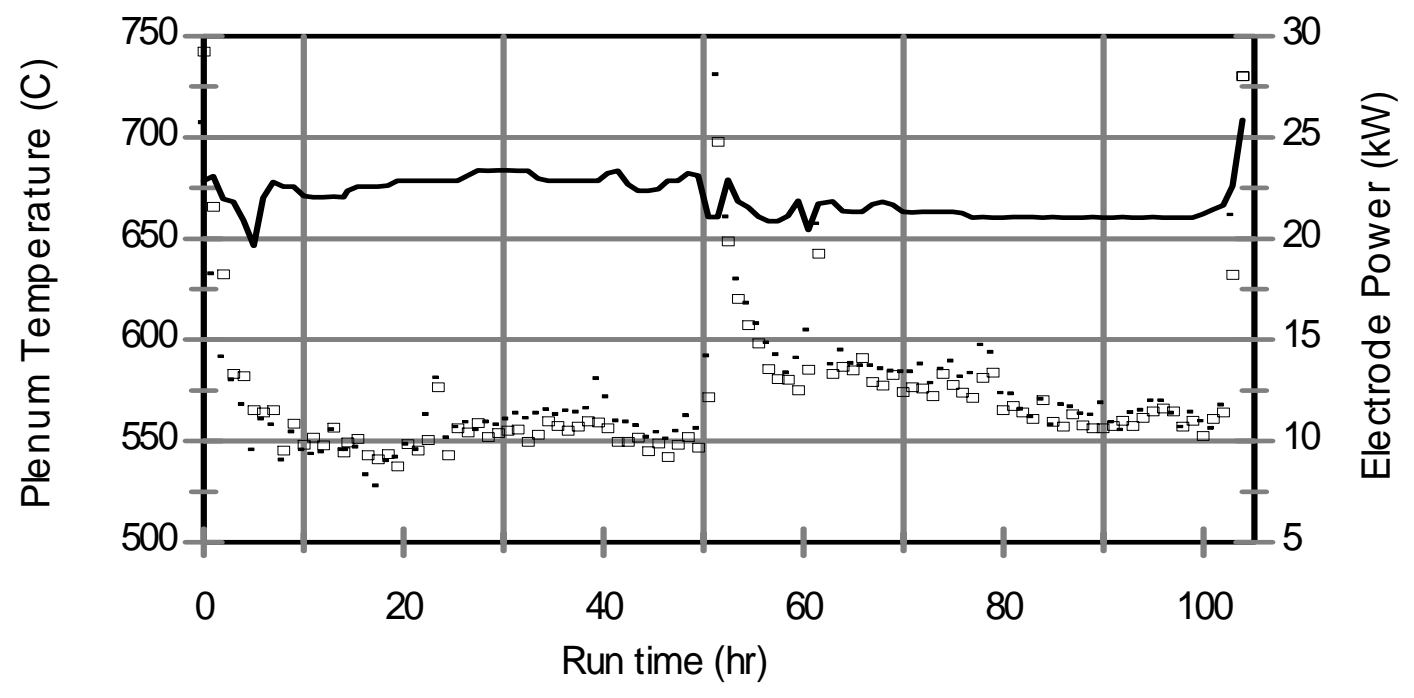

- 16" below ceiling (exposed)

$\square \quad 16$ " below ceiling (thermowell)

Electrode Power

Figure 3.5.f. Plenum temperatures and electrode power during Test 6 (hourly average data). 

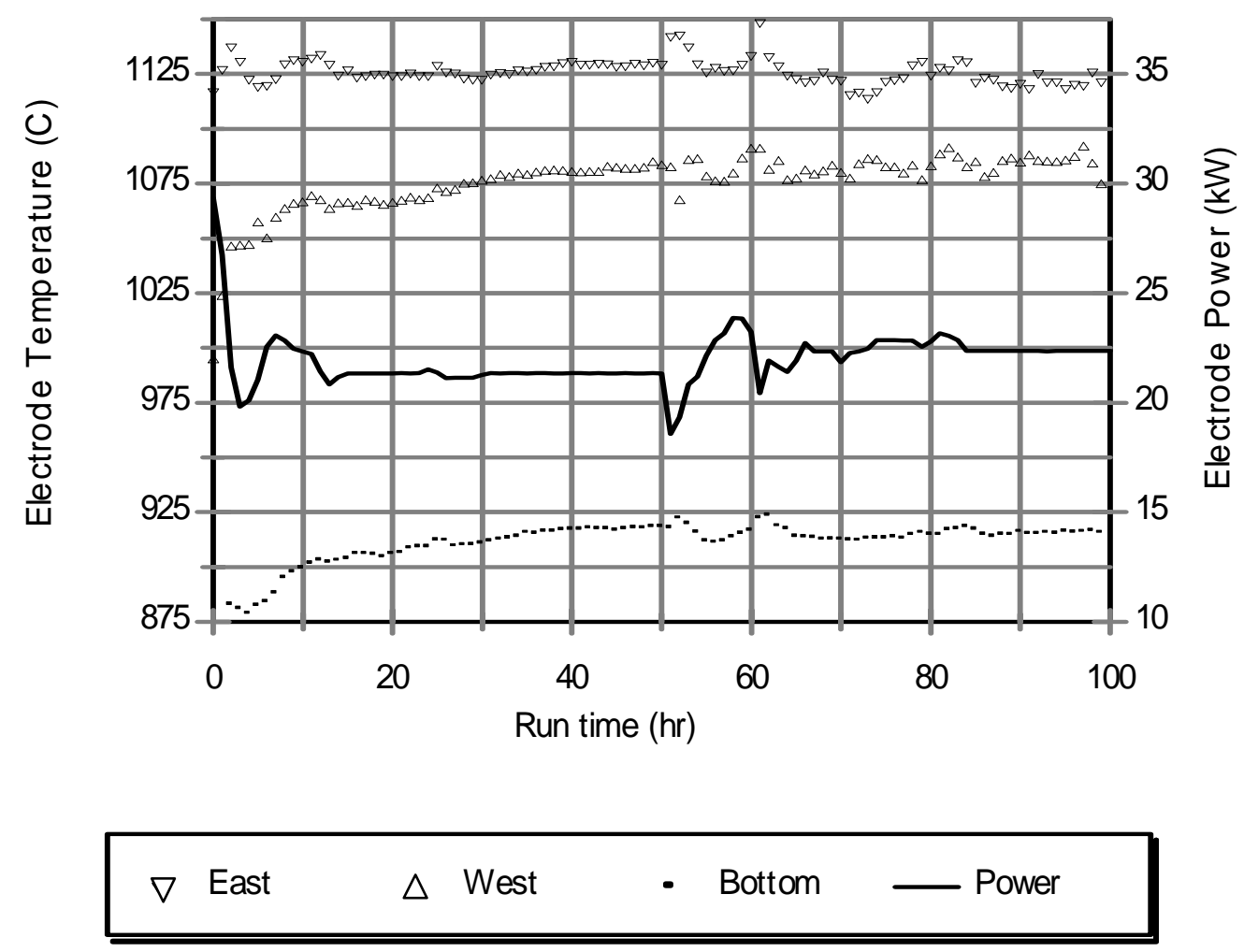

Figure 3.6.a. Electrode temperatures and power during Test 1 (hourly average data). 


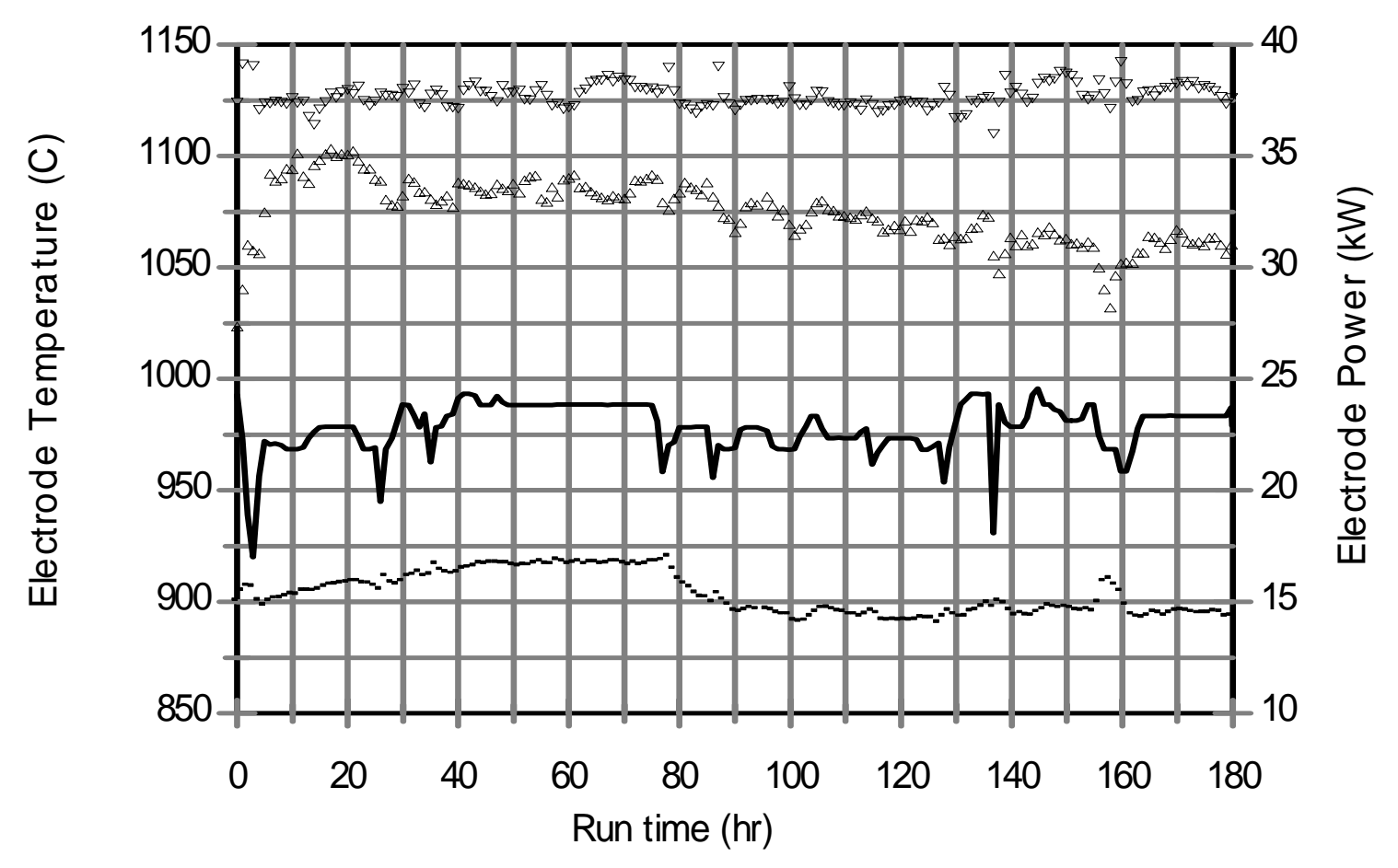
$\nabla$ East
$\triangle$ West
- Bottom
Power

Figure 3.6.b. Electrode temperatures and power during Test 2 (hourly average data). 


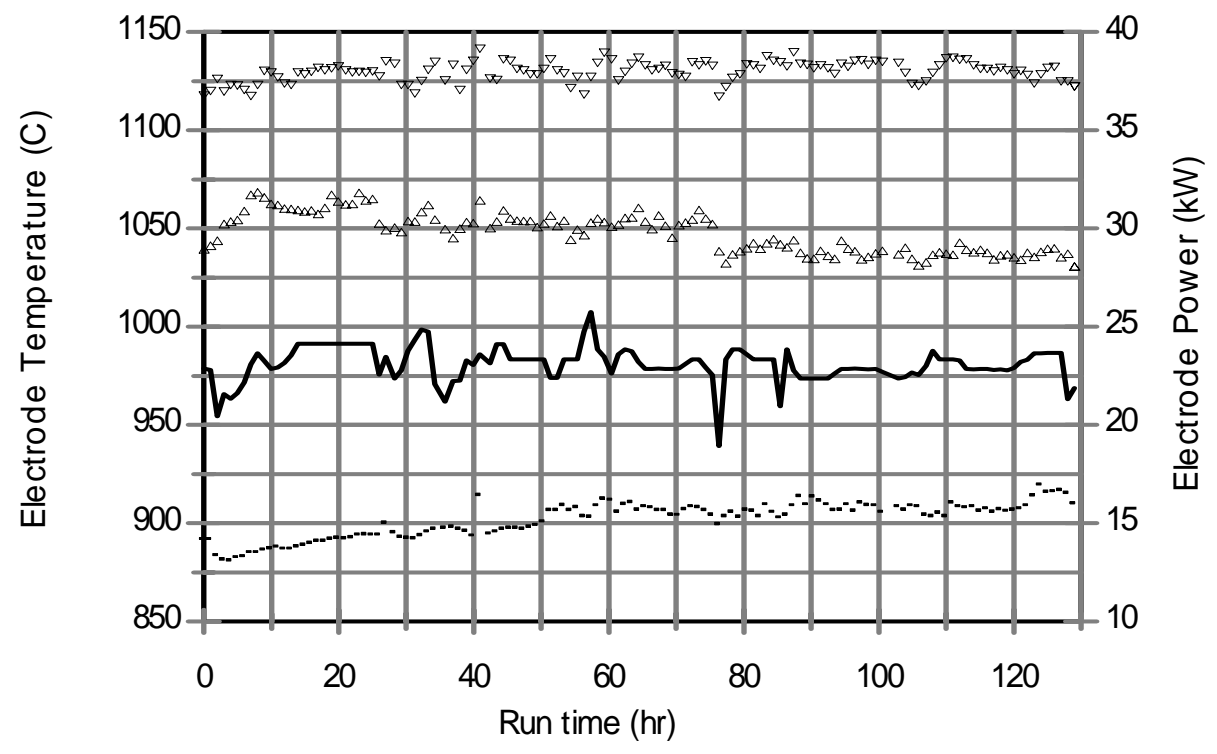

\begin{tabular}{llll}
\hline East $\quad \Delta$ West $\quad \cdot$ Bottom $\quad$ Power \\
\hline \hline
\end{tabular}

Figure 3.6.c. Electrode temperatures and power during Test 3 (hourly average data). 

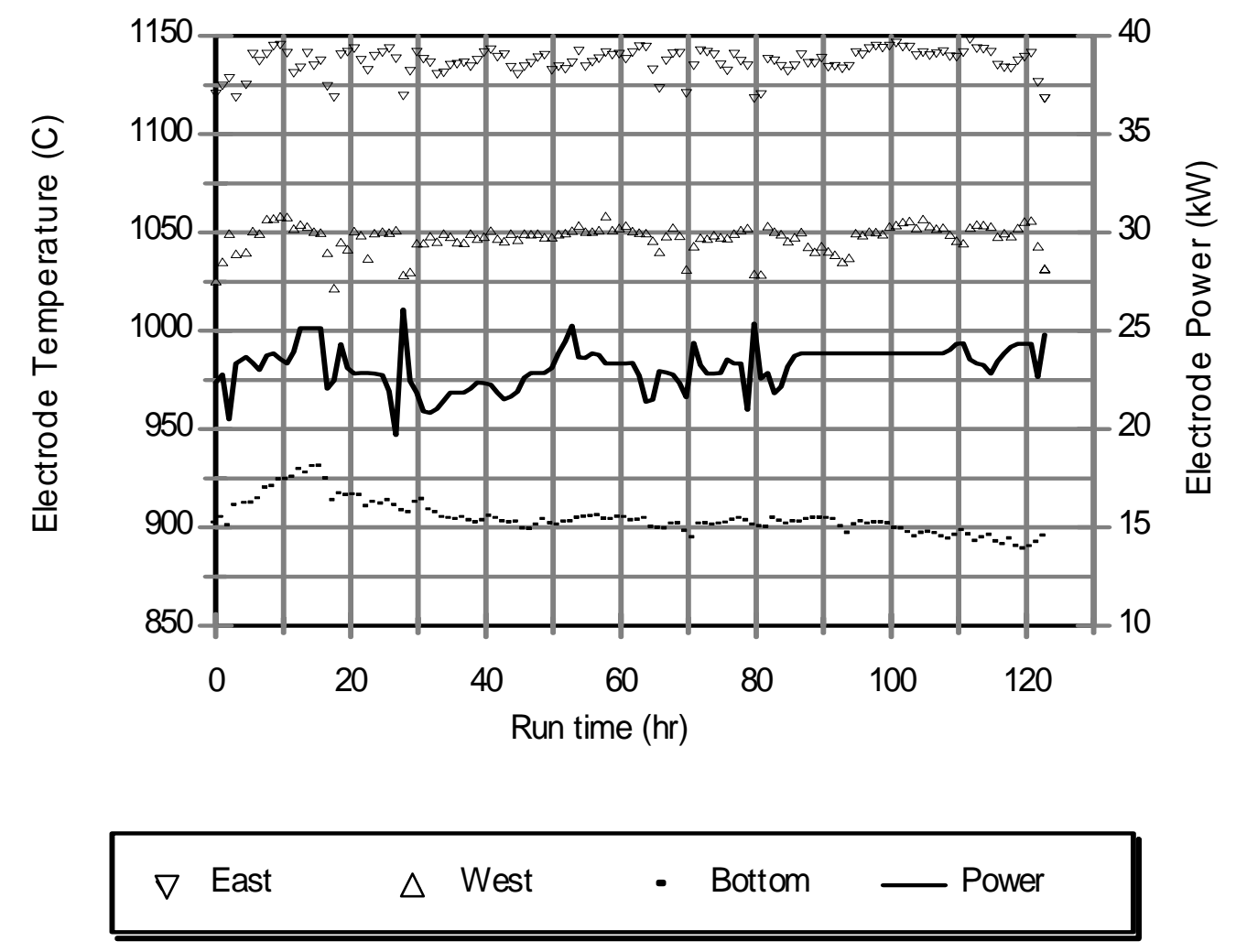

Figure 3.6.d. Electrode temperatures and power during Test 4 (hourly average data). 

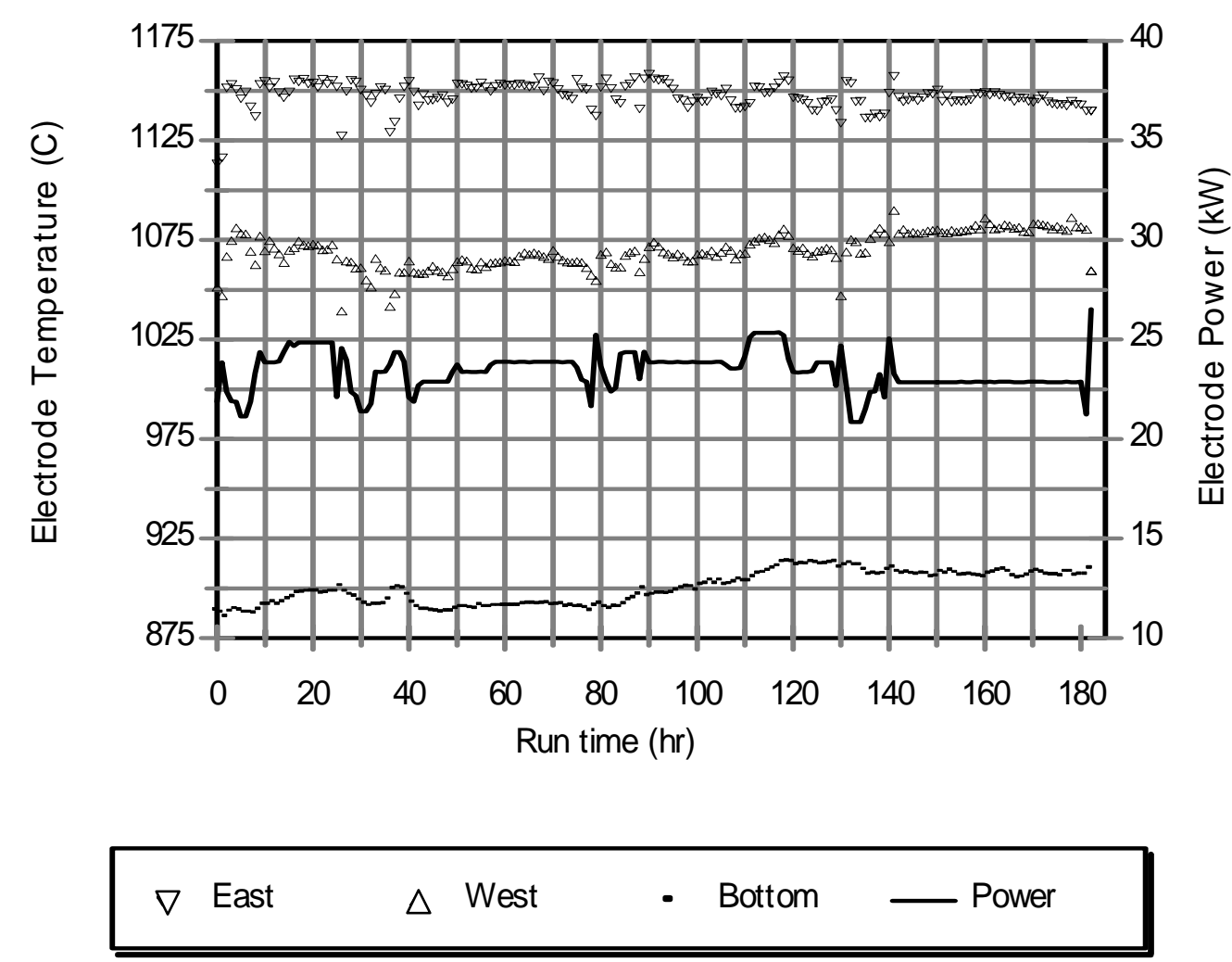

Figure 3.6.e. Electrode temperatures and power during Test 5 (hourly average data). 

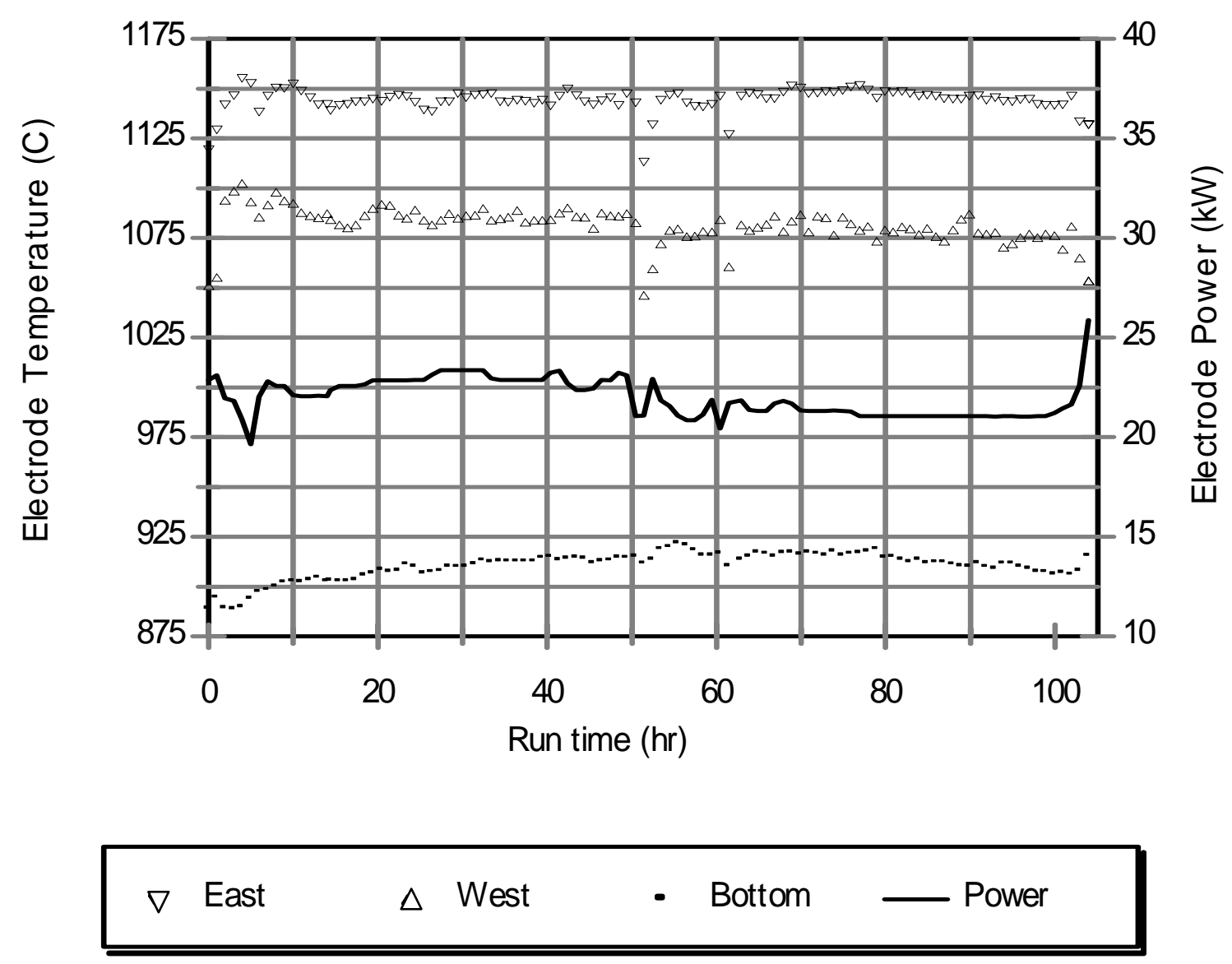

Figure 3.6.f. Electrode temperatures and power during Test 6 (hourly average data). 

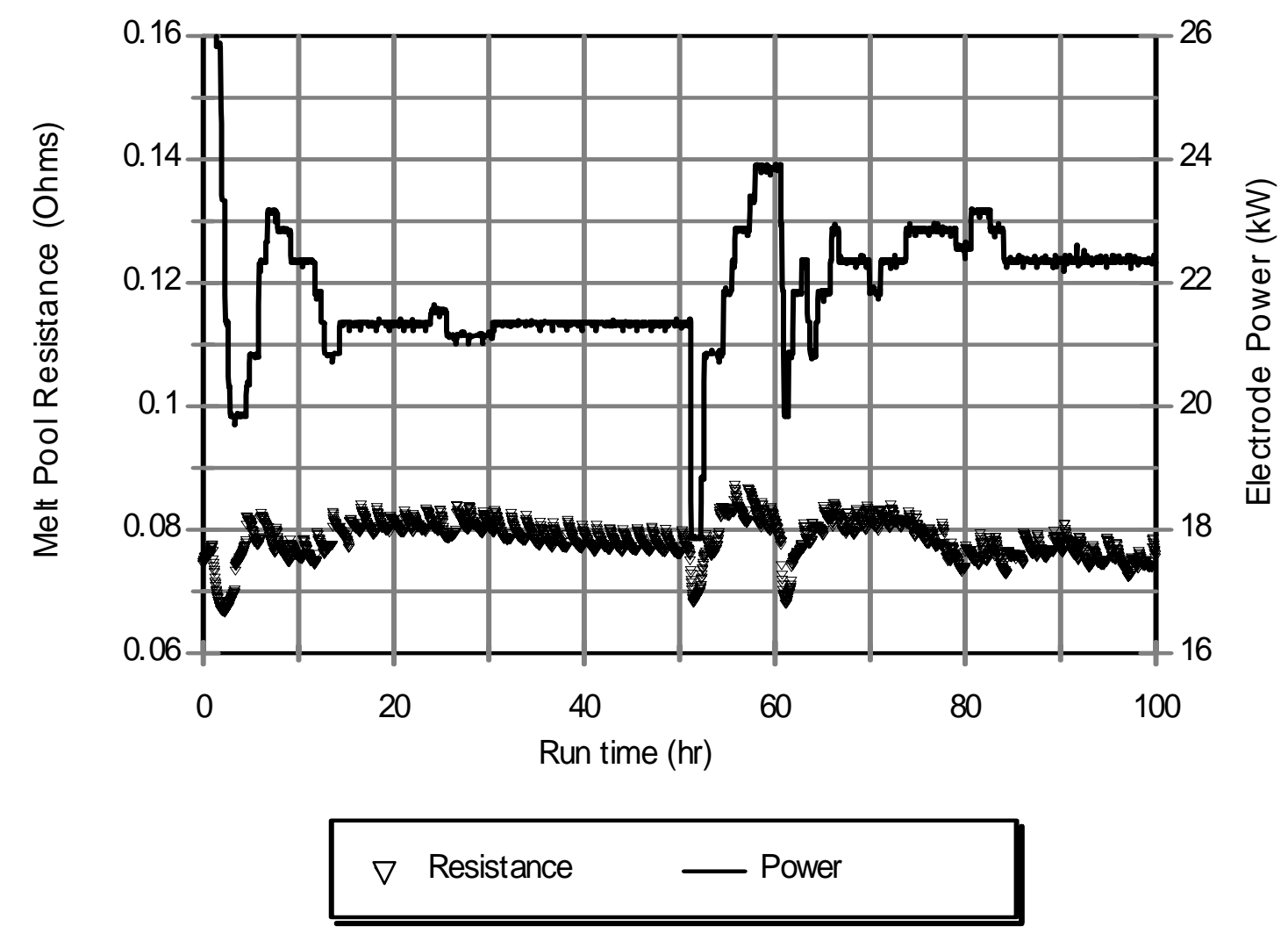

Figure 3.7.a. Melt pool resistance and electrode power during Test 1. 
The Catholic University of America Vitreous State Laboratory DuraMelter 100 Tests to Support LAW Glass Formulation Correlation Development Final Report, VSL-06R6480-1, Rev. 0

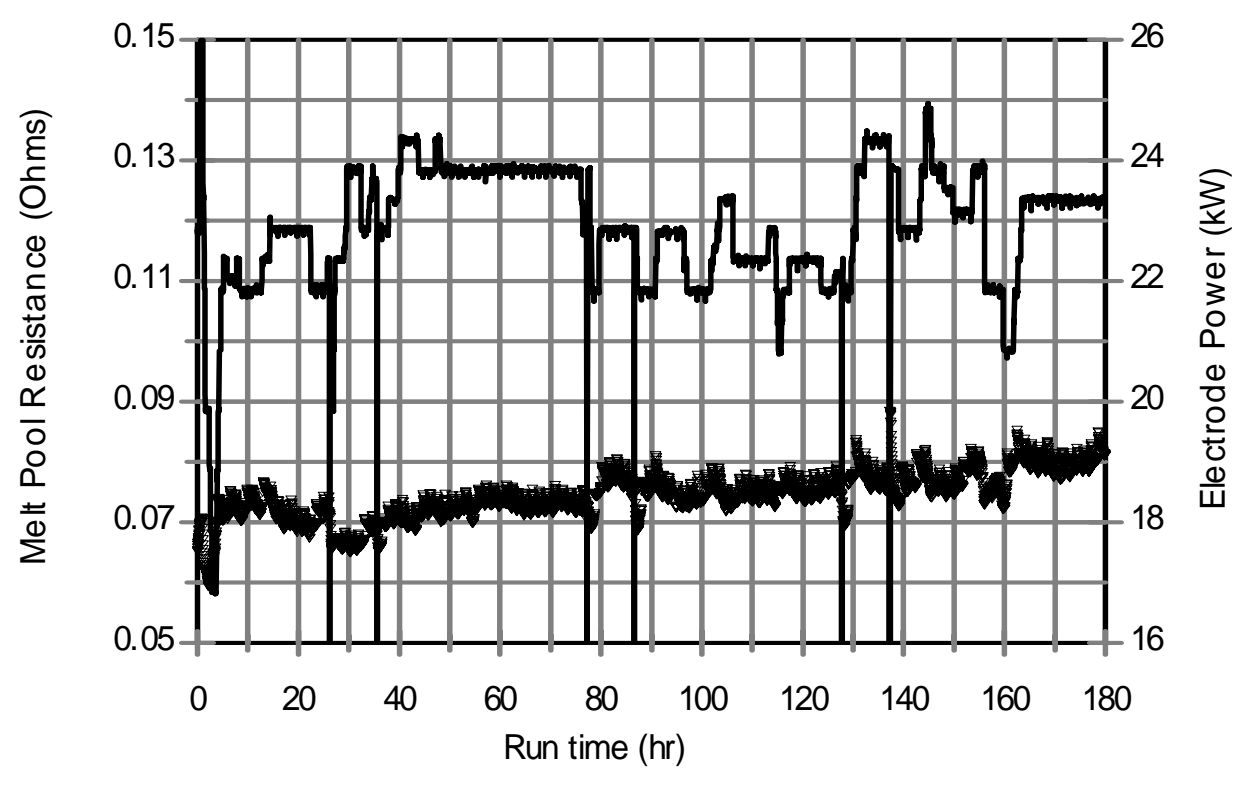

$\nabla$ Resistance

Power

Figure 3.7.b. Melt pool resistance and electrode power during Test 2. 


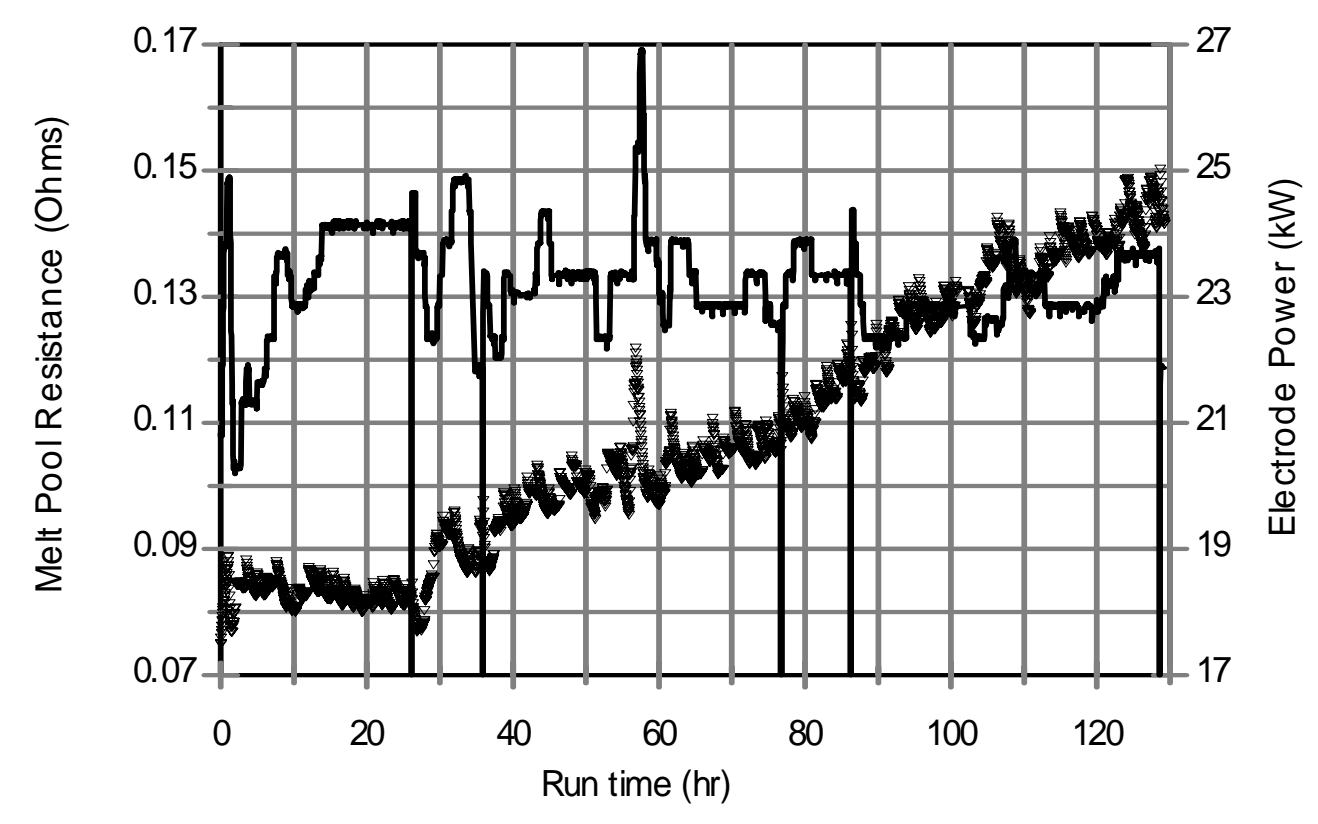

$\nabla$ Resistance $\quad$ Power

Figure 3.7.c. Melt pool resistance and electrode power during Test 3. 


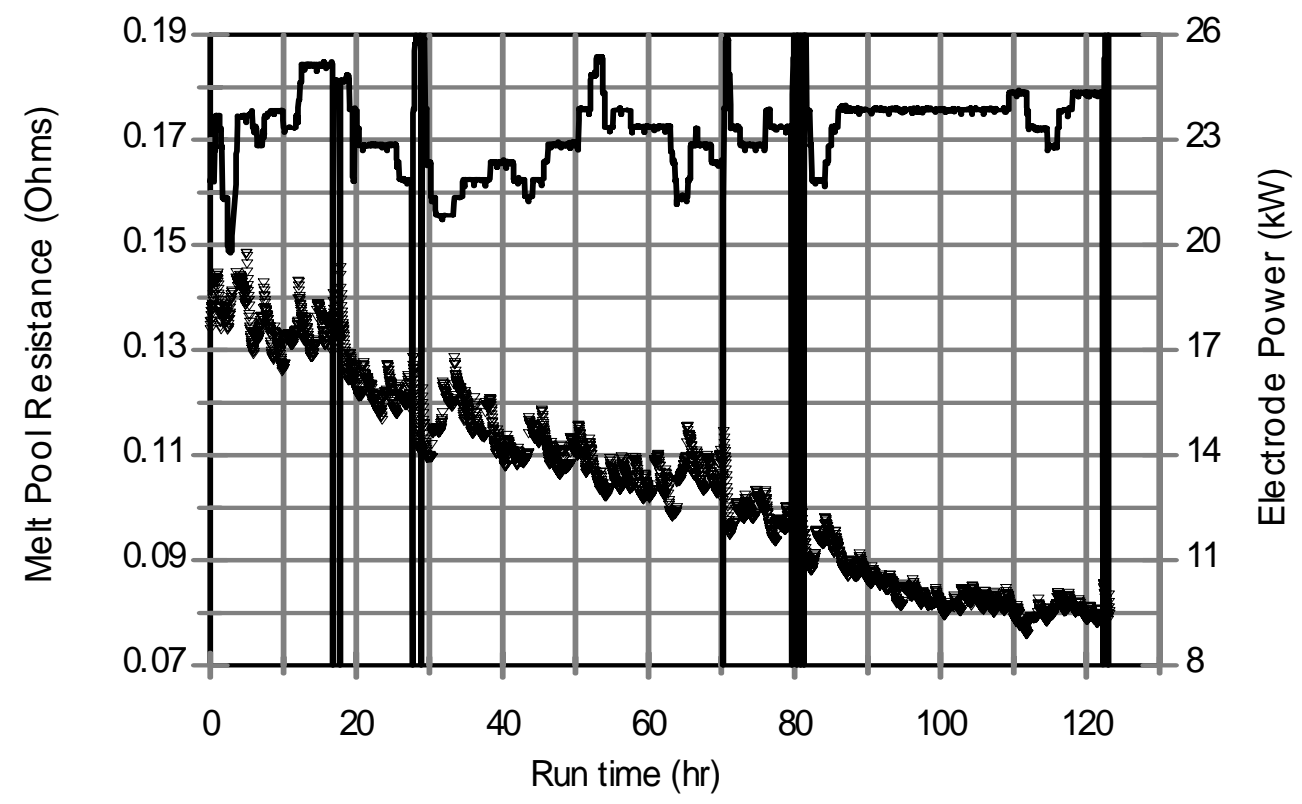

$\nabla$ Resistance $\quad$ Power

Figure 3.7.d. Melt pool resistance and electrode power during Test 4. 


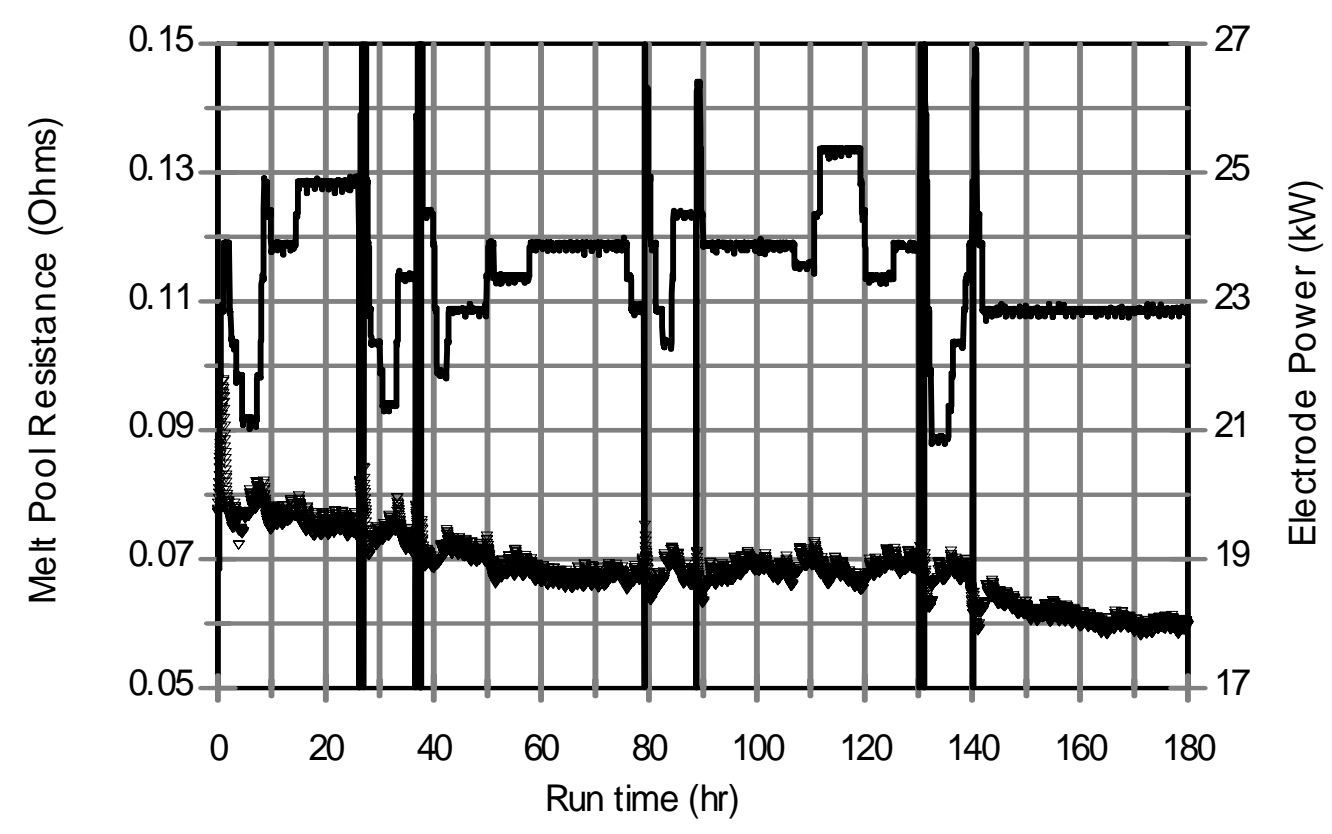

$\nabla$ Resistance Power

Figure 3.7.e. Melt pool resistance and electrode power during Test 5. 


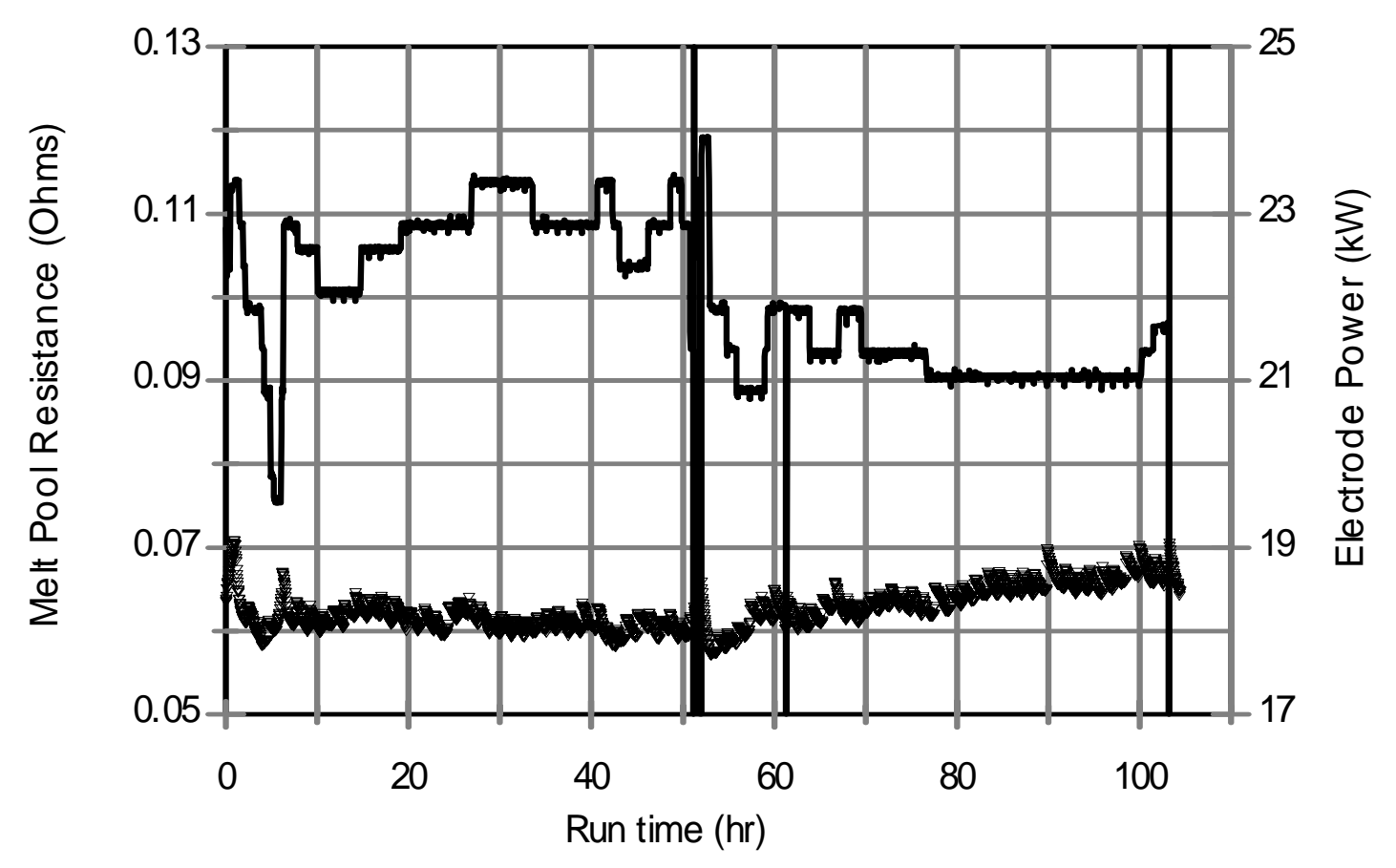

$\nabla$ Resistance - Power

Figure 3.7.f. Melt pool resistance and electrode power during Test 6. 

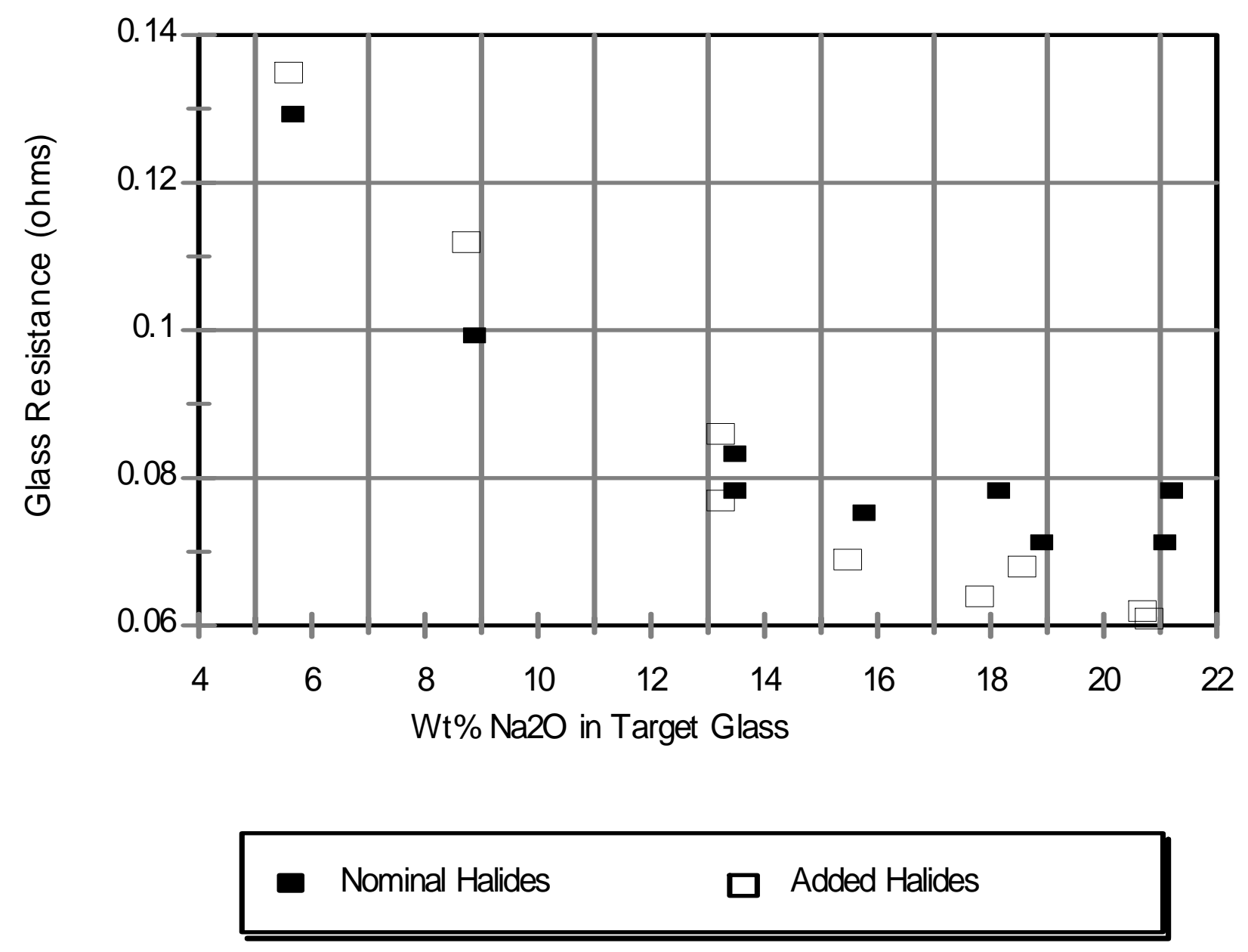

Figure 3.8. Melt pool resistance and feed sodium content. 


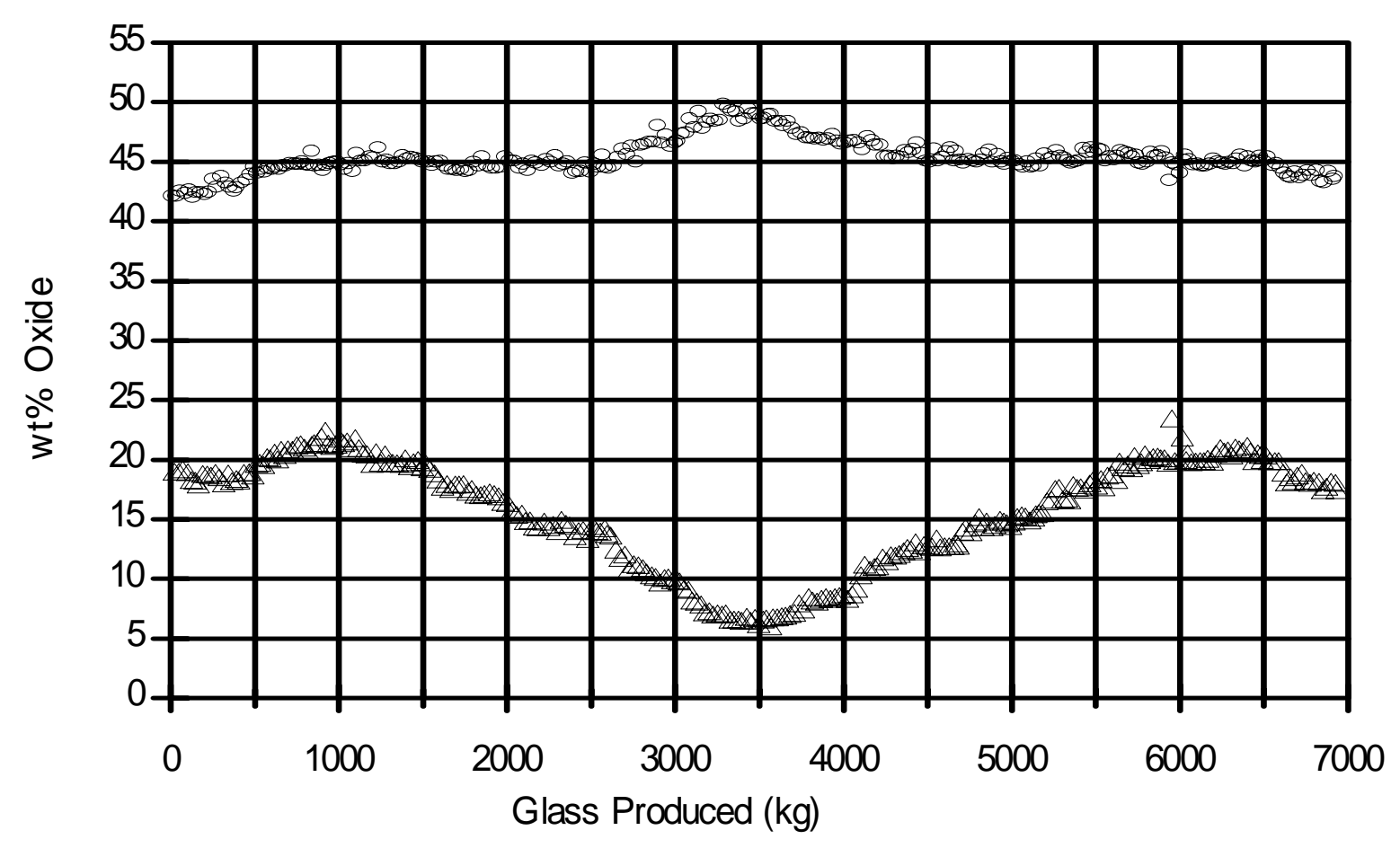
$\Delta \mathrm{Na} \quad 0 \quad \mathrm{Si}$

Figure 4.1. XRF analysis of $\mathrm{Na}_{2} \mathrm{O}$ and $\mathrm{SiO}_{2}$ in melter glasses. 

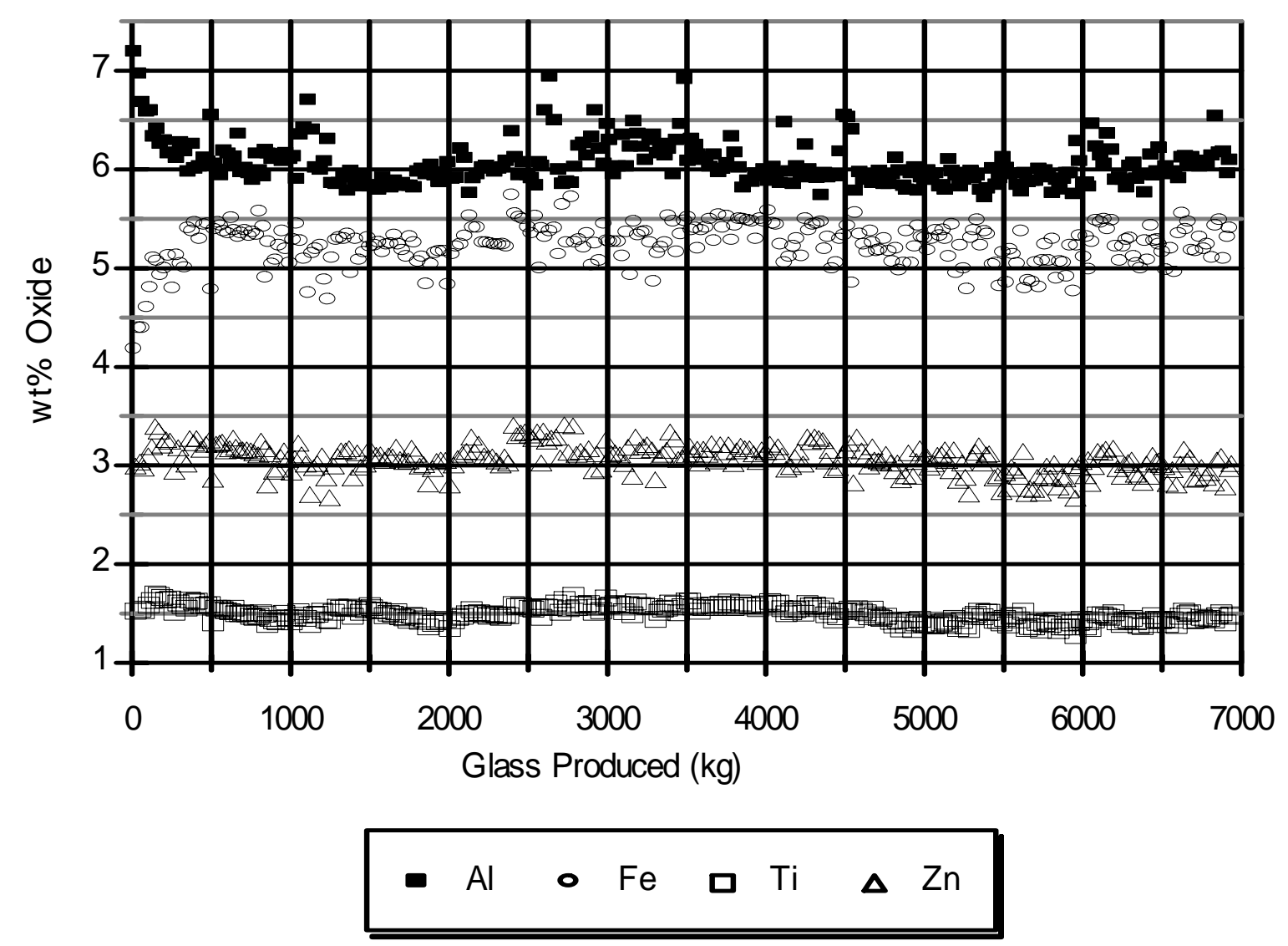

Figure 4.2. XRF analysis of oxides in product glasses which are constant during testing. 


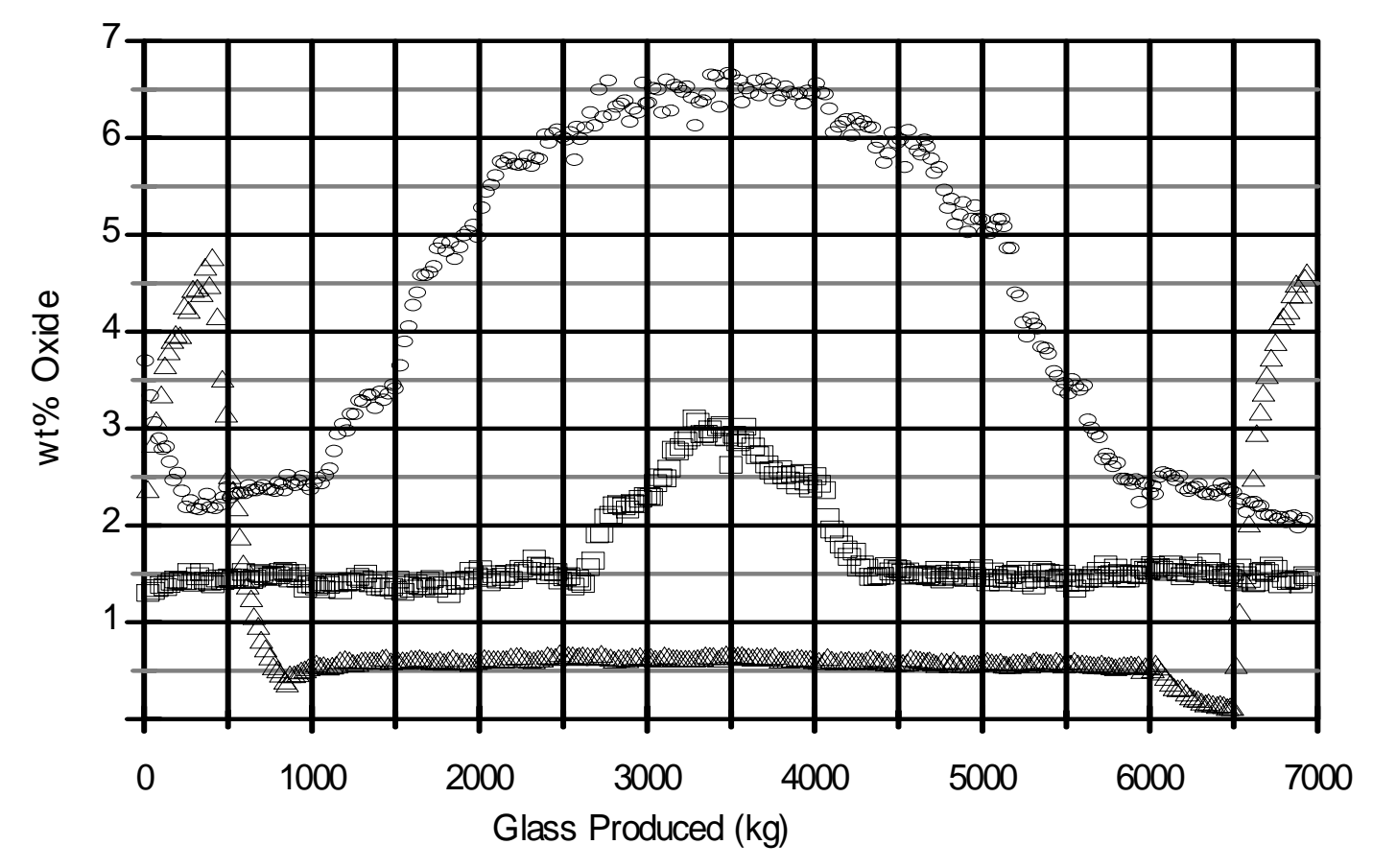

\section{- $\mathrm{Ca} \quad \boldsymbol{\Delta} \mathrm{K} \quad$ 口 $\mathrm{Mg}$}

Figure 4.3. XRF analysis of oxides in product glasses which vary during testing. 


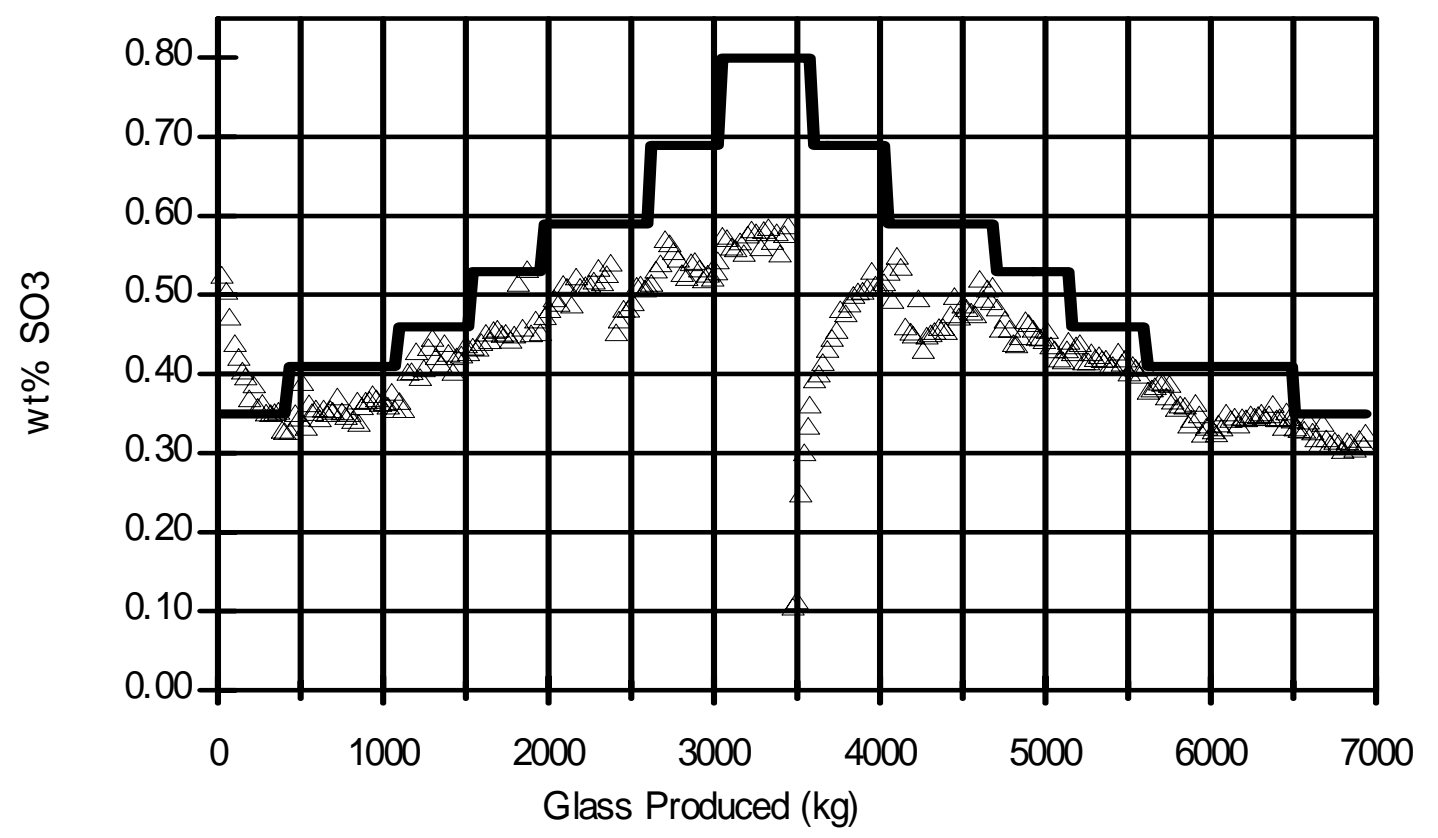

$\Delta$ Measured Target

Figure 4.4. XRF analysis of sulfur in melter glasses. 


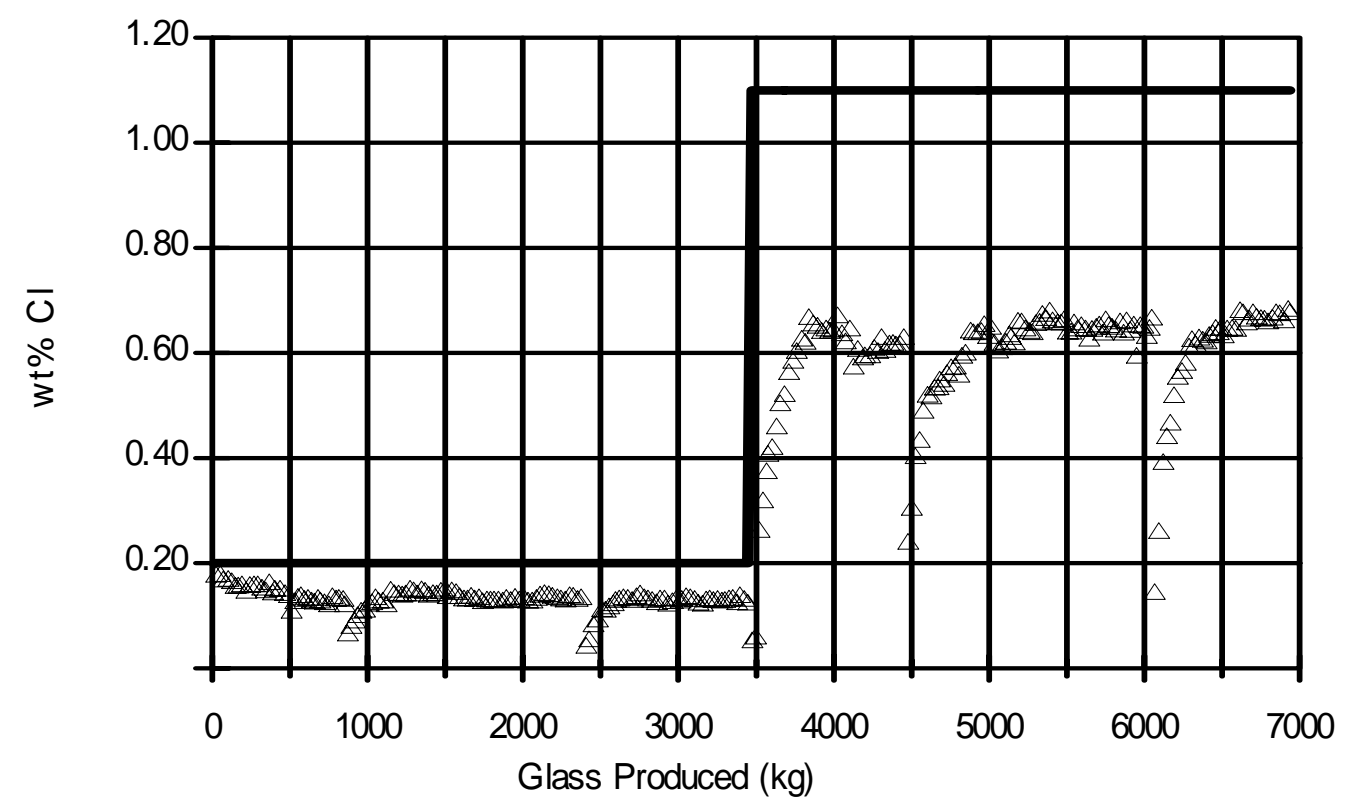

Figure 4.5. XRF analysis of chlorine in melter glasses. 

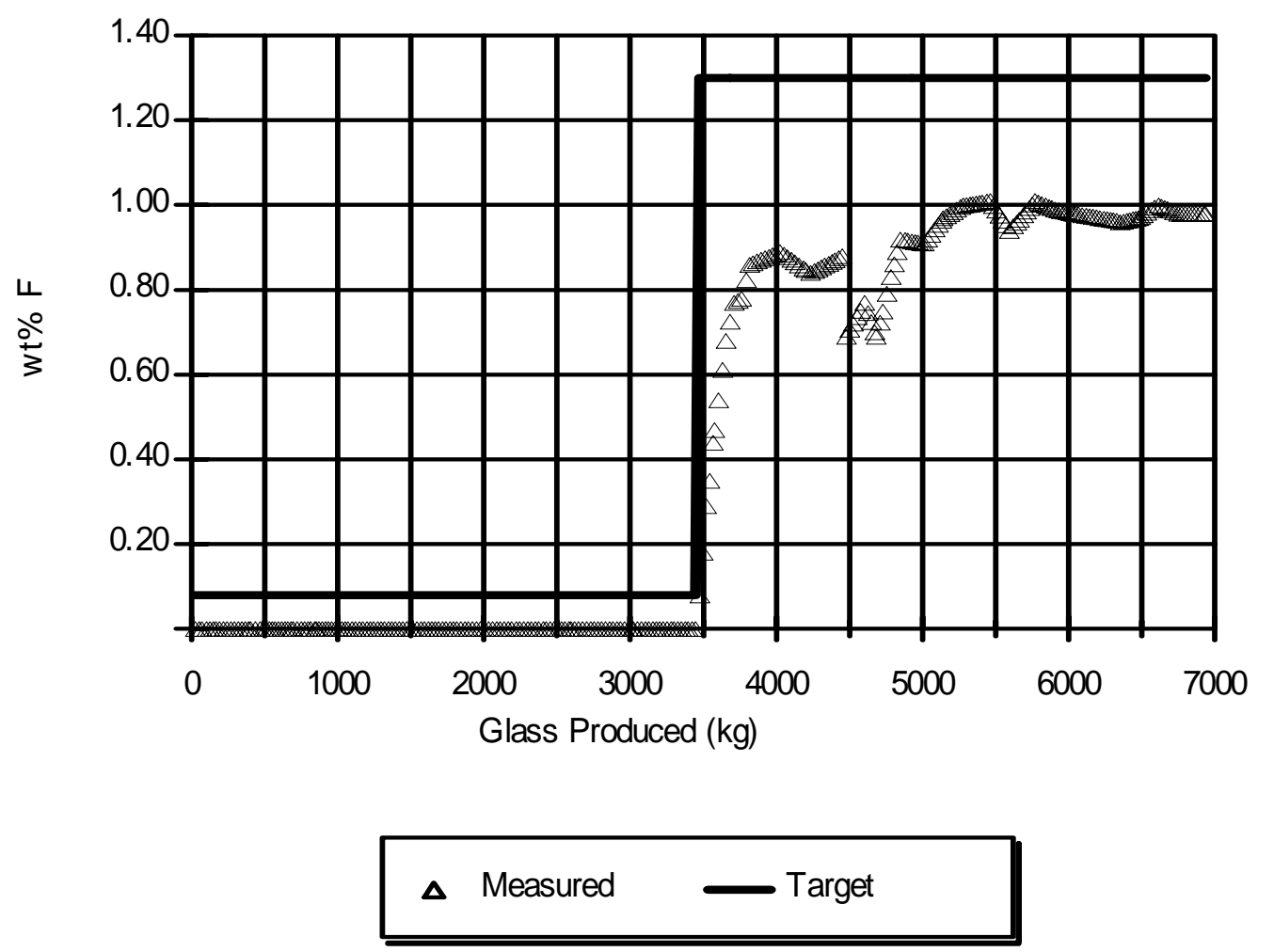

Figure 4.6. XRF analysis of fluorine in melter glasses. 

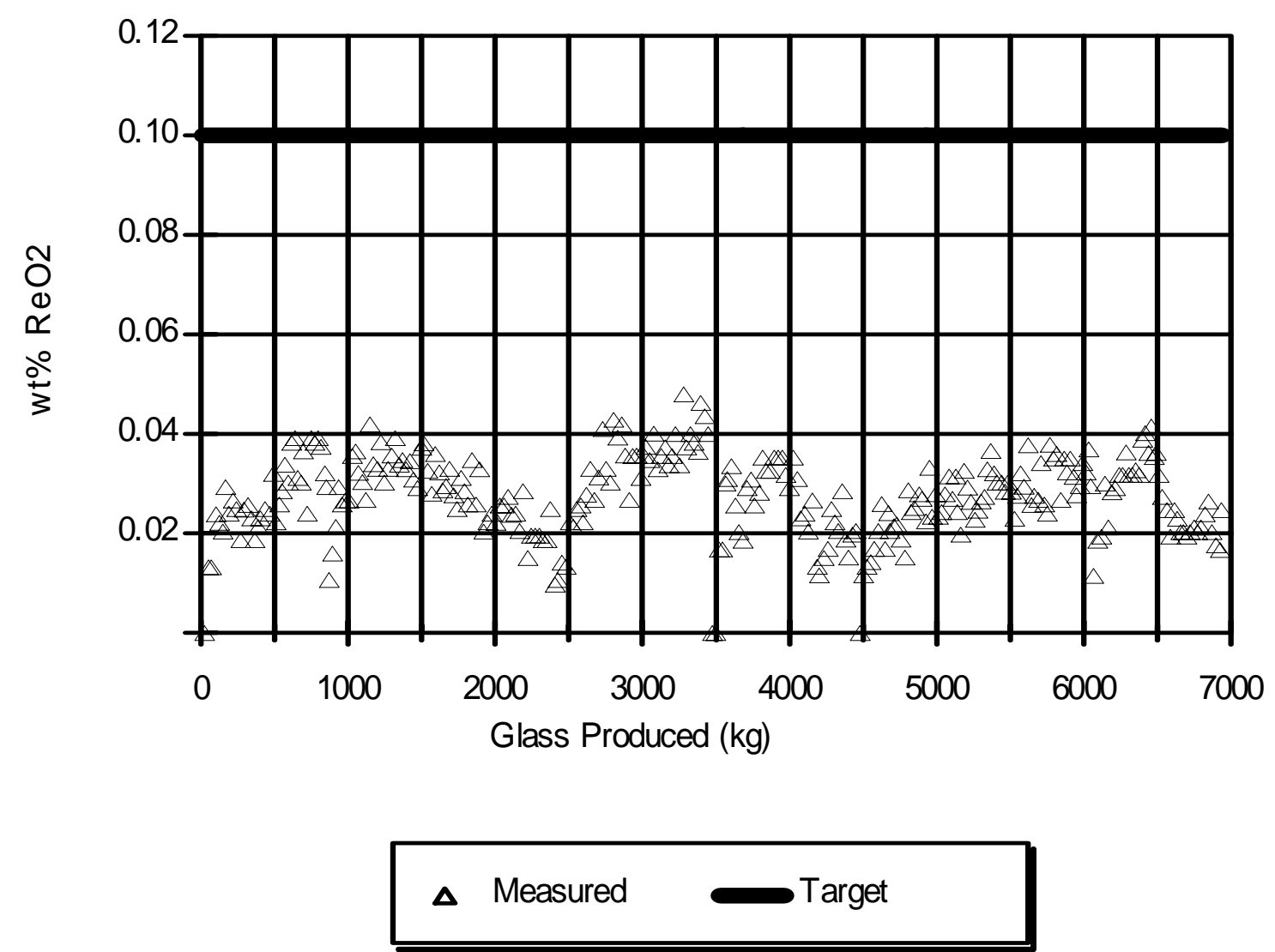

Figure 4.7. XRF analysis of rhenium in melter glasses. 


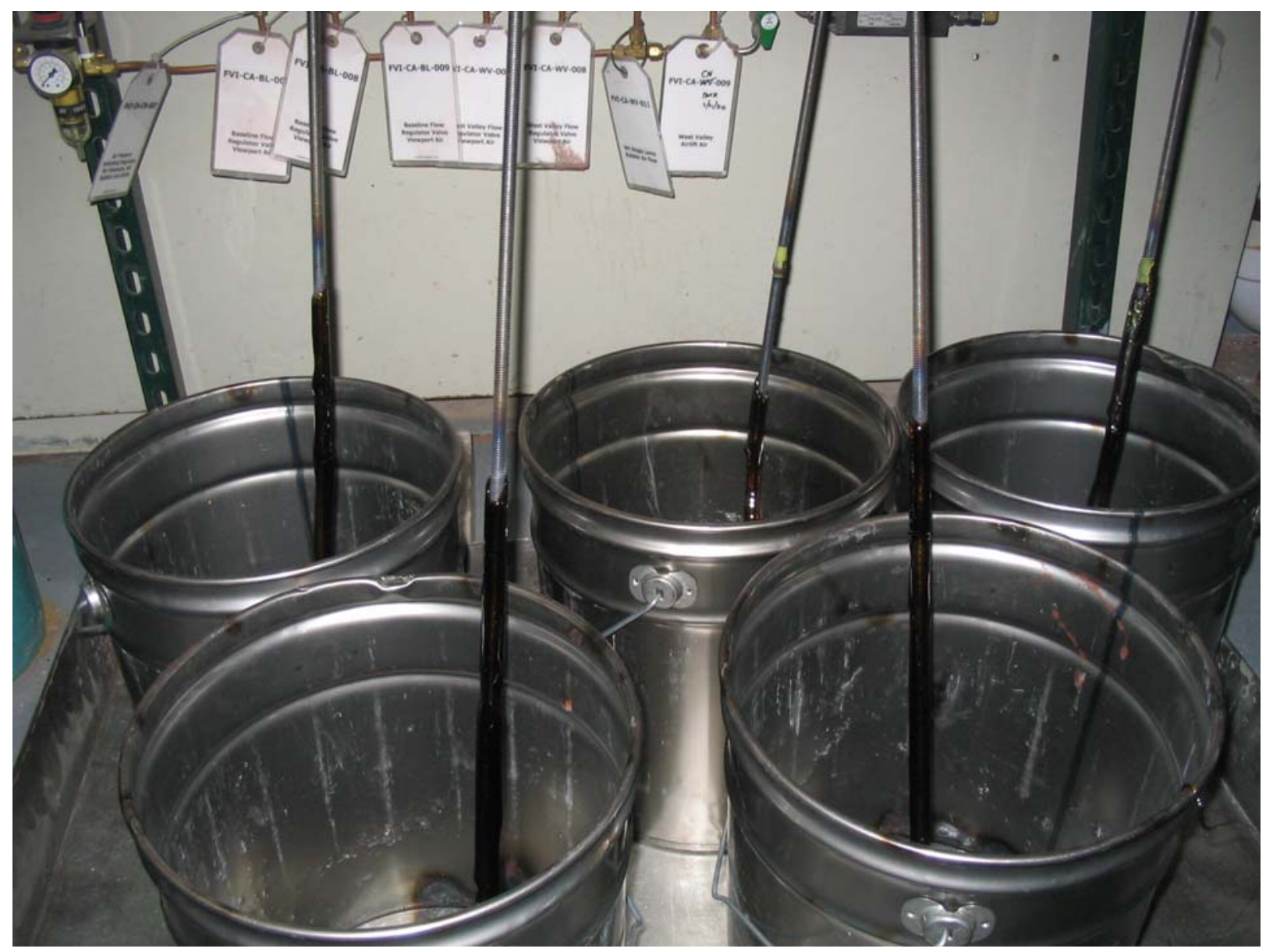

Figure 4.8. Dip samples from Test 6A. Note separated sulfur on two rods in the back right buckets. 


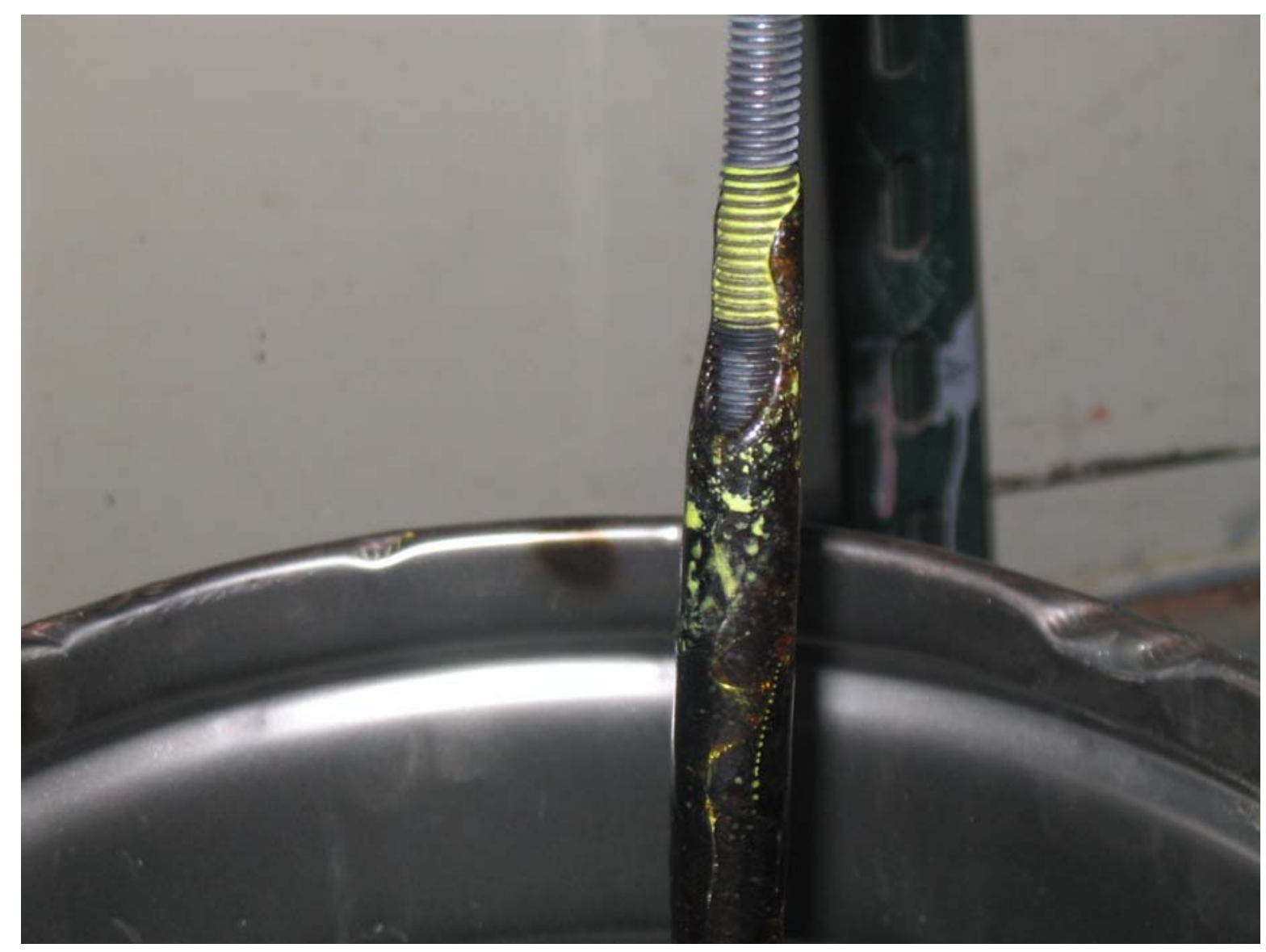

Figure 4.9. Separated sulfur on dip sample DWV-D-22A from Test 6A. 


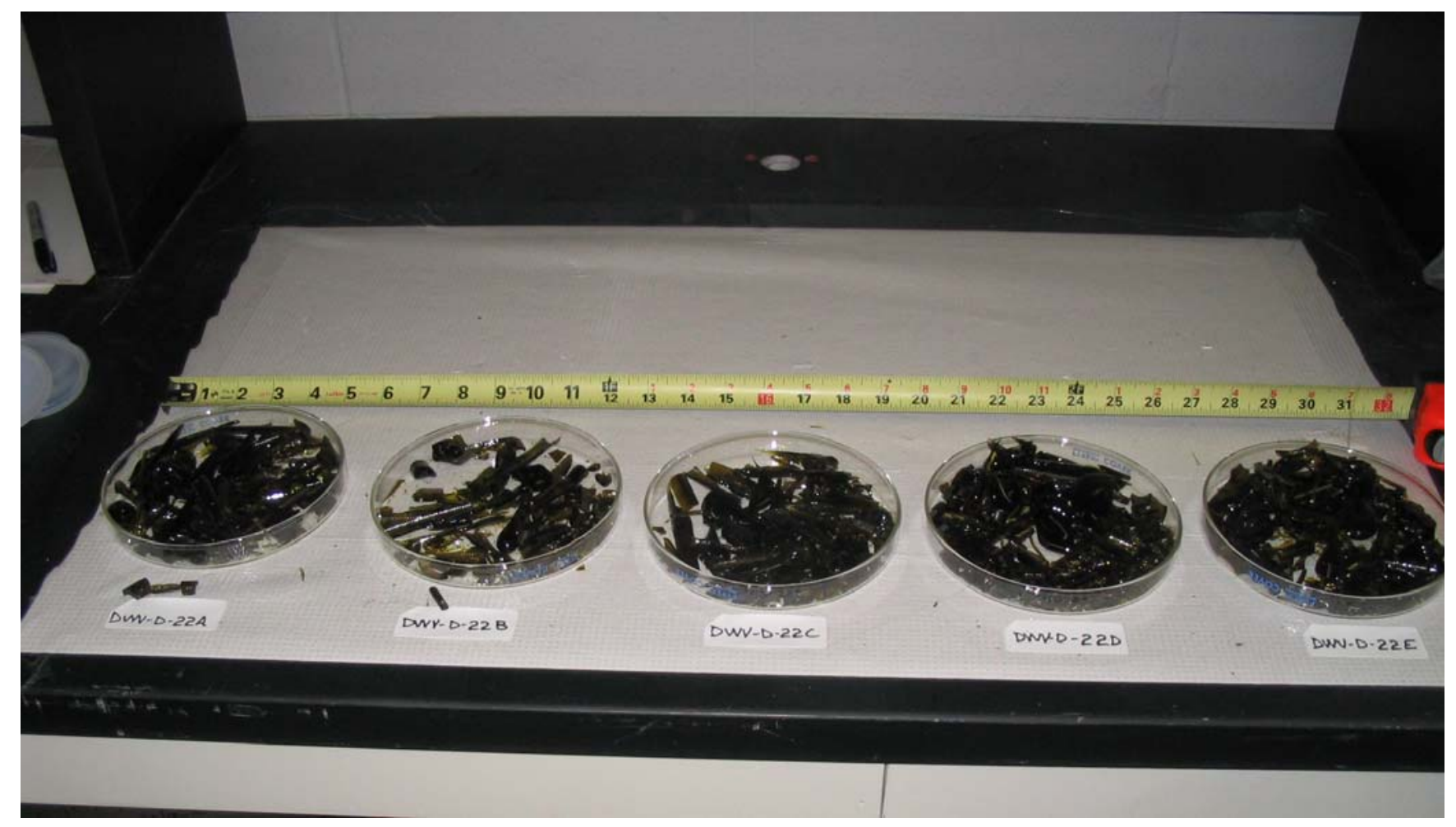

Figure 4.10. Dip samples from Test 6A removed from rods and placed in Petri dishes for examination. 


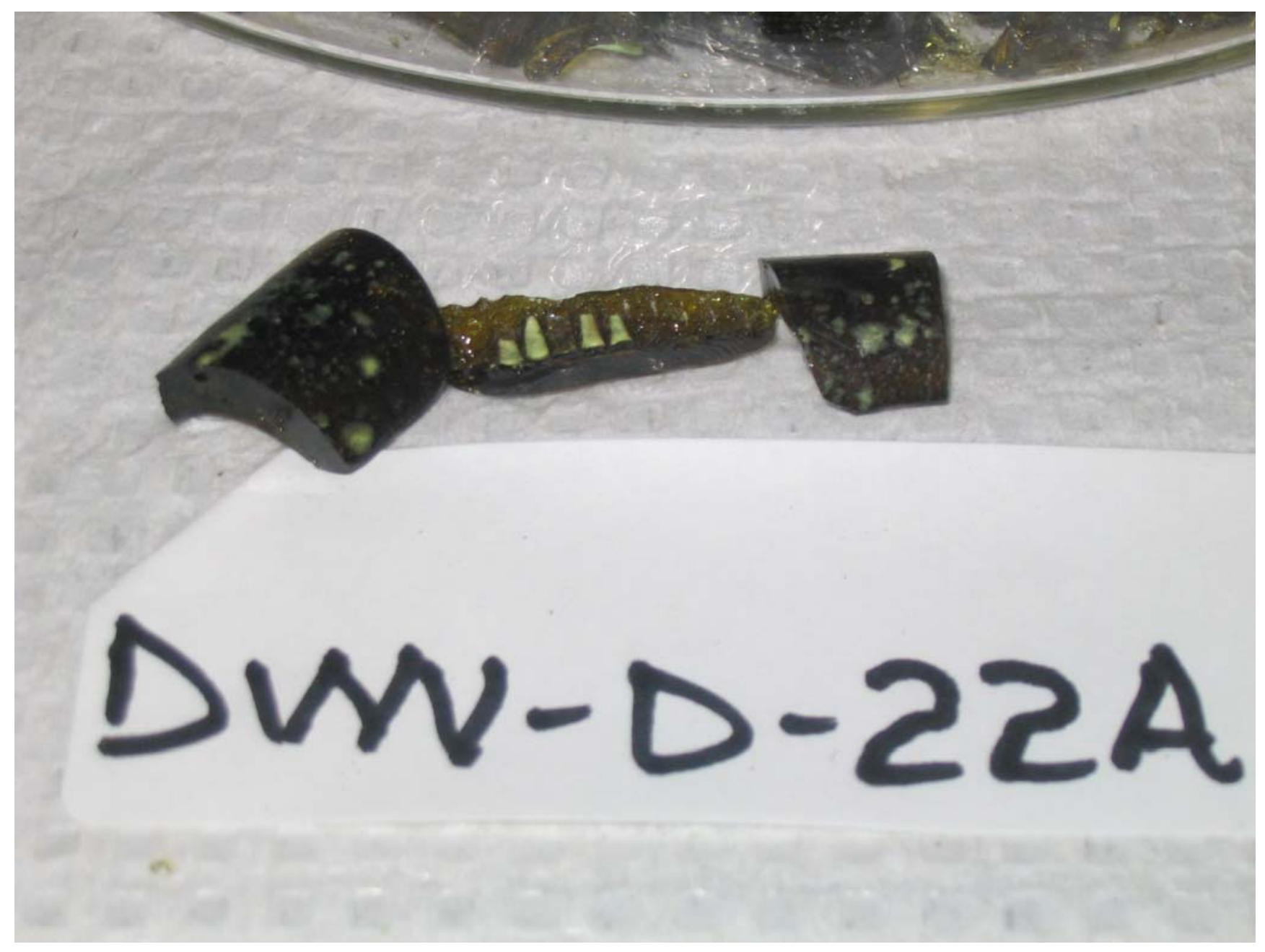

Figure 4.11. Separated sulfur on dip sample DWV-D-22A from Test 6A after removal from rod. 
The Catholic University of America Vitreous State Laboratory
DuraMelter 100 Tests to Support LAW Glass Formulation Correlation Dev24, Rev. 0 DuraMelter 100 Tests to Support LAW Glass Formulation Correlation Development Final Report, VSL-06R6480-1, Rev. 0

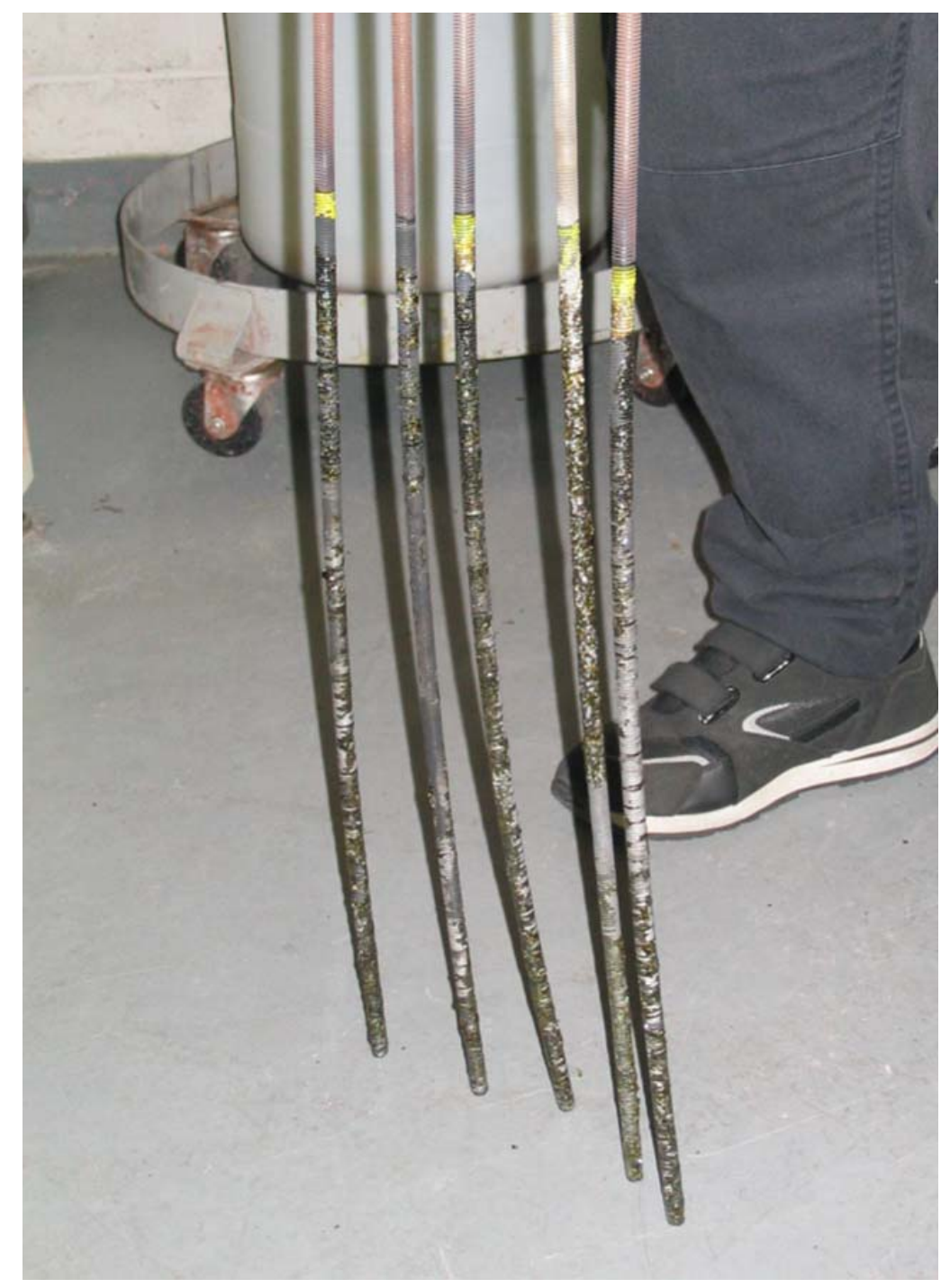

Figure 4.12. Separated sulfur remaining on rods after glass removal during Test 4B. 


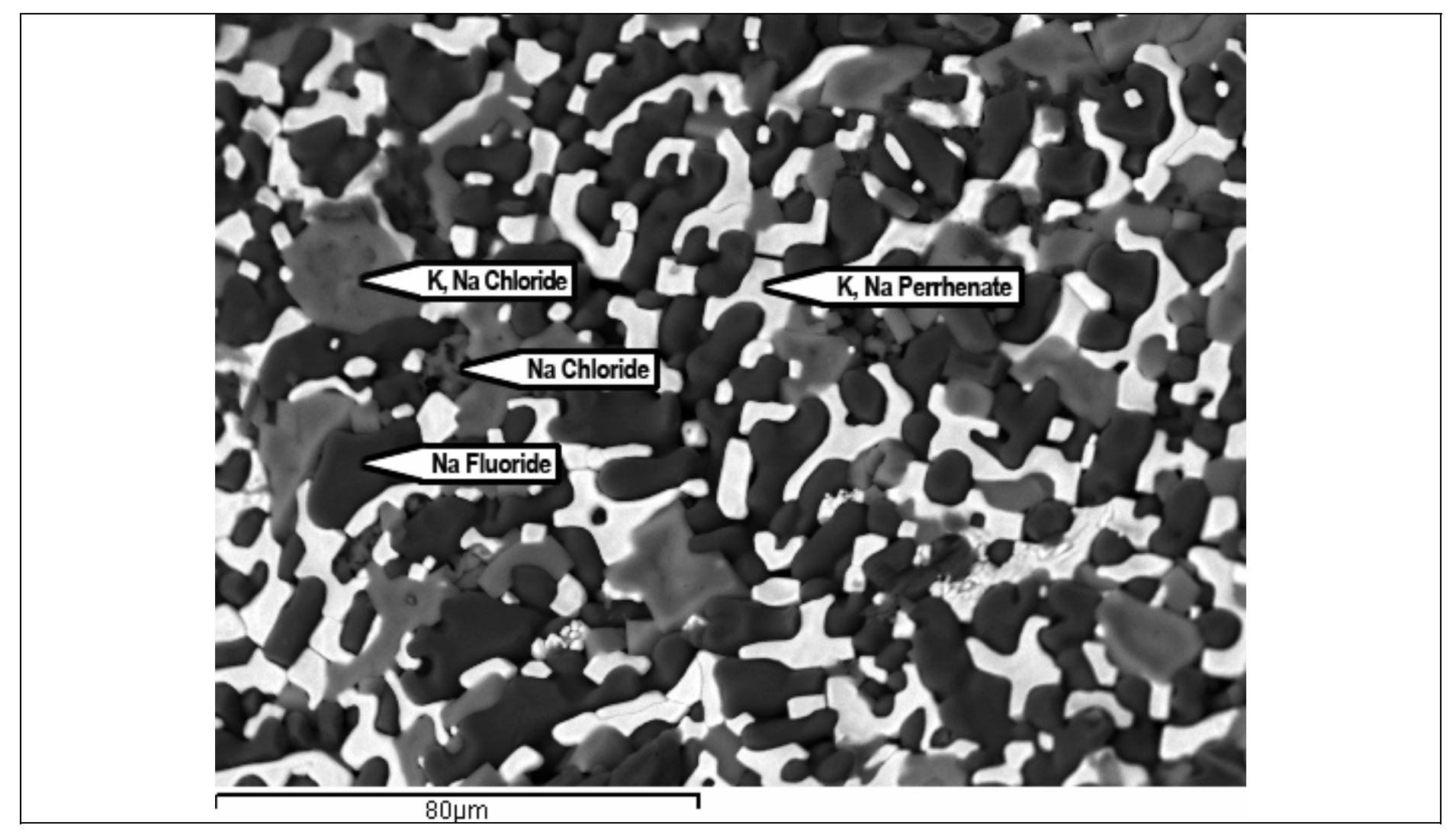

Figure 4.13.a. Back scattered electron image taken at 1000X magnification on WVZ-D-74C. 


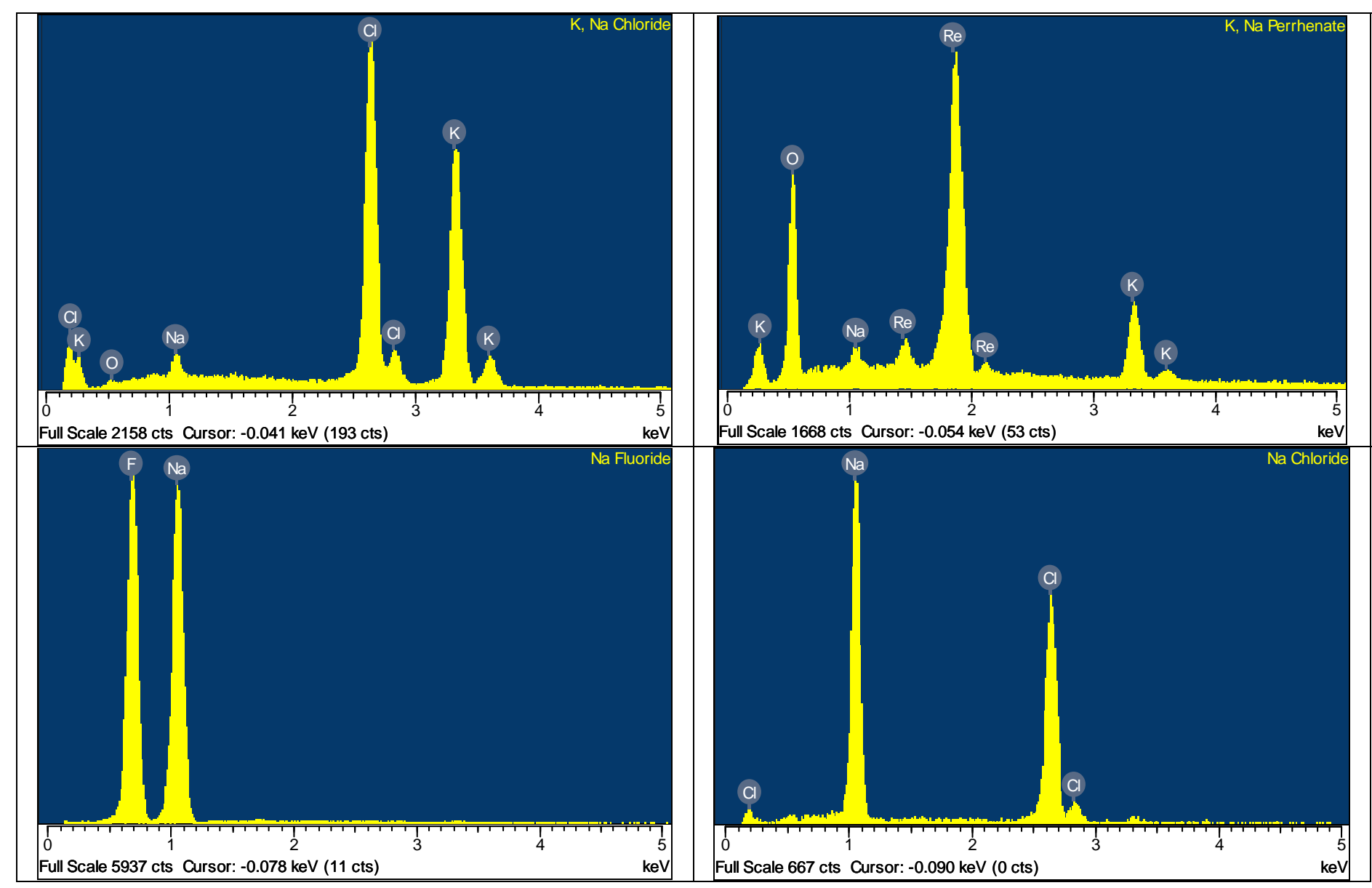

Figure 4.13.b. Spectra from secondary phases in WVZ-D-74C. 


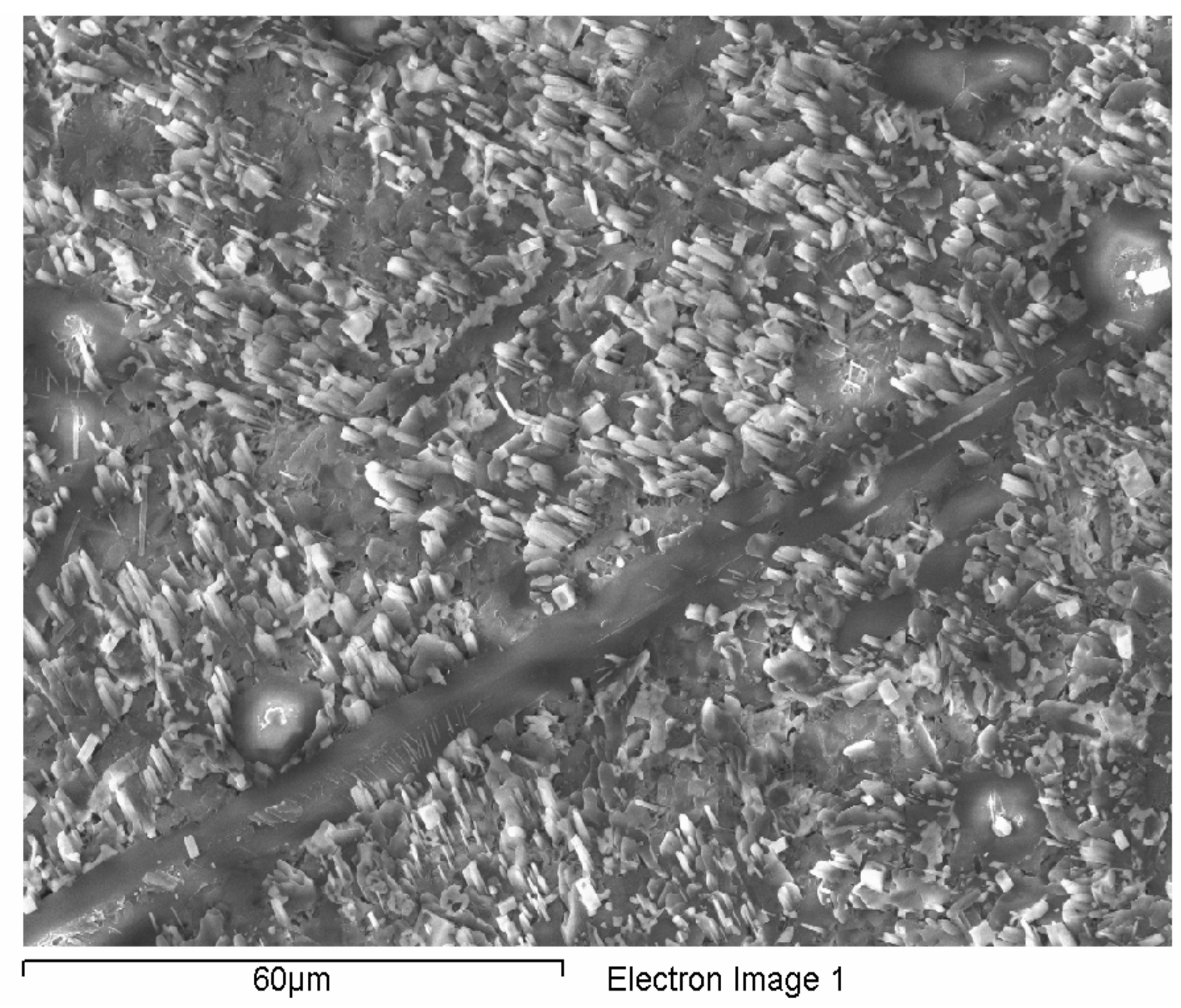

Figure 4.14.a. SEM micrograph of secondary phases in BWV-D-68E. Note that the material consists of two distinct phases: a substrate and crystals which protrude from the substrate. 


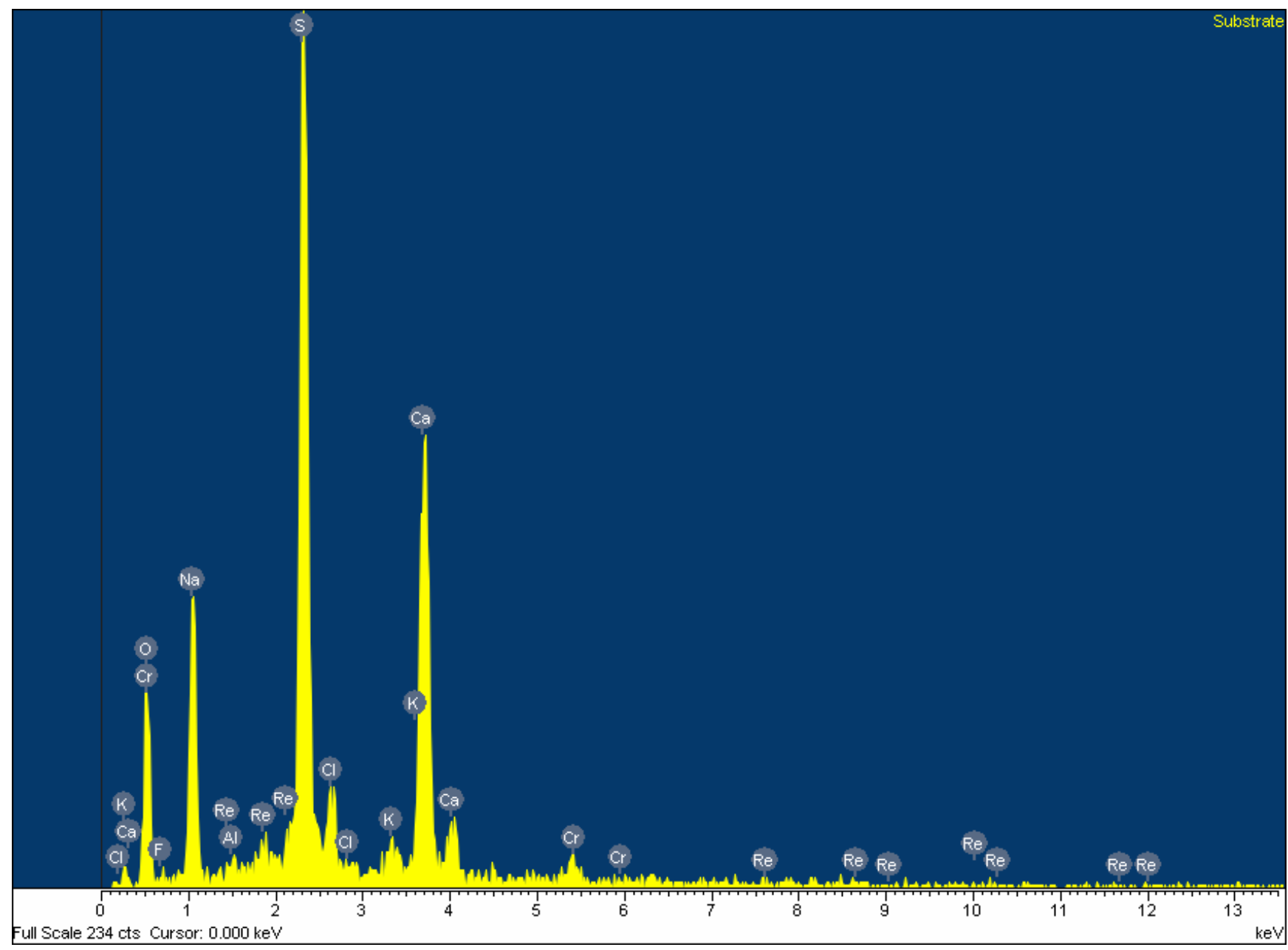

Figure 4.14.b. Spectrum from substrate of secondary phases in BWV-D-68E. 


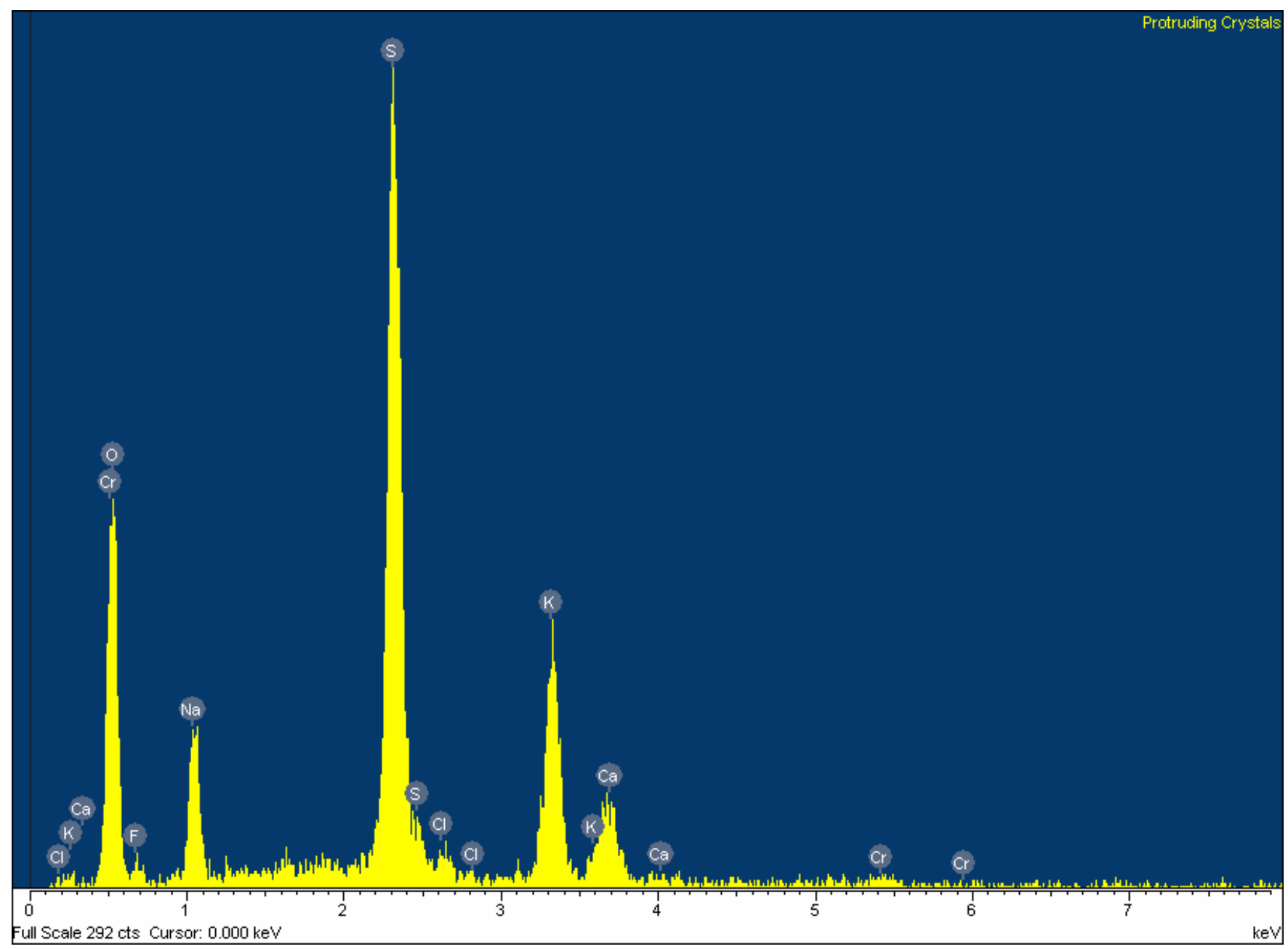

Figure 4.14.c. Spectrum from protruding crystals on secondary phases of BWV-D-68E. 


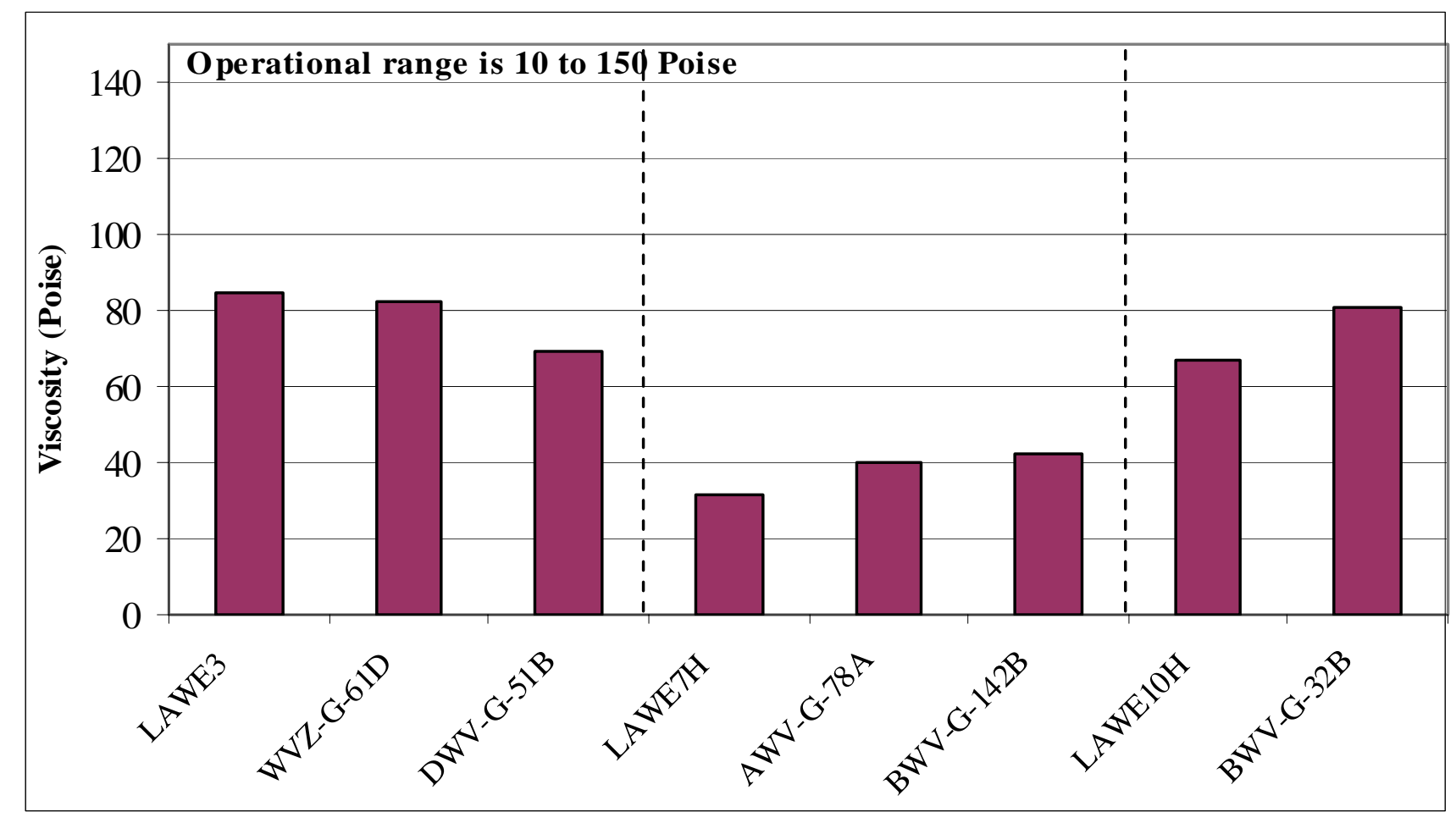

Figure 4.15 Melt viscosity (at $1100{ }^{\circ} \mathrm{C}$ ) for formulations tested as crucible melts (LAWE3, LAWE7H, and LAWE10H) and DM100 melter glasses with nominal (WVZ-G-61D, AWV-G-78A, BWV-G-32B) and increased halides (DWV-G-51B, BWV-G-142B). 


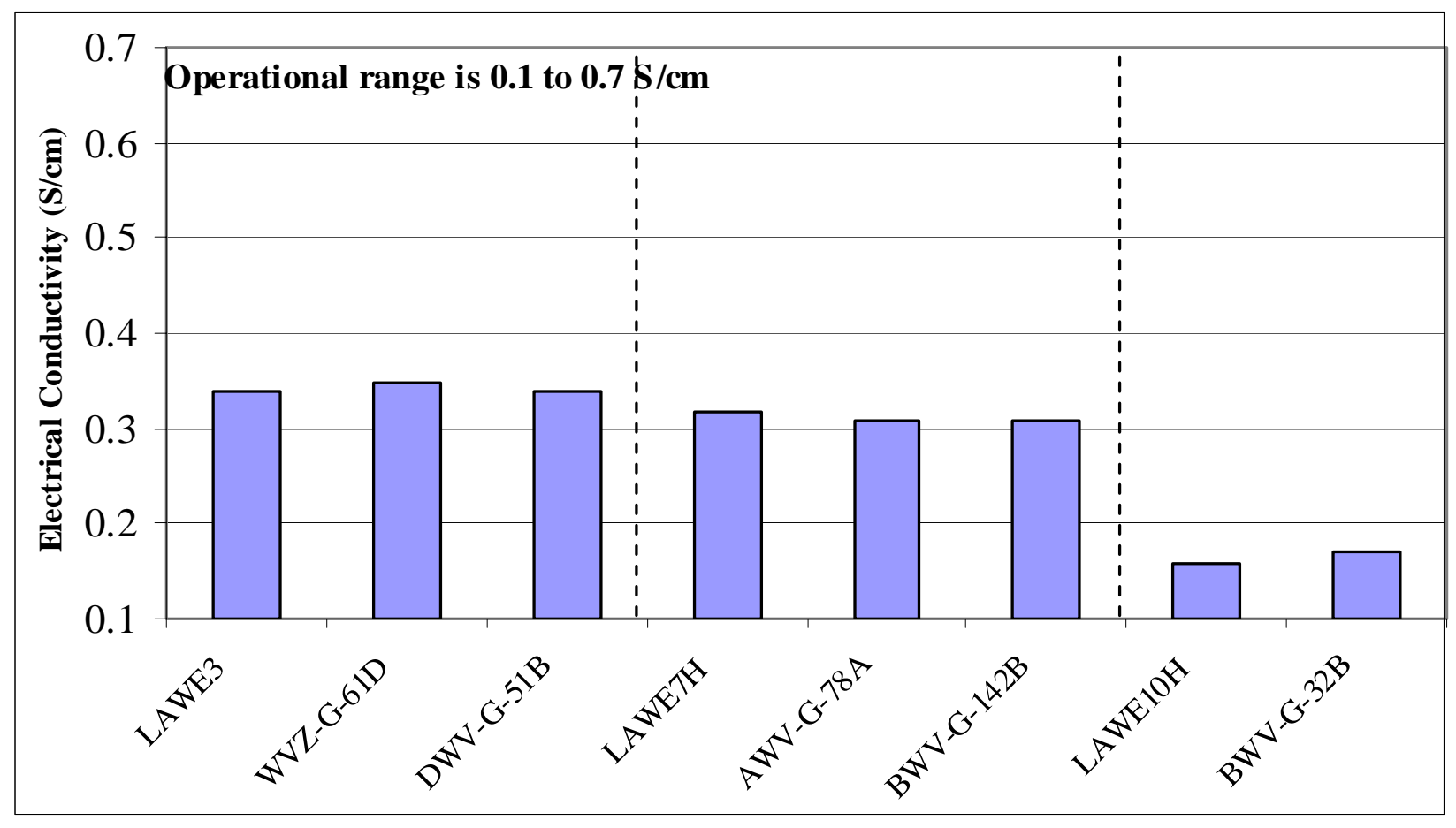

Figure 4.16 Melt electrical conductivity $\left(\right.$ at $1100^{\circ} \mathrm{C}$ ) for formulations tested as crucible melts (LAWE3, LAWE7H, and LAWE10H) and DM100 melter glasses with nominal (WVZ-G-61D, AWV-G-78A, BWV-G-32B) and increased halides (DWV-G-51B, BWV-G-142B). 


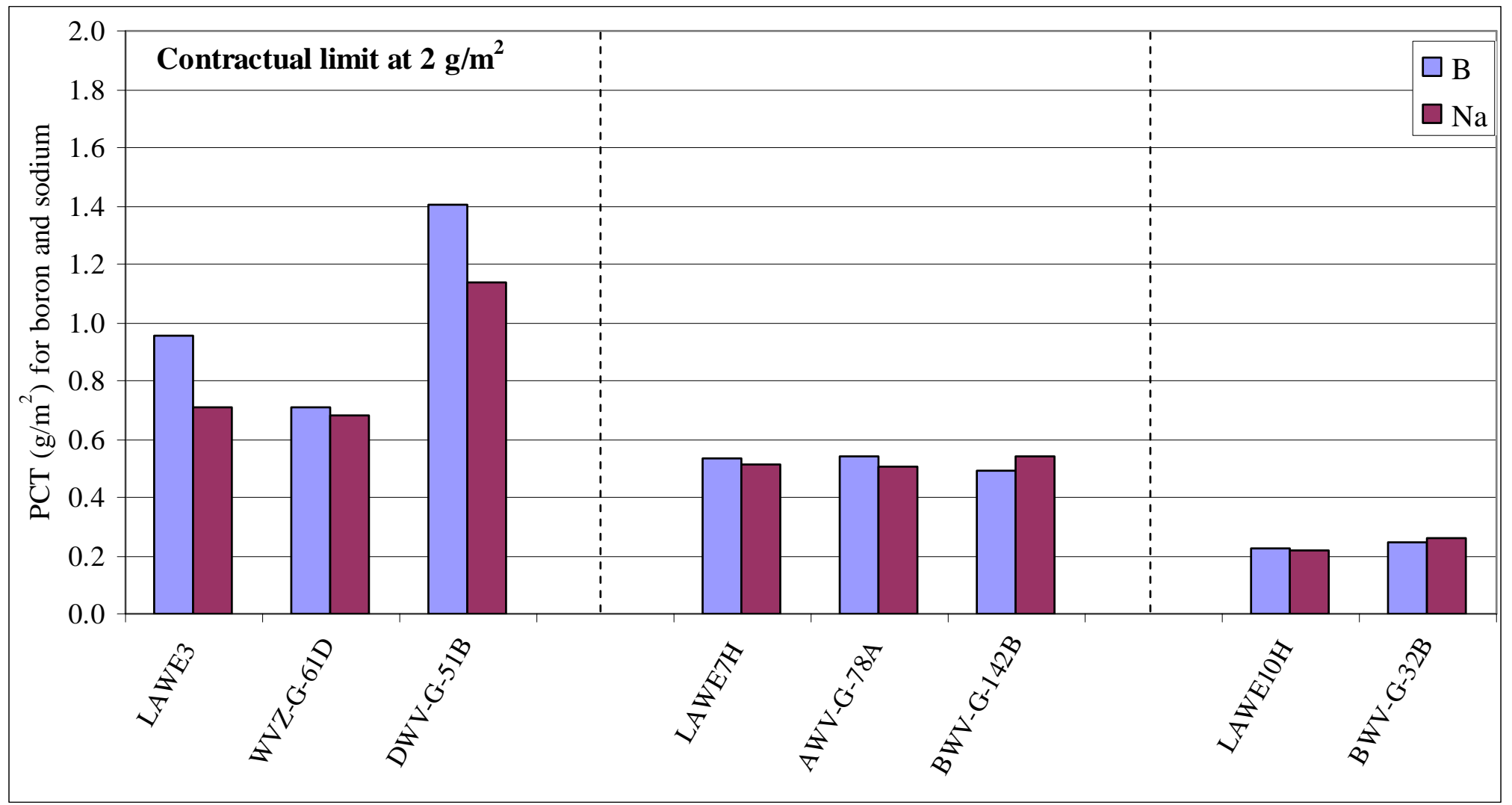

Figure 4.17 PCT normalized mass loss (for boron and sodium) for formulations tested as crucible glasses (LAWE3, LAWE7H, and LAWE10H) and DM100 melter glasses with nominal (WVZ-G-61D, AWV-G-78A, BWV-G-32B) and increased halides (DWV-G-51B, BWV-G-142B). 


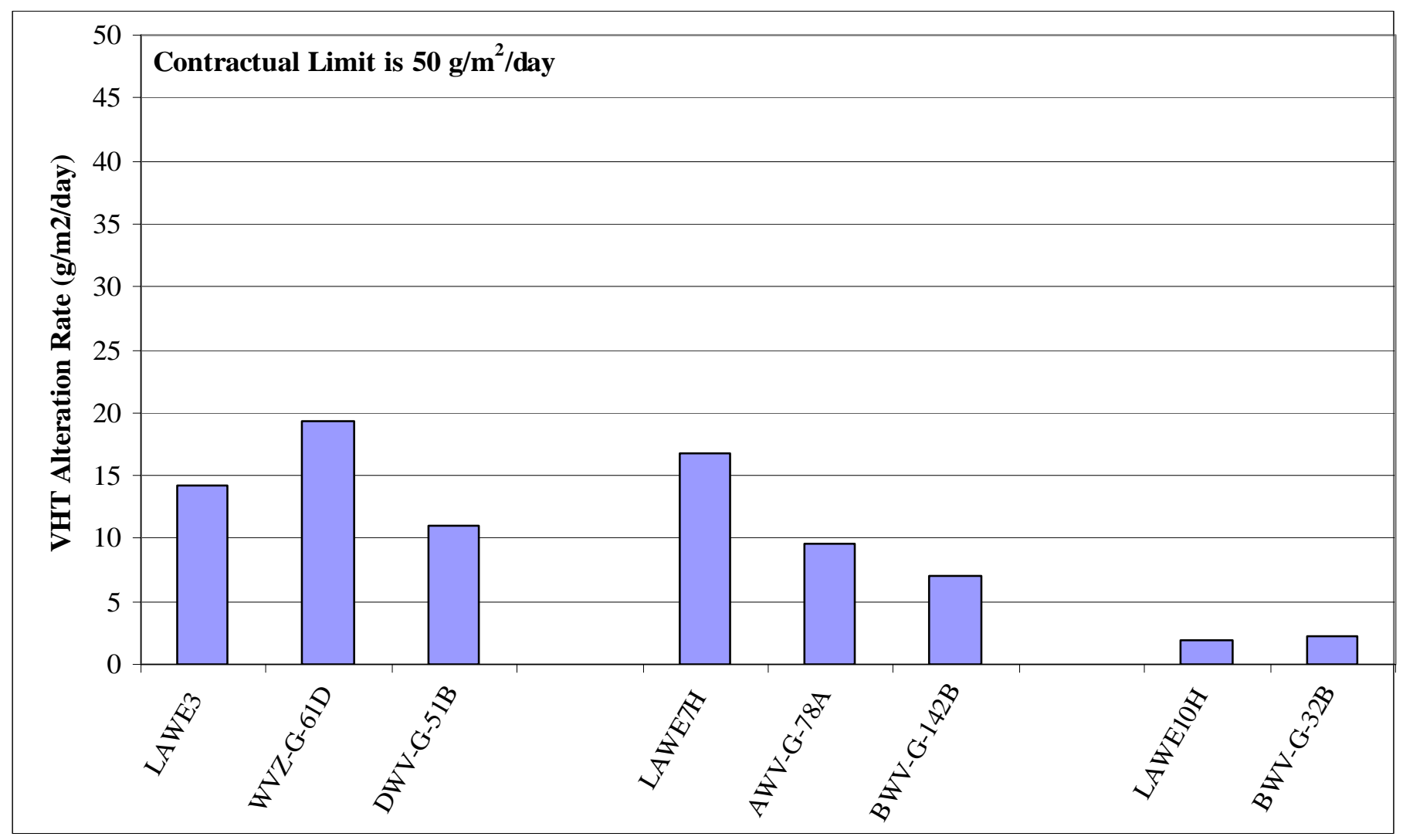

Figure 4.18 Alteration rate given by Vapor Hydration Test $\left(\right.$ at $200^{\circ} \mathrm{C}$ ) for formulations tested as crucible glasses (LAWE3, LAWE7H, and LAWE10H) and DM100 melter glasses with nominal (WVZ-G-61D, AWV-G-78A, BWV-G-32B) and increased halides (DWV-G-51B, BWV-G-142B). 


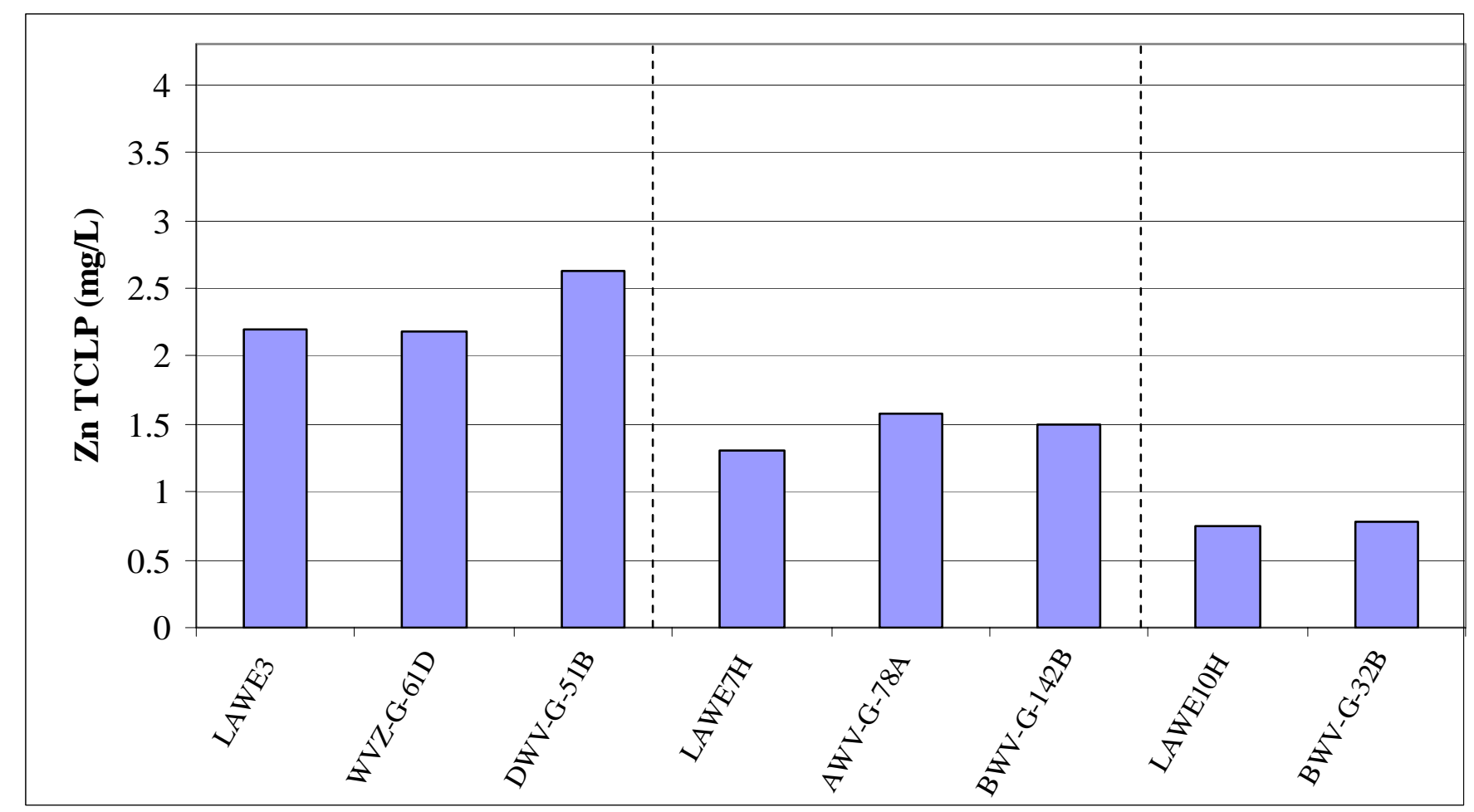

Figure 4.19. TCLP zinc concentration for formulations tested as crucible glasses (LAWE3, LAWE7H, and LAWE10H) and DM100 melter glasses with nominal (WVZ-G-61D, AWV-G-78A, BWV-G-32B) and increased halides (DWV-G-51B,

BWV-G-142B). 


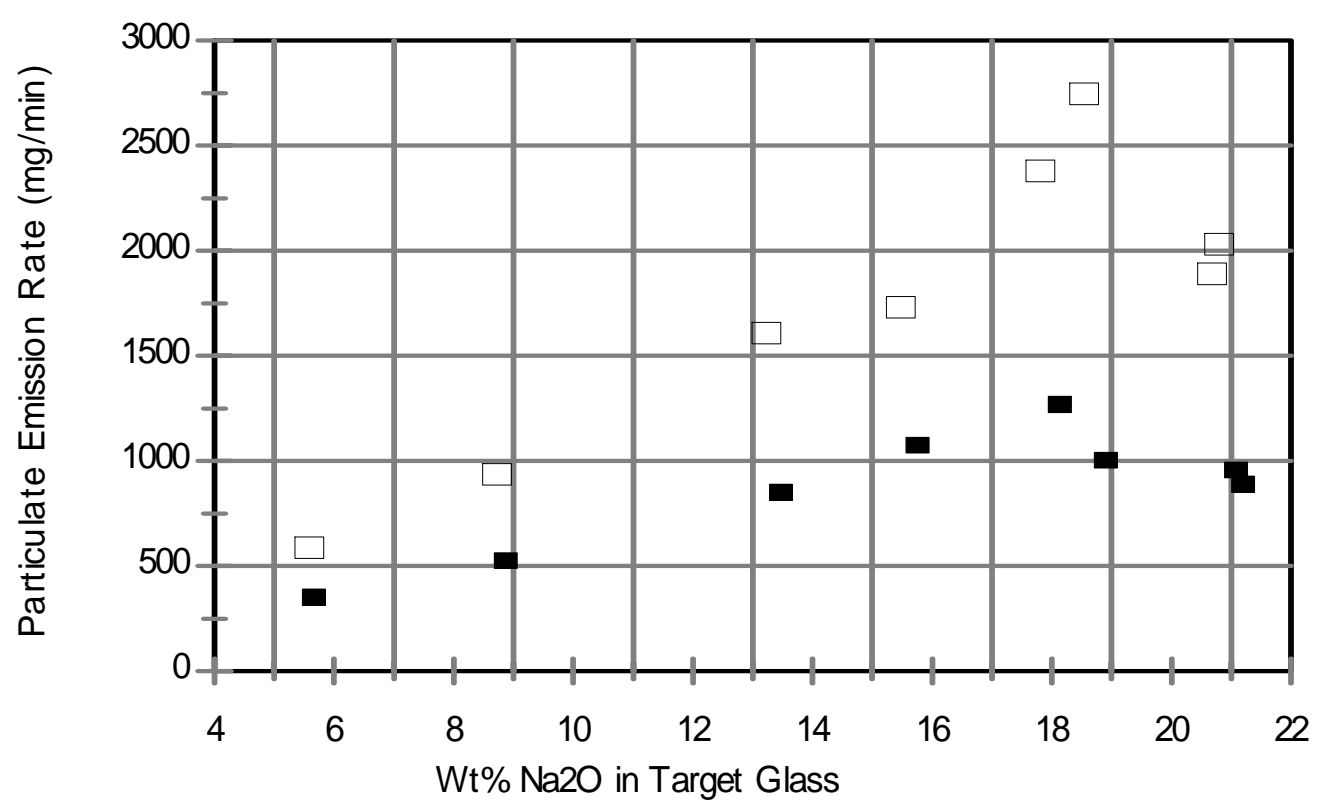

Figure 5.1. Measured particle emission rate from the DM100 as a function of feed sodium content. 


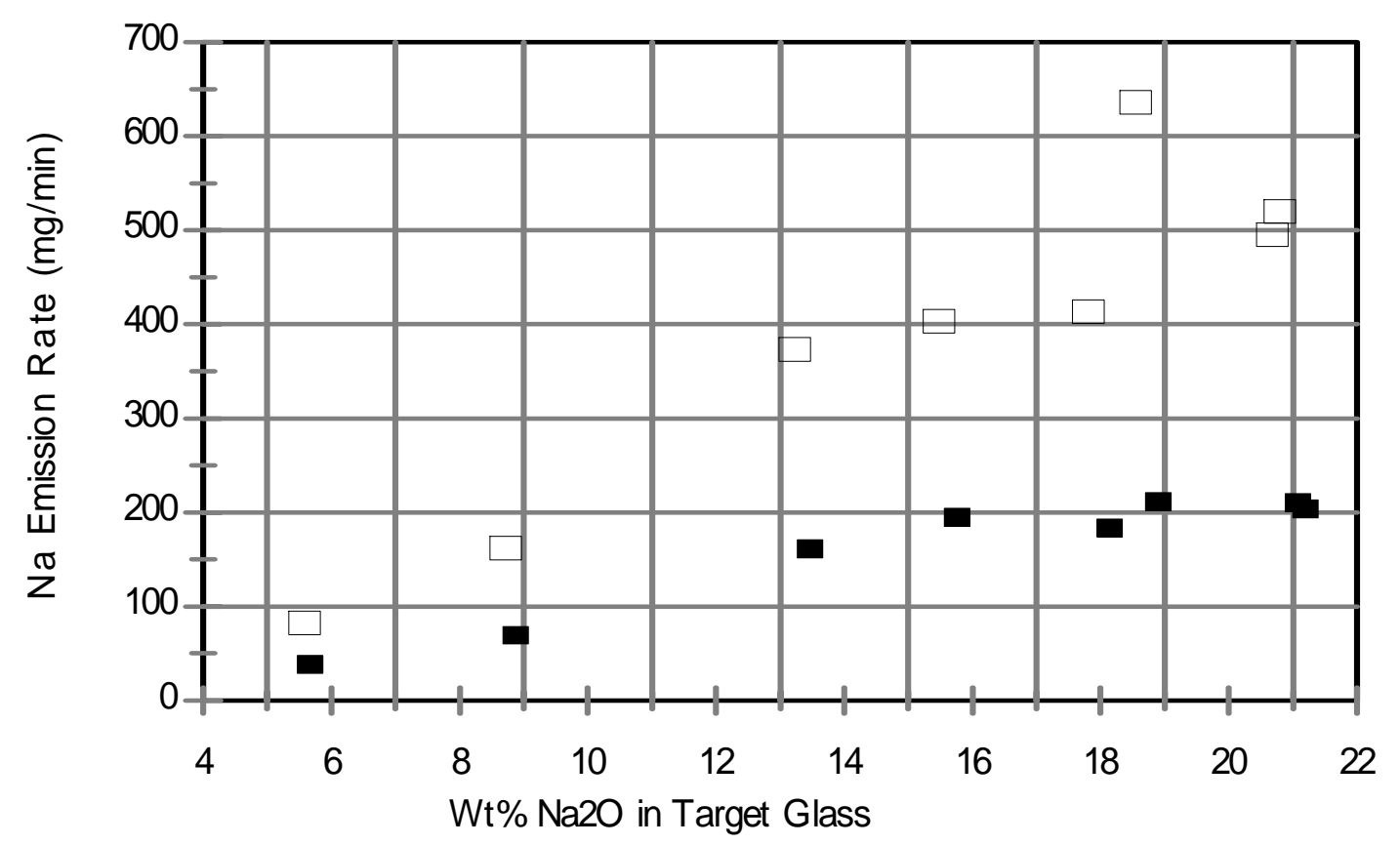
- Nominal Halides
$\square$ Added Halides

Figure 5.2. Measured sodium emission rate from the DM100 as a function of feed sodium content. 


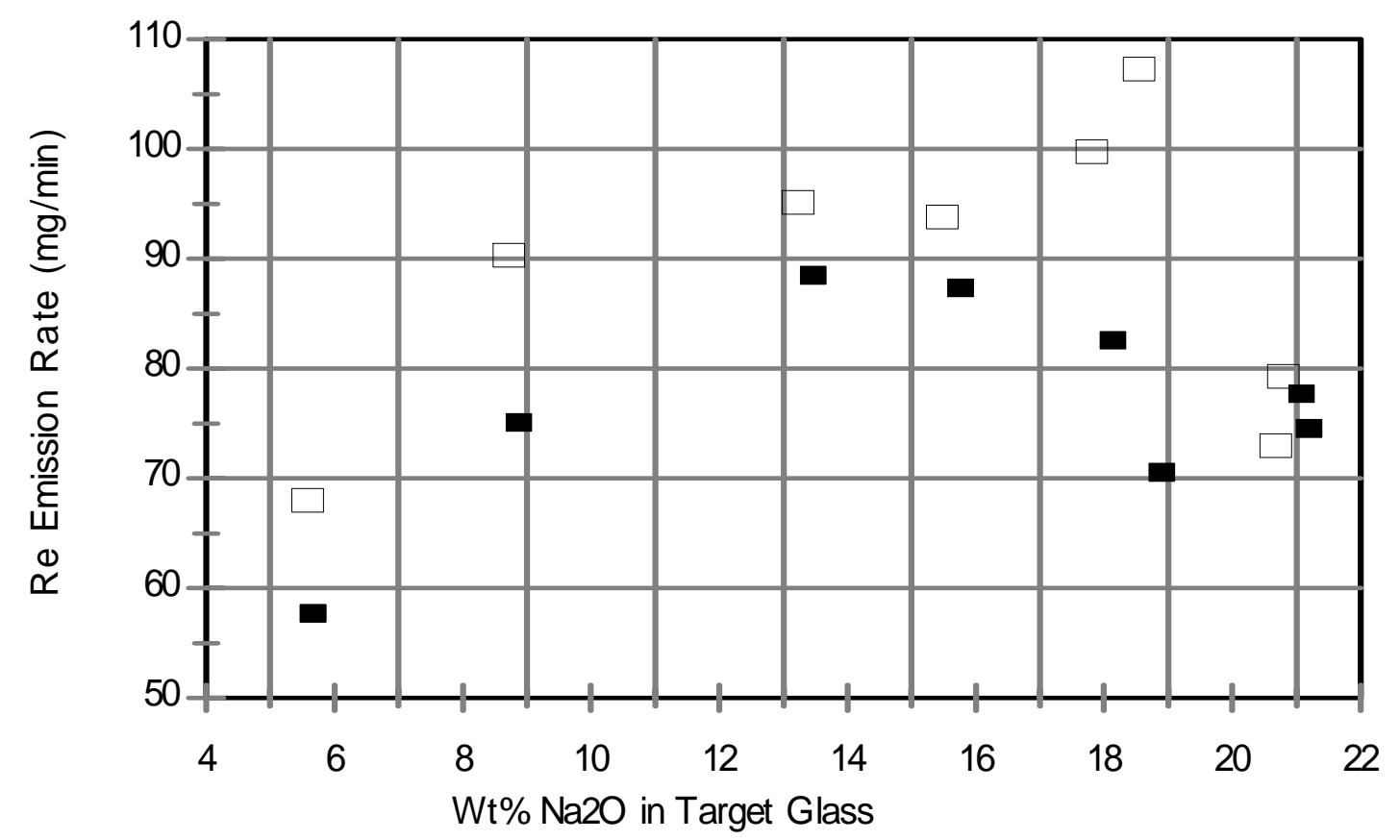

Figure 5.3. Measured rhenium emission rate from the DM100 as a function of feed sodium content. 


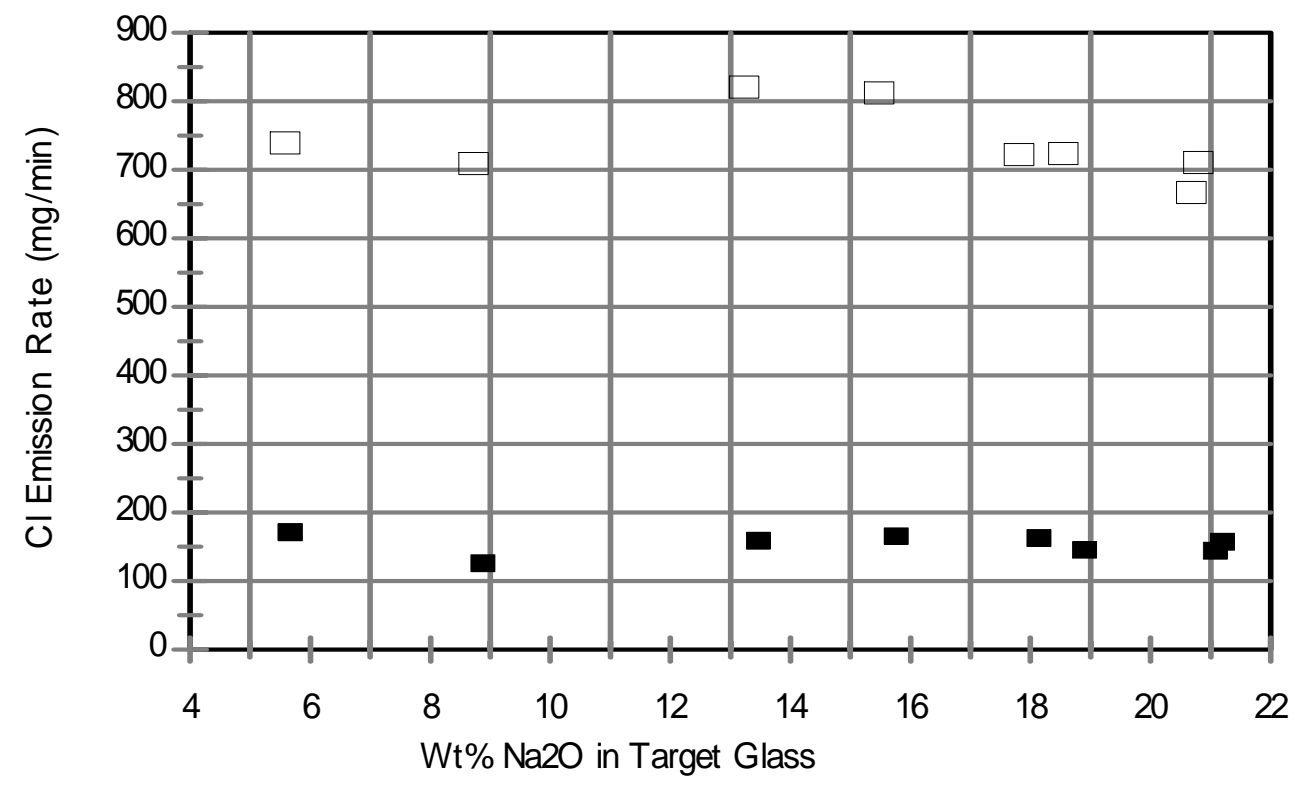

Figure 5.4. Measured chlorine emission rate from the DM100 as a function of feed sodium content. 

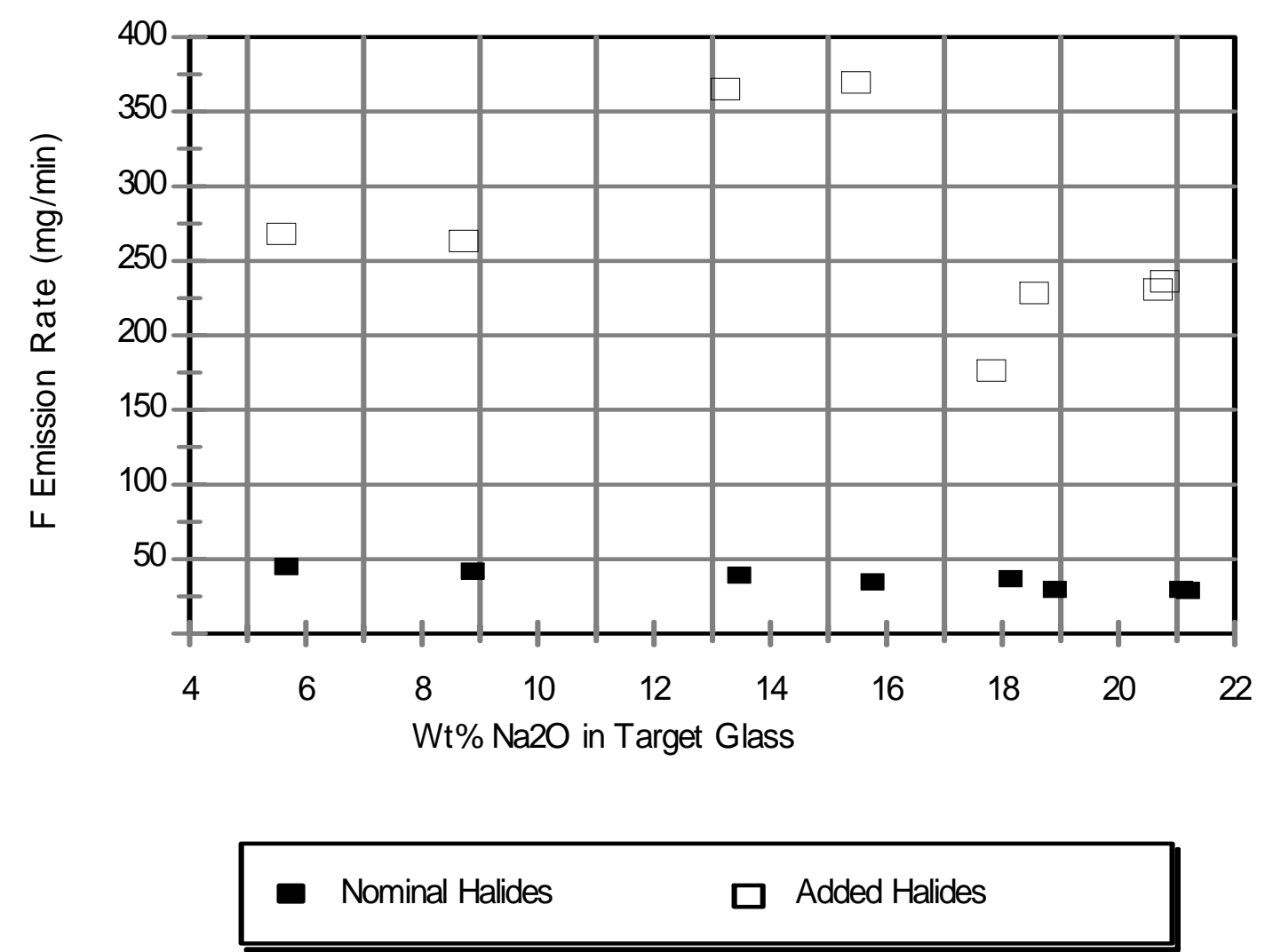

Figure 5.5. Measured fluorine emission rate from the DM100 as a function of feed sodium content. 

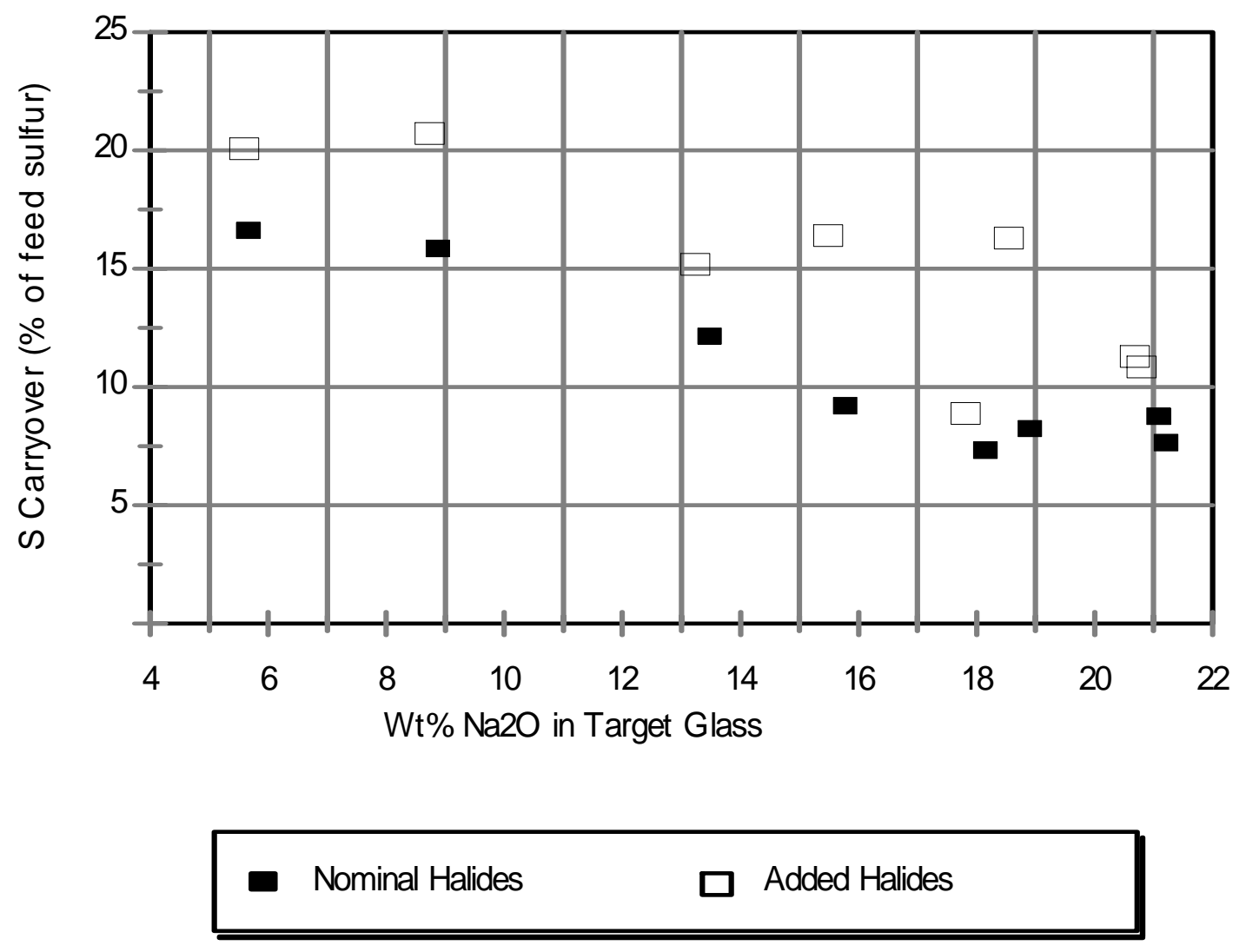

Figure 5.6. Percent sulfur carryover from the DM100 as a function of feed sodium content. 


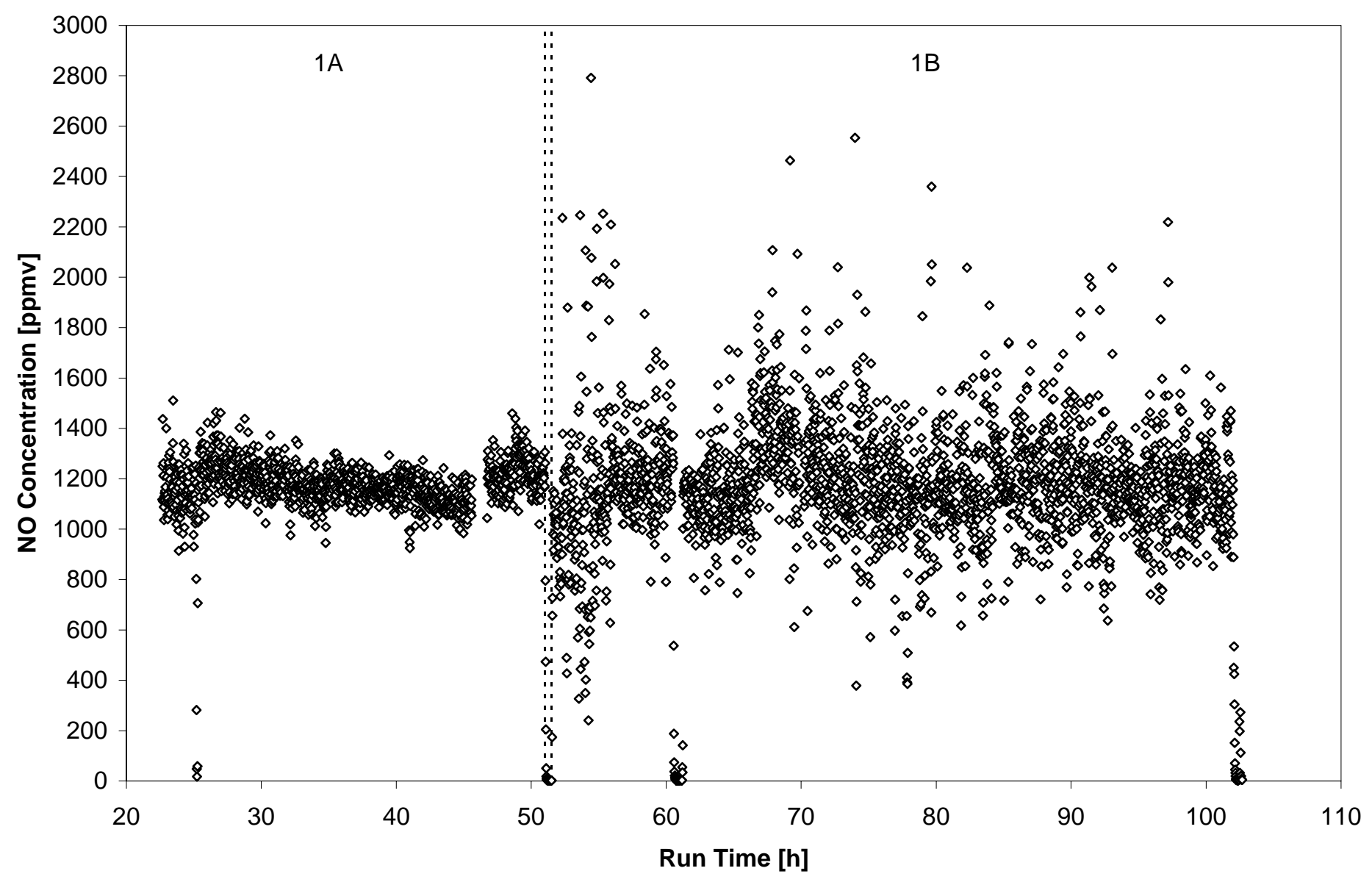

Figure 5.7.a. Nitric oxide concentrations in off-gas from FTIR during Test 1. 


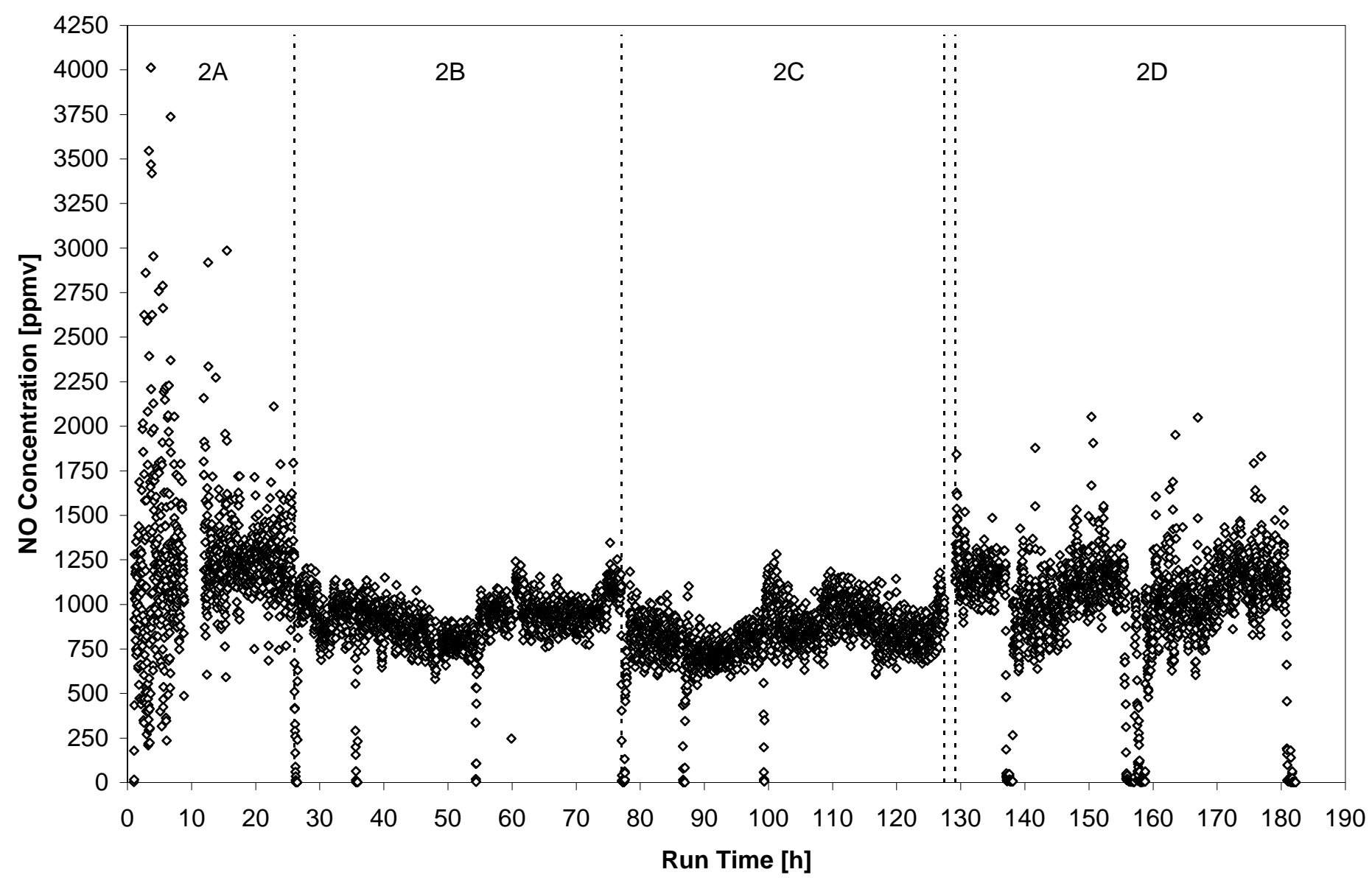

Figure 5.7.b. Nitric oxide concentrations in off-gas from FTIR during Test 2. 


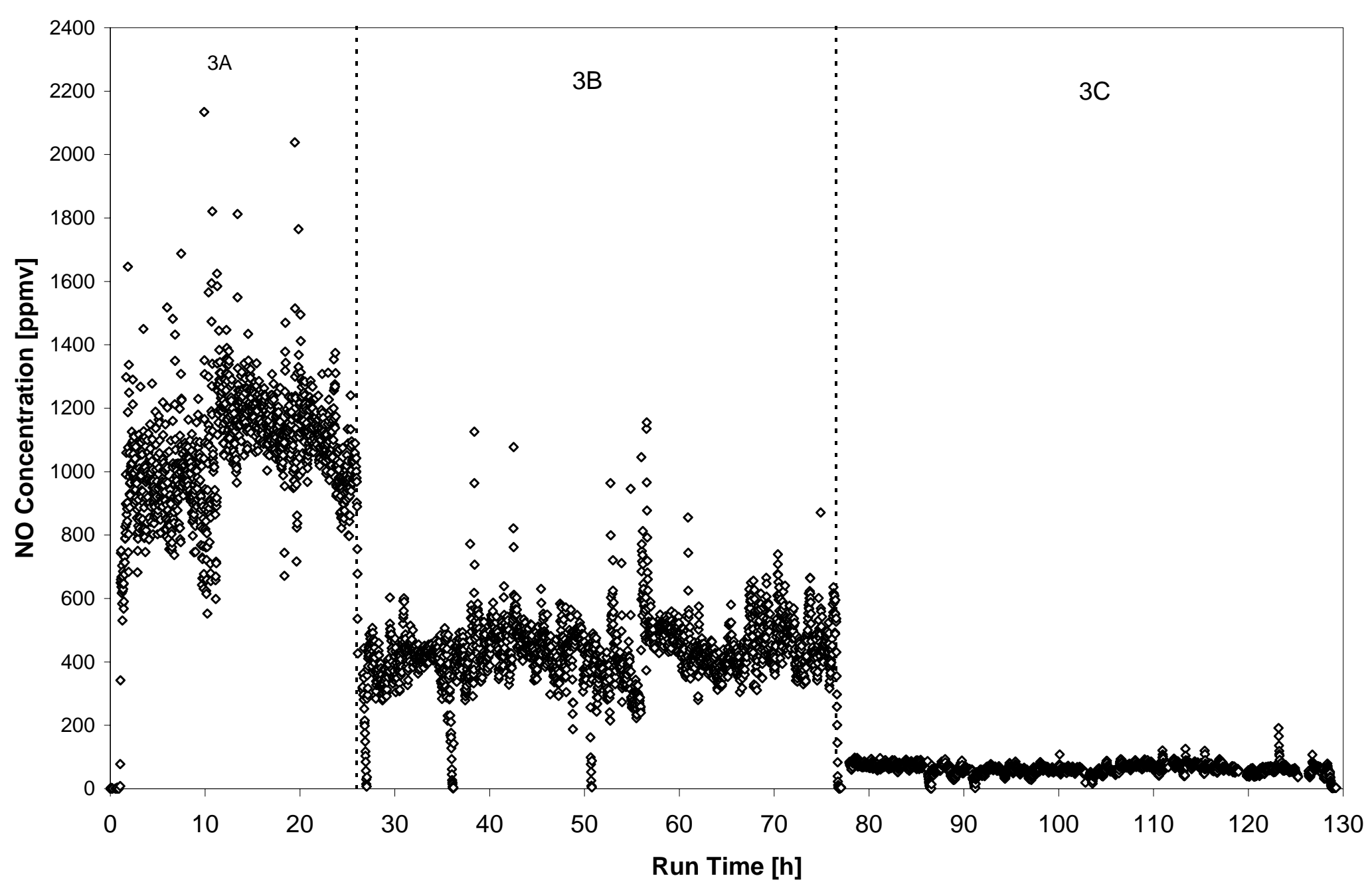

Figure 5.7.c. Nitric oxide concentrations in off-gas from FTIR during Test 3. 


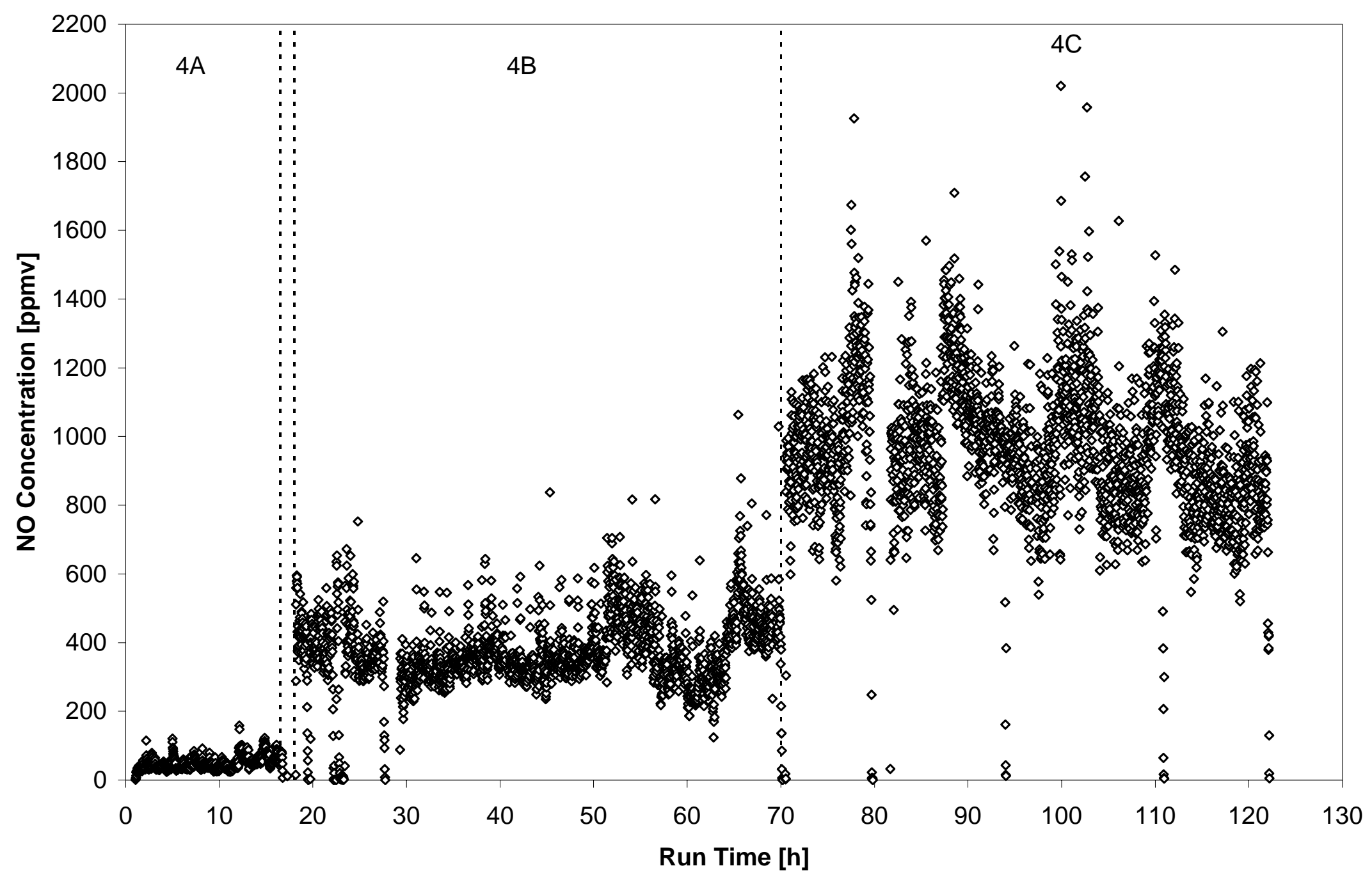

Figure 5.7.d. Nitric oxide concentrations in off-gas from FTIR during Test 4. 


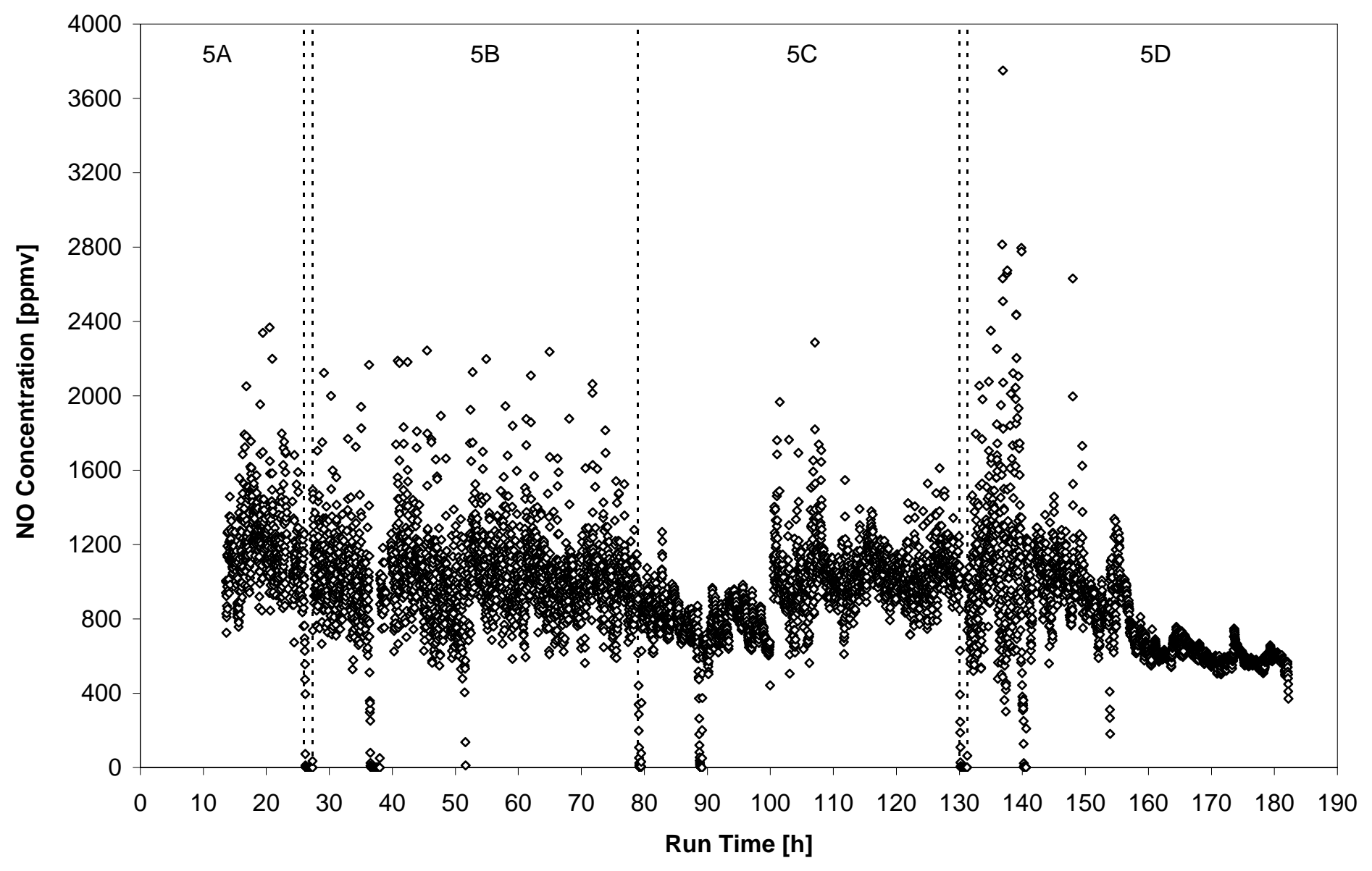

Figure 5.7.e. Nitric oxide concentrations in off-gas from FTIR during Test 5. 


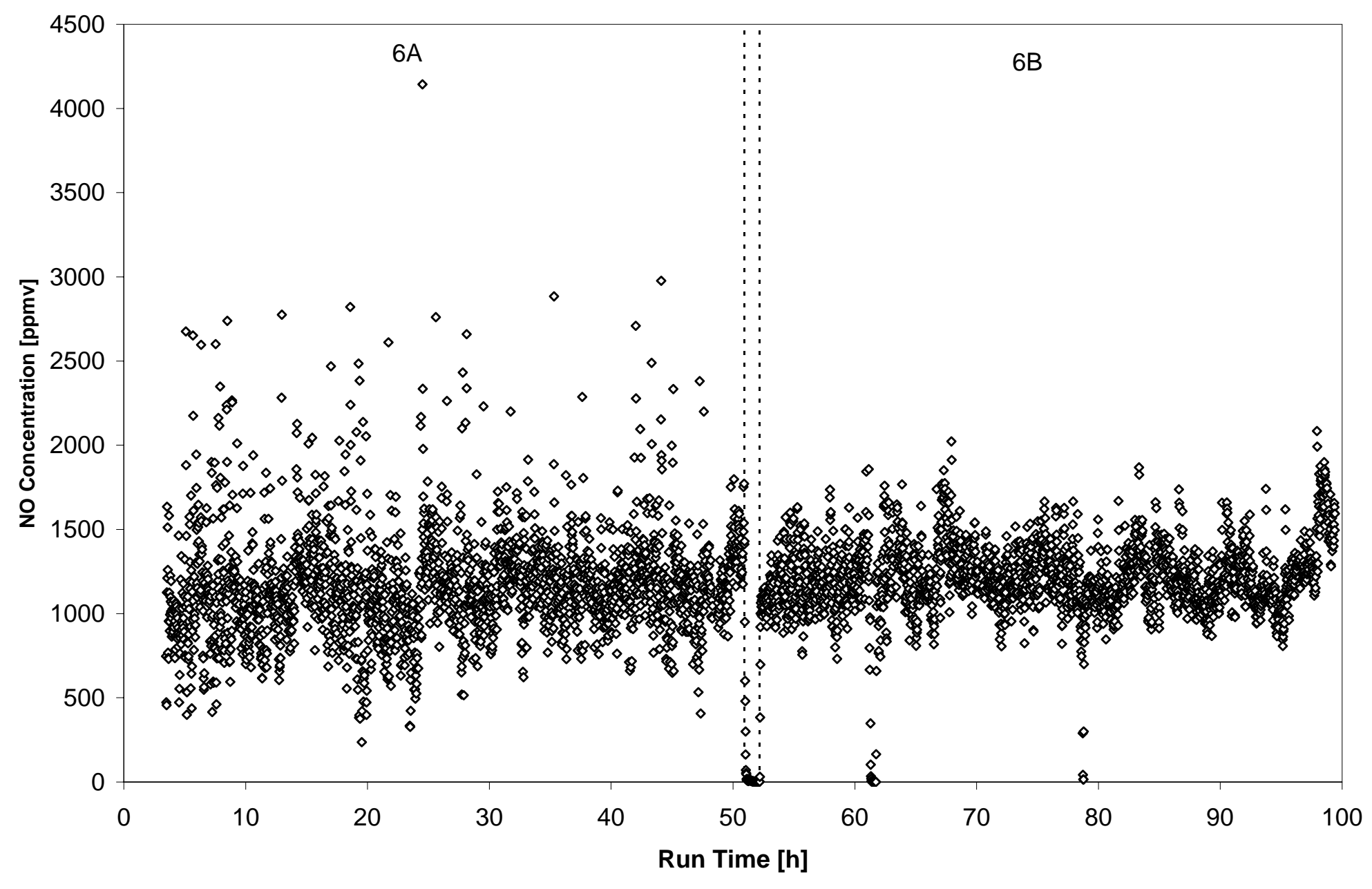

Figure 5.7.f. Nitric oxide concentrations in off-gas from FTIR during Test 6. 


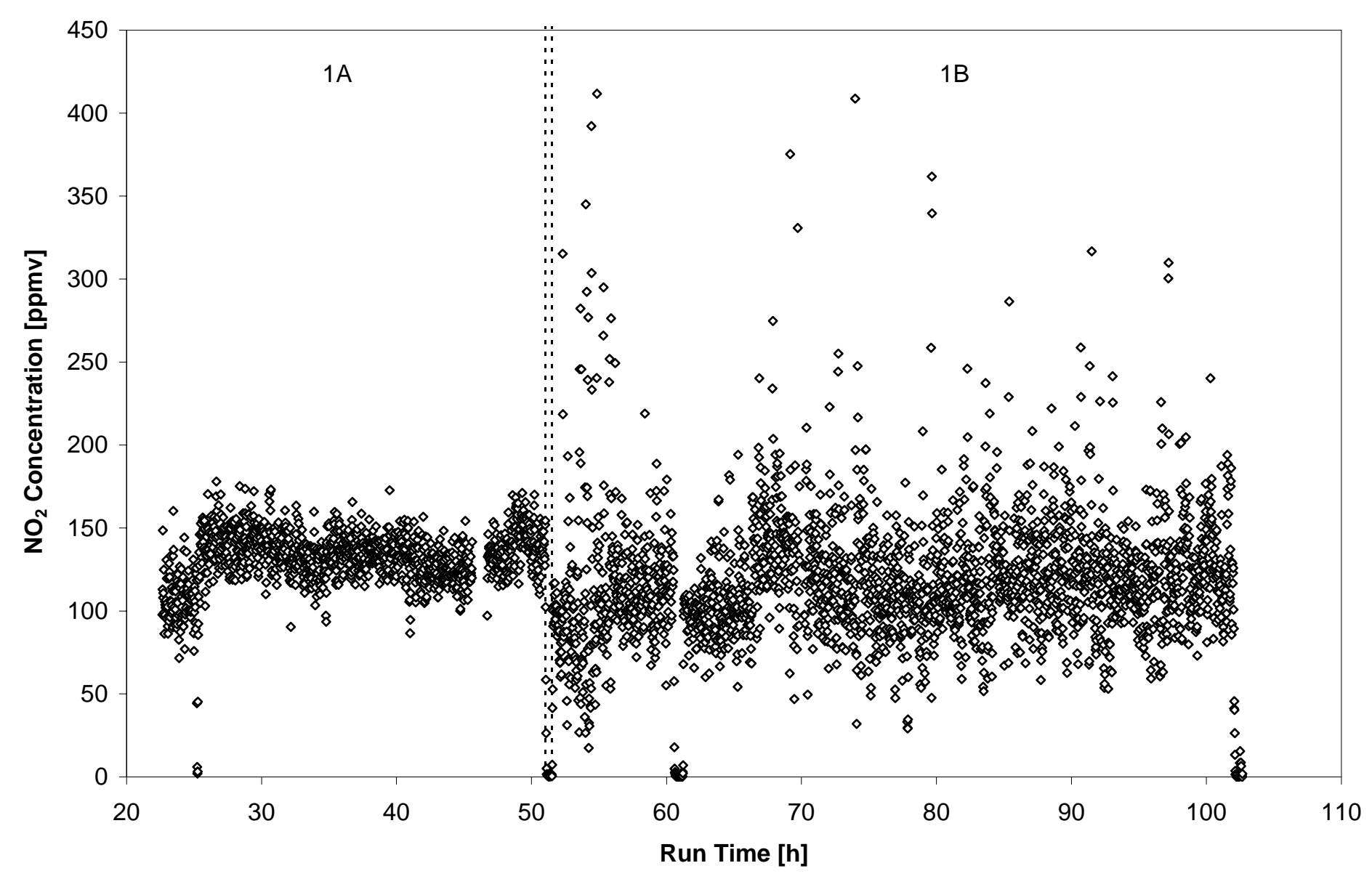

Figure 5.8.a. $\mathrm{NO}_{2}$ concentration in off-gas from FTIR during Test 1. 


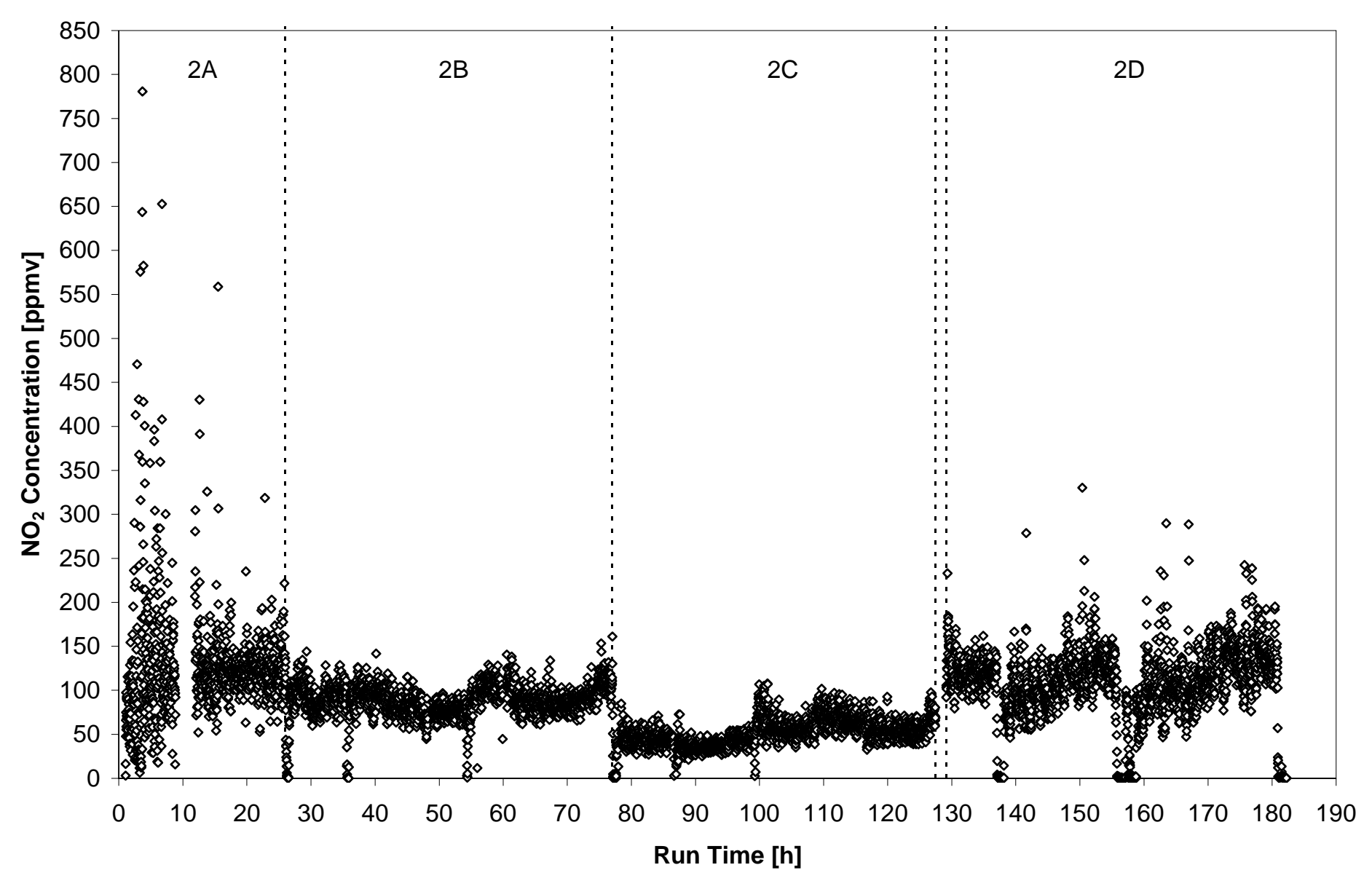

Figure 5.8.b. $\mathrm{NO}_{2}$ concentration in off-gas from FTIR during Test 2 . 


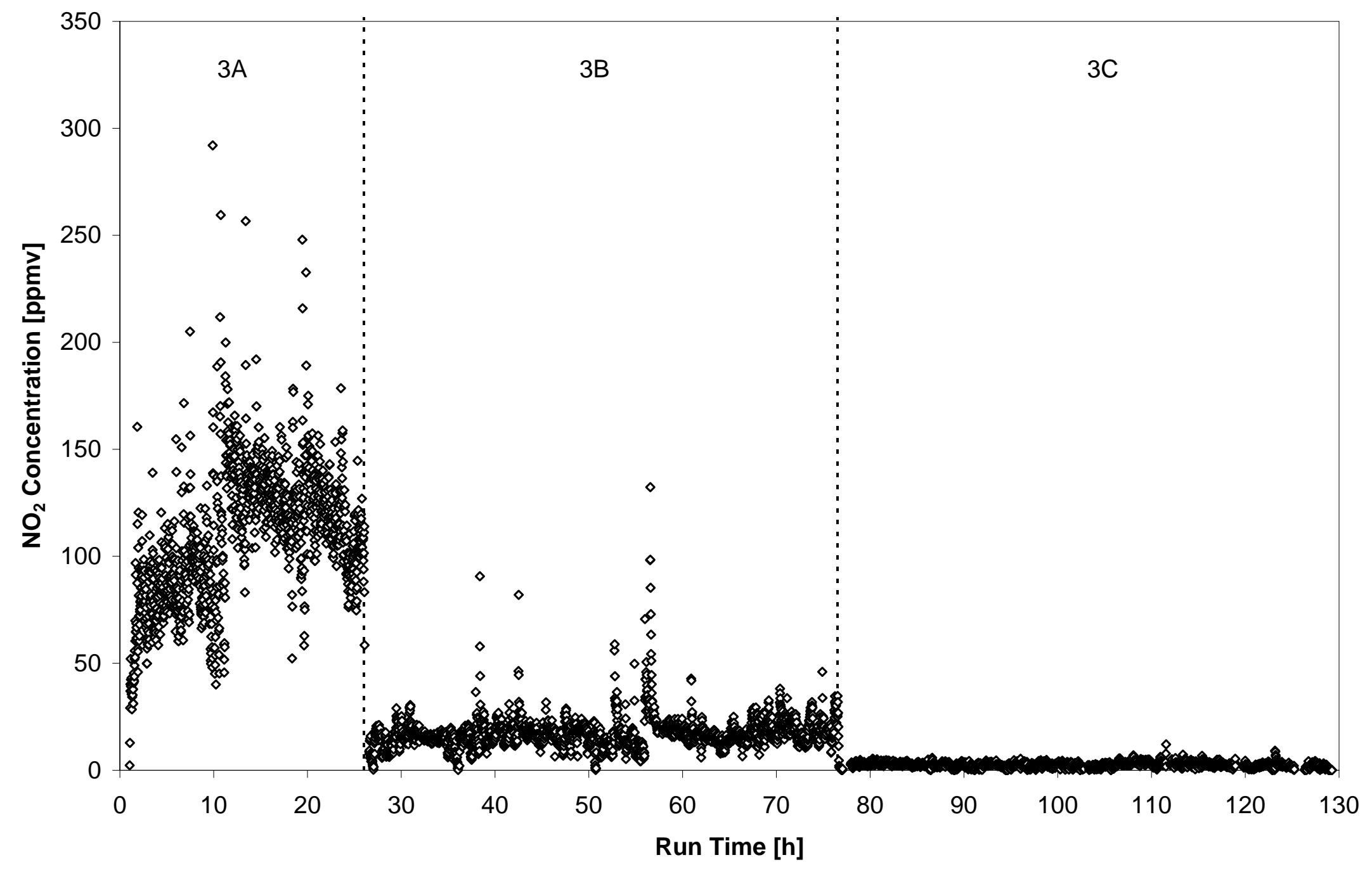

Figure 5.8.c. $\mathrm{NO}_{2}$ concentration in off-gas from FTIR during Test 3 . 


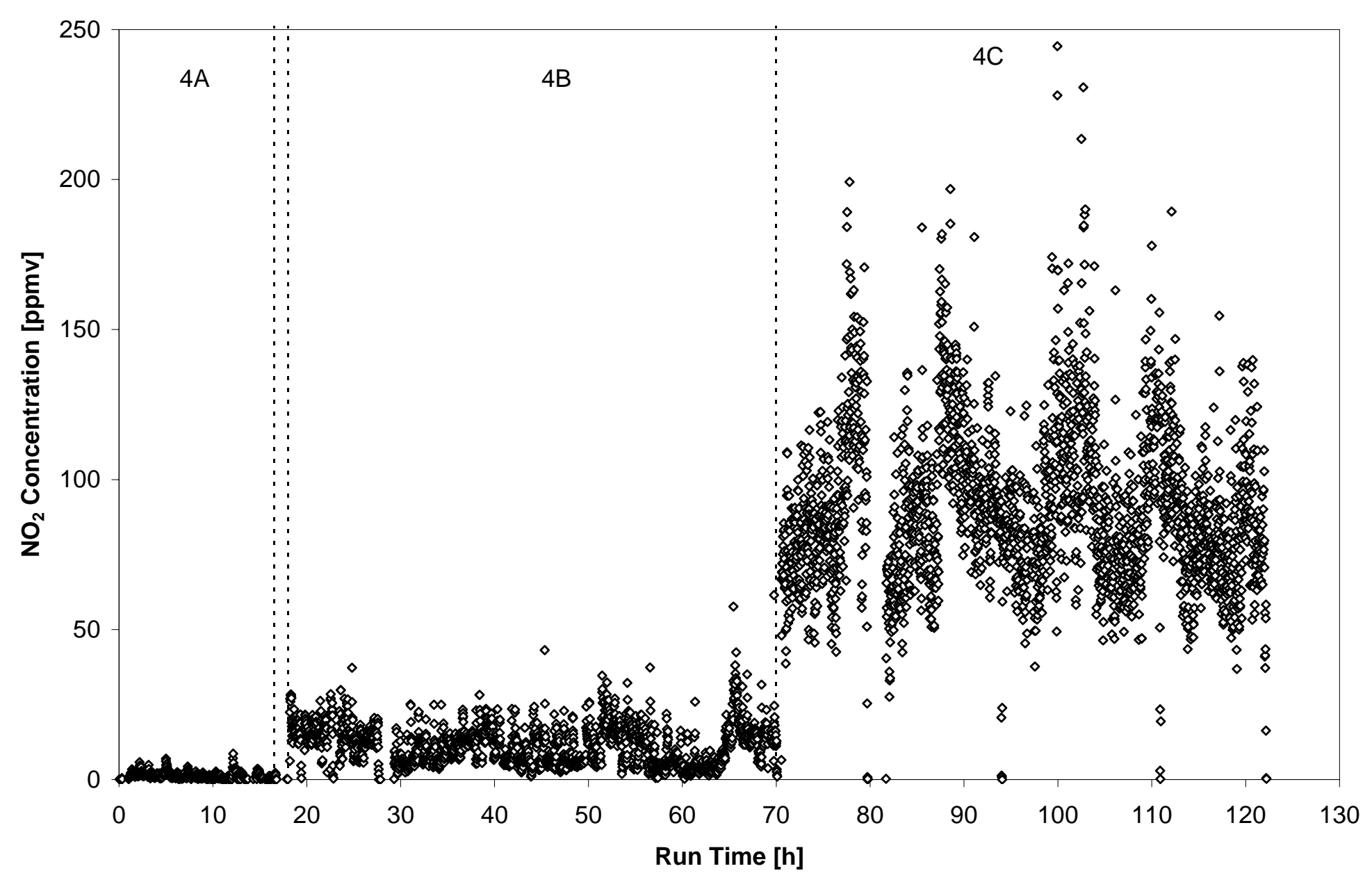

Figure 5.8.d. $\mathrm{NO}_{2}$ concentration in off-gas from FTIR during Test 4 . 


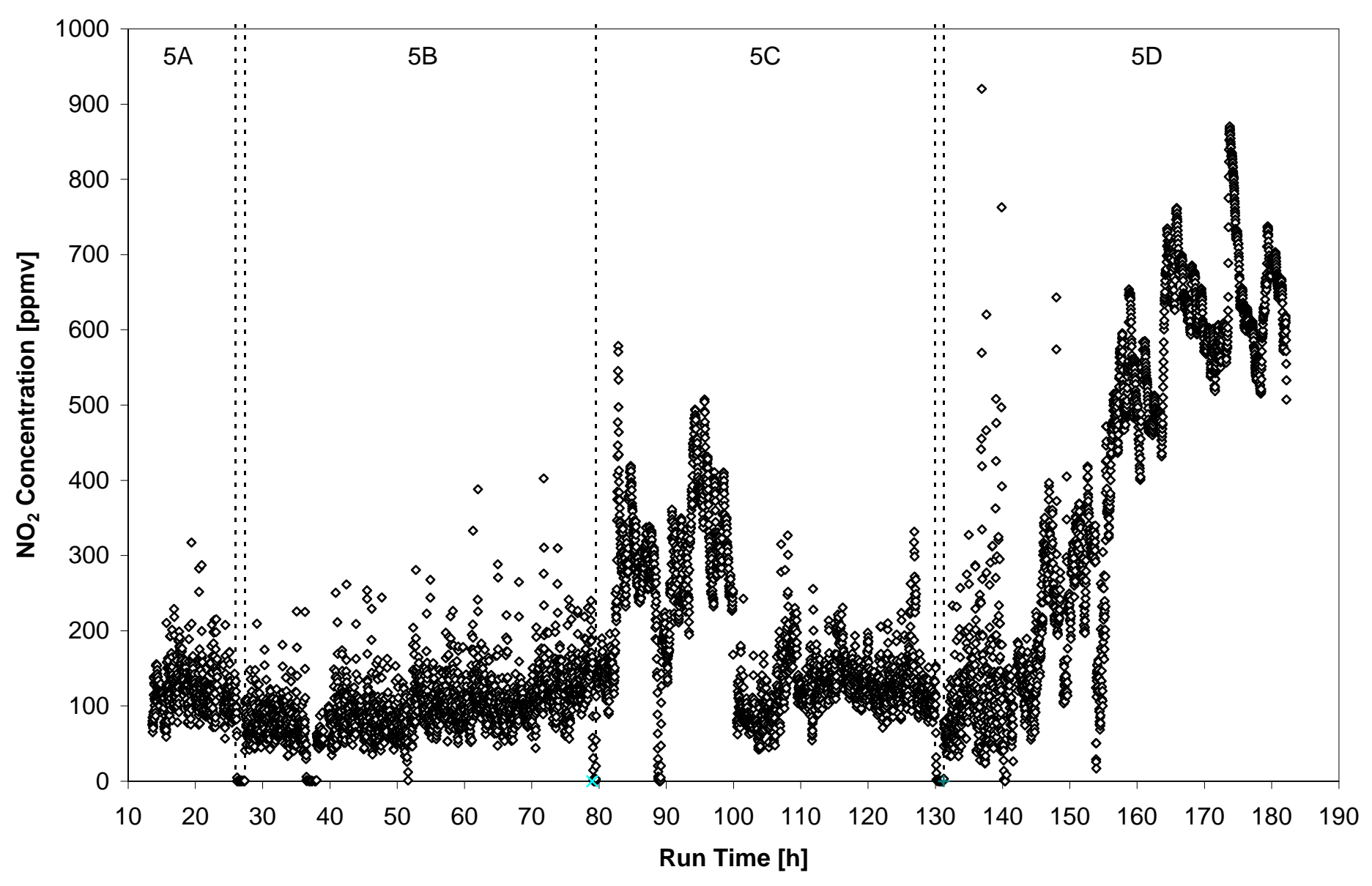

Figure 5.8.e. $\mathrm{NO}_{2}$ concentration in off-gas from FTIR during Test 5. 


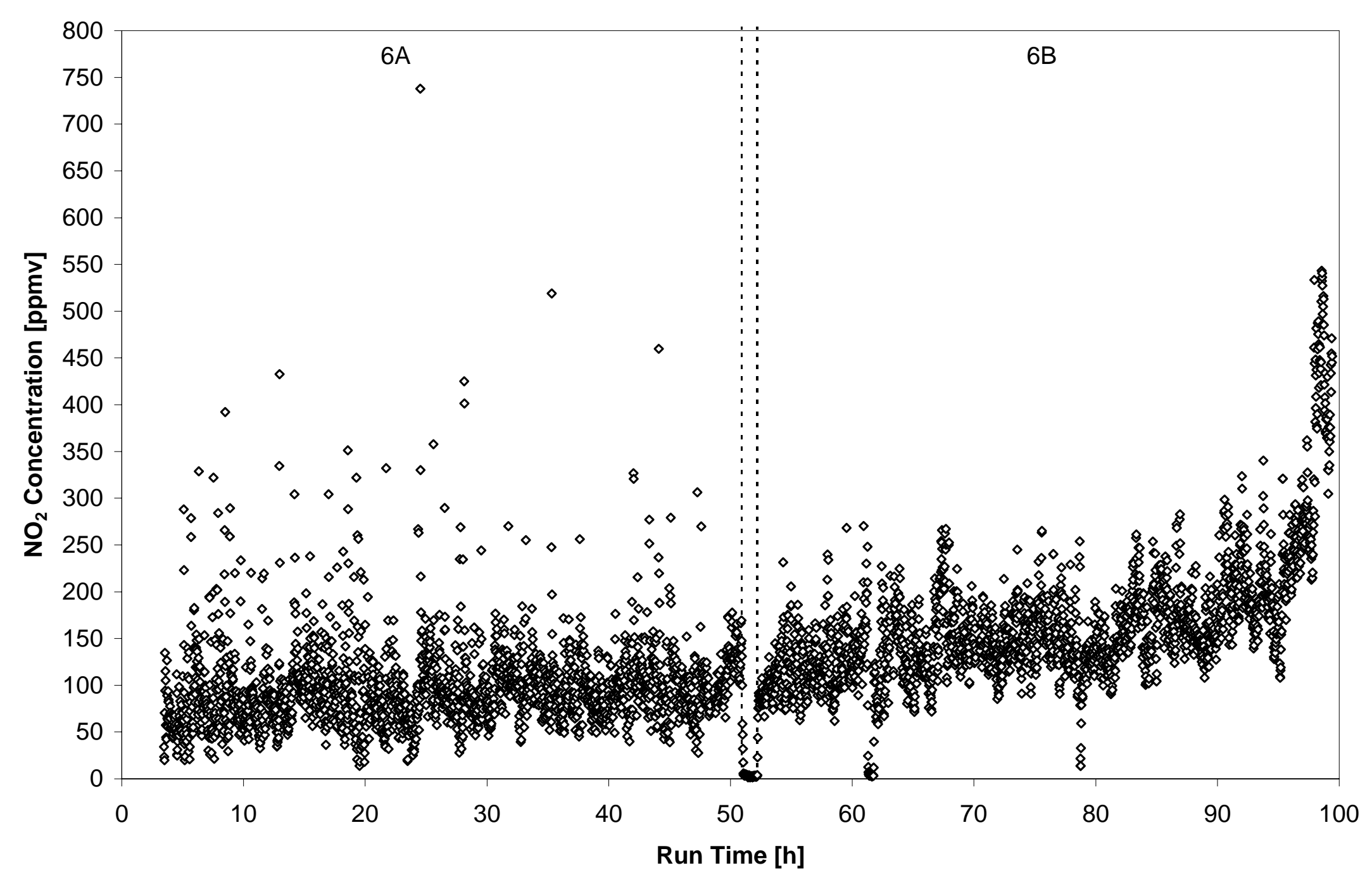

Figure 5.8.f. $\mathrm{NO}_{2}$ concentration in off-gas from FTIR during Test 6. 


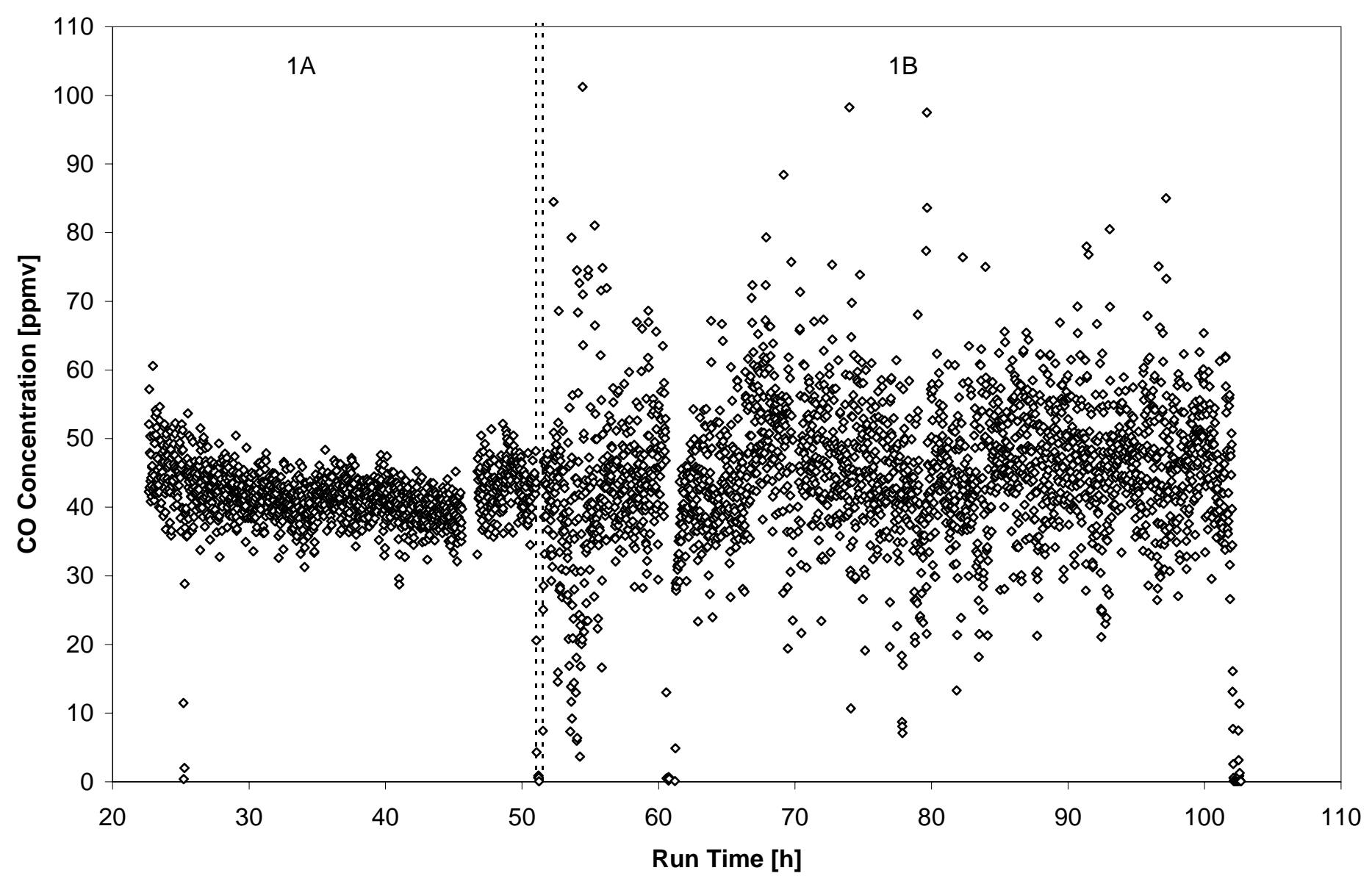

Figure 5.9.a. CO concentration in off-gas from FTIR during Test 1. 


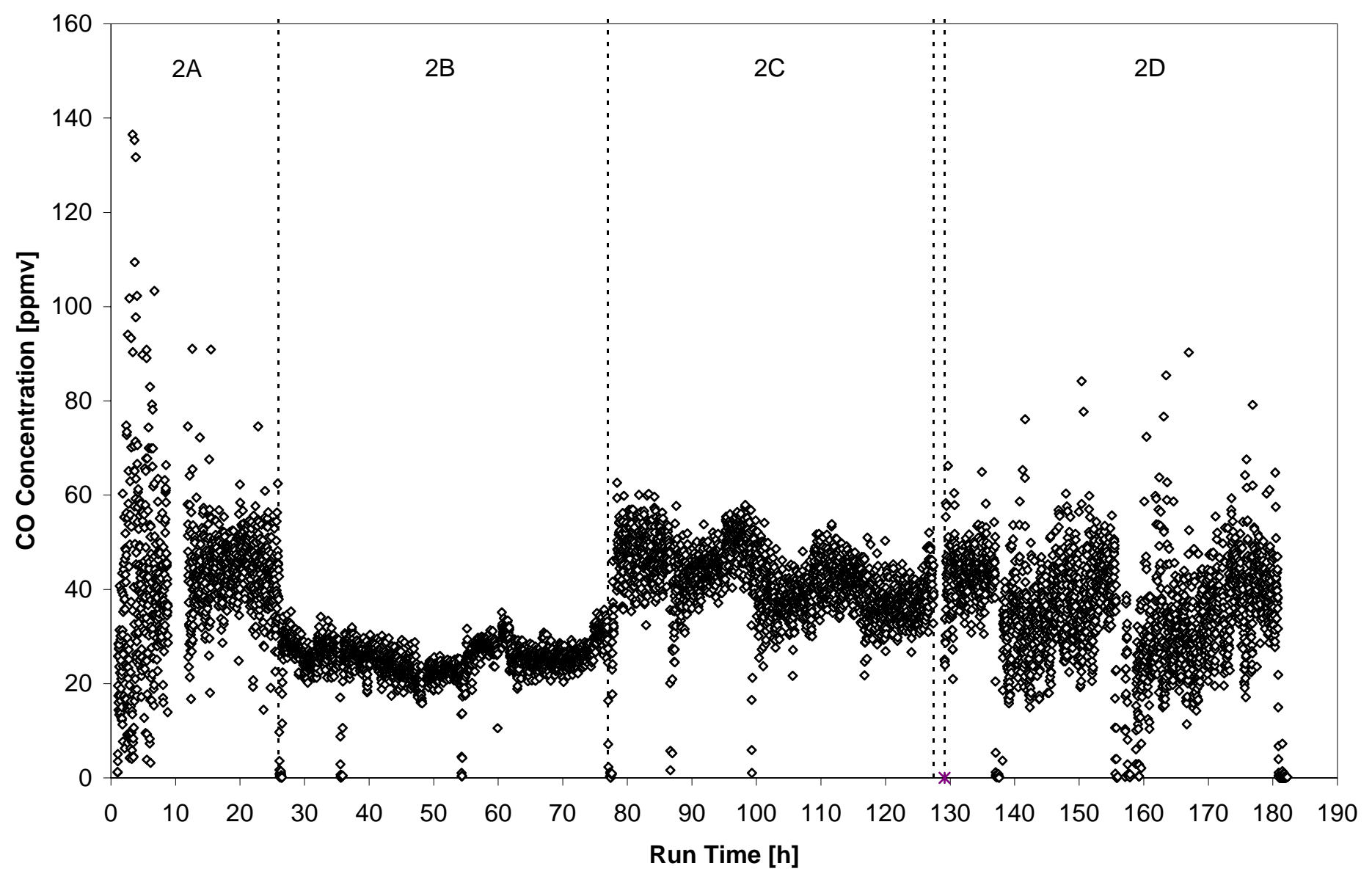

Figure 5.9.b. CO concentration in off-gas from FTIR during Test 2. 


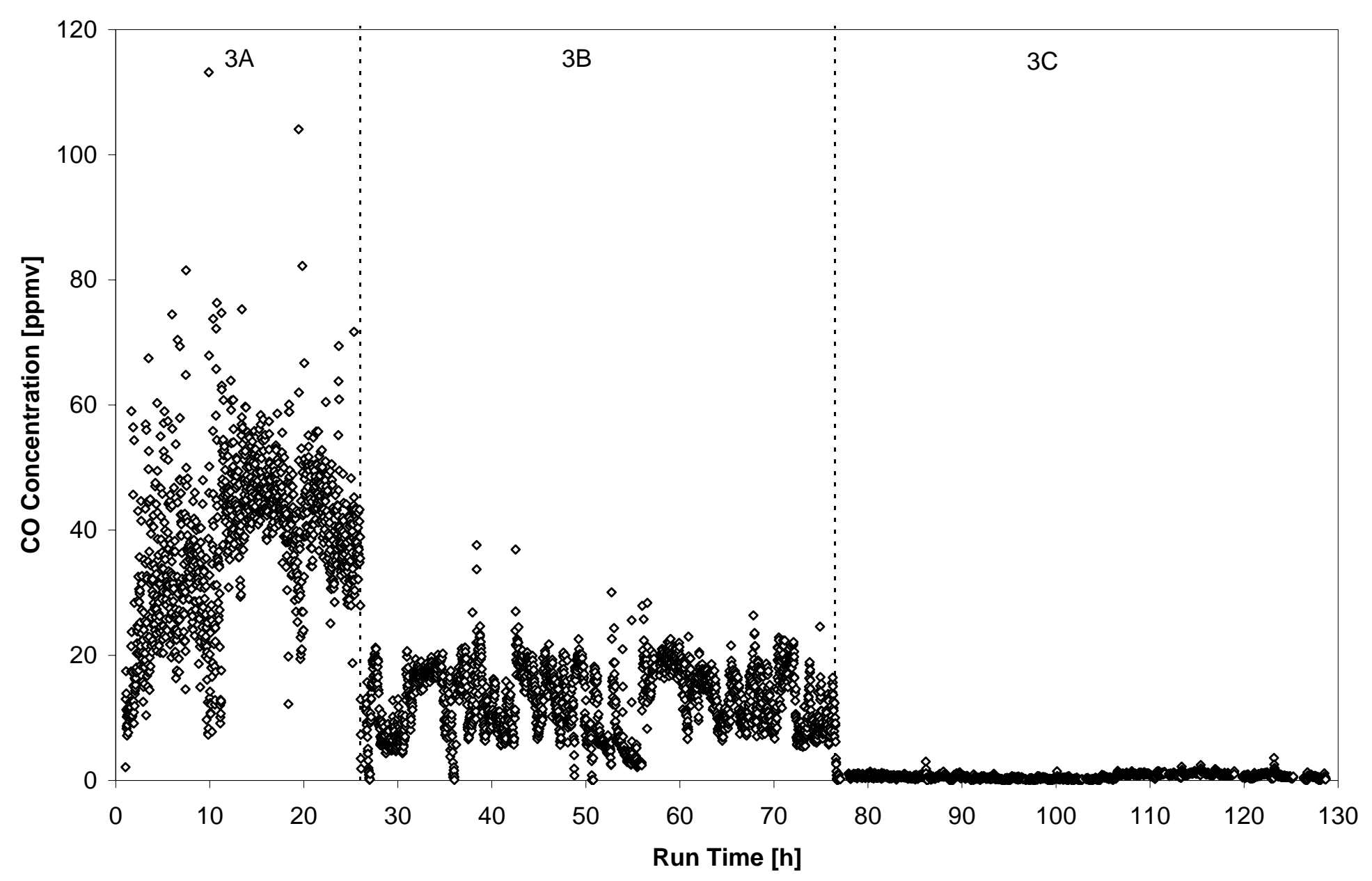

Figure 5.9.c. CO concentration in off-gas from FTIR during Test 3. 


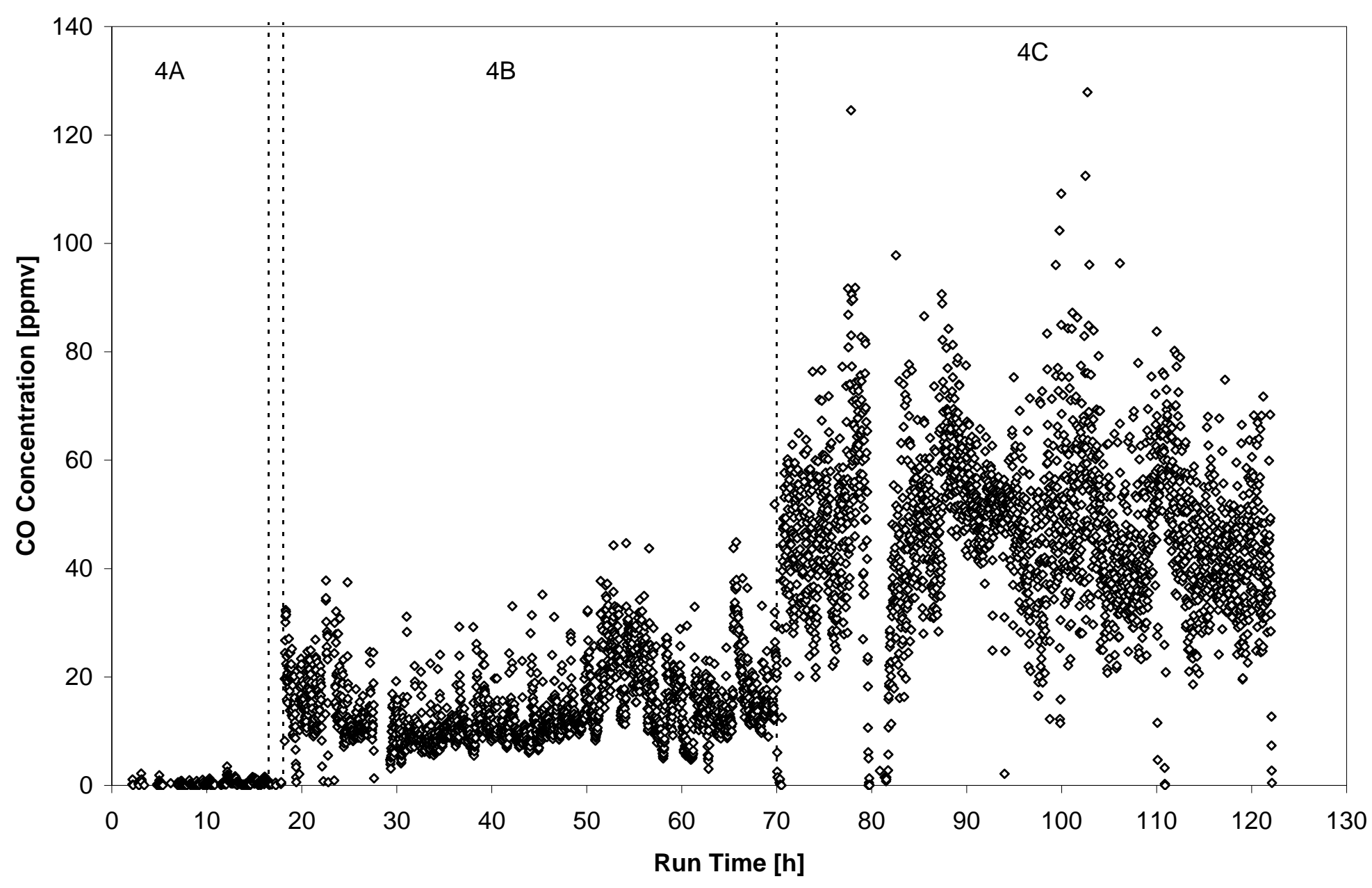

Figure 5.9.d. CO concentration in off-gas from FTIR during Test 4. 


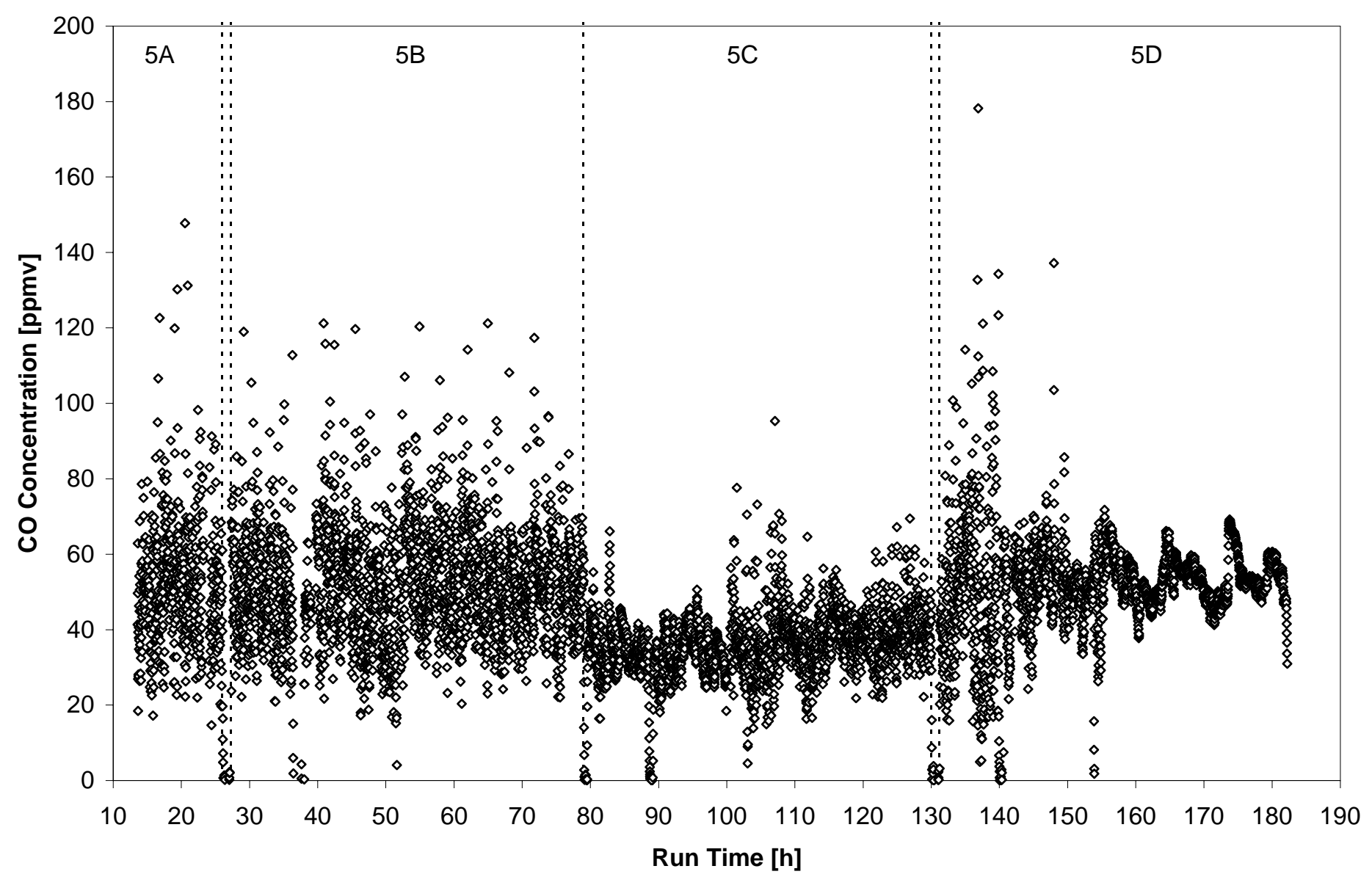

Figure 5.9.e. CO concentration in off-gas from FTIR during Test 5. 


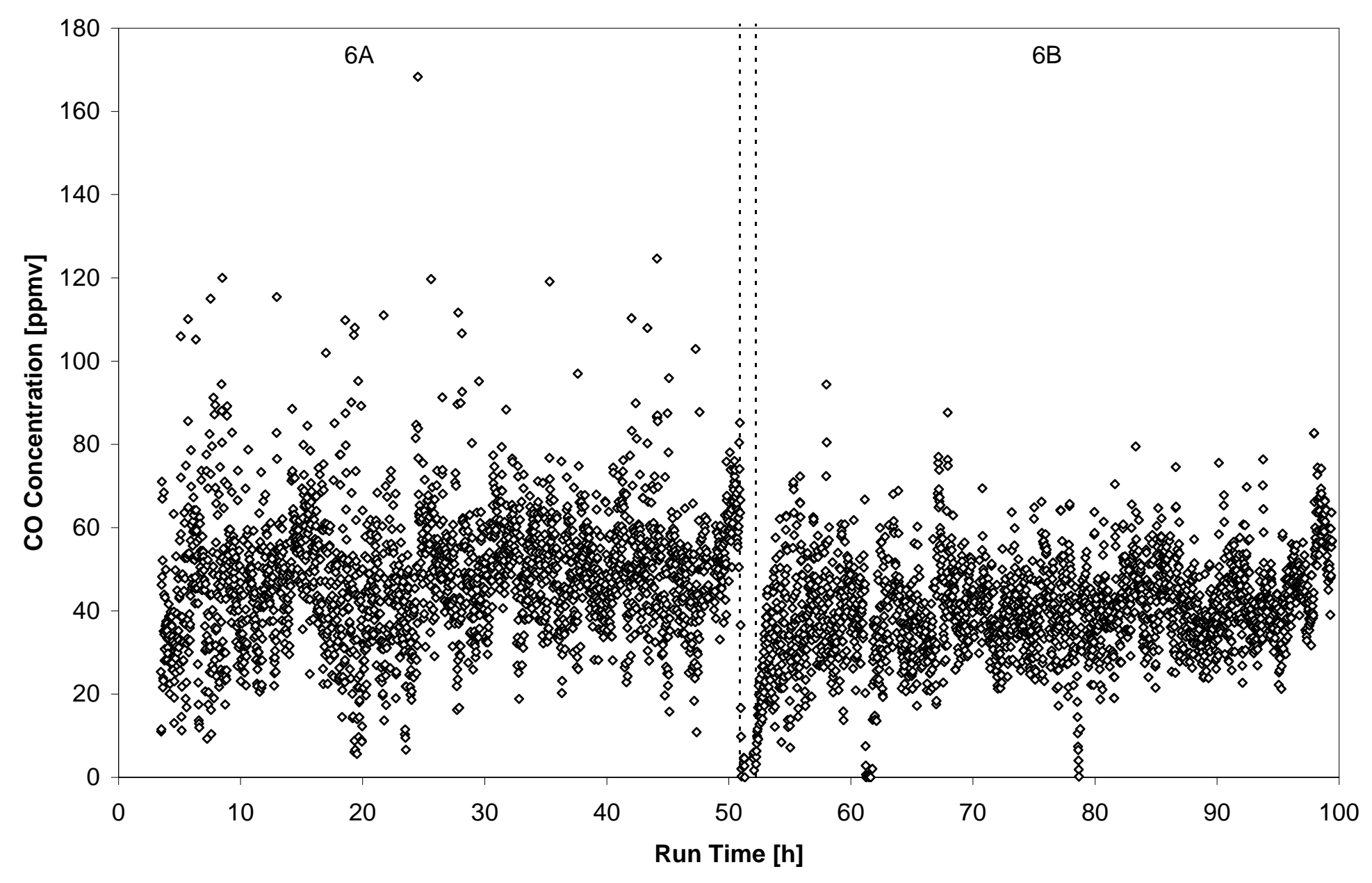

Figure 5.9.f. CO concentration in off-gas from FTIR during Test 6. 


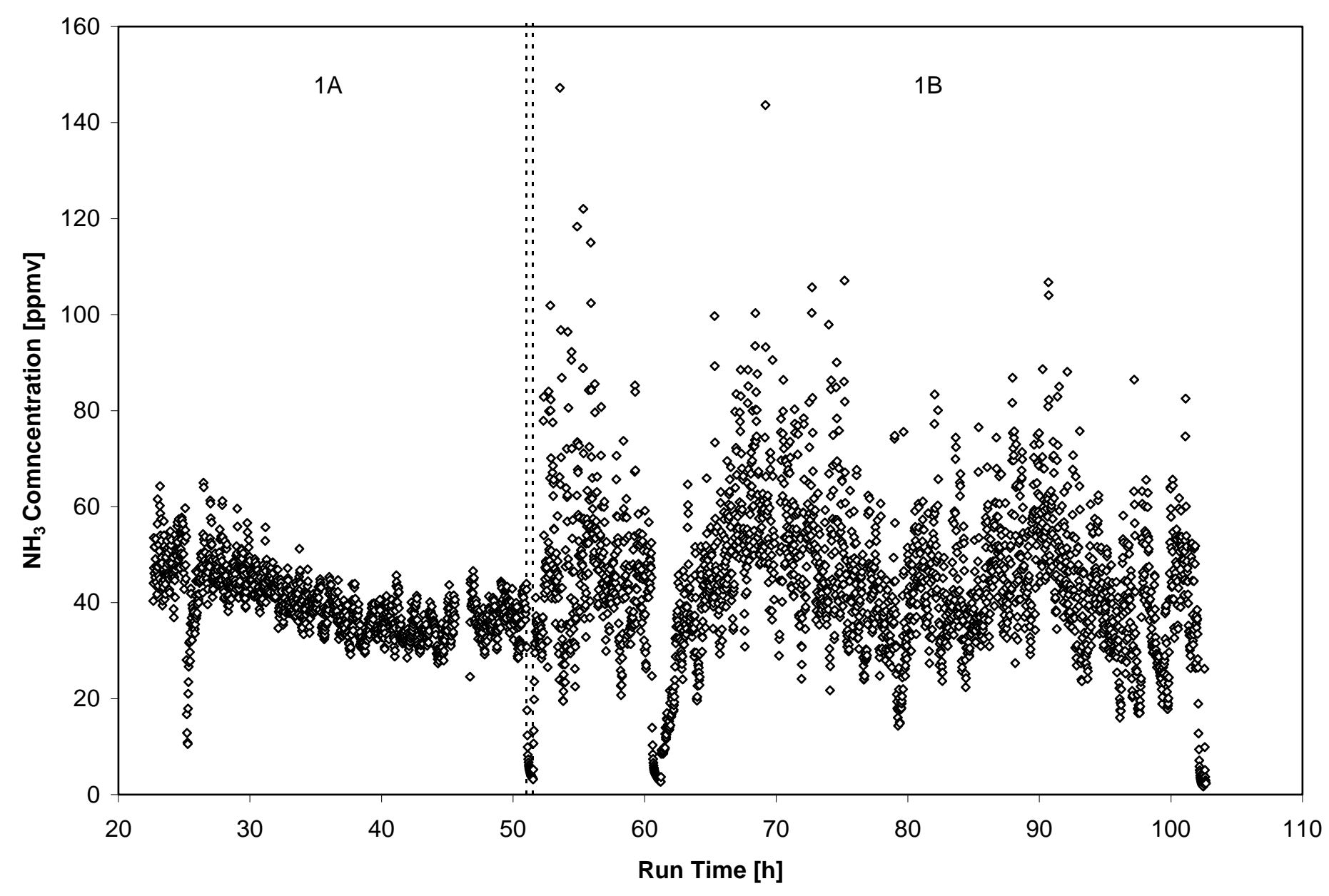

Figure 5.10.a. Ammonia concentration in off-gas from FTIR during Test 1. 


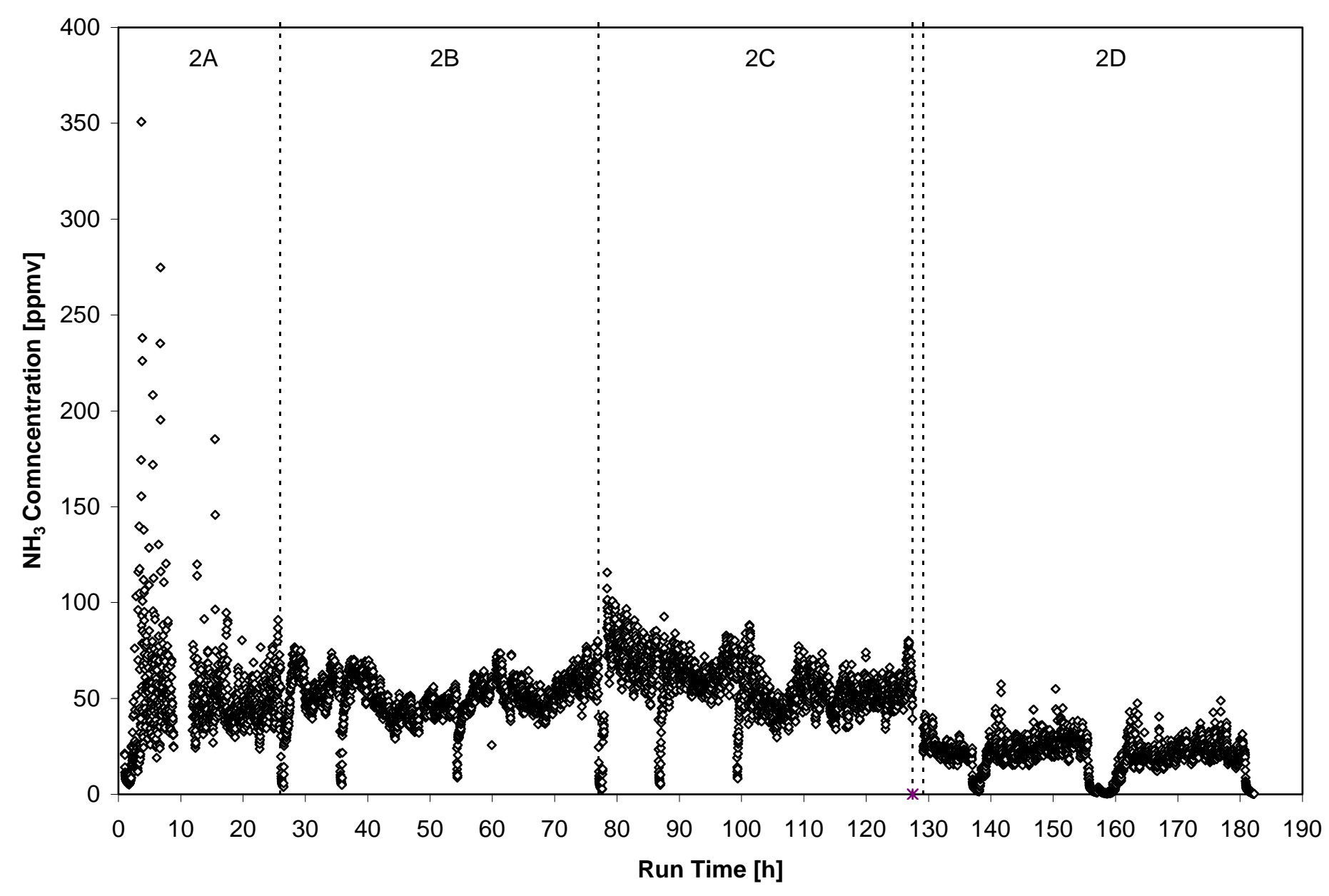

Figure 5.10.b. Ammonia concentration in off-gas from FTIR during Test 2. 


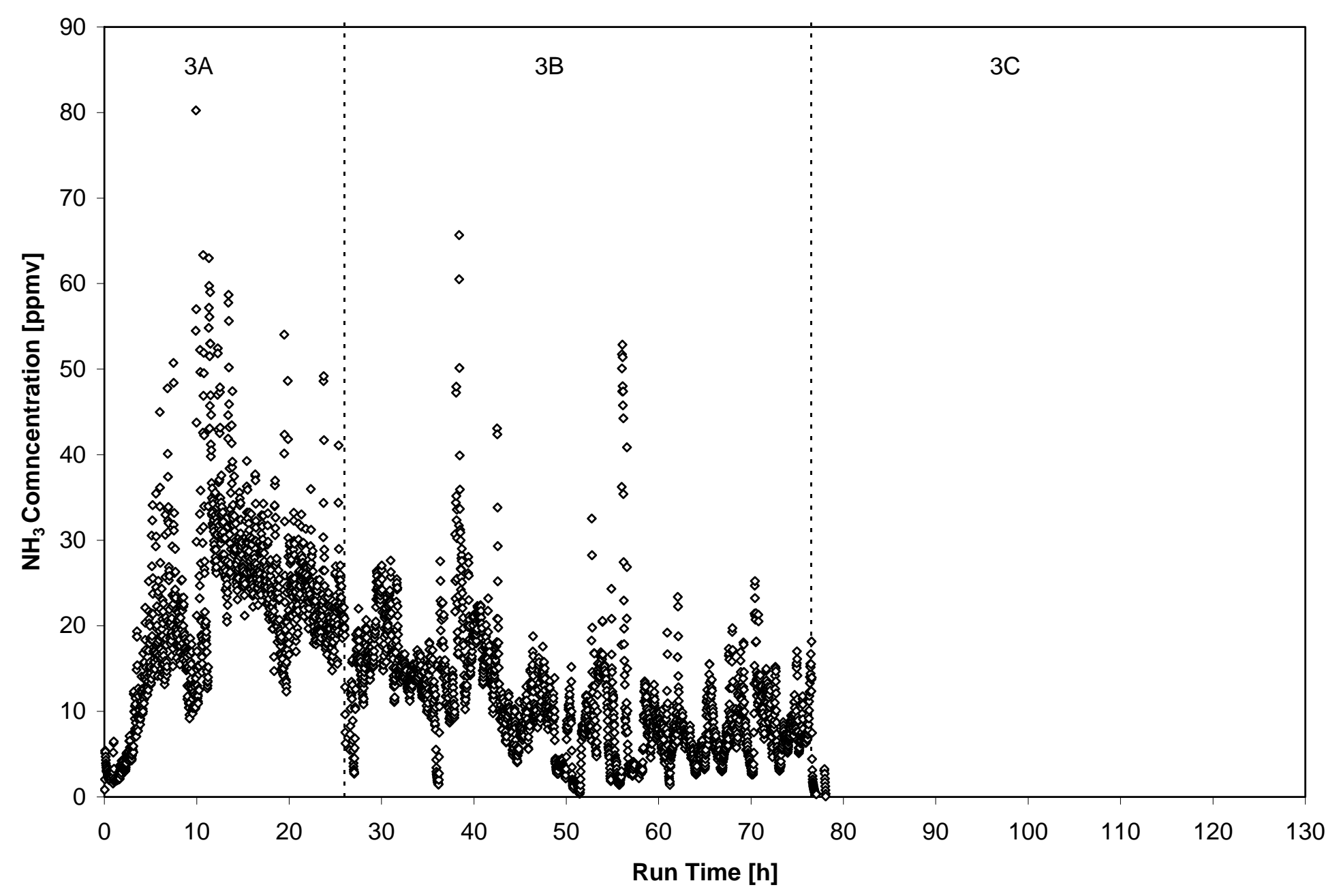

Figure 5.10.c. Ammonia concentration in off-gas from FTIR during Test 3. 


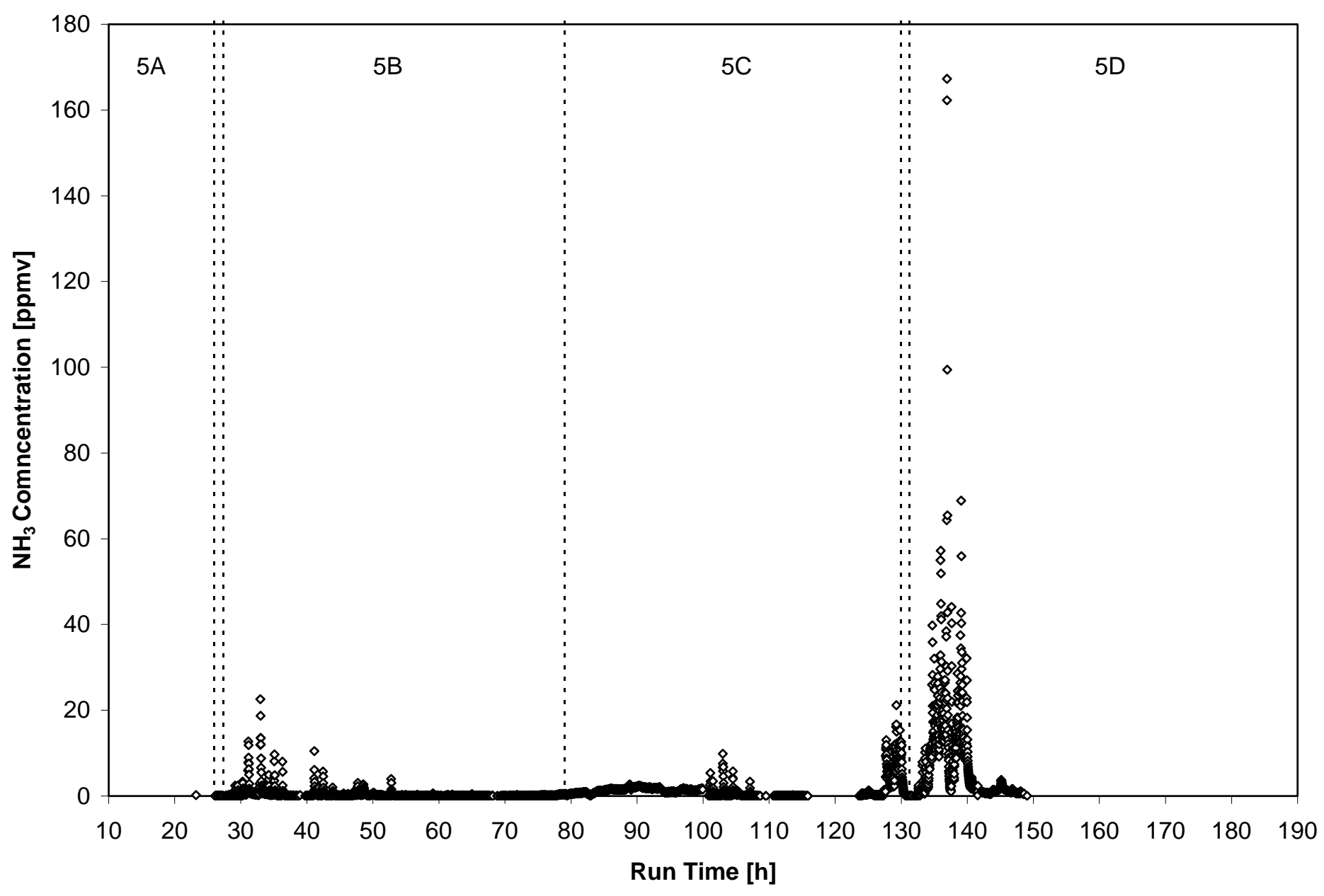

Figure 5.10.d. Ammonia concentration in off-gas from FTIR during Test 5. 


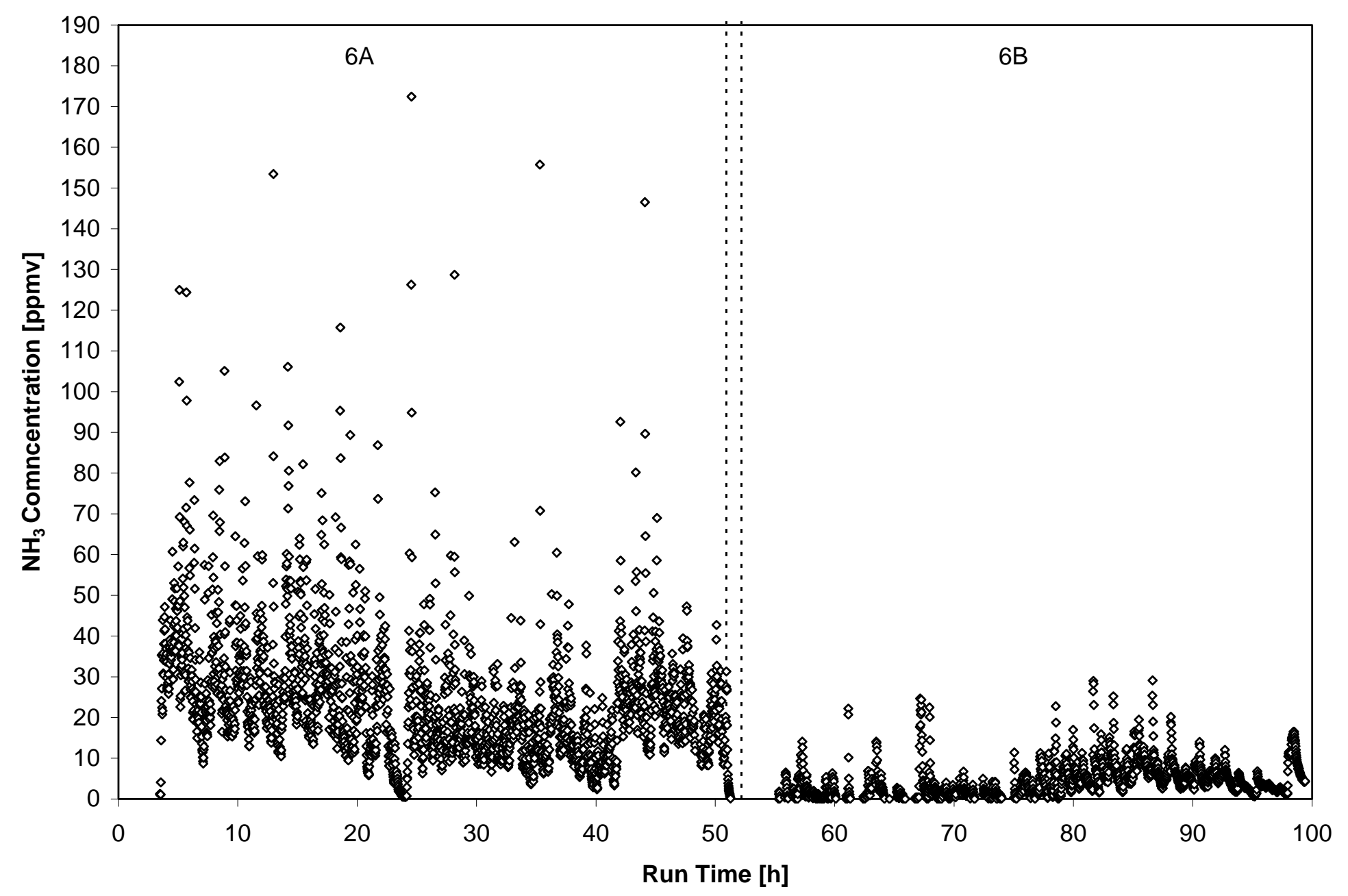

Figure 5.10.e. Ammonia concentration in off-gas from FTIR during Test 6. 


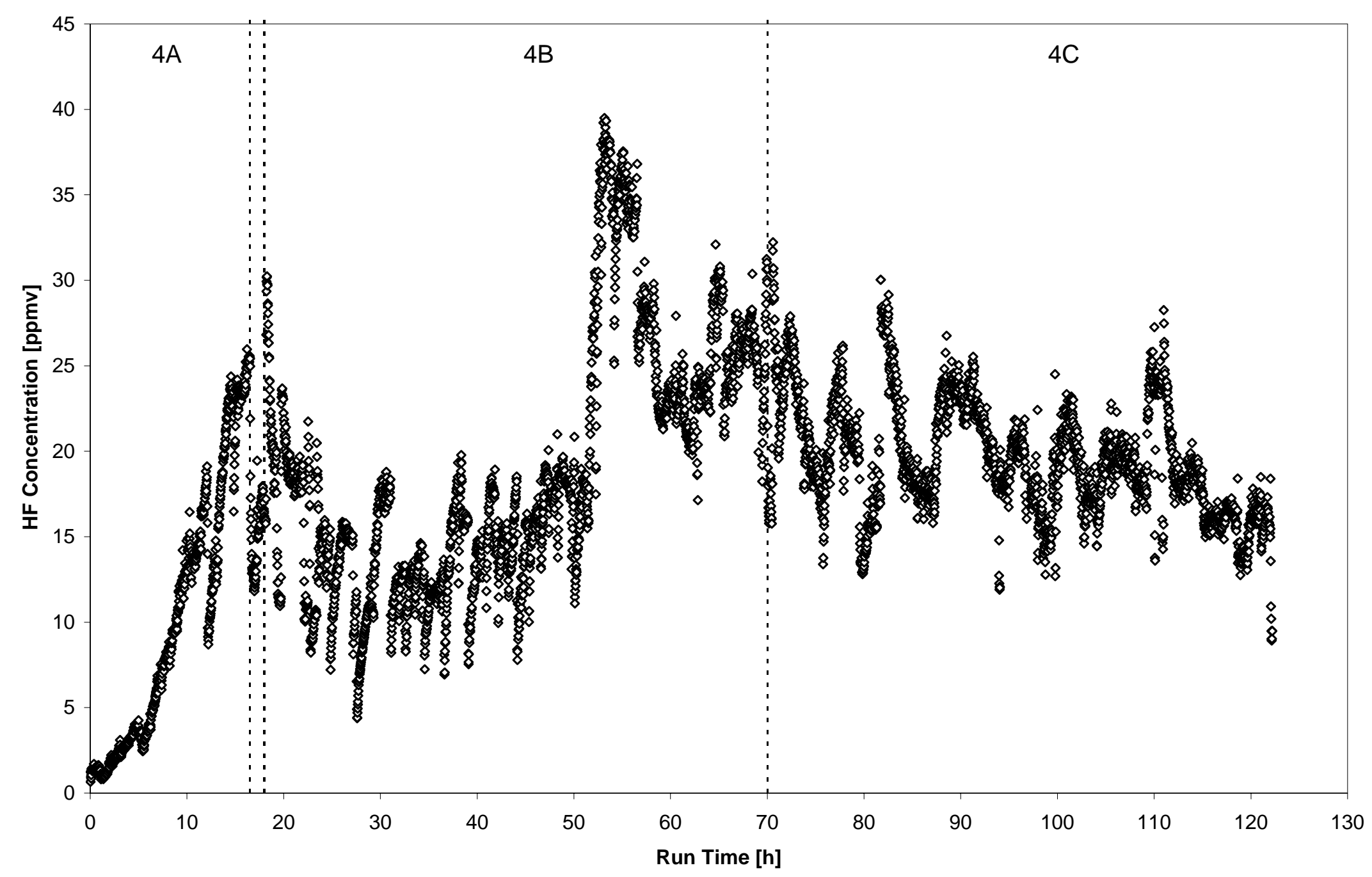

Figure 5.11.a. Hydrogen fluoride concentration in off-gas from FTIR during Test 4. 


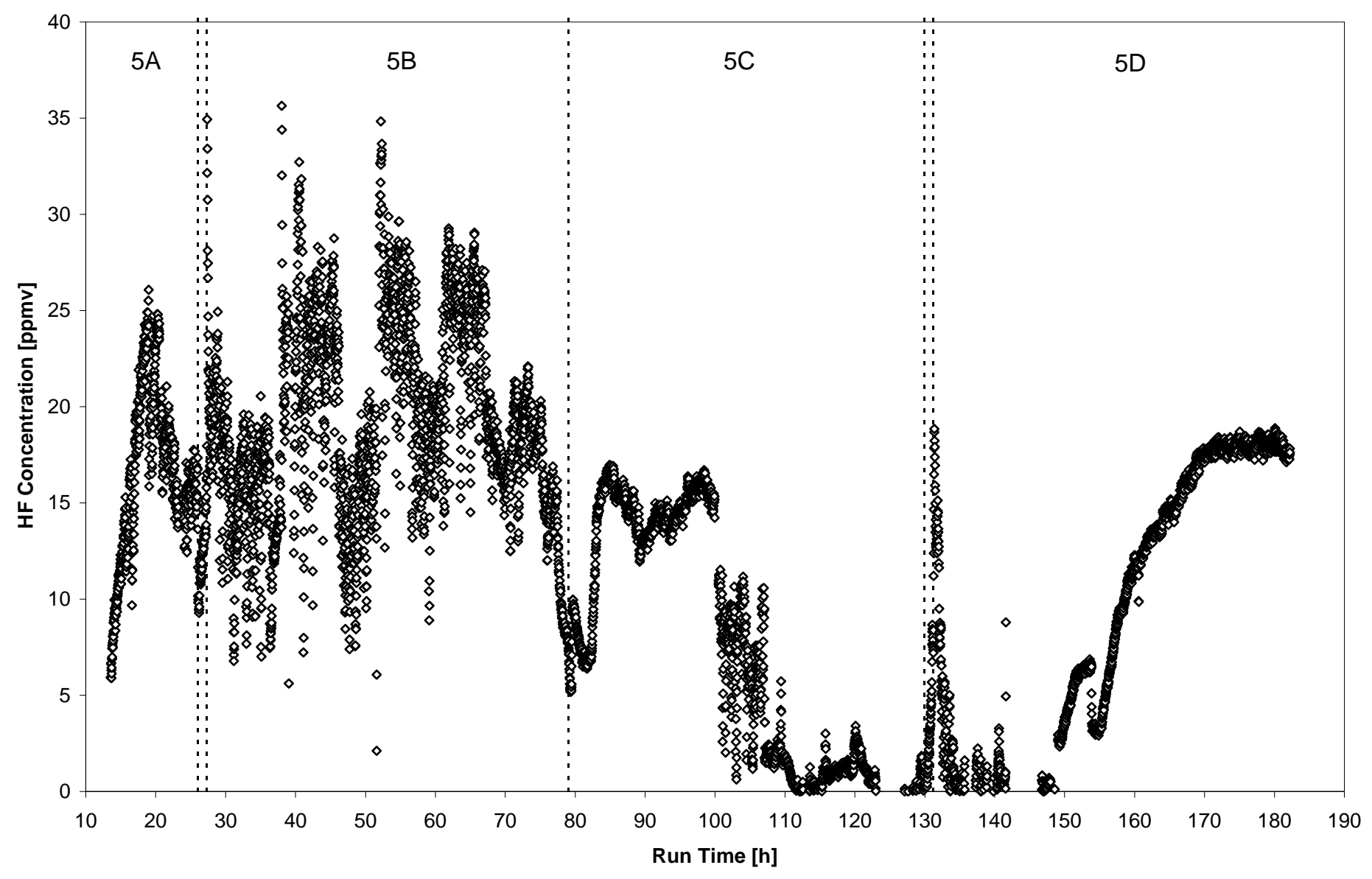

Figure 5.11.b. Hydrogen fluoride concentration in off-gas from FTIR during Test 5. 


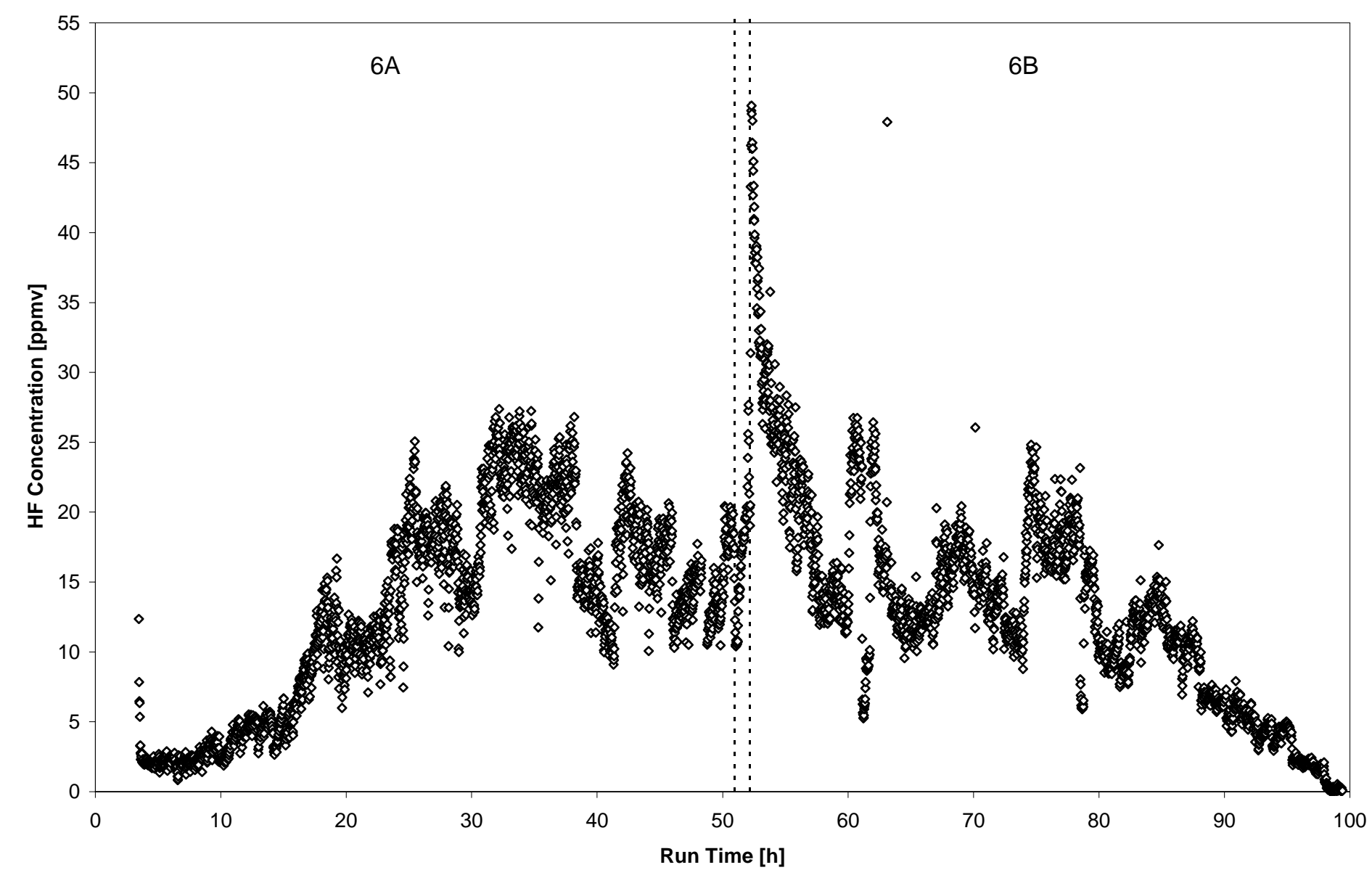

Figure 5.11.c. Hydrogen fluoride concentration in off-gas from FTIR during Test 6. 